Ulrike Hardt

\title{
Kommunale Finanzkraft
}

Die Problematik einer objektiven Bestimmung kommunaler Einnahmemöglichkeiten in der gemeindlichen Haushaltsplanung und im kommunalen Finanzausgleich 


\section{Ulrike Hardt}

\section{Kommunale Finanzkraft}

Die Finanzkraftmeßzahl erfüllt im kommunalen Finanzausgleich - genau wie die Bedarfsmeßzahl - zentrale Steuerungsfunktionen. Allerdings wird ihre Objektivität in Zweifel gezogen, weil sie die Einnahmemöglichkeiten der Gemeinden offensichtlich nicht genau wiedergibt. Die vorliegende Arbeit unterzieht deshalb die Verfahren der Finanzkraftmessung in den Bundesländern einer grundsätzlichen Überprüfung und vergleicht sie mit Meßkonzeptionen, die bei einer gemeindlichen Kalkulation von Einnahmemöglichkeiten im Rahmen der Haushaltsplanung zugrunde gelegt werden könnten. Die Gegenüberstellung zeigt, daß kommunaler Finanzausgleich und gemeindliche Haushaltsplanung völlig verschiedene Zwecke verfolgen und die angewendeten Meßkonzepte nicht nach den gleichen Kriterien bewertet werden dürfen. Eine Beurteilung der Finanzkraftmeßzahl kann also nicht anhand des Vergleichs mit einem Maß für die gesamten gemeindlichen Einnahmemöglichkeiten erfolgen, sondern erfordert andere Kriterien, die aus der jeweiligen Aufgaben-, Ausgaben- und Einnahmenzuweisung an die Ebenen des föderativen Staates abzuleiten sind.

Ulrike Hardt wurde 1955 in Gummersbach geboren. Von 1974-1979 Studium der Volkswirtschaftslehre an der Philipps-Universität Marburg. 1979 Dipl.-Volkswirt, bis 1987 wissenschaftliche Mitarbeiterin in der Abteilung Finanzwissenschaft am Fachbereich Wirtschaftswissenschaften der Universität Marburg. 1988 Promotion an der Universität Marburg. 


\section{Kommunale Finanzkraft}




\section{FINANZWISSENSCHAFTLICHE SCHRIFTEN}

Herausgegeben von den Professoren

Albers, Krause-Junk, Littmann, Oberhauser, Pohmer, Schmidt

\section{Band 38}

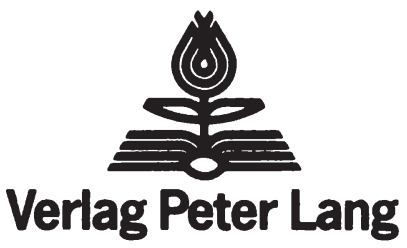

Frankfurt am Main · Bern · New York · Paris

Ulrike Hardt - 978-3-631-75265-4

Downloaded from PubFactory at 01/11/2019 06:31:57AM

via free access 


\section{Ulrike Hardt}

\section{Kommunale Finanzkraft}

Die Problematik einer objektiven Bestimmung kommunaler Einnahmemöglichkeiten in der gemeindlichen Haushaltsplanung und im kommunalen Finanzausgleich

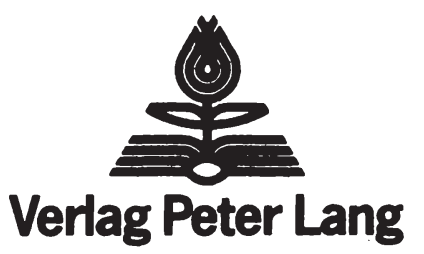

Frankfurt am Main - Bern - New York - Paris

Ulrike Hardt - 978-3-631-75265-4 
CIP-Titelaufnahme der Deutschen Bibliothek

Hardt, Ulrike:

Kommunale Finanzkraft : d. Problematik e. objektiven Bestimmung kommunaler Einnahmemöglichkeiten in d. gemeindl. Haushaltsplanung $u$. im kommunalen

Finanzausgleich / Ulrike Hardt. - Frankfurt am Main ; Bern ; Now York ; Paris : Lang, 1988

(Finanzwissenschaftliche Schriften ; Bd. 38)

Zugl.: Marburg, Univ., Diss., 1988

ISBN 3-631-40788-2

NE: GT

Open Access: The online version of this publication is published on www.peterlang.com and www.econstor.eu under the international Creative Commons License CC-BY 4.0. Learn more on how you can use and share this work: http://creativecommons. org/licenses/by/4.0.

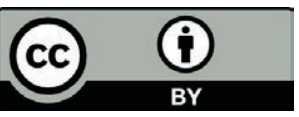

This book is available Open Access thanks to the kind support of ZBW - Leibniz-Informationszentrum Wirtschaft.

\author{
D 4 \\ ISSN 0170-8252 \\ ISBN 3-631-40788-2 \\ ISBN 978-3-631-75265-4 (eBook) \\ (c) Verlag Peter Lang GmbH, Frankfurt am Main 1988 \\ Alle Rechte vorbehalten.
}

Das Werk einschließlich aller seiner Teile ist urheberrechtlich geschützt. Jede Verwertung außerhalb der engen Grenzen des

Urheberrechtsgesetzes ist ohne Zustimmung des Verlages unzulässig und strafbar. Das gilt insbesondere für Vervielfältigungen, Übersetzungen, Mikroverfilmungen und die Einspeicherung und Verarbeitung in elektronischen Systemen.

Printed in Germany 
A. Zur Einordnung der Fragestellung in die Diskussion um den kommunalen Finanzausgleich und die Messung der gemeindlichenFinanzkraft

B. Konzeption und Aufbau der Untersuchung

2. Kapitel: Zur Beurteilung praktizierter und alternativ diskutierter Verfahren der Messung gemeindlicher Finanzkraft im Rahmen des kommunalen Finanzausgleichs

A. Die Fragestellung des Kapitels

B. Die Stellung der Finanzkraftmessung im System des kommunalen Finanzausgleichs

I. Die Zwecke des kommunalen Finanzausgleichs - Anforderungen an die Finanzkraftmessung im vertikalen und horizontalen Finanzausgleich -

II. Eingeschränkte Anforderungen an die Finanzkraftmessung aufgrund der Dominanz des horizontalen Ausgleichs im kommunalen Finanzausgleich der Bundesrepublik Deutschland?

III. Fiktive Grundlagen zur Beurteilung der Steuerkraftmessung in der weiteren Untersuchung

C. Die Verfahren der Finanzkraftmessung in den Bundesländern und ihre Beurteilung 
I. Anmerkungen zur Auswahl der im Finanzausgleich und der Finanzkraftmessung berücksichtigten Einnahmearten

a. Konzepte zur Ermittlung und zum Ausgleich gemeindlicher Finanzierungsmöglichkeiten in den Bundesländern

b. Argumente für eine umfassendere Messung der gemeindlichen Finanzkraft

II. Probleme der Wahl fiktiver Steuer und Abgabensätze für die Finanzkraftmessung

D. Alternative Verfahren der Steuerkraft- und Finanzkraftmessung als Ersatz der praktizierten Konzepte?

I. Die Neuberechnung der Steuerkraft von Grimme

II. Der "Representative Tax System Approach"

III. Der "Income Approach"

60

E. Zusammenfassung

3. Kapitel: Das "kommunale Einnahmepotential"- die Bestimmung der zu messenden Einnahmemöglichkeiten aus gemeindlicher Sicht

A. Die Ansatzpunkte der Betrachtung

I. Zum Aufbau des 3. Kapitels und dem Stellenwert empirischer Überlegungen in der Gesamtkonzeption

II. Möglichkeiten gemeindlicher Mitgestaltung des Budgets als Voraussetzung eines kommunalen Interesses an der Bestimmung des Einnahmepotentials 
III. Zwecke und Objekte der Messung bei gegebenem und variablem Angebot kommunaler Güter und Leistungen

B. Die Begrenzungen kommunaler Einnahmemöglichkeiten bei gegebenem gemeindlichen Angebot

I. Möglichkeiten der Einnahmesteigerung und Probleme ihrer Einschätzung bei verschiedenen Einnahmearten

II. Grenzen kommunaler Besteuerung

a. Zur sukzessiven Einführung der begrenzenden Faktoren in die Betrachtung

b. Einkommen und Vermögen als materielle Obergrenze kommunaler Besteuerung?

c. Die Begrenzung des Steuerpotentials durch die Reaktionen der Wirtschaftssubjekte

1. Die relevanten Reaktionen im Überblick

1.1. Ökonomische Verhaltensänderungen

1.2. Politische Reaktionen

2. Sachliche und räumliche Anpassung an kommunale Steuerpolitik unter den Bedingungen der interkommunalen Konkurrenz

2.1. "Monopolistische" Besteuerungsspielräume bei sachlicher Steuerausweichung und ihre Determinanten

2.2. Besteuerungsspielräume bei räumlicher Ausweichung unter den Bedingungen interkommunaler Konkurrenz 
3. Zusammenfassung

d. Begrenzungen kommunaler Besteuerungsmöglichkeiten durch staatliche Rahmensetzung

1. Festlegung von Steuerquellen

2. Festlegung von Höchst- und Mindeststeuersätzen

3. Festlegung von Kopplungsvorschriften

e. Gemeindliche Zielsetzungen als Begrenzung des eigenen Steuerpotentials?

III. Kommunale Besteuerungsspielräume im Gemeindefinanzsystem der Bundesrepublik Deutschland und ihre Determinanten im interkommunalen Vergleich

a. Die Ausgestaltung des kommunalen Steuersystems unter dem Aspekt gemeindlicher Besteuerungsmöglichkeiten

b. Die Gewerbesteuerbelastung als Determinante sachlicher und räumlicher Ausweichung im Unternehmenssektor

1. Die Belastung des betrieblichen Ertrags bei unterschiedlichen Gewerbesteuerhebesätzen

2. Die Mobilität der Betriebe als Ausdruck räumlicher Ausweichmöglichkeiten

3. Der Stellenwert der Steuerbelastung im $\mathrm{Zu}$ sammenhang mit anderen Faktoren der Standortwahl

4. Die Ergebnisse der betriebstypen-spezifischen Betrachtung 
c. Wirtschafts-, Bevölkerungs- und Raumstruktur als Determinanten der fiskalischen Wirkungen kommunaler Hebesatzpolitik bei der Gewerbesteuer

1. Zur fiskalischen Ergiebigkeit von Hebesatzanhebungen unter Status- quo- Bedingungen

2. Ausweicheffekte als Resultat einer Hebesatzanhebung und ihre Folgen in verschiedenen Gemeindetypen

d. Zusammenfassung

IV. Grenzen kommunaler Entgeltpolitik

a. Das kommunale Leistungsangebot als Ausgangspunkt der Betrachtung

b. Zur Entgeltfähigkeit kommunaler Leistungen

c. Grenzen der Entgeltfestsetzung

1. Die Preiselastizität der Nachfrage

2. Staatliche Begrenzungen bei unterschiedlichen Organisationsformen des kommunalen Angebots

d. Einige Ergebnisse für verschiedene Gemeindetypen

V. Die Bedeutung "preispolitischer" Spielräume und ihrer Erfassung für die Einnahmepolitik der Gemeinden

C. Determinanten des kommunalen Einnahmepotentials bei variablem Leistungsangebot 
I. Bemessungsgrundlagenpolitik bei verschiedenen gemeindlichen Einnahmearten und ihre Restriktionen

II. Strategien zur Ausdehnung des Einnahmepotentials

a. Anforderungen an eine einwohnerorientierte Entwicklungspolitik

b. Ziele und Ansatzpunkte einer kommunalen Gewerbepolitik

III. Möglichkeiten und Beschränkungen expansiver Bemessungsgrundlagenpolitik im Gemeindevergleich

D. Die Ergebnisse der Bestimmung des kommunalen Einnahmepotentials als Maßstab für die Finanzkraftmessung des kommunalen Finanzausgleichs?

4. Kapitel: Die Bestimmung der "kommunalen Finanzkraft" aus übergeordneter Sicht - der Gegenstand der Messung in Systemen mit unterschiedlichem föderativen Gehalt

A. Die vertikale Aufgabenverteilung als Ansatzpunkt der Betrachtung

B. Ausgabenbedarf und Finanzkraft der Gemeinden in Systemen mit unterschiedlicher vertikaler Kompetenzverteilung bei der Aufgabenerfüllung und der Einnahmebeschaffung

I. Die Auswahl der betrachteten Fälle

II. Das Extrem einer vollständig weisungsgebundenen Kommunalebene

III. Der Extremfall unbeschränkt autonomer Gemeinden 
IV. Grundfragen der Bestimmung von Ausgabenbedarf und Finanzkraft in Systemen mit geteilter Kompetenzzuweisung

a. Externe Effekte als Ursache für Eingriffe des Staates in die kommunale Haushaltspolitik

b. Meritorisierung lokaler öffentlicher Angebote als Ursache staatlicher Eingriffe in die kommunale Autonomie

1. Vollständige Zuordnung einzelner Aufga ben-, Ausgaben -und Einnahmekompetenzen zu einer Gebietskörperschaftsebene

2. Geteilte Entscheidungen bei der Aufgaben-, Ausgaben- und Einnahmetätigkeit

C. Einzelfragen der Normierung von Finanzbedarf und Finanzkraft in verschiedenen Einnahmesystemen mit geteilten Entscheidungskompetenzen

I. Die übergemeindliche Festlegung des normierten Ausgabenbedarfs

a. "Originäre" Bedarfsfestlegungen

b. Die Problematik einer pauschalierten Bedarfsfestlegung

c. Konsequenzen für die Finanzkraftmessung in der abstrakten Betrachtung

II. Möglichkeiten der Finanzierung des "Normfinanzbedarfs" und Konsequenzen für die Bestimmung der kommunalen Finanzkraft

a. Finanzierung über Zweckzuweisungen

b. Finanzierung über Schlüsselzuweisungen 
c. Finanzierung über gemeindeeigene Einnahmequellen

1. Die Festlegung der Einnahmequellen

2. Zur Frage der zieladäquaten Normierung gemeindlicher Entgelt- und Steuersatzgestaltung

2.1 Normierung von Entgeltsätzen

3. Zusammenfassung

D. Integration unterschiedlicher Kompetenzverteilungen und segmentierte Messung von Finanzbedarf und Finanzkraft

E. Ein Rückblick: "Kommunales Einnahmepotential" und "normierte Finanzkraft" - Ein Vergleich der gemeindlichen und der staatlichen Interessen bei der Bestimmung der kommunalen Einnahmemöglichkeiten

5. Kapitel: Probleme einer konsistenten Bestimmung der Finanzkraft im System des kommunalen Finanzausgleichs in der Bundesrepublik Deutschland

A. Ansatzpunkte der Finanzkraftbestimmung

B. Zur Abgrenzung normierter gemeindlicher Aktivitäten im geltenden Finanzausgleich

I. Die Gliederung der Aufgabenbereiche nach ihrem Autonomiegrad

II. Die Finanzierungsstruktur und die Kompetenzverteilung bei der Einnahmebeschaffung nach Aufgabenbereichen 
a. Eine Unterscheidung der gemeindlichen Einnahmen nach Autonomiespielräumen und Folgerungen für die Messung der Finanzkraft

b. Inkongruente Finanzierungsstrukturen in den Aufgabensegmenten als besonderes Problem der Finanzkraftmessung

C. Zur Neuformulierung der Kritik an der Steuerkraftmessung im System des kommunalen Finanzausgleichs 


\section{Verzeichnis der Abbildungen}

Abbildung 1: $\quad$ Steuerzahlungen und kommunale Einnahmen bei monopolistischer Stellung der Gemeinde

Abbildung 2: Bestimmung des optimalen Steuersatzes bei unterschiedlichem Umfang der Bemessungsgrundlagen

Abbildung 3: $\quad$ Fiskalisch optimaler Steuersatz bei unterschiedlichen Reaktionen der Zensiten auf Steuersatzanhebungen

Abbildung 4: $\quad$ Monopolistischer Steuersatzspielraum bei räumlicher und sachlicher Ausweichung

Abbildung 5: Steuersatz-Spielräume in verschiedenen Gemeindetypen bei staatlicher Festsetzung von Höchststeuersätzen

Abbildung 6: Wirkung einer Kopplungsvorschrift bei ähnlichem Reaktionsverlauf auf Steuersatzvariationen

Abbildung 7: Wirkungen einer Koppplungsvorschrift bei sehr unterschiedlichen Reaktionsverläufen auf Steuersatzvariationen

Abbildung 8: $\quad$ Nachfrage nach kommunalen Leistungen bei alternativen Entgeltsätzen 
Abbildung 9: $\quad$ Reaktionsverläufe bei Gebühren und Beiträgen auf unterschiedliche Entgelthöhe

Abbildung 10: $\quad$ Kostendeckungsgrade kommunaler Leistungen bei unterschiedlichen Kostenund Nachfrageverläufen

Abbildung 11: Wirkungen gemeindlicher Bemessungsgrundlagenpolitik über Steuersatzvariationen und über andere Parameter 


\section{Verzeichnis der Tabellen}

Tabelle 1: Die kommunalen Einnahmequellen und ihre Erträge, Bundesrepublik Deutschland 1984

Tabelle 2: Staatliche Zuweisungen an die Gemeinden, Bundesrepublik Deutschland 1983, in Mrd. DM

Tabelle 3: Gewogene landesdurchschnittliche Realsteuersätze, 1982, in \%

Tabelle 4: Belastung der um den Freibetrag gekürzten Erträge einer Personengesellschaft bei alternativen Gewerbesteuerhebesätzen

Tabelle 5: Belastung des konstanten Gewerbeertrags bei wachsendem Gewerbekapital und alternativen Hebesätzen

Tabelle 6: Gewerbesteuerbelastung des steuerfreien Gewerbeertrags bei wachsendem Gewerbekapital und alternativen Hebesätzen

Tabelle 7: Gewerbesteuerbelastung des Ertrags bei alternativen Hebesätzen nach Wirtschaftsbereichen

Tabelle 8: Kostendeckungsgrade in ausgewählten kommunalen Gebührenhaushalten, 1982, nach einer Umfrage des DST 
Tabelle 9: Entgelteinnahmen nach Arten und Haushaltsteilen in Gemeinden verschiedener Größenklassen, 1980

Tabelle 10: Aufgaben und Ausgaben der Gemeinden und Gemeindeverbände nach ihrem Autonomiegrad, Nordrhein-Westfalen 1983

Tabelle 11: Kommunale Einnahmen nach ihrem Autonomiegrad, Nordrhein-Westfalen 1983

Tabelle 12: Bereinigte Einnahmen nach Aufgabenbereichen, Nordrhein-Westfalen 1983

Tabelle 13: Relationen des Ausgleichs in steuerschwachen Gemeinden bei gemeinsamer und getrennter Finanzierung pflichtiger und normierter Aufgaben 
Verzeichnis der Übersichten

Übersicht 1: $\quad$ Verfahren der Messung kommunaler

Finanzkraft (ohne Stadtstaaten), 1984

Übersicht 2: $\quad$ Fiktive Hebesätze und Berechnungs-

modi der Steuerkraftzahlen in den Bun-

desländern (ohne Stadtstaaten), 1984, in \%

Übersicht 3: Das entgeltfähige kommunale Leistungsangebot

Übersicht 4: $\quad$ Einzelkomponenten der wichtigsten kommunalen Einnahmearten als Ansatzpunkt expansiver Einnahmepolitik 


\section{A. Zur Einordnung der Fragestellung in die Diskussion um den kommunalen Finanzausgleich und die Messung der gemeindlichen Finanzkraft}

Die Finanzierung der Kommunen in der Bundesrepublik Deutschland erfolgt über ein Gemeindesteuer- und abgabensystem, das durch ein Zuweisungssystem ergänzt wird. Diese Ergänzung soll Unzulänglichkeiten des Systems gemeindeeigener Einnahmen ausgleichen. Beide Komponenten des kommunalen Finanzsystems werden heute intensiv kritisiert:

Bezogen auf das Gesamtsystem wird in zunehmendem Maße der Vorwurf laut, daß die gemeindliche Finanzausstattung zumindest in der jüngsten Vergangenheit kaum mehr zur Deckung der erforderlichen Ausgaben ausreiche - der Finanzierungsspielraum wird nach Auffassung der Kritiker besonders durch die Verschiebung finanzieller Belastungen innerhalb des föderativen Staates, wie etwa im Bereich der sozialen Sicherung, eingeengt, da ihr keine entsprechende Aufstockung der Finanzausstattung, sondern insgesamt eher eingeschränkte Einnahmemöglichkeiten gegenüberstehen. ${ }^{1)}$

Aber auch einzelnen Bestandteilen des kommunalen Einnahmesystems werden unerwünschte Wirkungen zugesprochen; sowohl allokative und stabilitätspolitische Ziele als auch raumordnungspolitische Vorstellungen und Gerechtigkeitsnormen über die Mittelverteilung zwischen den Gemeinden werden durch das Finanzsystem verletzt. Aus dem Spektrum der eigenen kommunalen Einnahmen ist besonders die Gewerbesteuer umstritten, weil ihr Aufkommen im Konjunkturverlauf zu sehr schwankt und im interregionalen

1) Siehe auch Karrenberg, Hanns und Münstermann, Engelbert, Gemeindefinanzbericht 1984, in: Der Städtetag 2/1984, S. 81 u. S. 102. Schmidt-Jortzig konstatierte bereits 1981 eine deutliche "Unterfinanzierung der Fremdverwaltung" in den Kommunen, die sich natürlich auf die Finanzierung aller übrigen kommunalen Aktivitäten niederschlagen muB. Vgl. Schmidt-Jortzig, Edzard, Probleme der kommunalen Fremdverwaltung - Fehlentwicklungen in der Struktur einer bewährten Verwaltungsform - in: Die öffentliche Verwaltung, 34. Jg., Heft 11, Juni 1981, S. 393-401, hier S. 401. 
Vergleich zu stark streut. ${ }^{2)}$ Die Streuung der übrigen Gemeindesteuern fällt zwar geringer aus, es zeigen sich jedoch ebenfalls beträchtliche Aufkommensunterschiede zwischen den Gemeinden. Innerhalb des Zuweisungssystems werden vor allem die Zweckzuweisungen wegen mangelnder Transparenz der Vergabepraxis und wegen der Tendenz zur Verzerrung kommunaler Präferenzen angegriffen. ${ }^{3)}$ Die Verteilung von Schlüsselzuweisungen wird dagegen vorwiegend unter raumordnungspolitischen Aspekten kritisiert. ${ }^{4}$ )

Auch die vorliegende Arbeit widmet sich der Kritik eines Bestandteils des kommunalen Finanzausgleichs; Ansatzpunkt ist die Vermutung, daß die Messung der kommunalen Steuerkraft als wesentliche Determinante der Verteilung von Schlüsselzuweisungen die zu erfassende Finanzkraft der Gemeinden nicht "objektiv" abbildet und insofern unerwünschte Verteilungswirkungen erzeugt.

Unter anderem ausgelöst durch die vielfältige Kritik wurden das kommunale Finanzsystem insgesamt oder bestimmte seiner Bestandteile inzwischen häufiger einer grundsätzlichen Überprüfung unterzogen. Zu diesem Zweck werden aus den zu erfüllenden Zielen Anforderungen an das kommunale Einnahmesystem und seine Steuerungsgrößen abgeleitet. Daraus lassen sich wiederum "rationale Systeme" kommunaler Finanzierung oder umfassender: der Zuweisung von Aufgaben-, Ausgaben- und Einnahmekompetenzen auf die verschiedenen Gebietskörperschaften entwickeln, die den gesetzten Anforderungen weitestmöglich entsprechen sollen. ${ }^{5)}$ Häufig sind solche rationalen

2) So z.B. der Wissenschaftliche Beirat beim Bundesministerium der Finanzen. Gutachten zur Reform der Gemeindesteuern in der Bundesrepublik Deutschland. Schriftenreihe des B.MF. Heft 31. Bonn 1982, S. 47 ff.

3) So etwa Massat, Dieter, Die ökonomische Problematik von Zuweisungen. Thun-Frankfurt/Main 1984, S. 196.

4) Vgl. dazu den Sammelband "Räumliche Aspekte des kommunalen Finanzausgleichs". Veröffentlichungen der ARL. Forschungs- und Sitzungsberichte Bd. 159. Hannover 1985.

5) Zu diesem Fragenkomplex liegt ein breites Spektrum von Lntersuchungen mit sehr unterschiedlichem Abstraktionsgrad vor. Die Bandbreite der Arbeiten reicht von der grundsätzlichen Betrachtung der Gestaltungsmöglichkeiten und -probleme des Fiskalföderalismus bis hin etwa zur Entwicklung zieladäquater Gemeindesteuern für den konkreten Fall der Gemeinden in der Bundesrepublik Deutschland. Einen C̈berblick gibt Zimmermann. Horst. Allgemeine Probleme und Methoden des Finanzausgleichs. in: Handbuch der Finanzwissenschaft. 3., gänzlich neubearbeitete Auflage. Bd. IV. Tübingen 1983. S. 3-52.

Als Beispiel für die Entwicklung eines "rationalen" kommunalen Steuersystems sei verwiesen auf: Pohmer. Dieter und Saile. Peter. Das "ideale" Einnahmensystem. in: Handbuch der kommunalen Wissenschaft und Praxis. Bd. 6, Kommunale Finanzen. hrsg. von Günter Püttner. 2., völlig neu bearbeitete Auflage. Berlin u.a. 1985. S. 25-105. 
Konzepte letztlich nicht in die Praxis umsetzbar, entweder, weil ihre Realisierung auf politische Widerstände stößt, oder weil sich technische Schwierigkeiten ergeben, deren Überwindung nicht möglich oder unter Kostenaspekten nicht sinnvoll erscheint. Als Orientierungs- und Beurteilungsmaßstab für die praktisch realisierten Konzepte sind diese rationalen Systeme oder Konzepte jedoch durchaus von Bedeutung.

Um einen solchen Entwurf, wenn auch nur für eine einzelne Steuerungsgröße des kommunalen Finanzausgleichs, geht es in der vorliegenden Arbeit. Ihr Gegenstand ist ein - auf den ersten Blick relativ enger - Ausschnitt des institutionellen Finanzausgleichs: Die Bestimmung der kommunalen Finanzkraft, also der potentiellen gemeindlichen Einnahmen, die dem ebenfalls zu ermittelnden Finanzbedarf der Gemeinden zum Zweck der Zuteilung von Schlüsselzuweisungen gegenübergestellt wird und die in nicht unerheblichem Maße über die letzendliche Verteilung von Finanzmitteln auf die Gemeinden mitentscheidet. Die Fragestellung kann zunächst folgendermaßen umschrieben werden: Wie sind die Einnahmemöglichkeiten der Gemeinden für die Zwecke des kommunalen Finanzausgleichs theoretisch richtig zu bestimmen, damit von ihrer Messung keine eigenständigen unerwünschten Wirkungen auf die Verteilung der Schlüsselzuweisungen ausgehen?

Ziel dieser Arbeit ist also nicht die Messung der Einnahmemöglichkeiten, sondern nur die theoretische Bestimmung dessen, was gemessen werden sollte. Übereinstimmung herrscht in der Auffassung, daß die potentiellen Einnahmen mit den tatsächlich erzielten Einnahmen der Gemeinden nicht übereinstimmen. Darüber hinaus besteht in der Literatur jedoch wenig Einigkeit, was konkret mit der "Finanzkraft" der Gemeinden in der Bundesrepublik Deutschland gemeint ist. Die vorliegende Arbeit soll einige Ansatzpunkte liefern, um diese Lücke zu schließen.

Zweck der Zuteilung von Schlüsselzuweisungen ist zum einen eine Aufstockung der gemeindlichen Finanzmasse. Da den Gemeinden einerseits eine zur eigenverantwortlichen Aufgabenerfüllung ausreichende Finanzausstattung zugesichert werden soll, andererseits "die Unfähigkeit zur Selbstfinanzierung gegenwärtig der gesetzlich veranlaßte Regelfall" ${ }^{6)}$ ist, sind die Finanzierungs-

6) Kirchhof. Paul. Rechtliche Rahmenbedingungen des kommunalen Finanzausgleichs. in: Hoppe. Werner (Hrsg.). Reform des kommunalen Finanzausgleichs. Schriftenreihe des Landkreistages Nordrhein-Westfalen. Bd. 4. Köln u.a. 1985. S. 1-30; hier S. 11 
lücken durch eine Beteiligung an Einnahmen der übergeordneten Gebietskörperschaften abzudecken. Zum zweiten soll mit den Schlüsselzuweisungen Einfluß auf die horizontale Verteilung der Finanzierungsspielräume - immer gemessen am Finanzbedarf - zwischen den einzelnen Gemeinden genommen werden. Keine der beiden Zielsetzungen der Zuweisungsvergabe scheint aber derzeit in vollem Umfang erfüllt zu werden. Insbesondere die Ergebnisse des horizontalen Finanzausgleichs werden in wachsendem Maße kritisiert und eine Umgestaltung der Verteilungsschlüssel diskutiert. ${ }^{\text {T) }}$

Dazu bieten sich zwei Ansatzpunkte an, die jedoch sehr unterschiedliche Ursachen für die unerwünschten Wirkungen der Schlüsselzuweisungsverteilung voraussetzen und zu beseitigen versuchen:

- Der Ausgleich von Finanzbedarf und Finanzkraft selbst entspricht den Anforderungen nicht und wäre konsequenterweise anzupassen. Unter der Bedingung, daß Finanzbedarf und Finanzkraft der Gemeinden richtig ermittelt worden sind und daß die Anforderungen an den Ausgleich nicht miteinander im Konflikt stehen, bedarf es dazu lediglich einer politischen Entscheidung. Ein analytisches Problem stellt sich nur mit Blick auf die Wirkungen - etwa allokativer oder stabilitätspolitischer Art - der Umgestaltung des Ausgleichs. Darauf soll hier jedoch nicht näher eingegangen werden.

- Der Finanzbedarf bzw. die Finanzkraft der Gemeinden wird nicht "richtig" ermittelt und verursacht so ein verzerrtes Bild vom Zuweisungsbedarf der einzelnen Gemeinden. Wird etwa die Finanzkraft eines bestimmten Gemeindetyps durch das verwendete Meßkonzept systematisch zu niedrig ausgewiesen, so profitiert dieser Typ stärker von der Vergabe der Schlüsselzuweisungen als alle übrigen Gemeinden. Wie stark diese ungewollte Begünstigung ausfällt, ist allerdings überhaupt nicht abschätzbar, solange die tatsächliche Finanzkraft der Gemeinden nicht bestimmt werden kann. Ohne ein Konzept zur objektiven Erfassung dieser Größen ist eine zielgerechte Steuerung des Ausgleichs letztlich also kaum möglich; im Ex-

7) So wird z.B. häufig festgestellt, daß der Verteilungsschlüssel zentralörtlich bedingte $\mathrm{Zu}-$ satzbelastungen von Gemeinden nicht angemessen berücksichtige. Vgl. etwa Münstermann. Engelbert. Die Berücksichtigung zentralörtlicher Funktionen im kommunalen Finanzausgleich. Forschungsberichte des Landes Nordrhein-Westfalen, Nr. 2520. Opladen 1975. 
tremfall bleiben Fehlsteuerungen in ihrem Kern sogar unbemerkt und treten nur in ihren Wirkungen auf finanzpolitische Ziele zutage. Insofern kommt der Bestimmung sowohl von Finanzbedarf als auch der - bisher seltener untersuchten - Finanzkraft der Gemeinden zentrale Bedeutung im System des kommunalen Finanzausgleichs zu.

Für die Bedarfsermittlung liegen einige Untersuchungen vor, die sich der Kritik des zur Zeit verwendeten Ermittlungskonzepts widmen und in denen Alternativvorschläge zur Messung entwickelt werden. Insbesondere der Hauptansatz, der einen mit steigender Gemeindegröße wachsenden Ausgabenbedarf der Gemeinden unterstellt, wurde bereits mehrfach daraufhin überprüft, ob er den "tatsächlichen" Ausgabenbedarf zumindest im interkommunalen Vergleich richtig wiedergibt und vor allem, ob die dadurch bewirkte Verteilung von Schlüsselzuweisungen raumordnungspolitischen Anforderungen genügt. ${ }^{8)}$

Weitergehende Kritiken richten sich gegen die pauschalierende, auf den Einwohner bezogene Bedarfsermittlung und schlagen statt dessen eine "originäre", stärker an den konkreten Bedingungen in den einzelnen Gemeinden ansetzende Bedarfsmessung vor. ${ }^{9}$ )

Anders als die Bedarfsermittlung wurde die im kommunalen Finanzausgleich praktizierte Ermittlung der gemeindlichen Finanzkraft mittels der Steuerkraftmeßzahl bisher nur vereinzelt einer detaillierten Kritik unterzogen, obwohl sie ebenfalls bedeutenden Einfluß auf die Verteilung der Schlüsselzuweisungen haben dürfte. Daß der Finanzkraftermittlung weniger Aufmerksamkeit gewidmet worden ist, läßt sich möglicherweise darauf zurückführen, $\mathrm{da} ß$ sie als wesentlich unproblematischer als die Bedarfsermittlung erscheint und die Steuerkraftmeßzahl eher als objektive Maßgröße eingestuft wird als

8) Einen Überblick gibt z.B. Zabel, Gerhard, Ist die Hauptansatzstaffel noch zeitgemäß?, in: Informationen zur Raumentwicklung. hrsg. von der BfLR Heft 6/7, 1983, S. 445-459.

9) Vgl. etwa Hanusch, Horst und Kuhn, Thomas. Messung des kommunalen Finanzbedarfs Ein alternativer Ansatz für die Schlüsselzuweisungen. in: Räumliche Aspekte des kommunalen Finanzausgleichs, Veröffentlichungen der ARL, Forschungs- und Sitzungsberichte Bd. 159. Hannover 1985. S. 55-74, sowie Zimmermann. Horst: Hardt, Ulrike und Postlep. Rolf-Dieter. Determinanten der kommunalen Finanzsituation - unter besonderer Berücksichtigung der Gemeinden in Ballungsgebieten - Schriftenreihe der Gesellschaft für Regionale Strukturentwicklung e.V., Bd. 15, Bonn 1987. S. 377 ff. 
die Bedarfsmeßzahl. ${ }^{10)}$ Trotzdem werden auch hier einige Unzulänglichkeiten festgestellt. So ist beispielsweise nicht ohne weiteres einsichtig, warum die gemeindliche Finanzkraft allein über gemeindliche Steuern abgeschätzt wird und die übrigen Einnahmen, insbesondere die Entgelte von vornherein unberücksichtigt bleiben.

Ähnlich den Kritikansätzen bei der Bestimmung des kommunalen Finanzbedarfs lassen sich auch mit Blick auf die Betrachtung der Finanzkraftbestimmung verschiedene Kritikansätze unterscheiden:

- Zum einen wird über die Verteilungsergebnisse nach der Zuteilung von Schlüsselzuweisungen argumentiert. Es wird gezeigt, daß alternative Ansätze zur Bestimmung der gemeindlichen Finanzkraft andere, zum Teil als zieladäquater eingeschätzte Verteilungsergebnisse hervorbringen als das derzeitig angewendete Verfahren der Steuerkraftermittlung. ${ }^{11)}$ Solche Ansätze können dann hilfreich sein, wenn eine Verteilungsänderung gegenüber der vorhergehenden Situation angestrebt wird; allerdings muß sie nicht notwendig über die Ermittlung der Finanzkraft gesteuert werden, sondern könnte auch direkt über die Ausgleichsentscheidung beeinflußt werden.

Zur Feststellung und Beurteilung der "objektiven" Situation der einzelnen Gemeinden - ist eine Gemeinde "arm" oder "reich" - und der Höhe ihres Zuschußbedarfs trägt die auf diese Weise umgestaltete Bestimmung der Finanzkraft jedoch nicht bei; sie bleibt letztlich ebenso willkürlich wie die Beurteilung nach der ursprünglich verwendeten Finanzkraftmaßgröße.

- Andere Kritikansätze gehen von systematischen Überlegungen zur Gestaltung der Finanzkraftmessung aus, ohne damit direkt eine bestimmte veränderte Verteilung der Schlüsselzuweisungen bewirken zu wollen. So wird etwa überprüft, welche Einnahmearten überhaupt in die Bestimmung der Finanzkraft eingehen sollten, von welchen Steuer- und Abgabe-

10) So hält etwa Schmölders die Bedarfsbestimmung für schwieriger als die Finanzkraftmessung. Schmölders. Günter, "Finanzstarke" und "finanzschwache" Gemeinden. in: Archiv für Kommunalwissenschaften, 1. Halbjahresband 1965. S. 30-46, hier S. 32.

11) So gehen Grimme und auch Postlep vor: vgl. Grimme. Leonhard, Neuberechnung der Steuerkraft im kommunalen Finanzausgleich - dargestellt am Beispiel des Landes Bayern. in: ARL Hrsg., Räumliche Aspekte des kommunalen Finanzausgleichs. a.a.O.. S. 159-200. sowie Postlep. Rolf-Dieter, Räumliche Effekte der Steuerkraft- und Finanzbedarfsermittlung bei den Schlüsselzuweisungen. in: ebenda. S. 201-234. 
sätzen dabei auszugehen ist usw. ${ }^{12)}$ Dahinter steht der Anspruch, daß die zur Bedarfsdeckung erzielbaren Einnahmen der Gemeinden durch das Meßkonzept zumindest im Ansatz "objektiv" wiedergegeben werden sollen. Erst wenn dies gelingt, lassen sich die Positionen der Gemeinden im "Reichtumsvergleich" abschätzen und die Verteilungsergebnisse des kommunalen Finanzausgleichs genau beurteilen. Ob diese Ergebnisse positiv bewertet werden oder nicht, ist dann eine ganz andere Frage.

Mit dem Versuch einer theoretischen Bestimmung der kommunalen Einnahmemöglichkeiten ist die vorliegende Arbeit der zuletzt genannten Gruppe von Untersuchungen zuzuordnen. Konkrete Verteilungsergebnisse der Finanzkraftmessung interessieren hier zunächst also nicht; sie sind zumindest nicht Ausgangspunkt der Überlegungen; der besteht vielmehr in der Suche nach einem objektiven $\mathrm{Ma}$ für die gemeindliche Finanzkraft.

Verschiedentlich wird in der Literatur bestritten, daß, "die absolute, eindeutige und direkte Messung der Finanzkraft einer Gemeinde"13) überhaupt möglich sei. Dabei bestehen neben empirischen Problemen offensichtlich auch theoretische Schwierigkeiten. Darauf deutet jedenfalls die Uneinigkeit darüber hin, was denn eine solche Messung überhaupt erfassen soll.

Ein Hauptproblem der Bestimmung der gemeindlichen Einnahmemöglichkeiten scheint zunächst in der qualitativen Verschiedenartigkeit der kommunalen Einnahmequellen zu bestehen. Die Aufgabenfinanzierung erfolgt nach unterschiedlichen Prinzipien und ist von den Entscheidungen und Aktivitäten der Gemeinden und den jeweiligen Handlungsbedingungen mehr oder minder abhängig; daher erscheint es auch von vornherein unmöglich, die gemeindliche Finanzkraft mittels eines einheitlichen und zugleich umfassenden Maßstabes zu erfassen. So ist etwa das Aufkommen aus dem gemeindlichen Einkommensteueranteil für jede einzelne Gemeinde festgelegt und kurzfristig nicht beeinflußbar. Aufkommen und Einnahmemöglichkeiten stimmen zu einem gegebenen Zeitpunkt - von berechnungstechnisch bedingten Abweichun-

12) Als Beispiele für diesen Ansatz können etwa die Ausságen von Kirchhof und Schmölders gelten. Vgl. Kirchhof. Paul. Der Finanzausgleich als Grundlage kommunaler Selbstverwaltung. in: Deutsches Verwaltungsblatt. Jg. 1980. S. 711-719. sowie Schmölders, Günter. "Finanzstarke" und "finanzschwache" Gemeinden, a.a.O.

13) Gläser. Martin. Die staatlichen Finanzzuweisungen an die Gemeinden - Gestaltungskriterien. Effektivität. Reform -. Thun - Frankfurt/Main 1981. S. 155. 
gen einmal abgesehen - überein. Daran ändert sich auch dann nichts, wenn die Gemeinde ihre Ausgabetätigkeit umstrukturiert. Üblicherweise wird deshalb davon ausgegangen, daß der gemeindliche Einkommensteueranteil vollständig in die zu messende Gesamtsumme der Finanzierungsmöglichkeiten aufgenommen werden sollte.

Demgegenüber kann etwa das Entgeltaufkommen - ebenso wie das Aufkommen aus den Realsteuern - nicht ohne weiteres mit den Einnahmemöglichkeiten gleichgesetzt werden. Da Entgelte nur für eine spezifische Gegenleistung der Kommune gezahlt werden, dementsprechend also zum Teil entfallen, wenn die Gemeinden ihr Leistungsangebot stärker auf nicht entgeltfähige Leistungen ausrichten, ist hier zu klären, was als "richtige", in die Bestimmung der Einnahmemöglichkeiten aufzunehmende Bemessungsgrundlage von Entgeltpotentialen gelten kann. Darüber hinaus stellt sich die Frage nach der für die Zwecke der Finanzkraftmessung "richtigen" Entgelthöhe. Eine nähere Untersuchung dieser Probleme ist für die Zwecke des kommunalen Finanzausgleichs allerdings erst dann sinnvoll, wenn geklärt werden kann, was die Bestimmung der gemeindlichen Einnahmemöglichkeiten leisten und was gemessen werden soll. Gerade die Frage, ob nicht-steuerliche Kommunalabgaben überhaupt in die Ermittlung der gemeindlichen Finanzkraft einbezogen werden sollten oder nicht, wird bisher jedoch durchaus unterschiedlich beantwortet. ${ }^{14)}$

Diese Uneinigkeit ist insofern erst einmal verwunderlich, als die Entgelte unbestreitbar einen erheblichen Anteil zur Finanzierung kommunaler Ausgabetätigkeit beitragen. Zumindest aus gemeindlicher Sicht sind sie also bei der Ermittlung ihrer Einnahmemöglichkeiten zu berücksichtigen ${ }^{15)}$.

Weiterhin unbestritten ist auch, daß die gemeindlichen Einnahmemöglichkeiten insgesamt - alle Einnahmequellen einbezogen - begrenzt sind; das heißt, für jede Gemeinde existiert irgendwo eine Grenze, über die hinaus die

14) Während Schmölders ihre Einbeziehung befürwortet. wird sie etwa von Kirchhof abgelehnt. Vgl. Schmölders. Günter. "Finanzstarke" und "finanzschwache" Gemeinden, a.a.O.. S. 32: dagegen Kirchhof. Paul. Die kommunale Finanzhoheit, in: Handbuch der kommunalen Wissenschaft und Praxis. Bd. 6. Kommunale Finanzen. hrsg. von Günter Püttner. 2. völlig neu bearbeitete Auflage. Berlin u.a. 1985. S. 3-28. hier S. 25.

15) Gemeindliche Lntersuchungen der kommunalen Finanzierungsmöglichkeiten schließen die Entgelte daher selbstverständlich ein. Siehe etwa Karrenberg. Hanns und Münstermann. Engelbert. Gemeindefinanzbericht 1985. in: Der Städtetag 2/1985. S. 72-117, hier S. $92 f$. 
Einnahmen nicht mehr gesteigert werden können. Diese Grenze empirisch zu ermitteln, dürfte tatsächlich kaum möglich sein. Daraus kann jedoch nicht gefolgert werden, daß das so begriffene "Einnahmepotential" auch theoretisch nicht bestimmt werden könne.

Mit Blick auf den Finanzausgleich ist allerdings die Frage zu stellen, ob das aus gemeindlicher Sicht so bestimmte Einnahmepotential für übergeordnete Gebietskörperschaften überhaupt von Interesse ist. Mit beiden - der gemeindlichen und übergeordneten Sicht - beschäftigt sich die vorliegende Arbeit.

\section{B. Konzeption und Aufbau der Untersuchung}

In der vorliegenden Arbeit wird der kommunalen Finanzkraft das kommunale Einnahmepotential gegenübergestellt. Nun ist der Begriff des "kommunalen Einnahmepotentials" in der mit Kommunalfinanzen befaßten Literatur wenig gebräuchlich. Hier werden vor allem die Begriffe der Finanzkraft, der Steuerkraft, der kommunalen Einnahmemöglichkeiten, der Finanzierungsspielräume sowie weitere, hier nicht vollständig aufzulistende Ausdrücke verwendet, um jeweils eine Summe an Finanzierungsmitteln zu umschreiben, die die Gemeinden aufbringen können, um die anfallenden Aufgaben zu erfüllen und die dadurch entstehenden Kosten zu decken. Allen diesen Begriffen ist gemeinsam, daß sie nicht auf die Bestimmung der tatsächlich realisierten Einnahmen, sondern auf die Bestimmung potentiell erzielbarer Einnahmen ausgerichtet sind. Dies gilt auch für den hier neu eingeführten Begriff des Einnahmepotentials: Es soll eine Bestimmung möglicher, nicht tatsächlich erzielter Einnahmen erfolgen. Trotz dieser Übereinstimmung wird den verwendeten Begriffen zum Zwecke der Abgrenzung dieser weitere hinzugefügt, der die Gesamtheit der für eine Gemeinde beschaffbaren Einnahmen umschreiben soll. Diese Abgrenzung scheint notwendig, weil die üblicherweise verwendeten Begriffe, sofern sie nicht gänzlich unbestimmt sind, etwas anderes bezeichnen, als jene Größen, die hier als "Einnahmepotential" benannt werden. Dies gilt insbesondere für "Finanzkraft" und "Steuerkraft", deren Inhalt in den Finanzausgleichsgesetzen der Länder eindeutig definiert sind und die die 
übergeordnete Sicht im föderativen System der Bundesrepublik Deutschland widerspiegeln.

An der derzeit praktizierten Messung von Finanzkraft und Steuerkraft in den Bundesländern und ihrer Beurteilung im Gesamtzusammenhang des kommunalen Finanzausgleichs setzt die vorliegende Arbeit an.

Im zweiten Kapitel wird deshalb zuerst nach den Anforderungen an die Finanzkraft- und Steuerkraftmessung gefragt, die sich aus der Gesamtkonzeption des kommunalen Finanzausgleichs ergeben.

Daran schließt sich eine Betrachtung der Meßkonzepte in den Bundesländern an; die Stadtstaaten und Berlin bleiben wegen ihrer besonderen Situation dabei allerdings ausgeklammert. Beim Vergleich der Finanzausgleichsgesetze fällt zunächst auf, daß Finanzkraft und Steuerkraft in den Ländern sehr unterschiedlich bestimmt werden. Diese Abweichungen könnten etwa dann gerechtfertigt werden, wenn die Einnahmesysteme der Gemeinden in den Ländern ebenfalls deutliche Unterschiede aufweisen würden. Dies ist jedoch nicht der Fall, was vermuten läßt, daß die Meßkonzepte vor allem mit Blick auf die angestrebten Verteilungswirkungen des kommunalen Finanzausgleichs entwickelt worden sind.

Die Untersuchung der Komponenten von Finanz- und Steuerkraft und ihrer Zusammensetzung in einzelnen Bundesländern verdeutlicht Ungereimtheiten, von denen einige auch in der Literatur bereits - wenn auch mit unterschiedlichen Ergebnissen - herausgestellt worden sind, wie etwa die Beschränkung der Messungen auf einen Teil der gemeindlichen Steuern oder die mehr oder weniger willkürlichen Festsetzungen nivellierter Hebesätze. Diese Vorgehensweisen sind zumindest nicht ohne weiteres einsichtig und scheinen zu anderen Konstruktionsprinzipien des kommunalen Finanzausgleichs, wie beispielsweise der Bedarfsbestimmung, im Widerspruch zu stehen. Worin die Inkonsistenzen letztlich bestehen, läßt sich im 2. Kapitel allerdings noch nicht endgültig klären.

Abschließend werden alternativ vorgeschlagene oder in anderen Staaten praktizierte Verfahren der Finanz- und Steuerkraftbestimmung daraufhin betrachtet, ob sie sich in das Gesamtsystem des kommunalen Finanzausgleichs 
in der Bundesrepublik Deutschland besser einfügen als die derzeit verwendeten Konzepte. Dies ist im Ergebnis jedoch zu verneinen.

Einer der in der Literatur häufig vorgebrachten Kritikpunkte an den praktizierten Meßkonzepten stellt fest, daß für die Steuerkraftmessung deutlich niedrigere als die im Durchschnitt realisierten und realisierbaren Hebesätze zugrundegelegt werden. Dieser Einwand führt zu der Frage, welche Steuersätze eine Gemeinde durchsetzen, oder umfassender betrachtet: wieviele Einnahmen eine Gemeinde aus Steuern und aus anderen Einnahmequellen maximal erzielen kann. Ihr widmet sich das 3. Kapitel.

$\mathrm{Zu}$ diesem Zweck wird der Standpunkt der einzelnen Gemeinden eingenommen und die Grenzen kommunaler Einnahmetätigkeiten werden für verschiedene fiktive Typen kommunaler Einnahmesysteme genauer betrachtet. Dabei werden ökonomische, rechtliche und politische Grenzen unterschieden; das Hauptgewicht der Untersuchungen liegt hier auf der Analyse der ökonomischen Grenzen.

Um die Komplexität des Gegenstandes etwas zu reduzieren, vor allem aber, weil die Bestimmung des kommunalen Einnahmepotentials - wie auch der kommunalen Finanzkraft - für die gegebene Situation in den einzelnen Gemeinden vorgenommen werden soll, wird zunächst nur nach den Möglichkeiten der Einnahmesteigerung bei konstantem kommunalen Leistungsangebot, insbesondere also nach fiskalisch optimalen Steuer- und Abgabesätzen gefragt.

Unter dieser Bedingung werden nacheinander die relevanten Begrenzungen der kommunalen Einnahmebeschaffung eingeführt, deren Berücksichtigung sukzessive zu einer theoretischen Annäherung an die potentiellen gemeindlichen Einnahmen führt:

- $\quad$ die Begrenzung durch das verfügbare Einkommen und Vermögen in der Region,

- Begrenzungen durch sachliche und räumliche Anpassungen der Wirtschaftssubjekte an die Abgabeerhebung durch die Gemeinde

- $\quad$ staatliche Festsetzung von Abgabeobergrenzen.

In diesem Abschnitt kann gezeigt werden, daß das kommunale Einnahmepotential im wesentlichen durch die Stellung der Gemeinden in der inter- 
kommunalen Konkurrenz und die Ausweichmöglichkeiten der Wirtschaftssubjekte bestimmt wird; Determinanten, die auch dann großes Gewicht behalten, wenn der Staat zusätzliche Begrenzungen der Abgabegestaltung einführt.

Anschließend wird die Annahme eines vorgegebenen Leistungsangebots aufgegeben und - wenn auch nur in einer knappen Übersicht - der Fall der Budgetmaximierung durch die Gemeinde untersucht. Auch hier erweist sich ihre Position in der interkommunalen Konkurrenz als entscheidende Determinante des Einnahmepotentials.

Als Ergebnis kann festgehalten werden, daß für die Bestimmung des kommunalen Einnahmepotentials aus gemeindlicher Sicht politische Zielsetzungen, die nach übergeordneten Vorstellungen mit der Einnahmetätigkeit realisiert werden sollen, weitgehend unerheblich sind, es sei denn, sie werden mittels rechtlicher Einschränkungen kommunalen Handelns von übergeordneten Gebietskörperschaften durchgesetzt und werden tatsächlich als Begrenzung kommunaler Einnahmetätigkeit wirksam. Für die Bestimmung des Einnahmepotentials sind vielmehr solche Faktoren relevant, wie sie auch bei der Bestimmung unternehmerischer Handlungsspielräume eine Rolle spielen; Gemeinden können unter diesem Aspekt ähnlich wie private Anbieter mit mehr oder minder großen monopolistischen Handlungsspielräumen behandelt werden. Allerdings besteht der Zweck kommunaler Aktivitäten im Gegensatz zu unternehmerischer Tätigkeit nicht in der Gewinnerzielung, insofern kann gemeindliche Einnahmepolitik nicht aus ihren Einnahmemöglichkeiten abgeleitet werden. Dazu wären weitere Determinanten kommunalen Handelns in die Analyse einzubeziehen. Dies ist jedoch nicht die Zielsetzung der vorliegenden Arbeit.

Während für die einzelne Gemeinde die Abschätzung ihrer gesamten Einnahmemöglichkeiten durchaus sinnvoll sein kann - etwa dann, wenn mit stark steigenden Aufwendungen für die Aufrechterhaltung des Leistungsangebots gerechnet wird - ist die aus gemeindlicher Sicht vorgenommene Bestimmung des Einnahmepotentials nicht ohne weiteres für die Zwecke des kommunalen Finanzausgleichs verwendbar. Hier lautet die Frage vielmehr, wieviele Mittel die Gemeinden im Rahmen ihrer Möglichkeiten selbst auf- 
bringen sollen, um ihre Aufgaben zu erfüllen und die anfallenden Ausgaben zu decken. Diese normativen Probleme werden im 4. Kapitel behandelt.

Da die Antwort auf die Frage, was die Gemeinden selbst zur Finanzierung beitragen sollen, wesentlich mit ihrer Stellung im gesamten föderativen System und den ihnen übertragenen Kompetenzen zusammenhängt, werden im 4. Kapitel verschiedene Organisationsformen föderativer Systeme und die entsprechenden Aufteilungen von Aufgaben, Ausgaben und Einnahmen auf die Gebietskörperschaften genauer daraufhin untersucht, welche Anforderungen die Bestimmung gemeindlicher Finanzkraft jeweils erfüllen soll und wie die Messung im einzelnen auszugestalten ist. Die Ergebnisse sind je nach Gestalt des Staatsaufbaus höchst unterschiedlich.

So kann etwa gezeigt werden, daß für eine vollständig weisungsgebundene Gemeindeebene eine gesonderte Bestimmung der Finanzkaft aus übergeordneter Sicht überflüssig ist, sofern das Gesamtsystem rational konstruiert ist und die Finanzierung kommunaler Aktivitäten ebenfalls vom Staat übernommen wird. Für eine vollständig autonome Gemeindeebene ist eine Bestimmung der Finanzkraft hingegen aus übergeordneter Sicht sinnvoll; allerdings ist sie für diesen Fall anders zu bestimmen als das Einnahmepotential aus gemeindlicher Sicht, da für den Staat jene Begrenzungen kommunaler Einnahmemöglichkeiten, die aus den Präferenzen der angesiedelten Wirtschaftssubjekte und ihren Ausweichreaktionen auf potentielle kommunale Einnahmepolitik resultieren, nicht von Interesse sind. Vielmehr kommt es darauf an, wieviele Leistungen eine Gemeinde maximal finanzieren könnte, wenn die Abgabezahler - Bürger und Unternehmen - dies wünschen würden. Die finanzielle Leistungsfähigkeit der Gemeinden wäre also nach einem Einkommenskonzept zu bestimmen.

Interessanter als die Betrachtung solcher reiner Systeme ist die Untersuchung von zwischen diesen beiden Extremen angesiedelten Mischsystemen. Dies gilt vor allem mit Blick auf den Staatsaufbau in der Bundesrepublik Deutschland, in dem das Spektrum kommunaler Aktivitäten von der vollständig reglementierten Übertragung staatlicher Aufgaben auf die Gemeinden bis zur uneingeschränkt freiwilligen Wahrnehmung und selbständigen Finanzierung von Aufgaben reicht. 
Das Hauptaugenmerk liegt im 4. Kapitel daher auf verschiedenen Typen von Mischsystemen und den jeweils angemessenen Konzepten zur Bestimmung gemeindlicher Finanzkraft. Das Ergebnis lautet, daß diese Bestimmung theoretisch kaum Probleme aufweist, solange die Zuweisung von Aufgaben-, Ausgaben- und Finanzierungskompetenz kongruent ist, d.h.: für einzelne Aufgabenbereiche jeweils bei der gleichen Gebietskörperschaftsebene angesiedelt ist.

Von besonderer Bedeutung ist jener Fall, in dem von den Gemeinden ohne sie im einzelnen zu reglementieren - ein bestimmtes "Normalangebot" an Leistungen erwartet wird und ihnen zur Finanzierung solche Einnahme-, speziell Steuerquellen überlassen werden, die idealerweise mit dem "Normalbedarf" korrespondieren sollten. Nur für dieses Organisationsprinzip ist überhaupt eine Gegenüberstellung von Finanzbedarf und Finanzkraft, wie sie im bundesdeutschen kommunalen Finanzausgleich vorgenommen wird, notwendig und sinnvoll. Die Bestimmung der gemeindlichen Finanzkraft muß sich dann auf die - im 4. Kapitel intensiver betrachtete - Normfinanzkraft richten. Damit soll die Summe jener Einnahmen erfaßt werden, die eine Gemeinde zu erzielen im Stande ist, wenn ihre Aktivitäten staatlichen Normen entsprechen.

Im 5. Kapitel wird danach gefragt, welche Schlüsse aus den Ergebnissen des 4. Kapitels für die Gestaltung der Finanzkraftmessung im kommunalen Finanzausgleich der Bundesrepublik Deutschland zu ziehen sind. Sofern die kommunalen Aktivitäten in Segmente aufspaltbar wären, die jeweils einem der behandelten Organisationsprinzipien von Aufgaben-, Ausgaben- und Finanzierungskompetenz folgen, ergäbe sich daraus auch ein Grundraster für die Bestimmung der gemeindlichen Finanzkraft (wie auch des Finanzbedarfs), die ebenfalls segmentweise zu erfassen wäre. Aufgaben-, Ausgaben- und Einnahmezuweisungen an die Gemeinden folgen in den einzelnen Segmenten jeweils einer eigenen Logik und erfordern einen besonderen Typ von Finanzausgleich, und nur für den im 4. Kapitel eingehender betrachteten "Normbereich" hätte ein Ausgleich nach Maßgabe von Finanzbedarf und Finanzkraft, wie er derzeit praktiziert wird, zu erfolgen.

Eine solche Aufspaltung ist für das geltende System jedoch nur partiell möglich. So lassen sich schon die Aufgabenbereiche nur tendenziell nach ihrer Weisungsgebundenheit zuordnen; die darüber hinaus erforderliche Zuord- 
nung bestimmter Finanzierungsarten zu den jeweiligen Aufgabenbereichen ist - sofern keine Zweckbindung der Einnahmen vorliegt - kaum möglich. Damit bleibt der Normbereich kommunaler Einnahmetätigkeit jedoch unbestimmt. Auch für die Ermittlung der normierten Finanzkraft ist deshalb nicht festgelegt, welche Einnahmequellen zur Deckung des Normalbedarfs verwendet und wie weit sie von den Gemeinden ausgeschöpft werden sollen. So müssen etwa - je nach der konkreten Situation der Gemeinde - mehr oder minder große Teile der eigenen, allgemeinen Deckungsmittel zur Finanzierung des übertragenen Wirkungskreises verwendet werden, weil auch die unabweisbaren, von der jeweiligen Kommunalpolitik unabhängigen Kosten der Erfüllung dieser Aufgaben nicht vollständig von der für die Übertragung verantwortlichen Ebene erstattet werden.

Es wird deshalb zu zeigen versucht, daß die Probleme einer "objektiven" Bestimmung gemeindlicher Finanzkraft für die Zwecke des kommunalen Finanzausgleichs letztlich weniger aus der Differenziertheit des gemeindlichen Finanzsystems als aus der Inkongruenz der Kompetenzverteilung resultieren. Sachverhalte, die in ganz anderen Zusammenhängen diskutiert werden, wie etwa die angesprochene tendenzielle Unterfinanzierung pflichtiger Aufgabenerfüllung aufgrund pauschalierter Erstattungen, sind demnach auch dafür verantwortlich, daß im gegenwärtigen System des kommunalen Finanzausgleichs eine "richtige" Bestimmung der gemeindlichen Finanzkraft nicht konzipiert werden kann. Unter den gegebenen Voraussetzungen wäre Hanusch darin zuzustimmen, "daß im Grunde jedes Konzept des kommunalen Finanzausgleichs ein willkürlicher Ansatz bleiben muß." ${ }^{16)}$ Dies gilt allerdings - wie im 4. Kapitel nachgewiesen werden sollte - nur insofern, als die Zuweisung von Aufgaben-, Ausgaben- und Finanzierungskompetenzen willkürliche Elemente enthält.

16) Hanusch, Horst und Kuhn, Thomas, Messung des kommunalen Finanzbedarfs, a.a.O.. S. 70 . 
Ulrike Hardt - 978-3-631-75265-4

Downloaded from PubFactory at 01/11/2019 06:31:57AM

via free access 
2. Kapitel: Zur Beurteilung praktizierter und alternativ diskutierter Verfahren der Messung gemeindlicher Finanzkraft im Rahmen des kommunalen Finanzausgleichs

\section{A. Die Fragestellung des Kapitels}

Gegenstand dieses Kapitels ist noch nicht die Konstruktion eines "richtigen" oder "objektiven" Konzepts zur Bestimmung und Messung kommunaler Finanzkraft. Die Beurteilung der betrachteten Konzepte kann sich deshalb auch nicht auf ein solches "objektives" Verfahren berufen und gegebenenfalls Abweichungen vom Idealkonzept aufzeigen. Vielmehr wird eine rein immanente Kritik vorgebracht: Aus dem Zweck des kommunalen Finanzausgleichs lassen sich verschiedene Anforderungen ableiten, denen die Messung der gemeindlichen Finanzkraft gerecht werden muß, wenn die tatsächliche Situation der Gemeinden unverzerrt wiedergegeben und die Ausgleichs- und Verteilungswirkungen des kommunalen Finanzausgleichs überprüfbar und steuerbar sein sollen. Anhand dieser Anforderungen sind die jeweiligen Konzepte dann zu beurteilen.

Ergebnis ist zunächst eine Reihe negativer Aussagen: Keines der betrachteten Konzepte erfüllt die Anforderungen uneingeschränkt; für jedes von ihnen lassen sich Unzulänglichkeiten aufzeigen.

Aus den beschriebenen Mängeln ließe sich nun ableiten, um welche Komponenten die praktizierten Meßkonzepte zu erweitern oder zu verändern wären, wenn der Anforderungskatalog in den Regelungen des kommunalen Finanzausgleich der Bundesrepublik hinreichend konkretisiert wäre. Dies ist jedoch nicht der Fall. Deshalb liefert die Kritik allein noch keine konkreten Ansatzpunkte zur Neuformulierung des Finanzkraftbegriffs; es kann lediglich festgestellt werden, daß sich weder die verwendeten noch die alternativ vorgeschlagenen Konzepte "nahtlos" in die Gesamtkonzeption des kommunalen Finanzausgleich einfügen. Wo die Ursachen der aufgezeigten Inkongruenzen liegen und wie sie beseitigt werden könnten, ist daraus noch nicht ersichtlich. 
Allerdings stellen sich bei der näheren Betrachtung eine Reihe offener Fragen, sowohl zur Ausgestaltung des Finanzausgleichssystems als auch zur Konzeption einer angemessenen Finanzkraftmessung, die den weiteren Gang der Überlegungen vorstrukturieren.

\section{B. Die Stellung der Finanzkraftmessung im System des kommunalen Finanzausgleichs}

I. Die Zwecke des kommunalen Finanzausgleichs

- Anforderungen an die Finanzkraftmessung im vertikalen und horizontalen Finanzausgleich -

Der Begriff des kommunalen Finanzausgleichs deutet zunächst nur auf einen die Einnahmen der Gemeinden betreffenden Vorgang hin. Bereits Popitz wies jedoch darauf hin, daß bei der Betrachtung des Finanzausgleichs die Verteilung von Ausgaben und Aufgaben einzubeziehen sei. ${ }^{1)}$ Dieser Auffassung wurde später - u.a. von Albers ${ }^{2)}$ beigepflichtet. So weit gefaßt regelt der kommunale Finanzausgleich die Einbindung der untersten föderativen Ebene in den Gesamtstaat.

Üblicherweise werden drei Stufen des Finanzausgleichs unterschieden: ${ }^{3)}$

(1) Die Zuweisung von Aufgaben bzw. des Aufgabenspielraums (sowie der daraus resultierenden Ausgaben) auf die Gebietskörperschaftsebenen.

(2) Die Zuweisung eigener Einnahmequellen.

(3) Die Bestimmung der Ausgleichsintensität und der Form des Ausgleichs zwischen Ausgabenbedarf und eigenen Finanzierungsmöglichkeiten.

Der in Stufe 3 vorzunehmende Ausgleich setzt - wenn er nicht willkürlich erfolgen soll - eine Messung der auszugleichenden Größen voraus; der Gegen-

1) Vgl. Popitz, Johannes, Der künftige Finanzausgleich zwischen Reich, Ländern und Gemeinden, Berlin 1932. S. 1 und S. 196ff.

2) Vgl. Albers, Willi, Finanzausgleich (III), Deutschland. in: Handwörterbuch der Sozialwissenschaften, dritter Band, Tübingen-Göttingen 1961, S. 553-575, hier S. 553.

3) Vgl. z.B. Seiler, Gerhard, Ziele und Mittel des kommunalen Finanzausgleichs, in: Probleme des Finanzausgleichs II, von Karl-Heinrich Hansmeyer, Gerhard Seiler und Christian Smekal, hrsg. von Dieter Pohmer, Schriften des Vereins für Socialpolitik, N.F. Bd. 96/III, Berlin 1980, S. 11-82, hier S. 11. 
stand der Finanzkraftmessung wird hier umrissen als die Summe der zur Dekkung des Ausgabenbedarfs (der ebenfalls zu erfassen wäre) vorhandenen eigenen Finanzierungsmöglichkeiten. Über die konkrete inhaltliche Bestimmung des Gegenstandes ist damit allerdings nichts gesagt; darüber entscheiden allein die Ziele und die konkrete Ausgestaltung der drei Stufen des Finanzausgleichs. Wie die Aufgaben-, Ausgaben- und Einnahmenzuweisung auf die Gebietskörperschaften ausgestaltet und welche Ausgleichsentscheidungen gefällt werden, ist davon abhängig, welche Ziele erfüllt werden sollen und wie die Einzelziele bewertet werden. So wird das Gesamtsystem bei Dominanz eines interregionalen und interkommunalen Verteilungsziels anders konzipiert werden, als wenn Allokations- oder Wachstumsüberlegungen die Hauptrolle spielen. Eine "richtige" Aufgabenverteilung oder eine "richtige" Einnahmeautonomie sind wissenschaftlich ebensowenig begründbar wie die anzustrebende Ausgleichsintensität ${ }^{4}$. Genauso kann eine "richtige" Messung der kommunalen Einnahmemöglichkeiten nicht konzipiert werden, ohne auf das Gesamtsystem und seine Bestandteile zu rekurrieren.

Als grundlegende Minimalforderung - unabhängig von der Gestaltung des Finanzausgleichs - kann hier jedoch bereits die Forderung nach Neutralität der Messungen aufgestellt werden. Das heißt vor allem, daß die Messungen selbst die Ausgleichs- und Umverteilungsentscheidungen nicht vorwegnehmen dürfen, indem - mehr oder weniger geplant - solche Gemeinden als finanzschwach ausgewiesen werden, denen, aus welchen Gründen auch immer, vergleichsweise hohe Zuschüsse gewährt werden sollen. Ein solch gezielter Ausgleich sollte als Verteilungsentscheidung erkennbar bleiben und nicht durch den Meßvorgang verdeckt werden. Die Kontrollfunktion der Messung würde andernfalls erheblich beeinträchtigt.

Die Notwendigkeit einer Finanzkraft- (und Bedarfs)messung, aber auch eine erste inhaltliche Eingrenzung ihres Gegenstandes auf potentielle Einnahmen leitet sich aus dem Selbstverwaltungsrecht der Gemeinden in der Bundesrepublik Deutschland ab:

Selbstverantwortung bei der Aufgabenerfüllung und der Ausgabentätigkeit erfordert zum einen die Möglichkeit selbstverantwortlicher Einnahmebe-

4) Ebenda, S. 25. 
schaffung, zum anderen eine insgesamt ausreichende Finanzausstattung. ${ }^{5)}$ Daraus erklären sich die Versuche, ein Einnahme- und speziell ein Steuersystem zu entwickeln, das sich dem gemeindlichen Finanzbedarf jeweils optimal anpassen läßt, das also bei wachsenden Ausgabenerfordernissen gleichzeitig und in gleichem Umfang wachsende Einnahmemöglichkeiten bietet. Ein solches mit dem Bedarf korrespondierendes System scheint jedoch nicht konstruierbar zu sein.6)

In jedem Fall ist also mit Diskrepanzen zwischen Bedarf und Einnahmemöglichkeiten zu rechnen, die zunächst einmal zu quantifizieren - und soweit man dies für erforderlich hält - auszugleichen sind.

Gegenstand der Finanzkraftmessung sind - sofern den Gemeinden ein Mitspracherecht bei der Einnahmebeschaffung zugestanden wird - nun allerdings nicht die tatsächlichen Einnahmen, sondern potentielle Einnahmen. In der Bestimmung dieser Kraft liegt offenbar das Hauptproblem bei der Entwicklung eines geeigneten Meßkonzepts. Es muß jedoch gelöst werden, wenn man nicht mit der Verwendung tatsächlicher Einnahmen unerwünschte Anreizwirkungen auf die kommunale Einnahmepolitik induzieren will.

Der kommunalen Selbstverwaltungsgarantie entsprechend sieht das Gemeindefinanzsystem in der Bundesrepublik Deutschland auch bei der Einnahmebeschaffung einen Entscheidungsspielraum für die Gemeinden vor. Zumindest einige der gemeindlichen Einnahmequellen können - je nach Einschätzung des Finanzbedarfs durch die Gemeinde selbst - mehr oder weniger stark ausgeschöpft werden. Die aufgrund kommunalpolitischer Entscheidungen realisierten Einnahmen dürften daher nur in Ausnahmefällen mit den potentiell erzielbaren Einnahmen, die die Gemeinden zur Deckung des Bedarfs aufbringen könnten, übereinstimmen. Würde man die tatsächlichen Einnahmen anstelle eines "objektiven Maßstabs für die Zuweisung allgemeiner Deckungsmittel"7) verwenden, so könnte der Effekt auftreten, daß ein-

5) Vgl. etwa Flämig, Christian, Gemeindefinanzen und kommunale Wirtschaftsentwicklungsplanung, Baden-Baden 1974, S. 23 f.

6) Diese Forderung könnte nur durch eine generelle Äquivalenzfinanzierung erfüllt werden, die sich für das gegenwärtig von den Gemeinden wahrgenommene Aufgabenspektrum jedoch nicht vollständig realisieren läßt. Vgl. dazu auch: Wissenschaftlicher Beirat beim Bundesministerium der Finanzen, Gutachten zur Reform der Gemeindesteuern in der Bundesrepublik Deutschland, a.a.O., S. 31f.

7) Schmölders, Günter, "Finanzstarke" und "finanzschwache" Gemeinden, a.a.O., S. 37. 
zelne Gemeinden oder sogar die Gemeinden insgesamt ihre eigenen Einnahmequellen in besonderem Maße schonen, um bei einer Beurteilung ihrer Finanzsituation anhand der realisierten Einnahmen als zuschußbedürftig zu gelten und um auf diese Weise zusätzliche Mittel von übergeordneten Ebenen in Anspruch nehmen zu können. "Die effektive Höhe der Einnahmen sagt (deshalb) nichts darüber aus, inwieweit die vorhandenen Steuerquellen und ihre Ausnutzung den vorhandenen Erfordernissen entsprechen oder nicht." ${ }^{8)}$ Weder zur Kontrolle des kommunalen Einnahmesystems noch zur Feststellung eines möglicherweise auftretenden Zuschußbedarfs sind die tatsächlichen Einnahmen daher geeignet.

Die tatsächlichen Einnahmen einer Gemeinde können sich von den zu bestimmenden potentiellen Einnahmen zunächst einmal für jede einzelne Einnahmequelle in ihrer Höhe unterscheiden. Deshalb schließt sich an die Ablehnung der tatsächlichen Einnahmen als Maßstab für die Finanzkraft einer Gemeinde unmittelbar die Frage an, in welcher Höhe potentielle Erträge aus den gemeindlichen Einnahmequellen in die Ermittlung der Finanzierungsmöglichkeiten eingehen sollen, damit der Finanzkraftmaßstab als objektiv gelten kann.

Der Begriff der Finanzierungsmöglichkeiten deutet für sich genommen auf ein - wie auch immer bestimmtes - Maximum an erzielbaren Einnahmen hin; in der Literatur finden sich jedoch auch Aussagen, die eher auf Maßstäbe wie den der Zumutbarkeit oder Angemessenheit ${ }^{9)}$ oder eine interkommunal einheitliche Anspannung ${ }^{10)}$ der Quellen hindeuten. Auch diese Anforderungen bleiben also noch wenig konkret; zur Beurteilung des verwendeten Konzepts können sie aber dennoch herangezogen werden.

Eine weitere Eingrenzung des zu messenden Gegenstandes "Finanzkraft" erfolgt durch die Auswahl der einzubeziehenden Einnahmequellen. Zum ersten sollen nur gemeindeeigene Finanzierungsmöglichkeiten ermittelt werden,

8) Ebenda, S. 38.

9) So könnte etwa die auf S. 7 zitierte Aussage von Schmölders interpretiert werden.

10) So beispielsweise Kops, Manfred, Die regionalen Verteilungswirkungen des kommunalen Finanzausgleichs, Thun - Frankfurt 1983, S. 128 und Ehrlicher, Werner, Kommunaler Finanzausgleich und Raumordnung, Veröffentlichungen der ARL, Abhandlungen Bd. 51, Hannover 1967, S. 42. 
zum zweiten bezieht sich die Finanzkraftmessung explizit nur auf jene Einnahmemöglichkeiten, die zur Deckung des "Bedarfs" dienen können. Deshalb wäre es auch zu kurz gegriffen, wenn die Konzeption der Finanzkraftmessung allein mit Blick auf die kommunalen Einnahmequellen vorgenommen würde. ${ }^{11)}$ Entscheidend ist vielmehr der Zusammenhang von "Bedarf" im Sinne "notwendiger Ausgaben" ${ }^{12)}$ und vorgesehener Finanzierung; die Messungen sollen feststellen, ob die Finanzierungsmöglichkeiten der Gemeinden eine zielgerechte Aufgabenerfüllung erlauben. Der Zusammenhang zwischen Bedarf und Finanzierungsquelle könnte nur dann vernachlässigt werden, wenn es keine direkte - oder indirekte - Zweckbindung oder Zuordnung bestimmter kommunaler Einnahmequellen zu bestimmten Aufgaben und Ausgaben gäbe.

Muß die objektive Messung des so weit eingegrenzten Gegenstandes "gemeindliche Finanzierungsmöglichkeiten" nun jeweils alle relevanten Einnahmequellen in ihrer wirklichen Ergiebigkeit erfassen, oder reicht die Messung über einen Indikator nicht ebenfalls aus? Die Antwort auf diese Frage ist vor allem davon abhängig, ob die Messung für vertikale oder horizontale Ausgleichsmaßnahmen zugrunde gelegt werden soll.

Jede der drei oben beschriebenen Stufen des kommunalen Finanzausgleichs hat einen vertikalen und einen horizontalen Aspekt: Mit der Aufgaben-, Ausgaben- und Einnahmenverteilung auf die föderativen Ebenen einschließlich des Ausgleichs von Finanzbedarf und Finanzkraft der Gemeinden wird gleichzeitig immer auch über die Position der einzelnen Gemeinden im interkommunalen Vergleich entschieden. Eine Messung von Finanzbedarf und Finanzkraft kann mit Blick auf jede der beiden Komponenten vorgenommen werden; je nachdem, welcher Aspekt im Vordergrund steht, hat die Finanzkraftmessung unterschiedliche Anforderungen zu erfüllen, um als objektiver Maßstab gelten zu können.

11) Der Grundsatz, daß bei der Ermittlung der Finanzkraft von der Einnahmeseite auszugehen sei, darf also nicht so ausgelegt werden, daß nur die kommunalen Einnahmen zu betrachten seien. Vgl. Metz, Hans-Günter, Der Ausgleich von Finanzkraft- und Bedarfsunterschieden im föderalistischen Staat, Frankfurt 1979, S. 52.

12) Nach Kirchhof enthält der Rechtsbegriff "notwendig" keinen "für politisch beliebige Entscheidungen offenen Tatbestand, sondern ist durch langjährige Rechtsprechung zu einem justitiablen Rechnungsmaßstab geworden." Danach "bezeichnet die 'notwendige Maßnahme eine Relation zwischen Mittel und Zweck, die das taugliche und das erforderliche Mittel bezeichnet." Kirchhof, Paul, Der Finanzausgleich als Grundlage kommunaler Selbstverwaltung, a.a.O., S. 715. 
Der vertikale Aspekt steht im Vordergrund, wenn nach der Sicherung eines finanziellen Existenzminimums oder nach der Finanzierbarkeit einer "als notwendig" angesehenen Grundausstattung ${ }^{13)}$ gefragt wird. Allgemein wird anerkannt, daß die Mehrzahl der Gemeinden nicht in der Lage ist, diese Grundausstattung aus eigenen Mitteln zu finanzieren: "... die Unfähigkeit zur Selbstfinanzierung ist gegenwärtig der gesetzlich veranlaßte Regelfall."14) Insofern wird davon ausgegangen, daß der Zuschußbedarf zu ermitteln und abzudecken ist. "Bezugsgröße für eine angemessene Finanzangleichung ist (...) das Deckungsverhältnis von aufgabenabhängigem Bedarf und verfügbaren Finanzmitteln." ${ }^{15)}$ Die Messungen von Finanzbedarf und Finanzkraft haben in diesem Fall idealerweise absolute monetäre Größen zu ermitteln, aus deren Gegenüberstellung die auszugleichende Differenz unmittelbar ersichtlich ist. In die Finanzkraftmessung müßten dementsprechend alle Einnahmemöglichkeiten eingehen, die zur Deckung des Bedarfs verwendet werden können.

Mit der vertikalen Zuordnung von Aufgaben, Ausgaben und Einnahmen auf die Gebietskörperschaften werden gleichzeitig die horizontalen Positionen auf einer Gebietskörperschaftsebene festgelegt. Dabei treten sowohl im Bedarf als auch bei den Finanzierungsmöglichkeiten offenbar unvermeidliche regionale und interkommunale Disparitäten auf, die durch die Gestaltung des vertikalen Finanzausgleichs nicht vollständig vermieden werden können ${ }^{16)}$ und deren nachträglicher Ausgleich über die Sicherung einer Grundausstattung hinaus angestrebt wird. Diese horizontale Angleichung wird in der Bundesrepublik mit Hilfe der Zuweisungen von übergeordneten Gebietskörperschaften, im "vertikalen Finanzausgleich mit horizontalem Effekt", vorgenommen. Bezugsgröße der Angleichung ist ebenfalls das Deckungsverhältnis von Finanzbedarf und Finanzkraft in den verglichenen Gemeinden.

Betrachtet man diese beiden Größen unter horizontalem Aspekt zunächst isoliert, so würde jeweils ein relatives $\mathrm{Ma} ß$ genügen, um die Vertei-

13) Voigtländer. Hubert, Zur Problematik der Gestaltung eines selbstverwaltungsgerechten und aufgabenorientierten Finanzausgleichs, in: Informationen zur Raumentwicklung, hrsg. von der BfLR, Heft 2/3 1978. S. 185-192, hier S. 186.

14) Kirchhof, Paul, Der Finanzausgleich als Grundlage kommunaler Selbstverwaltung, a.a.O., S. 713.

15) Ebenda.

16) Vgl. Münstermann. Engelbert, Der Kommunale Finanzausgleich I - Begriff und Funktion, in: WISU 6/1976, S. 275-279, hier S. 275. 
lungspositionen für den interkommunalen Vergleich abzubilden. Es erfüllt das Kriterium der Neutralität bereits dann, wenn es die Verteilungsrelationen richtig wiedergibt, unabhängig vom Niveau der ausgewiesenen Werte. Allein in diesem Fall reicht eine Messung der Finanzierungsmöglichkeiten über einen Ausschnitt der Einnahmequellen aus; dann nämlich, wenn der gewählte Ausschnitt alle übrigen relevanten Einnahmemöglichkeiten im richtigen Verhältnis mit abbildet.

Zwar können mit einem solchen $\mathrm{Maß}$ die absoluten Unterschiede in den Ausgabenanforderungen oder in den Finanzierungsmöglichkeiten nicht ermittelt werden; für ein Ausgleichssystem, das nur eine Angleichung der Verteilungspositionen zum Ziel hat, ist diese Information aber auch nicht unbedingt erforderlich.

Strengere Anforderungen müssen erfüllt werden, wenn die horizontale Verteilung des Zuschußbedarfs untersucht werden soll. Da zu diesem Zweck Finanzbedarf und eigene Finanzierungsmöglichkeiten in den einzelnen Gemeinden gegenüberzustellen sind, müssen die Indikatoren für beide Größen auch im Niveau vergleichbar und saldierbar sein. Nur unter dieser Bedingung können überhaupt Aussagen über die Verteilung des Zuschußbedarfs gemacht werden.

Zusammenfassend sind noch einmal die Anforderungen an eine objektive Messung der gemeindlichen Finanzierungsmöglichkeiten festzuhalten:

Gegenstand der Messung sind die zur Deckung des Finanzbedarfs vorhandenen eigenen Einnahmemöglichkeiten der Gemeinden. Erfaßt werden sollen die potentiellen, nicht die tatsächlichen Erträge jener Einnahmequellen, die zur Deckung des "Bedarfs" dienen können, und zwar in einer Höhe, die angemessen und zumutbar erscheint und die eine Gleichbehandlung der Gemeinden sicherstellt.

- Die konkreten Anforderungen an die Ausgestaltung der Finanzkraftmessung hängen vom Verwendungszweck der erfaßten Größe ab.

So ist etwa in einem System, das lediglich eine Angleichung der Finanzkraft vorsieht und dazu eine vorgegebene Summe nach Maßgabe der Finanzkraftrelationen verteilt, ein anderes Meßkonzept erforderlich als in einem System, das in allen Gemeinden den Ausgleich des Defizits zwischen Ausgabenbedarf und Finanzierungsmöglichkeiten anstrebt. 
In der Literatur wird in der Regel davon ausgegangen, daß im kommunalen Finanzausgleich in der Bundesrepublik sowohl die vertikale Sicherung der "Grundausstattung" als auch eine darüber hinausgehende horizontale Angleichung angestrebt wird. Die Finanzausgleichsgesetze liefern auch einige Anhaltspunkte für diese Interpretation. Entsprechend hätte die Finanzkraftmessung die weitestgehende Anforderung nach absoluter und vollständiger Abbildung der kommunalen Finanzierungsmöglichkeiten zu erfüllen. Bevor die verwendeten und diskutierten Meßverfahren anhand dieser Anforderungen beurteilt werden, soll das Ausgleichsverfahren des praktizierten kommunalen Finanzausgleichs jedoch noch einmal näher betrachtet werden. Dabei wird deutlich, daß die Anforderungen innerhalb des Finanzausgleichs im engeren Sinne deutlich niedriger angesetzt werden könnten.

II. Eingeschränkte Anforderungen an die Finanzkraftmessung aufgrund der Dominanz des horizontalen Ausgleichs im kommunalen Finanzausgleich der Bundesrepublik Deutschland

Würde der Ausgleich von Finanzbedarf und Finanzkraft vor allem unter vertikalem Aspekt und mit der Zielsetzung vorgenommen, in allen Gemeinden die Finanzierung einer für notwendig gehaltenen Grundausstattung zu sichern, so wäre die Summe der zu verteilenden Mittel aus dem berechneten Zuschußbedarf der Gemeinden abzuleiten. ${ }^{17)}$ Dabei ließe sich der Zuschußbedarf für jede Gemeinde isoliert errechnen; ein Vergleich mit dem Zuschußbedarf anderer Gemeinden wäre nicht erforderlich. Durch die unterschiedliche Höhe der Zuschüsse würde zwar ein horizontaler Effekt erzielt - indem einige Gemeinden hohe, einige niedrigere und ein kleiner Teil der Gemeinden gar keine Zuschüsse erhielten, würden die Differenzen in der Leistungsfähigkeit der Gemeinden verringert - dies ist allerdings nicht Hauptzweck des vertikalen Finanzausgleichs.

In den Ländern der Bundesrepublik Deutschland wird jedoch von vornherein anders vorgegangen, wie am Beispiel des Gemeindefinanzierungsgeset-

17) Vgl. Voigtländer, Hubert, Zur Problematik der Gestaltung eines selbstverwaltungsgerechten und aufgabenorientierten Finanzausgleichs. a.a.O., S. 137. 
zes Nordrhein-Westfalen (NW) für $1984^{18)}$ gezeigt werden kann. Davon weichen die Regelungen in den übrigen Bundesländern zwar teilweise ab, der grundlegende Ablauf des Finanzausgleichs ist jedoch in etwa der gleiche.

Ausgangspunkt des Finanzausgleichsverfahrens sind nicht etwa die ermittelten Bedarfs- und Finanzkraftkennziffern, sondern ist die zur Verteilung verfügbare Finanzausgleichsmasse, die sich vor allem aus dem Aufkommen der Verbundsteuern ergibt und deshalb weitgehend festliegt (\$2). Verringert um jenen Teil der Gesamtmasse, der für zweckgebundene Zuweisungen verwendet werden soll, wird die verbleibende Schlüsselmasse nach Maßgabe der Bedarfsmeßzahl einerseits und einer Steuerkraftmeßzahl andererseits verteilt $(\S 6,2)$. Bereits dieses Verfahren deutet auf die Dominanz des horizontalen Ausgleichszwecks hin, denn es kann keineswegs als sicher gelten, daß die Schlüsselmasse zur Sicherung des Grundbedarfs in allen Gemeinden ausreicht, solange man dessen Kosten nicht errechnet hat. Würde die Schlüsselmasse von vornherein so hoch angesetzt, daß damit auf jeden Fall die Mindestausstattung in allen Gemeinden finanzierbar wäre, so könnten die Ziele des vertikalen Finanzausgleichs trotzdem erfüllt werden, in diesem Fall könnte es allerdings auch zu einem "Überausgleich" kommen. Angesichts der knappen verteilbaren Mittel kann davon jedoch nicht ohne weiteres ausgegangen werden. Das Hauptgewicht der horizontalen Angleichung wird jedoch noch deutlicher sichtbar, wenn man die Ermittlung der Verteilungsmaßstäbe, insbesondere der Bedarfsmeßzahl betrachtet.

Die Bedarfsmeßzahlen der Gemeinden werden aus dem Gesamtansatz, multipliziert mit dem einheitlichen Grundbetrag, gebildet (§ 8). Der gemeindeindividuelle Gesamtansatz wiederum setzt sich aus dem nach Einwohnerzahlen differenzierten Hauptansatz und einem auf besondere Belastungen abstellenden Nebenansatz (in NW einem Schüleransatz; andere Bundesländer beziehen andere Nebenansätze mit ein) zusammen. Beide, Hauptansatz und Nebenansätze, sind Gewichtungsziffern, die den Grundbetrag um einen bestimmten Prozentsatz erhöhen oder absenken. Hier werden die Bedarfe also nur interkommunal differenziert, nicht aber gemessen. Der Grundbetrag als zweiter Bestandteil der Bedarfsmeßzahl legt nicht etwa - wie der Begriff ver-

18) Gesetz zur Regelung der Zuweisungen des Landes Nordrhein-Westfalen an die Gemeinden und Gemeindeverbände im Haushaltsjahr 1984 vom 21. Februar 1984. 
muten lassen könnte - eine monetäre Mindestausstattung für die Gemeinden fest, sondern wird jeweils so angesetzt, daß entsprechend den im Gesetz aufgestellten Verteilungsregeln die Schlüsselmasse aufgebraucht wird $(\S 8,3)$. Der Grundbetrag ist also die eigentlich in den Finanzausgleichsgesetzen gesuchte Größe, die mit den "Kosten einer - wie auch immer bestimmten Grundausstattung" nichts zu tun hat.

Seine aus dem Aufteilungsmodus abzuleitende Höhe wird entscheidend durch die angestrebte Verteilungswirkung, deren Ausmaß aber wiederum durch die im Gesetz festgelegte Ausschüttungsquote beeinflußt, die festlegt, bis zu welchem Prozentsatz Differenzen zwischen Bedarfsmeßzahl und Steuerkraftmeßzahl ausgeglichen werden sollen. In Nordrhein-Westfalen wie in den meisten anderen Bundesländern wird die Ausschüttungsquote auf $50 \%$ festgelegt ( $\$ 10)$; um allen Gemeinden eine bestimmte Deckung der Bedarfsmeßzahl zu sichern, werden den Gemeinden in NW wie in allen Bundesländern Mindestgarantien gegeben $(\$ 10,2)$, die jedoch - wie die Ableitung der Bedarfsmeßzahl gezeigt hat - nicht etwa aus den Kosten einer Mindestausstattung errechnet werden. Sie erhöhen vielmehr nur die Ausschüttungsquote für finanzschwache Gemeinden. "Es ist offensichtlich, daß mit steigender Ausschüttungsquote das Umverteilungsmaß zunimmt. Bei unveränderter Zuweisungsmasse reduzieren sich dann nämlich die Transferzahlungen an finanzstarke Gemeinden. Umgekehrt kann die Ausschüttungsquote soweit gesenkt werden, daß auch die finanzstarken Gemeinden Schlüsselzuweisungen beanspruchen können." ${ }^{19)}$ Bei gegebener Ausschüttungsquote bestimmt die Festsetzung des Grundbetrags also auch darüber, wann eine Gemeinde als "abundant" gilt, das heißt, welche Gemeinden keine Schlüsselzuweisungen erhalten sollen.

Der beschriebene Vorgang kann anhand der formalisierten Darstellung noch einmal verdeutlicht werden:

$$
\text { Schlüsselmasse }=
$$

n

$\sum$ (Gesamtansatz x Grundbetrag - Steuerkraftzahl) x Ausschüttungsquote $\mathrm{G} 1$

19) Postlep, Rolf-Dieter, Räumliche Effekte der Steuerkraft- und Finanzbedarfsermittlung bei den Schlüsselzuweisungen, a.a.O., S. $207 \mathrm{ff}$. 
(1) Alle Größen mit Ausnahme des Grundbetrages und der Anzahl der berücksichtigten Gemeinden werden durch die im Gesetz festgelegten Regeln determiniert.

(2) Bei der Aufteilung der Schlüsselmasse werden nur jene Gemeinden berücksichtigt, in denen die Steuerkraftmeßzahl die Bedarfsmeßzahl nicht übersteigt. Ob dies der Fall ist, hängt von der Höhe des gesuchten Grundbetrages ab.

Die Verteilung der Schlüsselmasse erfolgt also mit Hilfe einer Gleichung mit zwei Unbekannten; Gleichungssysteme dieser Art sind aber bekanntlich nicht eindeutig lösbar, sondern bieten eine Vielzahl von Lösungsmöglichkeiten. Eine eindeutige Lösung kann nur in der Weise erfolgen, daß die angestrebten Verteilungsergebnisse bereits in die Berechnung eingeschlossen werden. In den praktizierten Verfahren wird dies erreicht, indem im Verlauf der iterativen Annäherung - völlig willkürlich - festgelegt wird, welche Gemeinden keine Schlüsselzuweisungen erhalten sollen.

Darin zeigt sich, daß selbst die in Abschnitt I erhobene, trivial erscheinende Minimalforderung nach einer durch das gewünschte Verteilungsergebnis unbeeinflußten Messung von Finanzbedarf und Finanzkraft im System des kommunalen Finanzausgleichs in der Bundesrepublik Deutschland nicht erfüllt wird. Hier kommt es gar nicht darauf an, die tatsächliche finanzielle Situation der einzelnen Gemeinden zwischen Ausgabenanforderungen und Finanzierungsmöglichkeiten transparent $\mathrm{zu}$ machen und den Zuschußbedarf $\mathrm{zu}$ ermitteln, sondern allenfalls darauf, gewisse "Reichtumsunterschiede" abzumildern. Sieht man einmal von dem Tatbestand $a b$, daß die Zuweisung von "oben" nach "unten" erfolgt und die allgemeinen Zuweisungen ebenso wie die Zuweisung anderer Einnahmen bzw. Einnahmequellen dem vertikalen Finanzausgleich zugeordnet werden kann, so wäre der beschriebene Vorgang weniger als "vertikaler Finanzausgleich mit horizontalem Effekt", denn als "horizontaler Finanzausgleich mit vertikalem Effekt" zu bezeichnen. Bei der Angleichung zwischen "armen" und "reichen" Gemeinden durch übergeordnete Gebietskörperschaften mittels der Verteilung allgemeiner Zuweisungen kommt es automatisch auch zu einem Aufstockungseffekt, der jedoch dem zu ermittelnden Zuschußbedarf keineswegs entsprechen muß. 
Ohne auch nur einen Blick auf die Messung der Finanzkraft der Gemeinden geworfen zu haben, kann also festgehalten werden, daß jene Zwecke, die dem kommunalen Finanzausgleich in der Literatur unterstellt werden, in der Praxis bestenfalls partiell angestrebt und verwirklicht werden. Eine Objektivierung der Finanzkraftmessung würde daran wenig ändern, solange die $\mathrm{Be}$ darfsmessung nicht ebenfalls umgestaltet und der Zuweisungsvorgang insgesamt den oben genannten Anforderungen angepaßt würde.

III. Fiktive Grundlagen zur Beurteilung der Steuerkraftmessung in der weiteren Untersuchung

Für die beabsichtigte Beurteilung der Finanzkraftmessung stellt sich nun die Frage, welche Zwecke dem kommunalen Finanzausgleich unterstellt und anhand welcher Kriterien die verwendeten Meßkonzepte überprüft werden sollen, da grundlegende Ziele und Maßstäbe des theoretisch behandelten kommunalen Finanzausgleichs im praktizierten Verfahren offenbar wenig gelten.

Weil sich die angestrebte objektive Bestimmung der Finanzkraft der Gemeinden ohnehin nicht in das Verteilungskonzept einfügt - weder unter vertikalem noch unter horizontalem Aspekt, da die Verteilungskriterien nicht exakt bestimmt sind - wird zunächst von den in der Literatur genannten Kriterien ausgegangen. Danach hat die Finanzkraftmessung die Kriterien der "Vollständigkeit, Vergleichbarkeit, Unmanipulierbarkeit, zeitlichen Kontinuität"20) zu erfüllen; als Zwecke der Messung werden vertikale und horizontale Angleichung von Finanzbedarf und Finanzkraft vorgegeben.

Ausgangspunkt der Überlegungen ist dabei das Ziel, die zu ermittelnden Einnahmemöglichkeiten dem Finanzbedarf gegenüberzustellen. Damit wird der Finanzkraftbegriff, wie beschrieben, durch den Bedarfsbegriff festgelegt: Als Finanzkraft einer Gemeinde müssen alle jene Mittel gelten, die zur Dekkung des Bedarfs aufgebracht und verwendet werden können. Das bedeutet umgekehrt, daß Mittel, die für solche Zwecke verwendet werden (müssen), die nicht als Bedarf definiert sind oder nicht in die Berechnung des Finanzbe-

20) Gläser, Martin, Die staatlichen Finanzzuweisungen an die Gemeinden - Gestaltungskriterien, Effektivität, Reform - a.a.O., S. 155. 
darfs eingehen, auch bei der Messung des Einnahmepotentials unberücksichtigt bleiben (müssen).

Hier zeigt sich, daß die Neukonzeption der Finanzkraftmessung auf einen stimmigen Bedarfsbegriff angewiesen ist. Angesichts der Kritik, der der Gesamtansatz und insbesondere der Hauptansatz als Indikatoren des Finanzbedarfs ausgesetzt sind, kann die konkrete Konzeption der Bedarfsmessung in der Bundesrepublik Deutschland im folgenden nicht zur Grundlage der Überlegungen gemacht werden. Allerdings soll das Konzept der pauschalen, auf den Einwohner bezogenen Bestimmung des Bedarfs hier noch nicht grundsätzlich in Frage gestellt werden. ${ }^{21)}$ Es wird zunächst davon ausgegangen, daß ein solches Konzept den Zielen des Finanzausgleichs angemessen sei und die Meßzahl des kommunalen Finanzbedarfs sowohl den Ausgabenbedarf für pflichtige Gemeindeausgaben als auch einen hinreichenden Spielraum zur freiwilligen Erfüllung kommunaler Bedarfe einschließt. Es wird also in Anlehnung an die praktizierte Bedarfsmessung ein Gesamtbedarfskonzept unterstellt, das nicht vollständig bestimmt, welche Aufgaben und Ausgaben in gemeindliche Kompetenz fallen. Soweit es sich bei den kommunalen Aktivitäten und den daraus resultierenden Ausgaben um öffentliche Aufgaben mit räumlich begrenztem Nutzenradius ${ }^{22)}$ als typischer gemeindlicher Aufgabe handelt, wird durch den Bedarfsbegriff also keine Unterscheidung nach der Bedarfsrelevanz getroffen.

Anhand dieser Vorauswahl soll überprüft werden, inwieweit die in den Bundesländern verwendeten Finanzkraftindikatoren systemadäquat sind. Dazu ist zum einen nach den einzubeziehenden Einnahmearten, darüber hinaus aber nach dem "richtigen" Niveau der berücksichtigten Komponenten zu fragen.

21) Im einzelnen wird darauf noch einmal im 4. Kapitel einzugehen sein.

22) Vgl. dazu Zimmermann, Horst, Allgemeine Probleme und Methoden des Finanzausgleichs, a.a.O., S. $11 \mathrm{ff}$. 


\section{Die Verfahren der Finanzkraftmessung in den Bundesländern und ihre Beurteilung}

I. Anmerkungen zur Auswahl der im Finanzausgleich und der Finanzkraftmessung berücksichtigten Einnahmearten

a. Konzepte zur Ermittlung und zum Ausgleich gemeindlicher Finanzierungsmöglichkeiten in den Bundesländern

Ausgangspunkt zur Messung gemeindlicher Finanzierungsmöglichkeiten ist in allen Ländern die Steuerkraftmeßzahl. Deren Ermittlung sowie die Einbettung in das Ausgleichsverfahren unterscheidet sich jedoch länderweise zum Teil erheblich. Innerhalb der Länder wird darüber hinaus in der Regel zwischen den Gemeinden und Gemeindeverbänden unterschieden, in einigen Ländern werden die Gemeinden selbst noch differenziert behandelt - z.B. in Nordrhein-Westfalen und in Rheinland-Pfalz.

Übersicht 1 listet die angewendeten Meßkonzepte in den Bundesländern auf. Danach sind vor allem zwei Verfahren der Messung und des Ausgleichs mit Schlüsselzuweisungen zu unterscheiden: Einstufige Verfahren, die aus der Gegenüberstellung von Steuer- bzw. Finanzkraftmeßzahl (in Schleswig-Holstein) und Bedarfsmeßzahl den Schlüssel zur Verteilung der Zuweisungsmasse errechnen, sowie zweistufige Verfahren, die zunächst unterdurchschnittliche Steuerkraft ohne Berücksichtigung des Bedarfs ausgleichen und dann erst eine Gegenüberstellung von Finanzkraft und Finanzbedarf zur weiteren Aufteilung der Schlüsselmasse vornehmen. In dieser Form wurde 1984 im Saarland und in Rheinland-Pfalz vorgegangen. Gemeinden, deren Steuerkraft weniger als $70 \%$ (Saarland) bzw. $75 \%$ (Rheinland-Pfalz) beträgt, erhalten zunächst den Unterschiedsbetrag als Schlüsselzuweisung A. Anschließend wird aus Steuerkraftmeßzahl und erhaltener Schlüsselzuweisung A die Finanzkraftmeßzahl gebildet und mit der Bedarfsmeßzahl verglichen.

Mit den aufgeführten Verfahren werden verschiedene Konzepte des horizontalen Ausgleichs verfolgt, nicht aber die vertikale Kontrolle der Aufgaben-, Ausgaben- und Einnahmenzuweisung. Dies wird schon daran deutlich, daß die Ermittlung der Steuer- und Finanzkraftmessung von vornherein nur 
auf die Aufteilung der Schlüsselmasse in den Ländern abzielt. Andere Zwecke der Messung werden in den jeweiligen Gesetzen nicht angestrebt.

\section{Übersicht 1: $\quad$ Verfahren der Messung kommunaler Finanzkraft (ohne Stadtstaaten), 1984}

\begin{tabular}{|c|c|c|}
\hline Land & Gebietseinheit & Meßkonzept \\
\hline \multirow[t]{2}{*}{$\begin{array}{l}\text { Schleswig- } \\
\text { Holstein }\end{array}$} & $\begin{array}{l}\text { kreisangehörige } \\
\text { Gemeinden }\end{array}$ & $\begin{array}{l}\text { Summe der Steuerkraft- } \\
\text { zahlen = Steuerkraftmeß- } \\
\text { zahl }\end{array}$ \\
\hline & $\begin{array}{l}\text { Kreise und } \\
\text { kreisfreie } \\
\text { Städte }\end{array}$ & $\begin{array}{l}2 / 3 \text { der Steuerkraft- } \\
\text { zahlen } \\
+2 / 3 \text { der allgemeinen } \\
\text { Gemeindeschlüsselzuwei- } \\
\text { sungen } \\
=\text { Finanzkraftmeßzahl }\end{array}$ \\
\hline \multirow[t]{2}{*}{$\begin{array}{l}\text { Nieder- } \\
\text { sachsen }\end{array}$} & $\begin{array}{l}\text { kreisangehörige } \\
\text { Gemeinden und } \\
\text { kreisfreie } \\
\text { Städte }\end{array}$ & $\begin{array}{l}\text { Summe der Steuerkraft- } \\
\text { zahlen } \\
=\text { Steuerkraftmeßzahl }\end{array}$ \\
\hline & Landkreise & $\begin{array}{l}30 \% \text { der Steuerkraftmeß- } \\
\text { zahlen der kreisangehöri- } \\
\text { gen Gemeinden } \\
+30 \% \text { von } 95 \% \text { der all- } \\
\text { gemeinen Schlüsselzuwei- } \\
\text { sungen der kreisangehö- } \\
\text { rigen Gemeinden } \\
=\text { Umlagekraftmeßzahl }\end{array}$ \\
\hline \multirow[t]{2}{*}{$\begin{array}{l}\text { Nordrhein- } \\
\text { Westfalen }\end{array}$} & $\begin{array}{l}\text { kreisangehörige } \\
\text { Gemeinden und } \\
\text { kreisfreie } \\
\text { Städte }\end{array}$ & $\begin{array}{l}\text { Summe der Steuerkraft- } \\
\text { zahlen } \\
\text { = Steuerkraftmeßzahl }\end{array}$ \\
\hline & Kreise & $\begin{array}{l}30 \% \text { der Umlagegrundlagen } \\
=\text { Umlagekraftmeßzahl }\end{array}$ \\
\hline \multirow[t]{2}{*}{ Hessen } & $\begin{array}{l}\text { kreisangehörige } \\
\text { Gemeinden und } \\
\text { kreisfreie } \\
\text { Städte }\end{array}$ & $\begin{array}{l}\text { Summe der Steuerkraft- } \\
\text { zahlen } \\
=\text { Steuerkraftmeßzahl }\end{array}$ \\
\hline & Kreise & $\begin{array}{l}32 \% \text { der Umlagegrundlagen } \\
=\text { Umlagekraftmeßzahl }\end{array}$ \\
\hline
\end{tabular}




\section{noch Übersicht 1:}

Land

Saarland

Rheinland-

Pfalz
Gebietseinheit

Gemeinden

Gemeinde-

verbände

Ortsgemeinden, verbandsfreie Gemeinden, große kreisangehörige Städte, kreisfreie Städte

Verbandsgemeinden, verbandsfreie Gemeinden und große kreisangehörige Städte

Landkreise

kreisfreie Städte
Meßkonzept

für Schlüsselzuweisungen

A:

Summe der Steuerkraftzahlen

$=$ SteuerkraftmeBzahl

für Schlüsselzuweisungen

B:

Steuerkraftmeßzahl

+ Schlüsselzuweisungen A

= Finanzkraftmeßzahl

Umlagegrundlagen vervielfältigt mit landesdurchschnittlichen Umlagesätzen = UmlagekraftmeBzahl

für Schlüsselzuweisungen

A:

Summe der Steuerkraftzahlen

= SteuerkraftmeBzahl

für Schlüsselzuweisungen

B:

$30 \%$ der SteuerkraftmeB-

zahl

$+30 \%$ der Schlüsselzu-

weisungen $\mathbf{A}$

= Finanzkraftmeßzahl

$70 \%$ der Steuerkraftmeß-

zahlen

$+70 \%$ der Schlüsselzu-

weisungen $\mathrm{A}$

+ Aufkommen der Grunder-

werbsteuer

= Finanzkraftmeßzahl

$100 \%$ der SteuerkraftmeBzahl

$+100 \%$ der Schlüsselzuweisungen $\mathbf{A}$

+ Aufkommen der Grunderwerbsteuer

= Finanzkraftmeßzahl 
noch Übersicht 1:

\begin{tabular}{|c|c|c|}
\hline Land & Gebietseinheit & Meßkonzept \\
\hline \multirow[t]{2}{*}{$\begin{array}{l}\text { Baden- } \\
\text { Württem- } \\
\text { berg }\end{array}$} & Gemeinden & $\begin{array}{l}\text { Summe der Steuerkraft- } \\
\text { zahlen } \\
+ \text { Schlüsselzuweisungen } \\
\text { nach Einwohnerzahl } \\
=\text { Steuerkraftmeßzahl }\end{array}$ \\
\hline & Landkreise & $\begin{array}{l}\text { landesdurchschnittliche } \\
\text { Umlagesätze x Kreisumlage } \\
\text { + Grunderwerbsteuer } \\
\text { + Zuschlag zur Grund- } \\
\text { erwerbsteuer } \\
\text { = Steuerkraftmeßzahl }\end{array}$ \\
\hline Bayern & $\begin{array}{l}\text { Gemeinden } \\
\text { und } \\
\text { Landkreise }\end{array}$ & $\begin{array}{l}\text { Summe der Steuerkraft- } \\
\text { zahlen } \\
=\text { Steuerkraftmeßzahl }\end{array}$ \\
\hline
\end{tabular}

Quellen: Finanzausgleichsgesetze der Länder 1984

Eine vertikale Betrachtung wäre anhand der Steuerkraftmeßzahlen aber ohnehin nicht durchführbar, da nur ein Teil der gemeindlichen Einnahmequellen berücksichtigt wird. Die Steuerkraftmeßzahl setzt sich in der Regel aus den Steuerkraftmeßzahlen der Grundsteuer A und B, der Gewerbesteuer nach Ertrag und Kapital sowie des gemeindlichen Einkommensteueranteils zusammen. Die in Rheinland-Pfalz zur Aufteilung der Schlüsselzuweisungen B verwendete Finanzkraftmeßzahl bezieht neben den Schlüsselzuweisungen A in kreisfreien Städten darüber hinaus das Aufkommen aus der Grunderwerbsteuer ein. Eine weitere Besonderheit enthält das FAG-Gesetz für BadenWürttemberg: hier werden vorab gewährte einheitliche Schlüsselzuweisungen nach der Einwohnerzahl in die Steuerkraftmeßzahl eingerechnet.

In der Mehrzahl der Bundesländer bleiben damit die "Bagatellsteuern" der Gemeinden unberücksichtigt, nichtsteuerliche eigene Einnahmemöglichkeiten werden überall vernachlässigt. 
b. Argumente für eine umfassendere Messung der gemeindlichen Finanzkraft

Die Verwendung von Steuerkraftmeßzahlen als Maß für die gemeindlichen Einnahmemöglichkeiten ist umstritten und soll deshalb anschließend genauer betrachtet werden. Dabei ist - entsprechend den in Abschnitt B genannten Anforderungen - nach den Zwecken des Finanzausgleichs zu differenzieren.

Als Maßstab für den vertikalen Finanzausgleich und als dem Finanzbedarf gegenüberzustellende Größe erscheint die Steuerkraftmeßzahl von vornherein ungeeignet, weil sie nur einen, wenn auch wichtigen Bestandteil der kommunalen Einnahmequellen erfaßt. Zwar werden in der Literatur verschiedene Argumente zur Verteidigung der Steuerkraftmessung vorgebracht; sie erscheinen jedoch nicht für alle betreffenden Einnahmequellen überzeugend. Sie werden im folgenden näher erläutert.

Für die Zwecke des horizontalen Ausgleichs könnte unter bestimmten Bedingungen ein relativer Maßstab ausreichen; hier ist die Steuerkraft also nicht von vornherein abzulehnen. Vorausgesetzt, daß sie selbst "richtig" gemessen worden ist, könnte argumentiert werden, daß sie die übrigen Einnahmemöglichkeiten "richtig" mitabbildet. Allerdings sind auch dieser Argumentation gegenüber Zweifel angebracht. Die Ausgrenzung der nicht einbezogenen Einnahmequellen wird deshalb auch unter dem Aspekt des horizontalen Ausgleichs noch einmal überprüft.

Geht man zunächst wieder von der in Abschnitt B vorgenommenen Eingrenzung des Gegenstandes einer Finanzkraftmessung aus, nach der jene Finanzierungsmöglichkeiten erfaßt werden sollen, die zur Deckung des Finanzbedarfs dienen können, so ist festzuhalten, daß über die Erfassung des Bedarfs keine der kommunalen Einnahmequellen von vornherein als irrelevant für die Finanzkraftmessung ausgeschlossen wird, weil der Finanzbedarf - wie oben bereits beschrieben - pauschal, d.h. ohne direkten Bezug auf bestimmte Aufgaben und Aufgabenerfordernisse ermittelt wird; insofern kann nicht argumentiert werden, daß einige kommunale Aktivitäten nicht als Bedarf im Sinne "notwendiger Aufgabenerfüllung" anzusehen seien und die zur Finanzierung herangezogenen Einnahmequellen dementsprechend auch bei der Finanzkraftmessung nicht berücksichtigt werden dürften. 
Die weitere Betrachtung hat deshalb von den einzelnen Einnahmequellen auszugehen und nach Argumenten für ihren Ausschluß aus der Finanzkraftmessung und möglichen Wirkungen - gemessen an den oben genannten Anforderungen - eines Ausschlusses zu fragen.

In Tabelle 1 sind die wichtigsten Einnahmequellen der Gemeinden einzeln aufgeführt. Um die Bedeutung abschätzen zu können, die ihnen bei der gegenwärtigen Finanzierungsstruktur der Gemeinden zukommt, werden zusätzlich Aufkommen und Haushaltsanteile aus dem Jahr 1984 ausgewiesen. Aufgrund verschiedener Einflüsse kann die Finanzierungsstruktur einzelner Gemeinden natürlich stark von der durchschnittlichen abweichen; überdies ersetzen die Einnahmezahlen die Erfassung potentieller Einnahmen nicht. Allerdings können die aufgeführten Zahlen einen Anhaltspunkt dafür abgeben, welche Dimension mögliche Verzerrungen in der Finanzkraftmessung aufgrund der Vernachlässigung der jeweiligen Einnahmequelle haben können.

\section{Tabelle 1: Die kommunalen Einnahmequellen und ihre Erträge, Bundesrepublik Deutschland 1984}

Einnahmeart

Aufkommen

1984 in

Mrd. DM

Anteil an den

Gesamtein-

nahmen in \%

\section{Verwaltungshaushalt}

1. Steuern und

steuerähnliche

Einnahmen

52,64

31,0

darunter:

Gemeindeanteil

an der Einkommen-

steuer

22,50

Gewerbesteuer (netto)

22,21

Grundsteuer A

Grundsteuer B

2. Zahlungen von Bund, Land. LAF. ERP

3. Sonstige Einnahmen aus Verwaltung und Betrieb 
darunter:

Einnahmeq aus

Gebühren $^{1)} \quad 32,63$

19,2

Summe I $\quad 134,98$

79,5

II. Vermögenshaushalt

1. Veräußerung von

Vermögen

2. Beiträge und ähn-

liche Entgelte

3. Inv. Zahlungen von

Bund, LAF, ERP, Land

10,72

6,3

Summe II

18,93

11,1

III. Besondere Finanzierungsvorgänge

1. Schuldaufnahme am Kreditmarkt

10,76

2. Entnahme aus

Rücklagen

3,00

1,7

Summe III 13,76

8,1

Bereinigte Einnahmen

169,64

100,0

1) Einschl. zweckgebundene Abgaben und Pflegesatzeinnahmen der Krankenhäuser

Quelle: Gemeindefinanzbericht 1987, in: Der Städtetag 2/1987, S. 93 und 95 sowie eigene Berechnungen.

Wichtigste Finanzierungsquelle im Verwaltungshaushalt der Gemeinden sind die Steuern. Als allgemeine Deckungsmittel sind sie von den Gemeinden frei zur Deckung des anfallenden Bedarfs verwendbar und müssen schon deshalb 
vollständig in die Erfassung der Finanzierungsmöglichkeiten eingehen. Ihre qualitative Vorrangstellung wird auch unter staatsrechtlichen Gesichtspunkten betont: "Die Grundsatzentscheidung des Grundgesetzes für den aus Steueraufkommen finanzierten Steuerstaat und gegen den aus staatseigener Erwerbswirtschaft finanzierten Unternehmerstaat verweist jedoch auch die Gemeinden auf die Besteuerung als vorrangige Einnahmequelle"23). Ob daraus eine Rechtfertigung abgeleitet werden kann, allein die Steuerquellen der Gemeinden in die Finanzkraftmessung einzubeziehen, erscheint jedoch zweifelhaft. Zum ersten werden zur Zeit im Gemeindedurchschnitt nur etwa ein Drittel aller kommunalen Ausgaben über Steuereinnahmen finanziert. Der Anteil an den eigenen Einnahmen fällt zwar wesentlich höher aus, aber auch hier bilden die Steuern nur einen Ausschnitt ab. Überdies nennen die Gemeindeordnungen im Gegensatz zur oben genannten Auffassung die Steuern erst an dritter Stelle; vorrangige Finanzierungsquelle sollen vielmehr spezielle Entgelte sein; Steuern nur, "soweit die sonstigen Einnahmen nicht ausreichen"24).

Unter vertikalem Aspekt erscheint die Steuerkraft-Konzeption daher bedenklich.

Zum zweiten schwankt die Ergiebigkeit der nichtsteuerlichen Einnahmen zwischen Gemeinden erheblich, weshalb für eine umfassendere Messung der Finanzkraft auch unter horizontalem Aspekt plädiert wird ${ }^{25}$.

Aber selbst die gemeindlichen Steuerquellen werden nicht vollständig einbezogen; in der Mehrzahl der Länder gehen nur die Gewerbesteuer, die Grundsteuern sowie der gemeindliche Einkommensteueranteil in die Steuerkraftmeßzahl ein.

Außer aus den aufgeführten Steuern beziehen die Gemeinden in einigen Bundesländern Einnahmen aus der landesgesetzlich festgelegten Grunderwerbsteuer sowie in allen Bundesländern aus örtlichen Aufwand- und Ver-

23) Kirchhof. Paul, Die kommunale Finanzhoheit, in: Handbuch der kommunalen Wissenschaft und Praxis. Bd. 6. Kommunale Finanzen. a.a.O.. S. 4.

24) So etwa die Gemeindeordnung in Nordrhein-Westfalen $\$ 63$ (2).

25) Vgl. etwa Gläser, Martin, Die staatlichen Finanzzuweisungen an die Gemeinden, a.a.O., S. 291: Kops. Manfred. Die regionalen Verteilungswirkungen des kommunalen Finanzausgleichs, a.a.O., S. 127 f.: Limbert-Michaelis. Helga. Neuere Entwicklung in der Theorie des Finanzausgleichs. Diss., Reinheim 1973, S. 28. 
brauchsteuern. Die Einnahmemöglichkeiten aus diesen Steuern wären prinzipiell ebenfalls in die Messung der Einnahmemöglichkeiten einzubeziehen ${ }^{26)}$.

Da diese Steuern auf der Gemeindeebene insgesamt ein recht geringes Aufkommen erbringen, sprechen Praktikabilitätsüberlegungen dafür, sie bei der Messung außer acht zu lassen. Unter horizontalem Aspekt erscheint dies insbesondere bezogen auf die Grunderwerbsteuer - jedoch als bedenklich, da die Einnahmemöglichkeiten ebenso oder noch stärker als die tatsächlichen Einnahmen im interkommunalen Vergleich beachtliche Unterschiede aufweisen können ${ }^{27)}$.

Der von Deubel für Nordrhein-Westfalen geltend gemachte Einwand, daß eine Einbeziehung der Grunderwerbsteuer, die den kreisfreien Städten und Kreisen zusteht, nicht sinnvoll sei, weil auf die beiden Gruppen keine gemeinsamen, sondern getrennte, vorneweg festgelegte Schlüsselmassen verteilt werden ${ }^{28)}$, erscheint nicht einsichtig, da es mit Blick auf die Zuweisungen entscheidend auf die Verteilung der Finanzierungsmöglichkeiten innerhalb der jeweiligen Gruppen ankommt.

Gegen die Berücksichtigung der in Tabelle 1 ausgewiesenen Übertragungen - im Verwaltungs- und Vermögenshaushalt zusammen machten sie 1984 fast $25 \%$ der gemeindlichen Einnahmen aus - spricht zunächst, daß es sich dabei nicht um gemeindeeigene Einnahmen handelt.

26) Kirchhof wendet dagegen ein. daß über das $\mathrm{Ob}$ und Wie der örtlichen Verbrauch- und Aufwandsteuern autonom von den Gemeinden entschieden werde; die Wirkungen solcher autonomer Entscheidungen sollten aber grundsätzlich nur die jeweilige Gemeinde selbst treffen. Die Einbeziehung der genannten Steuern in die Finanzkraftmessung würde über den Einfluß auf die Höhe der Schlüsselzuweisungen die Wirkungen über die Gemeindegrenzen hinaustragen und sei daher abzulehnen. Diese Argumentation ist durchaus plausibel und wird im 4. Kapitel noch einmal aufgenommen. Innerhalb des praktizierten Finanzausgleichssystems (im engeren Sinne) ist ihr jedoch nicht beizupflichten. Ausgehend von der Feststellung. daß auch die Einnahmen aus autonom zu nutzenden Quellen zur Dekkung des Bedarfs - der ebenfalls autonom entscheidbare Komponenten umschließt - verwendet werden können. ist dieser Auffassung zunächst einmal zu widersprechen. Vgl. dazu Kirchhof, Paul. Der Finanzausgleich als Grundlage kommunaler Selbstverwaltung, a.a.O., S. 717.

27) So stellte Grimme etwa für Bayern fest. daß "die Einnahmen aus der Grunderwerbsteuer schwerpunktmäßig den Großstädten in den Verdichtungsräumen zufließen und hinsichtlich ihres finanziellen Gewichts die Schlüsselzuweisungen an diese Städte um insgesamt mehr als das doppelte übersteigen." Vgl. Grimme. Leonhard. Neuberechnung der Steuerkraft im kommunalen Finanzausgleich - dargestellt am Beispiel des Landes Bayern, in: Räumliche Aspekte des kommunalen Finanzausgleichs. a.a.O.. S. 170.

28) Deubel, Ingolf, Der kommunale Finanzausgleich in Nordrhein-Westfalen - Eine ökonomische und statistische Analyse. Köln u.a. 1984. S. 120. 
In Tabelle 2 sind die Zuweisungen für 1983 noch einmal aufgegliedert und dabei zeigt sich, daß hier Einnahmen ganz unterschiedlicher Qualität zusammengefaßt werden, für die die Frage einer Einbeziehung in die Erfassung der kommunalen Finanzkraft nur jeweils gesondert gestellt werden kann. Kriterien für die Antwort sind zum einen die Maßstäbe und zum anderen die Zwecke der Zuweisungsvergabe.

\section{Tabelle 2: Staatliche Zuweisungen an die Gemeinden, Bundesrepublik Deutschland 1983, in Mrd. DM}

Zweckgebundene Zuweisungen

Erstattungen v. Ausgaben

für staatliche Aufgaben

Zuweisungen für laufende

Quelle: $\quad$ Finanzbericht 1987, hrsg. vom Bundesministerium der Finanzen, Bonn 1986, S. 120. 
Jene Zuweisungen, die nach Maßgabe des Vergleichs von Steuerkraft oder in einem umfassenderen Konzept von Einnahme- bzw. Finanzkraft - und Finanzbedarf vergeben werden, sind selbstverständlich nicht in die Erfassung einzubeziehen, sie sind vielmehr erst Ergebnis des anschließenden Vergleichs mit dem Finanzbedarf. Damit ist jedoch nur ein Teil der in Tabelle 2 ausgewiesenen allgemeinen Zuweisungen und auch nur ein Teil der hier dominierenden Schlüsselzuweisungen angesprochen. Sofern Schlüsselzuweisungen nach anderen als den genannten Kriterien, etwa pauschal nach der Einwohnerzahl, vergeben werden, sind sie bei der zusätzlichen Vergabe von $\mathrm{Zu}$ weisungen nach Maßgabe von Finanzbedarf und -kraft in die Erfassung der Finanzkraft einzubeziehen ${ }^{29}$. Genauso geht der Finanzausgleich in BadenWürttemberg vor.

Beim Kriterium des Zwecks der Vergabe von Zuweisungen geht es vor allem um die Unterscheidung zwischen gemeindlichem Verwendungszweck also insbesondere für Aufgaben mit begrenztem Nutzenradius (s.o.) - und darüber hinausgehenden staatlichen Zielsetzungen. Nach dieser Unterscheidung $^{30)}$ - wäre sie in der Realität durchgängig nachvollziehbar - ist zunächst der Auffassung zuzustimmen, daß jene Zuweisungen, die als Kostenbeteiligung des Staates an speziellen übergemeindlichen Aufgaben gelten können und nicht aus kommunalem Bedarf gerechtfertigt werden, nicht in die Erfassung der gemeindlichen Finanzkraft einzubeziehen sind ${ }^{31)}$. Ein Teil der Zweckzuweisungen - und zwar insbesondere die "Erstattungen für staatliche Aufgaben", aber auch andere Zweckzuweisungen - könnte damit aus der Messung ausgegrenzt werden.

Allerdings wird die Unterscheidung zwischen Zuweisungen für kommunale und Zuweisungen für staatliche Zwecke durch die herrschende Zuweisungspraxis erschwert ${ }^{32}$. Dies ist am deutlichsten für die Investitionszuweisungen erkennbar; außer mit Infrastrukturbedarfen werden die geförderten Investitionen zum Beispiel mit konjunkturellen und Wachstumszielen begrün-

29) So auch Kops. Manfred, Die regionalen Verteilungswirkungen des kommunalen Finanzausgleichs, a.a.O., S. 128.

30) Ursprünglich von Popitz. Johannes. Der künftige Finanzausgleich zwischen Reich. Ländern und Gemeinden. a.a.O.. S. 200 ff.

31) So Schmölders. Günter. "Finanzstarke" und "finanzschwache" Gemeinden, a.a.O., S. 37.

32) Ebenda. 
det - hier decken sich zwar nicht unbedingt die Zwecke, wohl aber die Instrumente. Eine Zuordnung ist deshalb kaum möglich ${ }^{33)}$.

Für die Erstattungen stellt sich hingegen das umgekehrte Problem: Aus der Qualität der erfüllten Aufgaben ließe sich klar ableiten, daß sie nicht in die Finanzkraftmessung aufzunehmen wären. Grundsätzlich wird die Auffassung vertreten, daß die Gemeinden in diesem Bereich nur als Vermittler staatlicher Aktivitäten fungieren (sollen); weder die erfüllten Aufgaben werden dem kommunalen Bedarf zugerechnet - obwohl eine explizite Ausgrenzung aus der Bedarfsmessung nicht vorgenommen wird - noch werden die entsprechenden Finanzströme der gemeindlichen Finanzierung zugeordnet, sondern wie "durchlaufende Posten" außerhalb kommunaler Zuständigkeit behandelt. Praktisch ist aber auch diese Abgrenzung kaum haltbar, da die Mittelzuweisung durch den Staat häufig nicht nach Maßgabe der entstandenen Kosten bei der Aufgabenerfüllung erfolgt, sondern pauschaliert, etwa nach der Einwohnerzahl oder anderen Kriterien ${ }^{34)}$.

Die Deckung der aus Aufgaben des übertragenen Wirkungskreises entstandenen Kosten über den allgemeinen Finanzausgleich wird von der Rechtsprechung gebilligt, allerdings ist es in Folge dieses Verfahrens nach Auffassung einiger Autoren zu einer deutlichen Unterfinanzierung der Aufgabenerfüllung für den Staat gekommen ${ }^{35)}$; die Gemeinden müssen zur Finanzierung zusätzliche allgemeine Deckungsmittel aufwenden. Dieser $\mathrm{Zu}$ schuß wäre von den gemeindlichen Finanzierungsmöglichkeiten abzuziehen. Allerdings können dann wiederum Abgrenzungsprobleme auftreten, wenn die Gemeinden eine über staatliche Anforderungen hinausgehende Aufgabenerfüllung betreiben. Als Alternative bietet sich jedoch nur die vollständige Einbeziehung der Erstattungen in die Erfassung der gemeindlichen Finanzkraft an. Beide Alternativen sind sowohl unter vertikalem als auch besonders unter horizontalem Aspekt - bestimmte Merkmalsausprägungen in den einzelnen Gemeinden bestimmen deren Kostenbelastung durchaus mit - unbefriedigend.

33) Metz schlägt daher eine gesamthafte Einbeziehung der speziellen Zuweisungen mit Ausnahme der Erstattungen vor. Metz, Hans-Günter, Der Ausgleich von Finanzkraft und Bedarfsunterschieden im föderalistischen Staat, a.a.O., S. 97 f. und S. 100.

34) Zu den Regelungen in Nordrhein-Westfalen vgl. etwa Deubel, Ingolf, Der kommunale Finanzausgleich in Nordrhein-Westfalen. a.a.O.. S. $171 \mathrm{ff}$.

35) So Schmidt-Jortzig, Edzard, Probleme der kommunalen Fremdverwaltung, a.a.O., S. 401. 
Die Praxis des Finanzausgleichs bietet jedoch kein Kriterium, nach dem eine befriedigende Antwort gefunden werden könnte.

Nach den Steuern sind die Einnahmen aus Verwaltung und Betrieb, einschließlich der Erwerbseinkünfte, die zur Zeit ergiebigste Einnahmequelle im Verwaltungshaushalt, die Beiträge spielen im Vermögenshaushalt hingegen eine untergeordnete Rolle (vgl. Tabelle 1). Über die Einbeziehung dieser auch unter der Bezeichnung "Entgelte" zusammengefaßten Einnahmen wird uneinheitlich geurteilt. Allein die Berücksichtigung der Gewinne aus wirtschaftlicher Betätigung der Gemeinden wird weitgehend für richtig gehalten.

Demgegenüber wird die Einbeziehung der Gebühren und Beiträge von einigen Autoren mit dem Hinweis auf das Äquivalenzprinzip abgelehnt; danach erbringen diese Einnahmen "keinen Wertzuwachs" und erweitern die Finanzkraft der Gemeinde nicht ${ }^{36}$, sondern neutralisieren nur eine Leistung bzw. ein Leistungsangebot der Gemeinden ${ }^{37}$ ). Abgesehen davon, daß zwischen den typischen Gemeindesteuern, den Beiträgen und den Gebühren nur graduelle Unterschiede in der Enge der Äquivalenzbeziehung bestehen ${ }^{38)}$ - sie können von individueller kosten- oder marktmäßiger Äquivalenz bis hin zur gruppenmäßigen oder lokalen, nicht mehr individuell zurechenbaren Äquivalenz reichen -, zielt das Argument auch am Zweck der Messung vorbei: Nicht die Erweiterung des finanziellen Spielraums, sondern die zur Bedarfsdeckung verfügbaren Finanzmittel sind Gegenstand der Messung. Ausgehend vom pauschalierenden Bedarfsbegriff gehören die Entgelte (brutto) genau wie die Steuern zu diesen zur Bedarfsdeckung verwendbaren Finanzmitteln. Diese Auffassung wird durch den Tatbestand gestützt, daß die Gemeindeordnungen die Entgelte als vorrangige gemeindliche Einnahmequelle noch vor den Steuern einstufen ${ }^{39)}$.

Unter vertikalem Aspekt würde eine Vernachlässigung der Einnahmemöglichkeiten aus Entgelten nur für einen Sonderfall keine verzerrten Ergeb-

36) Kirchhof, Paul, Der Finanzausgleich als Grundlage kommunaler Selbstverwaltung. a.a.O.. S. 717.

37) Derselbe, Die kommunale Finanzhoheit, a.a.O., S. 25; ähnlich äußert sich Metz, a.a.O., S. 98.

38) Bohley bezeichnet Gebühren und Beiträge sogar nur als Unterfall der Besteuerung; Bohley, Peter, Gebühren und Beiträge - ihre wirtschaftspolitischen Funktionen und ihr Platz im System der öffentlichen Einnahmen -, Tübingen 1977, S. 81 f.

39) Vgl. GO NW \$63(2). 
nisse liefern; dann nämlich, wenn entgeltfinanzierte Leistungen bei der Bedarfsermittlung unberücksichtigt blieben und gleichzeitig für alle diese Leistungen ein Kostendeckungsgrad von genau $100 \%$ erreicht werden könnte. Dann gilt das oben angeführte Neutralisierungsargument, und eine Vernachlässigung der Entgelthaushalte auf der Bedarfs- und auf der Finanzkraftseite ist lediglich gleichbedeutend mit einer Bilanzverkürzung. Der Zuschußbedarf bleibt davon unberührt, sofern auf beiden Seiten der Bilanz die gleiche Verkürzung vorgenommen wird. Ein Blick auf die kommunalen Entgelthaushalte in der Bundesrepublik läßt die Annahme, daß in allen Bereichen eine volle Kostendeckung durchsetzbar sei, jedoch als realitätsfern erscheinen.

Horizontale Effekte treten nicht auf, wenn davon ausgegangen werden kann, daß die Steuerkraftmeßzahlen die Einnahmemöglichkeiten aus Entgelten richtig mit abbilden. Als Argument für einen solchen Zusammenhang könnte angeführt werden, daß der Kostendeckungsgrad entgeltfähiger Leistungen die $100 \%$-Marke vielfach nicht erreicht und die Entgelthaushalte deshalb durch allgemeine Deckungsmittel ausgeglichen werden müssen; die Fähigkeit einer Gemeinde, diesen Ausgleich zu leisten, dürfte mit ihrer Steuerkraft zusammenhängen. Diese Argumentation vernachlässigt jedoch den entscheidenden Einfluß der Bedarfsstruktur auf die Möglichkeiten einer Gemeinde, den Finanzbedarf über Entgelte zu decken. Hier können zwischen Gemeinden etwa unterschiedlicher Größenklassen oder Zentralitätsstufe beträchtliche Streuungen auftreten, deren Ausmaß bisher allerdings kaum untersucht worden ist.

Läßt man die "Entgeltpotentiale" außer acht, so werden also die Möglichkeiten der Gemeinden zur Selbstfinanzierung wahrscheinlich verzerrt abgebildet. Bezieht man sie andererseits gesamthaft ein und verwendet als "Bemessungsgrundlage" die Summe der in den jeweiligen Gemeinden angebotenen entgeltfähigen Leistungen, so treten ebenfalls Probleme, wenn auch von anderer Art, auf. Dies sei an einem Beispiel verdeutlicht: Man kann sich etwa eine außerordentlich "reiche" Gemeinde vorstellen, für die genau wie für andere Gemeinden mit ähnlich bedarfswirksamen Merkmalen ein bestimmter Finanzbedarf errechnet worden ist. Man stelle sich nun weiter vor, daß diese reiche Gemeinde bei durchschnittlichen leistungsspezifischen Entgeltsätzen allein aus Entgelten mehr Einnahmen erzielen kann als zur Deckung des errechneten Finanzbedarfs notwendig sind. Da die durchschnittlichen Kosten- 
deckungsgrade unter $100 \%$ liegen, und überdies nicht alle Leistungen entgeltfähig sind, kann das hohe "Entgeltpotential" nur aus einem Angebot resultieren, das ein quantitativ dem Finanzbedarf entsprechendes Angebot bei weitem übersteigt. Die aus diesem überdurchschnittlichen Angebot erzielbaren Einnahmen können dem Finanzbedarf nicht ohne weiteres gegenübergestellt werden; ginge es nur um dessen Deckung, so wäre ein eingeschränktes Angebot für die Zwecke der Finanzkraftmessung zugrunde zu legen. Es sind also durchaus nicht alle möglichen Entgelteinnahmen in die Messung einzubeziehen, da hinter der Bedarfsmessung sehr wohl eine Vorstellung von Quantität und Qualität der Bedarfe und der Bedarfsdeckung steckt. Die damit befaßte Literatur ist von solchen Vorstellungen geprägt, das System des bundesdeutschen Finanzausgleichs mit seiner pauschalierenden Bedarfsfeststellung bietet jedoch kein Kriterium, nach dem ein Teil der entgeltfähigen Leistungen aus der Erfassung ausgegrenzt werden könnte.

Außer den Beiträgen und den Zuweisungen und Zuschüssen wurden bisher noch keine Einnahmen des Vermögenshaushalts betrachtet. $\mathrm{Zu}$ nennen sind hier Einnahmen aus der Veräußerung von Vermögen, interne und externe Schuldaufnahme sowie die Entnahmen aus Rücklagen. Diese Einnahmen weisen die Eigenart auf, daß die Quellen mit jeder daraus erzielten Einnahme schrumpfen: Vermögen können nur einmal veräußert und Rücklagen nur einmal verbraucht werden, die Verschuldungsmöglichkeiten werden - zumindest im Falle sonst unveränderter kommunaler Einnahmepolitik - durch jede Neuverschuldung für die Zukunft eingeschränkt. Insofern können sie nicht mit den laufenden Einnahmen gleichgesetzt werden. Für die Schuldaufnahme tritt als Besonderheit hinzu, daß sie - wenn auch nie gesamthaft - rückzahlbar ist und insofern nur eine vorläufige Einnahme darstellt. Überdies werden die genannten Quellen vor allem aus den Quellen des Verwaltungshaushalts gespeist und sind daher im Falle einer genauen Messung der Einnahmemöglichkeiten des Verwaltungshaushalts schon einmal berücksichtigt worden. Zwar ist insbesondere die Verschuldung inzwischen zu einem durchaus üblichen und - besonders in Großstädten - regelmäßig eingesetzten Instrument der Finanzierung von Ausgaben geworden. Trotzdem sprechen die 
genannten Argumente letztendlich dafür, sie bei der Messung kommunaler Einnahmemöglichkeiten unberücksichtigt zu lassen ${ }^{40}$.

Faßt man die Ergebnisse noch einmal zusammen, so ist festzuhalten, daß die Steuerkraftmessung des kommunalen Finanzausgleich zu eng erscheint, um die zur Bedarfsdeckung verfügbaren Finanzmittel richtig abbilden zu können. Unter vertikalem Aspekt wird die Finanzkraft der Gemeinden zu niedrig ausgewiesen. Auch unter horizontalem Aspekt ist die Vernachlässigung insbesondere der Entgelte, aber auch eines Teils der Zuweisungen, angreifbar: "Da anzunehmen ist, daß die interkommunale Streuung dieser Abgaben bzw. Einkünfte ungleichmäßig ist (...), dürfte sich beim derzeitigen Verfahren gegenüber der vollen Berücksichtigung aller Einnahmen ein mehr oder weniger stark abweichendes Meßergebnis einstellen. Dieses wird sich (tendenziell) dadurch auszeichnen, daß die Gruppe der finanzstärkeren Gemeinden einen Teil ihrer "wahren" Finanzkraft verdecken kann." ${ }^{41)}$

Eine umfassendere Messung der gemeindlichen Finanzkraft wäre daher angebracht. Aus dem Gesamtsystem des praktizierten kommunalen Finanzausgleichs kann eine konsistente Konzeption für eine solche umfassendere Messung jedoch nicht abgeleitet werden.

II. Probleme der Wahl fiktiver Steuer- und Abgabensätze für die Finanzkraftmessung

$\mathrm{Da}$ - wie oben ausgeführt - nicht die tatsächlichen, sondern die potentiellen Einnahmen der Gemeinden Gegenstand der Finanzkraftmessung sind, ist nicht nur zu klären, welche Einnahmearten berücksichtigt werden sollen, sondern auch, in welcher Höhe Steuern und Abgaben, über deren Sätze die Gemeinden mitentscheiden können, in die Berechnung eingehen sollen. Im Finanzausgleich der Bundesrepublik Deutschland tritt dieses Problem nur für die in die Steuerkraftmeßzahl einbezogenen Steuern auf, deshalb wird es anschließend auch nur mit Bezug auf Steuern diskutiert. Würden die Entgelte in die Messung einbezogen, so stellte es sich in ähnlicher Form.

40) Ähnlich argumentiert auch Deubel, Ingolf, Der kommunale Finanzausgleich in NordrheinWestfalen, a.a.O., S. 5.

41) Gläser, Martin, Die staatlichen Finanzzuweisungen an die Gemeinden, a.a.O., S. 291. 
Statt der realisierten Hebesätze werden in allen Bundesländern zur Ermittlung der Steuerkraftmeßzahlen fiktive, nivellierte Hebesätze verwendet. Dieses Vorgehen wird in der Regel damit gerechtfertigt, daß es um die Messung der "Steuerkraft" und nicht der tatsächlichen Einnahmen gehe und den Gemeinden mit der Nivellierung die Möglichkeit genommen werden soll, über besonders niedrige Hebesätze ihre eigenen Steuerquellen zu schonen und sich statt dessen über Schlüsselzuweisungen zu finanzieren ${ }^{42}$.

Damit ist wiederum ein vertikaler und ein horizontaler Aspekt angesprochen: Verhindert werden soll sowohl die Finanzierung der Gemeindeebene insgesamt auf Kosten übergeordneter Gebietskörperschaften als auch die "Sanierung" einiger Gemeinden auf Kosten anderer.

In Übersicht 2 wird aufgezeigt, wie dieses Problem in den Bundesländern zu lösen versucht wird. Mit Ausnahme von Nordrhein-Westfalen setzen die Bundesländer gleiche fiktive Hebesätze für alle Gemeinden fest, die sich allerdings zwischen den Bundesländern deutlich unterscheiden. Auffällig ist außerdem die Tatsache, daß in zwei Bundesländern die Einkommensteuer mit Sätzen unter $100 \%$ in die Steuerkraftmeßzahl eingeht, obwohl die Gemeinden bei der Einkommensteuer derzeit nicht über ein Hebesatzrecht verfügen; hier bedürfte es also gar keines fiktiven Hebesatzes.

Ein Blick auf die Hebesatzpolitik der Gemeinden zeigt, daß auch die fiktiven Hebesätze für Gewerbesteuer und Grundsteuer erstaunlich niedrig angesetzt sind. In Tabelle 3 sind die Realsteuerhebesätze für 1982 ausgewiesen. Der Zwei-Jahres-Abstand zu den für 1984 aufgeführten fiktiven Hebesätzen wurde gewählt, weil sich deren Festsetzung in einigen Ländern an Vorjahreswerten orientiert.

42) Vgl. z.B. Grimme, Leonhard, Neuberechnung der Steuerkraft im kommunalen Finanzausgleich, a.a.O., S. 161 f. und Münstermann, Engelbert und Becker, Hubert, Finanzausgleichsleistungen an Kommunen - Ein Vergleich der Finanzausgleichssysteme in den Bundesländern - DST-Beiträge zur Finanzpolitik, Heft 7, hrsg. vom Deutschen Städtetag, Köln 1978, S. 53. 


\section{Übersicht 2: $\quad$ Fiktive Hebesätze und Berechnungsmodi der Steuerkraftzahlen in den Bundesländern (ohne Stadtstaaten), 1984, in \%}

\begin{tabular}{|c|c|c|c|c|}
\hline Land & $\begin{array}{l}\text { Grund- } \\
\text { steuer } \\
\text { A }\end{array}$ & $\begin{array}{l}\text { Grund- } \\
\text { steuer } \\
\text { B }\end{array}$ & $\begin{array}{l}\text { Gewerbe- } \\
\text { steuer } \\
\text { nach Ertrag } \\
\text { und Kapital }\end{array}$ & $\begin{array}{l}\text { Geme } \\
\text { anteil } \\
\text { der Ei } \\
\text { menst }\end{array}$ \\
\hline $\begin{array}{l}\text { Schleswig- } \\
\text { Holstein }\end{array}$ & $\begin{array}{l}220 \% \text { der } \\
\text { Meßbeträge }\end{array}$ & $\begin{array}{l}240 \% \text { der } \\
\text { Meßbeträge }\end{array}$ & $\begin{array}{l}280 \% \text { der } \\
\text { Meßbeträge } \\
\text { - Meßbeträge } \\
\text { X Umlagever- } \\
\text { vielfältiger }\end{array}$ & $100 \%$ \\
\hline $\begin{array}{l}\text { Nieder- } \\
\text { sachsen }\end{array}$ & $\begin{array}{l}220 \% \text { der } \\
\text { Meßbeträge }\end{array}$ & $\begin{array}{l}220 \% \text { der } \\
\text { Meßbeträge }\end{array}$ & $\begin{array}{l}250 \% \text { von } \\
81 \% \text { der } \\
\text { Meßbeträge }\end{array}$ & $90 \%$ \\
\hline \multicolumn{5}{|l|}{$\begin{array}{l}\text { Nordrhein- } \\
\text { Westfalen }\end{array}$} \\
\hline $\begin{array}{l}\text { Gemeinden } \\
\text { bis } 150.000 \\
\text { Einwohner }\end{array}$ & $\begin{array}{l}135 \% \text { der } \\
\text { Grundbe- } \\
\text { träge }\end{array}$ & $\begin{array}{l}220 \% \text { der } \\
\text { Grundbe- } \\
\text { träge }\end{array}$ & $\begin{array}{l}300 \% \text { der } \\
\text { Grundbeträge } \\
-52 \% \text { der } \\
\text { Grundbeträge }\end{array}$ & $100 \%$ \\
\hline $\begin{array}{l}\text { Gemeinden } \\
\text { mit mehr } \\
\text { als } 150.000 \\
\text { Einwohnern }\end{array}$ & $\begin{array}{l}150 \% \text { der } \\
\text { Grundbe- } \\
\text { träge }\end{array}$ & $\begin{array}{l}250 \% \text { der } \\
\text { Grundbe- } \\
\text { träge }\end{array}$ & $\begin{array}{l}330 \% \text { der } \\
\text { Grundbeträge } \\
-52 \% \text { der } \\
\text { Grundbeträge }\end{array}$ & $100 \%$ \\
\hline Hessen & $\begin{array}{l}175 \% \text { der } \\
\text { Meßbeträge }\end{array}$ & $\begin{array}{l}200 \% \text { der } \\
\text { Meßbeträge } \\
\text { bis zur er- } \\
\text { sten Million } \\
\text { der Meßbeträ- } \\
\text { ge, darüber } \\
260 \%\end{array}$ & $\begin{array}{l}250 \% \text { der } \\
\text { Grundbeträge } \\
\text { - } 300 \% \text { des Um- } \\
\text { lagegrund- } \\
\text { betrags }\end{array}$ & $75 \%$ \\
\hline
\end{tabular}

Saarland

$85 \%$ der jeweiligen ge-

$85 \%$ der gewoge-

$85 \%$ wogenen landesdurchnen landesdurchschnittl. Hebesätze abzügl. des sätze (des zweitvorausgegangenen Jahres) $x$ Grundbetrag für die Umlage maßgeblichen Hebesatzes (im zweitvorausgegangenen Jahr) $x$ Grundbetrag 
noch Übersicht 2:

\begin{tabular}{|c|c|c|c|c|}
\hline Land & $\begin{array}{l}\text { Grund- } \\
\text { steuer } \\
\text { A }\end{array}$ & $\begin{array}{l}\text { Grund- } \\
\text { steuer } \\
\text { B }\end{array}$ & $\begin{array}{l}\text { Gewerbe- } \\
\text { steuer } \\
\text { nach Ertrag } \\
\text { und Kapital }\end{array}$ & $\begin{array}{l}\text { Gemeinde- } \\
\text { anteil an } \\
\text { der Einkom- } \\
\text { mensteuer }\end{array}$ \\
\hline $\begin{array}{l}\text { Rheinland- } \\
\text { Pfalz }\end{array}$ & $\begin{array}{l}240 \% \text { der } \\
\text { Grundzahl }\end{array}$ & $\begin{array}{l}270 \% \text { der } \\
\text { Grundzahl }\end{array}$ & $\begin{array}{l}270 \% \text { der } \\
\text { Grundzahl }\end{array}$ & $100 \%$ \\
\hline $\begin{array}{l}\text { Baden- } \\
\text { Württemberg }\end{array}$ & $\begin{array}{l}195 \% \text { der } \\
\text { Grundbe- } \\
\text { träge }\end{array}$ & $\begin{array}{l}185 \% \text { der } \\
\text { Grundbe- } \\
\text { träge }\end{array}$ & $\begin{array}{l}290 \% \text { der } \\
\text { Grundbe- } \\
\text { träge abzüg- } \\
\text { lich Umlage } \\
\text { des zweit- } \\
\text { vorausge- } \\
\text { gangenen } \\
\text { Jahres }\end{array}$ & $100 \%$ \\
\hline Bayern & $\begin{array}{l}250 \% \text { der } \\
\text { Grundbe- } \\
\text { träge }\end{array}$ & $\begin{array}{l}250 \% \text { der } \\
\text { Grundbe- } \\
\text { träge }\end{array}$ & $\begin{array}{l}300 \% \text { von } \\
731 / 3 \% \\
\text { der Grund- } \\
\text { beträge }\end{array}$ & $\begin{array}{l}65 \% \text {, } \\
\text { wenn der } \\
\text { Einkommen- } \\
\text { steueranteil } \\
\text { um } 50 \% \text { un- } \\
\text { ter dem Lan- } \\
\text { desdurch } \\
\text { schnitt liegt, } \\
\text { sonst } 100 \%\end{array}$ \\
\hline
\end{tabular}

Quellen: Finanzausgleichsgesetze der Länder 1984.

Abgesehen von einer Ausnahme - dem fiktiven Hebesatz der Grundsteuer A in Schleswig-Holstein - liegen die fiktiven Hebesätze in allen Bundesländern mehr oder weniger unter den landesdurchschnittlich realisierten Hebesätzen ${ }^{43)}$.

Dabei tritt zusätzlich noch ein Struktureffekt aufgrund der Ungleichbehandlung der einzelnen Steuern auf. So betrug in Baden-Württemberg der Nivellierungssatz der Grundsteuer A für 1984 nur $59 \%$ des Durchschnittssatzes

43) Münstermann und Becker stellen bereits für 1978 ein ähnliches Ergebnis fest. Vgl. Münstermann, Engelbert und Becker, Hubert, a.a.O., S. 54 f. 
von 1982, während er für die Gewerbesteuer nach Ertrag und Kapital bei etwa $88 \%$ des Durchschnittshebesatzes lag.

Tabelle 3: Gewogene landesdurchschnittliche Realsteuerhebesätze, 1982, in $\%$

Land

Grundsteuer

A

220

270

sachsen

Nordrhein-

Westfalen

Hessen

Saarland

Rheinland-

Pfalz

Baden-

Württemberg

Bayern

Bundesrepublik

Deutschland
296

278

147

251

217

252

277
247

301

312

253

279

281
Grundsteuer

B

Gewerbesteuer
305

337

342

351

386

338

329

356

339

Quelle: Statistisches Jahrbuch für die Bundesrepublik Deutschland, hrsg. vom Statistischen Bundesamt, Stuttgart und Mainz 1984, S. 455.

Diese Praxis wird insofern kritisiert, als zum ersten der Durchschnittshebesatz als "richtiges" Nivellierungsniveau angesehen wird ${ }^{44)}$ und man zum zweiten der Auffassung ist, daß die gemeindlichen Steuerquellen gleich behandelt

44) So z.B. Gläser, Martin, Die staatlichen Finanzzuweisungen an die Gemeinden, a.a.O., S. 165; Ehrlicher, Werner, Kommunaler Finanzausgleich und Raumordnung, a.a.O.. S. 80; Limbert-Michaelis, Helga, Neuere Entwicklungen in der Theorie des Finanzausgleichs, a.a.O.. S. 28. 
werden sollten. Werden die durchschnittlichen Hebesätze als Nivellierungssätze verwendet, so scheint die Gleichbehandlung gesichert; die Einkommensteuer ist dann mit $100 \%$ in Ansatz zu bringen. Sollten jedoch niedrigere Nivellierungshebesätze in die Steuerkraftmeßzahl eingehen, so "müßten sich der Anrechnungsfaktor für die Einkommensteuer und das Verhältnis aus fiktiven zu durchschnittlichen Hebesätzen entsprechen" ${ }^{45)}$.

Geht man jedoch von den durchschnittlichen Hebesätzen als "richtigem" Nivellierungsniveau aus, so sind die verwendeten fiktiven Hebesätze als bewußte Unterbewertung der gemeindlichen Einnahmemöglichkeiten zu verstehen. In vertikaler Richtung hätte diese Unterbewertung eine Bezuschussung der Gemeinden über das erforderliche Maß hinaus zur Folge - dieser Effekt tritt natürlich nur dann ein, wenn der Zuschußbedarf vollständig ausgeglichen wird.

Von größerer praktischer Bedeutung sind die horizontalen Verteilungseffekte. Es kann nachgewiesen werden, daß mit sinkenden nivellierten Hebesätzen jene Gemeinden besonders bevorzugt werden, die über hohe Steuerbemessungsgrundlagen verfügen, weil die ermittelten Steuerkraftunterschiede immer geringer ausfallen. Im Extremfall eines Nivellierungssatzes von 0 sind gar keine Finanzkraftunterschiede zwischen den Gemeinden zu erkennen, sie werden um so höher ausgewiesen, je höher der Nivellierungssatz ist. Zwar werden die Relationen zwischen den abgebildeten Steuerkraftpositionen außer bei einem Satz von 0 - dadurch nicht berührt, die Abstände zwischen den einzelnen Positionen werden jedoch verschieden groß eingeschätzt, und dadurch wird die Aufteilung der Schlüsselmasse beeinflußt. Je niedriger die Nivellierungssätze festgelegt werden, desto stärker fällt die Begünstigung steuerstarker Gemeinden aus ${ }^{46)}$. Hält man die Durchschnittshebesätze für das "richtige" Nivellierungsniveau, so muß die Schlußfolgerung natürlich lauten, daß mit der Wahl niedrigerer fiktiver Hebesätze eine bewußte Besserstellung steuerstarker Gemeinden vorgenommen wird ${ }^{47}$.

45) Deubel. Ingolf, Der kommunale Finanzausgleich in Nordrhein-Westfalen, a.a.O., S. 121.

46) Wie die Verteilungseffekte aufgrund der Wahl des Hebesatzniveaus im einzelnen zustande kommen, wird detailliert bei Postlep vorgeführt. Vgl. Postlep, Rolf-Dieter, Räumliche Effekte der Steuerkraft- und Finanzbedarfsermittlung bei den Schlüsselzuweisungen, a.a.O., S. $221 \mathrm{ff}$.

47) So äußert sich etwa Patzig, Werner, Strukturprobleme des kommunalen Finanzausgleichs. in: Deutsches Verwaltungsblatt, 94. Jg., Heft 13, Juli 1979, S. 477-490, hier S. 490. 
Allerdings ist die häufig in der Literatur geäußerte Präferenz für die Verwendung von Durchschnittshebesätzen ebenfalls angreifbar, und zwar sowohl unter vertikalen als auch unter horizontalen Gesichtspunkten. Unter vertikalem Aspekt impliziert die Verwendung gewichteter durchschnittlicher Hebesätze, daß man die Inanspruchnahme der Steuerquellen auf der Gemeindeebene insgesamt für angemessen hält. Darüber kann jedoch allein angesichts der tatsächlichen Einnahmen - ob nun gesamthaft oder gemeindeindividuell betrachtet - gar keine Aussage gemacht werden, außer der, daß die durchschnittlichen Sätze eben im Durchschnitt üblich sind. Zur Beurteilung, daß sie "richtig" oder "angemessen" seien, bedürfte es aber darüber hinausgehender Kriterien.

Vor allem aber wird die Nivellierung, also die Verwendung identischer fiktiver Hebesätze für alle Gemeinden, die auch im Fall der Durchschnittshebesätze vorliegt, angegriffen. Dabei werden Leistungsfähigkeit, aber auch Äquivalenzargumente vorgebracht.

Das Leistungsfähigkeitsargument zielt auf die Tatsache, daß die einzelnen Gemeinden durchaus unterschiedliche Hebesätze durchsetzen können. So kann etwa angenommen werden, daß Gemeinden in Ballungsräumen mit attraktiven Standortbedingungen und hoher Flächennachfrage für Wohn- und Produktionszwecke höhere Hebesätze fordern können als peripher gelegene Gemeinden oder Gemeinden in alten Industriegebieten, die sich mehr oder weniger ausgeprägten Abwanderungsbewegungen gegenübersehen und die Hebesatzpolitik (neben anderen Aktivitäten) als Instrument zur Wirtschaftsförderung einsetzen müssen.

Ähnlich der Einwohnerveredelung durch den Hauptansatz wird der Nivellierung deshalb ebenfalls ein "Veredelungseffekt" zugesprochen: "Dieser Veredelungseffekt ist höchst intransparent und in seinen Auswirkungen nur über aufwendige Simulationsrechnungen zu ermitteln. Grobe Ermittlungen haben jedoch schon gezeigt, daß in Bundesländern mit großem Stadt-LandGefälle der Wirtschaftskraft der Effekt aus der Nivellierung auf die tatsächliche Finanzausstattung der Gemeinden sogar denjenigen der HauptansatzStaffel übersteigt"48).

48) Zabel, Gerhard, Ist die Hauptansatzstaffel noch zeitgemäß?, a.a.O.. S. 454 f. 
Die äquivalenzorientierten Argumente zielen in eine andere Richtung: danach sollen sich die Hebesätze in den einzelnen Gemeinden nach den Kosten der Bedarfsdeckung richten; sofern also etwa in großen Gemeinden höhere Kosten der Bedarfsdeckung anfallen, sollen die Kosten über die Steuersätze an Bürger und Unternehmen weitergegeben werden. Die auf diese Weise normierten differenzierten Hebesätze sollten dann auch bei der Steuerkraftermittlung in Ansatz gebracht werden ${ }^{49)}$.

Bei beiden Formen der Differenzierung - nach Leistungsfähigkeits- und nach Äquivalenzkriterien - wäre zu klären, ob zwischen den Gemeinden eine Unterscheidung nach Klassen vorgenommen werden kann, oder ob die Leistungsfähigkeit bzw. die Kosten der Leistungserstellung für jede Gemeinde gesondert ermittelt werden müßten. Soll jede Verzerrung ausgeschlossen werden, so wäre allein eine einzelfallbezogene Ermittlung erforderlich. In einer klassierten Differenzierung könnten jedoch die Verzerrungen ebenfalls gering gehalten werden, wenn geeignete Indikatoren für die Klassifizierung gefunden werden. Dazu müßte aber zunächst geprüft werden, ob und welche Art der Differenzierung eine objektive bzw. neutrale Grundlage für die Messung des Zuschußbedarfs abgeben kann. Aus der Konzeption des praktizierten kommunalen Finanzausgleich läßt sich eine solche Entscheidung nicht ableiten.

Unter den Bundesländern differenziert allein Nordrhein-Westfalen die fiktiven Hebesätze nach der Gemeindegrößenklasse. Ob sie dem "Leistungsfähigkeits"- oder dem "Äquivalenz"-Argument Rechnung tragen soll, die durchaus nicht miteinander einhergehen müssen, ist aus dieser Differenzierung nicht ersichtlich. Deubel bezeichnet die Sprungstelle zwischen den beiden "Hebesatzgrößenklassen" überdies als "völlig unsystematisch und willkürlich" ${ }^{\text {"50) }}$.

Zusammenfassend läßt sich auch für die Wahl der fiktiven, in die Steuerkraftmessung eingehenden Hebesätze folgendes festhalten: Die Praxis der Steuerkraftermittlung in den Bundesländern wird sowohl unter verteilungspolitischen Aspekten kritisiert als auch mit systematischen Argumenten ange-

49) So argumentieren Kirchhof, Paul, Der Finanzausgleich als Grundlage kommunaler Selbstverwaltung, a.a.O., S. 718 und Deubel, Ingolf, Der kommunale Finanzausgleich in Nordrhein-Westfalen, a.a.O., S. 122.

50) Derselbe, S. 124. 
griffen. Allerdings läßt sich aus den gestellten Anforderungen allein das "richtige" Verfahren nicht ableiten. Die Vorstellungen über ein solches Verfahren weichen dementsprechend auch in der Frage nach den "richtigen" fiktiven Hebesätzen stark voneinander ab.

\section{Alternative Verfahren der Steuerkraft- und Finanzkraftmessung als Ersatz der praktizierten Konzepte?}

Alternative Konzepte zur Messung der gemeindlichen Steuerkraft werden zum einen direkt aus der Kritik des praktizierten Verfahrens abgeleitet, zum anderen werden sie aus der Praxis anderer föderalistisch aufgebauter Staaten zu übertragen versucht.

Im folgenden werden drei verschiedene Konzepte - zwei von ihnen verwenden fiskalische Indikatoren zur Bestimmung der gemeindlichen Steuerbzw. Finanzkraft, das dritte geht von ökonomischen Indikatoren aus ${ }^{51)}$ - näher betrachtet:

- die aus der Kritik der im deutschen kommunalen Finanzausgleich verwendeten Steuerkraftmeßzahl entwickelte Neuberechnung der Steuerkraft von Leonhard Grimme,

- $\quad$ der "Representative Tax System Approach" und seine Erweiterung der Advisory Commission on Intergovernmental Relations (ACIR) sowie

- die Schätzung kommunaler Einnahmemöglichkeiten über die regionale Einkommensentstehung (der "Income Approach").

I. Die Neuberechnung der Steuerkraft von Grimme ${ }^{52)}$

Verglichen mit den beiden anschließend betrachteten, in den USA entwickelten Konzepten ist der Gegenstand des Grimme-Konzepts am engsten eingegrenzt: Es zielt von vornherein nur auf die Erfassung steuerlicher Einnahme-

51) In der amerikanischen Literatur werden die beiden verschiedenen Ansätze mit "tax source or income approach" and "tax base or representative tax system approach" bezeichnet. Vgl. etwa Goetz, Charles, J., What is Revenue Sharing?, Washington D.C. 1972. S. 63.

52) Grimme, Leonhard, Neuberechnung der Steuerkraft im kommunalen Finanzausgleich. a.a.O., S. 159-200. 
möglichkeiten und ist daher zur Messung kommunaler Finanzkraft insgesamt nicht in der Lage. Für die Steuerkraftmessung enthält dieses Konzept jedoch den interessanten Vorschlag, die zur Errechnung der Steuerkraftzahlen verwendeten fiktiven Hebesätze nach Gemeindetypen zu differenzieren. Damit wird einer der wichtigsten Kritikpunkte am praktizierten Verfahren der Steuerkraftmessung angesprochen. Überdies könnte eine Differenzierung nach Gemeindetypen auch für andere Einnahmearten von Bedeutung sein.

Grimme setzt bei der Frage an, "inwieweit die Angleichung an die durchschnittlichen Hebesätze in der Praxis erfolgt, und ob der Landesdurchschnitt als geeigneter Maßstab sowohl für kleine Landgemeinden als auch für Großstädte anzusehen ist" (S. 161). Er kommt zu dem Ergebnis, "daß bei der fiktiven Steuerkraftermittlung die tatsächlichen Steuereinnahmen der Gemeinden zum Teil erheblich unterbewertet werden" (S. 170), wovon in Bayern insbesondere Verdichtungsräume profitieren, weil deren Gewerbesteuerkraft unterbewertet wird, sowie kleine Landgemeinden in strukturschwachen Gebieten, weil ihre Einkommensteuerkraft und die Steuerkraft aus der Grundsteuer A nicht angemessen berücksichtigt wird (S. 168). Er erarbeitet eine Reihe von Alternatiwvorschlägen für die Messung der Steuerkraft, von denen zwei als methodisch geeignet eingestuft werden, um die zuvor konstatierten Probleme zu lösen (S. 182).

Im ersten dieser beiden Alternativvorschläge (Modell 7) wird das Konzept eines einheitlichen Nivellierungssatzes für jede Einzelsteuer aufgegeben, und stattdessen werden für nach Zentralität und Gemeindegröße gebildete Gemeindegruppen neue, unterschiedliche Nivellierungssätze angesetzt. Diese Nivellierungssätze werden aus dem tatsächlichen Hebesatzverhalten der Gemeindetypen abgeleitet und orientieren sich daher weitgehend an den typenspezifischen Durchschnittshebesätzen. Allein für die "Siedlungsschwerpunkte" in den großen Verdichtungsräumen und die nichtzentralen Orte ab 4000 Einwohnern wird ein weit über dem Durchschnittshebesatz liegender Nivellierungshebesatz der Grundsteuer B festgesetzt; beiden Gemeindetypen wird damit unterstellt, daß sie ihre Einnahmemöglichkeiten aus der Grundsteuer B in geringerem Maße ausschöpfen als die übrigen Gemeindetypen (S. 174). Die Einkommensteuereinnahmen werden in voller Höhe berücksichtigt. 
Das Berechnungsmodell wird insbesondere deshalb als der praktizierten Steuerkraftberechnung überlegen angesehen, weil es damit "möglich ist, die Abweichungen zwischen fiktiver Steuerkraft und tatsächlichen Steuereinnahmen gering zu halten" (S. 175).

Der zweite für tauglich gehaltene Alternativvorschlag (Modell 8) kommt zu ähnlichen Ergebnissen wie Modell 7, ist darüber hinaus aber einfacher handhabbar: Als Nivellierungshebesätze werden die landesdurchschnittlichen Realsteuerhebesätze gewählt, zusätzlich werden den Gemeinden $50 \%$ der Realsteuereinnahmen zugerechnet, die sie über die fiktiv ermittelte Steuerkraft hinaus erzielen. Einen Abschlag für "Realsteuermindereinnahmen" gibt es dagegen nicht.

Die Einkommensteuereinnahmen werden wie in Modell $7 \mathrm{zu} 100 \%$ berücksichtigt.

Nun stellt sich allerdings die Frage, welche Beurteilungskriterien Grimme zugrundelegt, wenn er die dargestellten Alternativorschläge für besser geeignet hält, die "tatsächliche Steuerkraft" zu ermitteln, als die bisher angewendete Steuerkraftmeßzahl. Die von ihm präferierten Modelle zeichnen sich dadurch aus, daß sie den in den Gemeinden erzielten Einnahmen näher kommen als die Steuerkraftmeßzahl. Dieses Ziel hätte sich aber problemloser und besser erfüllen lassen, wenn die erzielten Einnahmen unmittelbar als Steuerkraftindikator verwendet worden wären.

Grimme führt aus, daß "... es bei der Steuerkraftermittlung nicht darauf ankommt, welche Steuereinnahmen eine Gemeinde tatsächlich erzielt, sondern welche Einnahmen sie erzielen kann, wenn sie ihre Hebesätze wie vergleichbare andere Gemeinden anspannt, ..." (S. 174). Verglichen werden hier also nur Gemeinden mit - hinsichtlich der betreffenden Steuerbemessungsgrundlagen - ähnlicher Struktur. Über das richtige Verhältnis der fiktiven Hebesätze beim Vergleich verschiedener Gemeindetypen kann damit allerdings wenig ausgesagt werden. So kann etwa der Fall eintreten, daß alle Gemeinden eines Typs eben aufgrund ihrer Strukturmerkmale dazu gezwungen sind, ihre Steuerquellen besonders stark auszuschöpfen, oder umgekehrt aufgrund ihres Reichtums in der Lage sind, die Steuerquellen mehr als Gemeinden anderen Typs zu schonen. 
Grimme erkennt weiterhin an, daß manche Gemeinden aufgrund "ihrer schwierigen Finanzlage gezwungen sind, überdurchschnittlich hohe Hebesätze festzusetzen" (S. 183), bestreitet aber, daß solche Gemeinden durch die vorgeschlagenen Meßkonzepte bestraft werden, weil "eine Anhebung der Hebesätze später in aller Regel auch dann nicht mehr rückgängig gemacht (wird), wenn sich die Finanzlage erheblich gebessert hat" (ebenfalls S. 183). Mit diesen Aussagen wird offenbar angezweifelt, daß eine schwierige Finanzlage zum Dauerzustand werden kann und daß deswegen Gemeinden bestimmten Typs auch auf Dauer gezwungen sein könnten, ihre Steuerquellen - relativ - stärker anzuspannen als andere. Oder mit anderen Worten: Die Gemeinden belasten ihre Steuerquellen langfristig in dem Maße, wie es ihren Möglichkeiten entspricht. Damit stände aber einer Anrechnung der erzielten Einnahmen nichts mehr entgegen. Im Gegenteil: Auch zwischen Gemeinden vergleichbaren Typs müßten Hebesatzunterschiede als Ausdruck unterschiedlicher Einnahmemöglichkeiten gewertet werden. Wenn Gemeinden mit situationsbedingt "zu hohen" Hebesätzen nicht benachteiligt werden, ist eine Bevorzugung von Gemeinden mit "zu niedrigen" Hebesätzen wohl ebensowenig vorstellbar.

Der Hauptvorwurf, der oben gegen das praktizierte Verfahren gerichtet wurde, daß nämlich die Festsetzung der in die Steuerkraftmessung einbezogenen Komponenten, insbesondere die Festsetzung der nivellierten Hebesätze, letztlich willkürlich erfolgt, ist damit auch gegen den Vorschlag von Grimme zu erheben.

Möglicherweise geben die realisierten Einnahmen der Gemeinden tatsächlich einen besseren Indikator für die potentiellen Einnahmen der Gemeinden $a b$ als die in den Ländern errechneten Steuerkraftmeßzahlen. Für den Vergleich und den Nachweis bedürfte es jedoch zunächst einmal einer Bestimmung und anschließenden Messung des Potentials. Daß es nicht um eine unmittelbare Messung der Einnahmen geht, wurde oben ja bereits angesprochen.

Für die theoretische Bestimmung des Potentials bietet der Grimme-Vorschlag jedoch keine weiterführenden Ansatzpunkte. 


\section{Der "Representative Tax System Approach"}

Das Konzept der $\mathrm{ACIR}^{53)}$ wurde 1962 zunächst nur zur Ermittlung der Steuerkraft der 50 US-Bundesstaaten entwickelt, dann aber um nicht-steuerliche Einnahmemmöglichkeiten erweitert. Ebenso wie im Konzept des Income Approach wird ein relativer Vergleich der Staaten angestrebt und nicht etwa deren absolute Einnahmemöglichkeiten zu messen versucht. Prinzipiell scheint dieses Verfahren auf die Gemeinden in der Bundesrepublik Deutschland übertragbar zu sein.

Hauptansatzpunkt der Messung durch den Representative Tax System Approach (RTS) sind die üblicherweise in den Bundesstaaten erhobenen Steuern. Die daraus zu berechnende Steuerkraft aus einer Steuer wird einem Staat dann auch zugerechnet, wenn er sie - obwohl dies rechtlich möglich und nach übergeordneten Vorstellungen angemessen wäre - gar nicht erhebt. Für die in der Steuerkraftmeßzahl des derzeit geltenden kommunalen Finanzausgleichs zusammengefaßten Steuern stellt sich dieses Problem allerdings in der Regel nicht, da die ergiebigsten Steuern - die Einkommensteuer, die Grundsteuer und die Gewerbesteuer - in fast allen Gemeinden erhoben werden. Es gewinnt erst dann an Bedeutung, wenn solche Einnahmemöglichkeiten in die Finanzkraftberechnung einbezogen werden sollen, die nicht in allen Gemeinden eines Landes in Anspruch genommen werden ${ }^{54)}$, wie dies bis 1979 für die Lohnsummensteuer der Fall war.

Die Frage nach den erzielbaren Steuersätzen in den einzelnen Staaten kann auch von der ACIR nicht befriedigend beantwortet werden. Als Gewichte der im RTS enthaltenen Steuern werden daher ersatzweise die durchschnittlich angewendeten Steuersätze verwendet. Gegen dieses Vorgehen wurden oben sowohl Leistungsfähigkeits-Argumente (die Gemeinden können unterschiedlich hohe Steuersätze durchsetzen) als auch Äquivalenz-Argu-

53) Ein Überblick findet sich in: Advisory Commission on Intergovernmental Relations, The Tax Capacity of the Fifty States: Methology and Estimates, Washington 1982, S. $11 \mathrm{ff}$.

54) Das Landesgesetz über den Finanzausgleich in Rheinland-Pfalz - hier wurden in einigen Gemeinden zeitweise nicht alle Realsteuern erhoben - enthält in $\S 12$ (4) eine dem RTS entsprechende Regelung. Danach werden die von den Finanzämtern festgesetzten Meßbeträge der nicht erhobenen Steuern mit zum Teil sogar höheren Nivellierungssätzen gewichtet. als wenn die Steuer erhoben worden wäre. 
mente (die Gemeinden sollten unterschiedliche Hebesätze fordern) vorgebracht, geklärt werden konnte die Kontroverse jedoch nicht.

Zusätzlich zu den Steuern wurden von der ACIR 1971 nicht-steuerliche Einnahmemöglichkeiten der Staaten berechnet und damit das RTS zum "Average System" ausgebaut. ${ }^{55)}$ Zur Entgeltschätzung wurden die durchschnittlichen Kostendeckungsgrade der öffentlichen Funktionen bzw. die durchschnittliche Rendite der öffentlichen Unternehmen ermittelt, um damit die entsprechenden Angaben bzw. Vermögen der betrachteten Gebietskörperschaften zu gewichten. Ein Rest von nicht auf diese Weise quantifizierbaren Entgeltmöglichkeiten wurde als Funktion der regionalen Einkommen berechnet, aber die tatsächlich erzielten Einnahmen wurden den Gebietskörperschaften direkt zugerechnet. Ähnlich wie bei der Steuerkraftberechnung ist die Verwendung eines durchschnittlichen Kostendeckungsgrades jedoch problematisch, weil davon ausgegangen wird, daß in allen Staaten (bzw. Gemeinden) pro Ausgabeneinheit einer Funktion gleich hohe Entgeltforderungen gestellt werden können und gestellt werden sollten, und zwar unabhängig von Bevölkerungs- und Wirtschaftsstruktur und vor allem unabhängig von der Effizienz der Ausgabentätigkeit. Ausgaben allein begründen aber dann nicht unbedingt entsprechende Entgelteinnahmemöglichkeiten, wenn die erstellte Leistung nicht nachgefragt wird, oder die Leistungserstellung mit besonders hohen Kosten verbunden ist, die die Nachfrager nicht tragen wollen oder können.

Außer den Entgelten bezieht die ACIR in einem nochmals erweiterten Ansatz die Verschuldungsmöglichkeiten in die Messung mit ein. ${ }^{56)}$ Abgesehen davon, daß die Frage, ob Schuldfinanzierung überhaupt bei der Ermittlung kommunaler Finanzkraft berücksichtigt werden sollte, nicht eindeutig zu beantworten ist - in der hier vorliegenden Untersuchung wurde dagegen plädiert - ist das Konzept der ACIR mit erheblichen Problemen behaftet. Die Möglichkeiten der Nettoneuverschuldung werden danach weitgehend der tatsächlichen Nettoneuverschuldung gleichgesetzt, wobei allerdings der künftige Schuldendienst abgezogen wird; wie hoch die Gebietskörperschaften sich hätten verschulden können, wenn sie gewollt hätten, wird damit nicht gezeigt. Im Gegenteil, wenn eine Gemeinde sich besonders hoch verschuldet, weil die

55) Siehe ACIR, Measuring the Fiscal Capacity and Effort of State and Local Areas, Washington 1971, S. 70 ff.; hier wird das "Average Financing System" im einzelnen dargestellt.

56) Ebenda, S. $111 \mathrm{ff}$. 
Summe ihrer endgültigen Einnahmen zur Deckung notwendiger Ausgabentätigkeit nicht ausreicht, wird ihre Verschuldungstätigkeit im Gegensatz zu anderen Gemeinden, die nicht auf Schuldaufnahme angewiesen sind, als Finanzkraft angerechnet.

Dem von der ACIR entwickelten Konzept ist zugute zu halten, daß hier eine wesentlich breitere Basis von Einnahmequellen bei der Ermittlung von staatlichen bzw. lokalen Einnahmemöglichkeiten zugrundegelegt wird als in der Steuerkraftmeßzahl. Prinzipiell wäre das Verfahren durchaus übertragbar; allerdings stellen sich dabei letztlich die gleichen Probleme wie bei der Steuerkraftmeßzahl: Ausgangspunkt der Ermittlungen sind die tatsächlichen Einnahmen - gemeindeindividuell in den "Bemessungsgrundlagen" und gesamthaft in den durchschnittlichen Steuer- und Entgeltsätzen abgebildet - Größen also, die man genau genommen nicht messen wollte.

\section{Der "Income Approach"}

Der vor allem in den USA diskutierte, ebenfalls für die Bundesstaaten konzipierte Income Approach geht von der Tatsache aus, daß zumindest mittelund langfristig alle öffentlichen Einnahmen aus der Einkommensentstehung zu zahlen sind, Zugriffsmöglichkeiten auf das Vermögen hingegen nur kurzfristig - bis zu seinem Verbrauch - bestehen. Insofern ist es naheliegend, zur Messung der finanziellen Leistungsfähigkeit einer Region an der regionalen Einkommensentstehung anzusetzen. Da den untergeordneten Gebietskörperschaften nicht das gesamte regionale Einkommen zur Verfügung steht, aber auch kein fester Anteil angegeben werden kann, auf den sie zurückgreifen können, eignet sich das Einkommenskonzept nur als relativer Maßstab zum interregionalen Vergleich; ein absolutes Einnahmepotential kann damit kaum ermittelt werden. Davon abgesehen, liegt der Vorteil dieses Meßkonzepts vor allem darin, daß es - indem es von der Zahlungsfähigkeit der in der Region angesiedelten Wirtschaftssubjekte ausgeht - Spielräume bei der Einnahmebeschaffung tendenziell eher transparent machen kann als jene Konzepte, die vorwiegend oder ausschließlich auf staatliche vorgegebene Einnahmequellen und deren übliche Ausschöpfung rekurrieren. 
Einer praktischen Anwendung stehen mit Blick auf das kommunale Finanzsystem in der Bundesrepublik Deutschland jedoch schwerwiegende Probleme entgegen:

Die Erfassung der regionalen Einkommensentstehung kann grundsätzlich am Ort der Produktion oder am Ort des Einkommenszuflusses erfolgen. ${ }^{57)}$ Die Entscheidung für den einen oder anderen Ansatzpunkt kann bezogen auf bundesdeutsche Verhältnisse aber kaum vorgenommen werden, ohne daß es zu gravierenden Verzerrungen kommen könnte. Die zur Zeit gewichtigsten Gemeindesteuern fallen am Ort der Produktion einerseits (Gewerbesteuer) und am Ort des Einkommenszuflusses (Einkommensteuer) andererseits an, andere Kommunalabgaben werden ebenfalls bei Unternehmen und bei Haushalten erhoben. Das Einkommenskonzept ist deshalb nur für Funktionalregionen anwendbar, in denen beide Seiten der Einkommensentstehung zusammengefaßt werden ${ }^{58)}$. Dazu bieten sich insbesondere Arbeitsmarktregionen an ${ }^{59)}$, mit deren Verwendung der Gemeinderaum als relevante räumliche Einheit jedoch verlassen wird.

- Unter den Bedingungen des geltenden Finanz- und Steuersystems sind die Komponenten der Einkommensentstehung aus gemeindlicher Sicht unterschiedlich ergiebig ${ }^{60)} \mathrm{Da}$ verschiedene Produktionen und Einkommensquellen den Gemeinden nicht im gleichen Maße Zugriffsmöglichkeiten bieten - etwa weil einige Einkommensquellen stärker als andere vom Zentralstaat beansprucht werden oder der gemeindliche Zugriff zugunsten bestimmer Einkommensempfänger eingeschränkt wird - wären

57) Vgl. Hessler. Heinz-Dieter, Finanzwissenschaftliches System der Besteuerung, Wisu-Texte, Tübingen/Düsseldorf 1976, S. $21 \mathrm{ff}$.

58) Vgl. dazu im einzelnen Zimmermann, Horst; Hardt, Ulrike und Postlep, Rolf-Dieter, Determinanten der kommunalen Finanzsituation, a.a.O., S. 77 ff. Für die Ebene der Bundesstaaten in den USA ist dieses Problem weniger gravierend, da die "grenzüberschreitenden" Einkommensströme hier von insgesamt geringem Gewicht sein dürften. Die Entscheidung für das "Inländerkonzept" kann daher nach Maßgabe der Verfügbarkeit und Einfachheit des Indikators getroffen werden. Vgl. Gläser, Martin, Die staatlichen Finanzzuweisungen an die Gemeinden, a.a.O., S. 156.

59) Zur Abgrenzung von Arbeitsmarktregionen in der Bundesrepublik Deutschland vgl. Klemmer, Paul, Abgrenzung strukturgefährdeter ländlicher Räume - Indikatoren für die Arbeitsmarktsituation, Arbeitsmaterialien der ARL, Nr. 8, Hannover 1978, S. 6.

60) Vgl. Zimmermann, Horst; Hardt. Ulrike; Postlep, Rolf-Dieter, a.a.O., S. 105 ff. 
die Einkommensarten gewichtet nach ihrem "tax-potential"61) oder allgemeiner nach ihrem Abgabepotential in den Index der kommunalen Einnahmemöglichkeiten aufzunehmen. Dabei wären auch die Möglichkeiten des Steuerexports durch Steuerüberwälzung und Einschränkungen infolge der Einkommensverteilung - die im deutschen Gemeindefinanzsystem aufgrund der Sockelregelung bei der Berechnung des gemeindlichen Einkommensteueranteils eine besondere Rolle spielt - zu berücksichtigen.

Aufgrund der genannten Probleme bei der Umsetzung erweist sich der Income-Approach als insgesamt zu weit, um die Einnahmemöglichkeiten der Gemeinden in der Bundesrepublik Deutschland (selbst nur für die Zwecke eines interkommunalen Vergleichs) richtig wiedergeben zu können. Zwar könnte der Versuch unternommen werden, das Konzept an die restriktiven Bedingungen des Gemeindefinanzsystems anzupassen. Allerdings stellt sich dann die Frage, wozu Einkommensquellen - und für eine kürzerfristige Betrachtung auch Vermögensbestände, die zeitweise durchaus ebenfalls der Abgabepflicht unterworfen werden könnten - überhaupt gemessen werden sollen, wenn sie von den Gemeinden nicht ausgeschöpft werden können.

Eher als für das hier geltende System wäre das Einkommenskonzept auf ein föderatives System anwendbar, in dem der gemeindliche Spielraum bei der Einnahmebeschaffung weniger geregelt und eingeschränkt ist.

\section{E. Zusammenfassung}

Gemessen an den Anforderungen, die im vertikalen und horizontalen Finanzausgleich an die Messung der Finanzkraft zu stellen sind, konnten einige Unzulänglichkeiten der Steuerkraftmessung in den Bundesländern herausgearbeitet werden.

- Zum ersten erscheint danach der alleinige Bezug auf einen - wenn auch vom Volumen her gewichtigen - Teil der gemeindlichen Steuerquellen als zu eng gewählt.

61) Gläser, Martin, Die staatlichen Finanzzuweisungen an die Gemeinden, a.a.O., S. 158. 
- Zum zweiten ist die Festlegung der fiktiven Hebesätze, mit denen die einzelnen Steuern in die Steuerkraftmeßzahl eingehen, umstritten.

Aus der Kritik der Steuerkraftmeßzahlen allein ließ sich jedoch kein Konzept ableiten, das den Anforderungen uneingeschränkt und unangefochten standhält, weil der Gegenstand der Messung aus dem Gesamtsystem des kommunalen Finanzausgleichs heraus nicht hinreichend konkretisiert werden kann. Insofern verwundert auch die Uneinigkeit der Kritiker - von notwendigen Vereinfachungen aufgrund der Schwierigkeiten der empirischen Umsetzung einmal abgesehen - nicht.

Eines der Hauptprobleme der Konkretisierung des Gegenstandes scheint dabei in der Frage zu liegen, ob gemeindliche Selbstverantwortung und Handlungsspielräume bei der Einnahmebeschaffung mit einer umfassenden, alle Spielräume einschließenden Messung der Finanzkraft überhaupt vereinbar sei. Anders ausgedrückt: Sollen jene Einnahmemöglichkeiten, über deren Ausschöpfung die Gemeinden autonom entscheiden können, in die Messung einbezogen werden oder nicht? Dieses Problem wurde etwa bei der Betrachtung der gemeindlichen Verbrauch- und Aufwandsteuern, besonders aber bei der Diskussion um die "richtigen" fiktiven Hebesätze deutlich.

Einige der vorgeschlagenen Erweiterungen der Steuerkraftmessung, aber auch alternativ diskutierte Konzepte wie etwa das des Average Financing System der ACIR zielen von ihrer Anlage darauf hin, genau zu erfassen, wieviele Mittel die einzelnen Gemeinden insgesamt einnehmen können. Daß letztlich etwas anderes gemessen wird, etwa indem die Anforderungen von vornherein auf einen relativen Vergleich der Einnahmemöglichkeiten eingeschränkt werden, resultiert eher aus technischen Problemen der Erfassung.

Diese Probleme sollen im anschließenden 3. Kapitel völlig außer acht gelassen werden, wenn gefragt wird, wie die gesamten Einnahmemöglichkeiten einer Gemeinde bestimmt werden müßten. Im 4. Kapitel schließt sich dann allerdings die Frage an, ob dieses umfassend bestimmte Einnahmepotential aus staatlicher Sicht von Interesse ist, oder ob für die Zwecke des Finanzausgleichs eine anders bestimmte Größe gemessen werden soll. 
Ulrike Hardt - 978-3-631-75265-4

Downloaded from PubFactory at 01/11/2019 06:31:57AM

via free access 
3. Kapitel: Das "kommunale Einnahmepotential" - die Bestimmung der zu messenden Einnahmemöglichkeiten aus gemeindlicher Sicht

\section{A. Die Ansatzpunkte der Betrachtung}

I. Zum Aufbau des 3. Kapitels und dem Stellenwert empirischer Überlegungen in der Gesamtkonzeption

Das gesamte Kapitel beleuchtet ausschließlich die gemeindeindividuelle Sicht des Gegenstandes "kommunale Einnahmemöglichkeiten". Allerdings wird dabei nicht unmittelbar von der konkreten Situation der Gemeinden in der Bundesrepublik Deutschland ausgegangen, sondern zunächst umfassender angesetzt und nach den Bedingungen gefragt, unter denen die Bestimmung des Einnahmepotentials für die untergeordneten Körperschaften eines föderativen Staates überhaupt ein lösungsbedürftiges Problem darstellt. Anschließend wird der Gegenstand der Bestimmung aus den kommunalen Interessen heraus näher eingegrenzt, wobei der allgemeine Charakter der Betrachtung noch beibehalten wird.

Ohne von vornherein auf ein bestimmtes kommunales Einnahmesystem abzustellen, kann gezeigt werden, daß die schwerwiegendsten Bestimmungsprobleme bei Einnahmen aus Abgaben auftreten, für die den Gemeinden ein Recht zur Preis- bzw. Steuersatzgestaltung zugestanden worden ist. An diese Probleme richtete sich auch die Hauptkritik an der Steuerkraftmeßzahl, wie im 2. Kapitel belegt wurde. Die Betrachtung der beiden genannten Einnahmetypen beansprucht allein deshalb schon breiten Raum im 3. Kapitel. Dabei wird zunächst ganz allgemein nach den Möglichkeiten kommunaler Besteuerung und Entgelterhebung gefragt und erst auf der daraus entwickelten Grundlage auf die besonderen Bedingungen der Gemeindefinanzierung in der Bundesrepublik eingegangen. Empirische Überlegungen werden daher jeweils erst relativ spät angestellt. 
Aber selbst dann ist es nicht Ziel der Untersuchung, das Einnahmepotential für eine oder mehrere Gemeinden zu berechnen. Vielmehr kommt es wie oben bereits angemerkt - darauf an, die ineinandergreifende Wirkungsweise verschiedener Grenzen kommunaler Einnahmepolitik in unterschiedlichen Gemeindetypen herauszuarbeiten, damit den Gegenstand einer gemeindeindividuellen Erfassung des Einnahmepotentials zu bestimmen und letztlich aufzuzeigen, wie es richtigerweise zu erfassen wäre.

\section{Möglichkeiten gemeindlicher Mitgestaltung des Budgets als Voraussetzung eines kommunalen Interesses an der Bestimmung des Einnahmepotentials}

Wenn Gemeinden - oder genereller: Gebietskörperschaften - versuchen, ihr Einnahmepotential abzuschätzen, dann in der Regel mit dem Zweck, Entscheidungen über die Gestaltung des Haushalts oder haushaltsrelevante Maßnahmen vorzubereiten. Die Erfassung des Einnahmepotentials soll eine Antwort auf die Frage liefern, wie hoch die Finanzierungskapazität der betreffenden Körperschaft ist.

Voraussetzung dafür, daß von gemeindlicher Seite die Frage nach den Einnahmemöglichkeiten überhaupt gestellt wird, ist ein gewisser Mitgestaltungsspielraum bei der Einnahmengestaltung. Für die untergeordnete Ebene eines Staates, in dem die Entscheidung über die kommunalen Einnahmen ausschließlich zentral getroffen wird, stellt sich das Problem der Abschätzung von Einnahmemöglichkeiten kaum. Hier liegen die Einnahmen fest und sind von den Gemeinden nicht zu beeinflussen; da aber kein von den zugewiesenen Einnahmen unterscheidbares Einnahmepotential existiert, das die Gemeinden mehr oder weniger stark ausschöpfen können, reduziert sich die Analyse der Einnahmen aus gemeindlicher Sicht allenfalls auf die Frage, ob die zugewiesenen Einnahmen zur Ausgabedeckung ausreichen, oder ob dies - etwa aufgrund besonderer regionaler Bedingungen, wie beispielsweise eines überdurchschnittlichen Niveaus der Faktorpreise - nicht der Fall ist.

Die Notwendigkeit einer Erfassung potentieller Einnahmen besteht für die einzelnen Gemeinden also von vornherein nur dann, wenn ihnen ein Spielraum zur Beeinflussung des Einnahmenniveaus oder der Einnahmestruktur 
zugestanden worden ist. Normalerweise impliziert dies auch eine - vollständige oder teilweise - Entscheidungskompetenz bei der Aufgabenerfüllung und der Ausgabentätigkeit. Andernfalls wäre die Erfassung des Einnahmepotentials durch die Gemeinden selbst wenig sinnvoll:

Zwar kann man sich ein föderatives System vorstellen, in dem Art und Umfang der Aufgabenerfüllung, die daraus resultierende Ausgabensumme und damit auch die Summe der zur Finanzierung notwendigen Mittel für die Gemeinden vorgegeben sind, die Aufbringung der Mittel jedoch nach Möglichkeit durch die Gemeinde erfolgen soll und nur im Falle zu geringer eigener Leistungsfähigkeit eine Aufstockung durch übergeordnete Ebenen vorgesehen ist. Die Messung des gemeindlichen Einnahmepotentials müßte dann ebenfalls von der übergeordneten Ebene vorgenommen werden, da eine Selbsteinschätzung der Gemeinden in der Regel auf eine Unterbewertung der eigenen Einnahmemöglichkeiten hinauslaufen würde. Da auf diese Weise erhöhte Zuweisungen zu bekommen wären, widerspräche es dem gemeindlichen Interesse, das Einnahmepotential offenzulegen; es gäbe überhaupt keine Veranlassung, eine objektive Messung anzustreben. Dieser Fall soll daher nicht weiter betrachtet werden.

Ein Interesse an der Erfassung des Einnahmepotentials kann hingegen unterstellt werden, wenn die Gemeinden ihr Budget beeinflussen oder mehr noch, wenn sie selbstverantwortlich über Ausgaben und Einnahmebeschaffung entscheiden können. Dann erst tritt bei der Budgetplanung die Notwendigkeit auf, die Finanzierungsmöglichkeiten abzuschätzen. Wie groß die den Gemeinden von übergeordneten Gebietskörperschaften belassenen Spielräume sind ob sie sich auf die gesamte kommunale Aktivität oder nur auf bestimmte Teilbereiche beziehen und ob eine direkte oder nur eine indirekte Einflußnahme möglich ist - ist für die Notwendigkeit der Messung letztlich unerheblich; sie berührt nur deren Ausgestaltung und die dabei auftretenden Probleme.

Nach dem Grad der Beeinflußbarkeit sind grundsätzlich vier Typen kommunaler Einnahmen zu unterscheiden:

- Einnahmen, bei denen die Gemeinden selbständig über Quelle und Inanspruchnahme entscheiden kann. Einnahmen dieses Typs weisen eine besonders große Flexibilität auf, stehen den Gemeinden in der Bundesre- 
publik Deutschland aber - abgesehen von ihrem eng begrenzten Steuerfindungsrecht - praktisch nicht $\mathrm{zu}$.

- Einnahmen mit übergemeindlich mehr oder weniger genau festgelegten Quellen, die von den Gemeinden durch eine Entscheidung über die Ausschöpfung der Quelle unmittelbar beeinflußt werden können. Hierzu zählen im Gemeindefinanzsystem der Bundesrepublik etwa die Realsteuern, ein großer Teil der Entgelte einschließlich der Konzessionsabgaben sowie die Schuldaufnahme. Diese Einnahmen lassen sich längerfristig auch durch eine entsprechende "Bemessungsgrundlagenpolitik" beeinflussen.

- Einnahmen, deren Höhe nur durch eine Bemessungsgrundlagenpolitik beeinflußt werden kann. Als Beispiel sei auf den gemeindlichen Einkommensteueranteil und die Schlüsselzuweisungen hingewiesen.

- Einnahmen, deren Höhe überhaupt nicht beeinflußt werden kann. Darunter hätte man sich etwa solche Zuweisungen vorzustellen, die unabhängig von gemeindeindividuellen Merkmalen vergeben werden. Einnahmen dieses Typs spielen im bundesdeutschen Gemeindefinanzierungssystem allerdings keine Rolle.

Allein im 4. der aufgeführten Fälle liegen die Einnahmen vollkommen fest, und die Überlegung, ob und wie weit das Einnahmevolumen ausgedehnt werden könnte, erübrigt sich deshalb. In allen anderen Fällen ist eine Abschätzung der Mittel, die erzielbar wären, sinnvoll. Sie kann kurzfristig angelegt sein und nach den unmittelbaren Möglichkeiten der Einnahmesteigerung - etwa über Steuersatzvariationen - fragen, sie kann die über eine Beeinflussung der Bemessungsgrundlagen erzielbaren Einnahmen zu ermitteln versuchen. So könnte etwa darüber nachgedacht werden, wie sich die Einnahmen durch eine gezielte Wirtschaftsförderungsstrategie steigern lassen. Dabei sind natürlich die Beziehungen zwischen verschiedenen Einnahmearten, wie sie im bundesdeutschen Kommunalfinanzsystem etwa zwischen Steuern und Schlüsselzuweisungen bestehen, zu berücksichtigen.

Gegenstand der Schätzversuche kann zunächst ausschließlich jener Teil der Einnahmen sein, der durch die kommunale Politik ausgedehnt werden soll. So wäre im Falle einer Anhebung der Gebührensätze die Veränderung 
des Gebührenaufkommens als ein Ausschnitt aus der gesamten Finanzierungskapazität zu betrachten.

Daran wird sich jedoch im nächsten Schritt die Analyse der gesamten Einnahmemöglichkeiten anschließen: Bleibt man bei dem Beispiel des Gebührenhaushalts, so reduziert sich hier durch die Gebührenanhebung unter bestimmten Bedingungen der Ausgleichsbedarf; die frei werdenden Mittel können also für andere Zwecke verwendet werden. Wieviele Mittel unter dieser Voraussetzung insgesamt erzielt werden können, wird dann im Vordergrund des Interesses stehen, wenn die Gemeinden in einer längerfristigen Haushaltsplanung Ausgabenvorhaben und Einnahmenerfordernisse zur Dekkung bringen müssen. Dann richtet sich das Interesse grundsätzlich auf alle Finanzierungsmöglichkeiten, unabhängig davon, ob sie von den Gemeinden im Planungszeitraum beeinflußt werden können oder sollen.

So sind in der Bundesrepublik Deutschland etwa die Einnahmen aus dem gemeindlichen Einkommensteueranteil nicht durch die Gemeinden selbst gestaltbar; trotzdem sind sie wichtiger Bestandteil der kommunalen Finanzierungskapazität und werden deshalb von den Gemeinden selbstverständlich in die Ermittlung des Einnahmepotentials eingeschlossen. Ähnliches gilt auch für Einnahmen aus Erstattungen, da sie in der Mehrzahl nicht nach Maßgabe der Kosten, sondern pauschal gewährt werden.

Eine Aufspaltung des Potentials und die Vernachlässigung bestimmter Einnahmen wäre nur dann gerechtfertigt, wenn vollständig durch übergeordnete Körperschaften geregelte Aufgabenbereiche allein mittels Kostenerstattungen finanziert würden. Man könnte diesen Bereich dann als insgesamt "nicht-kommunal" ausgrenzen und außer Betracht lassen. Dadurch ergäbe sich nur eine Bilanzverkürzung im geplanten Haushalt. Eine Vernachlässigung nicht mitbestimmbarer Einnahmen ist dagegen ausgeschlossen, wenn keine Zweckbindung vorliegt oder wenn insofern eine Beziehung zwischen den verschiedenen Einnahmearten besteht, als die Ausgaben für übergeordnete Aufgaben nicht mit den dafür zweckgebundenen Einnahmen deckungsgleich sind und entweder zufinanziert werden muß oder anderweitig verwendbare Überschüsse entstehen. Immer dann tritt die Notwendigkeit auf, die Gesamtheit der geplanten Ausgaben allen erzielbaren Einnahmen gegenüberzustellen. 
Aus dem dargestellten Zweck einer Ermittlung der Einnahmemöglichkeiten ergibt sich zuletzt, daß es für die Gemeinden vorrangig auf eine absolute Messung ankommt. Zwar sind unter Konkurrenzgesichtspunkten für die einzelnen Gemeinden auch Vergleiche mit anderen Gemeinden von Interesse, weil damit eine der wichtigsten Beschränkungen gemeindlicher Einnahmemöglichkeiten zusammenhängt; für die Haushaltsplanung sind solche Informationen über die relative Position allein jedoch unzureichend.

\section{Zwecke und Objekte der Messung bei gegebenem und variablem Angebot kommunaler Güter und Leistungen}

Die Frage nach den gemeindlichen Finanzierungsmöglichkeiten läßt sich auch folgendermaßen stellen:

Wieweit kann die betreffende Gemeinde ihre Einnahmen über die derzeit erzielten Einnahmen hinaus steigern? Für die Gemeinden in der Bundesrepublik Deutschland bieten sich nun grundsätzlich zwei der angesprochenen, sich nicht unbedingt ausschließenden Möglichkeiten, ihre zu einem gegebenen Zeitpunkt realisierten Einnahmen zu steigern: indem sie die vorhandenen Einnahmequellen stärker als bisher ausschöpfen oder indem sie versuchen, ein Wachstum dieser Quellen selbst zu induzieren.

Unter fiskalischem Gesichtspunkt könnte für beide Strategien untersucht werden, bis zu welcher Grenze sich die Einnahmen ausdehnen lassen. Die ermittelten "Einnahmepotentiale" unterscheiden sich jedoch grundlegend voneinander.

Betrachtet man zunächst den ersten Fall, in dem es allein um die potentielle Ergiebigkeit der vorhandenen Einnahmequellen geht, so ist zu unterstellen, daß die gemeindlichen Aktivitäten in der Aufgabenerfüllung keine Veränderung erfahren sollen, weil mit einer solchen Veränderung unvermeidlich auch die kommunalen Einnahmequellen einer Veränderung unterliegen würden. Dies gilt zumindest dann, wenn die zusätzlich eingenommenen Mittel für Zwecke verwendet werden, die ihrerseits wieder eine Ausdehnung der Einnahmemöglichkeiten zur Folge haben. Eine solche Wirkung tritt etwa auf, wenn das Angebot entgeltfähiger Leistungen ausgedehnt oder wenn zweckzuweisungsfähige Ausgaben getätigt werden. Zumindest langfristig kann aber 
darüber hinaus jede Anhebung des Leistungsangebots zu einer Attraktivitätssteigerung der Gemeinde und zur Ansiedlung zusätzlicher Wirtschaftssubjekte und Abgabenzahler führen.

Die geschilderten Wirkungen sollen jedoch im ersten Schritt der Untersuchung außer acht gelassen werden. Die Bedingung des gegebenen gemeindlichen Angebots wird hier also vor allem aus Gründen der Vereinfachung angeführt.

Allerdings könnten die daraus ableitbaren Informationen auch für die Gemeinden von Interesse sein, wenn etwa Kostensteigerungen erwartet werden oder wenn eine Ausgabe, die bisher von übergeordneten Gebietskörperschaften getragen wurde, nun nach unten verlagert wird und von den Gemeinden zu finanzieren ist. Sie kann weiterhin dann von Bedeutung sein, wenn Investitionen zum Ersatz abgenutzter Einrichtungen finanziert werden müssen, um das Leistungsangebot langfristig aufrecht zu erhalten.

Die Frage, die zu beantworten wäre, lautet dann: "Wieweit können die Einnahmen gesteigert werden, wenn allein der Ausschöpfungsgrad der Quellen angehoben wird?". Bei ihrer Untersuchung in Abschnitt B soll gezeigt werden, durch welche Faktoren die Einnahmeerzielung unter dieser Bedingung begrenzt wird.

Dieser Abschnitt hat in der Gesamtkonzeption des 3. Kapitels besonders großes Gewicht, weil mit den angeschnittenen Fragen noch einmal auf die Kritik der Steuerkraftmessung im 2. Kapitel eingegangen wird. Dort stellte sich u.a. die Frage, ob die Steuerkraftmeßzahlen in der Lage sind, die kommunalen Einnahmemöglichkeiten unter den jeweils vorliegenden, gegebenen Bedingungen richtig wiederzugeben. Dies wird anschließend vorrangig zu prüfen sein. Dabei wird zunächst allgemein und erst im zweiten Schritt für die besonderen Bedingungen der Gemeindefinanzierung in der Bundesrepublik Deutschland argumentiert.

Anders als im Unternehmensbereich steht hinter gemeindlicher Einnahmebeschaffung insgesamt nicht der Zweck der Gewinnerzielung. Einnahmen dienen hier letztlich immer der Finanzierung eines bestimmten Leistungsangebots; insofern wird im Gemeindebereich die Frage nach den Einnahme- 
möglichkeiten vor allem mit Blick auf eine Ausdehnung des Leistungsangebots gestellt.

Die Einbeziehung einer potentiellen Ausdehnung des kommunalen Angebots läuft auf die Frage hinaus, bis zu welchem Umfang ein kommunales Budget unter dem Aspekt der Finanzierung maximal ausgedehnt werden kann. Sofern die Gemeinden als "Budgetmaximierer" einzustufen sind ${ }^{1)}$, wäre eine solche Information sowohl für die einzelnen Gemeinden, aber auch für die übergeordnete Ebene von Interesse. Allerdings setzt sie wiederum Informationen über komplexe Entwicklungszusammenhänge voraus, die in ihrer Gesamtheit kaum beschafft werden können. So werden etwa die Möglichkeiten einer Gemeinde, Einnahmen aus Gebühren zu erzielen, durch die angebotene Menge an gebührenfähigen Leistungen einerseits und durch die Höhe der Gebühren, die die Gemeinde durchsetzen kann, andererseits begrenzt. Soll nun die Mengenkomponente erklärt werden, so kann der Gebührenhaushalt nicht isoliert betrachtet und die Grenze des Angebots dort lokalisiert werden, wo eine Kostendeckung gerade noch zustande kommt.

Vielmehr ist zu berücksichtigen, daß das Angebot auch ohne $100 \%$ Kostendeckung erstellt und Defizite aus anderen Quellen, etwa aus Steuermitteln, gedeckt werden können. Die Gebührenhaushalte lassen sich über diese "Querfinanzierung" weit über die nachfragebedingte Grenze des Angebots, wie sie für den Unternehmensbereich eher relevant wäre, hinaus ausdehnen und das Gesamtbudget damit erhöhen.

Darüber hinaus werden kommunale Leistungen, ob entgeltfähig oder nicht, als Instrument gemeindlicher Entwicklungspolitik eingesetzt. Eine Ausdehnung des Angebots kann also Entwicklungsprozesse induzieren, die außer zu gegebenenfalls steigenden Einnahmemöglichkeiten aus Gebühren auch zu

1) So ist etwa nach den Ergebnissen der ökonomischen Theorie der Politik davon auszugehen, $\mathrm{da} B$ Behörden bestrebt sind, ihren Einfluß allgemein über eine Ausdehnung des Budgets zu steigern. Vgl. z.B. Niskanen. W.A., Ein ökonomisches Modell der Bürokratie, in: Pommerehne, Werner W. und Frey, Bruno S., Hrsg.. Ökonomische Theorie der Politik, Berlin, Heidelberg, New York 1979, S. 349-368, hier S. 364 f.

Eine etwas anders fundierte These läuft darauf hinaus, daß Bürokraten die Differenz zwischen notwendigen Ausgaben bei kostenminimaler Produktion und Budgetansatz zu maximieren versuchen, um die finanziellen Spielräume für andere Ziele möglichst groß zu halten. Vgl. dazu Roppel, Ulrich. Ökonomische Theorie der Bürokratie, Freiburg 1979. S. $152 \mathrm{ff}$.

Empirische Belege für die genannten Thesen finden sich bei Massat. Dieter, Die ökonomische Problematik von Zuweisungen an die Kommunen, a.a.O., S. $181 \mathrm{ff}$. 
steigender Finanzierungsfähigkeit aus anderen Einnahmequellen führen. ${ }^{2}$ In diesem Fall sind deshalb auch jene Einnahmen als durch die Gemeinde beeinflußbar zu betrachten, die nur durch kommunale Bemessungsgrundlagenpolitik gesteigert werden können.

Die angesprochene Problemstellung läßt sich - wenn überhaupt - nur im Rahmen einer umfassenden Analyse kommunaler Entwicklungsmöglichkeiten bearbeiten und soll wegen ihrer Komplexität nur relativ kurz in Abschnitt C besprochen werden.

\section{B. Die Begrenzungen kommunaler Einnahmemöglichkeiten bei gegebenem gemeindlichen Angebot}

I. Möglichkeiten der Einnahmesteigerung und Probleme ihrer Einschätzung bei verschiedenen Einnahmearten

Bei gegebenem kommunalen Leistungsangebot entfällt die Möglichkeit, die bisher von der Gemeinde realisierten Einnahmen durch eine Ausdehnung des Angebots zu steigern. Zur Einschätzung des Einnahmepotentials bzw. der zusätzlich erzielbaren Einnahmen sind daher nur die folgenden Fragen zu beantworten:

- Bis zu welchem Aufkommen können die bereits in Anspruch genommenen Einnahmequellen zusätzlich belastet werden?

- Gibt es Einnahmequellen, die bisher nicht in Anspruch genommen wurden, und welche Einnahmen können daraus maximal erzielt werden?

Welche Einnahmequellen den Gemeinden zur Verfügung stehen, wird im einzelnen durch die Finanzverfassung bestimmt. Als mögliche Quellen kommen in Betracht:

- die Besteuerung der im Gemeindegebiet ansässigen Wirtschaftssubjekte oder im Gemeindegebiet angesiedelter Tatbestände,

2) Ähnliche Probleme sind zu lösen, wenn Maßnahmen der Wirtschaftsförderung unter fiskalischem Aspekt beurteilt werden sollen. Vgl. z.B. Glaser, Gundolf, Lohnt sich die Neuansiedlung von Industrie aus fiskalischer Sicht der Kommunen? in: Ballung und öffentliche Finanzen. Veröffentlichung der ARL. Forschungs- und Sitzungsberichte Bd. 134, Hannover 1980, S. 185-202. 
- die Erhebung von Gebühren und Beiträgen für die Inanspruchnahme kommunaler Leistungen,

- Zuweisungen von über- oder nebengeordneten Körperschaften,

- Schuldaufnahme,

- die Veräußerung von Vermögen,

- $\quad$ eigene erwerbswirtschaftliche Betätigung der Gemeinden.

Auch bei gegebenem gemeindlichem Angebot läßt die Mehrzahl der genannten Einnahmequellen prinzipiell durchaus Einnahmesteigerungen zu, soweit die Gemeinde noch nicht an der Grenze ihrer Finanzierungsmöglichkeiten angelangt ist. Die mit der Abschätzung der zusätzlich erzielbaren Einnahmen verbundenen Probleme sind jedoch von unterschiedlichem Gewicht:

Als einzige der aufgeführten Einnahmearten sind die nach angebotsunabhängigen Kriterien vergebenen Zuweisungen prinzipiell und unabhängig von ihrer konkreten Ausgestaltung nicht durch die Gemeinden beeinflußbar, es sei denn, die kommunale Einflußnahme richtet sich unmittelbar auf die Willensbildung der übergeordneten Körperschaften. Für die Zweckzuweisungen kann dagegen geprüft werden, ob zusätzliche Mittel durch bisher nicht genutzte Förderprogramme, die beim gegebenen Ausgabeverhalten hätten in Anspruch genommen werden können, beschaffbar sind.

Sofern die Gemeinden im Besitz von Vermögensgegenständen, insbesondere Grund und Boden, sind, können über Vermögensveräußerung zusätzliche Finanzierungsmittel beschafft werden. Wie hoch diese Einnahmen sein können, ist über die Vermögensbewertung zu Marktpreisen relativ einfach zu ermitteln.

Problematischer ist auch für ein gegebenes Ausgabenverhalten die Ermittlung möglicher Schuldaufnahme, die in der Regel vom Kreditgeber nach Maßgabe der Kreditwürdigkeit des Kreditnehmers begrenzt wird. Dabei ist zu berücksichtigen, daß die Fähigkeit zur Rückzahlung der Kredite nicht zuletzt auch vom konkreten Einnahmeverhalten der Gemeinden abhängt: Nicht nur das übrige Einnahmepotential, sondern auch dessen voraussichtliche bzw. geplante Ausschöpfung ist für die zukünftige Tilgungs- und Verzinsungsfähigkeit ausschlaggebend. Insofern ist die Verschuldungskapazität von anderen Be- 
standteilen und Determinanten kommunaler Einnahmepolitik abhängig und kann deshalb auch erst in der Gesamtbetrachtung näher bestimmt werden.

Hier sei nur kurz darauf hingewiesen, daß in der Bundesrepublik die Verschuldungsgrenze durch die Kommunalaufsicht vorgegeben wird. Wichtigste Bestimmungsgründe der zusätzlichen Verschuldungsmöglichkeiten sind die Steuerkraft und die Ausgabentätigkeit, aber auch die Einnahmepolitik der Gemeinden, die wesentlichen Einfluß auf die freie Spitze als Kriterium für die "Kreditwürdigkeit" der Gemeinden haben. ${ }^{3)}$

Die Hauptprobleme der Bestimmung des kommunalen Einnahmepotentials liegen im betrachteten Fall also zunächst in der Bestimmung von Obergrenzen der Besteuerung und kommunaler "Preissetzung" bei Gebühren und Beiträgen sowie den Erwerbseinkünften.

Bis zu gewissen Grenzen lassen sich diese Einnahmen ohne Veränderung des Angebots durch Anhebung der Steuer- und Entgeltsätze steigern. Wo diese Grenzen liegen, ist jedoch nicht unmittelbar ersichtlich. Im folgenden sollen die begrenzenden Faktoren deshalb näher untersucht werden.

\section{Grenzen kommunaler Besteuerung}

a. Zur sukzessiven Einführung der begrenzenden Faktoren in die Betrachtung

Die gemeindlichen Besteuerungsmöglichkeiten unterliegen verschiedenen Begrenzungen von unterschiedlicher Restriktivität, die aufeinander aufbauend wirksam werden. Die durch den Staat festgelegten Einschränkungen - dazu zählt auch der Ausschluß der Gemeinden von bestimmten Steuerquellen durch das Finanzsystem und die Einnahmebeschränkungen durch das Steuerrecht - bilden nur eine dieser Grenzen, die im Rahmen anderer, auch ohne staatliche Aktivität existierender Begrenzungen zusätzlich wirksam werden.

Die anschließende Betrachtung beginnt daher mit der weitesten Eingrenzung - dem Einkommen und Vermögen im Gemeindegebiet - und setzt analytisch in einer Situation an, in der von den Reaktionen der Wirtschaftssubjekte

3) Zu den Einzelheiten vgl. Klein. Richard R.. Kommunale Schuldenpolitik, Schriften des Deutschen Instituts für Urbanistik, Band 61, Stuttgart u.a. 1977. 
auf die Besteuerung und der Gesetzgebung des Staates zunächst abgesehen wird. Diese restriktiven Begrenzungen werden erst in den Folgeschritten eingeführt. Das bedeutet, daß auch nicht von vornherein von einem bestimmten kommunalen Steuersystem ausgegangen werden kann, sondern daß verschiedene Formen der Besteuerung zu betrachten sind.

Obwohl den Gemeinden in der Bundesrepublik Deutschland der Zugriff auf manche der in die Analyse einbezogenen Steuerquellen zur Zeit verwehrt wird, ist ihre Untersuchung insofern interessant, als nach wie vor ein Umbau des kommunalen Steuersystems diskutiert wird und hier einige Rückschlüsse darüber gewonnen werden können, wie die gemeindlichen Einnahmespielräume durch einen solche Umbau berührt würden. Auf der Grundlage der allgemeinen Betrachtung läßt sich außerdem das Gemeindesteuersystem und die den Gemeinden darin verbleibenden Besteuerungsspielräume leichter beurteilen.

\section{b. Einkommen und Vermögen als materielle Obergrenze kommunaler Besteuerung?}

Steuern sind Zwangsabgaben ohne einen Anspruch auf Gegenleistung. Sofern den Gemeinden also ein uneingeschränktes Recht der Besteuerung eingeräumt würde, sie über Gesetzgebungs-, Verwaltungs- und Ertragshoheit verfügten und mit einem entsprechenden Machtapparat ausgestattet wären, so könnten sie zumindest unter bestimmten Bedingungen kaum daran gehindert werden, den gesamten materiellen Reichtum innerhalb ihrer Grenzen wegzusteuern. Das Einkommen und darüber hinaus das vorhandene Vermögen bilden insofern die äußerste Grenze kommunaler - wie auch staatlicher - Besteuerung. ${ }^{4)}$ Voraussetzung für die Möglichkeit einer solchen "Total-Kommunalisierung" wäre zum einen die angesprochene Machtausstattung der Gemeinden, zum anderen wären aber unüberwindliche Gemeindegrenzen und somit geschlossene Wirtschaftsräume zu unterstellen, damit die Zensiten die Steuerobjekte nicht räumlich verlagern und sich damit der Besteuerung entziehen könnten.

4) Vgl. auch Schmölders, Günter und Hansmeyer, Karl-Heinrich, Allgemeine Steuerlehre, 5. Aufl., neu bearb. von Karl-Heinrich Hansmeyer, Berlin 1980, S. 97 ff. 
Der beschriebene Extremfall ist natürlich einschließlich seiner Bedingungen vollkommen wirklichkeitsfremd. Zum ersten würden weder der Staat noch die Zensiten unter den gegenwärtigen Bedingungen eine vollständige Aneignung aller Steuerquellen durch die Gemeinden hinnehmen. Zum zweiten kann davon ausgegangen werden, daß auch die Gemeinden kein Interesse an einer solchen "Kommunalisierung" haben, sondern nur einen - wenn auch möglichst großen Teil - des entstandenen Einkommens abschöpfen wollen. Dies impliziert eine längerfristige Perspektive bei der Einnahmebeschaffung: ein einmaliger Zugriff auf die Steuerquellen soll diese nicht zerstören oder deren Erträge dauerhaft mindern.

Aus dieser Einschränkung wird ersichtlich, daß das Einkommen als Indikator für das kommunale Steuerpotential immer nur als relativer Vergleichsmaßstab dienen kann. Weder für gemeindliche noch für übergeordnete Zwecke kann das Einkommenskonzept das absolute Steuer- oder Einnahmepotential der Gemeinden wiedergeben.

\section{c. Die Begrenzung des Steuerpotentials durch die Reaktionen der Wirtschaftssubjekte}

\section{Die relevanten Reaktionen im Überblick}

Weiterhin sei unterstellt, daß den Gemeinden von seiten übergeordneter Gebietskörperschaften keinerlei Einschränkungen für die Festlegung ihrer Steuersätze auferlegt werden. Unter dieser Voraussetzung bestehen für die Wirtschaftssubjekte mehrere - alternative oder sich ergänzende - Möglichkeiten, auf die erwartete oder praktizierte gemeindliche Abgabenpolitik zu reagieren und die damit einhergehenden Belastungen zu begrenzen.

\section{1. Ökonomische Verhaltensänderungen}

Sofern eine direkte Einflußnahme der Zensiten auf die Festlegung der Abgabesätze nicht in Betracht kommt, sind verschiedene Anpassungsreaktionen denkbar: Die Wirtschaftssubjekte können versuchen, der Abgabezahlung aus- 
zuweichen, sie zu überwälzen oder - sofern einer endgültigen Belastung nicht zu entgehen ist - sie zu kompensieren. ${ }^{5)}$

Betrachtet man zunächst die Möglichkeiten der Ausweichung, so kommen sachliche, zeitliche oder räumliche Ausweichstrategien in Frage. Je nach Typ der Kommunalsteuern sind sie von unterschiedlicher Relevanz.

Eine zeitliche Ausweichung kann, wenn überhaupt, nur kurzfristig vorgenommen werden, indem die Tatbestände, die zur Zahlung führen, zeitlich vorgezogen oder auf einen späteren Zeitpunkt verschoben werden. Für die Betrachtung längerfristiger kommunaler Finanzierungsmöglichkeiten ist diese Form der Ausweichung daher weniger bedeutsam als sachliche oder räumliche Ausweichung.

Sachliche Ausweichung kann darin bestehen, daß die Steuerobjekte zu substituieren versucht werden - etwa indem es bei der Besteuerung eines Produktionsfaktors zu Umstrukturierungen des Produktionsprozesses kommt oder daß bestimmte Aktivitäten ganz aufgegeben werden - indem z.B. die Produktion eingestellt wird, weil bereits vor der Steueranhebung an der Schwelle der Rentabilität produziert wurde.

Wie groß die Möglichkeiten einer sachlichen Ausweichung sind, hängt nicht zuletzt von der Ausgestaltung des gemeindlichen Steuersystems ab: Je umfassender der Steuergegenstand definiert wird, desto stärker werden dadurch zumindest die Substitutionsalternativen eingeschränkt.

Neben der sachlichen Ausweichung bietet sich jedoch als Reaktion auf die kommunale Besteuerung insbesondere eine räumliche Ausweichung ab. Diese Form der Ausweichung setzt Mobilität und häufig sogar eine Abwanderung voraus, das heißt, sie verursacht vergleichsweise hohe Kosten, die durch die erwarteten Vorteile zumindest kompensiert werden müssen. Oberhalb gewisser Belastungsunterschiede durch Kommunalsteuern wird diese Alternative jedoch durchaus interessant, zumal dann, wenn durch Mobilität andere Vorteile realisiert werden können, wenn also beispielsweise die Zielgemeinde für den Fall der Abwanderung günstigere Standort- oder Wohnortbedingungen bietet. Hier werden die Wirkungen der interkommunalen Konkurrenz

5) Vgl. dazu Zimmermann. Horst und Henke. Klaus-Dirk, Finanzwissenschaft - Eine Einführung in die Lehre von der öffentlichen Finanzwirtschaft -, 4., überarbeitete und ergänzte Auflage, München 1985, S. 138 ff. 
sichtbar: Die Steueranhebung einer Gemeinde - wird sie von den konkurrierenden Gemeinden nicht im gleichen Maße vollzogen - kann ihre Wettbewerbsposition um Steuergegenstände beeinträchtigen und zu einem von der Höhe der Steueranhebung abhängigen Verlust an Bemessungsgrundlagen an andere Gemeinden führen.

Wie schnell solche Verluste durch räumliche Ausweichung auftreten, hängt darüber hinaus aber von der Konstruktion des Steuersystems ab, weil damit die Mobilitätskosten beeinflußt werden. So sind die Kosten der räumlichen Ausweichung deutlich höher, wenn sie eine Standort- oder Wohnortverlagerung notwendig machen, als wenn nur die Verlagerung bestimmter Aktivitäten, etwa des Einkaufs im Falle einer kommunalen Umsatzsteuer, erforderlich ist. ${ }^{6)}$

Andererseits wird auf diese Weise die Notwendigkeit einer sachlichen Ausweichung berührt: Je leichter die räumliche Ausweichung fällt, desto stärker müssen die Steuersätze auf der Kommunalebene insgesamt angehoben werden, damit es zur sachlichen Ausweichung kommt. Je stärker die interkommunale Konkurrenz aufgrund der Konstruktion des Steuersystems ausgeprägt ist, desto eher wird die - gesamtwirtschaftlich möglicherweise unerwünschte - sachliche Ausweichung durch räumliche Ausweichung substituiert. Aus gemeindlicher Sicht wirkt diese Substitution jedoch einnahmebegrenzend: Je größer die Möglichkeiten räumlicher Ausweichung sind, desto stärker wird die interkommunale Konkurrenz wirksam und desto eher führen Steuersatzanhebungen zu Einnahmeverlusten.

Die angesprochenen Reaktionen schlagen sich in ihrer Mehrzahl nicht unmittelbar, sondern erst nach einer mehr oder weniger langen Anpassungsfrist in den kommunalen Einnahmen nieder. Sofern es der Gemeinde jedoch um die Ermittlung ihrer dauerhaften Finanzierungsmöglichkeiten geht, so sind diese Reaktionen - also die Determinanten der Elastizität - einzubeziehen. Nach Ablauf aller zu erwartenden Ausweichreaktionen wäre die maximale Finanzierungskapazität einer Gemeinde dann erreicht, wenn eine Steige-

6) Diese Probleme wurden im Zusammenhang mit der Ende der 70er Jahre geplanten Gemeindesteuerreform intensiv diskutiert. Vgl. etwa Neumark. Fritz, Gedanken zur Steuerund Finanzreform, in: Wirtschaftsdienst IX/1978, S. 446-450, hier S. 450 und Rehm, Hannes. Beteiligung der Gemeinden an der Umsatzsteuer? in: Wirtschaftsdienst XII/1978, S. 624-632. 
rung der Steuersätze keine steigenden, sondern sinkende Einnahmen zur Folge hätte, weil die zusätzlichen Einnahmen aus der "Preiserhöhung" niedriger sind als die Einnahmeverluste, die durch die Verminderung der Bemessungsgrundlagen verursacht werden.

Bis zu welchem Niveau die Abgaben erhöht werden können, ohne daß dieser Punkt überschritten wird, hängt mit davon ab, von wem die zu zahlenden Abgaben letztlich getragen werden. Aus gemeindlicher Sicht wäre von Vorteil, wenn - ausgehend von der Situation vor der Abgabenerhöhung - eine vollständige Überwälzung auf gemeindeexterne Wirtschaftssubjekte erfolgen könnte, ohne daß es innerhalb der Gemeinde zu Anpassungsreaktionen sachlicher oder räumlicher Art käme. Allerdings dürfte dieser Fall kaum relevant sein:

- Zum ersten erfordert ein außerhalb der Gemeindegrenzen liegender "Abgabenort" übergemeindliche Wirtschaftsbeziehungen. Da nicht Gavon auszugehen ist, daß alle Destinatare über solche Beziehungen verfügen, verbleibt auch bei gelungener vollständiger Überwälzung ein großer Teil der Belastung notwendig innerhalb der Gemeinde und ruft hier Einkommens- und Substitutionseffekte hervor, die die Zugriffsmöglichkeiten der Gemeinden ebenfalls schmälern.

- Zum zweiten setzt eine vollständige Überwälzung aber voraus, daß vor der Abgabenerhöhung bestehende Gewinnspielräume - zur Preissteigerung beim Absatz oder zur Verringerung von Vorleistungspreisen - nicht genutzt worden sind. Für diesen Fall wird durch die gelungene "Überwälzung" aber weder eine sachliche noch eine räumliche Anpassung ersetzt, da sie zusätzlich zur Ausnutzung von Verhandlungsspielräumen weitere Vorteile versprechen.

- Sind bereits vor der Abgabenanhebung im Rahmen der bestehenden Absatz- und Lieferverflechtungen alle Gewinnspielräume genutzt worden, so ist eine vollständige Überwälzung in keinem Fall möglich. Je nach Ausgestaltung der kommunalen Abgaben kommt eine teilweise Weiterwälzung der Last in Frage, die jedoch um so schwieriger wird, je intensiver die 
Konkurrenzbeziehungen zwischen "Abgabenexporteur" und Wirtschaftssubjekten aus weniger abgabenbelasteten Gemeinden sind."

Wenn auch die Möglichkeiten der übergemeindlichen Steuerüberwälzung begrenzt sind, so verringern sie doch in jenen Gemeinden, in denen - vor allem durch den Branchenbesatz und die Wettbewerbsfähigkeit der Unternehmen beding ${ }^{8)}$ - solche Möglichkeiten vorhanden sind, den Druck zu einer sachlichen oder räumlichen Anpassung an eine steigende Abgabenbelastung. Die Finanzierungsspielräume dieser Gemeinden werden damit erweitert, gleichzeitig die derjenigen Gemeinden, in denen die Abgabenbelastung getragen wird, geschmälert.

Ist die endgültige Abgabenbelastung einmal erfolgt, so können auf verschiedene Weise Anstrengungen zu ihrer Kompensation unternommen werden. In der Form der Steuereinholung - das heißt über Mehrarbeit im Bereich der privaten Haushalte und über Kostensenkung, etwa durch Prozeßinnovationen im Unternehmensbereich - verbreitert sie in der Regel die Abgabenbasis und damit die Einnahmemöglichkeiten der Gemeinden.

Eine Steuereinholung setzt voraus, daß auf dem Arbeitsmarkt bzw. im Unternehmen die entsprechenden Möglichkeiten bestehen; zumindest unter den heute gegebenen Bedingungen dürfte sie daher eine untergeordnete Rolle spielen. Zwar könnte im Unternehmensbereich der Fall auftreten, daß neue Techniken erst unter dem Druck zusätzlicher Abgabenbelastung eingeführt werden. Allerdings wird diese Umstrukturierung in der Regel mit einer sachlichen Anpassung einhergehen. Deshalb werden auch im folgenden die Wirkungen einer Abgabenkompensation nicht mehr gesondert betrachtet.

Kombinierte Anpassungsstrategien werden aber auch in den übrigen der beschriebenen Fälle häufig vorkommen. Da etwa eine Überwälzung der vollständigen Steuerlast nur unter bestimmten, eingeschränkten Bedingungen gelingt, wird auf die gegebenenfalls verbleibende Steuerlast mit einer Verschie-

7) Möglichkeiten der Steuerüberwälzung werden z.B. ausführlicher diskutiert bei Recktenwald. Horst Claus, Steuerüberwälzungslehre, 2., überarb. und erg. Aufl., Berlin 1966. Auf die Problematik regionaler Steuerinzidenz geht besonders ein Bertelmann, Brigitte D., Regionalpolitisch relevante Wirkungen von Steuern, Frankfurt 1982.

8) Dieses Ergebnis wurde auch für die besonderen Bedingungen der Finanzverfassung in der Bundesrepublik Deutschland bestätigt. Vgl. Bertelmann, Brigitte D., Regionalpolitisch relevante Wirkungen von Steuern, a.a.O., S. $188 \mathrm{f}$. 
bung der ökonomischen Aktivitäten reagiert werden. Trotzdem erfolgte hier eine getrennte Darstellung, um die Wirkungen der Anpassungsreaktionen auf die kommunalen Einnahmespielräume im einzelnen - wenn auch sehr kurz ansprechen zu können. Zwei der dargestellten Reaktionsmöglichkeiten werden unten noch einmal detaillierter beschrieben, um die resultierenden Beschränkungen des kommunalen Einnahmepotentials genauer herauszuarbeiten:

- die sachlichen Anpassungen an kommunale Steuererhöhungen und die

- räumliche Anpassung an kommunale Steuerpolitik.

Zunächst ist jedoch eine weitere, allerdings nicht auf eine Veränderung der ökonomischen Aktivitäten ausgerichtete Reaktionsmöglichkeit auf steigende Abgabenbelastung anzusprechen, die die ökonomischen Reaktionen ganz oder teilweise ersetzen kann.9)

\subsection{Politische Reaktionen}

Die Einnahmespielräume der Gemeinden resultieren im oben beschriebenen System aus der Immobilität der Zensiten und einer unelastischen Nachfrage nach bestimmten kommunalen Leistungen. Räumliche und sachliche Ausweichung sind also Maßnahmen, die mit teilweise sehr hohen Kosten verbunden sind und deshalb nur im "Notfall" ergriffen werden.

Zuvor werden die betroffenen Wirtschaftssubjekte versuchen, politischen Einfluß auf die Abgabengestaltung zu nehmen und die Erhöhung der Abgaben zu verhindern.

In demokratischen Systemen bietet sich vor allem der Wahlmechanismus als Instrument zur Verhinderung von Abgabenerhöhungen an. Die Wähler werden sich bemühen, diejenige Partei, die eine potentielle Abgabenerhöhung vertritt, zugunsten einer Partei abzuwählen, die keine Abgabenanhebung plant.

Sofern die Parteien sich in ihren Programmen ansonsten kaum unterscheiden und die Abwahl der herrschenden Partei durch die Abgabenzahler

9) Vgl. z.B. Hirschmann, Albert O., Abwanderung und Widerspruch, Tübingen 1974, S. 25 ff. 
jederzeit möglich ist, wäre deshalb eine Erhöhung der Abgaben kaum durchzusetzen. Bei einem gegebenen kommunalen Leistungsangebot würde durch die Konkurrenz der Parteien um die Wählerstimmen jeglicher gemeindliche Einnahmespielraum beseitigt: $\mathrm{Da}$ in einer Situation gegebenen Angebots immer dasjenige Programm vorgezogen wird, nach dem die niedrigsten Abgaben zu zahlen sind, ist klar, welche Partei den Konkurrenzkampf gewinnt.

Die sehr vereinfachten, auch der Grundform des Median-Wähler-Modells zugrundliegenden Bedingungen ${ }^{10)}$ beschrieben jedoch die Realität nicht hinreichend. Berücksichtigt man verschiedene Differenzierungen und Einschränkungen der Modellannahmen, so läßt sich auch in einem demokratischen System durchaus ein Einnahmespielraum der Gemeinden gegenüber ihren Bürgern ableiten.

Zum ersten hängt es von der Konstruktion des Abgabensystems ab, welche Wirtschaftssubjekte von einer Erhöhung der Abgaben überhaupt betroffen würden. Sind es nicht die Wähler der herrschenden Partei, sondern eine oppositionelle Minderheit, die die erhöhten Abgaben zu tragen hätten, so ist nicht unbedingt ein Machtwechsel zu erwarten. Als Beispiel kann man sich etwa ein kommunales Abgabensystem vorstellen, das ausschließlich die angesiedelten Unternehmen belastet. In diesem Fall wäre zunächst von einer geringen Merklichkeit einer Abgabenanhebung bei den Wählern auszugehen. Erst wenn die zusätzlichen Abgaben teilweise überwälzt worden sind oder sich der Unternehmenssektor auf andere Weise anzupassen beginnt, werden die Wirkungen der kommunalen Abgabenpolitik merklich und werden - sofern der Zusammenhang von den Bürgern gesehen wird - entsprechende politische Reaktionen hervorrufen. Ähnlich unmerklich dürften Anhebungen der Entgelte für nur von einem kleinen Nutzerkreis in Anspruch genommene, spezielle Leistungen sein. Sie werden unter Umständen von der Mehrheit der Wähler überhaupt nicht wahrgenommen.

Zum zweiten unterscheiden sich die Programme der Parteien in weit mehr als dem einen Punkt der Abgabenhöhe. Für die bisherigen Wähler der herrschenden Partei bringt daher ein Parteiwechsel andere, unerwünschte Veränderungen mit sich, die gegen die Abgabenanhebung abgewogen werden

10) Vgl. dazu Kirsch. Guy, Neue politische Ökonomie. 2.. neubearb. und erw. Auflage, Düsseldorf 1983, S. $140 \mathrm{ff}$. 
müssen und vermutlich erst $\mathrm{ab}$ einer gewissen Höhe der Zusatzbelastung in Kauf genommen werden.

Soll die Wahlentscheidung nach Maßgabe aller relevanten Konsequenzen eines Machtwechsels erfolgen, so sind die entsprechenden Informationen zu beschaffen. Dabei fallen ebenfalls Kosten an, die gegen die Zusatzbelastung durch erhöhte Abgaben aufgerechnet werden müssen.

Darüber hinaus ist zu berücksichtigen, daß Wahlen in der Regel keine permanenten Einrichtungen sind, sondern zwischen den Wahlen mehr oder weniger große Abstände liegen, die von der herrschenden Partei dazu genutzt werden können, um ihre Vorstellungen durchzusetzen. Innerhalb dieser Spanne könnte so versucht werden, die Abgaben zu erhöhen und dabei auf den Effekt der Gewöhnung oder der Dominanz anderer Maßnahmen bis zur Wahl zu setzen.

Schließlich könnte sogar der Fall eintreten, daß die Konkurrenzparteien in den verschiedensten Programmpunkten, nicht aber in der Frage der Abgabenerhöhung voneinander abweichen. In dieser Situation, die für die weitere Analyse der ökonomischen Restriktionen unterstellt werden soll, sind die Wirtschaftssubjekte gezwungen, ihren Widerstand in anderer Form als im Gang zur Wahlurne zu dokumentieren. Allerdings steht die Dringlichkeit des Widerspruchs hier im Gegensatz zu den Möglichkeiten seines Erfolgs: Während einerseits um so stärkerer Druck auf die Gemeindepolitiker ausgeübt werden kann, je realistischer eine Abwanderungsdrohung ist ${ }^{11)}$, "richtet sich die tatsächliche Intensität des Widerspruchs nach der unelastischen Nachfrage, d.h. nach dem Mangel an Abwanderungsmöglichkeiten"12), wird also um so dringlicher, je weniger Abwanderungsmöglichkeiten gegeben sind und je höher die Kostenbelastung bei einer Abwanderung wäre.

Die kurze Betrachtung gemeindeinterner Beziehungen zwischen Politik und Wirtschaftssubjekten zeigt, daß darin über die Wirkung ökonomischer Restriktionen hinausgehende Beschränkungen kommunaler Einnahmemöglichkeiten zu finden sind. Die Analyse der - höchst komplexen - innergemeindlichen Beziehungen soll jedoch im weiteren Verlauf der Untersuchung nicht weiter verfolgt werden. Vielmehr wird hier, wie häufig in der Theorie

11) Vgl. Hirschmann, Albert O., Abwanderung und Widerspruch, a.a.O., S. 47.

12) Ebenda, S. 28. 
des Föderalismus ${ }^{13)}$, allein auf die ökonomischen Reaktionen der Wirtschaftssubjekte und (in Punkt d.) auf Beschränkungen seitens übergeordneter Gebietskörperschaften abgestellt.

2. Sachliche und räumliche Anpassung an kommunale Steuerpolitik unter den Bedingungen der interkommunalen Konkurrenz

2.1. "Monopolistische" Besteuerungsspielräume bei sachlicher Steuerausweichung und ihre Determinanten

Die Reaktionen der Wirtschaftssubjekte auf kommunale Steuerpolitik verlaufen nach ähnlichen Mustern wie bei der Preisbildung im privaten Sektor: Mit der Besteuerung werden - je nach Steuerobjekt - bestimmte Aktivitäten verteuert; auf Steuererhöhungen wird daher mit einer Reduzierung jener Aktivitäten in der besteuernden Gemeinde zu reagieren versucht. Aus gemeindlicher Sicht führt eine Steuersatzanhebung also zu einer Verminderung der Bemessungsgrundlagen. Dies entspricht in seiner Wirkung einem Rückgang der Nachfargemenge bei Produktpreiserhöhungen des privaten Sektors. Insofern bietet es sich an, zur weiteren Analyse das Instrumentarium der Preistheorie zu verwenden.

Anders als im privaten Sektor ist eine mengenmäßige Veränderung der Bemessungsgrundlagen jedoch nicht unmittelbar mit einer Veränderung des Leistungsangebots bzw. der Absatzmenge verbunden.

Im privaten Sektor ist eine Ausdehnung der Mengenkomponente nur über eine Absatzsteigerung möglich, die in der Regel mit einer Veränderung der Grenzkosten verbunden ist, die bei der Preis- bzw. Gewinnpolitik in Rechnung zu stellen ist. Demgegenüber sind die Steuern hoheitliche Einnahmen, die zunächst keine Gegenleistung voraussetzen. Bemessungsgrundlage der Steuerpolitik ist kein gemeindlich erstelltes Produkt, deshalb ist eine mengenmäßige Veränderung der Bemessungsgrundlagen nicht direkt mit Kostenveränderungen verbunden. Gemeindliche Steuerpolitik kann also ohne Be-

13) Zur Kritik vgl. Kirsch, Guy, Hrsg., Föderalismus, Stuttgart - New York 1977, Einleitung. S. 1-14, hier S. $10 \mathrm{f}$. 
rücksichtigung von Kosten der Leistungserstellung betrieben und ebenso analysiert werden.

Unter der Bedingung eines konstanten Angebots und Grenzkosten von Null kann das kommunale Steuerpotential in der Terminologie der Preistheorie als Umsatzmaximum umschrieben werden, das sich aus dem Verlauf der "Steuerzahlungsfunktion" - analog zur "Preis-Absatz-Funktion" des privaten Sektors - ableiten läßt. Er hängt für die einzelne Gemeinde wesentlich von ihrer "Marktstellung" gegenüber etwaigen Konkurrenten ab.

Die Erhebung von Steuern steht allein den Gebietskörperschaften zu. Innerhalb ihrer Grenzen haben die Gemeinden also mit keinerlei Konkurrenz zu rechnen; vielmehr können als Konkurrenten nur andere Gemeinden auftreten. Daraus folgt, daß die gemeindlichen Einnahmemöglichkeiten insofern durch die interkommunale Konkurrenz begrenzt werden, als die Wirtschaftssubjekte der Besteuerung durch räumliche Ausweichung zu entgehen versuchen. Innerhalb der Gemeindegrenzen verbleibt als Ausweichmöglichkeit allein die sachliche Anpassung in Form der Vermeidung des Steuergegenstandes. Damit nimmt die Gemeinde die Stellung eines territorialen Monopols gegenüber den Wirtschaftssubjekten ein. ${ }^{14)}$

In dieser Stellung sieht sich die Gemeinde zunächst - das heißt solange keine räumliche Ausweichung einsetzt - einer quasi monopolistischen Einnahmefunktion gegenüber. Da die Wirtschaftssubjekte um so stärker versuchen werden, der Steuer durch Vermeiden des Steuergegenstandes zu entgehen, je höher der Steuersatz ist, ist die Funktion der Steuerzahlungen S (vgl. Abb. 1) durch einen mit steigendem Steuersatz zurückgehenden Umfang an besteuerbaren Bemessungsgrundlagen gekennzeichnet. ${ }^{15)}$

14) Die Auffassung. daß "the nature of government is monopolistic" wird überlicherweise für Nationalstaaten vertreten; sie liegt auch der Konzeption der "Laffer-Kurve" zugrunde. Vgl. Wanniski, Jude, Taxes, Revenues, and the "Laffer Curve". in: Laffer, Arthur B. und Seymour, Jan P., The Economics of the Tax Revolt: A Reader, New York u.a. 1979, S. 7-12, hier S. 10.

15) Diese Darstellung - die für die interkommunale Konkurrenz noch zu modifizieren ist lehnt sich weitgehend an die Laffer-Kurve an. Zu ihrer Erklärung vgl. Wanniski, Jude, Taxes. Revenues, and the "Laffer Curve", a.a.O., sowie Laffer, Arthur B. and Miles, Marc A., International Economics in an Integrated World. Dallas, Tex. u.a. 1982, S. 110 f.

Eine mikroökonomische Fundierung liefern z.B. Brennan. Geoffrey und Buchanan. James M.. The Power to Tax-Analytical foundations of a fiscal constitution. Cambridge u.a. 1980. insbes. S. $39 \mathrm{ff}$. 


\section{Abbildung 1: $\quad$ Steuerzahlungen und kommunale Einnahmen bei monopolitischer Stellung der Gemeinde}

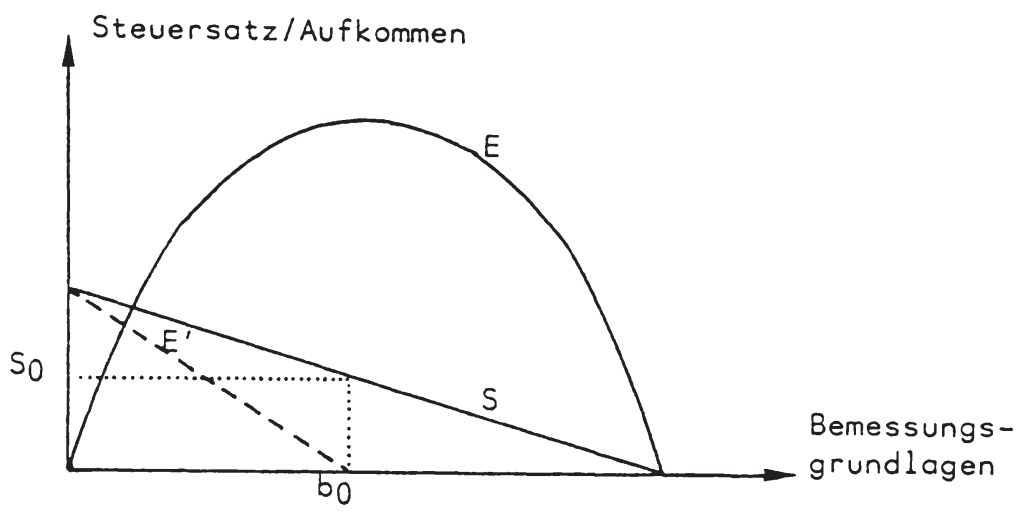

Die Gesamteinnahmen der Gemeinde werden durch die Funktion E abgebildet, die genau wie eine Umsatzfunktion als Produkt von jeweiligem Steuersatz und den zu diesem Steuersatz noch vorhandenen Bemessungsgrundlagen errechnet werden kann. Diese stark vereinfachte, in Anlehnung an das Cournot'sche Monopol ohne Produktionskosten konzipierte, von Laffer auf die staatlichen Steuereinnahmen übertragene Darstellung zeigt zunächst nur, daß die Gemeinde unter monopolistischen Bedingungen wrder beim "Maximalsteuersatz", der hinsichtlich der Bemessungsgrundlagen prohibitive Wirkung hat, noch beim Bemessungsgrundlagenmaximum, das bei einem Steuersatz von 0 erreicht wird, ihre Einnahmemöglichkeiten voll ausschöpft. Dies ist vielmehr dann der Fall, wenn die Gemeinde den Steuersatz bei $S_{0}$ ansetzt. Hier gilt:

$$
E^{\prime}=0 \text {, }
$$

das heißt, bei einer über $S_{0}$ hinausgehenden Anhebung des Steuersatzes werden die dadurch erzielten Mehreinnahmen von den durch die Reduktion

Eine verbale Beschreibung des "Steueroptimismus", das in seinen Grundgedanken als "Swiftsches Steuereinmaleins" bekannt wurde. findet sich bereits bei Gerloff. Wilhelm, Allgemeine Steuerlehre. in: Handbuch der Finanzwissenschaft, 2., völlig neubearbeitete Auflage, hrsg. von Wilhelm Gerloff und Fritz Neumark. zweiter Band, Tübingen 1956, S. 239325: hier S. 304 und 314 ff. 
der Bemessungsgrundlagen verursachten Mindereinnahmen überkompensiert.

Das kommunale Steuerpotential wird durch Lage und Steigung der Funktion, bei einem unterstellten linearen Verlauf also durch die Bemessungsgrundlagensumme bei einem Steuersatz von 0 und den Prohibitiv-Steuersatz bestimmt. Dabei wird der fiskalisch optimale Steuersatz - jener Steuersatz, bei dem das Steuerpotential voll ausgeschöpft wird - allein durch die Reaktionen der Wirtschaftssubjekte auf unterschiedliche Steuersätze determiniert.

Zur Illustration werden zunächst zwei Fälle verglichen, die sich allein durch die Summe der Bemessungsgrundlagen, also den Umfang der besteuerten wirtschaftlichen Aktivitäten unterscheiden sollen, ihre Struktur - und damit die Reaktionen auf Steuersatzerhöhungen - sei hingegen gleich. Das heißt, daß für alle Steuersätze die Summe der Bemessungsgrundlagen im Fall 1 doppelt so groß ist wie in Fall 2 (Abb. 2). Dabei sei angenommen, daß diese Situationen im Vergleich zweier Gemeinden festgestellt werden. Ebensogut könnte es sich aber auch um verschiedene Zeitpunkte oder verschiedene Steuergegenstände innerhalb der gleichen Gemeinde handeln.

\section{Abbildung 2: $\quad$ Bestimmung des optimalen Steuersatzes bei} unterschiedlichem Umfang der Bemessungsgrundlagen

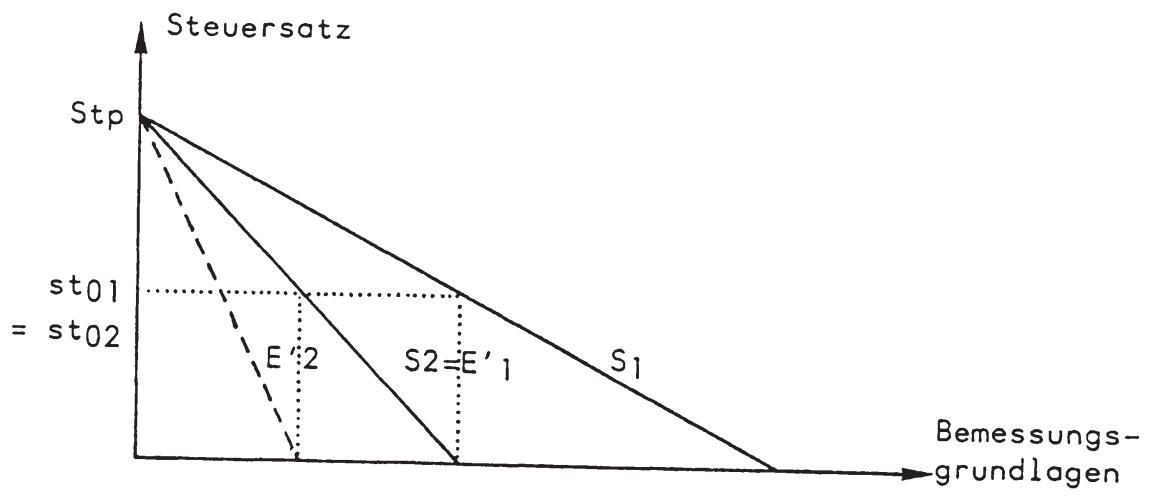


Strukturgleichheit der Bemessungsgrundlagen bedeutet hier, daß bei einer Anhebung des Steuersatzes z.B. um $10 \%$ in beiden Gemeinden $G_{1}$ und $\mathrm{G}_{2}$ ein gleichgroßer Prozentsatz der Bemessungsgrundlagen vermieden wird, der prohibitive Steuersatz ist deshalb in beiden Gemeinden identisch. Daraus kann abgeleitet werden, daß der fiskalisch optimale Steuersatz ebenfalls in beiden Gemeinden gleich hoch sein muß. Daß das bei diesem Steuersatz realisierbare Steuerpotential in Gemeinde 1 doppelt so groß ist wie in Gemeinde 2, ist allein auf den Bemessungsgrundlagen-Unterschied zurückzuführen.

Einen solchen, rein quantitativen Bemessungsgrundlagen-Unterschied wird man in der Realität kaum wiederfinden. Er träte etwa dann auf, wenn die eine Gemeinde doppelt so groß wäre wie die zweite, ohne daß damit Strukturunterschiede in den besteuerbaren Tatbeständen und Aktivitäten einhergingen. Dieser Fall ist jedoch höchst unrealistisch; Größen- und Struktureffekte dürften in der Regel miteinander gekoppelt sein. Allerdings soll zunächst auch der Struktureffekt gesondert betrachtet werden.

Abbildung 3: $\quad$ Fiskalisch optimaler Steuersatz bei unterschiedlichen Reaktionen der Zensiten auf Steuersatzanhebungen

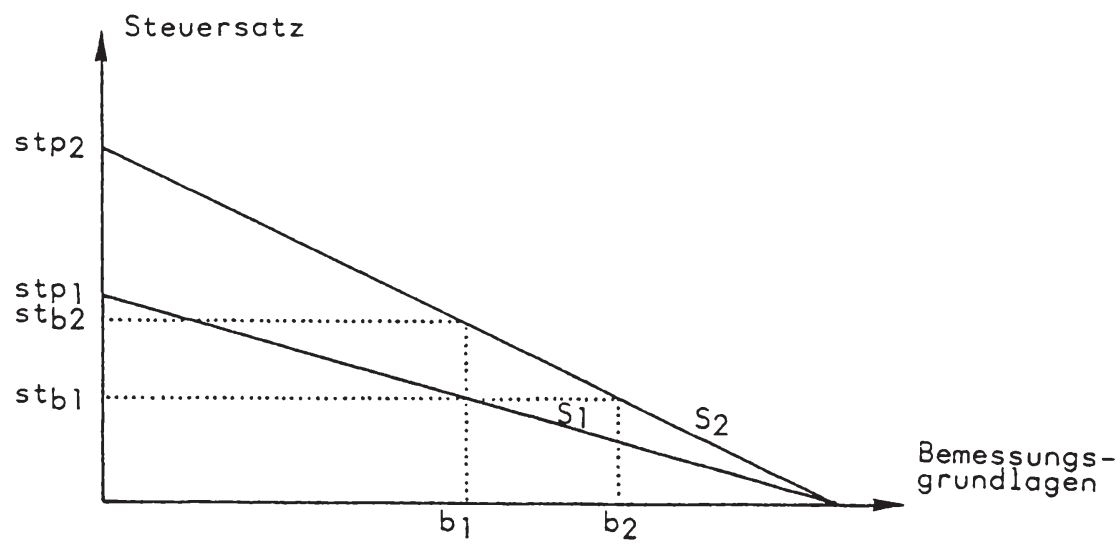

Die Steigungen der beiden Steuerzahlungs-Funktionen drücken aus, daß die Wirtschaftssubjekte im Fall 1 bei einer Anhebung des Steuersatzes (etwa um $10 \%$ ) in stärkerem Maße der Bemessungsgrundlage ausweichen als in Fall 2: So vermindern sich die Bemessungsgrundlagen bei einer Anhebung des 
Steuersatzes von 0 auf st ${ }_{01}$ in Gemeinde 1 mit der Steuerzahlungsfunktion $S_{1}$ auf $b_{1}$, in Gemeinde 2 mit der Steuerzahlungsfunktion $S_{2}$ hingegen nur auf $b_{2}$. Dem entspricht ein höherer Prohibitiv-Steuersatz $\left(\mathrm{st}_{02}\right.$ ), ein höherer fiskalisch optimaler Steuersatz $\left(\mathrm{st}_{02}\right.$ ), sowie ein ebenfalls höheres kommunales Einnahmepotential in Gemeinde 2.

Für die unterschiedliche Reaktionsstärke der Wirtschaftssubjekte in den abgebildeten Fällen können mehrere Ursachen verantwortlich sein:

Zum ersten ist die Reaktion davon abhängig, inwieweit das Steuerobjekt substituiert werden kann. Je leichter das Steuerobjekt ersetzt und damit die Steuer vermieden werden kann, desto flacher verläuft die Funktion der Steuerzahlungen, umgekehrt bedeutet Nicht-Substituierbarkeit aus gemeindlicher Sicht c.p. höherer Steuereinnahmemöglichkeiten.

Nicht-Substituierbarkeit kann einmal genereller Art sein, sie kann aber auch aus dem Umstand resultieren, daß das Substitut in der betreffenden Gemeinde nicht verfügbar ist. So ist etwa einer speziellen Verbrauchsteuer oder der Steuer auf einen bestimmten Produktionsfaktor noch eher durch Verzicht oder durch Substitution auszuweichen als einer Steuer auf Einkommen oder Gewinn. Aber auch der Steuer auf einen - wenn auch nur in gewissen Grenzen - substituierbaren Produktionsfaktor wie etwa Kapital kann nur dann durch Substitution ausgewichen werden, wenn innerhalb des regionalen Arbeitsmarktes genügend Substitute, d.h. hier, Arbeitskraftreserven vorhanden sind. Andernfalls ist eine sachliche Ausweichung nur über die Einschränkung der Produktion möglich.

Zum zweiten wird die Reaktionsstärke von den speziellen Wirtschaftsbedingungen sowie den Zielsetzungen und Verhaltensmustern der Zensiten beeinflußt. Hier ist zunächst auf die Merklichkeit der Steuer hinzuweisen; eine Eigenschaft, die nicht allein aus den Merkmalen des Steuerobjekts, sondern auch aus der Wirtschaftsweise der Zensiten erklärt werden muß. So werden etwa Verbrauchsteuern von den Haushalten, da diese in der Regel keine detaillierte Wirtschaftsrechnung durchführen, häufig erst ab einer bestimmten Höhe des Steuersatzes als Belastung wahrgenommen. ${ }^{16)}$ Unterhalb dieser Schwelle werden daher relativ geringfügige Ausweichreaktionen induziert.

16) Vgl. Schmölders, Günter, Finanzpolitik, 3., neu bearb. Aufl., Berlin-Heidelberg-New York 1970 , S. $327 \mathrm{ff}$. 
Wird sie überschritten, so sind jedoch um so heftigere Reaktionen zu erwarten.

Einer Substitution oder völligen Aufgabe des Steuerobjekts wird auch dann entgegengewirkt, wenn trotz prinzipieller Substituierbarkeit des Steuergegenstandes "Substitutionshemmungen" - etwa in Form gefühlsmäßiger Bindungen an einen hoch besteuerten Besitz - existieren. Insofern wird mit Blick auf die Stärke und Geschwindigkeit der Reaktion vor allem zwischen Unternehmen und Haushalten zu unterscheiden sein.

Eine der wichtigsten Determinanten des Reaktionsverlaufs dürfte die wirtschaftliche Lage der Zensiten sein. Sowohl bei Einkommen- als auch bei Gewinnsteuern wird umso eher das Existenzminimum der Wirtschaftseinheit erreicht, je niedriger deren Leistungsfähigkeit ist. Für den Haushaltssektor ist dieses Existenzminimum durch ein bestimmtes Mindesteinkommen, im Unternehmenssektor durch einen absoluten Mindestgewinn - etwa bei Einzelunternehmen oder Personengesellschaften - oder durch eine Mindestverzinsung des eingesetzten Kapitals vorgegeben. Diese Grenze kann auch bei der Unternehmensbesteuerung nicht überschritten werden, ohne daß Betriebsschließungen drohen. Diese besondere Form der "Steuerausweichung" wird um so stärker eintreten, je ungünstiger die Gewinnsituation in einer Gemeinde ist. Allerdings kann eine solche Reaktion auch bei der Besteuerung prinzipiell substituierbarer Produktionsfaktoren auftreten. Wenn etwa aufgrund von Liquiditätsengpässen eine zwar mögliche und auf Dauer lohnende Substitution nicht vollzogen werden kann, wird die Produktion ebenfalls eingeschränkt oder im Extrem sogar aufgegeben werden.

Die Einflüsse auf den Verlauf von Steuerzahlungs-Funktionen könnten noch wesentlich detaillierter untersucht werden. Allerdings reicht es hier zunächst aus, festzuhalten, welche Merkmale von Steuerobjekt und Steuerzahler aus gemeindlicher, rein fiskalischer Sicht besonders günstig erscheinen:

- Die Steuerobjekte sollten eine breite Bemessungsgrundlage abgeben und möglichst wenige Ausweichmöglichkeiten sachlicher Art offenlassen.

- Je leistungsfähiger die Wirtschaftssubjekte sind, desto größer ist der Umfang der Bemessungsgrundlagen und um so weniger dringlich ist eine Ausweichreaktion bei Steuersatzerhöhungen. 
Diese Kriterien gelten uneingeschränkt allerdings nur, solange die besteuerten Tatbestände die Gemeinde nicht verlassen, das heißt, solange die Zensiten die besteuerten Aktivitäten oder Gegenstände nicht räumlich verlagern. Ein Verlagerungsdruck tritt jedoch um so eher auf, je stärker die sachliche Ausweichung behindert wird - etwa durch die Besteuerung eines nicht substituierbaren Gegenstandes.

\subsection{Besteuerungsspielräume bei räumlicher Ausweichung unter den Bedingungen interkommunaler Konkurrenz}

Sobald für die Wirtschaftssubjekte Anreize zu räumlicher Ausweichung gegeben werden, tritt die Gemeinde in die Konkurrenz mit den möglichen Zielgemeinden ein, sie wird quasi als einer von mehreren Anbietern auf einem Markt aktiv, auf dem um die Verlagerung besteuerbarer Tatbestände in das eigene Territorium konkurriert wird.

Der Markt, auf dem die Gemeinden miteinander konkurrieren, weist nun einige Besonderheiten auf. Zum ersten handelt es sich um einen unvollkommenen Markt. ${ }^{17)}$ Die in den Gemeinden anzutreffenden Angebote sind nicht homogen, sondern aus der Sicht der Wirtschaftssubjekte von höchst unterschiedlicher Qualität, überdies dürften deutliche persönliche Präferenzen für die eine oder andere Gemeinde bestehen, ohne daß aber völlige Markttransparenz vorläge. Überdies verursacht die räumliche Ausweichung - je nach Typ des Ausweichvorgangs mehr oder weniger hohe - Kosten, die die Konkurrenzbeziehung zwischen den Gemeinden ebenfalls abschwächen.

Aus der gemeindlichen Konkurrenz resultieren daher nicht notwendig einheitliche Steuersatzspielräume und Steuersätze in allen Gemeinden, sondern es verbleibt jeder Gemeinde ein mehr oder weniger großer Steuersatzspielraum, innerhalb dessen sie eine monopolistische Stellung genießt.

Außerdem ist der betrachtete Markt insofern von besonderer Art, als besteuerbare Tatbestände nicht räumlich losgelöst sind, sondern immer an einen "Standort" gebunden sind, selbst wenn die Standorte leicht austauschbar sind;

17) Die Bedingungen für einen vollkommenen Markt werden im einzelnen dargestellt bei Ott, Alfred E., Preistheorie. in: Kompendium der Volkswirtschaftslehre, Bd. 1, 5., ergänzte und überarb. Aufl., Göttingen 1975, S. 114-181, hier S. 119. 
Standorte als solche sind also nicht substituierbar. Die Zahl möglicher Standorte ist jedoch - zumindest innerhalb des Staatsgebietes ${ }^{18)}$ - nicht vermehrbar und ihre Qualität ist von den Gemeinden selbst nur wenig, unter den Bedingungen eines gegebenen kommunalen Angebots an Gütern und Leistungen sogar überhaupt nicht beeinflußbar.

Die Zahl der Anbieter auf dem betrachteten Markt ist von vornherein fixiert; das Staatsgebiet ist flächendeckend auf die bestehenden Gemeinden aufgeteilt. Das heißt, es handelt sich um einen Markt mit absoluter Marktzutrittsbeschränkung und insofern ist das Auftreten weiterer Konkurrenten ausgeschlossen. Die beiden letzten Bedingungen können mit als Grund dafür angesehen werden, daß der Staat die kommunalen Einnahmespielräume über die durch private Reaktionen bedingten Grenzen hinaus einschränkt. Zunächst sollen jedoch weiterhin nur die Konkurrenzmechanismen ohne staatliches Eingreifen betrachtet werden.

Aufgrund des unterschiedlichen und deshalb nicht vollständig substituierbaren Angebots, der relativen, je nach Steuergegenstand mehr oder weniger ausgeprägten Immobilität der Zensiten und der besteuerten Aktivitäten und der in der Regel unvollständigen Markttransparenz, werden auch bei unterschiedlichen Steuersätzen in den Gemeinden nicht unmittelbar räumliche Ausweichprozesse in Gang gesetzt. Geht man einmal davon aus, daß zu einem bestimmten Zeitpunkt alle Wirtschaftssubjekte einen ihren Ansprüchen genügenden Standort für die besteuerten Tatbestände gefunden haben, so ist die räumliche Ausweichung mit zusätzlichen Kosten und insofern mit einer Verschlechterung der Situation verbunden. Räumliche Ausweichung lohnt sich deshalb erst dann, wenn die Differenz zwischen den zu zahlenden Steuern in verschiedenen Gemeinden größer ist als die monetären oder nicht-monetären Kosten der Verlagerung und eine eventuelle Standortverschlechterung durch die Steuerersparnis aufgewogen wird.

Im Anschluß an Gutenberg wird die Preis-Absatz-Funktion bei polypolistischer Konkurrenz mit monopolistischen Spielräumen üblicherweise mit zweifach geknicktem Verlauf dargestellt ${ }^{19)}$; diese Darstellungsweise läßt sich

18) Von den Möglichkeiten einer Verlagerung ins Ausland soll hier abgesehen werden.

19) Gutenberg, Erich, Zur Diskussion der polypolistischen Absatzkurve, in: Jahrbücher für Nationalökonomie und Statistik. Bd. 177, Heft 4 (1965), S. 289-303; zur Bewertung vgl. Ott, Alfred E., Preistheorie. a.a.O., S. 169 ff. 
auch auf die hier betrachtete interkommunale Konkurrenz bei sachlicher und räumlicher Steuerausweichung übertragen.

Abbildung 4: $\quad$ Monopolistischer Steuersatz-spielraum bei räumlicher und sachlicher Ausweichung

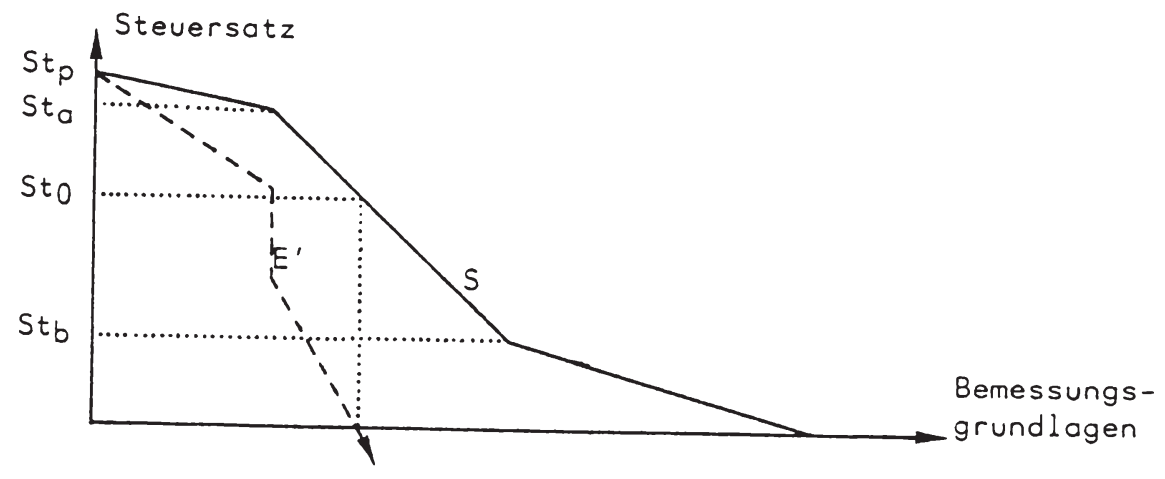

Innerhalb des in Abbildung 4 dargestellten Steuersatzspielraums der Gemeinde zwischen $\mathrm{St}_{\mathrm{a}}$ und $\mathrm{St}_{\mathrm{b}}$ führt eine Steuersatzerhöhung nicht zur räumlichen Verlagerung, sondern nur zur sachlichen Ausweichung. Eine Steuersenkung bewirkt aber andererseits auch keine räumliche Anziehung besteuerbarer Aktivitäten, sondern regt nur solche latenten Aktivitäten an, die innerhalb der Gemeinden bisher allein aus steuerlichen Gründen vermieden worden sind. Erst bei Über- oder Unterschreiten der Schwellensteuersätze $\mathrm{St}_{\mathrm{a}}$ nach oben bzw. $\mathrm{St}_{\mathrm{b}}$ nach unten kommt es zu einer Verlagerung der besteuerten Aktivitäten zusätzlich zur sachlichen Ausweichung. In diesem Moment beeinflussen die Strategien einer Gemeinde den fiskalischen Erfolg anderer Gemeinden; es kann von einer Marktbeziehung ${ }^{20)}$ zwischen den Gemeinden gesprochen werden.

Für die Gemeinden ist der Verlauf der Steuerzahlungsfunktion und insbesondere der monopolistische Bereich von besonderem Interesse. Zwar ist es nicht notwendig so, daß der fiskalisch optimale Steuersatz innerhalb des monopolistischen Bereichs liegt und daß das unter den Bedingungen der mögli-

20) Vgl. Willeke, Franz-Ulrich, Wettbewerbspolitik, Tübingen 1980, S. 13. 
chen sachlichen und räumlichen Steuerausweichung maximal erzielbare Aufkommen ohne Konkurrenzbeziehungen zu anderen Gemeinden erreicht werden kann. Dieser Fall ist jedoch um so wahrscheinlicher, je größer der monopolistische Spielraum ist. Für die betreffende Gemeinde ist damit insofern ein Vorteil verbunden, als sie bei der Gestaltung der Steuersatzpolitik - soweit sie allein fiskalischen Zwecken dient - keine Abwanderung und damit rapide sinkende Bemessungsgrundlagen befürchten muß. Deshalb wird jede Gemeinde bei ihrer Haushaltspolitik bestrebt sein, ihren "Monopolbereich" auszudehnen und sich als Standort der besteuerbaren Aktivitäten und Tatbestände möglichst unersetzbar zu machen. Auf diese Strategien wird in Abschnitt C einzugehen sein. Zunächst sollen jedoch die Bestimmungsgründe monopolistischer Steuersatz-Spielräume unter Status-quo-Bedingungen betrachtet werden.

Zunächst hängt die Bandbreite des monopolistischen Steuersatz-Spielraums von der Wahl des Steuergegenstandes ab. Die Bemessungsgrundlage bestimmt sowohl über Möglichkeiten der sachlichen Ausweichung und über den Anreiz zur räumlichen Verlagerung der besteuerten Aktivitäten oder Tatbestände als auch über den Typ der Verlagerung. So ist etwa der Verlagerungsdruck bei einer Einkommens- oder Gewinnbesteuerung hoch, weil kaum Möglichkeiten der sachlichen Ausweichung bestehen, die räumliche Ausweichung kann jedoch nur über einen Standortwechsel erfolgen und ist deshalb mit besonders hohen Kosten verbunden, die die Vorteile einer räumlichen Ausweichung schmälern. Demgegenüber sind die Möglichkeiten der räumlichen Ausweichung beispielsweise bei einer kommunalen Umsatzsteuer wesentlich größer und kostengünstiger. Die Steuersatz-Spielräume dürften hier deswegen deutlich kleiner als bei einer Gewinnsteuer sein. Im Falle einer kommunalen Besteuerung des Vermögens am Ort der Anlage ist dessen Mobilität entscheidend; Geldvermögen stellt das eine, völlig mobile, Grundvermögen das andere, vollkommen immobile Extrem dar.

Die zweite Determinante des monopolistischen Steuersatz-Spielraums ist die Marktstellung und das "akquisitorische Potential" ${ }^{\text {21) }}$ der Gemeinden gegenüber anderen potentiell um Steuergegenstände und Steuerzahler

21) Für den privaten Sektor bezeichnet Gutenberg damit die Fähigkeit eines Unternehmens, Nachfrager gegenüber potentiellen Konkurrenten an sich zu binden. Vgl. Gutenberg, Erich, Zur Diskussion der polypolistischen Absatz-Kurve, a.a.O., S. 295. 
konkurrierenden Gemeinden. Weder die konkurrierenden Gemeinden noch die standortsuchenden Wirtschaftssubjekte sind in ihren Angeboten bzw. ihren Ansprüchen an den erwünschten Standort oder den Ort ihrer Aktivitäten homogen. Daraus resultiert zunächst ein Aufspaltung des "Gesamtmarktes" in "Teilmärkte": Innerhalb der Teilmärkte ist die Konkurrenz zwischen den Gemeinden intensiver als über die Grenzen der Teilmärkte hinweg. Wird von den Wirtschaftssubjekten für ihre Aktivitäten etwa ein Ort mit einer gewissen Mindestverdichtung bevorzugt, so kommen als mögliche Ausweichziele nur solche Gemeinden in Frage, die diese Anforderungen ebenfalls erfüllen, nicht aber Gemeinden außerhalb von Verdichtungsgebieten.

Je spezifischer die Ansprüche in bezug auf Arbeitsmarkt, Infrastruktur, räumliche Lage oder Wohn- und Lebensqualität sind, die sich auf einen Gemeindetyp richten und den die jeweilige Gemeinde befriedigen kann desto weniger Wanderungsalternativen stehen den betreffenden Wirtschaftssubjekten offen. Für sie kommen nur wenige andere Standorte in Frage, die entweder die gleichen Qualitäten aufweisen wie der alte Standort oder die in räumlicher Nähe eines solchen Standorts liegen und so die Nutzung seiner Vorteile erlauben.

Innerhalb der Teilmärkte sind die Wanderungsbewegungen daher eher steuersatzabhängig als über die Grenzen der Teilmärkte hinweg. ${ }^{22)}$ Die monopolistischen Steuersatz-Spielräume hängen hier vor allem von den Verlagerungskosten (im weitesten Sinne) und von der Konkurrenz der Standortsuchenden $\mathrm{ab}$.

Beziehungen zwischen den Teilmärkten ergeben sich vor allem aus dem Vergleich der "Preis-Leistungs-Verhältnisse" zwischen verschiedenen Gemeindetypen. So ist etwa denkbar, daß der ursprüngliche Standort wegen seines vergleichsweise niedrigen Steuersatzes gewählt wurde. In diesem Fall kann die Anhebung des Steuersatzes zur Ausweichung in eine Gemeinde mit gleich hohem Steuersatz, aber qualitativ günstigeren Bedingungen für den be-

22) Diese These hat sich auch für Standortuntersuchungen für die konkreten Bedingungen der Bundesrepublik Deutschland bestätigt. Vgl. etwa Kaiser, Karl-Heinz, Industrielle Standortfaktoren und Betriebstypenbildung. Berlin 1979. S. 26. 
steuerten Tatbestand oder die besteuerte Aktivität führen. Der Teilmarkt wird dann verlassen.

Neben den Standortansprüchen spielt die Mobilitätsbereitschaft der Zensiten eine Rolle. Je stärker die Wirtschaftssubjekte an den derzeitigen Standort gebunden sind - sei es aufgrund hoher Mobilitätskosten, aufgrund persönlicher Bindungen oder anderer Ursachen - desto eher kann die Gemeinde ihnen gegenüber eine monopolistische Stellung einnehmen.

Zusammenfassend ist noch einmal festzuhalten, daß auch bei in allen Gemeinden gleichartigen Steuern die Einnahmespielräume und die fiskalisch optimalen Steuersätze in den einzelnen Gemeinden durchaus unterschiedlich hoch sein dürften. Ein überall weitgehend identisches kommunales Steuersystem unterstellt, werden diese Spielräume vor allem dadurch bestimmt, welche Ansprüche von den Wirtschaftssubjekten an die lokalen Bedingungen gestellt werden, was sie dafür zu zahlen bereit und fähig sind und welche Bedingungen die einzelne Gemeinde im Vergleich mit anderen Gemeinden zu bieten in der Lage ist.

\section{Zusammenfassung}

Ausgehend von der Gemeinde als territorialem Monopol als Ort für die - besteuerbaren - Aktivitäten der Wirtschaftssubjekte, werden die steuerlichen Einnahmemöglichkeiten einer Gemeinde von der Summe der Bemessungsgrundlagen und den Reaktionen der Zensiten auf Steuersatzerhöhungen bestimmt. Wichtigste Determinanten der sachlichen Ausweichung sind die Substituierbarkeit des Steuergegenstandes und die finanzielle Leistungsfähigkeit der Besteuerten.

Die Möglichkeiten der sachlichen Ausweichung werden im föderativen Staat jedoch auf kommunaler Ebene durch die Alternative der räumlichen Ausweichung ergänzt: Sie wird dann wahrgenommen, wenn sie aus der Sicht der Zensiten günstiger ist als eine sachliche Ausweichung und die Vorteile des neuen Standortes für die besteuerten Aktivitäten die Kosten des Standortwechsels überkompensieren. 
Aus der Sicht der Wirtschaftssubjekte ist ein Wechsel des Standorts um so schwieriger, je weniger Orte die gewünschten Qualitäten bieten; sofern die Nachfrage nach den betreffenden Standorten besonders hoch ist, resultiert für jene Gemeinden, die diesen Ansprüchen genügen, ein vergleichsweise großer monopolistischer Steuersatz-Spielraum. Gemeinden mit ubiquitärem Angebot sind hingegen der Konkurrenz um Steuerzahler weit intensiver ausgesetzt; ihre monopolistischen Spielräume sind entsprechend eingeschränkt.

Die Reaktionen der Wirtschaftssubjekte wurden jeweils beispielhaft für eine einzige Steuer und für den Vergleich verschiedener Steuern vorgeführt. Die Wirkungen der Reaktion auf das kommunale Steuerpotential konnten hier relativ leicht nachvollzogen werden. Die Analyse wird komplizierter, wenn die Gemeinden mehrere Steuern erheben. Die Steuersatzanhebung bei einer der Steuern wirkt sich dann nicht nur auf die Bemessungsgrundlagen dieser Steuer, sondern auch auf die Einnahmemöglichkeiten aus den übrigen Steuern aus. Der Zusammenhang zwischen den Einzelpotentialen kann direkter Art sein - wenn etwa die zu leistende Steuerzahlung einer Steuer unmittelbar die Bemessungsgrundlagen der anderen Steuer kürzt - oder indirekt durchschlagen - wenn über die sachlichen und räumlichen Ausweichreaktionen die Bemessungsgrundlagen anderer Steuern geschmälert werden. Beide Wirkungen sind im konkreten Fall einer Untersuchung gemeindlicher Einnahmemöglichkeiten zu berücksichtigen.

Greift man abschließend noch einmal die in Teil C. aufgestellte These auf, daß die Besteuerungsmöglichkeiten der Gemeinden durch das regionale Einkommen und Vermögen begrenzt würden, so ist zunächst noch einmal festzuhalten, daß die Reaktionen der Wirtschaftssubjekte die kommunalen Einnahmemöglichkeiten auf ein wesentlich niedrigeres Niveau begrenzen. Darüber hinaus erscheint der regionale Reichtum aber auch als Maßstab für den interkommunalen Vergleich nicht unbedingt geeignet:

Je nach dem angebots- und nachfrageseitig bestimmten akquisitorischen Potential und den daraus resultierenden monopolistischen Steuersatz-Spielräumen einer Gemeinde ist ein mehr oder weniger massiver Zugriff auf die regionalen Einkommen und Vermögen möglich, ohne daß die Zensiten räumlich ausweichen könnten. Insofern kann die weitergehende These aufgestellt werden, daß in den verschiedenen Gemeindetypen ein um so größerer Anteil 
des Reichtums für kommunale Zwecke weggesteuert werden kann, je stärker die monopolistische Stellung der Gemeinde ausgeprägt ist und je leistungsfähiger die angesiedelten Wirtschaftssubjekte sind, wodurch der Zwang zur sachlichen Ausweichung abgemildert wird.

\section{d. Begrenzungen kommunaler Besteuerungsmöglichkeiten durch staatliche Rahmensetzung}

Die interkommunalen Marktbeziehungen eröffnen den Gemeinden trotz der begrenzenden Wirkungen insgesamt Besteuerungsspielräume, die aus gesamtstaatlicher Sicht als zu umfangreich angesehen werden könnten und die gegebenenfalls noch weitergehend einzuschränken wären.

Für eine Einschränkung der gemeindlichen Besteuerungsmöglichkeiten können aus staatlicher Sicht eine ganze Reihe von Argumenten sprechen. So kann etwa angeführt werden, daß bestimmte Objekte gänzlich unbelastet bleiben sollten oder die Belastung zumindest auf staatlicherseits erhobene Abgaben beschränkt bleiben soll, weil die betreffenden Tatbestände aus gesamtwirtschaftlicher Sicht für besonders wünschenswert gehalten werden und daher nicht zurückgedrängt werden sollen. Als weiteres Argument kann eine besondere Empfindlichkeit der Zensiten - etwa aufgrund von Liquiditätsproblemen - gegenüber der Abgabenerhebung gelten. Als Beispiele können die Herausnahme einzelner Produktionszweige oder -typen aus der Besteuerung oder Steuerbefreiungen für bestimmte ökonomische Aktivitäten dienen.

Neben der Eingrenzung der mit Steuern belastbaren Tatbestände kann eine Festlegung von Steuerobergrenzen vorgenommen werden, etwa weil die Kosten der Anpassung in Grenzen gehalten werden sollen. Sowohl sachliche als auch räumliche Ausweichungen sind mit Wohlfahrtsverlusten verbun$\mathrm{den}^{23)}$, d.h., daß im Wege der Steuererhebung nicht nur eine Umverteilung von erwirtschafteten Mitteln vom privaten zum öffentlichen Sektor vorgenommen wird, sondern dabei eine gesamtwirtschaftliche Zusatzlast in Form unterlassener wirtschaftlicher Aktivitäten entsteht. Der öffentliche Sektor

23) Ausführlicher wird dieses Problem untersucht von Musgrave, Richard Abel, Musgrave, Peggy B. und Kullmer, Lore, Die öffentlichen Finanzen in Theorie und Praxis, 3. Bd., 1. Aufl., Tübingen 1977, S. 144 ff. 
kann sich daher nur einen Teil dessen aneignen, was ohne die Abgabenbelastung produziert und an Vermögen gebildet worden wäre. Diese - nicht im Aufkommen abgebildete - Zusatzlast gering zu halten kann ebenfalls Aufgabe einer Festlegung von Abgabenobergrenzen sein. Daneben könnten eine Reihe weiterer Begründungen für staatliche Einschränkungen kommunaler Steuerpolitik angesprochen werden.

Zwar haben auch die Gemeinden nicht-fiskalische Interessen; unter dem hier ausschließlich betrachteten Interesse der Gemeinden an den fiskalischen Möglichkeiten sind jedoch nur die Wirkungen, nicht aber die aus staatlicher Sicht vorgebrachten Begründungen für eine Einschränkung der Besteuerungsmöglichkeiten von Bedeutung. Diese Argumente sind hingegen im 4. Kapitel näher zu betrachten, wenn die aus staatlicher Sicht zu ermittelnde, weitgehend normativ bestimmte Steuerkraft der Gemeinden untersucht wird.

\section{Festlegung von Steuerquellen und Bemessungsgrundlagen}

Eine Einschränkung kommunaler Steuerpolitik kann darin bestehen, daß den Gemeinden von übergeordneten Gebietskörperschaften bestimmte Steuerquellen zur Ausschöpfung zugewiesen werden und darüber hinausgehende Besteuerungsmöglichkeiten eng begrenzt oder sogar ausgeschlossen werden.

Mit solchen Einschränkungen können sowohl die Bemessungsgrundlagen als auch die Reaktionsverläufe auf kommunale Steuerpolitik und die Konkurrenzsituation der Gemeinden untereinander entscheidend beeinflußt werden. Mit der Auswahl der Steuerobjekte wird über die Möglichkeiten der sachlichen sowie der räumlichen Ausweichung entschieden. Auf objektbedingte Ausweichhemmnisse wurde oben bereits hingewiesen. Darüber hinaus wird eine Selektion über die Zensiten vorgenommen, deren subjektiv bedingte Reaktionsmuster durchaus unterschiedlich sein können.

Als Beispiel für den "Bemessungsgrundlagen-Effekt" sei auf die Regelungen der Gewerbesteuer in der Bundesrepublik Deutschland hingewiesen, die isoliert betrachtet "Produktionsgemeinden" besserstellt als Wohngemeinden, darüber hinaus kleinbetriebliche Produktionsweise begünstigt, also die Einnahmemöglichkeiten jener Gemeinden, in denen kleinbetriebliche Produktion 
vorherrscht, stärker einschränkt als die großbetrieblich strukturierter Gemeinden. ${ }^{24)}$

Außer dem Bemessungsgrundlagen-Effekt tritt insofern eine Wirkung auf den Reaktionsverlauf ein, als Großbetriebe anders auf Steuersatzerhöhungen reagieren dürften als Kleinbetriebe. Werden letztere aus der Besteuerung herausgenommen, so wird damit auch der "gesamtgemeindliche" Reaktionsverlauf verändert.

Ohne solche Einschränkungen der Besteuerungsmöglichkeiten könnte jede Gemeinde diejenigen Aktivitäten und Tatbestände besteuern, von denen die höchsten Steuereinnahmen zu erwarten wären. Eine übergemeindliche Vorgabe der ausschöpfbaren Steuerquellen beschränkt diese Möglichkeit; dabei treten natürlich interkommunal um so größere "Verzerrungen" auf, je selektiver die Auswahl der Steuerquellen ist und je unterschiedlicher die Gemeinden strukturiert sind.

\section{Festlegung von Höchst- und Mindeststeuersätzen}

In Abschnitt c wurde zu zeigen versucht, daß sich die Gemeinden je nach Konkurrenzposition und nach der Leistungskraft der angesiedelten Wirtschaftssubjekte unterschiedlichen Steuerzahlungs-Funktionen gegenübersehen und die fiskalisch optimalen Steuersätze deshalb ebenfalls unterschiedlich hoch ausfallen.

Werden nun in dieser Situation staatlicherseits Höchststeuersätze festgelegt, so werden die Gemeinden dadurch nicht gleichermaßen betroffen; allerdings kommt es auch nicht zu einer vollständigen Angleichung der Steuersatz-Spielräume, sofern nicht bestimmte Bedingungen erfüllt werden.

Abbildung 5 zeigt die Steuerzahlungs-Funktionen $S_{1}$ und $S_{2}$ in zwei verschiedenen Gemeindetypen.

24) Vgl. dazu auch Karrenberg, Hanns. Die Bedeutung der Gewerbesteuer für die Städte, Neue Schriften des Deutschen Städtetages, Heft 52, Stuttgart u.a. 1985, S. 64 f. 


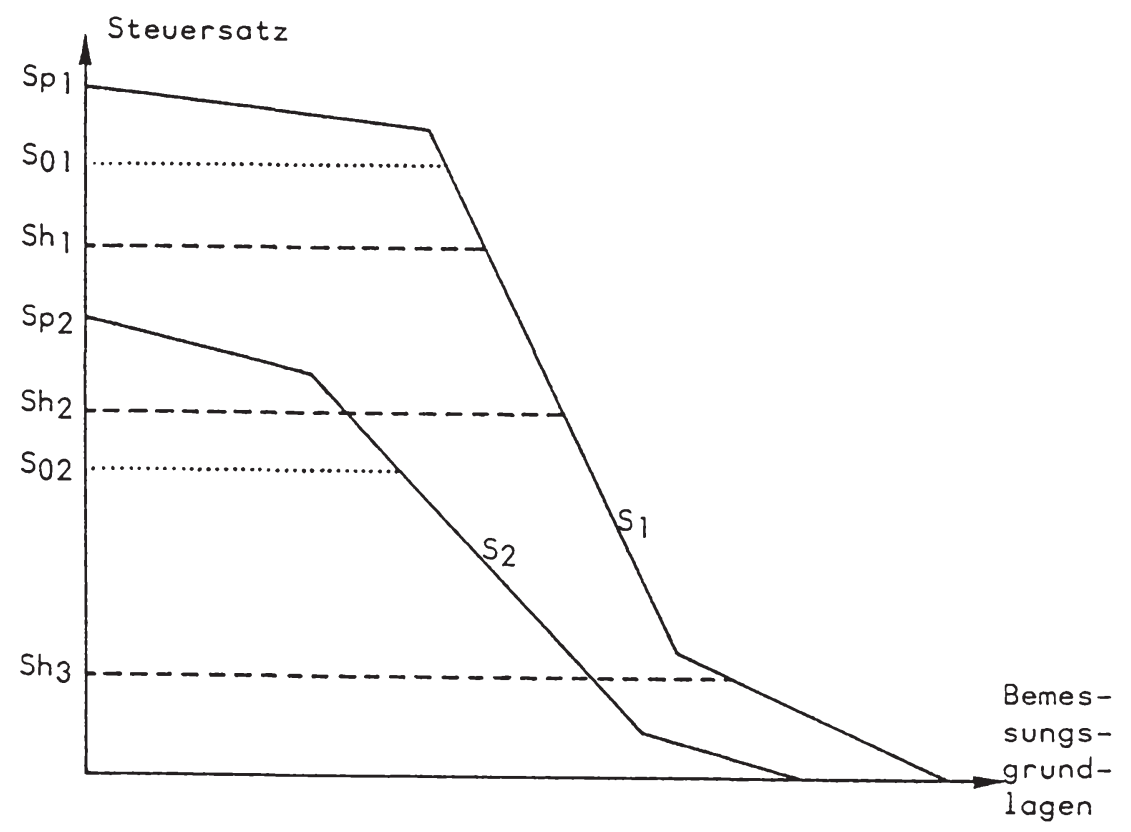

In beiden Gemeinden existieren monopolistische Steuersatz-Spielräume. Werden sie überschritten, so setzen wie dargestellt verstärkt Abwanderungstendenzen zu konkurrierenden Gemeinden ein; wird ein bestimmter Steuersatz unterschritten, so werden Wirtschaftssubjekte aus anderen Gemeinden angezogen. Die fiskalisch optimalen Steuersätze liegen in beiden Gemeinden innerhalb des monopolistischen Bereichs, und zwar in Gemeinde 1 bei $S_{01}$ und in Gemeinde 2 bei $\mathrm{S}_{02}$.

Wird der Höchststeuersatz in dieser Konstellation bei $S_{h 1}$ angesetzt, so wird das Einnahmepotential der Gemeinde 2 davon überhaupt nicht berührt, da er oberhalb des Prohibitivsteuersatzes $\mathrm{Sp}_{2}$ liegt. In Gemeinde 1 wird das Einnahmepotential jedoch verringert, da der fiskalisch optimale Steuersatz $\mathrm{S}_{01}$ nicht mehr erreicht werden kann. Durch die Festlegung des Höchsthebe- 
satzes werden so die Unterschiede in den Steuersatz-Spielräumen zwar verringert, nicht aber beseitigt.

Ähnliches gilt für die Begrenzung des Steuersatzes auf $\mathrm{S}_{\mathrm{h} 2}$, der $z w a r$ in beiden Gemeinden innerhalb des monopolistischen Bereichs liegt, aber immer noch oberhalb des fiskalisch optimalen Steuersatzes der schwächeren Gemeinde 2 .

Erst dann, wenn der Höchststeuersatz $\mathrm{S}_{02}$ unterschreitet - oder allgemeiner formuliert: niedriger oder gleich dem optimalen Steuersatz der schwächsten Gemeinde ist - stoßen die Möglichkeiten, über Steuersatzerhöhungen zusätzliche Einnahmen zu erzielen, in beiden Gemeinden an die gleiche Grenze. Allerdings kann nun der Fall eintreten, daß die steuerstärkere Gemeinde in jenen Bereich ihrer Steuerzahlungsfunktion hineingedrängt wird, in dem über "Steuersatzkonkurrenz" Wirtschaftssubjekte angezogen werden, die bisher in anderen Gemeinden angesiedelt waren. Gemeinde 2 sähe sich in diesem Moment einer veränderten Steuerzahlungsfunktion gegenüber, die möglicherweise einen fiskalisch optimalen Steuersatz unterhalb des optimalen Steuersatzes von Gemeinde 1 aufwiese.

Daraus folgt, daß eine Festlegung von Steuersatzobergrenzen allein noch nicht garantiert, daß das Einnahmepotential in allen Gemeinden beim gleichen Steuersatz erreicht wird. Vielmehr muß dieser Höchststeuersatz innerhalb eines bestimmten Bereichs festgelegt werden, der durch den Verlauf der Steuerzahlungsfunktionen, also durch die Konkurrenzpositionen der einzelnen Gemeinden und die sachlichen Ausweichmöglichkeiten auf die Besteuerung bestimmt wird. In extremen Fällen wäre sogar denkbar, daß ein solcher Bereich nicht existiert.

Für die einzelne Gemeinde ist die individuelle Situation entscheidend, in der die Steuersatzobergrenze greift: Gemeinden mit hohen Steuersatz-Spielräumen werden tendenziell eher und stärker eingeschränkt als Gemeinden mit engeren und niedrigeren Steuersatz-Spielräumen. Aber auch jene Gemeinden, die durch die Steuersatzobergrenzen nicht direkt eingeschränkt werden, weil ihr fiskalisch optimaler Steuersatz ohnehin unterhalb des Maximalsteuersatzes liegt, können durch eine Verschärfung der Konkurrenzsituation betroffen werden. Da die räumliche Ausweichung in besser ausgestattete Gemeinden für die Wirtschaftssubjekte kostengünstiger wird, werden Verlagerungsten- 
denzen gestärkt und die monopolistischen Spielräume des gesamten "Teilmarktes" eingeengt.

Neben der staatlichen Festlegung von Steuersatzobergrenzen ist auch die Festsetzung von Mindeststeuersätzen möglich. Je nach Niveau der Mindestbesteuerung kann eine solche Regelung ebenfalls unterschiedliche Wirkungen hervorbringen. Sie verhindert zunächst, daß die Gemeinden über besonders niedrige Steuersätze - oder sogar die Nicht-Erhebung von Steuern - in eine verschärfte Konkurrenz um Steuerzahler eintreten. Je höher der Mindeststeuersatz angesetzt wird, desto eher ist jedoch damit zu rechnen, daß Gemeinden mit ungünstiger "Marktposition" und ohnehin eingeschränkten monopolistischen Steuersatz-Spielräumen zur Festsetzung eines aus ihrer Sicht überhöhten Steuersatzes gezwungen und damit nach oben in die Konkurrenz mit besser ausgestatteten Teilmärkten gedrängt werden.

\section{Festlegung von Kopplungsvorschriften}

Kopplungsvorschriften berühren die Steuersatz-Spielräume der Gemeinden nur indirekt über die unterschiedlichen Reaktionsmöglichkeiten der Zensiten auf die kommunale Steuerpolitik. Geht man zunächst von einer Kopplungsvorschrift aus, die die Steuersätze zweier Steuern mit einem ähnlich Reaktionsverlauf miteinander verbindet, so werden die gemeindlichen Einnahmemöglichkeiten dadurch kaum eingeschränkt.

Abb. 6 stellt diesen Fall dar, wobei angenommen wird, daß für beide Steuern identische Steuersätze vorgeschrieben werden. Der neue fiskalisch optimale Steuersatz für beide Steuern läßt sich nun aus der Addition der beiden Steuerzahlungsfunktionen ermitteln. Im dargestellten Beispiel liegt er bei $\mathrm{S}_{0 \mathrm{~g}}$, also unterhalb des optimalen Steuersatzes der Steuer $S_{1}$ und oberhalb des fiskalisch optimalen Steuersatzes von $S_{2}$. Bei beiden Steuern müssen also Beschränkungen der Einnahmemöglichkeiten hingenommen werden, weil die fiskalisch optimalen Steuersätze der Einzelsteuern bei rationaler kommunaler Steuerpolitik nicht mehr verwirklicht werden. Allerdings sind diese Einbußen im ersten abgebildeten Fall relativ gering, weil die optimalen Steuersätze für beide Steuern nicht weit auseinanderliegen. 


\section{Abbildung 6: Wirkung einer Kopplungsvorschrift bei ähnlichem}

Reaktionsverlauf auf Steuersatzvariationen

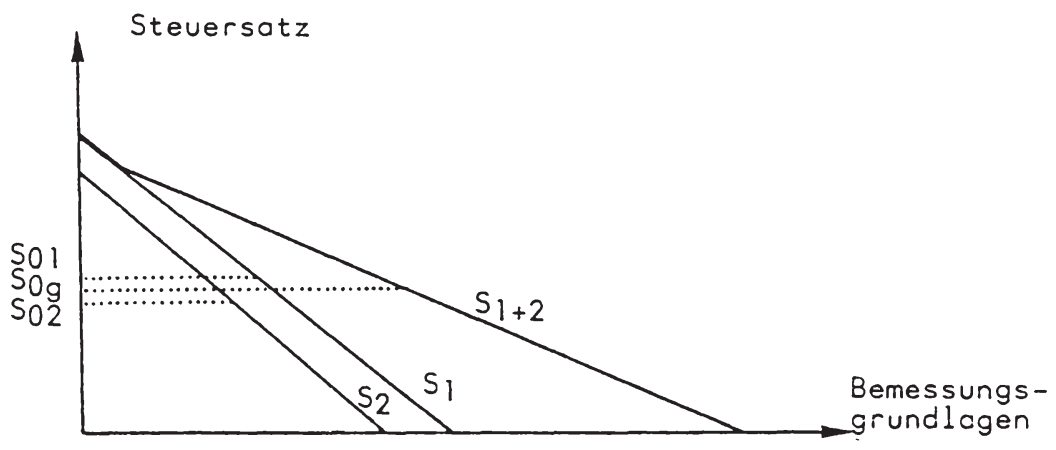

Anders im 2. abgebildeten Fall. Abb. 7 zeigt die Reaktionsverläufe für zwei Steuern mit sehr unterschiedlichen Möglichkeiten der räumlichen und sachlichen Anpassung.

Abbildung 7: Wirkung einer Kopplungsvorschrift bei sehr unterschiedlichen Reaktionsverläufen auf

Steuervariationen

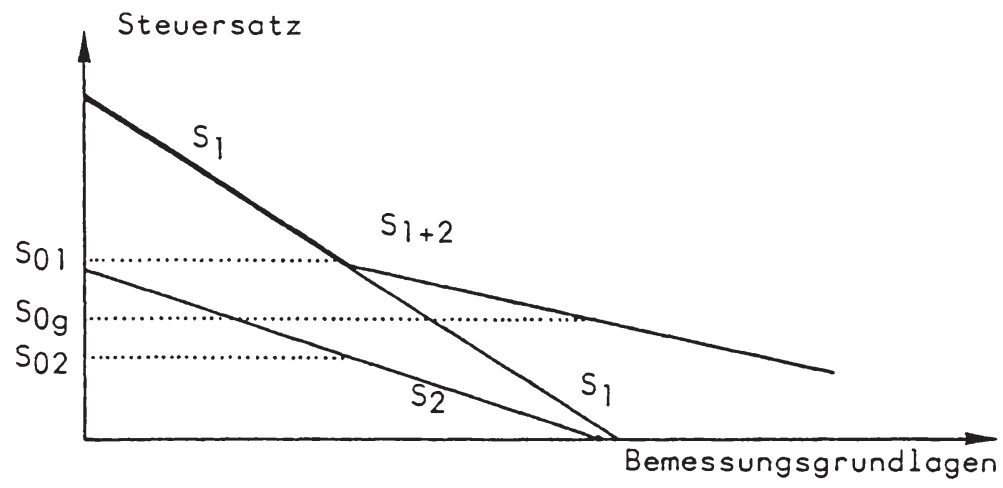


Der sehr viel flachere Verlauf der Funktion $\mathrm{S}_{2}$ drückt aus, daß die Bemessungsgrundlagen der Steuer 2 eher substituierbar oder mobiler sind als die der Steuer 1. Durch die Kopplungsvorschrift werden nun die Bemessungsgrundlagen der Steuer 1 quasi "geschützt". Weil der fiskalisch optimale Steuersatz der Steuer 1 über dem Prohibitivsteuersatz 2 liegt, wären bei einer Festsetzung des gemeinsamen Steuersatzes in dieser Höhe nur Einnahmen aus der Steuer 1 zu erzielen. Fiskalisch günstiger ist es jedoch, den Steuersatz niedriger, nämlich bei $S_{0 g}$, der wiederum aus der Addition von $S_{1}$ und $S_{2}$ ermittelt werden kann, anzusetzen, um die Summe der Steuereinnahmen zu maximieren.

Als Ergebnis kann festgehalten werden, daß Kopplungsvorschriften die Einnahmespielräume um so stärker einschränken, je weiter die fiskalisch optimalen Steuersätze auseinanderliegen und je enger die Kopplungsvorschrift die Steuersätze der verschiedenen Steuern aneinander bindet.

Betrachtet man die Wirkung von Kopplungsvorschriften im Vergleich der Gemeindetypen oder "Teilmärkte", so kommt man zu folgendem Ergebnis:

Eine in allen Gemeindetypen gleichermaßen einschränkende Wirkung tritt nur dann ein, wenn die Reaktionsverläufe der im Tarif gekoppelten Steuern überall etwa im gleichen Verhältnis zueinander stehen. Differenzierende Wirkungen gehen von Kopplungsvorschriften hingegen dann aus, wenn die Gemeindetypen sich vor allem durch den Reaktionsverlauf einer Steuer unterscheiden. Knüpft eine Steuer etwa in allen Gemeinden an immobilen Objekten an, die andere an eher mobilen Objekten, so ist der Reaktionsverlauf für letztere stärker von der individuellen Ausstattung und der Konkurrenzposition der Gemeinde gegenüber anderen Gemeinden abhängig; hier dürften sich daher stärkere Unterschiede zeigen als bei der ersten Steuer. Die Kopplungsvorschrift schränkt in diesem Fall die Besteuerungsmöglichkeiten jener Gemeinden am stärksten ein, die sich in einer besonders vorteilhaften Konkurrenzposition befinden und deren monopolistischer Spielraum ohne staatliche Beschränkung vergleichsweise groß ist. Durch die Verschärfung der Konkurrenz werden letztlich jedoch auch konkurrenzschwächere Gemeinden betroffen. 


\section{e. Gemeindliche Zielsetzungen als Begrenzung des eigenen Steuerpotentials?}

Für die gemeindliche Steuerpolitik wie für die Einnahmepolitik insgesamt sind natürlich nicht nur fiskalische Motive ausschlaggebend. Vielmehr werden eine Reihe ökonomischer, sozialer, kultureller und anderer Ziele verfolgt, die die Einnahmepolitik faktisch über die bisher betrachteten Begrenzungen der Mittelbeschaffung hinaus einengen.

Dazu gehören nicht zuletzt wahlkampftaktische Überlegungen, die es ratsam erscheinen lassen können, das innerhalb der ökonomischen und gesetzlichen Restriktionen erzielbare Einnahmepotential nicht voll auszuschöpfen.

Mit Blick auf die Besteuerung können soziale Überlegungen etwa dann eine Rolle spielen, wenn immobile Tatbestände nur bis zu einem gewissen für erträglich gehaltenen Grad belastet werden. Wichtiger noch sind vermutlich Wachstumszielsetzungen der gemeindlichen Politik, die nicht allein einem langfristig orientierten fiskalischen Interesse dienen. So kann es etwa um die Arbeitsmarktsituation gehen, wenn bestehende Steuersatzspielräume auch langfristig für nicht ausschöpfbar gehalten werden. Deshalb ist davon auszugehen, daß in der Regel keine fiskalisch optimale Steuerpolitik betrieben wird, weil sie wichtigen kommunalen Zielsetzungen widerspräche. ${ }^{25)}$

Häufiger noch als im Falle der Besteuerung werden den Gemeinden bei der Entgeltpolitik eigene Zielsetzungen zugesprochen, die die Einnahmebeschaffung begrenzen. Hier wird insbesondere auf die Gebühren als Instrument zur Nachfragelenkung hingewiesen.

Weitere Zielsetzungen und Begründungen für die Nicht-Ausschöpfbarkeit des ökonomisch und gesetzlich begrenzten Steuer- bzw. allgemeiner: des Einnahmepotentials - ließen sich finden und aufzählen. Allerdings ist die Frage zu stellen, ob die genannten - und andere - gemeindlichen Zielsetzungen als Begrenzung der Einnahmemöglichkeiten mit den bisher betrachteten

25) Nach Postlep sind solche Zielverluste, die durch die Einnahmebeschaffung verursacht werden. als Kosten der Einnahmebeschaffung zu interpretieren. Sie sind eine der Determinanten kommunaler Haushaltspolitik. Vgl. Postlep. Rolf-Dieter. Wirtschaftsstruktur und großstädtische Finanzen, Veröffentlichungen der ARL. Abhandlungen Bd. 87, Hannover 1985, S. $81 \mathrm{ff}$. 
Restriktionen überhaupt vergleichbar und als weitere Einschränkung des Einnahmepotentials anzusehen sind.

In der Literatur werden die genannten Zielsetzungen zum Teil ebenfalls unter die Grenzen der Besteuerung subsumiert: "Die Frage nach den Grenzen der Besteuerung ist also ihrem Wesen nach eine Frage des Sollens, nicht eine des Könnens." ${ }^{26)}$ Nur für extreme Notsituationen wird der Frage nach dem "Maximum der möglichen Inanspruchnahme der Volkswirtschaft" überhaupt eine gewisse Berechtigung zugesprochen. ${ }^{27}$ Dem ist allerdings entgegenzuhalten, daß hier bereits der Übergang zur Betrachtung der "optimalen Besteuerung" vollzogen ist ${ }^{28)}$; Im Vordergrund stehen dabei normative Aspekte, die ohne eine nähere Zieldiskussion nicht sinnvoll analysiert werden können. Die Frage nach den objektiven Rahmenbedingungen kommunaler - oder auch staatlicher - Steuerpolitik kann davon gesondert gestellt werden.

Überdies müßte man - würde man die gemeindlichen Ziele und Motive als Grenzen der Besteuerungsmöglichkeiten anerkennen - zwangsläufig zu dem Ergebnis kommen, daß das gemeindliche Steuerpotential den tatsächlichen Steuereinnahmen gleichzusetzen wäre. Unter der Bedingung eines gegebenen Angebots würde damit entweder jede Möglichkeit bestritten, etwa steigende Preise oder dringlicher werdende Erhaltungsmaßnahmen im Bereich der kommunalen Infrastruktur zu finanzieren, oder man müßte so argumentieren, daß mit jeder neuen Disposition einer Gemeinde auch deren Einnahmepotential einer Veränderung unterliegt. Dieser Auffassung kann jedoch nicht zugestimmt werden.

Die Praxis zeigt, daß in den genannten Fällen oft genug gemeindeinterne Zielvorstellungen durchbrochen und die Abgabenbelastung entgegen vorausgegangenen Äußerungen und Begründungen erhöht wird. Diese Praxis soll nicht kritisiert werden - sicherlich ist sie oft notwendig - allerdings zeigt sich daran ein qualitativer Unterschied zwischen "gemeindeexternen", vorgegebenen Begrenzungen und "gemeindeinternen", selbst gesetzten Grenzen der Einnahmebeschaffung: Letztere sind durch Entscheidung zu beseitigen oder hinauszuschieben, die erstgenannten hingegen nicht.

26) Heller, Wolfgang. Die Grenzen der Besteuerung, in: Festgabe für Georg Schanz, Bd. II, hrsg. von Hans Teschemacher, Tübingen 1928, S. 87-111, hier S. 89.

27) Ebenda, S. 90.

28) Ebenda, S. 90. 
Insofern erscheint es gerechtfertigt, gemeindliche Zielsetzungen auch aus der Sicht der Gemeinden selbst als für die Bestimmung des Einnahmepotentials irrelevante Größen einzustufen. Allein die außerhalb des gemeindlichen Entscheidungsprozesses anzusiedelnden Begrenzungen determinieren das Einnahmepotential; gemeindliche Ziele und Vorstellungen entscheiden hingegen über dessen Ausschöpfung und sind deshalb erst zur Erklärung tatsächlicher Einnahmepolitik relevant.

III. Kommunale Besteuerungsspielräume im Gemeindefinanzsystem der Bundesrepublik Deutschland und ihre Determinanten im interkommunalen Vergleich

Die in Abschnitt II. allgemein beschriebenen Begrenzungen kommunaler Besteuerung gelten grundsätzlich auch im Gemeindefinanzsystem der Bundesrepublik Deutschland. Besondere Bedeutung kommt hier den staatlicherseits gesetzten Rahmenbedingungen zu, die die gemeindlichen Besteuerungsrechte qualitativ weitgehend festlegen und auch quantitativ begrenzen.

Umstritten ist hingegen die Wirksamkeit ökonomischer Verhaltensänderungen der Zensiten - sachlicher und insbesondere räumlicher Ausweichreaktionen - und der interkommunalen Konkurrenz als Einschränkung fiskalisch sinnvoller, d.h. einnahmesteigernder Steuerpolitik. Diesem Aspekt wird daher anschließend besondere Aufmerksamkeit gewidmet. Ausgangspunkt ist hier nun die konkrete Ausgestaltung des kommunalen Steuersystems.

a. Die Ausgestaltung des kommunalen Steuersystems unter dem Aspekt gemeindlicher Besteuerungsmöglichkeiten

Die für gemeindliche Einnahmespielräume relevanten Regelungen der Finanzverfassung und des Steuerrechts beziehen sich zum einen auf die Gesetzgebungshoheit, oder - etwas weiter gefaßt - auf die Gestaltungskompetenz hinsichtlich der Festlegung von Steuerbemessungsgrundlagen und Steuersätzen, zum anderen auf die Ertragshoheit und die Verteilung des Steueraufkommens auf die Gebietskörperschaften. Regelungen über die Verwaltungshoheit sind hier hingegen kaum von Bedeutung. 
Mit der Festlegung von Steuerquellen und Bemessungsgrundlagen werden Steuerobjekte und Zensiten bestimmt und damit sowohl der Umfang der "besteuerbaren Masse" als auch die zu erwartenden Reaktionen der Zensiten auf Steuerveränderungen eingegrenzt.

Im föderativen System der Bundesrepublik Deutschland liegt die Gesetzgebungskompetenz zunächst allein beim Bund oder den Ländern; die gemeindlichen Steuerquellen und die Bemessungsgrundlagen stimmen daher zumindest länderweise - in allen Gemeinden weitgehend überein. Wichtigste Steuern sind die Realsteuern - Gewerbe- und Grundsteuern - sowie der Gemeindeanteil an der Einkommensteuer; ihre Bemessungsgrundlagen sind bundeseinheitlich geregelt. Seit 1983 ebenfalls bundeseinheitlich festgelegt sind die Bemessungsgrundlagen der Grunderwerbsteuer, von deren Erträgen den Gemeinden bzw. Gemeindeverbänden jedoch nicht in allen Ländern ein Anteil des örtlichen Aufkommens überlassen wird. ${ }^{29}$ )

Eine gewisse Ausnahme stellen allein die sog. "Kleinen Gemeindesteuern", insbesondere die örtlichen Aufwand- und Verbrauchsteuern dar. Zwar liegt die Gesetzgebungskompetenz hier bei den Ländern, nach Art. 105 III a GG haben die Länder jedoch das Recht, die Befugnis zur Gesetzgebung an die Gemeinden zu übertragen. Von diesem Recht haben alle Flächenländer Gebrauch gemacht: Die Gemeinden können "Kleine-Gemeindesteuern-Satzungen" erlassen, die allerdings genehmigungspflichtig sind und deren Gestaltung durch die Vorschriften der Kommunalabgaben-Gesetze der Länder mehr oder weniger reglementiert wird. ${ }^{30)}$ In den Bereich der "Kleinen Gemeindesteuern" fällt auch das gemeindliche "Steuerfindungsrecht".

Sowohl das gemeindliche Steuerfindungsrecht als auch die Option, bestimmte kleine Steuern zu erheben oder nicht zu erheben, scheint für die Ge-

29) Die Beteiligung der Gemeinden und Gemeindeverbände erfolgt länderweise nach verschiedenen Quoten:

- Rheinland-Pfalz und Baden-Württemberg: $100 \%$

- Bayern: $80 \%$, - Nordrhein-Westfalen: 9/14.

- Hessen, Saarland und Niedersachsen: 4/7.

Vgl. dazu: Unsere Steuern von A-Z. hrsg. vom Bundesminister der Finanzen, Ausgabe 1986, S. 80.

30) Die Einzelheiten werden dargelegt bei Bayer, Herrmann-Willfried, Die Kleinen Gemeindesteuern, in: Handbuch der kommunalen Wissenschaft und Praxis, Bd. 6, Kommunale Finanzen, hrsg. von Günter Püttner. 2., völlig neu bearb. Aufl., Berlin u.a. 1985, S. 156-245, hier S. 171. 
meinden insgesamt jedoch von untergeordneter Bedeutung zu sein. ${ }^{31)}$ Als Ausnahme wird allerdings die kommunale Zweitwohnungsteuer angesehen ${ }^{32}$, die bisher ausschließlich in Fremdenverkehrsgemeinden erhoben wird und deren Aufkommen in Einzelfällen durchaus beträchtlich ist. ${ }^{33}$ )

Insgesamt sind die gemeindlichen Möglichkeiten, steuerliche Bemessungsgrundlagen selbst zu wählen oder direkt zu verändern, damit stark eingeschränkt. Demgegenüber dürfte die Möglichkeit kommunaler Hebesatzpolitik wesentlich bedeutsamer sein, also das Recht der Gemeinden, die bundeseinheitlichen Steuersätze zu vervielfältigen.

Mit Blick auf die fiskalischen Wirkungen kommunaler Steuersatzpolitik sind die konkreten steuerrechtlichen Gestaltungsmerkmale der mit einem Hebesatzrecht ausgestatteten Steuern von besonderer Relevanz; nur für sie stellt der Steuersatz bzw. der Hebesatz einen Parameter der interkommunalen Konkurrenz dar. Bei der Hebesatzgestaltung ist gegebenenfalls auf räumliche Ausweichreaktionen zu achten. Diese hängen nun entscheidend von den Einzelregelungen des Steuerrechts ab: Inwieweit ist das Steuerobjekt substituierbar, wer sind die Zensiten und durch welche mobilitätsrelevanten Merkmale zeichnen sie sich aus?

Unter dem Aspekt der interregionalen oder interkommunalen Aufkommensverteilung sind steuerrechtliche Regelungen natürlich auch für die nicht mit einem gemeindlichen Hebesatzrecht ausgestatteten Steuern wesentlich. Allerdings kann weder das gemeindliche Aufkommen noch die Steuerschuld bei den jeweiligen Zensiten durch gemeindliche Steuersatzpolitik beeinflußt werden. Für die Frage nach fiskalisch sinnvoller gemeindlicher Steuersatzpolitik ist die steuerrechtliche Ausgestaltung daher zunächst irrelevant.

$\mathrm{Zu}$ diesen direkt nicht mitgestaltbaren Einnahmen zählen im kommunalen Steuersystem der Bundesrepublik Deutschland vor allem der gemeindliche Einkommensteueranteil und die Grunderwerbsteuer. Für die Einkommen-

31) Diese Auffassung leitet sich zunächst aus dem vergleichsweise niedrigen Aufkommen der kleinen Gemeindesteuern ab. Sie wird ebenfalls vertreten von Klein, Richard R., Kommunale Schuldenpolitik, Schriften des Deutschen Instituts für Urbanistik, Bd. 61, Stuttgart u.a. 1977 , S. 36 f.

32) Ebenda.

33) So wurde nach Bayer das Aufkommen der Gemeinde Westerland aus der Zweitwohnungsteuer für 1985 auf 1.2 Mio DM veranschlagt (zum Vergleich: Hundesteuer = 54.000 DM); Bayer, Herrmann -Willfried, Die Kleinen Gemeindesteuern, a.a.O., S. 208. 
steuer wird im Grundgesetz zwar ein gemeindliches Hebesatzrecht vorgesehen, zur Zeit wird es den Gemeinden jedoch nicht zugestanden. Vielmehr erhält die Gemeindeebene insgesamt $15 \%$ des jeweiligen Länderaufkommens, das nach dem örtlichen Aufkommen bis zum Sockelbetrag (seit 198532.000 DM bei Alleinveranlagten und 64.000 DM bei Zusammenveranlagten) verteilt wird. Auf die Verteilung der Grunderwerbsteuer wurde oben bereits hingewiesen.

Damit reduzieren sich die Möglichkeiten gemeindlicher Steuerpolitik weitgehend auf die Hebesatzgestaltung bei der Gewerbesteuer und den Grundsteuern A und B.

Die Gewerbesteuer wird in der Regel als Soll-Ertrag-Steuer interpretiert $^{34)}$; allerdings knüpft sie nur teilweise direkt am Ertrag - und zwar am IstErtrag an. Steuerobjekt ist der Gewerbebetrieb. Darunter fallen stehende und Reisegewerbebetriebe, nicht aber freie Berufstätigkeit und Vermögensverwaltung, sofern sie nicht aufgrund ihrer Rechtsform (als Mitunternehmerschaften oder Kapitalgesellschaften) steuerpflichtig werden.

Die Bemessungsgrundlage der Gewerbesteuer - der Gewerbesteuermeßbetrag - setzt sich aus den mit den Steuermeßzahlen gewichteten Faktoren Gewerbeertrag und Gewerbekapital zusammen und wird wie folgt ermittelt:

34) So etwa Hessler, Heinz-Dieter, Finanzwissenschaftliches System der Besteuerung, a.a.O., S. 99 ff. Vgl. dazu auch Tipke. Klaus, Steuerrecht - Ein systematischer Grundriß, 9., völlig überarb. Aufl., Köln 1983, S. 388 ff. 
Steuerbilanzgewinn

+ Hinzurechnungen

z.B. Zinsen für

- Kürzungen

- Freibetrag (36.000 DM

für natürliche Perso-

nen und Personenge-

sellschaften)

$=$ zu versteuernder

Gewerbeertrag x

Steuermeßzahl (5 v. H.)
Einheitswert des

Betriebsvermögens

im Inland

+ Hinzurechnungen

(z.B. Dauerschulden)

- Kürzungen

- Freibetrag (120.000

DM) $=\mathrm{zu}$ versteuerndes

Gewerbekapital x

Steuermeßzahl

(2v.T.)

\section{Gewerbesteuermeßbetrag}

Auf den so bestimmten Gewerbesteuermeßbetrag wendet die Gemeinde ihren individuellen Hebesatz an.

Steuerobjekt der ebenfalls als Soll-Ertrag-Steuern konzipierten Grundsteuern A und B ist der Grundbesitz im Gemeindegebiet. Besteuert wird jeweils die wirtschaftliche Einheit; bei der Grundsteuer A ist dies die gesamte landwirtschaftliche Betriebseinheit einschließlich der Wohngebäude.

Anders als die Gewerbesteuer kennt die Grundsteuer keine persönlichen Freibeträge. Steuerbefreit sind nur der Grundbesitz der öffentlichen Hand sowie Grundbesitz, der Zwecken des öffentlichen Interesses dient.

Die Bemessungsgrundlage - der Steuermeßbetrag - errechnet sich ähnlich wie bei der Gewerbekapitalsteuer aus dem Einheitswert per a. 1964, mul- 
tipliziert mit nach der Flächennutzung differenzierten Steuermeßzahlen. Sie sind zur Zeit folgendermaßen gestaffelt: ${ }^{35}$ )

Für Betriebe der Land- und Forstwirtschaft 6,0 v.T. für bebaute Grundstücke allgemein 3,5 v.T. für Einfamilienhäuser

a) für die ersten $75.000 \mathrm{DM}$ des Einheitswerts 2,6 v.T.

b) für den Rest des Einheitswerts 3,5 v.T. für Zweifamilienhäuser 3,1 v.T. für unbebaute Grundstücke

Auf diese Steuermeßbeträge wendet die Gemeinde dann ebenfalls einen ggf. für Grundsteuer A und B unterschiedlichen - Hebesatz an.

Mit Gewerbe- und Grundsteuer wird das Recht der Gemeinden zur Steuersatzgestaltung auf Steuern auf die Einkommensentstehung am Ort der Produktion eingegrenzt.

Diese Charakterisierung ist für die Gewerbesteuer unmittelbar einsichtig, sie gilt aber zumindest von ihrer Konzeption her auch für die Grundsteuer: Besteuert werden soll der Ertrag aus der produktiven Nutzung von Boden und Gebäuden, ein Tatbestand also, der zur Produktion zählt und deshalb von einigen Autoren auch dem Unternehmenssektor zugerechnet wird. ${ }^{36)}$ Betrachtet man die Flächennutzung jedoch von ihrem Zweck her, so ist ein Teil - die Wohnnutzung - eher dem Konsum zuzurechnen, die Steuerdestinatare sind dementsprechend die den Wohnraum nutzenden Haushalte. ${ }^{37}$

Die Gesamthöhe der Bemessungsgrundlagen wird entscheidend durch die wirtschaftlichen Bedingungen, d.h. durch den Umfang der Einkommensentstehung bestimmt. ${ }^{38)}$ Dabei handelt es sich um Bemessungsgrundlagen, die

35) Vgl. Tipke, Klaus, Steuerrecht, a.a.O., S. 401.

36) So etwa Littmann, Konrad, Überblick über die Ertragsteuern, in: Handbuch der Finanzwissenschaft, Bd II, 3. gänzl. neu bearb. Aufl., hrsg. von Fritz Neumark, Tübingen 1980, S. 565-574, hier S. 566.

37) Dies setzt allerdings eine vollständige Überwälzung der Grundsteuer auf die jeweiligen Nutzer voraus.

38) Vgl. Zimmermann, Horst, Hardt, Ulrike und Postlep, Rolf-Dieter, Bestimmungsgründe der kommunalen Finanzsituation, a.a.O., S. 89 ff. 
kaum substituiert werden können und für die eine räumliche Verlagerung unmöglich oder sehr kostenintensiv ist, weil sie einen Standortwechsel voraussetzt.

Allerdings wirken die staatlichen Bemessungsgrundlagen-Regelungen selektiv; der Zugriff auf die Einkommensentstehung wird je nach Produktionsstruktur mehr oder weniger eingegrenzt: Nach der Abschaffung der Lohnsummensteuer werden insbesondere arbeitsintensive Produktionen begünstigt, innerhalb der fortbestehenden Gewerbesteuer werden vor allem kleinund mittelbetriebliche ${ }^{39}$ ) und Produktionen des tertiären Sektors vor der gemeindlichen Besteuerung "geschützt". Für die Grundsteuer wird, wie oben gezeigt wurde, ebenfalls nach "Produktionstypen" differenziert. ${ }^{40)}$ So kann sich die Festlegung der Bemessungsgrundlagen auch auf die Hebesatzspielräume der Gemeinden auswirken:

Wenn davon ausgegangen werden kann, daß die nicht-gewerbesteuerzahlenden Betriebe stärker standortgebunden sind und letztlich eher durch Betriebsaufgabe als durch Abwanderung auf die Steuerlast reagieren, so würde durch die Eingrenzung der Zensiten auf die eher mobilen Bereiche der Produktion der monopolistische Spielraum der Gemeinden eingeschränkt (vgl. dazu Abschnitt b.). Für die verbleibenden mobileren Betriebe wäre zu untersuchen, welche Rolle die Hebesätze bzw. Steuersätze im Vergleich mit anderen Standortfaktoren bei der Standortwahl spielen. Je geringer die Bedeutung des Steuersatzes und je wichtiger die qualitative Ausstattung des Standortes ist, desto unelastischer dürfte die Reaktion auf Steuersatzerhöhungen sein. Je eher eine Gemeinde solche spezifischen Standortanforderungen erfüllen kann, desto größer ist dementsprechend ihr Steuersatzspielraum, während Gemeinden ohne besonderes Profil eher den Steuersatz als Konkurrenzparameter einsetzen müssen.

39) Diese Wirkung war von den im Steueränderungsgesetz 1979 für die Gewerbesteuer beschlossenen Erleichterungen durchaus intendiert; es wurde damit gerechnet, daB weitere 200.000 mittelständische Unternehmen durch die Neuregelung steuerfrei werden. Vgl. Finanzbericht 1980, hrsg. vom Bundesministerium der Finanzen. Bonn 1979, S. 150 f.

40) Über die Differenzierung der Steuermeßzahlen hinaus wird schon bei der Einheitswertbestimmung zwischen Nutzungsarten unterschieden. So gelten etwa die Einheitswerte landwirtschaftlicher Betriebe als deutlich unterbewertet. Vgl. dazu Köhne, Manfred, die steuerliche Sonderstellung der Landwirtschaft, in: Beiträge zur Agrarökonomie, Festschrift zum 80. Geburtstag von Emil Woermann, Hamburg und Berlin 1979, S. 153-168, hier S. 154. 
Staatlicherseits kann die kommunale Hebesatzpolitik durch Hebesatzgrenzen beschränkt werden; aufgrund bundesrechtlicher Ermächtigungen sind solche Regelungen den Ländern vorbehalten. Sie können darüber bestimmen, "in welchem Verhältnis die Hebesätze für die Grundsteuer A, für die Grundsteuer B, für die Gewerbesteuer nach dem Gewerbeertrag und dem Gewerbekapital ... zueinander stehen müssen, welche Höchstsätze nicht überschritten werden dürfen und inwieweit mit Genehmigung der Gemeindeaufsichtsbehörde Ausnahmen zugelassen werden können. ${ }^{41)}$ Von dieser Möglichkeit haben Niedersachsen, Schleswig-Holstein, Rheinland-Pfalz, Nordrhein-Westfalen und das Saarland Gebrauch gemacht. In den beiden letztgenannten Ländern wurden sie inzwischen jedoch wieder abgeschafft, bzw. die Regelungen wurden nie angewendet. ${ }^{42)}$

Aber selbst in jenen Ländern, in denen solche Vorschriften weiter bestehen, scheinen sie kaum Wirkungen gehabt zu haben, vielmehr wurden sie auf breiter Ebene durch Sondergenehmigungen umgangen. So zeigt Haverkamp auf, daß 1980 in einigen Ländern sogar die durchschnittlichen Hebesätze weit über den Maximalhebesätzen lagen. ${ }^{43)}$ Eine Einschränkung der kommunalen Besteuerungsrechte liegt jedoch allein schon in der Genehmigungspflicht der satzungsmäßig festzulegenden Hebesätze. Darüber hinaus ist die kommunale Steuersatzpolitik insofern inflexibel, als die Hebesätze jeweils bis zur Mitte eines Jahres für das Folgejahr festgelegt werden müssen; bei später erfolgenden Änderungen darf der Vorjahreshebesatz nicht überschritten werden. ${ }^{44)}$

Für die Gewerbesteuer sind darüber hinaus zwei Besonderheiten mit ebenfalls einschränkender Wirkung zu beachten: der einheitliche Hebesatz auf den aus den Faktoren Gewerbeertrag und Gewerbekapital summierten Steuermeßbetrag und die Verpflichtung zur Abführung der Gewerbesteuerumlage.

41) Haverkamp, Franz, Die Grundsteuer, in: Handbuch der kommunalen Wissenschaft und Praxis, Bd. 6, Kommunale Finanzen, hrsg. von Günter Püttner, Berlin u.a. 1985, S. 117-127, hier S. 124.

42) Ebenda.

43) Haverkamp, Franz, Die Gemeindefinanzreform, in: Der Bundesminister der Finanzen, Hrsg., Die Finanzbeziehungen zwischen Bund, Ländern und Gemeinden, Bonn 1982, S. 527-560, hier S. 552.

44) Vgl. Haverkamp, Franz, Die Grundsteuer, a.a.O., S. 124 sowie Milbradt, Georg H., Die Gewerbesteuer, ebenda, S. 128-140, hier S. 135. 
Die gemeinsame Bemessungsgrundlage für Gewerbeertrag- und Gewerbekapitalsteuer - die durch keinerlei Sondergenehmigung getrennt werden können - entspricht im Prinzip einer Kopplungsvorschrift. Allerdings unterscheidet sie sich von jenen Kopplungsvorschriften, die die Steuerbelastung verschiedener Zensiten betreffen: Eine räumliche Verlagerung kommt normalerweise nur für das gemeinsame Steuerobjekt der Gewerbesteuer in Frage. $^{45)}$

Da die Gewerbekapitalsteuer letztlich auch den Ertrag - als wichtigsten Zweck unternehmerischer Tätigkeit - belastet, sind kapitalintensive Produktionen bei gleicher Ertragskraft gegenüber anderen Produktionen benachteiligt und daher tendenziell steuerreagibler, sofern das Kapital nicht stark standortgebunden ist. Die Hebesatzspielräume sind deshalb in jenen Gemeinden stärker eingeschränkt, in denen kapitalintensive Produktionen von besonderer Bedeutung sind.

Die zweite Regelung, die die Abführung der Gewerbesteuerumlage betrifft, ähnelt in ihrer Wirkung einem Mindeststeuersatz ${ }^{46}$, dessen Anwendung für die Gemeinden jedoch keinerlei Erträge einbringt. Die Gemeinden sind verpflichtet, jenen Anteil aus der Gewerbesteuer an die übergeordneten Gebietskörperschaften abzuführen, der sich aus dem Meßbetrag, multipliziert mit dem Umlagevervielfältiger von $80 \%$ ergibt. Bis zu einem Hebesatz von $80 \%$ erzielen die Gemeinden damit keinerlei Einnahmen aus der Gewerbesteuer; bleiben sie mit ihrem Hebesatz unterhalb dieser Grenze, so haben sie die Umlage aus anderen Quellen zu finanzieren. Oberhalb dieser Schwelle kommen die Erträge aus Hebesatzsteigerungen dagegen allein den jeweiligen Gemeinden zugute.

Mit den genannten Regelungen wird das Besteuerungsrecht der Gemeinden insgesamt stark beschnitten. Die Bedeutung des gemeindlichen Hebesatzrechts wird daher mitunter als recht gering eingeschätzt. ${ }^{47}$ )

45) Von den Möglichkeiten der Teilauslagerung und den Wirkungen der in diesem Fall greifenden Zerlegungsmaßstäben soll hier abgesehen werden.

46) In einigen Ländern wird jenen Gemeinden, die eine Realsteuer nicht erheben, sogar ein höherer Betrag als Steuerkraft zugerechnet, als er sich bei normaler Berechnung der Steuerkraftmeßzahl ergeben hätte. Vgl. etwa das Gesetz über den kommunalen Finanzausgleich in Baden-Württemberg 1984, $\$ 6$ (4).

47) Ähnlich äußert sich etwa Klein, Richard R., Kommunale Schuldenpolitik, a.a.O., S. 31 ff. 
Aus der rechtlichen Einschränkung läßt sich die quantitative Bedeutung der verbleibenden Spielräume jedoch kaum abschätzen. Zumindest im interkommunalen Vergleich wird sie - wie im 2. Kapitel bereits angesprochen - für durchaus nicht unwesentlich gehalten.

Zwischen den beiden mit einem Hebesatzrecht ausgestatteten Steuern der Grundsteuer und der Gewerbesteuer - sind in dieser Hinsicht aber sicher Unterschiede zu machen. Zum ersten sind die Bemessungsgrundlagen der Grundsteuer deutlich niedriger als die der Gewerbesteuer, Hebesatzerhöhungen bringen daher viel geringere Erträge als gleich große Hebesatzsteigerungen bei der Gewerbesteuer. Zum zweiten scheinen gegen eine Erhöhung der Grundsteuerhebesätze auch in stärkerem Maße politische Restriktionen - die hier nicht näher betrachtet wurden - zu sprechen, als dies bei der Gewerbesteuer der Fall ist. ${ }^{48)}$

Anschließend soll beispielhaft nur noch die Gewerbesteuer betrachtet werden, ohne allerdings mögliche Auswirkungen der Hebesatzpolitik auf andere steuerliche Einnahmemöglichkeiten ganz zu vernachlässigen.

Hauptgegenstand der anschließenden Abschnitte sind die Auswirkungen einer Hebesatzanhebung, bzw. - bezogen auf den Gemeindevergleich - eines interkommunalen Hebesatzgefälles. Sie werden zunächst aus betrieblicher, dann aus gemeindlicher Sicht untersucht.

b. Die Gewerbesteuerbelastung als Determinante sachlicher und räumlicher Ausweichung im Unternehmenssektor

1. Die Belastung des betrieblichen Ertrags bei unterschiedlichen Gewerbesteuerhebesätzen

Der in den Kommunen angesetzte Hebesatz der Gewerbesteuer entscheidet über die Belastung der Produktionen; Hebesatzsteigerungen erhöhen natürlich diese Belastung. Allerdings sind die Wirkungen einer Hebesatzanhebung

48) Vgl. etwa Schaab, Bodo, Örtliche Wirtschaftsstruktur und kommunale Steuerkraft, Diss., Konstanz 1972, S. 27. Dabei ist allerdings noch einmal darauf hinzuweisen, daß hier eine Trennung zwischen tatsächlichen Restriktionen kommunaler Politik und den Zielen eben dieser Politik vorgenommen werden müßte. 
unterschiedlich, je nach Ertragslage und Faktoreinsatzkonstellationen in den Betrieben.

Daraus folgt zum einen eine mehr oder weniger große Empfindlichkeit der Produktionen gegenüber Hebesatzanhebungen, zum anderen wird - direkt und indirekt - die Ergiebigkeit der gemeindlichen Hebesatzpolitik beeinflußt. Dies soll anhand einiger Beispiele erläutert werden.

Als Ausgangspunkt soll die Belastung bzw. die Belastungssteigerung einer Personengesellschaft ermittelt werden, die keine Gewerbekapitalsteuer zahlt. Es wird angenommen, daß es sich um eine ausgeprägt arbeitsintensive Produktion handelt und das zu versteuernde Gewerbekapital insgesamt niedriger als 120.000 DM ist und somit voll unter den Freibetrag für Personengesellschaften fällt. Weiterhin sei angenommen, daß der zu versteuernde Gewerbeertrag vor Abzug der als Betriebsausgabe geltenden Gewerbesteuerschuld 136.000 DM betrage.

Nach Abzug des Freibetrages von 36.000 DM sind somit 100.000 DM zu versteuern.

Die Steuerschuld errechnet sich aus folgender Formel, in der die Gewerbesteuerschuld als Betriebsausgabe berücksichtigt ist:

Steuerschuld $=$

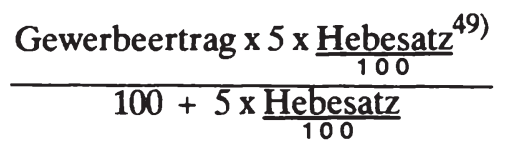

und beträgt beim unterstellten Gewerbeertrag und einem Hebesatz von $350 \%$ 14.893 DM. Dies entspricht einer Belastung des Gewinns vor Gewerbesteuern von $10,9 \%$.

49) Die verwendete Formel ist mit der von Rose verwendeten identisch. Sie lautet:

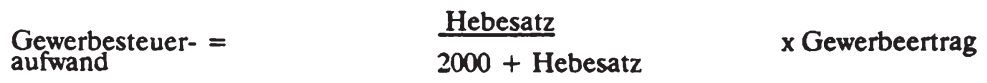

Durch die Umformung wird jedoch die Einbeziehung der Gewerbekapitalsteuer im 2. Schritt erleichtert. Vgl. Rose, Gerd, Betrieb und Steuer, 1. Buch, Die Ertragsteuern, 9., erhebl. überarb. Aufl., Wiesbaden 1986, S. 228 ff. 
Dabei ist nicht berücksichtigt, daß die Gewerbesteuer auch bei Einkommen- und Körperschaftsteuer abzugsfähig ist; die Gesamtbelastung fällt deshalb - je nach Verwendung des Gewinns und nach dem jeweiligen Grenzsteuersatz bei der Einkommensteuer entsprechend geringer aus. Für die Belastungsrechnung im Falle einer Kapitalgesellschaft wäre andererseits zu berücksichtigen, daß der Freibetrag von 36.000 DM nicht in Anspruch genommen werden kann. Von diesen Zusatzproblemen soll hier jedoch abgesehen werden.

Wird nun eine Hebesatzsteigerung um 50 Prozentpunkte vorgenommen und der Hebesatz von $350 \%$ auf $\mathbf{4 0 0} \%$ angehoben (dies entspricht einer Steigerungsrate von etwa $14,3 \%$ ), so errechnet sich für den oben betrachteten Fall eine Gewerbeertragsteuerschuld von 16.666 DM und eine Belastung des Gewinns vor Steuern (136.000 DM) von 12,3\%. Mit der Hebesatzsteigerung wird der Gewinn also um 1,4 \% stärker belastet als in der Ausgangssituation. Aus gemeindlicher Sicht ist dabei der Tatbestand von Interesse, daß der Steigerungsrate des Hebesatzes um 14,3\% eine Aufkommenssteigerung der Gewerbeertragsteuer von nur $11,9 \%$ gegenübersteht.

Tabelle 4 zeigt, wie sich die Belastung der Gesamterträge vor Abzug des Freibetrags bei steigendem Ertrag und für alternative Hebesätze entwickelt. Hier wird folgendes deutlich: 
Tabelle 4: Belastung der um den Freibetrag gekürzten Erträge einer Personengesellschaft bei alternativen Gewerbesteuerhebesätzen

\begin{tabular}{|c|c|c|c|c|c|}
\hline $\begin{array}{l}\text { zu versteu- } \\
\text { ernder Ge- } \\
\text { werbeertrag } \\
\text { nach Abzug } \\
\text { des Freibe- } \\
\text { trags } \\
\text { in DM }\end{array}$ & $\begin{array}{l}\text { Steuers } \\
\text { in DM } \\
\text { Hebe- } \\
\text { satz } \\
350 \%\end{array}$ & $\begin{array}{l}\text { huld } \\
\text { ei } \\
\text { Hebe- } \\
\text { satz } \\
400 \%\end{array}$ & $\begin{array}{l}\text { Belast } \\
\text { Ertrag } \\
\text { Hebe- } \\
\text { satz } \\
350 \%\end{array}$ & $\begin{array}{l}\text { g des } \\
\text { in \% bei } \\
\text { Hebe- } \\
\text { satz } \\
400 \%\end{array}$ & $\begin{array}{c}\text { Steigerungs- } \\
\text { rate } \\
\text { in } \%\end{array}$ \\
\hline 100.000 & 14.893 & 16.666 & 10,9 & 12,3 & 1,4 \\
\hline 200.000 & 29.792 & 33.333 & 12,6 & 14,1 & 1,5 \\
\hline 300.000 & 44.670 & 49.999 & 13,3 & 14,9 & 1,6 \\
\hline 400.000 & 59.572 & 66.666 & 13,7 & 15,3 & 1,6 \\
\hline 500.000 & 74.465 & 83.333 & 13,9 & 15,5 & 1,6 \\
\hline 600.000 & 89.361 & 99.999 & 14,1 & 15,7 & 1,6 \\
\hline
\end{tabular}

- Aufgrund der Freibetragsregelung nimmt die Belastung des Gewerbeertrags mit Gewerbeertragsteuer bei steigendem Ertrag zu, allerdings mit sinkenden Raten.

- Hebesatzanhebungen haben eine mit wachsendem Ertrag steigende Zusatzbelastung zur Folge; die durch die Freibetragsregelung verursachte indirekte Progression flacht aber auch hier mit steigendem Ertrag ab und geht in einen linearen Verlauf über.

Weitet man die Betrachtung auf die Gewerbekapitalsteuer aus, so sind die Wirkungen je nach Verhältnis von Gewerbeertrag zu Gewerbekapital in den einzelnen Betrieben unterschiedlich.

In diesem zweiten Fall soll zunächst wieder von einer Personengesellschaft mit einem zu versteuernden Gewerbeertrag von 100.000 DM (136.000 DM vor Abzug des Freibetrags der Gewerbeertragsteuer) ausgegangen werden. Das Gewerbekapital betrage 1.120.000 DM, nach Abzug des Freibetrags 
der Gewerbekapitalsteuer verbleibt ein zu versteuernder Betrag von 1 Million DM.

Die Formel zur Berechnung der Gewerbesteuerschuld ist nun zu erweitern. Dabei ist zu berücksichtigen, daß auch die gezahlte Gewerbekapitalsteuer als Betriebsausgabe abzugsfähig ist und den zu versteuernden Gewerbeertrag mindert.

Steuerschuld $=\frac{\text { Gewerbeertrag } \times 5 \times \frac{\text { Hebesatz }}{100}}{100+5 \times \frac{\text { Hebesatz }}{100}}+\frac{\text { Gewerbekapital } \times 2 \times \frac{\text { Hebesatz }}{100}}{1000+50 \times \frac{\text { Hebesatz }}{100}}$

Im betrachteten Fall beträgt die Steuerschuld der Gewerbesteuer bei einem Hebesatz von $350 \%$ 20.851 DM, davon entfallen 7.000 DM auf die Gewerbekapitalsteuer, 13.851 auf die Gewerbeertragsteuer. Wiederum bezogen auf den Gewinn vor Abzug der Gewerbesteuer beträgt die Gesamtbelastung $15,3 \%$.

Unterstellt man eine Hebesatzanhebung auf $400 \%$, so errechnet sich eine Steuerschuld von 23.332 DM und eine Belastung des Ertrags von 17,2\%. Die Belastungssteigerung liegt hier also knapp unter $2 \%$. Allerdings ist die Ausgangsbelastung aufgrund der fälligen Gewerbekapitalsteuer insgesamt wesentlich höher als im Falle jenes Betriebs, der allein zur Gewerbeertragsteuer herangezogen wird. 
Tabelle 5: Belastung des konstanten Gewerbeertrags bei wachsendem Gewerbekapital $^{1)}$ und alternativen Hebesätzen

\begin{tabular}{|c|c|c|c|c|c|}
\hline $\begin{array}{l}\text { zu versteu- } \\
\text { erndes Ge- } \\
\text { werbekapital } \\
\text { in } 1000 \mathrm{DM}\end{array}$ & $\begin{array}{l}\text { Steuers } \\
\text { in DM } \\
\text { Hebe- } \\
\text { satz } \\
350 \%\end{array}$ & $\begin{array}{l}\text { huld } \\
\text { Hebe- } \\
\text { satz } \\
400 \%\end{array}$ & $\begin{array}{l}\text { Belastu } \\
\text { Ertrags } \\
\text { Hebe- } \\
\text { satz } \\
350 \%\end{array}$ & $\begin{array}{l}g^{2)} \text { des } \\
\text { in \% bei } \\
\text { Hebe- } \\
\text { satz } \\
400 \%\end{array}$ & $\begin{array}{c}\text { Steigerungs- } \\
\text { rate } \\
\text { in } \%\end{array}$ \\
\hline 1.000 & 20.851 & 23.332 & 15,3 & 17,2 & 1,9 \\
\hline 2.000 & 26.807 & 29.999 & 19,7 & 22,1 & 2,4 \\
\hline 3.000 & 32.765 & 36.665 & 24,1 & 27.0 & 2,9 \\
\hline 4.000 & 38.722 & 43.332 & 28,5 & 31,9 & 3,4 \\
\hline 5.000 & 44.680 & 49.999 & 32,8 & 36,8 & 4,0 \\
\hline 6.000 & 50.637 & 56.665 & 37,2 & 41,7 & 4,5 \\
\hline
\end{tabular}

1) Abzüglich des Freibetrags von $120.000 \mathrm{DM}$.

2) Wie in Zeile 1 von Tabelle 4 wurde die Belastung des zu versteuernden Ertrags für einen Ertrag vor Gewerbesteuerabzug von 136.000 DM ermittelt.

In Tabelle 5 wird weiterhin von einem konstanten Gewinn von 136.000 DM ausgegangen und der Kapitaleinsatz - der Freibetrag von 120.000 DM schon jeweils abgezogen - hypothetisch variiert. Daraus lassen sich folgende Ergebnisse ableiten:

- Die Belastung des konstanten Ertrags steigt mit wachsendem Kapitaleinsatz proportional - mit einer Rate von etwa 4,4\% - an; die progressive Wirkung der Freibeträge und die regressive Wirkung des Abzugs des "Gewerbesteueraufwands" vom zu versteuernden Gewerbeertrag gleichen sich in etwa aus. Die Steigerungsrate der Belastung ist um so höher, je höher der Hebesatz ist. 
- Dementsprechend ist auch die Zinsbelastung des Ertrags aufgrund einer Hebesatzanhebung um so größer, je höher das zu versteuernde Gewerbekapital ist. Mit anderen Worten: Kapitalintensive Produktionen werden durch Hebesatzsteigerungen wesentlich stärker betroffen als arbeitsintensive, insbesondere dann, wenn sie als ertragsschwach einzustufen sind.

Die Belastungswirkungen einer Hebesatzsteigerung sind noch gravierender, wenn "steuerlich ertraglose", aber mit hohem Kapitaleinsatz produzierende Betriebe der Besteuerung unterliegen. Bleibt man beim oben gewählten Beispiel eines zu versteuernden Gewerbekapitals von 1 Million DM, unterstellt aber einen Gewinn von nur 36.000 DM, der damit voll der Freibetragsregelung unterliegt, so kann die Gewerbekapitalsteuerschuld nicht steuermindernd vom Gewinn abgezogen werden. Die Gewerbesteuerschuld errechnet sich als

Gewerbekapital x Steuermeßzahl x Hebesatz.

Sie beträgt bei einem Hebesatz von $350 \% 7.000 \mathrm{DM}$ und entspricht einer Belastung des Gewinns vor Steuern von 19,4\%. Bei einem Hebesatz von $400 \%$ steigt die Steuerschuld auf 8.000 DM und die Belastung um 2,8\% auf $22,2 \%$.

Allein in diesem Fall steigt das Steueraufkommen für die Gemeinde mit der gleichen Rate wie der Hebesatz.

Tabelle 6 zeigt, daß die relative Belastung des Gewinns mit wachsendem Gewerbekapital ansteigt. Die Zusatzbelastung nach einer Anhebung des Hebesatzes ist - verglichen mit den bisher betrachteten Fällen relativ groß, und sie ist um so höher, je schlechter das Verhältnis von Gewinn zu Gewerbekapital im einzelnen Betrieb ausfällt. Ertragsschwache Betriebe dürften von daher besonders empfindlich auf kommunale Hebesatzanhebungen reagieren, wenn sie kapitalintensiv produzieren. 
Tabelle 6: Gewerbesteuerbelastung des steuerfreien Gewerbeertrags ${ }^{1)}$ bei wachsendem Gewerbekapital und alternativen Hebesätzen

\begin{tabular}{|c|c|c|c|c|c|}
\hline $\begin{array}{l}\text { zu versteu- } \\
\text { erndes Ge- } \\
\text { werbekapital } \\
\text { in } 1000 \mathrm{DM}\end{array}$ & $\begin{array}{l}\text { Steuers } \\
\text { in DM } \\
\text { Hebe- } \\
\text { satz } \\
350 \%\end{array}$ & $\begin{array}{l}\text { huld } \\
\text { bei } \\
\text { Hebe- } \\
\text { satz } \\
400 \%\end{array}$ & $\begin{array}{l}\text { Belastu } \\
\text { Ertrags } \\
\text { Hebe- } \\
\text { satz } \\
350 \%\end{array}$ & $\begin{array}{l}\text { g des } \\
\text { in \% } \\
\text { Hebe- } \\
\text { satz } \\
400 \%\end{array}$ & $\begin{array}{l}\text { Steigerungs- } \\
\text { rate }\end{array}$ \\
\hline 1.000 & 7.000 & 8.000 & 19,4 & 22,2 & 2,8 \\
\hline 2.000 & 14.000 & 16.000 & 39,0 & 44,4 & 5,4 \\
\hline 3.000 & 21.000 & 24.000 & 58,3 & 66,7 & 8,4 \\
\hline 4.000 & 28.000 & 32.000 & 77,8 & 88,9 & 11,1 \\
\hline 5.000 & 35.000 & 40.000 & 97,2 & 111,1 & 13,9 \\
\hline 6.000 & 42.000 & 48.000 & 116,7 & 133,3 & 16,6 \\
\hline
\end{tabular}

1) Die Belastung wurde auf der Basis eines Gewerbeertrags von 36.000 DM ermittelt.

Aus diesen modellhaften Betrachtungen kann abgeleitet werden, daß die Belastung des Ertrags bei einheitlichem Hebesatz im Gemeindegebiet je nach Kostenstruktur und Ertragslage durchaus unterschiedlich ausfällt. Die einzelnen Wirtschaftszweige werden daher bereits bei einem gegebenen Gewerbesteuerhebesatz mehr oder weniger stark belastet; Hebesatzsteigerungen bringen entsprechend höhere oder niedrigere Zusatzbelastungen mit sich. Das heißt aber auch, daß Hebesatzunterschiede für die verschiedenen Produktionen von unterschiedlicher Bedeutung hinsichtlich der Standortwahl sein dürften.

Wichtige Determinanten dieser Belastung sind die Betriebsgröße (gemessen an der absoluten Höhe von Gewerbeertrag und -kapital), die Rechtsform, das Verhältnis von steuerlichem Gewerbeertrag und -kapital; außerdem 
ist wegen der gewerbesteuerlichen Behandlung von Dauerschulden die Finanzierung der Unternehmen zu beachten.

Wie die Steuerbelastung nach Wirtschaftszweigen und Betriebstypen aktuell verteilt ist, läßt sich indessen empirisch kaum ermitteln, da die letzte Gewerbesteuerstatistik für das Jahr 1970 erstellt wurde. Aktuellere Informationen lassen sich also nur durch originäre Erhebungen beschaffen oder aus groben Schätzungen anhand verschiedener Indikatoren ableiten. Darauf soll hier jedoch verzichtet werden; die grundsätzlichen Überlegungen lassen sich auch anhand der Daten von 1970 verdeutlichen.

In Tabelle 7 wurde die Steuerbelastung nach Wirtschaftsbereichen bei unterschiedlichen Hebesätzen anhand der Gewerbesteuerstatistik geschätzt. Die berechnete prozentuale Belastung des Ertrags wird dabei aus verschiedenen Gründen zu hoch ausgewiesen: Zum ersten wurde die Steuerbelastung auf den zu versteuernden Gewerbeertrag bezogen; Freibeträge und ihre belastungsmindernden Wirkungen bleiben außer Betracht, ebenso wie die Abzugsmöglichkeiten der Gewerbesteuerschuld bei Einkommen- und Körperschaftsteuer. Eine weitere Fehlerquelle liegt in dem Tatbestand, daß die als Ausgangspunkt der Berechnungen gewählten Steuermeßbeträge bereits für bestimmte Hebesätze ermittelt worden sind; bei einem unrealistisch niedrigen Hebesatz von $100 \%$ wären sie tatsächlich höher ausgefallen.

Trotz dieser Verzerrungen kann anhand der Berechnungen deutlich gemacht werden, daß Hebesatzsteigerungen oder interkommunale Hebesatzunterschiede nach Wirtschaftszweigen mehr oder weniger gravierende Belastungswirkungen haben. Bereits bei einem Hebesatz von $100 \%$ fällt die prozentuale Belastung des Gewerbeertrags sehr unterschiedlich aus, sie war 1970 extrem hoch im Bereich Energiewirtschaft, Wasserversorgung, unterdurchschnittlich dagegen in den Bereichen Holz-, Papier- und Druckgewerbe, im Leder-, Textil- und Bekleidungsgewerbe sowie besonders im Baugewerbe.

Bei einem Hebesatz von $200 \%$ steigt die relative Belastung des Ertrags nun gegenüber einem Hebesatz von $100 \%$ für jene Produktionszweige stark an, in denen das Gewerbekapital in Relation zum Gewerbeertrag extrem hoch ist. Diese Relation wird in Spalte II ausgewiesen; je kleiner die Kennziffer, de- 
sto höher ist der Meßbetrag der Gewerbekapitalsteuer im Vergleich mit dem Meßbetrag der Gewerbeertragsteuer. Spalte V zeigt, daß die Mehrbelastung des Gewerbeertrags nach einer Hebesatzanhebung in den "kapitalintensiven" Bereichen Energiewirtschaft, Wasserversorgung und Bergbau wesentlich höher ausfallen würde als in den unterdurchschnittlich "kapitalintensiven" Bereichen Elektrotechnik und Baugewerbe. Damit werden die oben modellhaft abgeleiteten Ergebnisse tendenziell noch einmal bestätigt.

Außer der Branchenzugehörigkeit wurde oben vor allem die Betriebsgröße als entscheidend für die Gewerbesteuerbelastung bezeichnet. Neuere Untersuchungen ${ }^{50)}$ kommen hier zu dem Ergebnis, daß - bezogen auf die Produktionskosten - insbesondere Unternehmen mittlerer Größe hohe Belastungen zu tragen haben, Großunternehmen hingegen geringer belastet werden. Für Kleinunternehmen wurde eine mittlere Belastung ermittelt.

Dieses Ergebnis scheint den oben abgeleiteten Wirkungen zunächst zu widersprechen. Dabei ist allerdings zu berücksichtigen, daß mit der Freibetragsanhebung inzwischen ein großer Teil der Kleinunternehmen keine Gewerbesteuer mehr zu entrichten hat.

Überdies ist kritisch anzumerken, daß aus betrieblicher Sicht weniger die Wirkung der Gewerbesteuer auf die Kosten als ihre Wirkung auf den verbleibenden Gewinn interessiert. Aus einzelbetrieblicher Perspektive kommt man zwar über beide Fragestellungen zum gleichen Ergebnis, Vergleiche zwischen verschiedenen Produktionen hätten jedoch das Verhältnis von Kosten und Gewinn zu berücksichtigen, um die Belastungswirkung der Gewerbesteuer auf den Gewinn abschätzen zu können. Dies ist in der zitierten Untersuchung nicht geschehen; der reine Größeneffekt wird in den betrachteten Unternehmen vermutlich durch strukturelle Effekte überdeckt.

50) Vgl. Kirsch, Bernd und Schußmann, Klaus, Kommunale Gewerbesteuerpolitik: Hebesatzpolitik versus gewerbesteuerorientierte kommunale Steuerpolitik, in: Informationen zur Raumentwicklung, hrsg. von der BfLR, Heft 2/3, 1978, S. 105-114, hier S. 107. 


\section{Tabelle 7: Gewerbesteuerlastung des Ertrages bei alternativen Hebesätzen nach Wirtschaftsbereichen 1970}

\begin{tabular}{|c|c|c|c|c|}
\hline I & II & III & IV & V \\
\hline $\begin{array}{l}\text { Wirtschafts- } \\
\text { bereiche }\end{array}$ & $\begin{array}{l}\text { Verhältnis } \\
\text { der Meßbe- } \\
\text { träge von } \\
\text { Gewerbeer- } \\
\text { trag- und } \\
\text { Gewerbeka- } \\
\text { pitalsteuer }\end{array}$ & $\begin{array}{l}\text { Steuerbe- } \\
\text { lastung } \\
\text { des Ertrags } \\
\text { beim Hebe- } \\
\text { satz von } \\
100 \%\end{array}$ & $\begin{array}{l}\text { Steuerbe- } \\
\text { lastung } \\
\text { des Ertrags } \\
\text { beim Hebe- } \\
\text { satz von }\end{array}$ & $\begin{array}{l}\text { Steige- } \\
\text { rungs- } \\
\text { rate } \\
\text { (IV-III) }\end{array}$ \\
\hline $\begin{array}{l}\text { Produzierendes } \\
\text { Gewerbe }\end{array}$ & 6,84 & 4,9 & 12,0 & 7,1 \\
\hline $\begin{array}{l}\text { Energiewirtschaft, } \\
\text { Wasserversorgung }\end{array}$ & 1,38 & 9,1 & 25,3 & 16,2 \\
\hline Bergbau & 1,77 & 5,0 & 22,6 & 17,6 \\
\hline $\begin{array}{l}\text { Verarbeitendes } \\
\text { Gewerbe }\end{array}$ & 7,58 & 5,0 & 11,7 & 6,7 \\
\hline $\begin{array}{l}\text { Chem. Indust rie, } \\
\text { Mineralölberarb. }\end{array}$ & 5,96 & 7,7 & 12,8 & 7,1 \\
\hline $\begin{array}{l}\text { Kunstst off }- \text {, } \\
\text { Gummi- und } \\
\text { Asbestverarb. }\end{array}$ & 6,81 & 5,4 & 12,3 & 6,9 \\
\hline Steine u. Erden u.ä. & 6,85 & 5,2 & 12,1 & 6.9 \\
\hline $\begin{array}{l}\text { Metallerzeugung } \\
\text { und -verarbeitung }\end{array}$ & 7,04 & 4,9 & 11,9 & 7,0 \\
\hline $\begin{array}{l}\text { Stahl-, Mascinen- } \\
\text { und Fahrzeugbau }\end{array}$ & 7,81 & 5,2 & 11,8 & 6,6 \\
\hline Elektrotechnik u.ä. & 9,95 & 5,1 & 11,2 & 6,1 \\
\hline $\begin{array}{l}\text { Holz-, Papier- } \\
\text { Druckgewerbe }\end{array}$ & 7,90 & 4,6 & 11,5 & 6,9 \\
\hline $\begin{array}{l}\text { Leder-, Textil-, } \\
\text { Bekleidungsgewerbe }\end{array}$ & 6,13 & 4,6 & 12.1 & 7,5 \\
\hline $\begin{array}{l}\text { Nahrungs- und } \mathrm{Ge}- \\
\text { nußmittelgewerbe }\end{array}$ & 7,04 & 5,2 & 12,0 & 6,8 \\
\hline Baugewerbe & 10,77 & 4,2 & 10,7 & 6,5 \\
\hline
\end{tabular}

Quelle: Statistisches Bundesamt, Gewerbesteuer nach Ertrag und Kapital 1970, Wiesbaden 1978, und eigene Berechnungen. 
Insgesamt bleibt festzuhalten, daß die Gewerbesteuerbelastung und die Wirkungen von Hebesatzunterschieden je nach Typ des Einzelbetriebs sehr unterschiedlich ausfallen und von daher als Argument für eine sachliche oder räumliche Ausweichung ebenfalls von unterschiedlichem Gewicht sind. Vor allem für die räumliche Ausweichung sind darüber hinaus aber andere Faktoren von Bedeutung, denen in der Regel größere Relevanz für die Standortwahl zugesprochen wird als der Steuerbelastung. Deshalb wird anschließend zu untersuchen sein, welche Standortforderungen hier eine Rolle spielen, welcher Stellenwert der Steuerbelastung zugemessen wird und ob dabei ebenfalls nach Produktions- und Betriebstypen differenziert werden kann.

Erst auf dieser Grundlage können Schlußfolgerungen über die Wirkungen kommunaler Hebesatzpolitik auf das Steueraufkommen in verschiedenen Gemeindetypen gezogen und Aussagen über Hebesatzspielräume gemacht werden.

2. Die Mobilität der Betriebe als Ausdruck räumlicher Ausweichmöglichkeiten

Je höher die Belastung des betrieblichen Ertrags durch die Gewerbesteuer bzw. durch die Anhebung des Hebesatzes ausfällt, desto eher ist mit einer Reaktion auf diese Belastung zu rechnen. Ob es dazu kommt und welcher Art räumlicher oder sachlicher - sie ist, hängt außer von der Steuerbelastung selbst von einer Reihe von Faktoren ab:

- Zum ersten ist der Reaktionsdruck insgesamt entscheidend für das Verhalten der Betriebe; eine hohe Steuerbelastung wird um so eher in Kauf genommen, je besser der gewählte Standort mit Blick auf die übrigen Standortfaktoren eingeschätzt wird und je eher standortbedingte Kostennachteile verkraftet werden können.

- Zum zweiten wird es auch bei hohem Reaktionsdruck nur dann zu einer räumlichen Ausweichung kommen, wenn Standortalternativen bekannt sind, die den gestellten Anforderungen insgesamt besser gerecht werden als der alte Standort. 
- Zum dritten wird eine räumliche Ausweichreaktion aber nur vollzogen, wenn keine mobilitätshemmenden Bindungen an den gewählten Standort bestehen. Diese Bindungen können persönlicher Art, aber auch sachlicher Art sein, etwa wenn der Kontakt zum regionalen Absatzmarkt für besonders wichtig gehalten wird oder wenn physische Bindungen des $\mathrm{Ka}$ pitals an den einmal gewählten Standort vorliegen, die eine Verlagerung stark verteuern oder sogar unmöglich machen.

Betrachtet man zunächst die Entwicklung der betrieblichen Mobilität in der Bundesrepublik, so ist ein deutlicher Rückgang zu verzeichnen: Während von 1960-1969 4.800 "echte Mobilitätsfälle" geschätzt werden, sind es für den Zeitraum von 1970-1979 nur noch etwa 3.050. ${ }^{51)}$ Dieser Rückgang wird vor allem auf die stark nachlassende Tendenz zur Gründung von Zweigbetrieben zurückgeführt. ${ }^{52)}$ Für die Zukunft wird allerdings mit wieder ansteigender Mobilität gerechnet, insbesondere deshalb, weil sich bei verlangsamendem Wirtschaftswachstum und wachsendem Konkurrenzdruck die verstärkte Notwendigkeit ergibt, standortbedingten Kostennachteilen auszuweichen. ${ }^{53)}$ Mobilitätshemmend könnte sich die verschlechterte Ertragslage allerdings insofern auswirken, als Mobilität mit Kosten und mit Risiken verbunden ist, die von wachsenden ertragstarken Unternehmen eher in Kauf genommen werden. ${ }^{54)}$

Das Mobilitätsverhalten der Betriebe war in der Vergangenheit nach Wirtschaftszweigen sehr unterschiedlich. Dabei spielte neben der physischen Kapitalbindung und der Bindung an Absatz- und Beschaffungsmärkte vor allem die Art der Verlagerungsmöglichkeiten eine Rolle. Die höchste Mobilität wurde für jene Wirtschaftszweige des Verarbeitenden Gewerbes ermittelt, in denen Zweigstellengründungen von größerer Bedeutung sind: In der Elektround Kunststoffindustrie sowie in den Bereichen Leder und Bekleidung. ${ }^{55)}$ Diese Zweigstellengründungen scheinen mit vergleichsweise geringem finan-

51) Sälzer, Bruno E., Standortdynamik von Industrieunternehmungen, Frankfurt-Bern-New York 1985, S. 49.

52) Ebenda, S. 50.

53) Ebenda, S. 53.

54) Vgl. Bade, Franz-Josef, Sektorale und räumliche Aspekte der Mobilität industrieller Betriebe, discussion papers des International Institute of Management - Wissenschaftszentrum Berlin, 1977, S. 29.

55) Vgl. ebenda, S. $9 \mathrm{f}$. 
ziellen Risiko verbunden zu sein; da es sich häufig um Zweigbetriebe mit eher provisorischem Charakter handelt, lassen sie sich ebenso leicht schließen, wie sie eröffnet wurden, und die Stillegungsquoten sind entsprechend hoch. ${ }^{56}$ ) Insofern dürften für die Mobilität von Zweigbetrieben bereits leichtere Standortmängel ausreichen als für die Verlagerung von Stammbetrieben. ${ }^{57}$

Eine vergleichsweise hohe Verlagerungsquote für Stammbetriebe ermittelte Bade für die Bereiche Kunststoffverarbeitung, Maschinenbau, Gummiund Asbestverarbeitung, als tendenziell eher immobil stellten sich die Bereiche Steine, Erden, Holz, Druck und Nahrung sowie der Bereich Eisen, Metall heraus. ${ }^{58)}$

Ursache dieser relativen Immobilität können sowohl geringer Reaktionsdruck, aber auch eine hohe Standortbindung sein. Im letzteren Fall wäre bei steigender Steuerbelastung dementsprechend eher mit einer sachlichen Ausweichung - das heißt im Extrem mit Betriebsschließung - zu rechnen.

3. Der Stellenwert der Steuerbelastung im Zusammenhang mit anderen Faktoren der Standortwahl

Standortfaktoren werden definiert als "Kostenvorteil, der für eine wirtschaftliche Tätigkeit dann eintritt, wenn sie sich an einem bestimmten Ort ... vollzieht. ${ }^{59)}$ Solche Kostenvorteile lassen sich durch die Standortwahl auf verschiedene Weise realisieren:

(1) Kostenvorteile können daraus resultieren, daß externe Effekte bereits an einem Standort vorhandener Aktivitäten und Einrichtungen internalisiert werden. Dabei wird überlicherweise zwischen localization economies und urbanization economies unterschieden. ${ }^{60)}$ Localization economies ergeben sich aus der regionalen Konzentration eines Wirtschaftszweiges und

56) Ebenda. S. $30 \mathrm{ff}$.

57) Ebenda, S. 20.

58) Ebenda, S. 10.

59) Weber, Alfred, Über den Standort der Industrien, Teil 1: Reine Theorie des Standorts, Tübingen 1909, S. 16.

60) Vgl. Lauschmann, Elisabeth, Grundlagen einer Theorie der Regionalpolitik, 3., völlig neu bearb. Aufl., Hannover 1976, S. 44. 
den darauf ausgerichteten Einrichtungen wie etwa den Anschlüssen an spezielle Transportwege, der Existenz organisierter Märkte (Börse) oder eines spezialisierten Arbeitsmarktes.

Urbanization economies resultieren demgegenüber aus der örtlichen Konzentration verschiedener Wirtschaftszweige und können auf vielfältige Weise entstehen: ${ }^{61)}$

- $\quad$ aus Vorteilen der räumlichen Nähe zu Beschaffungs- und Absatzmärkten,

- durch die Vorteile großer, differenzierter Arbeitsmärkte,

- durch ein breites Dienstleistungsangebot,

- aus dem Vorhandensein öffentlicher Infrastruktureinrichtungen und Leistungen,

- $\quad$ aus dem "Wirtschaftsgeist" eines Ortes oder einer Region,

- $\quad$ aus dem Einkommenspotential der angesiedelten Bevölkerung.

(2) Kostenvorteile können weiterhin dann realisiert werden, wenn Produktionsfaktoren zu besonders günstigen Preisen beschafft werden können. Diese Möglichkeit besteht insbesondere beim Faktor Boden, dessen Preise regional starke Unterschiede aufweisen, aber auch für den Faktor Arbeit. Die Kosten des Kapitaleinsatzes streuen wegen der vollständigen Mobilität hingegen nicht.

(3) Kostenvorteile aufgrund der Standortwahl können zum Dritten aus der Minimierung von Transportkosten zum Absatz- und Beschaffungsmarkt und hier insbesondere zum Wohnort der benötigten Arbeitskräfte und bei direkter Beziehung zum Endverbrauch - zum Wohnort der Nachfrager realisiert werden. Die Bedeutung der Transportkosten für den Güterverkehr nimmt wegen der Verbesserung der Verkehrsinfrastruktur und der Verringerung der ökonomischen Entfernungen hingegen ab. ${ }^{62}$ )

(4) Darüber hinaus lassen sich Kostenvorteile aus der regional unterschiedlichen öffentlichen Förderung bzw. aus dem Gefälle in der Abgabenbelastung ziehen. Insbesondere die Gewerbesteuer belastet den Gewinn bzw.

61) Ebenda, S. 47.

62) Vgl. Lauschmann. Elisabeth, Grundlagen einer Theorie der Regionalpolitik, a.a.O., S. 38. 
bei fehlendem Gewinn die Substanz; regionale Steuersatzunterschiede wirken daher prinzipiell ebenso wie andere Kostenunterschiede auch.

In einer Zusammenstellung von Freund und Zabel werden die wichtigsten Standortanforderungen des sekundären Sektors, wie sie in verschiedenen empirischen Untersuchungen ermittelt wurden, aufgelistet und in eine Rangfolge gebracht. ${ }^{63)}$ Danach stehen die Anforderungen an die Verfügbarkeit ausreichender Flächen zu möglichst günstigen Preisen und die Verfügbarkeit von Arbeitskräften eindeutig im Vordergrund, weiter werden in der Regel die Infrastrukturausstattung, insbesondere die Verkehrsanbindung sowie die Lage zum Absatzmarkt, aber auch die Förderung durch die öffentliche Hand als Ansiedlungskriterien genannt. Letztere, wie auch das Hebesatzgefälle zwischen den Gemeinden rangieren jedoch - sofern sie überhaupt genannt werden - an untergeordneter Stelle.

Für die niedrige Bewertung der Hebesätze als Standortfaktor dürfte nicht zuletzt das vergleichsweise schwache Gefälle zwischen den Gemeinden verantwortlich sein - 1985 lag der höchste Hebesatz bei $480 \%$ in Frankfurt, der niedrigste bei $200 \%$ in Ahaus (NW). Würden die Gemeinden ihren Hebesatzspielraum allein unter fiskalischem Gesichtspunkt ausschöpfen, so käme es wahrscheinlich zu einem größeren Gefälle und einer entsprechenden Höherbewertung der Steuersätze durch die Unternehmen.

Überdies lassen sich die Ergebnisse der in der Regel nicht nach Wirtschaftszweigen differenzierenden und allein auf den sekundären Sektor bezogenen Standortstudien nicht vorbehaltlos verallgemeinern.

Welche Bedeutung die einzelnen Standortfaktoren haben, hängt sowohl von den spezifischen Faktoreinsatzkonstellationen und Kostenstrukturen als auch von den Wettbewerbsbedingungen ab, "d.h. davon, inwieweit die Konkurrenz unter den Produzenten dazu zwingt, regionale und lokale Kostenvorteile effektiv zu nutzen. Die optimalen Standortbedingungen der Betriebe müssen also - je nach Branchenzugehörigkeit - entsprechend unterschiedlich

63) Freund, Ulrich und Zabel, Gerhard, Zur Effizienz der regionalpolitischen Industrieförderung, in: Raumforschung und Raumordnung, Heft 3, 36. Jg. 1978. S. 99-106, hier S. 101. 
sein."64) Dabei werden Steuern als Standortfaktor nur dann für relevant gehalten, wenn andere wesentliche Standortfaktoren an einem Ort gegeben sind. $\left.{ }^{65}\right)$

Mit anderen Worten; je unspezifizierter die Anforderungen an den Standort sind und je häufiger ein entsprechendes Standortangebot anzutreffen ist, desto wichtiger werden Hebesatzdifferenzen für die Standortentscheidung. Von nachrangiger Bedeutung sind sie hingegen dann, wenn die Produktion an das Vorhandensein besonderer Bedingungen gebunden ist und deshalb andere Standortfaktoren als der Hebesatz für wichtiger gehalten werden.

Unterschiede in den jeweiligen Standortanforderungen der Wirtschaftsbereiche lassen sich nun zunächst zwischen sekundärem und tertiärem Sektor feststellen. ${ }^{66)}$

- Folgt man der "klassischen" Sektorabgrenzung, so zeichnet sich der tertiäre Sektor vor allem durch die Anbindung des Standorts an die - der Reichweite seines Angebots entsprechende - räumliche Verteilung der Nachfrage aus. Nach der output-orientierten Abgrenzung der Sektoren werden im tertiären Sektor Produktionen zusammengefaßt, deren Produkte direkt an die Endverbraucher weitergegeben werden und weder lagerfähig noch transportierbar sind (Handel und Dienstleistungen). ${ }^{67)} \mathrm{Ko}$ sten der Raumüberwindung treten daher bei den Nachfragern auf, die zum Angebotsort hinpendeln müssen. Aus der Transportkostenminimierung der Nachfrager einerseits und bestimmter, den Rang des Angebots konstituierender Mindestabsatzmengen ergibt sich die Standortverteilung

64) Lauschmann, Elisabeth. Grundlagen einer Theorie der Regionalpolitik, a.a.O., S. 35.

65) Vgl. Amedick, Klaus, Die Besteuerung der gewerblichen Unternehmen als Standortfaktor, Diss. Köln 1959, S. 126, sowie Kaiser, Karl-Heinz, Industrielle Standortfaktoren und Betriebstypenbildung, a.a.O., S. 26.

Diese Bedingung wird deshalb häufig auch bei theoretischen Untersuchungen zur interregionalen Steuerkonkurrenz einführend zugrunde gelegt. Vgl. etwa Grewal, B.S. und Mathews, R.L., Intergovernmental Tax Competition and Co-Ordination, in: Mathews, R.L., Hrsg., State and Local Taxation, Canberra 1977, S. 83-93. hier S. 83.

66) Der primäre Sektor bleibt hier unberücksichtigt, weil sein Hauptbereich - die Land- und Forstwirtschaft - überwiegend nicht gewerbesteuerpflichtig ist und überdies eine Standortwahl im eigentlichen Sinne hier kaum getroffen wird.

67) Zur Abgrenzung des tertiären Sektors vgl. auch Postlep. Rolf-Dieter, Wirtschaftsstruktur und großstädtische Finanzen, a.a.O., S. 18 ff. 
des tertiären Sektors: das zentralörtliche System. ${ }^{68)}$ Die potentiellen Standorte für ein tertiäres Angebot richten sich demnach nach seinem Rang; sie sind um so zahlreicher, je niederrangiger das Angebot und je kleiner sein Einzugsbereich ist.

Die Bindung des tertiären Sektors an zentrale Standorte bestätigt sich in verschiedenen Untersuchungen zur Standortwahl des Dienstleistungsbereichs. Danach hängt die räumliche Verteilung der Betriebe eng mit der Bevölkerungsdichte zusammen ${ }^{69)}$; bei der Suche eines Standorts - sowohl bei Verlagerungen als auch bei Neugründungen - spielt der Kontakt zum Absatz- und Beschaffungsmarkt vor allem für private Dienstleistungsunternehmen eine wesentlich größere Rolle als Standortfaktor als im sekundären Sektor, insbesondere dem Produzierenden Gewerbe. ${ }^{70)}$ Daraus dürfte eine relative Immobilität dieses Bereiches abzuleiten sein. Unter den Ursachen für eine Standortverlagerung steht - ebenso wie im sekundären Sektor - die Grundstücks- und Gebäudesituation im Vordergrund $^{71)}$, allerdings löst sie vor allem Nahverlagerungen aus, eine zentrale Lage des Standortes und/oder eine günstige Verkehrsanbindung sollen weiterhin gegeben sein. ${ }^{72)}$ Daraus erklärt sich auch die Einschätzung verschiedener Raumtypen durch den tertiären Sektor: Stärker noch als Betriebe des sekundären Sektors lehnen Betriebe des Dienstleistungsbereiches periphere Räume ab, und zwar sowohl Kleingemeinden als auch - obwohl weniger strikt- Mittelstädte; Metropolen und Großstädte werden hingegen mit großer Häufigkeit als geeignete Standorte angesehen. ${ }^{73}$ ) Diese Beurteilung wird besonders deutlich von Großunternehmen vertreten.

68) Zum Aufbau und zur empirischen Erfassung zentralörtlicher Verflechtungen und Angebotsschwerpunkte vgl. auch Zimmermann, Horst. Hardt, Ulrike und Postlep, Rolf-Dieter, Bestimmungsgründe der kommunalen Finanzsituation, a.a.O., S. 169 ff.

69) Bloch, Arthur, Entwicklung und Standortorientierung des Dienstleistungsbereichs aus der Sicht der Landesplanung, Schriftenreihe Landes- und Stadtentwicklungsforschung des Landes Nordrhein-Westfalen, Bd. 1.021, ILS Dortmund 1980, S. 49 ff.

70) Dietrich, H.P. und Straus, H.-G., Das Standortverhalten im Dienstleistungsbereich - Ergebnisse von Betriebs- und Expertenbefragungen - 3. Bericht zum Forschungsprojekt "Raumordnerische Standortbeeinflussung bei Arbeitsstätten des Dienstleistungsbereichs, hrsg. von der Gesellschaft für Wohnungs- und Siedlungswesen mbH Hamburg (Gewos), Manuskript. Hamburg 1979, S. 17 und S. 30 ff.; so auch die Ergebnisse von Bloch, Arthur, a.a.O.. S. $69 \mathrm{ff}$.

71) Vgl. Dietrich. H.P. und Straus, H.-G., Das Standortverhalten im Dienstleistungsbereich, a.a.O., S. 17 .

72) Vgl. Dietrich, H.P. und Straus, H.-G., a.a.O., S. 37 und 42.

73) Ebenda, S. 57. 
Die Bedeutung von Steuern und Abgaben als Standortfaktor wird allgemein niedrig eingeschätzt; isoliert betrachtet scheinen sie kaum eine Rolle zu spielen, es sei denn, daß Standortalternativen ansonsten für gleichwertig gehalten werden. ${ }^{74)}$ Daraus läßt sich umgekehrt der Schluß ableiten, daß die Steuer- und Abgabenbelastung um so eher eine Abwanderung induziert, je weniger auch die übrigen Standortbedingungen den Anforderungen entsprechen.

Bemerkenswert ist allerdings, daß das Hebesatzgefälle tendenziell für die Bereiche Handel und Verkehr von größerem Gewicht zu sein scheint als für private Dienstleistungsunternehmen ${ }^{75)}$ und eher für die Errichtung und Verlagerung von Zweigbetrieben als wichtig angesehen wird. ${ }^{76)}$

An den dargestellten Ergebnissen ändert sich wenig, wenn statt der Output-orientierten Abgrenzung des tertiären Sektors eine funktionale Abgrenzung zugrunde gelegt wird. Vielmehr gewinnt dann ein weiterer Standortfaktor an Bedeutung, der die Anziehungskraft von Ballungsgebieten als potentiellen Standort zusätzlich stärkt: die Verfügbarkeit qualifizierter Arbeitskräfte.

Für die in den sekundären Sektor integrierten tertiären Produktionen entfällt zunächst die direkte Anbindung an die Nachfrager nach dem Endprodukt, die Zahl der Standortalternativen erscheint insofern zunächst größer. In vielen Fällen werden die Standorte an den Sitz des Gesamtunternehmens gekoppelt sein, es sei denn, daß aus Gründen unterschiedlicher Standortansprüche der einzelnen Teilproduktionen funktionale Standortspaltungen vorgenommen und insbesondere höherwertige Dienstleistungen räumlich ausgegliedert werden. $\left.{ }^{77}\right) \mathrm{Da}$ sich diese Dienstleistungen durch hohe Qualifikation der Beschäftigten auszeichnen ${ }^{78)}$, dürften die Arbeitsmarktbedingungen, also die Verfügbarkeit hoch qualifizierter Arbeitskräfte, die vor allem in Ballungs-

74) Ebenda, S. 46.

75) Dieser Effekt läßt sich möglicherweise aus dem hohen Anteil von Kleinbetrieben und freiberuflichen Tätigkeiten im Bereich der privaten Dienstleistungsunternehmen erklären, die vielfach gar keine Gewerbesteuer zahlen. Vgl. oben S. sowie Zimmermann, Horst, Hardt, Ulrike und Postlep, Rolf-Dieter, Bestimmungsgründe der kommunalen Finanzsituation, a.a.O., S. 109 f.

76) Vgl. Dietrich, H.P. und Straus, H.-G., Das Standortverhalten im Dienstleistungsbereich, a.a.O., S. 23.40 und 47.

77) Vgl. zum Überblick Bade, Franz-Josef, Funktionale Aspekte der regionalen Wirtschaftsstruktur, in: Raumforschung und Raumordnung, Heft 4, 37. Jg., 1979, S. 253-268.

78) So Postlep, Rolf-Dieter, Wirtschaftsstruktur und großstädtische Finanzen, a.a.O., S. 52. 
gebieten gegeben ist, die wesentliche Anforderung an den Standort darstellen. ${ }^{79)}$ Sowohl aufgrund der Arbeitnehmerpräferenzen als auch aufgrund der Internalisierbarkeit besonderer urbanization und localization economies ${ }^{80}$ ) bieten sich hier besonders jene Ballungsräume als potentielle Standorte an, in denen der Tertiäranteil bereits hoch ist.

Die Steuerbelastung dürfte für diese Produktionen hingegen nahezu unerheblich und daher als Standortfaktor unbedeutend sein.

Für die Bestimmungsgründe der Standortwahl des sekundären Sektors liegt eine Vielzahl von Untersuchungen vor, die jedoch zu teilweise recht unterschiedlichen Ergebnissen gelangen. ${ }^{81)}$ Diese Divergenzen werden zum einen darauf zurückgeführt, daß bei Unternehmensbefragungen Standortanforderungen genannt und als "wichtig" bezeichnet werden, die quasi ubiquitär erfüllt werden und insofern keine Standortlenkung bewirken. So wird etwa der Lenkungseffekt der Verkehrsinfrastruktur von einigen Autoren für weit geringer gehalten, als er von den Unternehmen selbst eingeschätzt wird. ${ }^{82)}$ Darüber hinaus werden Diskrepanzen aus der räumlichen und zeitlichen Abgrenzung der Untersuchungen sowie ihrer Fragestellung erklärt. ${ }^{83)}$ So sind etwa bei Befragungen in Fördergebieten andere Ergebnisse als bei Untersuchungen in Ballungsgebieten fast selbstverständlich zu erwarten, Befragungen im Falle von Betriebsverlagerungen kommen zu anderen Resultaten als im Falle

79) Schliebe kommt deshalb zu dem Ergebnis. daß etwa die Voraussetzungen für Forschungsund Entwicklungstätigkeiten von der Arbeitsplatzstruktur her in Peripherräumen relativ ungünstig sind. Schliebe. Klaus. Zur regionalen Verteilung höherwertiger Unternehmensfunktionen und Arbeitskräfteressourcen, in: Informationen zur Raumentwicklung, hrsg. von der BfLR. Heft 7/1978, S. 545-554, hier S. 552.

Bade belegt dies durch seine Feststellung, "daß die räumliche Arbeitsteilung bei höherwertigen Funktionen an Intensität zunimmt: je höherwertiger eine Funktion (zum Beispiel gemessen am Einkommen oder an der dafür notwendigen Ausbildung), desto stärker konzentriert sie sich auf die Ballungskerne." Bade. Franz-Josef. Die Beschäftigungsentwicklung in den Regionen der Bundesrepublik Deutschland 1976-1983, 2. Zwischenbericht zum Gutachten "Die regionale Verteilung von Wirtschaftsaktivitäten" im Auftrage des Niedersächsischen Ministers für Wirtschaft und Verkehr, Schriften des Deutschen Instituts für Wirtschaftsforschung. Berlin 1985. S. 64.

80) Vgl. Postlep, Rolf-Dieter, Wirtschaftsstruktur und großstädtische Finanzen, a.a.O., S. $108 \mathrm{ff}$.

81) Vgl. noch einmal die Übersichten von Freund. Ulrich und Zabel, Gerhard, Zur Effizienz der regionalpolitischen Industrieförderung ..., a.a.O.. S. 101, sowie Kaiser, Karl-Heinz, Industrielle Standortfaktoren und Betriebstypenbildung, a.a.O., S. 42 ff.

82) Ebenda, S. 102.

83) Kaiser, Karl-Heinz, Industrielle Standortfaktoren und Betriebstypenbildung, a.a.O., S. $28 \mathrm{ff}$. 
von Neugründungen usw. Trotzdem kristallisieren sich einige Ergebnisse als durchaus verallgemeinerbar heraus.

Das erste bezieht sich noch einmal auf einen wesentlichen Unterschied zur Standortwahl des tertiären Sektors: Sofern überhaupt eine Abwägung von Standortalternativen unter betrieblichen Kostengesichtspunkten vorgenommen wird und sich die Standortentscheidung nicht als Wohnortentscheidung des Unternehmers entpuppt ${ }^{84}$, ist sie räumlich insgesamt weit weniger an $\mathrm{Ab}$ satz- oder Beschaffungsmärkte gebunden als der auf Nachfragezentren hin orientierte tertiäre Sektor. Mit der Verbesserung der Verkehrsinfrastruktur und der Verringerung der ökonomischen Entfernungen sowie mit der Produktion weniger rohstoffintensiver Erzeugnisse nimmt der Einfluß der Transportkosten ab, damit verringert sich für die Mehrzahl der Betriebe des sekundären Sektors die Standortgebundenheit der Produktion, und andere Standortfaktoren, wie insbesondere die Verfügbarkeit von geeigneten Arbeitskräften und von kostengünstigen Flächen, gewinnen an Bedeutung.

Differenziert man jedoch nach Wirtschaftszweigen, so zeigen sich auch hier deutliche Unterschiede.

Als stark an Rohstoffquellen oder Beschaffungsmärkte angebunden haben sich die Bereiche Bergbau, Steine und Erden, Nahrungs- und Genußmittel sowie Teile der holz-, eisen- und metallverarbeitenden Industrie erwiesen. ${ }^{85)}$ Diese Produktionen wurden oben bereits als überwiegend immobil charakterisiert. Selbst wenn die Steuerbelastung von den Betrieben als zu hoch angesehen wird - oben wurde etwa ermittelt, daß gerade der Bergbau stark belastet wird - bestehen hier kaum räumliche Ausweichmöglichkeiten.

- Der Faktor Boden ist für die Bereiche Eisen, Metall, die chemische Industrie sowie den Maschinen-, Stahl- und Fahrzeugbau als entscheidender Standortfaktor anzusehen. ${ }^{86)}$ Für die drei zuletzt genannten ist darüber

84) Diese Art der "Standortwahl" scheint für Unternehmensneugründungen die Regel zu sein. Vgl. Fürst. Dietrich und Zimmermann, Klaus (unter Leitung von Karl-Heinrich Hansmeyer), Standortwahl industrieller Unternehmen, Schriftenreihe der Gesellschaft für Regionale Strukturentwicklung. Bd. 1, Bonn 1973. S. 79 f.

85) Freund, Ulrich und Zabel, Gerhard, Zur Effizienz der regionalpolitischen Industrieförderung .... a.a.O., S. 102.

86) So Kaiser, Karl-Heinz. Industrielle Standortfaktoren und Betriebstypenbildung, a.a.O., S. 81. 
hinaus die Verfügbarkeit qualifizierter Arbeitskräfte von besonderer Bedeutung. ${ }^{87)}$

- Wenig ausgeprägte Standortansprüche außer dem Bedarf an unqualifizierten Arbeitskräften zeigen sich für die Bereiche Elektro- und Feinmechanik sowie für die Textil- und Bekleidungsindustrie. ${ }^{88)} \mathrm{Da}$ diese Industrien als vergleichsweise mobil zu kennzeichnen sind, dürfte der Steuerbelastung tendenziell größeres Gewicht für eine Verlagerung oder Teilverlagerung zukommen.

Weitere Differenzierungen lassen sich mit Blick auf die Betriebsgröße sowie für Stamm- gegenüber Zweigbetrieben treffen:

Während kleine Betriebe eher auf Absatzmarktfaktoren achten, findet sich eine mit der Betriebsgröße positiv korrelierende Bedeutungseinschätzung von Arbeitsmarktfaktoren und umfangreichen Arbeitsmärkten. $^{89)}$ Dies erklärt sich vor allem daraus, daß die Vorteile großer und differenzierter Arbeitsmärkte in der Regel von Großbetrieben besser als von Kleinbetrieben genutzt werden können, da letztere in der Konkurrenz um Arbeitskräfte den Großbetrieben unterlegen sind und mögliche Einsparungen durch die Verfügbarkeit qualifizierter Mitarbeiter durch die Fluktuation von Arbeitskräften eher kompensiert werden können. ${ }^{90)}$ Nach Kaiser hat außerdem die Steuerbelastung für Großbetriebe größeres Gewicht als für Kleinbetriebe ${ }^{91)}$, wie aus der Konstruktion der Gewerbesteuer nicht anders zu erwarten war.

- Für die Verlagerung von Stammbetrieben scheint vor allem die Verfügbarkeit von Grund und Boden, für Zweigbetriebsgründungen oder Verlagerung dagegen besonders die "Billigkeit" der Faktoren, das Angebot an bezugsferatigen Hallen und die Anbindung an das überregionale Verkehrsnetz ins Gewicht zu fallen. ${ }^{92)}$ Aufgrund der sehr hohen Kostenrea-

87) Ebenda, S. 81 .

88) Ebenda, S. 81.

89) Ebenda, S. 82.

90) Vgl. Lauschmann. Elisabeth. Grundlagen einer Theorie der Regionalpolitik, a.a.O., S. 50.

91) Kaiser, Karl-Heinz, Industrielle Standortfaktoren und Betriebstypenbildung, a.a.O., S. 84.

92) Ebenda, S. 89, sowie Freund, Ulrich und Zabel, Gerhard, Zur Effizienz der regionalpolitischen Industrieförderung ..., a.a.O., S. 102. 
gibilität dürften für Zweigbetriebe Steuerbelastungsunterschiede ebenfalls von größerer Bedeutung sein.

\section{Die Ergebnisse der betriebstypen-spezifischen Betrachtung}

Zusammenfassend ist noch einmal festzuhalten, daß die Wirkungen kommunaler Hebesatzpolitik je nach Betriebstyp als mehr oder weniger gravierend gelten können und deshalb unterschiedlichen Reaktionsdruck verursachen. Entscheidende Bedeutung kommt dabei zum einen der Belastung des betrieblichen Ertrags bei alternativen Gewerbesteuerhebesätzen, zum anderen dem Gewicht der Steuerbelastung, verglichen mit anderen Standortfaktoren, zu. Dabei wurde die Frage der Überwälzungsmöglichkeiten hier ausgeklammert, weil sie in der Regel nicht standortabhängig sein dürften.

Bei einem modellhaften Belastungsvergleich wurde abgeleitet, daß die Belastung vor allem von der Betriebsgröße und der Höhe des Kapitaleinsatzes in Relation zum Gewerbeertrag abhängt. Die letzte der beiden Determinanten wurde für die Bereiche des produzierenden Gewerbes - anhand der Daten aus dem Jahr 1970 - aufgeschlüsselt. Hohe Belastungsunterschiede bei alternativen Hebesätzen errechneten sich erwartungsgemäß in jenen Bereichen, in denen hoher Kapitaleinsatz relativ niedrigen Gewinnen gegenübersteht, wie 1970 vor allem im Bergbau, aber auch der Textil- und Bekleidungsindustrie. Wegen der zum Teil starken Veränderung der Gewinn-Kapitalrelationen, aber auch der absoluten Gewinne im Zuge der seit 1970 eingetretenen Entwicklung lassen sich die ermittelten Ergebnisse nicht ohne weiteres für die einzelnen Wirtschaftszweige extrapolieren; Schätzungen anhand von Indikatoren wären jedoch zu grob, um zu einem brauchbaren Belastungsvergleich zu kommen. Allerdings erfolgte die Berechnung für 1970 nur exemplarisch; das grundsätzliche Ergebnis gilt nach wie vor und ist aus der Sicht der einzelnen Gemeinde in Kenntnis der jeweiligen wirtschaftsstrukturellen Bedingungen durchaus umsetzbar.

Der durch die Steuerbelastung erzeugte Reaktionsdruck führt, wenn er stark genug ist, zu sachlicher oder räumlicher Ausweichung; da eine sachliche Ausweichung im Falle der Gewerbesteuer aber nur sehr eingeschränkt mög- 
lich ist, wird es, sofern es sich als lohnend erweist, zu einer räumlichen Ausweichung kommen.

Entscheidend für die räumliche Ausweichung anläßlich einer Steuererhöhung ist zum einen die Standortbindung bzw. die Mobilität der Betriebe, zum anderen die Relevanz der Steuerbelastung im Vergleich mit anderen Standortfaktoren. Bei der Betrachtung betrieblicher Mobilität in der Vergangenheit erwiesen sich erwartungsgemäß Zweigbetriebe, aber auch Stammbetriebe in einigen Branchen, wie etwa der Kunststoffverarbeitung und im Maschinenbau als überdurchschnittlich mobil. Dabei ist jedoch zwischen solchen Betrieben, die keine besonderen Standortansprüche aufweisen und auch aufgrund hoher Steuerbelastung zur Abwanderung motiviert werden können, und Betrieben, die bestimmte spezifische Standortanforderungen stellen und aufgrund von Faktorengpässen mobil werden, zu unterscheiden.

Allgemein kann davon ausgegangen werden, daß das Steuerlastgefälle mit um so geringerer Wahrscheinlichkeit Abwanderung auslöst, je höher die Standortanforderungen eines Betriebs sind und je besser der gewählte Standort diese Ansprüche erfüllt.

Solche spezifischen Ansprüche und Standortbindungen finden sich vielfach im auf den Absatzmarkt ausgerichteten tertiären Sektor, der damit dem Zugriff der gemeindlichen Besteuerung in besonderem Maße "ausgeliefert" wäre, wenn die steuerrechtlichen Regelungen - Steuerpflicht, Freibetragsregelungen - nicht andererseits einen gewissen Schutz gerade dieses Wirtschaftsbereichs bewirken würden. Aber auch im sekundären Sektor finden sich Bereiche mit spezifischen Standortbedingungen.

Hier handelt es sich vor allem um Bereiche, die von Rohstoffquellen und Beschaffungsmärkten abhängig sind, wie etwa den Montanbereich oder die Nahrungs- und Genußmittelindustrie sowie jene Produktionen, die auf hochqualifizierte Arbeitskräfte angewiesen sind.

Wenig ausgeprägte Standortansprüche und entsprechend höheres Gewicht des Standortfaktors "Steuerlast" findet sich hingegen wieder im Bereich Textil, Bekleidung sowie einigen anderen Bereichen, die sich besonders durch niedrige Anforderungen an den Faktor "Arbeit" auszeichnen. 
Die Frage nach der Steuerbelastung und den räumlichen Ausweichmöglichkeiten einzelner Betriebstypen wurde deshalb so ausführlich behandelt, weil daraus abgeleitet werden kann, daß die gemeindlichen Besteuerungsspielräume im wesentlichen von der Wirtschaftsstruktur und den jeweiligen Standortbedingungen in einer Gemeinde bestimmt werden.

Der Analyse dieser Spielräume in unterschiedlichen Gemeindetypen und den Folgewirkungen kommunaler Gewerbesteuer-hebesatzpolitik auf andere Einnahmen wendet sich der folgende Abschnitt zu.

c. Wirtschafts-, Bevölkerungs- und Raumstruktur als Determinanten der fiskalischen Wirkungen kommunaler Hebesatzpolitik bei der Gewerbesteuer

1. Zur fiskalischen Ergiebigkeit von Hebesatzanhebungen unter Status-quoBedingungen

Die Gemeinden wenden ihren Gewerbesteuer-Hebesatz auf die innerhalb ihrer Grenzen ermittelten Steuermeßbeträge an; das Aufkommen an Gewerbesteuer errechnet sich als Summe der Meßbeträge x Hebesatz.

Oberhalb des Umlagesatzes von $80 \%$ ergibt sich das zusätzliche Aufkommen aus einer Hebesatzanhebung - vereinfacht dargestellt - als Hebesatz x Bemessungsgrundlagen. Mit anderen Worten: Die fiskalische Ergiebigkeit einer Hebesatzanhebung hängt zunächst - läßt man die Reaktionen der Steuerzahler noch außer Betracht - von der Summe der Bemessungsgrundlagen ab. Eine Hebesatzanhebung ist um so ergiebiger, je breiter die Bemessungsgrundlagenbasis einer Gemeinde ist. Betrachtet man beispielhaft die etwa gleich großen Gemeinden Düsseldorf und Dortmund, so errechnet sich aus den Gewerbesteuereinnahmen (brutto) und den angewendeten Hebesätzen für 1985 eine Bemessungsgrundlagensumme von 174.050.000 DM insgesamt und $310 \mathrm{DM}$ je Einwohner in Düsseldorf, von nur 63.913.000 DM bzw. 112 DM je Einwohner in Dortmund. Unterstellt man für beide Gemeinden eine Hebesatzanhebung von $400 \%$ auf $450 \%$, so würde Düsseldorf nach der vereinfachten Rechnung zusätzlich 87.025.000 DM einnehmen, Dortmund hinge- 
gen nur 31.956.500 DM. Dieser Unterschied ist rein wirtschaftsstrukturell bedingt. $^{93)}$

Der Bemessungsgrundlageneffekt wirkt sich natürlich umgekehrt auch auf diejenige Hebesatzsteigerung aus, die notwendig ist, um ein bestimmtes zusätzlich benötigtes Aufkommen zu erzielen. Für den Länder-Durchschnittsvergleich wird so etwa festgestellt, daß die Gemeinden in Baden-Württemberg bei durchschnittlicher Hebesatzanspannung überdurchschnittliche Einnahmen erzielen können, die strukturschwachen Gemeinden des Saarlandes trotz höchster Hebesätze jedoch nur unterdurchschnittliche Einnahmen zu verzeichnen haben. ${ }^{94)}$

Allgemein gilt, daß die Gewerbesteuer - bei gleichen Hebesätzen - tendenziell um so mehr Einnahmen erbringt, je größer die Produktionsleistung in einer Gemeinde ist; dabei ist allerdings von entscheidender Bedeutung, wie diese Produktionsleistung zustande kommt und aus welchen Komponenten sie sich zusammensetzt. Wie bereits beschrieben, unterliegen der Gewerbesteuer nur bestimmte Bestandteile der Wertschöpfung bzw. der Wert nur eines Produktionsfaktors, des Kapitals, und das auch nur dann, wenn die Produktion im Gewerbebetrieb erfolgt und die Freibeträge der Gewerbeertrag- und -kapitalsteuer überschritten werden.

Allein mit Blick auf die Bemessungsgrundlagen der Gewerbesteuer sind daher Gemeinden im Vorteil, in denen sich ertragsstarke Großbetriebe des sekundären Sektors mit hoher Kapitalintensität finden. Empirische Untersuchungen bestätigen, daß die steuerliche Ergiebigkeit des Bruttoinlandsprodukts in industriell geprägten Gemeinden am größten ist ${ }^{95)}$, entsprechend lohnend sind hier auch Hebesatzanhebungen.

Diese Darstellung hat allerdings verschiedene Aspekte vernachlässigt. Zum einen blieb unberücksichtigt, daß die Gewerbesteuerschuld als Betriebsausgabe vom zu versteuernden Gewinn abzugsähig ist und deshalb bereits die

93) Die wirtschaftsstrukturellen Merkmale beider Städte werden im einzelnen beschrieben bei Postlep, Rolf-Dieter, Regionale Effekte höherwertiger Dienstleistungen, Schriftenreihe der GRS, Bd. 10, Bonn 1982, S. 130 ff. sowie bei Zimmermann, Horst, Hardt, Ulrike und Postlep, Rolf-Dieter, Bestimmungsgründe der kommunalen Finanzsituation, a.a.O., S. $118 \mathrm{ff}$.

94) Strauß, Wolfgang, Räumliche Wirkungen der Gewerbesteuer, in: Informationen zur Raumentwicklung, Heft 6/7, 1983, S. 405-418, hier S. 410 f.

95) Zimmermann, Horst, Hardt, Ulrike und Postlep, Rolf-Dieter, Bestimmungsgründe der kommunalen Finanzsituation, a.a.O., S. $118 \mathrm{ff}$. 
Bemessungsgrundlage der Gewerbeertragsteuer schmälert. Zum zweiten wurde außer acht gelassen, daß sie als Betriebsausgabe auch die zu versteuernden Gewinne bei der Einkommensteuer mindert und insofern bei einer Hebesatzanhebung Folgewirkungen auf andere kommunale Einnahmequellen eintreten.

Der zuerst genannte Effekt bewirkt, daß bei Hebesatzanhebungen die Bemessungsgrundlagen der Gewerbesteuer sinken, ohne daß irgendeine Ausweichreaktion stattgefunden hätte. Wie oben gezeigt wurde, ist diese Minderung der Bemessungsgrundlagen am ausgeprägtesten, wenn die Betriebe keine Gewerbekapitalsteuer zahlen, er schlägt hingegen um so schwächer durch, je kapitalintensiver die Produktion ist und je niedriger die Gewinne sind. Diese Konstellation wirkt sich insgesamt negativ auf die Bemessungsgrundlagen in einer Gemeinde aus; die Konstruktion der Gewerbesteuer gewährleistet jedoch, daß die Gemeinden selbst bei Gewinnlosigkeit der 'ngesiedelten Betriebe noch Einnahmen aus der Gewerbekapitalsteuer erhalten und außerdem eine Hebesatzanhebung einen größeren prozentualen - wenn auch absolut geringeren - Aufkommenszuwachs erbringt als bei Gewinn erwirtschaftenden Betrieben. Dieser höheren relativen Ergiebigkeit einer Hebesatzsteigerung entspricht andererseits allerdings auch eine stärkere zusätzliche Steuerbelastung der Zensiten und damit ein höherer Reaktionsdruck bei den Belasteten.

Welche Wirkung der Abzug der Gewerbesteuerschuld von den zu versteuernden Gewinnen auf die kommunalen Einnahmen hat, hängt zum einen von der Höhe der Einkommen ab. Die Einnahmen aus dem gemeindlichen Anteil an der Einkommensteuer - und in der Folge die Einnahmen aus Schlüsselzuweisungen nach mangelnder Steuerkraft - bleiben nahezu unberührt, solange die Einkommensminderung die zu versteuernden Einkommen nicht unter die für die Aufteilung des Gemeindeanteils relevante Sockelgrenze absenkt. In diesem Fall reduziert sich zwar die zu verteilende Finanzmasse, für die einzelne Gemeinde dürfte die Wirkung auf die Einnahmen jedoch kaum merklich sein. Sie wird erst dann gewichtiger, wenn Einkommen unterhalb der Sockelgrenze vermindert werden.

Zum zweiten wird die betrachtete Gemeinde natürlich dann nicht von der Einkommensminderung betroffen, wenn die Bezieher der Gewinneinkommen 
selbst nicht innerhalb der Gemeindegrenzen ansässig sind. Die negativen fiskalischen Folgen der Hebesatzanhebung können unter dieser Bedingung auf andere Gemeinden abgewälzt werden.

2. Ausweicheffekte als Resultat einer Hebesatzanhebung und ihre Folgewirkungen in verschiedenen Gemeindetypen

Sobald sich die Steuerzahler an eine Anhebung der Hebesätze anzupassen beginnen, so bedeutet dies aus gemeindlicher Sicht in der Regel einen Verlust an Bemessungsgrundlagen. Solche Anpassungen sind im Falle der Gewerbesteuer in Form der Substitution von Kapital, als Betriebsschließung oder vollständiger oder teilweiser - Verlagerung möglich. Allein bei der Substitution von Kapital können aufgrund der Reaktion positive fiskalische Effekte auftreten, wenn über zusätzliche Einstellung von Arbeitskräften die Einnahmen aus dem Einkommensteueranteil wachsen. Diesem Wirkungsverlauf den die Gemeinden auch strategisch einsetzen könnten - sind jedoch wahrscheinlich enge Grenzen gesetzt.

Betriebsaufgaben und Verlagerungen mindern die Bemessungsgrundlagen der Kommunalsteuern jedoch in jedem Fall: Zunächst kommt es zu einer Schrumpfung der Bemessungsgrundlagen bei den unmittelbar reagierenden Zensiten; die betroffene Gemeinde hätte zu überprüfen, ob die Zusatzeinnahmen aus der Hebesatzsteigerung die Mindereinnahmen aufgrund sinkender Bemessungsgrundlagen noch übersteigen. Zusätzlich sind jedoch die durch die originären Reaktionen ausgelösten Folgewirkungen im Unternehmens- und im Haushaltsbereich und deren fiskalische Konsequenzen $\mathrm{zu}$ berücksichtigen. Im Unternehmensbereich können sich über Absatz- und Lieferverflechtungen Schrumpfungsprozesse fortsetzen, im Bereich der Haushalte kommt es zu Einkommensminderungen aufgrund von Beschäftigungseinbußen. ${ }^{96)}$ Die daraus resultierenden fiskalischen Effekte wären den hebesatzbedingten Mehreinnahmen ebenfalls gegenzurechnen.

96) Der umgekehrte Wirkungszusammenhang wird beschrieben bei Spehl, Harald; Töpfer, Klaus, und Töpfer, Peter, Folgewirkungen von Industrieansiedlungen, Schriftenreihe der Gesellschaft für Regionale Strukturentwicklung, Bd. 3, Bonn 1975. 
Das Ausmaß der zu erwartenden Veränderungen hängt nun vor allem von den Standortbedingungen und den wirtschaftsstrukturellen Gegebenheiten, darüber hinaus aber von den funktionalen Verflechtungen mit anderen Gemeinden ab.

Diese Bedingungen hätte jede Gemeinde - wollte sie ihr Gewerbesteuerpotential ermitteln, für sich selbst zu untersuchen. Hier können natürlich nur einige prinzipielle Überlegungen angeführt werden, die sich insbesondere auf den Vergleich verschiedener Gemeindetypen beziehen.

Oben wurde bereits beschrieben, in welch unterschiedlichem Maße die einzelnen Produktionen von einer Hebesatzerhöhung betroffen werden und welche Rolle die Steuersätze für diese Produktionszweige gegenüber anderen Standortfaktoren spielen.

Die Verteilung der einzelnen Produktionen auf den Gesamtraum läßt sich - sieht man von den Beharrungstendenzen auch bei unternehmerischem Standortverhalten einmal $a b^{97}$ - aus den jeweiligen Standortansprüchen ableiten. Dementsprechend unterschiedlich haben sich die einzelnen Raum- bzw. Gemeindetypen entwickelt; sie stehen in mehr oder weniger ausgeprägten Konkurrenzbeziehungen zueinander, weil bei Standortverlagerungen der angesiedelten Betriebe nur jeweils bestimmte neue Standorte in Frage kommen.

Unter diesem Aspekt läßt sich zunächst eine grobe Unterscheidung zwischen Ballungsräumen und nicht verdichteten Räumen treffen. Innerhalb der Ballungsräume ist noch einmal zwischen den Ballungskernen und den Umlandgemeinden zu differenzieren. Aber auch die Ballungskerne weisen im Vergleich noch einmal deutliche Unterschiede in ihrer Wirtschaftsstruktur auf.

Betrachtet man zunächst einmal die nicht verdichteten peripheren Räume, so ist wegen der schwach ausgeprägten Zentralität ein unterdurchschnittlicher Besatz mit tertiären Produktionen festzustellen. ${ }^{98)}$ Unter

97) Die Ausführungen Bades zum Standortverhalten von Industriebetrieben lassen vermuten, daß sich ein großer Teil der Betriebe zumindest nicht am optimalen Standort befindet, da Standortverlagerungen aufgrund der organisatorischen Komplexität vermieden werden, sofern nicht gravierende Engpaßsituationen auftreten. Bade, Franz-Josef, Sektorale und räumliche Aspekte der Mobilität industrieller Betriebe, a.a.O., S. 5 ff.

98) Vgl. die Ergebnisse für Nordrhein-Westfalen bei Zimmermann, Horst, Hardt, Ulrike und Postlep, Rolf-Dieter, Bestimmungsgründe der kommunalen Finanzsituation, a.a.O., S. 124 ff. 
steuerlichem Aspekt ist dieser Tatbestand nicht unbedingt von großer Bedeutung, weil ein nicht unerheblicher Teil dieser Produktionen ohnehin keine Gewerbesteuer zahlt. Allerdings kann sich die mangelnde Ausstattung durchaus als Standortnachteil für die betreffenden Gemeinden bemerkbar machen.

Innerhalb des sekundären Sektors zeigt sich ein Ansiedlungsverhalten mit offensichtlich niedrigen Standortansprüchen: Die betrachteten Gemeinden können vor allem preisgünstige Flächen, zum Teil bezugsfertige Betriebshallen sowie insbesondere die Verfügbarkeit unqualifizierter Arbeitskräfte anbieten, eine ausgebaute Verkehrsinfrastruktur und ein Anschluß an das überregionale Verkehrsnetz sind ebenfalls vielerorts vorhanden. Da die kostensenkenden Angebote aber von sehr vielen Gemeinden gemacht werden können, sehen sich diese einer harten Konkurrenz um ansiedlungswillige bzw. abwanderungsbereite Betriebe ausgesetzt. Dabei kann der Gewerbesteuerhebesatz als Konkurrenzparameter eingesetzt werden; allerdings dürfte diese Konkurrenz die Hebesatzspielräume stark einschränken.

Nach Kaiser "erweist sich die übergroße Mehrzahl der Betriebe als für die Ansiedlung in wirtschaftsschwachen Gebieten wenig geeignet, da die dortigen Bedingungen einfach nicht die Voraussetzungen in sich bergen, um für die große Mehrzahl industrieller Betriebe einen ökonomisch vertretbaren Standort ermöglichen zu können".99) Das bedeutet, daß solche Betriebe, sofern sie noch in den betrachteten Räumen angesiedelt sind, zu den potentiellen Abwanderern zählen. Eine Steuererhöhung kann hier im Zweifel den Ausschlag zur Mobilität geben.

Mit niedrigen Steuersätzen werden aber gleichzeitig Betriebe angezogen, die einerseits wenig leistungsfähig, andererseits stärker steuerreagibel sind. "Finanzielle Förderung von Betriebsansiedlungen dürfte in der Regel eher aus anderen Standorten verdrängte Betriebe anziehen, die aus Kostengründen den alten Standort nicht halten können, als expandierende Unternehmen". ${ }^{100)}$

99) Kaiser, Karl-Heinz, Industrielle Standortwahl und Betriebstypenbildung, a.a.O., S. 166.

100) Gee, Colin; Keller, Ulrike und Treuner, Peter, Infrastrukturelle und wirtschaftsstrukturelle Bestimmungsgründe der industriellen Standortwahl, Schriftenreihe des IREUS, Bd. 4, Stuttgart 1980, S. 153. 
Als geeigneten Betriebstyp zur Ansiedlung in wirtschaftsschwachen Regionen beschreibt Kaiser jenen, der besonderen Wert auf die Angebote der betrachteten Gemeinden legt: Boden und Gebäude sowie billige, insbesondere weibliche Arbeitskräfte. Dazu zählen vor allem Betriebe bzw. Zweigbetriebe der Konsumgüterindustrie, speziell der Textilindustrie. ${ }^{101)}$ Betriebe dieser Art, die oben als relativ steuerreagibel und mobil gekennzeichnet wurden, finden sich auch tatsächlich gehäuft in wirtschaftsschwachen Regionen. ${ }^{102)}$

Aufgrund ihrer Wirtschaftsstruktur stehen den betrachteten Gemeinden damit vergleichsweise eingeschränkte Besteuerungsspielräume offen. Dabei sind nicht nur die Bemessungsgrundlagen relativ niedrig, sondern auch die Hebesatzspielräume enger als in anderen Gemeindetypen. Dies resultiert zum einen aus den Merkmalen der angesiedelten Betriebe - ihrer Leistungsfähigkeit, der Bewertung von Steuern als Standortfaktor sowie ihrer teilweise höheren Mobilität - zum anderen aber auch aus der Ubiquität eines entsprechenden Standortangebots.

Gegenüber den wirtschaftsschwachen peripheren Gemeinden sind Ballungsraumgemeinden in der Regel aufgrund der realisierbaren urbanization economies sowohl mit Blick auf die steuerlichen Bemessungsgrundlagen ${ }^{103)}$ als auch hinsichtlich ihrer Steuersatzspielräume im Vorteil. Die hier angesiedelten Betriebe haben im Durchschnitt spezifischere, qualitativ höhere Ansprüche an ihren Standort als jene, die einen peripheren Standort gewählt haben. Dafür sind vor allem die Arbeitsmarktbedingungen verantwortlich; Verdichtungsräume bieten sowohl größere als auch spezialisiertere Arbeitsmärkte, Betriebe aus Branchen mit qualitativ hochwertigen Arbeitsplätzen bevorzugen solche Standorte. ${ }^{104)}$ Dabei dürfte es sich eher um Großbetriebe handeln, da diese die Vorteile großer Arbeitsmärkte - wie beschrieben - besser nutzen können. Zwar werden Großbetriebe tendenziell stärker durch die Gewerbesteuer belastet als Kleinbetriebe und sind deshalb

101) Vgl. Kaiser, Karl-Heinz, Industrielle Standortwahl und Betriebstypenbildung, a.a.O., S. 165 ff.

102) Vgl. Schliebe, Klaus, Industrieansiedlungen, Forschungen zur Raumentwicklung, Bd. 11, hrsg. von der BfLR, Bonn 1982, S. $101 \mathrm{f}$.

103) Vgl. die Ergebnisse für Nordrhein-Westfalen bei Zimmermann, Horst, Hardt, Ulrike und Postlep, Rolf-Dieter, Bestimmungsgründe der kommunalen Finanzsituation, a.a.O., S. 118 ff.

104) Vgl. Gee, Colin u.a., Infrastrukturelle und wirtschaftsstrukturelle Bestimmungsgründe der industriellen Standortwahl, a.a.O., S. 152 ff. 
tendenziell steuerreagibler, aus gemeindlicher Sicht bieten sie jedoch zunächst eine breitere Bemessungsgrundlagenbasis.

Aber auch andere wesentliche Standortansprüche - etwa an die Infrastruktur oder das Dienstleistungsangebot - werden insgesamt besser erfüllt als in peripheren Regionen. Deshalb war in der Vergangenheit die überwiegende Zahl der Verlagerungen auf Verdichtungsräume, nicht auf periphere Gebiete gerichtet. $^{105)}$

Teilweise treten jedoch auch Engpässe auf - insbesondere im Umweltbereich und im Angebot an preisgünstigen Flächen - die eine Segregation der Produktionen innerhalb der Verdichtungsräume verursachen. Insofern ist vor allem zwischen Ballungskernen und Umlandgemeinden, darüber hinaus aber aufgrund des unterschiedlichen Unternehmensbesatzes - zwischen verschiedenen Typen von Ballungskernen zu differenzieren.

Zunächst ist für so gut wie alle Ballungskerne eine direkte Konkurrenzbeziehung mit ihrem Umland auszumachen. Diese Konkurrenzbeziehung ist je nach Wirtschafts- und Sozialstruktur der Ballungsräume mehr oder weniger ausgeprägt. Dabei dürfte der Gewerbesteuerhebesatz zumindest für die Ballungskerne von untergeordneter Bedeutung als Konkurrenzparameter sein. Eher relevant ist er für die untereinander konkurrierenden Umlandgemeinden, sofern die übrigen Standortanforderungen etwa gleichmäßig erfüllt werden.

Betrachtet man zuerst wieder den tertiären Sektor, so sind hier besondere Präferenzen für die Ballungskerne, speziell auf Seiten höherrangiger Produktionen ${ }^{106)}$ festzustellen. Traditionell abgegrenzte tertiäre Produktionen sind in hohem Maße auf die Internalisierung externer Effekte - urbanization economies - und auf die Verbindung zum Absatzmarkt angewiesen; Abwanderung ist daher häufig mit Nachfrageproblemen verbunden. Überdies tritt der Abwanderungsdruck aufgrund von Flächenengpässen wegen der "Stapelbarkeit von Arbeitsplätzen" hier weniger ausgeprägt auf als im sekundären Sektor.

105) So Schliebe, Klaus, Zum Standortverhalten der Industriebetriebe, a.a.O., S. 357.

106) Nach Christaller bestimmt sich der Rang eines tertiären Angebots aus seiner Reichweite, dieses wiederum bestimmt über die Standortanforderungen; je weiter die Reichweite, desto zentraler muß der als geeignet anzusehende Standort sein. 
Mit einer Umfrage ${ }^{107)}$ im tertiären Sektor wurde ermittelt, daß insbesondere in "Metropolen" angesiedelte Betriebe den gewählten oder einen vergleichbaren, ebenfalls metropolitanen Standort für geeignet, nur noch $56 \%$ einen großstädtischen Standort und $25 \%$ einen Standort in einer Mittelstadt im Einzugsbereich von Metropolen oder Großstädten für geeignet halten. Trotzdem scheint sich der Kostendruck in zentralen Standorten auch hier bemerkbar zu machen: "Zwar hat die Mehrzahl der erfaßten Betriebe nach wie vor der Verlagerung ihren Standort in den Kernstädten. Dieser Standorttyp weist aber zugleich als einziger negative Wanderungssalden auf. Eindeutiger Wanderungsgewinner sind die Randgemeinden der Stadtregionen. ${ }^{108)}$ Die Abwanderungen erfolgen jedoch kaum aufgrund der in den Kernstädten nach wie vor höchsten Hebesätze, sondern aufgrund anderer Standortengpässe. Als Konkurrenten mittels des Hebesatzes treten daher vor allem die Umlandgemeinden gegeneinander an. Dabei dürften die Steuersatzspielräume um so weiter eingeschränkt sein, je größer die Entfernungen zum Zentrum sind, da zum ersten die Ausstrahlungseffekte des Zentrums mit wachsender Entfernung sinken und zum anderen die Zahl konkurrierender, ähnlich ausgestatteter Gemeinden steigt.

Im sekundären Sektor - speziell wenn er bedeutende tertiäre Produktionen enthält und daher auf Ballungsvorteile angewiesen ist - zeigt sich ein noch stärkerer Abwanderungsdruck, vor allem aufgrund des Bodenpreisgefälles. Innerhalb der Ballungsräume wird der sekundäre Sektor infolge der Vorteile der Horizontalproduktion und des daraus resultierenden hohen Flächenbedarfs in zunehmendem Maße "nach außen", teilweise sogar über die Ballungsränder hinaus gedrängt ${ }^{109}$ ), ein Vorgang, der von Richardson als Prozeß der dezentralen Konzentration bezeichnet wird. "Dieser Prozeß der dezentralen Konzentration beschreibt die Bedeutungszunahme von Städten der mittleren Größenklasse in eher ländlichen Gebieten und die Ausdehnung der großen Verdichtungsräume auf die angrenzenden Gemeinden, also die Suburbanisierung. Tatsächlich zeigt eine nähere Analyse der Beschäftigungsentwicklung

107) Dietrich, H.P. und Strauf, H.-G., Das Standortverhalten im Dienstleistungsbereich, a.a.O., S. 59.

108) Ebenda, S. 21.

109) Vgl. auch Schliebe, Klaus, Industrieansiedlungen - Das Standortwahlverhalten der Industriebetriebe, a.a.O., S. 127. 
insbesondere Vorteile in solchen Regionen, die unmittelbar an Regionen mit großen Verdichtungsräumen angrenzen." ${ }^{110)}$

Verantwortlich für diese Form der Ausweichung sind nach Bade vor allem push-Faktoren, d.h. die Industrien werden nicht durch das attraktivere Angebot alternativer Standorte angezogen, sondern von Engpässen verdrängt. ${ }^{111)}$ D.h., nicht ein Kostengefälle, sondern vielmehr ein als zu hoch erachtetes Kostenniveau am gewählten Standort ist der Abwanderungsauslöser.

Dabei spielen die Steuersätze in den Kernstädten als Abwanderungsort wahrscheinlich eine geringere Rolle als am Zuwanderungsort. Die durch push-Faktoren bedingte "Flucht aus den Agglomerationen"112) dürfte darüber hinaus aber auch von der Gewinnsituation der Unternehmen mitbestimmt werden. Zwar ist das finanzielle Risiko einer Verlagerung von leistungsfähigen Betrieben eher tragbar als von gewinnschwachen, die eher mit Betriebsschließung reagieren werden, andererseits werden gewinnstarke Betriebe durch Hebesatzsteigerungen weniger betroffen als gewinnschwache und können sich überdies einen suboptimalen Standort eher leisten. ${ }^{113)}$ Gemeinden mit gewinnstarken Produktionen ziehen daraus in zweierlei Hinsicht Vorteile: Zum ersten können sie auf höhere Bemessungsgrundlagen zurückgreifen, zum zweiten werden hohe Hebesatzforderungen mit weniger ausgeprägten ökonomischen Verhaltensänderungen verbunden sein.

Gegenüber den eigenen Umlandgemeinden haben die Kernstädte aus den aufgeführten Gründen vermutlich größere Hebesatzspielräume, zwischen den Kernstädten bzw. zwischen den Ballungsräumen insgesamt bestehen hier jedoch deutliche, vor allem wirtschaftsstrukturell bedingte Unterschiede.

Zwar dürften auch die internalisierten urbanization economies differenzierend wirken, weil ihr Umfang mit der Größe der Kernstadt zunimmt. So kann beispielsweise davon ausgegangen werden, daß die Ballungsvorteile -

110) Sälzer, Bruno E., Standortdynamik von Industrieunternehmungen, a.a.O., S. 129.

111) Bade, Franz-Josef, Sektorale und räumliche Aspekte der Mobilität industrieller Betriebe, a.a.O., S. 7.

112) Ebenda, S. 15.

113) So bemerkt Lösch bereits: "Wer gut verdient, kann sich eine schlechte Standortwahl leisten. Er zahlt nicht mit seiner Existenz, sondern nur mit einem Teil des möglichen Gewinns dafür." Lösch, August, Die räumliche Ordnung der Wirtschaft, 2. Aufl., Jena 1944, S. 236. 
aber auch die Ballungsnachteile - in einer Stadt wie München mit weit über einer Million Einwohnern stärker ausgeprägt sind als etwa in Saarbrücken. ${ }^{114)}$

Gravierende Unterschiede zwischen den Ballungen werden aber offensichtlich durch das Auftreten von localization economies, d.h. durch die Konzentration eines Wirtschaftszweiges oder Wirtschaftsbereichs bewirkt. ${ }^{115}$ ) Lokalisationsvorteile können natürlich bedingt sein - etwa aufgrund von Rohstoffvorkommen - oder nach und nach mit dem Konzentrationsprozeß entstehen, indem etwa eine besonders spezialisierte, nur bestimmten Anforderungen entsprechende Infrastruktur aufgebaut wird und ein auf spezielle Produktionen ausgerichteter Arbeitsmarkt heranwächst. Daneben scheint inzwischen auch die Umweltqualität als Resultat von Produktionsspezialisierungen bzw. der Abwesenheit von umweltbelastenden Produktionen eine solche Differenzierung hervorzurufen. ${ }^{116)}$

Localization economies ziehen jeweils ähnliche Produktionen an und bewirken insofern eine Produktionsspezialisierung in den jeweiligen Ballungsräumen. Diese Produktionsspezialisierung kann sich positiv, aber auch negativ auf die gemeindlichen Besteuerungsspielräume auswirken. Negative Wirkungen können insbesondere dann eintreten, wenn aufgrund der besonderen Standortbedingungen eine regionale Monostruktur entsteht. Solange die angesiedelten Betriebe hohe Erträge erzielen, sind die Besteuerungsspielräume der Gemeinden sowohl hinsichtlich der Bemessungsgrundlagen als auch der Hebesatzspielräume relativ weit. Mit der Verschlechterung der Ertragslage werden beide jedoch abrupt und massiv eingeschränkt, selbst wenn die Steuerzahler vollkommen immobil sind und eine räumliche Ausweichung für sie nicht in Frage kommt.

Als Beispiele für einen solchen Fall können die Gemeinden des durch den Montanbereich dominierten Ruhrgebiets gelten, von denen hier einmal die Stadt Duisburg herausgegriffen wird.

Die Wirtschaftsstruktur der Stadt Duisburg wird durch den sekundären Sektor geprägt, der tertiäre Sektor ist nicht zuletzt wegen der polyzentrischen

114) Vgl. Postlep, Rolf-Dieter, Regionale Effekte höherwertiger Dienstleistungen, a.a.O., S. 109.

115) Ebenda.

116) Vgl. ebenda, sowie Kaiser, Karl-Heinz, Industrielle Standortwahl und Betriebstypenbildung, a.a.O., S. 171. 
Lage und der unmittelbaren Nachbarschaft zum Finanz- und Dienstleistungszentrum Düsseldorf vergleichsweise schwach vertreten - gegenüber dem für einen Ballungsraumvergleich 1978 ermittelten Durchschnitt von 58,3\% aller Beschäftigten waren in Duisburg nur $41 \%$ im tertiären Sektor beschäftigt, der Anteil des sekundären Sektors war mit fast $59 \%$ extrem hoch. ${ }^{117}$ Innerhalb des sekundären Sektors nahmen vor allem die Bereiche Energiewirtschaft, Wasserversorgung, Bergbau mit $12 \%$ und die Herstellung von Eisen- und Metallerzeugnissen mit $21,2 \%$ weit überdurchschnittliche Anteile ein. ${ }^{118)}$ Diese Bereiche wurden in Abschnitt b als standortgebunden gekennzeichnet, außerdem wurde gezeigt, daß sie aufgrund des niedrigen Gewinns bei gleichzeitig - trotz weitgehender Abschreibung - immer noch hohem Kapitaleinsatz von Hebesatzunterschieden besonders stark belastet werden. Für die Stadt Duisburg resultierten daraus zunächst weit unterdurchschnittliche Bemessungsgrundlagen der Gewerbesteuer. Darüber hinaus dürften die Hebesatzspielräume stärker eingeschränkt sein als in anderen Kernstädten. Eine Abwanderung der betroffenen Produktionen ist kaum möglich, als Reaktionsalternative seitens der Betriebe böten sich nur Betriebsschließungen mit ebenfalls gravierenden fiskalischen Folgen für die Stadt an. Zwar wird einer solchen Reaktion im Extrem staatlicherseits, d.h. mittels Unterstützung der Betriebe vorgebeugt werden; in diesem Fall wäre jedoch mit massivem politischen Druck auf die Hebesatzpolitik der Stadt zu rechnen. Aber auch aus dem fiskalischen Interesse der Gemeinden heraus scheint eine äußerst vorsichtige Hebesatzpolitik angemessen: Da schon bei vergleichsweise gemäßigten Hebesatzsteigerungen - etwa verglichen mit der Hebesatzpolitik der Stadt Frankfurt - mit gravierenden Bemessungsgrundlagenverlusten und zusätzlich induzierten Einkommensteuerverlusten aufgrund von Beschäftigungseinbußen gerechnet werden muß, ist die Hebesatzpolitik hier vermutlich auf wesentlich niedrigerem Hebesatzniveau fiskalisch nicht mehr lohnend, zumal sie aufgrund der schmalen Bemessungsgrundlagenbasis ohnehin weniger ergiebig ist als in besser strukturierten Gemeinden.

117) Postlep, Rolf-Dieter, Regionale Effekte höherwertiger Dienstleistungen, a.a.O., S. $121 \mathrm{ff}$. 118) Ebenda. 


\section{d. Zusammenfassung}

Die Betrachtung des kommunalen Steuersystems in der Bundesrepublik Deutschland in Punkt a hat ergeben, daß gemeindliche Besteuerungsspielräume vor allem bei der Unternehmensbesteuerung, und zwar der Gewerbesteuer nach Ertrag und Kapital bestehen.

Punkt $b$ befaßte sich daher näher mit der Belastung verschiedener Betriebstypen - die vor allem nach Größe, Faktoreinsatzkonstellation und Gewinnsituation gebildet wurden - und versuchte nachzuweisen, daß Reaktionsdruck und Reaktionsmöglichkeiten eng mit der Branchenzugehörigkeit zusammenhängen, die ihrerseits von besonderer Bedeutung für die Standortanforderungen und die Alternativen der räumlichen Steuerausweichung ist.

Die Betrachtung der Standortwahl hat gezeigt, daß die Ansprüche bestimmter Produktionen nicht in allen Gemeinden gleichermaßen befriedigt werden können. Je nach Anforderungen, die an den Ansiedlungsort gestellt werden, kommen vielmehr nur ganz bestimmte Gemeinden als Standort in Frage. Diese Substitutionsmöglichkeiten bestimmen letztlich über die Wirtschaftsstruktur in den Gemeinden und die Konkurrenzbeziehungen zwischen ihnen. Vereinfacht ausgedrückt: Nur jene Gruppe von Gemeinden konkurriert um die Ansiedlung miteinander, die auch als Ansiedlungsort für Unternehmen (und Haushalte) substituierbar sind, weil sie die gestellten Ansprüche insgesamt etwa gleichermaßen erfüllen können.

In der Marktanalyse bedeutet dies, daß der Gesamtmarkt, auf dem die Gemeinden als Anbieter von Standorten auftreten, in Teilmärkte aufgespalten werden kann, die durch "Lücken in der Kette der Substitution"119) voneinander abzugrenzen wären.

Bei der Analyse stellte sich heraus, daß die Bestimmungsgründe betrieblicher Reaktion nach Branchen sehr unterschiedlich geprägt sind und von wenig spezifischen Standortansprüchen, hoher Mobilität bei gleichzeitig hoher

119) Ebenso wie im privaten Unternehmensbereich bereitet eine Marktabgrenzung natürlich auch für den Kommunalbereich kaum lösbare Probleme, weil praktisch zwischen allen Teilmärkten Substitutionsbeziehungen bestehen, wenn auch in unterschiedlicher Intensität. Insofern kommt es nur darauf an, Kommunen mit möglichst geringer Konkurrenzintensität voneinander abzugrenzen. Vgl. etwa Willeke, Franz-Ulrich, Wettbewerbspolitik, a.a.O., S. 25. 
Merklichkeit von Hebesatzunterschieden in bestimmten Produktionszweigen bis hin zu speziellen Standortansprüchen bei hoher Leistungsfähigkeit und geringer Hebesatzreagibilität reichen.

Für die einzelnen Gemeinden spielen daher unter fiskalischem Aspekt der Unternehmensbesatz und seine Struktur sowie die Ubiquität des eigenen Standortangebots die Hauptrolle bei der Bestimmung der Konkurrenzposition und des Gewerbesteuerpotentials.

Im Gemeindevergleich kommt man zu dem Ergebnis, daß die steuerlichen Einnahmemöglichkeiten - bei konstantem kommunalen Leistungsangebot - nicht nur aufgrund der zu einem gegebenen Zeitpunkt vorhandenen Bemessungsgrundlagen, sondern zumindest bei einer längerfristigen Planungsperspektive auch aufgrund unterschiedlicher Hebesatzspielräume starke Disparitäten aufweisen. Dabei stehen wenige konkurrenzstarke Gemeinden am einen Ende der Skala, die mittels hoher Hebesätze eine fiskalisch ergiebige Steuerpolitik betreiben können, ohne daß sie für ihren Haushalt gewichtige Reaktionen zu erwarten hätten, wirtschaftsschwachen Gemeinden am anderen Ende der Skala bzw. im "untersten Teilmarkt" gegenüber, die aufgrund ihrer ungünstigen Standortbedingungen einer hebesatzbegrenzenden Konkurrenz ausgesetzt sind und insofern von den ohnehin niedrigen Bemessungsgrundlagen entsprechend weniger abschöpfen können.

Wie intensiv die interkommunale Konkurrenz ist und welche Hebesatzspielräume sich daraus für die einzelnen Gemeinden ergeben, hängt nicht zuletzt auch von den gesamtwirtschaftlichen Bedingungen ab: Eine positive gesamtwirtschaftliche Lage stärkt die Bemessungsgrundlagen in den Gemeinden und vermindert auf diese Weise den interkommunalen Konkurrenzdruck. Demgegenüber schränkt vermindertes Wirtschaftswachstum die gemeindlichen Möglichkeiten der Einnahmesteigerung über ein Wachstum der Bemessungsgrundlagen unweigerlich ein, zusätzlich erhöht sich der Zwang für die Betriebe, auf Hebesatzsteigerungen bzw. auf interkommunale Hebesatzunterschiede zu reagieren. Für die Gemeinden folgt daraus eine Verschärfung der Konkurrenz, zumal neben fiskalischen Interessen auch andere Ziele, wie vor allem eine Bekämpfung regionaler Arbeitslosigkeit, verfolgt werden. Die Verschärfung der Konkurrenzsituation, insbesondere zwischen Kernstädten und ihrem Umland, mindert die Chancen der peripheren, wirtschaftsschwachen 
Räume noch weiter. ${ }^{120)}$ Diese Konkurrenz wird natürlich nicht nur über die Hebesatzpolitik, sondern vor allem über die - hier noch außer acht gelassene Angebotspolitik betrieben. Welche interkommunalen Konkurrenzbeziehungen und welche Einnahmespielräume daraus in verschiedenen Gemeindetypen resultieren, wird in Abschnitt $C$. näher betrachtet.

Mit der Bestimmung von Determinanten kommunaler Besteuerungsspielräume ist deren Quantifizierung natürlich noch nicht gelungen; sie wirft wegen der zu beschaffenden Information in der Tat massive Probleme auf. Die einzelne Gemeinde sieht sich hier ähnlichen Bedingungen und ähnlichen Schwierigkeiten gegenübergestellt wie ein Unternehmen, das seine Marktposition und den Verlauf der konjekturalen Preis-Absatz-Funktion zu ermitteln versucht. ${ }^{121)}$ Die Abschätzung der Besteuerungsspielräume wie auch die dann realisierte Hebesatzpolitik dürfte sich deshalb eher an einer groben Analyse der Konkurrenzbeziehungen und der Steuersatzpolitik der Konkurrenten orientieren. Dieser Tatbestand ändert jedoch nichts an der theoretischen Bestimmung des Steuerpotentials: Sowohl aus gemeindlicher Sicht als auch aus staatlicher Sicht ist dies der zu ermittelnde Gegenstand, den eine praktizierte Messung möglichst genau erfassen soll, wenn danach gefragt wird, wieviele Steuereinnahmen die einzelnen Gemeinden unter der Bedingung eines gegebenen Leistungsangebots erzielen können. Aus gemeindlicher Sicht ist die Beantwortung dieser Frage also durchaus von Bedeutung; ob und unter welchen Voraussetzungen sie auch für übergeordnete Gebietskörperschaften von Interesse ist, wird im 4. Kapitel genauer untersucht.

\section{Grenzen kommunaler Entgeltpolitik}

a. Das kommunale Leistungsangebot als Ausgangspunkt der Betrachtung

In der neueren finanzwissenschaftlichen Literatur werden Entgelte - die in der Praxis als Gebühren, Beiträge und Erwerbseinkünfte auftreten - z.T. nicht

120) Vgl. Strauß, Wolfgang, Räumliche Wirkungen der Gewerbesteuer, a.a.O., hier S. 416.

121) Zu den Problemen der Quantifizierung vgl. Kilger, Wolfgang, Die quantitative Ableitung polypolistischer Preisabsatzfunktionen aus den Heterogenitätsbedingungen atomistischer Märkte, in: Zur Theorie der Unternehmung, Festschrift zum 65. Geburtstag von Erich Gutenberg, hrsg. von Helmut Koch, Wiesbaden 1962, S. 269-312. 
mehr von den Steuern abgegrenzt, sondern als Spezialfall der Besteuerung angesehen. ${ }^{122)}$ Bereits Neumark konstatierte, daß "als einziger - offensichtlich nicht allzu bedeutsamer - Unterschied" zwischen der Gebühr und der Steuer die Tatsache übrigbleibt, "daß die Gebühr gelegentlich (aus Anlaß) einer individuellen Inanspruchnahme bestimmter öffentlicher Leistungen eingefordert wird, während die Steuererhebung ohne jede Bezugnahme auf solche Leistungen an gewisse generelle Tatbestände (...) anknüpft, die sich rein aus der Existenz und bzw. oder der Tätigkeit von Einzelwirtschaften ergeben". ${ }^{123)}$ Diese Spezialität der Entgelte gegenüber den Steuern, nämlich das Vorliegen einer öffentlichen Leistung, an die das Entgelt anknüpft, rechtfertigt es nun aber, die Entgelte hier gesondert von den Steuern zu betrachten: Die Möglichkeit der Gemeinden, Entgelteinnahmen zu erzielen, hängt zunächst eimmal davon $\mathrm{ab}$, wieviele entgeltfähige Leistungen sie überhaupt anbietet. Hier ist es also erstmals notwendig, die kommunale Aufgabenerfüllung im einzelnen in die Betrachtung einzubeziehen, was für die Steuerfinanzierung nicht notwendig war.

Bereits an dieser Stelle lassen sich zwei das kommunale Entgeltpotential begrenzende Tatbestände nennen:

- die Summe der entgeltfähigen Leistungen

- $\quad$ und die Höhe der gegenüber den Wirtschaftssubjekten durchsetzbaren

Entgeltsätze.

Das Hauptaugenmerk liegt hier in Abschnitt B noch ausschließlich auf der Frage, wie die Gemeinden das Entgeltpotential über die Variation der Entgeltsätze beeinflussen können. Eine Einflußnahme über die Ausweitung des entgeltfähigen Angebots bleibt zunächst hingegen ausgeklammert.

Bei der Analyse des kommunalen Entgeltpotentials kann ebenfalls das preistheoretische Instrumentarium eingesetzt werden. Wegen der Ähnlichkeit der Mengenkomponente mit jener bei der Preisbildung im privaten Sektor der angebotenen und der nachgefragten Leistungen - ist es hier sogar noch einfacher übertragbar als auf die Untersuchung des Steuerpotentials. Allerdings ergibt sich nun folgendes Problem:

122) Zur ausführlichen Begründung vgl. Bohley, Peter, Gebühren und Beiträge, a.a.O., S. 81 f.

123) Neumark, Fritz, Vom Wesen der Besteuerung, in: Voigt, Fritz, Hrsg., Beiträge zur Finanzwissenschaft und zur Geldtheorie, Festschrift für Rudolf Stucken, Göttingen 1953, S. 7-20, hier S. 18. 
Preisänderungen induzieren eine Veränderung der nachgefragten Leistungsmenge und des Absatzes. Auch für den Fall der Besteuerung konnte ein Zusammenhang zwischen Preis (Steuersatz) und Menge (der Bemessungslagenbasis) aufgezeigt werden. Die Mengenkomponente war bei der statischen Betrachtung jedoch zunächst völlig unabhängig vom kommunalen Leistungsangebot analysierbar; letzteres wurde als gegeben vorausgesetzt und allein die Reaktion der Privaten auf Steuersatzvariationen untersucht, soweit sie die Menge der Bemessungsgrundlagen berührten. Anders bei den Entgelten; die Mengenkomponente ist hier die Inanspruchnahme des kommunalen Leistungsangebots, die mit der Variation der Entgelthöhe zu- oder abnimmt. Nur beim Angebot rein öffentlicher Güter, die durch Nicht-Rivalität des Konsums gekennzeichnet sind ${ }^{124)}$, erfordert die Nachfrageveränderung keine Angebotsveränderung; die oben eingeführte Annahme eines konstanten kommunalen Leistungsangebots könnte in diesem Fall also uneingeschränkt aufrecht erhalten werden. Bei der Mehrzahl der kommunal angebotenen Leistungen handelt es sich jedoch um Mischgüter, bei denen eine Ausdehnung der Leistungsabgabe - zumindest sobald eine Kapazitätsgrenze erreicht wird - eine Verbreiterung des Angebots erforderlich macht. So kann man sich etwa vorstellen, daß nach einer Senkung der Benutzungsgebühren für Schwimmbäder deren Auslastung ansteigt und die Eintstellung zusätzlichen Aufsichtspersonals erforderlich macht. Damit kann die Kapazitätsgrenze verschoben werden, wenn auch nur unter Inkaufnahme zusätzlicher, hier sprungfixer Kosten, die bei der Abschätzung der zusätzlichen Einnahmen in Rechnung gestellt werden müssen.

Aus gemeindlicher Sicht hat hier natürlich eine Angebotsausdehnung stattgefunden, die Annahme des konstanten Angebots ist durchbrochen worden. Diese Annahme wurde jedoch gemacht, um "preisinduzierte" Ausweich-

124) Nach der normativen Theorie der öffentlichen Güter sollen solche Angebote gerade deswegen vom Staat erbracht werden, damit ihre Inanspruchnahme ohne Zahlung eines Entgelts erfolgen kann. Das heißt jedoch nicht, daß eine Entgelterhebung prinzipiell nicht möglich sei. Dies gilt nur insofern, als über die Nicht-Rivalität des Konsums hinaus ein Ausschluß einzelner Konsumenten nicht möglich ist. Das Allokationsargument der NichtRivalität ist jedoch aus gemeindeindividueller, einnahmenorientierter Sicht nicht unbedingt von Bedeutung.

Zur Theorie der öffentlichen Güter vgl. Musgrave, Richard Abel; Musgrave, Peggy und Kullmer, Lore, Die öffentlichen Finanzen in Theorie und Praxis, 1. Band, 3., völlig überarbeitete Auflage. Tübingen 1984. S. 60 ff. sowie Münch, Klaus v., Kollektive Güter und Gebühren-Elemente einer Gebührentheorie für Kollektivgüter, Göttingen 1976, S. 22 ff. 
effekte theoretisch von attraktivitätsbedingten Veränderungen der Mengenkomponenten des Steuer- und Entgeltpotentials, also von Verschiebungen der Preis-Absatz-Funktion, trennen zu können. Entscheidend ist die Sicht der Nachfrager. Für sie ist die Qualität des Angebots gleich geblieben, es bestehen also weder größere Auwahlmöglichkeiten noch veranlaßt eine verbesserte Bedarfsdeckung zur Ausweitung der Nachfrage. Die rein mengenmäßige Anpassung des Angebots an die Nachfrage kann deshalb noch unter die Betrachtung des Einnahmepotentials "bei konstantem Leistungsangebot" subsumiert werden.

\section{b. Zur Entgeltfähigkeit kommunaler Leistungen}

Wendet man sich der Frage zu, welche der kommunalen Leistungen entgeltfähig sind, so ist als erste Voraussetzung die Anwendbarkeit des Ausschlußprinzips oder des Verursacherprinzips zu nennen. Die Anwendung dieser Prinzipien muß zunächst nur technisch möglich sein, darüber hinaus darf sie aber nicht gegen geltende Regeln - darunter fallen auch allgemein anerkannte sittliche Normen - verstoßen. Aus gemeindlicher Sicht muß sich der Ausschluß oder die Belastung des Verursachers darüber hinaus fiskalisch lohnen, d.h. die Kosten der Entgelterhebung dürfen das erzielbare Entgeltaufkommen nicht übersteigen.

Die im Zeitablauf allesamt Veränderungen unterworfenen Möglichkeiten des Ausschlusses beziehen sich dabei immer nur auf einen Teil der Nutzen der erbrachten Leistungen. Dies gilt sowohl für die Erhebung von Gebühren als auch von Beiträgen. Ihre Erhebung "setzt das Vorliegen einer besonderen Art von Kollektivgütern voraus, deren Effekte partiell an die Allgemeinheit und partiell an die Adresse einzelner gerichtet sind ....". ${ }^{125)}$

Darüber hinaus muß es sich nicht notwendig um einen individuellen Ausschluß handeln. So unterscheiden sich beitragsfähige von den übrigen entgeltfähigen Leistungen "dadurch, daß die Ausschließbarkeit nicht für einen einzelnen möglich oder gewollt ist, sondern nur für eine Gruppe, die eine

125) Bohley, Peter, Gebühren und Beiträge, a.a.O., S. 34. 
Teilmenge der Gesamtheit der Wirtschaftssubjekte darstellt". ${ }^{126)}$ Anders ausgedrückt: "Es liegt ein geschlossener Klub von Betroffenen vor". ${ }^{127}$ )

Betrachtet man unter diesem Aspekt das traditionelle kommunale Leistungsspektrum, so finden sich nur wenige Bereiche, die nicht in der einen oder anderen Form entgeltfähig sind. Nicht entgeltfähig sind vor allem Geldund Naturaltransfers an Unternehmen und private Haushalte, wie etwa die Sozialhilfe, aber auch jene Bereiche, die grundsätzlich allen Wirtschaftssubjekten in der Gemeinde zugute kommen, wie etwa die allgemeine Verwaltung. ${ }^{128)}$ Im letzten Fall kann eine Anwendung des Äquivalenzprinzips nur in Form einer lokalen Äquivalenz und im Wege der Besteuerung erfolgen, da besonders bevorteilte Individuen oder Gruppen nicht ausgemacht und ein Ausschluß vom Nutzen der Leistung nicht vorgenommen werden kann. Demgegenüber sind Transfers nicht entgeltfähig, weil die Entgeltzahlung allein auf eine Leistungskürzung hinauslaufen würde. Alle übrigen Leistungsbereine und damit die Mehrzahl der kommunalen Angebote - sind zumindest technisch entgeltfähig. Anders als auf übergeordneten Ebenen kommt den Möglichkeiten der Entgeltfinanzierung auf Gemeindeebene deshalb relativ große Bedeutung zu. Dies spiegelt sich auch in den Gemeindeordnungen wieder, die spezielle Entgelte als vorrangige kommunale Einnahmequelle vor den Steuern nennen. ${ }^{129)}$

Außer durch die technische Entgeltfähigkeit werden die Möglichkeiten der Entgelterhebung durch die Regelungen übergeordneter Gebietskörperschaften eingeschränkt, die sich - wie etwa in den Gemeindeordnungen und den Kommunalabgabengesetzen - vorwiegend auf die Bemessung der Entgelt-, speziell der Gebührenhöhe beziehen, in speziellen Fällen aber auch auf die Abgrenzung entgeltfähiger Tatbestände. Die Frage, inwieweit es im Ermessen einer Gemeinde steht, ob sie auf einen Tatbestand Gebühren oder Beiträge erhebt, ist zwar nicht abschließend geklärt ${ }^{130}$, bestimmte Angebote werden jedoch explizit aus dem Kreis der entgeltfähigen Leistungen herausgenommen. Dazu zählen insbesondere Leistungen, deren Inanspruchnahme po-

126) Ebenda, S. 43.

127) Ebenda.

128) Vgl. Kentmann, Konrad, Das Äquivalenzprinzip in den Gemeinden, Frankfurt 1978, S. 68.

129) Vgl. etwa die Gemeindeordnung Nordrhein-Westfalen, § 63, Abs. 2.

130) So Scholler, Heinrich und Broß, Siegfried, Grundzüge des Kommunalrechts in der Bundesrepublik Deutschland, 3., neubearb. Aufl., Heidelberg 1984, S. 190. 
sitive externe Effekte hervorbringt oder von denen aus Gründen der Verteilungsgerechtigkeit niemand ausgeschlossen werden soll. Bohley verweist in diesem Zusammenhang etwa auf die landesverfassungsrechtlichen Vorschriften zur Schulgeld-, Lehrmittel- und Lernmittelfreiheit. ${ }^{131)}$ Nach den Kriterien der technischen Ausschließbarkeit könnten die Gemeinden durchaus Entgelte für die Vorteile des Schulbesuchs erheben, aus politisch-ethischen Gründen wird ihnen dies jedoch von übergeordneter Seite verwehrt.

Als drittes Entgeltfähigkeitskriterium wurde oben die Erhebungsbilligkeit genannt: Auch aus gemeindlicher Sicht wird vom Ausschluß nicht zahlungswilliger Individuen und Gruppen von der Inanspruchnahme zumindest unter dem Gesichtspunkt der Einnahmebeschaffung dann abgesehen werden, wenn die Erhebungskosten die Zusatzeinnahmen übersteigen. Dies scheint bei der Mehrzahl der prinzipiell entgeltfähigen, bisher aber nicht entgeltfinanzierten kommunalen Leistungen der Fall zu sein. Deshalb plädiert Seiler dafür, bei Verwaltungsgebühren den Grundsatz beizubehalten, daß sie nur für besonders personal- oder sachkostenintensive Verwaltungsleistungen erhoben werden sollten. "Andernfalls würde in einen kleinlichen Gebührenfiskalismus verfallen werden, der weniger als Belastung als vielmehr als Belästigung empfunden würde; das Aufkommen würde zudem finanzwirtschaftlich unbedeutend sein."132)

Ebenso wird einer auf dem Verursacherprinzip basierenden Entgeltfinanzierung kommunaler Umweltpolitik kaum eine Chance eingeräumt ${ }^{133}$ ), weil die Verursacher nicht oder nur mit hohen Kosten zu ermitteln sind.

Insgesamt scheinen die Möglichkeiten, die Entgeltpflicht auf weitere Tatbestände und über die derzeit entgeltfinanzierten Bereiche hinaus auszuweiten, eng begrenzt zu sein. Aus kommunaler Sicht ist damit die Entgeltfähigkeit weitgehend auf die bisher bereits ublicherweise entgeltfinanzierten Bereiche beschränkt. Sie sind in Übersicht 3 aufgelistet.

Über die Einordnung der einzelnen entgeltfähigen Leistungen nach dem Grad der Entgeltfähigkeit besteht dabei zum Teil Uneinigkeit. So könnten

131) Bohley, Peter, Gebühren und Beiträge, a.a.O., S. 32.

132) Seiler, Gerhard, Zur Bedeutung der Gebühren, Beiträge und anderer Verwaltungsausgaben für den Haushaltsausgleich der Gebietskörperschaften, Institut "Finanzen und Steuern" Hrsg., Brief 180, Bonn 1978, S. 33.

133) Ebenda, S. 34. 
etwa die Bereiche Bücherei oder Erwachsenenbildung durchaus auch in der Spalte "Teilentgeltfähigkeit" angesiedelt werden, weil eine individuelle $\mathrm{Zu}$ rechnung der Leistungsinanspruchnahme möglich ist. Ähnliches gilt für die Jugend- und Altenbewegung sowie das gesamte auf den Freizeitbereich gerichtete Angebot. Eine exakte Zuordnung kann hier letztlich nur unter Berücksichtigung der jeweiligen Länderregelungen erfolgen.

\section{Übersicht 3: $\quad$ Das entgeltfähige kommunale Leistungsangebot}

$\begin{array}{llll}\text { Funktions- } & \text { exakte } & \text { Teilent- } & \text { pauschale } \\ \text { bereich } & \text { Entgelt- } & \text { geltfähig- } & \text { Entgelt- } \\ & \text { fähigkeit } & \text { keit } & \text { fähigkeit }\end{array}$

I. Technische Gemeindeleistungen

1. Ver- und

Energie-

Entwässe-

Entsor-

und Wasser-

rung von

gung

versorgung,

Entwässe-

Straßen,

rung von

Straßenrei-

Grundstük-

nigung

ken, Müllab-

fuhr u.ä.

2. Verkehr

sämtliche

Leistungen

3. Feuerwehr

sämtliche

Leistungen

II. Gemeindeleistungen im sozialen Bereich

1. Gesund-

Kranken-

heit

hauslei-

stungen

2. Bildung

und

Museen, Thea-

Bücherei,

Kultur

ter u. ähnliche

Kulturlei-

stungen

Erwachse-

nenbildung

3. Soziales

Jugend-

und Alten-

begegnung 


\section{noch Übersicht 3:}

4. Freizeit

Zoo, Bota-

Erholung,

nische Gär-

Freizeitbe-

ten u.ä.

schäftigung,

Kommunika-

tionslei-

stungen

5. Verwaltung

spezielle

Verwaltungs-

leistungen

Quelle: Zusammengestellt nach Kentmann, Konrad, Das Äquivalenzprinzip in den Gemeinden, a.a.O., S. 67.

\section{c. Grenzen der Entgeltfestsetzung}

In der finanzwissenschaftlichen Literatur wird mitunter davon ausgegangen, daß die Höhe der Entgelte, speziell der Gebühren und Beiträge, durch die kostenmäßige Äquivalenz begrenzt wird oder begrenzt werden sollte. ${ }^{134)}$ In diesem Fall entsprächen die maximal erzielbaren Einnahmen den mit der Leistungserstellung und -abgabe verursachten Kosten. Daraus leitet sich auch das Argument $a b, d a ß$ die entsprechenden Einnahmen keinen zusätzlichen Finanzierungsspielraum für die Gemeinden eröffnen. ${ }^{135)}$ Diese Interpretation resultiert möglicherweise aus den Regelungen der Kommunalabgabengesetze oder der Gemeindeordnungen der Länder und läßt die Möglichkeiten einer Bemessung von Entgelten nach marktmäßiger Äquivalenz von vornherein außer acht. ${ }^{136)}$ Allerdings ist damit nur eine der Grenzen kommunaler Entgeltpolitik angesprochen, die überdies nur in bestimmten Fällen - in denen nämlich kostendeckende Entgelte durchgesetzt werden könnten -wirksam wird.

134) So weist etwa Seiler darauf hin, $\mathrm{da} B$ der über die Kostendeckung hinausgehende Teil des Gebührenaufkommens rechtssystematisch einer Steuer gleicht. Seiler, Gerhard, Zur Bedeutung der Gebühren, Beiträge..., a.a.O., S. 14.

135) So etwa Kirchhof, Paul, Der Finanzausgleich als Grundlage kommunaler Selbstverwaltung, a.a.O., S. 717.

136) Vgl. dazu Haller, Heinz, Die Steuern, 3., überarbeitete Auflage, Tübingen 1981, S. 16 ff. 


\section{Die Preiselastizität der Nachfrage}

Zunächst wird zum Zweck der theoretischen Ableitung unterstellt, daß der Gebührenzweck die Gebührenhöhe regiert. ${ }^{137}$ Geht man hier einmal hypothetisch davon aus, daß der Zweck ein rein fiskalischer sei und daß die Höhe der Entgelte staatlicherseits nicht eingeschränkt wird, so ist diejenige Höhe der Entgelte, bei denen ein maximales Aufkommen erzielt wird, allein durch die Reaktionen der betroffenen Wirtschaftssubjekte bestimmt. Wie bei der Besteuerung sind wiederum vor allem sachliche und räumliche Anpassungen denkbar. In kurzfristiger Betrachtung wären außerdem die Möglichkeiten zeitlicher Anpassungen, also etwa die vorgezogene Inanspruchnahme kommunaler Leistungen bei erwarteten Entgeltanhebungen zu berücksichtigen. Beim unterstellten längerfristigen Planungshorizont der Gemeinden spielen solche Ausweichreaktionen jedoch eine untergeordnete Rolle. Überdies sind temporale Reaktionen aufgrund der Qualität kommunaler Leistungen - es handelt sich vielfach um nicht lagerfähige Leistungen der Daseinsvorsorge, die regelmäßig in Anspruch genommen werden müssen und deren Konsum nicht aufschiebbar oder vorziehbar ist von untergeordneter Bedeutung. Sie werden deshalb hier vernachlässigt.

Die Anpassungsreaktionen lassen sich in Form einer konjekturalen PreisAbsatz-Kurve veranschaulichen. Dabei werden auf der x-Achse die abgesetzten Mengen der kommunalen Leistung abgetragen.

137) Kirchhof, Ferdinand, Die Höhe der Gebühr - Grundlagen der Gebührenbemessung Schriften zum öffentlichen Recht, Bd. 399, Berlin 1981, S. 56 und 60. 
Abbildung 8: $\quad$ Nachfrage nach kommunalen Leistungen bei alternativen Entgeltsätzen

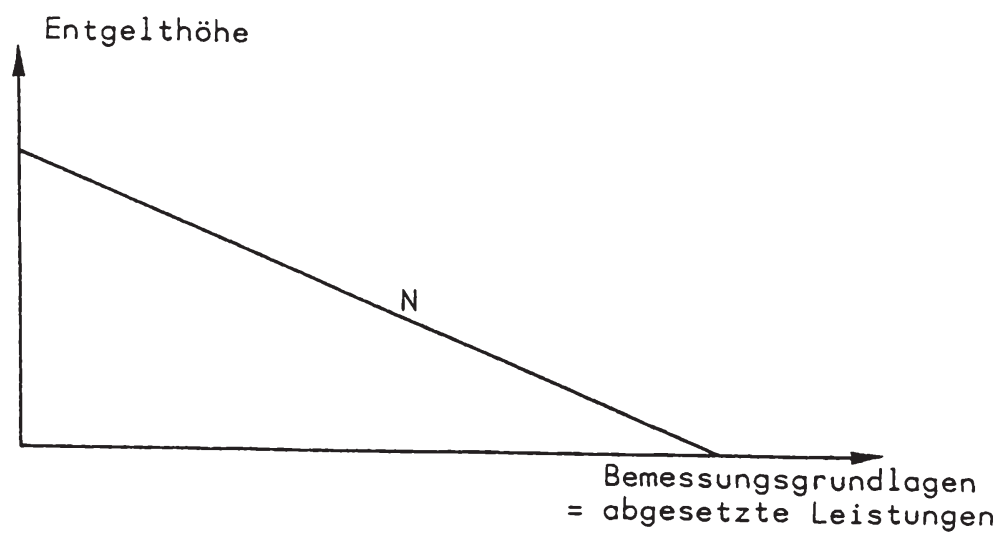

Der Verlauf der "Nachfragekurven" hängt wie bei der Besteuerung davon ab, welche Substitutionsmöglichkeiten den Wirtschaftssubjekten offenstehen. Entscheidend ist hier zum einen die Art des Ausschlusses von der Inanspruchnahme des Angebots, zum anderen die Substituierbarkeit der kommunalen Leistung. Die Art des Ausschlusses und der Zurechnung der gebotenen Leistung unterscheidet sich - wie angesprochen - vor allem zwischen Beiträgen und den übrigen Entgelten. Beitragszahlungen erfordern lediglich einen gruppenmäßigen Ausschluß, für die Zahlung des einzelnen Wirtschaftssubjekts kommt es also nicht auf die tatsächliche Nutzung an. Dementsprechend kann der Zahlung nicht durch einen Verzicht auf die Inanspruchnahme ausgewichen werden, sondern nur durch den Verzicht oder die Substitution jenes Tatbestandes, anhand dessen die Gruppe abgegrenzt wird - praktisch also durch den "Austritt aus dem Klub der Betroffenen". Ein solcher Austritt kann - je nach Abgrenzungsmerkmal - auf verschiedene Weise vonstatten gehen, etwa durch den Verkauf bestimmter Vermögensteile oder auch durch räumliche Ausweichungen innerhalb der Gemeinde oder über die Gemeindegrenzen hinaus.

Die Kurve $\mathrm{N}_{2}$ in Abbildung 9 bildet den Reaktionsverlauf auf die Beitragserhebung ab. Das Angebot liegt bei a und ermöglicht einem fest umrisse- 
nen Kreis potentiell Begünstigter die Nutzung. Da die Ausweichung mit relativ hohen Kosten verbunden ist, speziell wenn nur eine räumliche Ausweichung möglich ist wie etwa bei Anliegerbeiträgen, wird die Zahlung bis zu einer bestimmten Beitragshöhe in Kauf genommen, ohne daß eine Reaktion erfolgt. Erst wenn der geforderte Beitrag die Kosten der Ausweichung übersteigt, setzt die Reaktion der Wirtschaftssubjekte ein.

\section{Abbildung 9: $\quad$ Reaktionsverläufe bei Gebühren und Beiträgen auf unterschiedliche Entgelthöhe}

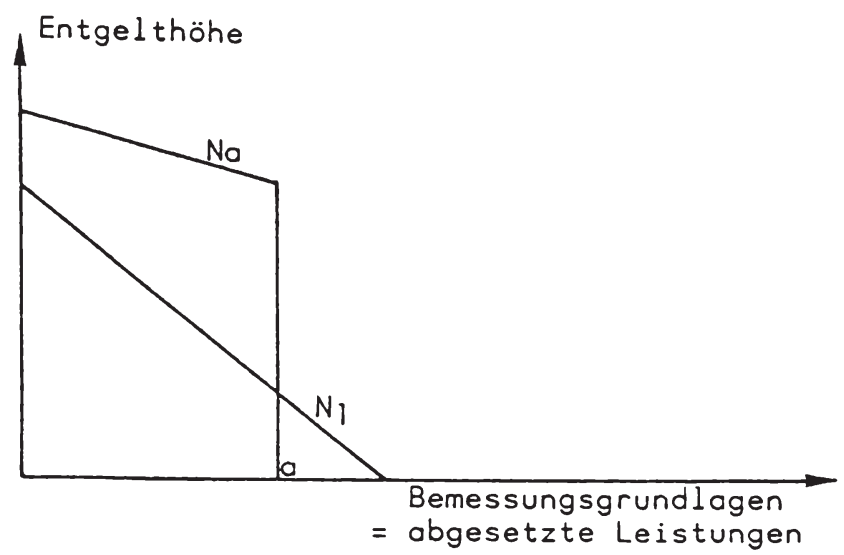

Anders als die Beiträge knüpfen Gebühren und in der Regel auch die Erwerbseinkünfte an der individuellen Inanspruchnahme einer kommunalen Leistung an. Der Zahlung kann deshalb - wie durch den Reaktionsverlauf von $\mathrm{N}_{1}$ dargestellt - prinzipiell dadurch ausgewichen werden, daß auf die Nutzung verzichtet wird. Für die faktischen Ausweichmöglichkeiten ist darüber hinaus jedoch entscheidend, ob ein Zwang zur Inanspruchnahme besteht und - sofern dies nicht der Fall ist - in welchem Umfang auf private Angebote oder die Angebote der Nachbargemeinden ausgewichen werden kann.

Hansmeyer und Fürst unterscheiden drei Typen von Nachfrageverläufen für verschiedene kommunale Leistungen: Für den Typ 1 wird von einer mit steigenden Gebühren kontinuierlich abnehmenden Nachfrage, wie mit $\mathrm{N}_{1}$ 
dargestellt, ausgegangen. "Tendenziell kann auf die Weise die Nachfrage bei Schwimm- und Badeanstalten, Parkgebührenhaushalten, Verkehrsbetrieben, Litfaßsäulen und Aussichtstürmen wiedergegeben werden." ${ }^{138)}$

Ein zweiter, nicht in Abbildung 9 aufgenommener Typ weist einen einoder mehrfach geknickten Verlauf auf. Dabei werden die Knickstellen als "Reizschwellenphänomen" interpretiert ${ }^{139)}$, d.h. erst ab einer bestimmten Preishöhe wenden sich die Verbraucher substituierenden Gütern zu. Hansmeyer und Fürst nennen als Beispiele für einen solchen Verlauf "Entwässerungsgebühren, Müllabfuhrgebühren, ..., aber auch Parkgebühren, Gebühren für öffentliche Verkehrsmittel, Theater, Schlachthöfe, Marktstände, Abdeckerei etc." ${ }^{140)}$ Allerdings gilt dieser Nachfrageverlauf nur, sofern für die genannten Leistungen kein Zwang zur Inanspruchnahme besteht.

Typ 3 beschreibt einen weitgehend preisunelastischen, dem Fall der Beitragserhebung ähnlichen, Nachfrageverlauf, der nach Hansmeyer und Fürst relativ selten auftritt. Als Beispiel wird ein kommunales Wassermonopol genannt; dabei wird auch hier davon ausgegangen, daß, wie durch $\mathrm{N}_{2}$ beschrieben, ab einer bestimmten Gebührenhöhe die Substitution über private Wasserlieferanten aus anderen Regionen einsetzt. ${ }^{141)}$

Preisunelastische Preis-Absatz-Funktionen liegen vor allem dann vor, wenn durch die Gemeinde ein Anschluß- oder Benutzungszwang angeordnet wird. Die Möglichkeit solcher Anordnungen ist in allen Gemeindeordnungen vorgesehen, allerdings müssen sie durch "Gründe des öffentlichen Wohls" gerechtfertigt sein. ${ }^{142)}$ Fiskalische Erwägungen können als Nebenzweck durchaus eine Rolle spielen, etwa wenn mit dem Anschlußzwang eine kommunale Einrichtung finanziell gesichert oder ihr rentabler Betrieb gewährleistet werden soll. ${ }^{143)}$

138) Hansmeyer, Karl-Heinrich und Fürst, Dietrich, Die Gebühren - Zur Theorie eines Instrumentariums der Nachfragelenkung bei öffentlichen Leistungen - Schriften des Vereins für Kommunalwissenschaften e.V., Bd. 18, Stuttgart u.a. 1968, S. 67.

139) Ebenda.

140) Ebenda, S. 68.

141) Ebenda.

142) Scholler, Heinrich und Broß, Siegfried, Grundzüge des Kommunalrechts..., a.a.O., S. 62.

143) Ebenda, S. 63. 
In welchen Bereichen die Gemeinden Anschluß- oder Nutzungszwang anordnen können, ist in den Gemeindeordnungen nicht abschließend geregelt, üblich sind sie jedoch nur insoweit, als sie der Volksgesundheit dienen. ${ }^{144)}$ Damit kann vor allem für Einrichtungen der Wasserver- und entsorgung, Entwässerungsanlagen, Schlachthöfe sowie Leichenhallen und Friedhöfe ein Zwang zur Inanspruchnahme ausgesprochen werden, der eine Substitution ausschließt.

Zusammenfassend ist noch einmal festzuhalten, daß die Preiselastizität der Nachfrage nach kommunalen Leistungen von den - durch verschiedene Größen beeinflußten - Substitutionsmöglichkeiten abhängt.

Ähnlich wie für die Besteuerung bereits ausführlicher dargestellt, entspricht das gemeindliche Entgeltpotential aus einer entgeltfähigen Leistung dem Umsatzmaximum aus dem Leistungsabsatz. Das Umsatzmaximum muß keinesfalls mit den Kosten der Leistungserstellung und -abgabe übereinstimmen; dieser Fall wird vielmehr nur zufällig und in Ausnahmen auftreten.

In Abbildung 10 sind die möglichen Relationen von Kosten und maximal erzielbaren Einnahmen noch einmal graphisch dargestellt. Dabei wird - der Einfachheit halber - davon ausgegangen, daß die Grenzkosten des Angebots gleich 0 sind und die Totalkosten gedeckt werden sollen. ${ }^{145)}$ Damit wird unterstellt, daß das gegebene Angebot unabhängig von der Nutzung immer die gleichen Kosten verursacht, Mehreinnahmen durch Variationen der Entgeltsätze sind daher für die Gemeinden in ihrer Gesamtheit frei verfügbar. Anders, wenn Auslastungsveränderungen Kostenveränderungen verursachen: In diesem Fall sind unter der hier betrachteten rein fiskalischen Fragestellung Versorgungsunterschiede und ihre Bewertung bleiben noch völlig ausgeklammert - nur die zusätzlichen Nettoeinnahmen von Interesse. Nicht die Ge-

144) Ebenda, S. 64.

145) Damit wird die Forderung nach kostenloser Abgabe der Leistung, wie sie unter allokativem Gesichtspunkt gefordert werden würde, natürlich mißachtet. Zum ersten handelt es sich hier jedoch immer um einen übergeordneten Standpunkt: Weder für einzelne Unternehmen im Marktgeschehen noch für die einzelne Gemeinde bei der Abschätzung von Einnahmemöglichkeiten ist dieser Standpunkt von Interesse. Zum zweiten stellt die Definition der Grenzkosten ein Sonderproblem dar: Bei langfristiger Kalkulation und unter Berücksichtigung von Kosten der Kapazitätserweiterungen nähem sich Grenzkosten und Durchschnittskosten stark aneinander an. Vgl. dazu etwa Alonso, William, Zur Ökonomie der Stadtgröße, in: Fürst, Dietrich, Hrsg., Stadtökonomie, Stuttgart, New York 1977, S. 50-67. 
samtkosten, sondern allein jene Kosten, die durch den Versuch der fiskalischen Optimierung entstehen, wären entsprechend den Mehreinnahmen gegenzurechnen.

\section{Abbildung 10: Kostendeckungsgrade kommunaler Leistungen bei unterschiedlichen Kosten- und Nachfrageverläufen}

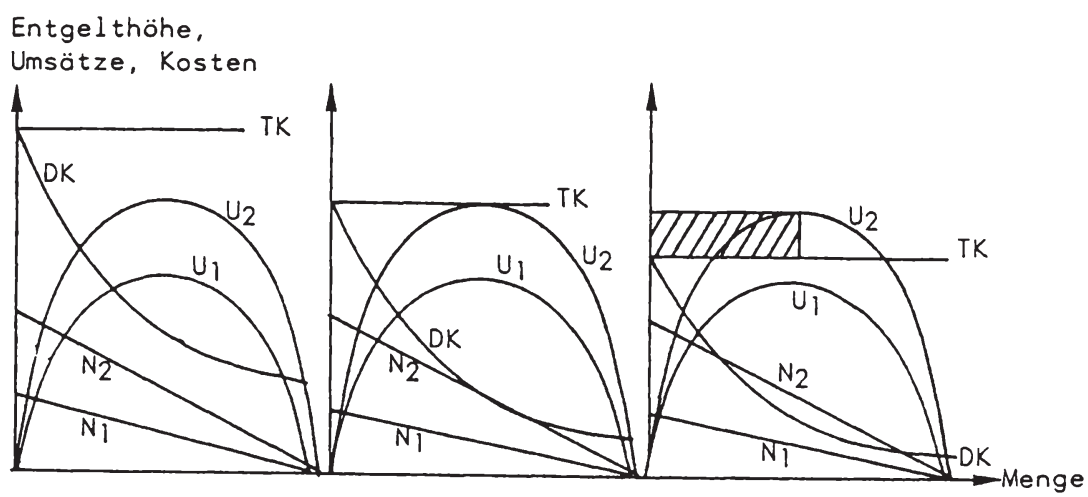

In Abb. 10 wird diese Schwierigkeit jedoch vernachlässigt. Hier wird zunächst nur deutlich, daß das kommunale Einnahmepotential aus Entgelten bei gegebenem Angebot ausschließlich vom jeweiligen Nachfrageverlauf abhängt, der Kostendeckungsgrad aber darüber hinaus durch die Höhe der anfallenden Kosten mitbestimmt wird.

Die erzielbaren Entgelteinnahmen und die realisierbaren Kostendekkungsgrade dürften sich vor allem zwischen den kommunalen Leistungsbereichen, teilweise aber auch im interkommunalen Vergleich identischer oder ähnlicher Leistungen unterscheiden.

Hohe Entgelteinnahmen und weitgehende Kostendeckung lassen sich zunächst in jenen Bereichen erzielen, in denen eine starre Nachfrage vorliegt; also insbesondere in den technischen Leistungen der Ver- und Entsorgung, zum zweiten in Bereichen, in denen das zu zahlende Entgelt von Dritten über- 
nommen wird und preisbedingte Ausweichreaktionen der Konsumenten deshalb unterbleiben, wie etwa im Gesundheitsbereich. Darüber hinaus sind hier diejenigen Leistungsbereiche anzuführen, in denen die Entgeltzahlung aufgrund einer pauschalen, d.h. nutzungsunabhängigen Zurechnung auf bestimmte Gruppen angeordnet werden kann, und der sich die Zahler deshalb durch den Verzicht auf die Nutzung nicht entziehen können, wie im Fall der Erschließungsbeiträge. Dem stehen entgeltfähige Leistungsbereiche gegenüber, in denen das Angebot in der Regel auf eine elastische Nachfrage trifft, wie vor allem im Freizeit- und Bildungsbereich.

Hier lassen sich zumindest dann nur geringere Kostendeckungsgrade realisieren, wenn das Angebot im Vergleich zu den erzielbaren Einnahmen mit hohen Kosten verbunden ist.

\section{Tabelle 8: Kostendeckungsgrade in ausgewählten kommunalen Gebührenhaushalten, 1982, nach einer Umfrage des DST}

Gebührenhaushalt

Abfallbeseitigung

Abwasserbeseitigung

Altenwohnheime

Friedhöfe

Schlachthöfe

Musikschulen

Volkshochschulen

Bäder

Theater

Kindergärten

Museen

Büchereien
Kostendeckungsgrad

in \%

93,0

83,4

74,3

55,0

52,4

25,5

18,8

18,2

11,3

10,9

8,1

1,1

Quelle: Karrenberg, Hanns und Münstermann, Engelbert, Gemeindefinanzbericht 1984, a.a.O., S. 98.

Der Vergleich der Kostendeckungsgrade für verschiedene kommunale Gebührenhaushalte in Tabelle 8 bestätigt diese Untergliederung noch einmal, wenn auch darauf hinzuweisen ist, daß die Entgeltfestsetzung nicht nur nach fiskalischen Kriterien vorgenommen wird und deshalb das realisierte Auf- 
kommen nicht mit dem Entgeltpotential übereinstimmen dürfte. Trotzdem ist davon auszugehen, daß in bestimmten Bereichen wie etwa bei Theatern und Museen kostendeckende Entgelte nicht nur aus Gründen der Kultur- und der Sozialpolitik weit unterschritten werden, sondern daß sie prohibitive und damit auch fiskalisch unerwünschte Wirkungen hätten. Einen Hinweis auf die erzielbaren Einnahmen könnte zum einen eine Analyse der Privatisierungsbemühungen, zum anderen der Vergleich mit ähnlichen Angeboten des privaten Sektors liefern: Angebote im kulturellen Bereich sind wegen der hohen Preiselastizität der Nachfrage hier ebenfalls häufig nur mit massiver öffentlicher Förderung existenzfähig. ${ }^{146)}$

2. Staatliche Begrenzungen bei unterschiedlichen Organisationsformen des kommunalen Angebots

Würde die kommunale Entgeltpolitik allein durch die Reaktionen der privaten Abnehmer gemeindlicher Leistungen begrenzt, so ständen den Gemeinden in diesem Bereich weitgehende Einnahmemöglichkeiten offen. Sie resultieren vor allem aus der für einige Leistungen gegebenen Monopolstellung des gemeindlichen Angebots und aus preisunelastischen Nachfrageverläufen, die besondere Vorteile bei der Preisbildung hervorbringen. Eine Einschränkung dieser Spielräume durch übergeordnete Ebenen erfolgte deshalb auch nicht zuletzt aufgrund der Tatsache, daß die Gemeinden ihre wirtschaftlichen Aktivitäten zeitweise vorwiegend mit rein fiskalischen Zielsetzungen betrieben und die genannten Spielräume weitgehend ausgenutzt haben. ${ }^{147}$ )

Aufgrund der damit verbundenen unerwünschten Wirkungen - insbesondere allokativer und verteilungspolitischer Art - greift der Staat hier begrenzend ein.

146) Vgl. etwa Tofaute, Hartmut, Die Übertragung öffentlicher Leistungen und Funktionen auf Private (Privatisierung) - Eine Darstellung politischer, ökonomischer, gesellschaftlicher und arbeitnehmerorientierter Gesichtspunkte - Schriften zur Privatisierung öffentlicher Dienstleistungen Nr. 5, hrsg. vom Hauptvorstand der Gewerkschaft OTV. Reutlingen 1977, S. 70, sowie Handwerkskammer Trier - Industrie- und Handelskammer Trier Hrsg., Sparen und rationalisieren? ... privatisieren!, Trier 1983, S. 16.

147) Vgl. Popitz, Johannes, Der künftige Finanzausgleich zwischen Reich, Ländern und Gemeinden, a.a.O., S. 46. 
Die einzelnen Begrenzungen sind vorwiegend in den Kommunalabgabegesetzen der Länder festgelegt und regeln die Entgelthöhe zum Teil direkt, zum Teil über die Vorgabe von "Preisbildungsrichtlinien" oder -obergrenzen.

Direkte Preissetzung erfolgt vor allem für jene Leistungen, die ohnehin eher den übergeordneten Gebietskörperschaften zuzurechnen sind: "... wenn und soweit eine Abgabe durch Bundes- oder Landesgesetze geregelt ist, wie z.B. ... die Erschließungsbeiträge ... und die Verwaltungsgebühren auf dem Gebiet der Auftragsangelegenheiten und der weisungsgebundenen Pflichtaufgaben, sind die Gemeinden zu einer eigenen Regelung nicht befugt". ${ }^{148)}$

Für die übrigen Leistungen werden weniger strikte Regelungen getroffen, die den Gemeinden je nach Art des Angebots mehr oder weniger große Spielräume belassen.

Relativ stark eingeengt wird der gemeindliche Spielraum bei der Festsetzung von steuerähnlichen Verwaltungsgebühren; hier gilt, daß die Gebühr die Kosten der Leistungserstellung und -abgabe im Abrechnungszeitraum nicht überschreiten dürfen. Anders als bei preisähnlichen Benutzungsgebühren ${ }^{149)}$ werden dabei als Kosten nur die tatsächlichen Geldausgaben anerkannt. ${ }^{150}$ )

Bei der Entgeltfestsetzung zur Finanzierung von Einrichtungen und Anlagen verbleibt den Gemeinden - sieht man von einigen Ausnahmen einmal ab - ein größerer Gestaltungsspielraum. Zunächst können die Gemeinden, wo dies möglich ist, zwischen Beitragsfinanzierung und der Erhebung eines nutzungsabhängigen Entgelts wählen ${ }^{151)}$, letztere können als privatrechtliches Entgelt oder als öffentlich-rechtliche Gebühr festgesetzt werden.

Beiträge werden als Gegenleistung für die "Herstellung, Anschaffung und Erweiterung öffentlicher Einrichtungen und Anlagen" ${ }^{152)}$ gekennzeichnet; Kosten der Inanspruchnahme können durch Beiträge dementsprechend nicht ab-

148) VVO zu § $1 \mathrm{KAG} \mathrm{NW.}$

149) Zur Abgrenzung von Verwaltungs- und Benutzungsgebühren vgl. etwa Schmölders, Günter, Finanzpolitik, a.a.O.. S. 299.

150) Vgl. Wilke, Dieter, Gebühren, in: Handwörterbuch der kommunalen Wissenschaft und Praxis, Bd. 6, Kommunale Finanzen, hrsg. von Günter Püttner, 2., völlig neu bearbeitete Auflage. Berlin u.a. 1985. S. 246-259, hier S. 254.

151) Vgl. VVO zu \& 8 KAG .NW.

152) $\S 8$, abs. 2 KAG . NW. 
gedeckt werden. ${ }^{153)}$ Das Beitragsrecht ist landesrechtlich normiert, soweit nicht das Bundesbaugesetz durchgreift, und schreibt für bestimmte Fälle die Beitragserhebung vor: "Bei den dem öffentlichen Verkehr gewidmeten Straßen, Wegen und Plätzen sollen Beiträge erhoben werden, ...."154), die Erhebung von Erschließungsbeiträgen ist nach dem BBauG zwingend vorgeschrieben. ${ }^{155)}$

Andererseits ist eine Beitragsfinanzierung in jenen Bereichen ausgeschlossen, in denen die Vorteile aus der Anlagenerrichtung nicht unmittelbar bestimmten Gruppen zugerechnet werden können. So könnte man sich etwa vorstellen, daß der Bau eines Theaters über Beiträge von Beziehern höherer Einkommen finanziert werden solle, weil angenommen wird, daß sie von dieser Einrichtung eher profitieren als Bezieher niedriger Einkommen. Ein solcher Versuch wäre jedoch kaum zulässig, weil die potentiellen Vorteile auf diese Weise nicht klar zurechenbar sind. Damit wird die Beitragsfinanzierung prinzipiell auf Leitungs- und Netzinfrastruktur mit direktem Anschluß der Beitragspflichtigen begrenzt. ${ }^{156)} \mathrm{Da}$ der Beitragszahlung kaum ausgewichen werden kann, sind die Prinzipien zur Beitragsbemessung vergleichsweise strikt geregelt: zunächst sollen Beiträge nach den Kosten der Einrichtung bemessen werden ${ }^{157}$ ), dabei ist landesrechtlich normiert, welche Kosten beitragsfähig sind. Im Gegensatz zu den Gebühren wird - entsprechend dem Wortsinn - dabei keine volle Kostendeckung intendiert. ${ }^{158)}$ Die Verteilung der beitragsfähigen Kosten auf die einzelnen Beitragszahler soll dann in der Regel nach dem Vorteilsprinzip erfolgen. ${ }^{159)}$

Soll die Finanzierung kommunaler Angebote über Entgelte für die Nutzung der Einrichtungen vorgenommen werden - was nach den Vorschriften der Kommunalabgabengesetze geschehen muß, wenn bestimmte Gruppen ge-

153) Vgl. VVO zu §8 KAG NW.

154) §8 Abs. 1 KAG NW.

155) Vgl. dazu Pagenkopf, Hans, Kommunalrecht, Bd. 2. Wirtschaftsrecht, 2., neubearb. u. erw. Auflage, Köln u.a. 1976, S. 95; sowie Lehmann, Matthias, Beiträge, in: Handbuch der kommunalen Wissenschaft und Praxis, Bd. 6. Kommunale Finanzen, hrsg. von Günter Püttner, 2., völlig neu bearbeitete Auflage, Berlin u.a. 1984, S. 260-279, hier S. 270.

156) Vgl. auch Lehmann, Matthias, Beiträge, a.a.O., S. 270.

157) Vgl. § 8, Abs. 2 KAG NW.

158) So Pagenkopf, Hans, Kommunalrecht, Band 2, Wirtschaftsrecht, a.a.O., S. 95.

159) Vgl. §8 Abs. 6 KAG NW; auch Lehmann, Matthias, Beiträge, a.a.O. S. 271. 
genüber der Allgemeinheit Sondervorteile aus der Nutzung ziehen ${ }^{160)}$ - so steht den Gemeinden innerhalb bestimmter Grenzen frei, ob sie für die Inanspruchnahme ein privatrechtliches Entgelt oder eine öffentlich-rechtliche Gebühr nach Maßgabe einer Gebührensatzung erheben wollen. ${ }^{161)}$ Dabei sind für die Gebühren zumindest formal engere Grenzen festgelegt als für privatrechtliche Entgelte aus wirtschaftlichen Unternehmen der Gemeinden: Während die Einnahmen aus Benutzungsgebühren die Kosten der Einrichtung nicht übersteigen sollen ${ }^{162)}$, sollen die wirtschaftlichen Unternehmen "einen Ertrag für den Haushalt der Gemeinde abwerfen, soweit dadurch die Erfüllung des öffentlichen Zwecks nicht beeinträchtigt wird". ${ }^{163)}$ Allerdings verliert diese Unterscheidung bei näherer Betrachtung an Präzision.

Pagenkopf differenziert zwischen Benutzungsgebühren und Erwerbseinkünften nach dem Zwang zur Inanspruchnahme der Leistungen: "Von den Erwerbseinkünften unterscheiden sich die Gebühren dadurch, daß bei diesen ein Element öffentlich-rechtlichen Zwanges in Erscheinung tritt, indem eine öffentlich-rechtliche Körperschaft bestimmte Tatbestände erfüllende Personengruppen zur Entgegennahme ihrer dargebotenen Leistungen zwingt, für die dann Gebühren zu entrichten sind". ${ }^{164)}$

Dieses Prinzip wird in der Praxis jedoch mitunter durchbrochen, insbesondere wenn man nicht auf den rechtlichen, sondern den faktischen Zwang zur Inanspruchnahme kommunaler Leistungen, also auf die Preiselastizität der Nachfrage abstellt. Als Beispiele seien etwa kommunale Einrichtungen der Energie- und Wasserversorgung genannt, die durchaus als wirtschaftliche Unternehmen geführt werden können.

Eine Einschränkung der gemeindlichen Wahlmöglichkeit zwischen Gebühr und privatrechtlichem Entgelt und damit auch der Preisgestaltungsspielräume erfolgt in den Gesetzen zunächst durch die Abgrenzung jener Angebote, die nicht durch wirtschaftliche Unternehmen erstellt werden dürfen.

160) Vgl. §5 und §6 KAG NW.

161) Vgl. Pagenkopf, Hans, Kommunalrecht, Band 2, Wirtschaftsrecht, a.a.O., S. 94; sowie Wilke, Dieter, Gebühren, a.a.O., S. 253.

162) So $\$ 6$, Abs. 1 KAG NW.

163) $\$ 94$ Abs. 1 GO NW.

164) Pagenkopf, Hans, Kommunalrecht, Bd. 2, Wirtschaftsrecht, a.a.O., S. 90. 
Dies sind zum einen jene Angebote, zu denen die Gemeinden gesetzlich verpflichtet sind, sowie nach $\S 67$ Abs. 1 und 2 DGO, an die sich die meisten Gemeindeordnungen anlehnen, "Einrichtungen des Unterrichts- und Erziehungsund Bildungswesens, der körperlichen Ertüchtigung, der Kranken-, Gesundheits- und Wohlfahrtspflege...". ${ }^{165}$ ) Die Gemeindeordnung in NordrheinWestfalen schließt darüber hinaus Einrichtungen der Abfall- und Abwasserbeseitigung, der Straßenreinigung sowie "Einrichtungen ähnlicher Art" als Bereich wirtschaftlicher Unternehmen aus. ${ }^{166)}$ In diesen Bereichen unterliegt die Preissetzung damit voll den Regelungen zur Gebührenbegrenzung.

Die erste dieser Regelungen bezieht sich auf die Kosten der jeweiligen Einrichtung, die durch das Gebührenaufkommen nur gedeckt, nicht aber überschritten werden sollen. ${ }^{167)}$ Anders als bei den Verwaltungsgebühren sind damit bei den Benutzungsgebühren jedoch "die nach betriebswirtschaftlichen Grundsätzen ansatzfähigen Kosten" ${ }^{168)}$ umschrieben.

Der betriebswirtschaftliche Kostenbegriff - für die Zwecke gewinnorientierter Unternehmensrechnung entwickelt - schließt nun auch Kostenbestandteile ein, deren "Gebührenfähigkeit" unter den auf den öffentlichen Sektor bezogenen Zielsetzungen umstritten ist, wie etwa die "angemessene Verzinsung des aufgewandten Kapitals"169) 170), und der die Begrenzung gegenüber den Einnahmen aus wirtschaftlichen Unternehmen aufweicht. Die Ermittlung dieses Kostenbestandteils bietet die Möglichkeit, die Höhe der in Ansatz gebrachten Gesamtkosten mehr oder weniger stark zu beeinflussen. Empirische Untersuchungen über "Kommunale Gebührenhaushalte" zeigen, wie weit die Ansätze zur Kostenermittlung interkommunal differieren. ${ }^{171)}$ Zwar geht ein Teil dieser Unterschiede auf die länderweise voneinander abweichenden Regelungen zurück, trotzdem verbleibt den Gemeinden ein (nicht zu unterschätzender) Spielraum, innerhalb dessen die Kostenhöhe beeinflußt werden kann. Budäus weist darauf hin, daß unter diesen

165) Scholler, Heinrich und Broß, Siegfried, Grundzüge des Kommunalrechts..., a.a.O., S. 200.

166) §88 Abs. 2 GO NW.

167) §6 Abs. 1 KAG NW.

168) §6 Abs. 2 KAG NW.

169) Ebenda.

170) Zur Kritik dieser Kostendefinition vgl. Budäus, Dietrich, Betriebswirtschaftliche Instrumente zur Entlastung kommunaler Haushalte, Baden-Baden 1982, S. 162.

171) Siehe ebd., S. 162 ff.: sowie Seiler, Gerhard, Zur Bedeutung der Gebühren, Beiträge und anderer Verwaltungsabgaben..., a.a.O., S. $18 \mathrm{ff}$. 
Bedingungen die Einnahmen dadurch maximiert werden können, daß jene Kosten maximiert werden, denen keine Ausgaben gegenüberstehen. ${ }^{172)}$

Einer solchen fiskalisch motivierten "Kostenaufblähung" steht jedoch der zweite Grundsatz der Äquivalenz bzw. der Verhältnismäßigkeit des Entgelts entgegen, mit dem eine Beziehung zwischen Gebührenhöhe und der finanziellen Belastung des Schuldners unabhängig von den Kosten der Einrichtung hergestellt wird. ${ }^{173)}$ Es besagt, daß zwischen "der Höhe der Gebühr einerseits und der Bedeutung, dem wirtschaftlichen Wert oder dem sonstigen Nutzen der Benutzung für den Kostenschuldner andererseits... ein angemessenes Verhältnis zu bestehen"174) habe. Damit wird allerdings keine konkrete Begrenzung vorgenommen, sondern nur eine Richtlinie festgelegt, über deren Einhaltung im Einzelfall entschieden werden muß. Diese Regelung wird im übrigen auch dort wirksam, wo der Kostendeckungsgrundsatz keine Anwendung findet, also bei den Erwerbseinkünften.

Eine dritte Begrenzung kommunaler Gebührenpolitik resultiert aus der Vorgabe, daß mittels Gebühren nur Sondervorteile aus der Nutzung der gebührenpflichtigen Einrichtung abgegolten werden sollen. Vorteile für die Allgemeinheit, also externe Effekte, sollen hingegen auch bei der Finanzierung berücksichtigt und die entsprechenden Kosten durch allgemeine Deckungsmittel abgedeckt werden. Diese Regelung setzt ebenfalls keine konkreten Grenzen und muß für den Einzelfall entschieden werden, sofern in den die jeweilige Aufgabenerfüllung regelnden Vorschriften keine Grenzen festgesetzt sind. Als Beispiel für eine solche Regelung kann etwa der § 4a des preußischen Gesetzes über die Reinigung öffentlicher Wege vom 1.7.1912 gelten, wonach die Gebührensätze so zu bemessen sind, daß maximal $75 \%$ der Verwaltungs- und Unterhaltskosten der "Straßenreinigungsveranstaltung" einschließlich der Ausgaben für Verzinsung und Tilgung des aufgewendeten Kapitals gedeckt werden. ${ }^{175)}$

Insgesamt bleibt festzuhalten, daß die Begrenzungen kommunaler Gebührenpolitik relativ weit sind und von den Gemeinden durchaus mit beeinflußt werden können. Für die Erwerbseinkünfte sind sie noch weniger strikt.

172) Budäus. Dietrich, Betriebswirtschaftliche Instrumente ..., a.a.O., S. 155.

173) Vgl. Wilke. Dieter, Gebühren. a.a.O.. S. 254 f.

174) Vgl. § 3 NW GebG; § 8 Abs. 2 KAG Bad.-Württ.

175) Vgl. Pagenkopf, Hans, Kommunalrecht, Bd. 2, Wirtschaftsrecht, a.a.O., S. 91. 
Pagenkopf charakterisiert kommunale wirtschaftliche Unternehmen als solche, die prinzipiell auch von einem Privatunternehmer mit der Absicht der Gewinnerzielung betrieben werden könnten. ${ }^{176)}$ Dementsprechend besteht für kommunale Unternehmen gegenüber privaten Unternehmen zunächst keine preisrechtliche Sonderstellung. ${ }^{177)}$ Eine Ausnahme bilden hier verschiedene in Spezialgesetzen vorgesehene Preisgenehmigungsbefugnisse übergeordneter Ebenen für die Bereiche Verkehrs- und Versorgungswirtschaft wie z.B. die "Bundestarifordnungen für Strom- und Gastarife, die kostenbedingte Preiserhöhungen einem energiepreisrechtlichen Genehmigungsvorbehalt unterwerfen". 178)

Davon abgesehen unterliegen kommunale Unternehmen den auch im privaten Sektor greifenden Vorschriften zur Verhinderung des Monopolmißbrauchs, die hier insofern besonders wirksam werden, als kommunale Unternehmen häufig eine Monopolstellung einnehmen. ${ }^{179)}$

Anders als für private Unternehmen ist für kommunale wirtschaftliche Unternehmen und deren Preisbildung die öffentliche Zweckbindung relevant ${ }^{180)}$; wirtschaftliche Tätigkeit der Gemeinden ist überhaupt nur zulässig, sofern sie dem "Gemeinwohl" dient, womit unter anderem eine von privaten Unternehmen nicht zu erwartende, sozial orientierte Tarifgestaltung angesprochen ist. ${ }^{181)}$ Allerdings handelt es sich hier um eine sehr unpräzise Begrenzung ${ }^{182)}$, deren Konsequenzen für die kommunalen Preissetzungsspielräume kaum einschätzbar sind. Zumindest in jenen Bereichen, in denen kommunale Unternehmen mit dem privaten Sektor konkurrieren, scheint sich die Preisbildung der kommunalen Unternehmen an den Preisen der privaten Unternehmen zu orientieren. So weist Bullinger auf Überlegungen in einzelnen Gemeinden hin, die darauf abzielen, "das eine

176) Ebd. S. 149; zur Kritik dieser Auffassung vgl. Scholler, Heinrich und BroB, Siegfried, Grundzüge des Kommunalrechts..., a.a.O., S. 199 f.

177) Vgl. Hidien, Jürgen, Staatliche Wirtschaftsaufsicht und Besteuerung kommunaler Unternehmen, in: Handbuch der kommunalen Wissenschaft und Praxis, Bd. 5, Kommunale Wirtschaft, hrsg. von Günter Püttner, 2., völlig neu bearb. Aufl., Berlin u.a. 1984, S. 98-118, hier S. 101.

178) Ebenda.

179) Vgl. Pagenkopf, Hans, Kommunalrecht, Bd. 2, Wirtschaftsrecht, a.a.O., S. 154.

180) Vgl. auch Hidien, Jürgen, Staatliche Wirtschaftsaufsicht, a.a.O., S. 107.

181) So Pagenkopf, Hans, Kommunalrecht, Bd. 2, Wirtschaftsrecht, a.a.O., S. 153.

182) Vgl. auch Hidien, Jürgen, Staatliche Wirtschaftsaufsicht, a.a.O., S. 108. 
oder andere Angebot zu privatisieren (z.B. eines von mehreren Hallenbädern), um die dort dann notwendigen (privaten) Preise als Argument auch für eine Erhöhung der Preise in den kommunalen Einrichtungen anführen zu können." ${ }^{183)}$

Zusammenfassend ist noch einmal festzuhalten, daß die Gemeinden nur bei Entgelten für die Nutzung kommunaler Einrichtungen über die Kostendeckung hinausgehende Einnahmespielräume haben, und das auch nur dann, wenn ein erwerbswirtschaftliches Angebot mit privatrechtlicher Preisfestsetzung erfolgt. Bei Verwaltungsgebühren und Beiträgen sind die Einnahmespielräume hingegen stärker begrenzt und an die Normen übergeordneter Gebietskörperschaften gebunden.

Aber auch für einen Teil jener Leistungen, die prinzipiell mit erwerbswirtschaftlicher Zielsetzung angeboten werden könnten, wird ein Angebot durch kommunale wirtschaftliche Unternehmen untersagt, insbesondere in den Bereichen Kultur, Bildung, Sport sowie für pflichtige Angebote. Hier liegt die Obergrenze der Einnahmen bei der Deckung der entstehenden Kosten. Die Wirkung dieser Begrenzung hängt vor allem vom jeweiligen Nachfrageverlauf ab: Liegt ein flacher, preiselastischer Verlauf der Nachfragekurve vor, so kann in bestimmten Fällen die rechtliche Obergrenze der Kostendeckung faktisch gar nicht erreicht werden. So dürften die Ausweichreaktionen der Nutzer z.B. bei Theatern und Museen eine kostendeckende Preisfestsetzung von vornherein ausschließen. In Bereichen mit preisunelastischer Nachfrage, wie vor allem der kommunalen Ver- und Entsorgung, kann dagegen in der Regel ein hoher Kostendeckungsgrad oder - sofern nicht rechtlich ausgeschlossen - sogar ein Überschuß erwirtschaftet werden. Hier liegt deshalb das Schwergewicht kommunaler wirtschaftlicher Betätigung, die sich darüber hinaus aber auch auf Bereiche, wie den öffentlichen Nahverkehr sowie die Veranstaltung von Märkten erstreckt. ${ }^{184}$ )

183) Bullinger, Dieter, Die Einnahmesituation und Strategien zur Einnahmesteigerung, in: Handbuch der kommunalen Wissenschaft und Praxis, Bd. 6, Kommunale Finanzen, hrsg. von Günter Püttner, 2., völlig neu bearb. Aufl., Berlin u.a. 1984, S. 106-116, hier S. 113.

184) Vgl. Scholler, Heinrich und Broß, Siegfried, Grundzüge des Kommunalrechts ..., a.a.O., S. 199. 


\section{d. Einige Ergebnisse für verschiedene Gemeindetypen}

Aus dem vorangegangenen Abschnitt ging hervor, daß sich die Entgelt- und Einnahmespielräume je nach Leistungsbereich stark unterscheiden; an einem Ende der Skala stehen Leistungen, die gar nicht entgeltfähig sind, am anderen Ende solche, aus deren Angebot ein Gewinn erzielt werden kann. Bei unterstelltem gegebenen Angebot in den Gemeinden hängt deren Entgeltpotential daher zunächst und vor allem von der Struktur dieses Angebots ab. Deshalb ist zu untersuchen, ob mit Blick auf das Entgeltpotential bestimmte gemeindetypenspezifische Angebotsstrukturen erkennbar sind.

Darüber hinaus ist anzunehmen, daß sich die Nachfrageverläufe auch für identische Leistungen im Gemeindevergleich unterscheiden und die kommunale Entgeltpolitik auf unterschiedlichem Niveau begrenzen. Wichtige Determinanten für die Preiselastizität der Nachfrage sind zum einen bestimmte Merkmale der Nachfrager wie etwa ihre Präferenzen und Ansprüche und vor allem ihre finanzielle Leistungsfähigkeit. So kann etwa davon ausgegangen werden, daß in Gemeinden mit einkommensstarker Bevölkerung von hohem Bildungsniveau ein Theaterangebot auf eine breitere und zahlungskräftigere Nachfrage trifft als in Gemeinden mit einer ungünstigeren Sozialstruktur. ${ }^{185)}$ Zum anderen ist für die Nachfrage nach kommunalen Leistungen der Umfang und die Qualität konkurrierender, insbesondere privater Angebote entscheidend.

Damit ist bereits eine Vielfalt von Einflußgrößen angesprochen, die in ihrer Gesamtheit hier nicht näher betrachtet werden können. Die einzelne Gemeinde hätte sie jedoch bei der Abschätzung ihres Entgeltpotentials vollständig zu berücksichtigen.

Im folgenden soll allein auf die Angebotsstruktur in verschiedenen Gemeindetypen eingegangen werden.

Für den Gemeindevergleich ist zunächst natürlich auch der Umfang des Angebots von Bedeutung: man kann annehmen, daß das Entgeltpotential insgesamt desto höher ist, je umfangreicher das kommunale Angebot ist. Fragt man hingegen aus einzelgemeindlicher Sicht nach den Möglichkeiten der Ent-

185) Vgl. etwa Postlep, Rolf-Dieter, Regionale Effekte höherwertiger Dienstleistungen, Schriftenreihe der Gesellschaft für Regionale Strukturentwicklung, Bd. 10, Bonn 1982, S. 83. 
geltfinanzierung im Gesamthaushalt, so kommt es vor allem auf die Angebotsstruktur an. Dies gilt prinzipiell für jedes Angebotsvolumen.

Gemeindetypenspezifische hohe bzw. niedrige Entgeltfinanzierungsmöglichkeiten ergäben sich dann, wenn sich in bestimmten Gemeinden hohe Anteile nicht entgeltfähiger Leistungen oder - im Gegenteil dazu - von Leistungen mit weiten Entgeltspielräumen fänden. Insbesondere mit Blick auf zwei Leistungsarten kann eine solche gemeindetypenspezifische Konzentration tatsächlich nachgewiesen werden, und zwar zum einen für die Transferzahlungen im Rahmen der sozialen Sicherung - die grundsätzlich nicht entgeltfähig sind -, und zum anderen für die Mehrzahl der zentralörtlichen Angebote.

Die Leistungen der Sozialhilfe steigen sowohl absolut als auch in ihrem Haushaltsanteil mit der Gemeindegröße an. ${ }^{186)}$ Dafür sind verschiedene Ursachen verantwortlich: Da mit dem Verdichtungsgrad die Kosten der Lebenshaltung in der Regel ansteigen, wachsen auch die kommunalen Ausgabenerfordernisse für jeden auftretenden Sozialfall. Darüber hinaus ist die Zahl der Sozialhilfeempfänger in den Städten und speziell in den Großstädten wesentlich höher als in kleinen Gemeinden im eher ländlichen Raum, in dem Fälle materieller Notsituation offenbar noch eher durch Familien und Nachbarn aufgefangen werden und der Bezug von Sozialhilfe überdies meist als gesellschaftlich diskriminierend gilt, weswegen sie weniger selbstverständlich in Anspruch genommen wird.

Im Gemeindevergleich innerhalb einer Größenklasse stellen sich die Wirtschafts- und Sozialstruktur als Hauptdeterminanten der Ausgaben für die soziale Sicherung heraus. ${ }^{187)}$ Die Gemeinden müssen desto mehr dieser nicht entgeltfähigen Leistungen erbringen, je ungünstiger die Produktionsstruktur insbesondere mit Blick auf die Beschäftigungssituation ist. Alle übrigen Leistungsangebote einmal gleichgesetzt, sinkt damit die "Entgeltergiebigkeit" bzw. die Möglichkeit der Ausgabendeckung aus Entgelten mit wachsender Gemeindegröße ab. In einer besonders nachteiligen Lage befinden sich dabei strukturschwache Großstädte, zumal hier auch das Steuerpotential vergleichsweise niedrig ausfällt.

186) Vgl. Zimmermann, Horst, Hardt, Ulrike und Postlep, Rolf-Dieter, Bestimmungsgründe der kommunalen Finanzsituation, a.a.O., S. $270 \mathrm{ff}$.

187) Vgl. ebenda, S. 82 ff. 
Aber auch das Leistungsangebot außerhalb des Bereichs der sozialen Sicherung wird mit wachsender Gemeindegröße und vor allem mit wachsender Zentralität tendenziell immer weniger entgeltergiebig.

Betrachtet man noch einmal die realisierten Kostendeckungsgrade der verschiedenen entgeltfähigen Leistungen in Tabelle 8 auf S. , so ist festzustellen, daß es sich bei jenen Einrichtungen, die einen hohen Kostendeckungsgrad aufweisen und für die geringe Nachfrageelastizitäten vermutet wurden, um solche Einrichtungen der Daseinsvorsorge - Wasserver- und Entsorgung, Abfallbeseitigung, Energieversorgung und kostenrechnende Einrichtungen des Gesundheitswesens - handelt, die prinzipiell in allen Gemeinden vorhanden sind. Mitunter werden diese Aufgaben von Zweckverbänden oder von einzelnen Gemeinden für das überörtliche Angebot wahrgenommen; hier resultiert daraus ein überdurchschnittlich hohes Entgeltpotential (bezogen auf die abgegebenen Leistungen insgesamt oder auf die Ausgabensumme bei gegebenem Leistungsangebot).

Das empirische Bild zeigt, daß die Gemeinden mit wachsender Einwohnerzahl und Zentralitätsstufe ${ }^{188)}$ zusätzliche, über die Grundversorgung hinausgehende Aufgaben für die überörtliche Inanspruchnahme übernehmen, die jedoch zum Teil gar nicht entgeltfähig sind oder für die nur relativ niedrige Kostendeckungsgrade realisiert werden können. Als Beispiel können etwa die typisch mittelzentralen Angebote des Schulbereichs, die aufgrund rechtlicher Beschränkungen nicht entgeltfähig sind sowie die oberzentralen Kulturangebote wie Theater und Museen, die gemessen an den Ausgaben nur niedrige Entgeltsätze erbringen, herangezogen werden.

Sofern sich also der Aufgabenschwerpunkt auf solche Leistungen verlagert, wächst das Entgeltpotential mit den Ausgaben nicht mit; ein immer kleinerer Anteil der Ausgabentätigkeit kann mit Entgelten abgedeckt werden.

Tabelle 9 bestätigt noch einmal den vermuteten Zusammenhang zwischen Gemeindegröße und den Entgelt-Deckungsquoten des Gesamthaushalts. Zwar ist hier wiederum darauf zu verweisen, daß die von den Gemein-

188) Aufgrund der besonderen Bedingungen, unter denen sich Zentralität entwickelt, hängen Zentralität und Gemeindegröße in der Regel eng miteinander zusammen. Vgl. dazu Zimmermann, Horst, Hardt, Ulrike und Postlep. Rolf-Dieter, Bestimmungsgründe der kommunalen Finanzsituation a.a.O., S. 248 ff. 
den realisierten Entgelte auch unter anderen als fiskalischen Gesichtspunkten festgelegt werden und insofern das Entgeltpotential nicht unbedingt in seiner wirklichen Höhe abbilden. Allerdings deuten die empirischen Ergebnisse in diesem Fall darauf hin, daß die vorhandenen Spielräume die Entgeltfestsetzung maßgeblich mitbestimmen. ${ }^{189)}$

\section{Tabelle 9: Entgelteinnahmen nach Arten und Haushaltsanteilen in Gemeinden verschiedener Größenklassen, 1980}

\begin{tabular}{|c|c|c|c|c|c|}
\hline \multirow{2}{*}{\multicolumn{2}{|c|}{$\begin{array}{l}\text { Kreisangeh. } \\
\text { Gemeinden }\end{array}$}} & \multicolumn{3}{|c|}{$\begin{array}{c}\text { Entgelteinnahmen, } \\
\text { DM je Einwohner } \\
\text { darunter }\end{array}$} & \multirow[t]{2}{*}{$\begin{array}{l}\text { Anteil der Entgelt- } \\
\text { einnahmen an den } \\
\text { Gesamteinnahmen } \\
\text { (netto) }\end{array}$} \\
\hline & & insgesamt & $\begin{array}{l}\text { Gebühren, } \\
\text { Mieten, } \\
\text { Verwaltungs- } \\
\text { einnahmen }\end{array}$ & $\begin{array}{l}\text { Beiträge } \\
\text { u.ä. } \\
\text { Entgelte }\end{array}$ & \\
\hline unter & 5000 & 291 & 201 & 90 & 26,5 \\
\hline 5000 & -10000 & 211 & 125 & 86 & 22,7 \\
\hline 10000 & - 20000 & 208 & 137 & 71 & 19,2 \\
\hline 20000 & -50000 & 227 & 173 & 54 & 18,3 \\
\hline 50000 & -100000 & 245 & 210 & 35 & 17,2 \\
\hline 100000 & -200000 & & & & 18,0 \\
\hline $\begin{array}{l}\text { Kreisf re } \\
\text { Städte }\end{array}$ & & 279 & 252 & 27 & \\
\hline 100000 & -200000 & & & & 17,5 \\
\hline 200000 & -500000 & 337 & 305 & 32 & 19,1 \\
\hline über & 500000 & 371 & 353 & 18 & 17,8 \\
\hline
\end{tabular}

Quelle: Zimmermann, Horst, Hardt, Ulrike und Postlep, Rolf-Dieter, Bestimmungsgründe der kommunalen Finanzsituation, a.a.O., S. 323.

189) Diese These wird durch den Hinweis von gemeindlicher Seite, daß die Gebührenspielräume der Kommunen inzwischen weitgehend ausgeschöpft seien, bestärkt (vgl. oben, 2. Kapitel). 
V. Die Bedeutung "preispolitischer" Spielräume und ihrer Erfassung für die Einnahmepolitik der Gemeinden

Die vorausgegangene Betrachtung für ein gegebenes kommunales Leistungsangebot hat gezeigt, daß gemeindliche Einnahmepolitik durch verschiedene Faktoren begrenzt wird, die mit der Verteilung der Bemessungsgrundlagen und der staatlichen Normensetzung bei der Steuer- und Entgeltpolitik nur zum Teil beschrieben sind. Sie legen überdies weder in gleicher Weise für alle untersuchten Steuer- und Entgelteinnahmen, noch für identische Einnahmearten in unterschiedlichen Gemeindetypen gleichermaßen die Obergrenze der Einnahmebeschaffung fest.

Von entscheidender Bedeutung sind vielmehr auch die Reaktionen der Wirtschaftssubjekte auf die Abgabenerhebung, insbesondere wenn sie das gemeindliche Einnahmepotential in einigen oder sogar in allen Gemeinden unterhalb der staatlich festgesetzten Abgabenobergrenze limitieren. Dadurch wird eine Differenzierung der Einnahmemöglichkeiten zwischen den Gemeindetypen verursacht, die durch eine Erfassung der Bemessungsgrundlagen allein nicht abgebildet wird.

So führt die Besteuerung zu Ausweichreaktionen, die soweit wie möglich in der Substitution des Steuergegenstandes bestehen, oberhalb einer Grenze, die durch die Kosten und Nutzen der räumlichen Ausweichung bestimmt wird, aber eine Verlagerung des Steuergegenstandes in konkurrierende Gemeinden zur Folge hat. Welches die konkurrierenden Gemeinden sind, hängt wiederum vom Steuergegenstand und von der daraus abzuleitenden Art der räumlichen Ausweichung ab.

Im oben betrachteten Fall der Gewerbesteuer bestehen neben der Substitution von Kapital kaum andere Ausweichmöglichkeiten als die der Standortverlagerung. Da die oberhalb der beschriebenen Schwelle der Besteuerung mobilitätsbereiten Wirtschaftssubjekte bestimmte Ansprüche an den alten wie den neuen Ansiedlungsort haben, kommen als Abwanderungsort nicht alle übrigen Gemeinden, sondern nur Gemeinden mit bestimmten Merkmalen in Frage. Je höher und spezifischer die jeweiligen Ansprüche sind, desto geringer ist die Zahl der Gemeinden, die sie befriedigen könnten. Niedrigere Ansprüche können hingegen in einer Mehrzahl von Gemeinden, die entsprechend 
hart miteinander konkurrieren, abgedeckt werden. Daraus resultiert ein Gefälle in den Steuersatzspielräumen zwischen besonders gut ausgestatteten und - gemessen an den Ansprüchen der Wirtschaftssubjekte - weniger gut ausgestatteten Räumen.

Stärker noch als für die Besteuerung gilt für die Erhebung bestimmter Entgelte, daß die Gemeinden die Entgeltsätze selbst ohne staatliche Einschränkungen nicht folgenlos und unbegrenzt anheben können, weil neben der interkommunalen Konkurrenz, die in der Regel erst bei sehr hohen Entgeltsätzen zu wirken beginnt, eine Konkurrenzbeziehung zu privaten Anbietern besteht. Die Intensität dieser Konkurrenzbeziehungen und damit die Entgeltspielräume hängen vor allem vom Typ der entgeltfähigen Leistungen ab. Für das gesamte Entgeltpotential sind deshalb die Struktur des Leistungsangebots und die für die einzelnen Leistungen vorhandenen Nachfrageverläufe entscheidend.

Die Finanzkraftmessung des kommunalen Finanzausgleichs berücksichtigt die genannten Wirkungen nicht. Ob dies gerechtfertigt ist oder nicht, wird in Abschnitt D und im 4. Kapitel, das die Sicht übergeordneter Gebietskörperschaften analysiert, genauer betrachtet. Aus gemeindlicher Sicht ist eine umfassende Erfassung des Einnahmepotentials auch für ein gegebenes Angebot jedoch auf jeden Fall von Interesse:

- Zum ersten spielt die Kenntnis des Einnahmepotentials für die Planung des Einnahme- und Ausgabeverhaltens eine Rolle. Wie beschrieben, setzen die Ausweichreaktionen auf die Abgabenerhebung zum Teil nicht unmittelbar, sondern erst nach mehr oder weniger langen Anpassungsfristen ein und sind daher im Augenblick der Abgabenerhöhung nicht direkt im Aufkommen spürbar. Wären die quantitativen Auswirkungen der Einnahmepolitik auf den Umfang der Bemessungsgrundlagen bekannt oder zumindest tendenziell abschätzbar, so könnten nachträgliche Überraschungen über mittel- und langfristig sinkende Einnnahmen vielleicht vermieden werden. Überdies könnte eher kalkuliert werden, inwieweit Einnahmesteigerungen allein über eine "Preisanhebung" realisierbar wären und ab welcher Belastungsgrenze eine ausgabeseitige Attraktivitätspolitik einsetzen müßte, um das Budget weiter auszudehnen. 
Außer den direkten Wirkungen kommunaler Preispolitik auf die Steuerund Entgelteinnahmen wären die indirekten Wirkungen der kommunalen "Preispolitik" auf andere Einnahmearten, wie etwa die Schlüsselzuweisungen (vgl. oben) und die Möglichkeiten der Schuldaufnahme, die durch Abgaben-Mehreinnahmen erweitert werden können, besser abschätzbar.

- Zum zweiten wird die Kenntnis der jeweiligen Reaktionsverläufe die Einnahmepolitik aber selbst dann beeinflussen, wenn das Einnahmepotential nicht vollständig ausgeschöpft werden soll, was in der kommunalen Praxis die Regel sein dürfte. ${ }^{190)}$ Wie oben bereits angesprochen, verfolgen die Gemeinden bei ihrer Einnahmepolitik auch außerfiskalische Ziele wie etwa eine Gewerbepolitik zum Schutz der innergemeindlichen Arbeitsplätze oder speziell mittels der Entgelte eine Politik der Nachfragelenkung, die die Nutzung meritorisierter Güter wie etwa im Kultur- und Bildungsbereich anregen und die Nachfrage nach knappen Gütern wie etwa Wasser oder Energie zurückdrängen soll. Jedoch erst die Kenntnis der Reaktionsverläufe bei verschiedenen Steuerund Abgabesätzen erlaubt die Abstimmung der fiskalischen mit den nicht-fiskalischen Interessen, weil die Steuer- und Entgeltausweichung eben nicht-fiskalische Zielverluste bzw. -gewinne bedeutet. Werden in einer bestimmten Situation Einnahmesteigerungen notwendig, so kann in etwa abgeschätzt werden, auf welche Weise die zusätzlich erforderlichen Einnahmen mit den am wenigsten bedenklichen Nebenwirkungen beschafft werden können.

Abgabe- und Einnahmeplanung werden von den Gemeinden jeweils simultan und letztlich gesamthaft vorgenommen, weil die Kosten entgeltfähiger, aber nicht mit voller Kostendeckung absetzbarer sowie nicht entgeltfähiger Leistungen mit den Einnahmen aus speziellen und allgemeinen Deckungsmitteln einschließlich der Überschüsse aus kommunalen wirtschaftlichen Unternehmen zur Deckung gebracht werden müssen. Da die einzelnen Ausgabeund Einnahmepositionen über diese "Querfinanzierung" zusammenhängen,

190) So bezeichnet auch Gandenberger das Streben nach höchstmöglichem Steuerertrag als seltenen Grenzfall. Gandenberger, Otto, Das Finanzmonopol - Fiskalische und außerfiskalische Wirkungen im Vergleich zur Verbrauchssteuer - Veröffentlichungen des Forschungsinstituts für Wirtschaftspolitik an der Universität Mainz, Bd. 24, Heidelberg 1968, S. 61. 
haben die Gemeinden in jedem Fall ein Interesse an der Ermittlung aller Einnahmemöglichkeiten, unabhängig davon, ob sie sie direkt im Wege der "Preisgestaltung" beeinflussen können oder nicht. Abtrennbar und gesondert zu planen sind Bereiche, in denen eine Nettobudgetierung vorgenommen und nur der Überschuß oder das entstandene Defizit in den Haushalt übernommen wird. Damit kommt es allerdings lediglich zu einer Bilanzverkürzung; innerhalb der jeweiligen Einrichtung bleibt das Interesse an den Einnahmemöglichkeiten aus der Leistungsabgabe insgesamt bestehen, das Ergebnis wird nur "vorgefiltert" an die Haushaltsplanung weitergegeben.

Unter den Bedingungen eines gegebenen Angebots unterscheiden sich jene Einnahmen, bei denen die Gemeinden einen "Preissetzungsspielraum" haben, jedoch von rein bemessungsgrundlagenabhängigen - wie die Bemessungsgrundlage auch immer gestaltet ist - Einnahmen in der Ermittlung ihres potentiellen Aufkommens und dessen Determinanten. Hier steht die Gemeinde vor dem Problem, die Grenzen der Einnahmeerzielung aufzuspüren. Insofern befindet sie sich in einer ähnlichen Lage wie ein Unternehmen, das seine Preisspielräume, die durch die Nachfrage und die Konkurrenzsituation bestimmt werden, festzustellen versucht. Aus der Sicht der Gemeinde handelt es sich bei der Ermittlung des Einnahmepotentials im Grunde ebenfalls um eine "unternehmerische Kalkulation". Daran ändert auch die Tatsache massiver staatlicher Eingriffe - denen sich Unternehmen z.T. ebenfalls ausgesetzt sehen - nichts, wenn auch die Spielräume der Einnahmebeschaffung im Kommunalbereich enger begrenzt sein dürften.

Ebenso wie im Unternehmensbereich ist die Messung des Einnahmepotentials bzw. die Schätzung des möglichen Umsatzes natürlich problematisch und schon für ein vorgegebenes Angebot wegen fehlender Informationen praktisch kaum realisierbar.

Theoretisch sind diese Größen trotzdem bestimmbar und für die Gemeinden lassen sie sich über die Eruierung der eigenen Konkurrenzsituation und der wirtschaftlichen Lage sowie der Dispositionen der durch die Abgabeerhebung betroffenen Wirtschaftssubjekte durchaus annähern oder in Erfahrungswerten ausdrücken.

Allerdings muß hier nochmals darauf hingewiesen werden, daß zwar die Bedingungen, nicht aber die Ziele kommunalen Handelns denen des privaten 
Sektors ähnlich sind: Ziel gemeindlicher Aktivität ist nicht die Erwirtschaftung von Gewinnen, sondern die Einnahmeerzielung dient als Mittel anderer Zwecke. Solche Zwecke können sich bei der Einnahmebeschaffung als weitere Restriktionen der Hebesatz- und Entgeltpolitik erweisen, die von den externen Restriktionen jedoch zu unterscheiden sind.

Wo die selbst gesetzten Grenzen liegen sollen, ist von den Gemeinden in den jeweiligen Fällen zu entscheiden. Damit wird aber nicht das "Einnahmepotential" festgelegt, sondern nur dessen Ausschöpfung; diese Grenzen drükken aus, welche Einnahmepolitik eine Gemeinde betreiben will, nicht wieviele Einnahmen sie erzielen könnte, wenn sie wollte.

\section{Determinanten des kommunalen Einnahmepotentials bei variablem Leistungsangebot}

I. Bemessungsgrundlagenpolitik bei verschiedenen gemeindlichen Einnahmearten und ihre Restriktionen

In Abschnitt B wurde das Einnahmepotential unter der Bedingung eines konstanten Leistungsangebots betrachtet, deshalb standen jene Einnahmearten im Zentrum der Analyse, bei denen die Gemeinden allein über die Steuersatzund Preisgestaltung bei entgeltfähigen Leistungen Einfluß auf die Höhe der individuellen Zahlungen nehmen können. Für die übrigen Einnahmequellen liegt das Einnahmepotential unter der genannten Bedingung fest und war nachträglich nur zu erfassen und hinzuzurechnen.

Die weitergehende Fragestellung des Abschnitts C lautet nun, mittels welcher Maßnahmen und bis zu welchen Grenzen eine Ausdehnung des Gesamtbudgets der Gemeinden, also der Einnahmesteigerung mit Hilfe einer entsprechenden Ausgaben- bzw. Leistungspolitik möglich ist. Damit werden jene Einnahmearten, die unter den Bedingungen eines konstanten Leistungsangebots als festgelegt aus der Analyse ausgeklammert wurden, ebenfalls einbezogen; es ist zu untersuchen, wie eine über die Preisvariation hinausgehende Bemessungsgrundlagenpolitik gestaltet werden kann und an welche Grenzen sie gegebenenfalls stoßen wird. 
Diese umfassendere Fragestellung wird der praktischen gemeindlichen Finanzpolitik insofern eher gerecht als die isolierte Sichtweise des Abschnitts B, weil zusätzliche Einnahmen vor allem zur Finanzierung zusätzlicher Angebote dienen. Die qualitativen Möglichkeiten der Angebotsausweitung und der über Attraktivitätspolitik angelegten Einnahme-, bzw. Bemessungsgrundlagenpolitik werden letztlich jedoch wiederum durch die aktuelle Finanzierungskapazität begrenzt.

Über die bisher untersuchte kommunale "Preispolitik" hinaus wird Budgetmaximierung hauptsächlich im Wege einer breiter angelegten "Bemessungsgrundlagenpolitik" betrieben. Nach der Analyse in B läßt sich jetzt zeigen, daß die dort im Zentrum stehende Variation von Steuer- und Entgeltsätzen einen Spezialfall umfassender Bemessungsgrundlagenpolitik darstellt: In dem Maße, wie mit der "Preisvariation" Ausweichreaktionen einsetzen, verändern sich die Bemessungsgrundlagen der jeweiligen kommunalen Einnahmequelle.

\section{Abbildung 11: Wirkungen gemeindlicher Bemessungsgrundlagenpolitik} über Steuersatzvariation und über andere Parameter

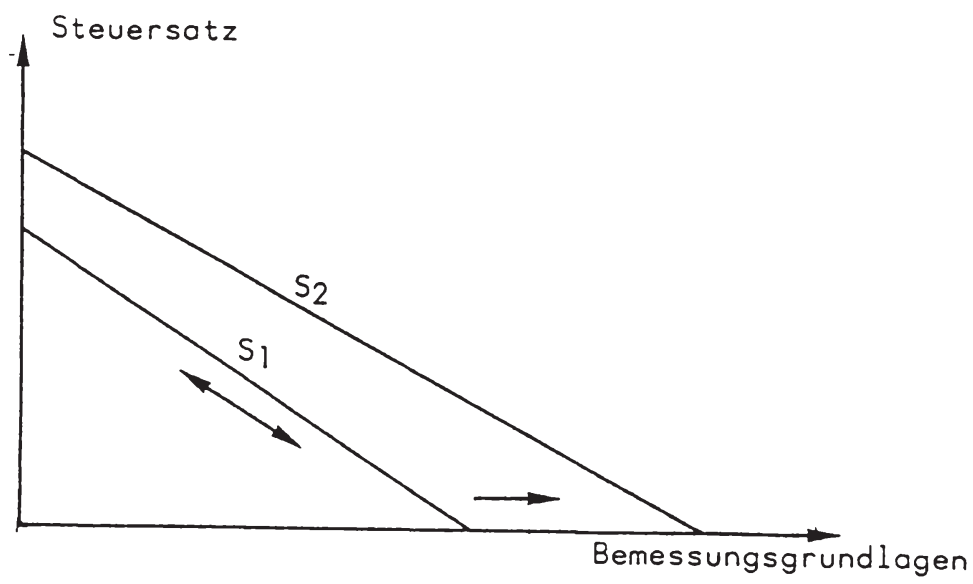

In Abbildung 11 kann dieser Vorgang für den Fall der Besteuerung als Bewegung auf der Kurve $S_{1}$ dargestellt werden. Weitergehende Möglichkeiten 
der Einnahmesteigerung bestehen jedoch in einer anders gearteten Beeinflussung der Einnahmequellen, insbesondere in ihrer Verbreiterung über eine konkurrenzwirksame Ausgabenpolitik. Diese Strategie entspricht einer Verschiebung der Steuerzahlungs-Funktion von $\mathrm{S}_{1}$ nach $\mathrm{S}_{2}$, wobei gegebenenfalls außer verbreiterten Bemessungsgrundlagen auch erweiterte Steuersatzspielräume geschaffen werden können.

Da eine solche umfassendere Bemessungsgrundlagenpolitik oder allgemeiner "Determinantenpolitik" nicht an Preisvariationen gebunden ist, kann sie also auch nicht nur an den mit einem Hebesatzrecht ausgestatteten Gemeindesteuern und an Entgelten, sondern prinzipiell an allen gemeindlichen Einnahmearten ansetzen. Die anschließende Betrachtung muß deshalb alle kommunalen Einnahmequellen auf Möglichkeiten und Bedingungen von Einnahmesteigerungen überprüfen.

Übersicht 4 listet die wichtigsten kommunalen Einnahmequellen noch einmal auf und beschreibt die Komponenten, an denen gemeindliche Einnahmepolitik ansetzen kann.

Hier zeigt sich, daß letztlich alle kommunalen Einnahmen - wenn auch größtenteils nur langfristig - beeinflußbar sind. Dabei kann bei der Mehrzahl der Einnahmequellen auf verschiedene Mengen und Wertkomponenten einzuwirken versucht werden. Wird etwa eine Verbreiterung der Bemessungsgrundlagen der Gewerbesteuer angestrebt, so kann sich die Kommunalpolitik sowohl auf die Zahl, Größe und Produktionsstruktur der angesiedelten bzw. der anzuwerbenden Unternehmen richten als auch auf deren Ertragskraft. Sollen zusätzliche Zweckzuweisungen beansprucht werden, so wäre bei der Zahl und dem Umfang der zuweisungsfähigen Projekte einerseits und der Zuweisungshöhe - oder umgekehrt formuliert - bei der Eigenbeteiligung der Kommunen anzusetzen. Letztere kann allerdings kaum direkt, sondern nur indirekt über die Struktur der verwirklichten Projekte, allenfalls über die Interessenvertretung durch kommunale Spitzenverbände auf Landesebene beeinflußt werden. Ähnliches gilt für die Entgelte, bei denen der Kostendekkungsgrad bzw. der erzielbare Gewinn durch die in Abschnitt B beschriebenen Grenzen limitiert wird; eine Ausweitung des Entgeltpotentials erfordert daher eine Ausweitung oder eine Umstrukturierung des Angebots hin zu Leistungen mit höherem Kostendeckungsgrad. 
Die Verschuldungsmöglichkeiten nehmen insofern eine Sonderstellung ein, als ihre Determinanten von der Ausgabenpolitik sowie von den übrigen Einnahmemöglichkeiten und deren Ausschöpfung festgelegt werden. Eine Ausweitung des Verschuldungspotentials kann deshalb nur durch vorgelagerte Maßnahmen zur Umstrukturierung des Budgets oder zur Verbesserung der finanziellen Leistungsfähigkeit bei anderen Quellen erfolgen.

\section{Ubersicht 4:}

\section{Einzelkomponenten der wichtigsten kommunalen \\ Einnahmearten als Ansatzpunkt expansiver Einnahmepolitik}

Einnahmeart

Steuern
Determinanten potentieller Einnahmen
Ertrag und Kapital der ansässigen Unternehmen, Steuersatz

Einheitswerte landwirtschaftlicher Betriebseinheiten, Steuersatz

Einheitswerte von Grund und Gebäuden, Steuersatz

Einkommen der ansässigen Bevölkerung

Entgelte

Gebühren

Beiträge

Erwerbseinkünfte
Deckung der Kosten angebotener Leistungen

Deckung der Erstellungskosten von Einrichtungen

Preise der abgegebenen Leistungen

Zuweisungen

Schlüsselzu-

Differenz von Steuerkraft weisungen und Finanzbedarf, der Einwohnerzahl, Nebenansätze 
Zweckzuweisungen

Schuldaufnahme

Vermögensveräußerungen
Zuschuß zur Finanzierung zuweisungsfähiger Ausgaben

Summe der Investitionen, InvestitionsförderungsmaBnahmen und Umschuldungen unter der Bedingung der dauernden Leistungsfähigkeit

Marktwert des veräußerlichen Vermögens

Die genannten Quellen eignen sich jedoch nicht gleichermaßen als Ansatzpunkte zur Ausdehnung des Budgets. Hier ist zunächst zwischen allgemeinen und speziellen Deckungsmitteln zu unterscheiden:

Bei der Mehrzahl der speziellen Deckungsmittel ist zur Finanzierung der jeweils zusätzlichen Ausgaben ein Zuschuß aus allgemeinen Deckungsmitteln erforderlich; die isolierte Ausweitung dieser Einnahmequellen findet deshalb aus gemeindlicher Sicht ihre Grenze in der Fähigkeit, nicht durch spezielle Deckungsmittel ausgleichbare Defizite abzudecken. In der Regel werden diese Einnahmequellen deshalb im Anschluß an eine steuerpolitische Wachstumsstrategie auszudehnen versucht werden.

Eine Ausnahme unter den speziellen Deckungsmitteln bilden die Erwerbseinkünfte. $\mathrm{Da}$ in diesem Bereich Gewinne erwirtschaftet werden können und eine Bezuschussung aus allgemeinen Deckungsmitteln in der Regel nicht erforderlich ist, könnten mit dem vermehrten Angebot erwerbswirtschaftlicher Leistungen zusätzliche Einnahmen ohne fiskalische Restriktionen erzielt werden. Allerdings wird der Umfang solcher privatwirtschaftlichen und rechtlichen Aktivitäten durch den Gesetzgeber begrenzt. Voraussetzung für die Einrichtung eines wirtschaftlichen Unternehmens durch die Kommune ist zunächst die Erfüllung eines "öffentlichen Zwecks".191) Darüber hinaus legen einige Gemeindeordnungen fest, daß jener Zweck "nicht ebenso gut und wirtschaftlich durch einen anderen erfüllt werden kann". ${ }^{192)}$ Backhaus vermutet

191) Vgl. dazu ausführlicher Schmidt-Jortzig, Edzard, Die Zulässigkeit kommunaler wirtschaftlicher Unternehmen in einzelnen; in: Handbuch der kommunalen Wissenschaft und Praxis, Bd. 5, Kommunale Wirtschaft, hrsg. von Günter Püttner, 2., völlig neu bearb. Aufl., Berlin u.a. 1984, S. 50-70, hier S. 56 ff.

192) Scholler, Heinrich und Broß, Siegrid, Grundzüge des Kommunalrechts ..., a.a.O., S. 199. 
jedoch, daß sich in jeder Gemeinde zahllose Gelegenheiten dieser Art finden lassen ${ }^{193)}$; ähnlich wie im privaten Sektor dürfte vor allem die zahlungskräftige Nachfrage die Erschließung zusätzlicher Einnahmequellen begrenzen.

Aber auch die allgemeinen Deckungsmittel sind nicht gleichermaßen als Ansatzpunkt zur Ausdehnung des Budgets geeignet. So können z.B. nur dann höhere Schlüsselzuweisungen in Anspruch genommen werden, wenn entweder ein steigender Bedarf in der Bedarfsmeßzahl oder sinkende Steuerkraft nachgewiesen werden können. Daß dabei nur der Bedarf als Ansatzpunkt der Budgetpolitik in Frage kommt, ist unmittelbar einsichtig. Eine solche Strategie ist jedoch nur in Sonderfällen fiskalisch sinnvoll, etwa dann, wenn ohnehin ansässige Einwohner dazu bewegt werden sollen, ihren Hauptwohnsitz in der Gemeinde anzumelden, damit sie bei der Berechnung des Hauptansatzes bedarfswirksam berücksichtigt werden, oder wenn Tatbestände zuweisungswirksam beeinflußt werden können, die über die Nebenansätze in die Beda fsermittlung eingehen.

Ansonsten sind die Schlüsselzuweisungen - ähnlich wie die Verschuldungsmöglichkeiten - eher als abhängige Einnahmegröße zu betrachten. Sie verändern sich in der Folge einnahmepolitischer, insbesondere auf das Steuerpotential gerichteter Maßnahmen, jedoch anders als die Verschuldungsmöglichkeiten in gegenläufiger Richtung zum Steuerpotential. Ein Teil der steuerpolitischen Erfolge der Gemeinde wird daher durch den Verlust von Schlüsselzuweisungen kompensiert.

Als wichtigste Zielgrößen expansiver kommunaler Budgetpolitik verbleiben damit die Steuern und deren Determinanten, also vor allem der Umfang und die Struktur von Wirtschaft und Bevölkerung im Gemeindegebiet. Ein signifikantes Wachstum der übrigen Einnahmequellen kann in der Regel erst im Zusammenhang mit einer gezielten Gewerbe- und Einwohnerpolitik induziert oder - wie im Fall der speziellen Deckungsmittel - über eine entsprechende Ausgabenpolitik als Instrument eingesetzt werden. Dabei kann die Gemeinde zusätzlich eine Maximierungsstrategie anwenden, indem sie sich darum bemüht, die Ausgabenstruktur so zu gestalten, daß sich möglichst viele Ausgaben über spezielle Deckungsmittel "selbst finanzieren".

193) Backhaus, Jürgen. Offentliche Unternehmen, 2. durchgesehene u. erweiterte Aufl., Frankfurt 1980, S. 168 . 
Die Bestimmung des Einnahmepotentials einer Gemeinde hätte nun die Grenzen aufzuspüren, an die eine solche Politik stößt:

- Dies sind zum ersten Entwicklungsgrenzen, die sowohl durch die gesamtwirtschaftliche Entwicklung und die nationale Bevölkerungsentwicklung, aber auch durch die Position der Gemeinde in der Konkurrenz mit anderen Gemeinden beeinflußt werden.

- Zum zweiten wird die Budgetpolitik durch staatliche Rahmensetzung begrenzt, indem die kommunalen Aktivitäten eingeschränkt werden und die Mittelvergabe eher restriktiv erfolgt.

- Eine weitere Begrenzung ergibt sich aus den angesprochenen Beziehungen zwischen den Einnahmequellen, die bewirken, daß die Budgetmaximierungspolitik letztlich nur an einer Bemessungsgrundlagenpolitik bei den gemeindlichen Steuern ansetzen kann, während auf andere Quellen gerichtete Strategien sehr schnell an fiskalische Grenzen stoßen.

Eine Ermittlung dieser Grenzen für den Einzelfall der Gemeinde ist praktisch nicht durchführbar, sie dürfte darüber hinaus aber auch theoretisch kaum möglich sein, weil sie voraussetzt, daß gesamtgesellschaftliche und einzelwirtschaftliche Entwicklungsmöglichkeiten prognostizierbar und quantifizierbar sind. Dies ist jedoch nicht der Fall. ${ }^{194)}$

Die anschließenden Ausführungen beschränken sich deshalb auf den interkommunalen Vergleich von Entwicklungsperspektiven nach Maßgabe des heute verfügbaren Wissens. Sie sollen vor allem zeigen, daß die Erfolgschancen kommunaler "Budgetausweitungspolitik" sehr unterschiedlich sind und daß die gegenwärtigen Finanzierungsmöglichkeiten eine der Restriktionen einer erfolgversprechenden Strategie darstellen.

Mit der Beschränkung auf die interkommunal vergleichende Perspektive wird die gemeindeindividuelle Sicht nicht etwa verlassen: Wie oben bereits dargestellt, spielt auch für die Einzelgemeinde ihre Stellung im Vergleich und in der Konkurrenz mit anderen Gemeinden eine große Rolle für die Einschätzung des Einnahmepotentials. Aufgegeben wird hier lediglich der Anspruch,

194) Die Problematik der Bestimmung von Entwicklungspotentialen wird eingehender betrachtet bei Biehl, Dieter und Mitarbeitern, Bestimmungsgründe des regionalen Entwicklungspotentials, Kieler Studien Nr. 133, hrsg. von Herbert Giersch, Tübingen 1975. 
das Einnahmepotential langfristig in seiner absoluten Höhe zu bestimmen. Diese eingeschränkte Fragestellung entspricht jedoch immer noch dem gemeindlichen Interesse und der gemeindlichen Sichtweise.

Darüber hinaus soll die umfassendere Analyse noch einmal - zusätzlich zu den in Abschnitt B herausgearbeiteten Ergebnisse - nachweisen, daß die tatsächlichen kommunalen Einnahmemöglichkeiten auch im vor allem übergemeindlich interessierenden Gemeindevergleich durch die Steuerkraftmeßzahlen des kommunalen Finanzausgleichs kaum objektiv abgebildet werden. Orientiert man den Anspruch an die Messung an den längerfristigen Entwicklungsmöglichkeiten und den daraus resultierenden Budgetspielräumen, so müßte die interkommunal "verzerrende" Wirkung der praktizierten Messung als noch gravierender eingeschätzt werden, als dies im Vergleich mit dem in Abschnitt B behandelten Einnahmepotential bei gegebenem Leistungsangebot bereits sichtbar wurde.

Abschnitt II beschäftigt sich zunächst mit den möglichen Strategien der Gewerbe- und Einwohnerpolitik; Abschnitt III fragt daran anschließend nach den Erfolgschancen dieser Strategien in verschiedenen Gemeindetypen.

\section{Strategien zur Ausdehnung des Einnahmepotentials}

\section{a. Anforderungen an eine einwohnerorientierte Entwicklungspolitik}

Eine erfolgreiche Einwohnerpolitik ist für die Gemeinden in verschiedener Hinsicht von Vorteil: Auf der Einnahmeseite schlägt sie sich vor allem in einem Wachstum des Anteils an der Einkommensteuer nieder, ausgabeseitig können mit der Verbesserung der Sozialstruktur Einsparungen etwa in den Ausgaben für soziale Sicherung oder zumindest eine Verschiebung in der Ausgabenstruktur hin zu attraktiveren Aufgaben erreicht werden. Darüber hinaus dient eine gezielte Einwohnerpolitik auch wirtschaftspolitischen Zwekken, indem sie zur qualitativen und quantitativen Bereicherung des Arbeitskräftepotentials beiträgt und gleichzeitig mit der Anziehung zusätzlichen Einkommens die örtliche Nachfrage stärkt.

Daraus folgt, daß sich kommunale Einwohnerpolitik nicht auf die Ansiedlung von Einwohnern schlechthin richtet, sondern daß es um die Anzie- 
hung ganz bestimmter Haushaltstypen, insbesondere von Unternehmerhaushalten und qualifizierten Arbeitskräften geht. ${ }^{195)}$ Deren Ansprüche an den Wohnort entscheiden über die Instrumente, mittels derer eine Gemeinde gezielte Ansiedlungspolitik betreiben kann.

Die Ansprüche der mobilen bzw. mobilitätsbereiten Haushalte lassen sich vereinfachend zusammenfassen als das Streben nach hohem Lohnwert, hohem Wohnwert und hohem Freizeitwert. ${ }^{196)}$ Dies bestätigen eine Reihe von Untersuchungen zum Wanderungsverhalten der privaten Haushalte. ${ }^{197}$

Mit dem Begriff des Lohnwerts wird dabei nicht allein das erzielbare Einkommen in einem bestimmten Arbeitsverhältnis umschrieben, sondern er umfaßt die beruflichen Perspektiven insgesamt, wie etwa Aufstiegsmöglichkeiten innerhalb und außerhalb eines Unternehmens, die Arbeitsmöglichkeiten für die übrigen Familienmitglieder und ähnliches mehr.

Bevölkerungsorientierte Entwicklungspolitik erfordert daher in der Regel eine korrespondierende gemeindliche Arbeitsmarkt- und insbesondere Gewerbepolitik, sofern nicht von externen Effekten erfolgreicher wirtschaftspolitischer Aktivitäten in Nachbargemeinden profitiert werden kann.

Die Ansprüche an den Wohnwert beziehen sich zum einen auf ein attraktives Wohnungsangebot in günstiger Lage - dabei erfolgt die Lagebeurteilung sowohl nach dem engeren Wohnumfeld als auch nach den ökonomischen Entfernungen zu Arbeits-, Einkaufs- und Freizeitorten - zum anderen auf die Möglichkeiten, Wohneigentum zu erwerben. ${ }^{198)}$

Auf diese Anforderungen können die Gemeinden mit einer gezielten Wohnungspolitik eingehen; insbesondere der Stadtsanierung wird dabei nicht

195) Vgl. etwa Tetsch, Friedemann, Raumwirkungen des Finanzsystems in der Bundesrepublik Deutschland, Frankfurt 1978, S. 96 f.

196) Vgl. dazu Lauschmann, Elisabeth, Grundlagen einer Theorie der Regionalpolitik, a.a.O., S. 64.

197) So etwa Färber, Robert und Schneider, Ekkehard, Wanderungsmotivuntersuchung in der Region Oberfranken-Ost und im Landkreis Kronach, in: Tendenzen der Wanderungen und der Bevölkerungs- und Siedlungsentwicklung in Bayern, Veröffentlichungen der ARL, Forschungs- und Sitzungsberichte, Band 136, Hannover 1981, S. 35-66, sowie Prognos AG, Struktur und Motive der Wanderungsbewegungen in der Bundesrepublik Deutschland, hrsg. vom Bundesminister des Innern, Basel 1968, S. 90 und Zimmermann, Horst und Mitarbeiter, Regionale Präferenzen, Schriftenreihe der Gesellschaft für Regionale Strukturentwicklung, Bd. 2, Bonn 1973.

198) Ebenda, S. 201. 
zu unterschätzende Relevanz zugesprochen. ${ }^{199)}$ Wichtigste Voraussetzung für eine erfolgreiche Wohnungs- und Siedlungspolitik scheint jedoch die Bereithaltung und Erschließung von Baulandreserven zu sein ${ }^{200)}$, damit den gestiegenen Flächenansprüchen und den zahlungskräftigen Wohnpräferenzen für Einfamilienhäuser mit Gärten, wie sie vor allem in mittleren und höheren Einkommensschichten bestehen, entsprochen werden kann. Neben dem quantitativen und qualitativen Flächenangebot spielen dabei natürlich auch die Flächenpreise eine entscheidende Rolle. Allerdings haben die Gemeinden hier nur wenig Einflußmöglichkeiten.

Der dritte der genannten Anforderungsbereiche wird mit dem Begriff des "Freizeitwerts" ebenfalls sehr eng eingegrenzt, da sich die hier subsumierten Ansprüche auf sämtliche Bereiche der materiellen und immateriellen Reproduktion beziehen. Sie richten sich zum einen auf die natürlichen Bedingungen, wie etwa Klima, Landschaft und Vegetation, die von den Gemeinden nur unmaßgeblich beeinflußt werden können und insofern als Datum zu betrachten sind, zum anderen auf kommunale und private Angebote zum Konsum und zur Daseinsvorsorge. In der Literatur werden hier vor allem zentralörtliche Güter und Dienste genannt: "Bildungs-, und kulturelle Einrichtungen, Verkehrs-, Verwaltungs-, Kommunikations- und Gesundheitsdienste, gute Einkaufsmöglichkeiten, Restaurationen, Sport- und Parkanlagen, Banken sowie last not least ausreichende ärztliche Versorgung...". ${ }^{201)}$ Von den üblicherweise kommunal angebotenen Leistungen hebt Postlep mit Blick auf die Zielgruppe der qualifizierten Arbeitskräfte noch einmal die Angebote im Bereich der weiterführenden Schulen, der Wissenschaft, Forschung und Kulturpflege sowie des Sports und der Erholung heraus. ${ }^{202)}$ Sofern das Angebot privat erfolgen kann, scheint mit Blick auf die angestrebte Attraktionswirkung eine kommunale Förderung angezeigt.

Mit der Betrachtung der für die Wohnortwahl relevanten Ansprüche konnten eine Reihe von Ansatzpunkten für kommunale Einwohnerpolitik aufgezeigt werden. Allerdings ist zu vermuten, daß mit Ausnahme der Woh-

199) Postlep, Rolf-Dieter, Regionale Effekte höherwertiger Dienstleistungen, a.a.O., S. 95.

200) Vgl. Fischer, Ralf Joachim, Gschwind, Friedemann und Henkel, Dietrich, Siedlungsstrategien und kommunale Einnahmen, Schriftenreihe "Städtebauliche Forschung" des Bundesministers für Raumordnung, Bauwesen und Städtebau, Bonn 1980, S. 34.

201) Lauschmann, Elisabeth, Grundlagen einer Theorie..., a.a.O., S. 65.

202) Postlep, Rolf-Dieter, Regionale Effekte höherwertiger Dienstleistungen, a.a.O., S. 98 f. 
nungs- und Wohnumfeldpolitik, die in besonderem Maße in der kleinräumigen Konkurrenz zwischen Kern und Umland im Ballungsgebiet wirksam wird, die Lenkungseffekte einzelner Maßnahmen ähnlich wie bei der unternehmerischen Standortwahl recht gering eingeschätzt werden müssen.

Da sich die Ansprüche der mobilen und mobilitätsbereiten Haushalte eher auf Leistungsbündel bzw. auf ein bestimmtes, mit dem sozialen Status ansteigendes Ausstattungsniveau beziehen ${ }^{203}$, muß eine entsprechende kommunale Strategie ebenso umfassend und - zumindest im gesamten Funktionalraum - kombiniert mit einer gezielten Gewerbe- und Arbeitsmarktpolitik ansetzen. ${ }^{204)}$

\section{b. Ziele und Ansatzpunkte einer kommunalen Gewerbepolitik}

Ziel kommunaler Gewerbepolitik sind zum einen die wirtschafts- und arbeitsmarktpolitischen Entwicklungen im Gemeindegebiet, zum anderen fiskalische Vorteile. Letztere ergeben sich zunächst aus den erwarteten Gewerbesteuereinnahmen von den direkt geförderten Betrieben, darüber hinaus aber auch aus verflechtungsbedingten Gewerbesteuermehreinnahmen, einem höheren Einkommensteueranteil sowie zusätzlich erzielbaren Entgelten aus der wachstumsbedingten Mehrnachfrage nach entgeltfähigen kommunalen Leistungen. Aus diesen Zielsetzungen können die Merkmale anzusiedelnder oder zu haltender "Optimalbetriebe" abgeleitet werden.

(1) Unter entwicklungspolitischen Aspekten sollte Produktionen des BasisBereichs besondere Aufmerksamkeit gewidmet werden. Diese Produktionen zeichnen sich durch regionsüberschreitende Absatzverflechtungen und im besten Fall durch gemeinde- oder regionsinterne Lieferverflechtungen aus und induzieren auf diese Weise die Entstehung von Folgeproduktionen und -einkommen mit einem besonders hohen Multiplikator-

203) Darauf deuten zumindest die Ergebnisse von Zimmermann hin. Vgl. Zimmermann, Horst und Mitarbeiter, Regionale Präferenzen, a.a.O., S. 231.

204) In neueren Untersuchungen zur kommunalen Wirtschaftspolitik wird deshalb regelmäBig auf die Notwendigkeit eines Rahmenkonzepts verwiesen, welches die Gesamtheit dieser Ansprüche berücksichtigt. Vgl. dazu etwa Göb, Rüdiger, Kommunale Wirtschaftspolitik, in: Archiv für Kommunalwissenschaften, 26. Jahrgang, 1987, Erster Halbjahresband, S. 6687, hier S. 72 f. 
effekt. Produktionen dieser Art finden sich vor allem in der Industrie ${ }^{205)}$, aber auch im tertiären Sektor. $\left.{ }^{206}\right)$

(2) Unter fiskalischen Aspekten sollte die betriebliche Zielgruppe eine hohe "Gewerbesteuerproduktivität"- bezogen auf die Zahl der Arbeitsplätze und/oder auf die notwendige Gewerbefläche - aufweisen. Empirische Untersuchungen zeigen, daß je $\mathrm{m}^{2}$ der in Anspruch genommenen Fläche und je Arbeitsplatz nach Branchenzugehörigkeit und Betriebsgrößen sehr unterschiedliche Gewerbesteuer-Zahlungen für die Gemeinden abfallen. ${ }^{207)}$ Für 1975 lagen, bezogen auf den durchschnittlichen Arbeitsplatz, die Bereiche Bergbau, Energie, Wasserversorgung sowie die Kreditinstitute und Versicherungen an der Spitze, auf die Fläche bezogen waren ebenfalls Kreditinstitute und Versicherungen, mit weitem Abstand der Handel die Spitzenreiter.

(3) Weiterhin ist die Integrationsmöglichkeit des Betriebs unter umweltpolitischen und stadtplanerischen Aspekten von Bedeutung. Zwar mögen auch Betriebe mit hoher Emissionsintensität oder mit wenig ansehnlichen Gebäudeformationen speziell in Gemengelagen hohe Gewerbesteuereinnahmen versprechen, unter entwicklungspolitischen, insbesondere auf die einwohnerorientierte Politik bezogenen Aspekten erscheint ihre Anwerbung jedoch wenig vorteilhaft.

(4) Last not least sollten die umworbenen Betriebe insgesamt niedrige Nettoausgaben im kommunalen Budget verursachen, um "fiskalisch lohnend"208) zu sein. Betriebe, deren Ansiedlung oder Haltung hohe Vorleistungen, insbesondere im Bereich spezieller unternehmensorientierter kommunaler Infrastrukturangebote erfordern, sind insofern als Objekt gemeindlicher Einnahmepolitik weniger geeignet.

205) So zeigte sich in der empirischen Untersuchung von Pohl, "daB der EinfluB der Industrie auf den regionalen Arbeitsmarkt größer ist als sein Beschäftigtenanteil. Dies liegt an ihrer Ausstrahlung auf Teile des Dienstleistungssektors". Pohl, Martha, Wirtschaftsförderung in Großstädten, Teil I: Arbeitsmarkt und Wirtschaftsstruktur, in: Bremer Zeitschrift für Wirtschaftspolitik, Heft 5/1982, S. 5-199, hier S. 39.

206) Dabei handelt es sich vor allem um zentralörtliche Produktionen. Vgl. auch Klemmer, Paul, Ermittlung von Basisdienstleistungsbereichen, hrsg. von der ARL, Beiträge Bd. 38, Hannover 1980.

207) Kirsch, Bernd und Schußmann, Klaus, Kommunale Gewerbesteuerpolitik ..., a.a.O., S. 111.

208) Vgl. dazu Glaser, Gundolf, Lohnt sich die Neuansiedlung von Industrie aus fiskalischer Sicht der Kommunen?, a.a.O. 
Mit den genannten Anforderungen wird das Spektrum der unter fiskalischen Aspekten unmittelbar "ansiedlungs- und förderwürdigen" Betriebe stark eingegrenzt. In der Regel kann daraus jedoch nicht folgen, daß sich kommunale Gewerbepolitik allein auf den beschriebenen Betriebstypus konzentriert: Zum ersten erfüllen auch fiskalisch wenig ergiebige Produktionen wichtige kommunale Zielsetzungen und können der Entwicklung insgesamt durchaus förderlich sein. Zum zweiten bestehen für die Mehrzahl der Kommunen - besonders seitdem die Mobilität der Betriebe zurückgegangen ist - aber nur beschränkte Möglichkeiten zur gezielten Auswahl ansiedlungswilliger Produktionen; es hat sich eher durchgesetzt, jede Gelegenheit zur Gewerbeförderung zu ergreifen und in zunehmendem Maße Bestandspolitik zu betreiben. "Standorte, die durch Abwanderung einheimischer Betriebe ins Defizit geraten, werden diesen Verlust immer weniger durch Neuansiedlungen kompensieren können". ${ }^{209)}$ Die Pflege der ansässigen Produktionen rückt deshalb mehr und mehr ins Zentrum kommunaler Wirtschaftspolitik. ${ }^{210}$ )

Wie in Abschnitt B eingehender besprochen, erfolgt die Produktion und als eine ihrer Funktionen auch die unternehmerische Standortwahl vorwiegend kostenorientiert; kommunale Gewerbepolitik muß insofern Kostenvorteile des Standorts innerhalb des Gemeinderaums anbieten oder zumindest darauf bedacht sein, Kostennachteile zu begrenzen, damit sie nicht zu Abwanderungsfaktoren bzw. zu Aufgabefaktoren werden. Die Standortfaktoren wurden oben bereits genannt; es sind vor allem:

- ein kostengünstiges, ausreichendes Flächenangebot,

- die Bereitstellung qualifizierter - bzw. für manche Branchen wichtiger unqualifizierter Arbeitskräfte,

- die Pflege und qualitative Verbesserung der Infrastrukturausstattung, insbesondere im Verkehrsbereich, aber auch in anderen kommunalen Vorleistungsangeboten sowie im Dienstleistungsbereich,

- $\quad$ eine günstige Lage zu Absatz- und Beschaffungsmärkten und, wenn auch nachrangig, öffentliche Förderung und kostengünstige, d.h. niedrige Ausgestaltung der Steuer- und Abgabesätze.

209) Heuer, Hans, Instrumente kommunaler Gewerbepolitik - Ergebnisse empirischer Erhebungen - Schriften des Deutschen Instituts für Urbanistik, Bd. 73, Stuttgart u.a. 1985, S. 28.

210) So auch Göb, Rüdiger, Kommunale Wirtschaftspolitik, a.a.O., S. 73. 
Diese Faktoren lassen sich von den Gemeinden allerdings nicht gleichermaßen beeinflussen. Wirtschaftsförderung im herkömmlichen Stil setzte deshalb vorwiegend an den Faktoren Fläche und finanzielle Förderung an; die gebräuchlichsten Förderungsarten waren

- die subventionierte Bereitstellung von Baugelände,

- die Gebühren- und Beitragsstundung und

- die besondere Steuervereinbarung mit einzelnen Unternehmen. ${ }^{211)}$

Förderungsmaßnahmen dieser Art werden inzwischen jedoch für zu eng gehalten, weil sie sich zu stark auf die Ansiedlung von Unternehmen, nicht aber auf die Bestandspflege richten. Letztere erfordert vor allem Informationen über die Lage der angesiedelten Betriebe und ihre Entwicklungsmöglichkeiten am alten Standort. Dabei wird wiederum dem Flächenproblem, aber auch den Wirkungen immissionsschutzrechtlicher Auflagen besondere $\mathrm{Be}$ deutung zugemessen. ${ }^{212}$ )

Eine erfolgreiche Entwicklungspolitik hätte jedoch noch umfassender, im besten Falle an allen der genannten Standortfaktoren anzusetzen, um die Voraussetzung für eine diversifizierte Wirtschaftsentwicklung und damit für die Herausbildung eines günstigen Gewerbeklimas mit entsprechenden Anziehungseffekten zu schaffen. Besondere Bedeutung kommt dabei der Kombination von Flächenbevorratung einerseits und Arbeitsmarktpolitik andererseits in Verbindung mit einer sowohl unternehmens- als auch haushaltsorientierten Infrastrukturpolitik zu.

Damit ist eine langfristige Entwicklungsstrategie angesprochen, die vielfältige kommunale Vorleistungen erfordert und deshalb in vielen Gemeinden allein aufgrund finanzieller Restriktionen nicht durchführbar zu sein scheint. Darüber hinaus lassen die gegebenen Bedingungen eine solche Politik in den meisten Gemeinden nicht zu: So kann etwa der verdrängungswirksamen Flächenverknappung in den Kernstädten kaum in einer Weise entgegengewirkt werden, daß sie für flächenintensive Produktionen des sekundären Sektors wieder attraktiv würden; andererseits dürften in ballungsfernen, wenig ver-

211) So Müller, Wolfgang-Hans, Wirtschaftsförderung, in: Handbuch der kommunalen Wissenschaft und Praxis, Bd. 4, Die Fachaufgaben, hrsg. von Günter Püttner, 2., völlig neubearb. Aufl., Berlin u.a. 1983, S. 625-641, hier S. 628.

212) Vgl. Heuer, Hans, Instrumente kommunaler Gewerbepolitik, a.a.O., S. 37, auch Müller, Wolfgang-Hans, Wirtschaftsförderung, a.a.O., S. 634. 
dichteten Gebieten wenig Chancen zum Aufbau großer und differenzierter Arbeitsmärkte bestehen (vgl. dazu Abschnitt B). Neben einigen sehr punktuellen Ansätzen zur Wirtschaftsförderung - wie etwa der Bildung von Gründerund Technologiezentren ${ }^{213)}$ - wird den Gemeinden deshalb die "Nutzung komparativer Standortvorteile" angeraten. ${ }^{214)}$

Da die Standortanforderungen nach Betriebstypen - nach Sektor, Branche, Betriebsgröße usw. - recht unterschiedlich ausfallen, bestünde theoretisch die Möglichkeit einer selektiven Gewerbepolitik (vgl. dazu ebenfalls oben, Abschnitt B). Wie die anschließenden Überlegungen zeigen sollen, sind die Erfolgschancen einer solchen Politik jedoch in jenen Gemeinden, die sowohl unter fiskalischen als auch wirtschafts- und sozialpolitischen Aspekten eine erfolgreiche Entwicklungspolitik am nötigsten hätten, gleichzeitig am geringsten.

Diese grobe Einschätzung ergibt sich zum einen daraus, daß ihre "komparativen Standortvorteile" - sofern sie überhaupt noch wirksam sind - tendenziell jene Produktionen anziehen, die wenig geeignet erscheinen, um einen sich selbst tragenden Wachstumsprozeß zu induzieren. Eine Verbesserung der Standortbedingungen würde den massiven Einsatz finanzieller Ressourcen erfordern, die den genannten Gemeinden aber gerade aufgrund ihrer wirtschaftsstrukturellen Lage und der vergleichsweise niedrigen Finanzkraft in der Regel fehlen; werden trotzdem Erfolge bei der Entwicklungspolitik erzielt, so werden die positiven fiskalischen Wirkungen darüber hinaus durch den Finanzausgleich geschmälert. Daraus folgt die Einschätzung, daß in Gemeinden mit hohen aktuellen Einnahmemöglichkeiten die Bedingungen zur Ausweitung des Potentials und des Budgets tendenziell besser sind als in Gemeinden mit niedrigem aktuellen Potential.

Diese Aussage ist natürlich insofern abzuschwächen, als der Ausgabenbedarf, der den Einnahmemöglichkeiten gegenübersteht, ebenfalls zu berücksichtigen ist: Maßnahmen zur örtlichen Entwicklung und zur Bemessungsgrundlagenpolitik sind desto leichter finanzierbar, je größer der Úberschuß der Einnahmemöglichkeiten über den Ausgabenbedarf - der hier nicht weiter betrachtet wurde - ist. Dies ist auch längerfristig zu beachten, da sich gleich-

213) Heuer, Hans, Instrumente kommunaler Gewerbepolitik, a.a.O., S. 162.

214) Kirsch, Bernd und Schußmann, Klaus, Kommunale Gewerbesteuerpolitik ..., a.a.O., S. 112. 
zeitig mit der Bemessungsgrundlagenpolitik die Ansprüche an kommunale Leistungen ausweiten.

Mit den darzustellenden Bedingungen gemeindlicher Bemessungsgrundlagenpolitik wird außerdem aber auch keine präzise Aussage über die tatsächliche zukünftige Entwicklung verschiedener Gemeinden oder Gemeindetypen angestrebt; selbst unter gleichen Ausgangsbedingungen kann - nicht zuletzt durch eigene gemeindliche Anstrengungen - die Weiterentwicklung mehr oder weniger positiv beeinflußt werden. Allerdings werden die Einflußmöglichkeiten durch die Restriktionen der vorgefundenen Ausgangslage eingeschränkt.

Diese Restriktionen sollen im folgenden Abschnitt nun noch einmal näher betrachtet werden.

III. Möglichkeiten und Beschränkungen expansiver

Bemessungsgrundlagenpolitik im Gemeindevergleich

In Abschnitt B wurden mit Blick auf die Standortwahl vereinfachend Ballungsgebiete und periphere Räume gegenübergestellt, innerhalb der Ballungsgebiete wurde noch einmal zwischen Kernstädten und Umlandgemeinden unterschieden. Für jeden dieser Gemeindetypen konnten besondere Standortvorteile, aber auch -nachteile herausgearbeitet werden:

- Die Kernstädte zeichnen sich durch die Vorteile der Verdichtung sowie durch die Konzentration zentralörtlicher Leistungen und die entsprechende Nachfrage aus, nachteilig machen sich sowohl für die Standortals auch die Wohnortwahl vor allem die Flächenverknappung, das Bodenpreisniveau und inzwischen in verstärktem Maße Umweltprobleme wie Lärm oder Luftbelastung aus.

- Die Umlandgemeinden können als besonderen Vorteil für sich verbuchen, daß die urbanization economies der Verdichtung hier noch genutzt werden können, gleichzeitig aber noch ausreichende Flächen für Industriestandorte und zur Wohnnutzung bereitstehen.

- Wenig verdichtete Regionen wurden durch ein großes Flächenangebot und die Verfügbarkeit billiger, insbesondere weiblicher Arbeitskräfte charakterisiert; dem stehen häufig jedoch die Nachteile mangelnder In- 
frastrukturausstattung, gerade im Bereich zentralörtlicher Leistungen sowie ein Mangel an qualifizierten Arbeitskräften gegenüber.

Kommunale Entwicklungspolitik wird nun durch die Disposition und die Einschätzungen der gesamten Bedingungskonstellationen im privaten Unternehmens- und Haushaltsbereich je nach Gemeindetyp mehr oder weniger stark eingeschränkt. Dies gilt sowohl für exogen als auch für endogen ansetzende Entwicklungsstrategien.

Exogen ansetzende Entwicklungsstrategien richten sich auf die Anziehung von Unternehmen und Haushalten aus anderen Regionen und sind insofern auf deren Mobilität angewiesen. Allerdings ist diese Mobilität seit Mitte der 70er Jahre stark zurückgegangen; auf nennenswerte Zuwanderungen können inzwischen nur noch Gemeinden im Umland der Ballungskerne rechnen. ${ }^{215)}$ Kernstädte und periphere Gemeinden müssen sich deshalb von vornherein auf endogene Entwicklungsmaßnahmen - also auf die Stärkung und Pflege des bereits ansässigen Potentials - konzentrieren.

Aber selbst, wenn - wie in Abschnitt B erwartet wurde - die Wanderungsbewegungen wieder zunehmen, muß bezweifelt werden, daß die peripheren Regionen unter entwicklungspolitischen und damit letztlich auch unter fiskalischen Aspekten davon profitieren können, weil in ihrer Ausgangslage die relevanten Ansprüche nur partiell erfüllt werden können: Expandierende, entwicklungsfähige Unternehmen bevorzugen offensichtlich die Ballungsregionen als Standort; die "komparativen Standortvorteile" der peripheren Regionen haben indessen trotz zusätzlicher staatlicher Förderung eher die schwächeren Wirtschaftsbereiche angezogen. Gee, Keller und Treuner vermuten, daß diese Segregationstendenzen dem Abbau räumlicher Disparitäten entgegenwirken. ${ }^{216)}$ Damit dürfte auch eine weitere Auseinanderentwicklung der Einnahmepotentiale einhergehen.

Die Anziehungskraft der Ballungsräume läßt sich bei den Bevölkerungsbewegungen ebenfalls feststellen. Dafür dürfte vor allem die "Lohnwertorientierung" der Erwerbstätigen verantwortlich sein, deren Ansprüche an den Arbeitsmarkt in Ballungsgebieten besser erfüllt werden. Darüber hinaus ist von

215) So Heuer, Hans, Instrumente kommunaler Gewerbepolitik, a.a.O., S. 29.

216) Gee, Colin, Keller, Ulrike und Treuner, Peter, Infrastrukturelle und wirtschaftsstrukturelle Bestimmungsgründe der industriellen Standortwahl, a.a.O., S. 153. 
Bedeutung, daß mit dem sozialen Status offenbar das Niveau der Erwartungen an die gesamte Ausstattung des gewählten Wohnortes steigt und zunehmend ein "urbaner Lebensstil" präferiert wird ${ }^{217}$, selbst wenn die vorhandenen Angebote letztlich kaum genutzt werden.

Damit sind auch die Möglichkeiten der Arbeitsmarkt- und Bevölkerungspolitik in peripheren Räumen stark eingeschränkt ${ }^{218)}$; Gewerbepolitik und Bevölkerungspolitik werden durch die gleichen Ursachen gehemmt; von Ausnahmefällen - wie etwa Fremdenverkehrs- und Kurorten einmal abgesehen, dürfte hier eine fiskalisch lohnende Bemessungsgrundlagenpolitik in der Regel kaum möglich sein.

Zumindest innerhalb der gut strukturierten, modernen Ballungsräume "alte Industriegebiete" nehmen eine Sonderstellung ein, die hier jedoch nicht intensiver behandelt werden soll - stellen sich die Möglichkeiten extern ansetzender Bemessungsgrundlagenpolitik wegen der intensiven interkommunalen Konkurrenzbeziehungen besser dar. Dabei können zwischen Kernstadt und Umlandgemeinden durchaus komparative Standort- aber auch Wohnortvorteile herausgestellt werden. Ihre Wirksamkeit hat sich in der Vergangenheit in der Umlandwanderung der Bevölkerung in Gemeinden mit größeren Baulandreserven ${ }^{219)}$, in der Umlandwanderung sekundärer Produktionen, andererseits in der Konzentration hochrangiger tertiärer Produktionen in einigen Kernstädten niedergeschlagen.

In der Konkurrenz um Produktion und Einwohner waren bisher die Ballungsrandgemeinden eindeutige Gewinner: Wie in Abschnitt B gezeigt, waren nur hier noch Zuwanderungsgewinne zu verzeichnen. Soweit Produktionsstätten mitsamt ihren Mitarbeitern abgewandert sind, haben die Kernstädte wenige Möglichkeiten, diese Entwicklung umzukehren, weil sie insbesondere die von sekundären Produktionen beanspruchten Flächen nicht oder zumindest nicht zu ebenso günstigen Preisen bieten können wie die Gemeinden im

217) Zimmermann. Horst und Mitarbeiter, Regionale Präferenzen, a.a.O., S. 231.

218) Vgl. auch Fischer, Ralf Joachim, Gschwind, Friedemann und Henckel, Dietrich, Siedlungsstrategien und kommunale Einnahmen, a.a.O., S. 34.

219) Vgl. Rohr, Hans-Gottfried von, Intraregionale Wanderungen in ihrem EinfluB auf die Entwicklung in Verdichtungsräumen - Erklärungsansätze und Trendszenarien - Schriftenreihe "Raumordnung" des Bundesministers für Raumordnung, Bauwesen und Städtebau, Nr. 06029, Bonn 1978, S. 21 ff. 
Umland; weder die Produktion noch die Arbeitskräfte werden zurückwandern.

Die Chancen der Kernstädte, Pendler aus dem Umland, die im Zentrum arbeiten, zurückzugewinnen, sind dagegen größer. Da ein Teil der Abgewanderten die Kernstadt aus Gründen der Wohn- und Wohnumfeld-Situation verlassen hat, bieten sich hier über entsprechende Maßnahmen der Siedlungspolitik Ansatzpunkte zur Trendumkehr.

Anders als die beschriebene konkurrenzorientierte Entwicklungspolitik setzt eine Politik der Nutzung endogener Potentiale an den in der Region bzw. den innerhalb der Gemeindegrenzen bereits vorhandenen, nicht ausgeschöpften Ressourcen an. ${ }^{220)}$

Arbeitsmarktpolitische Strategien müßten dabei vor allem auf die Qualifikation der Arbeitskräfte ausgerichtet sein; die Mobilisierung zusätzlicher nicht-qualifizierter Arbeitskräfte aus der Bevölkerung wäre unter den derzeitigen Bedingungen dagegen wenig sinnvoll. Da der Hauptanteil der bildungspolitischen Aufgaben jedoch nicht bei den Kommunen liegt, bieten sich hier kaum Ansatzpunkte für gemeindliche Aktivitäten. Überdies hätten die Gemeinden mit relativ hohen externen Effekten ihrer Politik zu rechnen; zumindest kann nicht gesichert werden, daß die Arbeitskräfte nach ihrer Weiterqualifikation im Gemeindegebiet oder der Wirtschaftsregion verbleiben. Dieses gemeindliche Risiko ist natürlich um so größer, je unbefriedigender die Arbeitsmarktsituation gemessen an den "lohnwertorientierten" Ansprüchen der Arbeitnehmer ist.

Endogene kommunale Entwicklungsstrategien werden sich deshalb vorwiegend auf den Unternehmensbereich beziehen. Im Unternehmensbereich kann hier zum einen die Bestandspflege intensiviert werden, indem ein Ausbau des Kontakts zwischen Unternehmen und Gemeinde mit dem Ziel vorgenommen wird, Schwierigkeiten schneller zu erkennen und gegebenenfalls abzubauen und Expansionspläne soweit wie möglich zu unterstützen. Die erforderlichen Voraussetzungen bestehen prinzipiell in allen Gemeinden; sofern die erforderlichen Personalkapazitäten in der Gemeinde selbst nicht vorhan-

220) Aus regionalpolitischer Sicht wird diese Politik beschrieben bei Hahne, Ulf, Regionalentwicklung durch Aktivierung intraregionaler Potentiale, Schriften des Instituts für Regionalforschung der Universität Kiel, Bd. 8, hrsg. von Karin Peschel, München 1985. 
den sind, kann auf die Beratungsangebote privater Firmen zurückgegriffen werden. Wie erfolgreich eine solche Politik sein kann, hängt natürlich nicht zuletzt auch von der wirtschaftlichen Situation der zu "pflegenden" Wirtschaft und ihren speziellen Problemen ab; Gemeinden, die mit gesamtwirtschaftlich schrumpfenden Branchen besetzt sind, werden hier von vornherein weniger wirksam eingreifen können.

Unter Entwicklungsgesichtspunkten kommt der Förderung von Neugründungen besondere Bedeutung zu. Als geeignete Maßnahme werden auch in diesem Bereich die gezielte Beratung von Existenzgründungs- und Erweiterungsvorhaben sowie die Schaffung institutionalisierter Einrichtungen der Informationsbeschaffung und -verwertung, wie dies etwa durch "Gründerund Technologiezentren" geleistet werden soll ${ }^{221)}$, genannt.

Aus mehreren Gründen dürften solche kommunalen Strategien ebenfalls in jenen Gemeinden besonders erfolgreich sein, die bereits im Ausgangszeitpunkt relativ gut strukturiert sind:

- Zum ersten liegt der sektorale Schwerpunkt des Ansiedlungspotentials heute im tertiären Sektor ${ }^{222}$ ), der, wie beschrieben, in hohem Maße auf urbanization economies und auf eine ausreichend große kaufkräftige Nachfrage im Einzugsbereich angewiesen ist. Diese Bedingungen können die Gemeinden um so eher bieten, je höher ihr zentralörtlicher Status ist und je höher die erzielten Einkommen im Funktionalraum sind.

- Als zweiter expandierender Wirtschaftszweig, in dem Neugründungen große Erfolgschancen zugesprochen werden, gilt die innovative Technikentwicklung und -verwendung. ${ }^{223)}$ Gleichzeitig wird jedoch vermutet, daß eine darauf ausgerichtete Förderpolitik - selbst von übergeordneten Ebenen - nur an wenigen Standorten wirksam werden kann, an denen bereits eine gewisse Konzentration innovativer technologieorientierter Unternehmen vorhanden ist oder an denen entsprechende Forschungsinstitutionen existieren und sich insofern ein Transfer neuen technischen Wissens anbietet. 224 )

221) Vgl. dazu Heuer, Hans, Instrumente kommunaler Gewerbepolitik, a.a.O., S. 165 ff.

222) So Müller, Wolfgang-Hans, Wirtschaftsförderung, a.a.O., S. 633.

223) Siehe Hahne, Ulf, Regionalentwicklung, a.a.O., S. 185.

224) Ebenda, S. 184. 
Als Fazit der bisher angestellten Überlegungen ist festzuhalten, daß die sozioökonomischen und raumstrukturellen Bedingungen bestimmte Gemeinden bei ihren Anstrengungen, die steuerlichen Bemessungsgrundlagen zu verbreitern, begünstigen, andere eher hemmen. Nimmt man einmal an, daß dabei alle Gemeinden in etwa die gleichen Mittel (je Einwohner oder bezogen auf die Kosten der zu fördernden Projekte) einsetzen würden, so wären die Erfolgschancen ihrer Politik also trotzdem höchst unterschiedlich.

Die Annahme, daß im Gemeindevergleich gleich viele Mittel eingesetzt werden könnten, ist jedoch schon unzutreffend. Vielmehr können gut strukturierte Ballungsraumgemeinden zusätzlich wesentlich höhere Summen einsetzen, um die angestrebten Ziele zu erreichen. Sie verfügen sowohl über wesentlich breitere Bemessungsgrundlagen als auch - wie in Abschnitt B gezeigt über höhere Hebesatzspielräume, überdies kann hier ein höherer Anteil der Einnahmen in attraktivitätssteigernde Ausgabenbereiche geleitet werden, da der Haushalt prozentual weniger durch Pflichtaufgaben belastet wird. ${ }^{225}$ )

Allerdings sind noch weitere Einschränkungen zu berücksichtigen. Aus dem Erfolg wirtschafts- und einnahmeorientierter Bemessungsgrundlagenpolitik ist ein Wachstum des Einnahmepotentials der Gemeinden noch keineswegs garantiert. Gelingt die Erweiterung der Bemessungsgrundlagenbasis trotz der eingeleiteten Maßnahmen in einer Gemeinde nicht, so ist - vor allem aufgrund der Schwächung der "dauerhaften Leistungsfähigkeit" und der daraus folgenden Einschränkung des zukünftigen Verschuldungspotentials - eher mit verminderten Einnahmemöglichkeiten zu rechnen; werden die angestrebten Entwicklungsziele erreicht, so kann trotzdem nicht unbedingt mit gleichermaßen erhöhten Einnahmemöglichkeiten kalkuliert werden. Zuvor sind vor allem die konterkarierenden Wirkungen der Zuweisungspraxis im System des kommunalen Finanzausgleichs einzubeziehen.

Im 2. Kapitel wurde die Ermittlung der Schlüsselzuweisungen genauer beschrieben. Danach werden Schlüsselzuweisungen dann gewährt, wenn die Differenz zwischen Finanzbedarf und Finanzkraft eine gewisse Höhe übersteigt; ihr Umfang bemißt sich nach der Größe der Differenz. Eine erfolgreiche Bemessungsgrundlagenpolitik schmälert nun - bei unterstelltermaßen

225) Ausführlicher dazu Tetsch, Friedemann, Raumwirkungen des Finanzsystems in der Bundesrepublik Deutschland, a.a.O., S. $98 \mathrm{ff}$. 
gleichbleibenden Bedarfen - den Anspruch auf Zuweisungen (ebenso wie sinkende Steuerkraft zu erhöhten Zuweisungen führt). Der Erfolg der eigenen Anstrengungen bleibt nur in den ambudanten Gemeinden - jenen, die bereits im Ausgangszeitpunkt keine Zuweisungen beanspruchen konnten - voll erhalten.

Für die übrigen Gemeinden hängt die fiskalische Nettoergiebigkeit von den Einzelregelungen der Zuweisungspraxis, insbesondere von der Existenz und der Ausgestaltung von Mindest- oder Sockelgarantien ab: Gemeinden, deren Finanzkraft nach dem Empfang regulärer Schlüsselzuweisungen noch einmal bis zu einem bestimmten Prozentsatz des Finanzbedarfs aufgestockt wird ${ }^{226)}$, gewinnen durch die Bemessungsgrundlagenpolitik nur dann, wenn die Steuersatzspielräume größer sind als in der Steuerkraftmessung mit dem dort angewendeten fiktiven Hebesatz unterstellt wird. Der eigentliche Bemessungsgrundlageneffekt wird durch den Wegfall eines Teils der Aufstc kung hingegen fiskalisch weitgehend kompensiert oder in Extremfällen sogar überkompensiert.

Proberechnungen für verschiedene Strategien der Bemessungsgrundlagenpolitik bestätigen diese Ergebnisse weitgehend. So kommen Fischer, Gschwind und Henckel zum Beispiel zu dem Schluß, daß die Einnahmewirkungen "strategiebedingter Verschiebungen der Wohnungsteilmärkte" durch das kommunale Finanzsystem zu mindestens der Hälfte wieder ausgeglichen werden und folgern: "Siedlungspolitische Maßnahmen, die auf die Struktur des Wohnungsmarktes abzielen, sind deshalb nicht geeignet, finanzpolitische "Nebenziele" zu erreichen". 2 27)

Deubel bezieht sich bei seinen Untersuchungen direkt auf den Zusammenhang zwischen Steuerkraft und Einnahmemöglichkeiten und deckt damit weitergehende, auch die Gewerbepolitik umfassende Strategien der Bemessungsgrundlagenpolitik ab. Sein Ergebnis fällt noch prägnanter aus: "Zusammenfassend läßt sich also feststellen, daß eine Erhöhung der Steuerkraft per Saldo nur für die bereits besonders steuerstarken Gemeinden eine weitere Erhöhung der Finanzkraft zur Folge hat. ... entsprechend liegt der Wert von

226) Dies ist z.B. in Nordrhein-Westfalen vorgesehen. Zu den Einzelheiten vgl. Deubel, Ingolf, Der kommunale Finanzausgleich in Nordrhein-Westfalen, a.a.O., S. $135 \mathrm{ff}$.

227) Fischer, Ralf-Joachim, Gschwind, Friedemann und Henckel, Dietrich, Siedlungsstrategien und kommunale Einnahmen, a.a.O., S. 36. 
Wirtschaftsförderungsmaßnahmen im Normalfall auch nicht mehr darin, daß sich die kommunalen Einnahmen erhöhen, sondern ausschließlich darin, daß Arbeitsplätze geschaffen oder erhalten werden. Aus finanzieller Sicht stellen so Wirtschaftsförderungsmaßnahmen gerade in den Gemeinden, wo sie am nötigsten sind, nämlich in den struktur- und steuerschwachen Gemeinden mit einem großen Arbeitsplatzdefizit ein ausgesprochenes Verlustgeschäft dar".228)

Die Probleme finanzschwacher Gemeinden, ihr Einnahmepotential auszuweiten, resultieren damit vor allem aus den objektiven Bedingungen, denen sie sich bei ihren Aktivitäten gegenübersehen. Mitunter wird darauf hingewiesen, daß eine auf wirtschafts- und sozialpolitische wie auf fiskalische Zielsetzungen ausgerichteten Politik eine zuverlässige Analyse der jeweiligen Gegebenheiten $^{229)}$ sowie ein fundiertes Entwicklungskonzept ${ }^{230}$ ) voraussetze, daß beides aber häufig vor allem in kleinen Gemeinden fehle. ${ }^{231}$ )

Nach den oben gewonnenen Ergebnissen ist dazu allerdings anzumerken, $\mathrm{da}$ es in den betreffenden Gemeinden zumindest zum gegenwärtigen Zeitpunkt wenig zu planen und zu konzipieren gibt. Für die Gesamtheit peripherer wirtschaftsschwacher Gemeinden sind die Chancen einer Bemessungsgrundlagenpolitik recht gering, die möglichen fiskalischen "Erfolge" letztlich sogar negativ; die Entwicklung einer gezielten, schlagkräftigen Strategie dürfte darüber hinaus auch in einzelnen dieser Gemeinden und allein mit Blick auf sozioökonomische Zielsetzungen nur wirksam sein, solange sie die einzigen sind, die eine solche Strategie entwerfen. Sobald langfristig angelegte Konzepte zur Nutzung komparativer Standort- und Wohnortvorteile ubiquitär erarbeitet werden, dürfte aus einzelgemeindlicher Sicht ihr Lenkungseffekt, ähnlich wie bei der Bereitstellung bestimmter Infrastruktureinrichtungen, verloren gehen.

Zusammenfassend kann die Einschätzung festgehalten werden, daß die Anstrengungen zur Budgetausweitung in verschiedenen Gemeindetypen in Abhängigkeit von der jeweiligen Ausgangslage höchst unterschiedliche Erfolgsaussichten haben dürften:

228) Deubel, Ingolf, Der kommunale Finanzausgleich in Nordrhein-Westfalen, a.a.O., S. 141.

229) Heuer, Hans, Instrumente kommunaler Gewerbepolitik, a.a.O., S. 33.

230) Bullinger, Dieter, Die Einnahmesituation und Strategien zur Einnahmesteigerung, a.a.O., S. 115 .

231) Heuer, Hans, Instrumente kommunaler Gewerbepolitik, a.a.O., S. 31. 
- Zum ersten wird die auf die Steuern gerichtete Bemessungsgrundlagenpolitik als Grundlage weiterer Strategien zur Ausdehnung des Budgets in jenen Gemeinden am aussichtsreichsten sein, für die bereits in Abschnitt B hohe Steuersatz-Spielräume bei breiter Bemessungsgrundlagen-Basis vermutet wurden. Diese Gemeinden wurden durch eine vergleichsweise günstige Wirtschafts- und Sozialstruktur gekennzeichnet - die sowohl für das höhere Einnahmepotential bei gegebenem Leistungsangebot als auch für das endogene und das exogene Entwicklungspotential entscheidend sind. Die vorteilhaftere fiskalische Ausgangslage verschafft diesen Gemeinden darüber hinaus die Möglichkeit, größere Summen für Entwicklungsmaßnahmen aufzuwenden als andere Gemeinden aufbringen können. ${ }^{232)}$

- Speziell in den reichsten, daß heißt: den ambundanten Gemeinden, entfallen zum zweiten die konterkarierenden Wirkungen des Systems allgemeiner Zuweisungen.

- Zum dritten ist davon auszugehen, daß aus der Verfügbarkeit von allgemeinen Deckungsmitteln, besonders von Steuereinnahmen, ein höheres Verschuldungspotential als auch eine größere Fähigkeit zum Ausbau der Ausgleichshaushalte, also des Potentials an speziellen Deckungsmitteln resultiert, vor allem weil die Zufinanzierung des entgeltfähigen und des zuweisungsfähigen Angebots eher gesichert werden kann.

Aus diesen Ergebnissen leitet sich die These ab, daß die realisierbaren Budgets der Gemeinden noch weiter von den durch die Steuerkraftmeßzahlen des kommunalen Finanzausgleichs abgebildeten Einnahmemöglichkeiten der Gemeinden abweichen, als dies bereits für die Einnahmemöglichkeiten bei konstantem Leistungsangebot konstatiert wurde.

232) Diesen Effekt des geltenden kommunalen Einnahmesystems konstatiert auch Reissert, Bernd, Finanzielle Spielräume für kommunale Beschäftigungspolitik? in: Maier, Hans E. und Wollmann, Hellmut, Hrsg., Lokale Beschäftigungspolitik, Basel-Boston-Stuttgart 1986, S. 35-63, hier S. 39. 
D. Die Ergebnisse der Bestimmung des kommunalen Einnahmepotentials als Maßstab für die Finanzkraftmessung des kommunalen Finanzausgleichs?

Das 3. Kapitel setzte an der Frage an, wieviele Mittel eine Gemeinde einnehmen kann. Dabei sollte unter anderem auch deutlich werden, daß die Steuerkraftmeßzahlen, wie sie in den Bundesländern zum Zwecke des Finanzausgleichs ermittelt werden, die gesuchte Größe der potentiellen Einnahmen kaum "objektiv" wiederzugeben vermögen.

Ausgangspunkt und zugleich Hauptgegenstand des Kapitels waren die Finanzierungsmöglichkeiten der Gemeinden bei jeweils zum Beobachtungszeitpunkt gegebenem Angebot an kommunalen Leistungen. Unter diesen Voraussetzungen sind die potentiellen Erträge aus nur wenigen Quellen nicht eindeutig festgelegt und bedürfen der genaueren, allerdings recht aufwendigen - Bestimmung. Dies sind vor allem die Realsteuern, bei denen der Hebesatzspielraum zu ermitteln ist sowie die potentiellen Entgelteinnahmen.

Für die Hebesatzspielräume wurde deren Abhängigkeit von der Wirtschafts- und Sozialstruktur sowie der Einbindung der Gemeinde in das räumliche Gefüge, die ihre Konkurrenzposition gegenüber anderen Gemeinden im wesentlichen bestimmt, abzuleiten versucht. Danach erscheint es plausibel, wenn für verschiedene Gemeindetypen von sehr unterschiedlichen Hebesatzspielräumen ausgegangen wird. Die Steuerkraftmeßzahlen vernachlässigen dies fast vollständig, sie bilden weder die erzielbaren Steuereinnahmen absolut richtig oder im Gemeindevergleich relativ richtig ab und beschreiben auch keineswegs in allen Gemeinden gleiche Ausschöpfungsgrade des Steuerpotentials. Die Entgeltpotentiale - für die die Struktur des kommunalen Leistungsangebots sowie die jeweiligen Nachfrageelastizitäten und die staatliche Rahmensetzung als Hauptdeterminanten herausgearbeitet wurden - werden in der Steuerkraftmeßzahl völlig vernachlässigt, ebenso wie die übrigen unter der Bedingung des konstanten Leistungsangebots festgelegten Einnahmen.

Mit Blick auf die Verteilung der Schlüsselzuweisungen dürften diese "Auslassungen" wegen der Dominanz der Steuereinnahmen per Saldo tendenziell eine Begünstigung steuerstarker Gemeinden, zumindest aber interkom- 
munal starke Verzerrungen zwischen unterschiedlich strukturierten Gemeinden bewirken.

In einer Erweiterung der Fragestellung wurde das kommunale Einnahmepotential im Sinne einer Budgetausweitung bei variablem Leistungsangebot - allerdings mit wesentlich geringerer Intensität - betrachtet. Hier wurde eine längerfristige Entwicklungsperspektive gewählt und danach gefragt, auf welche Weise und mit welchen Erfolgschancen eine Gemeinde ihre gegebene, in Abschnitt B abgeleitete Finanzierungskapazität über eine gezielte ausgabenorientierte "Bemessungsgrundlagenpolitik" ausweiten könnten. Als Hauptansatzpunkt wurde hier eine auf das Steuerpotential gerichtete Politik herausgestellt, weil alle anderen Einnahmequellen und deren Erträge in der einen oder anderen Form durch die erzielbaren Steuereinnahmen begrenzt werden oder von ihnen abhängen.

Für den vereinfachten Vergleich zwischen (gut strukturierten) Gemeinden im Ballungsgebiet und peripheren Regionen lautete das Ergebnis, daß erstere ihre ohnehin wesentlich höheren, durch die Steuerkraftmeßzahl nicht vollständig abgebildeten Einnahmemöglichkeiten durch eine gezielte endogene und exogene Enwicklungspolitik leichter und erfolgreicher ausbauen können als sozioökonomisch ungünstig strukturierte, steuerschwache Gemeinden. Ist die Steuerkraft als Indikator für die "stationären" Einnahmemöglichkeiten schon kritisch einzuschätzen, so muß sie als Indikator für die längerfristigen Einnahmemöglichkeiten bei einer gesteuerten Weiterentwicklung der Bedingungen, unter denen die Kommune wirtschaftet, als völlig ungeeignet gelten, weil sie bei der Messung der Finanzierungsmöglichkeiten vom Nominalprinzip ausgeht: Eine erzielbare DM (soweit sie erfaßt wird) wird unabhängig von den Verwendungsnotwendigkeiten und vom Erfolg der durchgeführten Maßnahmen überall gleich bewertet, obwohl ihre "Ergiebigkeit" im Sinne ihres Beitrags zur Budgetausweitung im Gemeindevergleich höchst unterschiedlich ausfällt.

Allerdings scheinen die genannten Schwierigkeiten der Potentialmessung kaum lösbar zu sein. Eine exakte Messung der Einnahmepotentiale ist schon aus der gemeindlichen Position heraus nicht möglich, aus staatlicher Sicht stößt sie - allein wegen der Vielzahl der zu berücksichtigenden Gemeinden auf schier unüberwindliche Probleme. Als eine Annäherung an die exakte 
Messung könnte ein typisierendes, nach sozioökonomischen und raumstrukturellen Merkmalen klassifizierendes Meßsystem eingeführt werden, wie es etwa von Schmölders vorgeschlagen wurde. ${ }^{233)}$

Eine solche näherungsweise Bestimmung kommunaler Einnahmepotentiale wäre, ähnlich wie eine umfassende Marktanalyse für den Unternehmenssektor, allerdings immer noch mit erheblichem empirischen Aufwand verbunden, zumal wenn sie aus staatlicher Sicht für alle Gemeinden vorgenommen werden soll. Deshalb erscheint die Frage angemessen, wer, d.h. welche Gebietskörperschaften denn überhaupt ein Interesse an der Ermittlung der oben betrachteten Einnahmepotentiale haben und welchen Zwecken diese Ermittlung dienen könnte.

Das Interesse der Gemeinden an ihren Einnahmemöglichkeiten - kurzfristig und unter Status-quo-Bedingungen wie auch unter der langfristigen Entwicklungsperspektive - liegt auf der Hand und muß nicht ausführlich erläutert werden. Auch wenn die Potentiale nicht vollständig ausgeschöpft werden sollen, weil andere, etwa wirtschafts- und sozialpolitische Ziele im Vordergrund kommunalen Handelns stehen, erhellt die Analyse der potentiellen Möglichkeiten der Einnahmeerzielung die Rahmenbedingungen gemeindlicher Aktivitäten und macht die Wirkungen der praktizierten oder geplanten Einnahmepolitik durchschaubar und leichter kalkulierbar.

Welches Interesse könnten aber übergeordnete Gebietskörperschaften an der Ermittlung der gemeindlichen Einnahmepotentiale haben? Hier kommt es vor allem auf die Zwecke der Messung an, die sich von den Zwekken gemeindlicher Messungen zum Teil unterscheiden und dementsprechend auf andere Gegenstände als den oben beschriebenen gerichtet sind.

Zunächst kommt der genauen Ermittlung der gemeindlichen Einnahmemöglichkeiten aus staatlicher Sicht dann Bedeutung zu, wenn gemeindliches Handeln erklärt und gegebenenfalls im Sinne staatlicher Aufgabenerfüllung instrumentiert werden soll. Als Beispiele können etwa konjunktur- und wachstumspolitische Strategien herangezogen werden, deren Erfolg ohne zielgerechtes Verhalten der Gemeinden in Frage gestellt ist. In diesem Fall wären die gemeindlichen Einnahmemöglichkeiten als einer der Bestimmungsfaktoren kommunaler Aktivitäten zu ermitteln und, soweit notwendig,

233) Vgl. dazu die Ausführungen im 2. Kapitel. 
zieladäquat zu beeinflussen. Unter der konjunkturpolitischen Zielsetzung wären dabei eher die kurzfristig erzielbaren Einnahmen zu bestimmen, unter wachstumspolitischem Zielbezug wären die langfristigen Entwicklungs- und Einnahmepotentiale der Gemeinden von größerem Interesse.

Weiterhin können regional- und raumordnungspolitische Zielsetzungen eine Messung kommunaler Einnahmemöglichkeiten erforderlich machen, wenn etwa unerwünschte Disparitäten in der Einkommenssituation oder der Versorgung auftreten und diese Disparitäten über die Einschaltung der Kommunalebene und ihrer Haushalte abgebaut werden sollen.

In allen genannten Beispielen geht es vorrangig um eine Erklärung kommunaler Aktivitäten; hier wird die Politik der Gemeinden beobachtet und unter anderem aus ihren gesamten Finanzierungsmöglichkeiten abgeleitet.

Der kommunale Finanzausgleich - und als seine Bestandteile auch die Messung von Finanzkraft und Finanzbedarf - ist hingegen stärker normativ ausgerichtet; hier legen die übergeordneten Gebietskörperschaften ihre Erwartungen an kommunales Handeln fest, die aber nicht notwendig mit den Grenzen kommunaler Aktivität übereinstimmen müssen. Die Konsequenz kann etwa für die Frage, welche Einnahmequellen in die Messung gemeindlicher Einnahmemöglichkeiten einbezogen werden sollen, beispielhaft verdeutlicht werden: Im vertikalen Finanzausgleich wird eine Aufteilung von Aufgaben, Ausgaben und Einnahmen bzw. den entsprechenden Kompetenzen vorgenommen. Danach sollte jede Gebietskörperschaftsebene jene Aufgaben übernehmen, die sie am effizientesten erfüllen kann und dazu ausreichende Finanzierungskompetenzen erhalten. Ist diese Aufteilung - in welcher Form auch immer - vorgenommen worden, so sind für die Kommunalebene der Ausgabenbedarf aus den zugewiesenen Aufgaben und die Finanzierungsmöglichkeiten aus den für die Aufgabenerfüllung vorgesehenen Quellen zu messen und gegebenenfalls auszugleichen.

Darüber hinausgehende Aktivitäten sind unter der ausgleichsorientierten Zielsetzung jedoch anders zu bewerten und auch anders zu messen.

So kann man sich etwa vorstellen, daß den Gemeinden jenseits der für notwendig gehaltenen Bedarfsdeckung und deren Finanzierung ein Gestaltungsspielraum für das Angebot zusätzlich erwünschter Leistungen belassen 
wird. Sofern solche "Zusatzbedarfe" auftreten und die Gemeinde zu ihrer Deckung Finanzierungsquellen erschließen kann, sind diese Bedarfe und Einnahmemöglichkeiten aus staatlicher Sicht jedoch den explizit zugewiesenen Aufgaben und den speziell dafür vorgesehenen Einnahmequellen nicht gleichzusetzen.

Aus gemeindlicher Sicht stellt sich ein solches Problem der Festlegung eines "normierten Bedarfs" hingegen nicht. Natürlich wird jede Gemeinde im eigenen Interesse bemüht sein, den Präferenzen der angesiedelten Unternehmen und Einwohner im Rahmen ihrer Möglichkeiten zu entsprechen; ob diese Präferenzen mit den staatlichen Normen - sofern diese näher konkretisiert sind - übereinstimmen, ist dabei für die Einschätzung ihrer Dringlichkeit oder ihrer Bedeutung zumindest so lange unerheblich, wie die Gemeinden nicht zur Deckung bestimmter normierter Bedarfe verpflichtet werden. Ebenso ist für die Gemeinden weniger wichtig, welche übergeordneten Zielsetzungen letztlich hinter der Konstruktion des Einnahmesystems gestanden haben; sie sind hier nur insofern von Interesse, als dadurch die Grenzen der Finanzierungskapazitäten mitbestimmt werden.

Aus staatlicher Sicht sind diese Zielsetzungen hingegen von entscheidender Bedeutung. Im 4. Kapitel wird deshalb der Gegenstand staatlicher Messungen der "kommunalen Finanzkraft" noch einmal neu zu bestimmen und von dem aus gemeindlicher Sicht zu ermittelnden Einnahmepotential abzugrenzen sein. 
Ulrike Hardt - 978-3-631-75265-4

Downloaded from PubFactory at 01/11/2019 06:31:57AM

via free access 
4. Kapitel: Die Bestimmung der "kommunalen Finanzkraft" aus übergeordneter Sicht - der Gegenstand der Messung in Systemen mit unterschiedlichem föderativen Gehalt

\section{A. Die vertikale Aufgabenverteilung als Ansatzpunkt der Betrachtung}

Im 3. Kapitel wurde abgeleitet, wie das Einnahmepotential aus der Sicht der einzelnen Gemeinde zu bestimmen ist. Würde aus staatlicher Sicht nach dem gleichen Gegenstand gefragt, so wäre die gegenwärtig praktizierte Messung der kommunalen Steuerkraft in der Tat als wenig objektiv zu bezeichnen, vor allem weil sie für die Kommunen bedeutsame Einnahmearten vernachlässigt und interkommunal unterschiedliche Preissetzungsspielräume unberücksichtigt läßt.

Allerdings ist das für die gemeindliche Sicht entwickelte Konzept zur Bestimmung des Einnahmepotentials nicht ohne weiteres auf die staatliche Sicht übertragbar - und dies nicht allein wegen seiner geringen Praktikabilität.

Zweck einer gemeindlichen Bestimmung des Einnahmepotentials ist es, abzuschätzen, was unter den jeweiligen konkreten Bedingungen gemeindlichen Handelns tatsächlich eingenommen werden kann und welche Grenzen eine darüber hinausgehende Steigerung der Einnahmen verhindern.

Die Zwecke einer staatlichen Messung im Rahmen des kommunalen Finanzausgleichs sind hingegen andere:

- Zum ersten kann die Messung dazu dienen, die Funktionstüchtigkeit des Finanzausgleichssystems insgesamt in Hinblick auf die verschiedenen staatlichen Zielsetzungen zu überprüfen. So könnte etwa danach gefragt werden, ob die den Gemeinden zugewiesenen Einnahmequellen, insbesondere Steuern, in allen Gemeinden zusammengenommen eine der Aufgaben- und Ausgabenbelastung angemessene und kontinuierlich ergiebige Einnahmequelle sind. Fällt die Antwort negativ aus, so wäre in 
der Konsequenz eine Anpassung in der Aufgaben-, Ausgaben- oder der Einnahmenzuweisung auf die Gebietskörperschaften vorzunehmen.

- Zum zweiten kann die Messung auf den Gemeindevergleich abstellen. Dieser Ansatzpunkt ist dann von Bedeutung, wenn erwartet wird, daß die gemeindeeigenen Einnahmequellen in bestimmten Gemeinden bzw. Gemeindetypen, gemessen am Ausgabenbedarf, weniger ergiebig sind als in anderen, und wenn aus versorgungs-, verteilungs- oder regionalpolitischen Gründen ein Ausgleich vorgenommen werden soll.

Mit diesen beiden Fragestellungen sind der vertikale und der horizontale Finanzausgleich, wie sie im 2. Kapitel näher betrachtet wurden, beschrieben.

In beiden Fällen geht es letztlich darum zu überprüfen, inwieweit die konkrete Ausgestaltung des föderativen Systems staatliche Zielsetzungen erfüllt.

Diese Zielsetzungen schlagen sich bereits in der jeweiligen besonderen Form des Staatsaufbaus nieder, in der festgelegt ist, welche Stellung die Gemeinden im Gesamtsystem des föderativen Staates einnehmen, welche Funktionen sie übernehmen und welche Zielsetzungen mit ihren Aktivitäten erfüllt werden sollen. Hier werden die kommunalen Handlungsspielräume umrissen, und damit wird auch das "kommunale Einnahmepotential", wie es aus gemeindlicher Sicht zu bestimmen versucht wurde, eingegrenzt.

Darüber hinaus richten sich die staatlichen Zielsetzungen aber auch auf die innerhalb dieses Rahmens mögliche gemeindliche Aufgaben-, Ausgabenund Einnahmenpolitik. Anhand der staatlichen Zielsetzungen wird kommunale Politik bewertet und eine "Optimalpolitik" bzw. eine "Normalpolitik", die jene Zielsetzungen erfüllt, festgelegt. Die für gegebene staatlich gesetzte Rahmenbedingungen formulierte Optimalpolitik drückt sich unter anderem in den Größen "Finanzbedarf" und "Finanzkraft" aus. Beide schließen staatliche Politikzielsetzungen ein und sind insofern als normative Größen zu kennzeichnen. Sie beschreiben jene monetären Summen, die eine Gemeinde zur Aufgabenerfüllung einsetzen und die sie im Rahmen ihrer finanziellen Möglichkeiten einnehmen wird, wenn Aufgabenwahrnehmung, Ausgabentätigkeit und Einnahmengestaltung aus staatlicher Sicht zieladäquat durchgeführt werden. 
Wie stark die normativ bestimmte gemeindliche "Optimalpolitik" inhaltlich konkretisiert werden muß und wie sie im einzelnen konzipiert wird, hängt zunächst von der Gestaltung des föderativen Staatsaufbaus und den dahinter stehenden Zielsetzungen ab. Mit der Zuweisung von Aufgaben-, Ausgabenund Einnahmekompetenzen auf die Gebietskörperschaften werden die Gemeinden - je nach Ausgestaltung des Gesamtsystems - in der einen oder anderen Weise in die Erfüllung dieser Zielsetzungen eingebunden. Dabei werden unterschiedliche Mechanismen, die vom uneingeschränkt wirkenden dezentralen Preismechanismus über verschiedene Mischformen bis zur vollständigen staatlichen Planung kommunaler Aktivitäten reichen können, zur Steuerung der Zielerreichung eingesetzt. Die Funktionsweise des gewählten Mechanismus bestimmt nun darüber, wie die Optimalpolitik bestimmt werden kann und ob und wie die kommunale Finanzkraft und der Finanzbedarf gemessen und gegebenenfalls ausgeglichen werden müssen.

Voraussetzung für eine zieladäquate kommunale Haushaltspolitik ist vor allem ein konsistenter Aufbau des gesamten föderativen Systems und des Finanzausgleichs. Deshalb werden im 4. Kapitel zunächst nur "rationale" Systeme betrachtet, und es wird gefragt, was jeweils unter "zielgerechtem Wirtschaften" zu verstehen ist und welche Konsequenzen sich daraus für den gesuchten Gegenstand der "kommunalen Finanzkraft" ergeben. Die Bestimmung des Ausgabenbedarfs wird dabei - wenn auch nur am Rande - mit untersucht.

$\mathrm{Da}$ die Suche nach einem geeigneten Staatsaufbau und die Zuordnung von Aufgaben, Ausgaben und Einnahmen auf die Gebietskörperschaftsebenen vor allem das Ziel verfolgt, einen hohen Stand der staatlichen Güter- und Leistungsproduktion und die Ausrichtung ihrer Struktur an den Präferenzen der Wirtschaftssubjekte zu erreichen ${ }^{1)}$, ist der Ausgangspunkt der Überlegungen die Aufgabenerfüllung und die Aufgabenverteilung innerhalb des Gesamtstaates; die Zuordnung von Einnahmen bzw. von Einnahmekompetenzen ist dem nachgeordnet und hat sich an den Zielen der Aufgabenverteilung zu orientieren. ${ }^{2)}$

1) Vgl. Zimmermann, Horst, Allgemeine Probleme und Methoden des Finanzausgleichs, a.a.O., S. 11.

2) Ebenda, S. 4, sowie derselbe, Föderalismus und "Einheitlichkeit der Lebensverhältnisse", in: Beiträge zu ökonomischen Problemen des Föderalismus, von Guy Kirsch, Christian Smekal, Horst Zimmermann, hrsg. von Kurt Schmidt, Schriften des Vereins für Sozialpolitik, Neue Folge, Band 166, Berlin 1987, S. 35-70, hier S. 51. 
Ob und auf welche Weise der kommunale Ausgabenbedarf zu messen ist, wird schon mit der Kompetenzverteilung hinsichtlich Aufgabenerfüllung und Ausgabentätigkeit vorbestimmt. So ist die Berechnung des Ausgabenbedarfs aus einem quantitativ und qualitativ festgelegten Leistungsbündel etwa dann nicht angebracht, wenn die Gemeinden völlig autonom und allein nach Maßgabe der Präferenzen über ihre Aktivitäten befinden, sondern nur dann, wenn die gemeindliche Aufgabenerfüllung von übergeordneten Gebietskörperschaftsebenen in irgendeiner Form mitbestimmt und dabei eben dieses Leistungsbündel als Norm zugrunde gelegt wird.

Mit der Entscheidung darüber, was eine "angemessene" Aufgabenerfüllung sein soll und wie weit sie durch die übergeordnete Ebene des Staates qualitativ und quantitativ festgelegt werden soll, werden außerdem aber die Möglichkeiten bei der Gestaltung eines adäquaten Einnahmesystems eingeschränkt. So wäre es beispielsweise völlig unzweckmäßig, der Gemeindeebene einerseits weitgehende Freiheit bei der Aufgabenerfüllung zuzusichern, die Finanzierung der Aufgabentätigkeit aber durch Zweckzuweisungen vorzusehen. Vielmehr entspräche einer selbstverantwortlichen Aufgabenerfüllung auch eine selbstverantwortliche Einnahmebeschaffung durch die Gemeinden.

Sind die Ausgangsentscheidungen über die Zuweisung der Aufgabenund Ausgabenkompetenzen sowie das entsprechende Finanzsystem einmal getroffen, so ist damit auch festgelegt, inwieweit kommunale "Optimalpolitik" zu konkretisieren ist und welche Informationen die Finanzkraftmessung erbringen soll. So muß im Extremfall vollständig selbstverantwortlicher Aktivität davon ausgegangen werden, daß aus staatlicher Sicht jede mögliche kommunale Politik zu billigen ist, weil sie die jeweiligen Präferenzen der angesiedelten Wirtschaftssubjekte widerspiegelt. Eine "Optimalpolitik" kann also insofern gar nicht bestimmt und der Ausgabenbedarf nicht gemessen werden. Mit Blick auf die Finanzkraft interessieren hier nicht die Einschränkungen der kommunalen Einnahmemöglichkeiten durch die - aus staatlicher Perspektive überdies gar nicht ermittelbaren - Präferenzen der Nachfrager, mit denen die einzelnen Gemeinden konfrontiert sind, sondern allein die finanzielle Leistungsfähigkeit der Wirtschaftssubjekte unabhängig von den jeweiligen, örtlich unterschiedlichen Verwendungswünschen. 
Aus den genannten Beispielen wird deutlich, daß mit der staatlicherseits zu ermittelnden kommunalen Finanzkraft ein ganz anderer Gegenstand angesprochen ist als mit dem im 3. Kapitel betrachteten "gemeindlichen Einnahmepotential", wie es für die Gemeinden selbst von Interesse ist.

Das 4. Kapitel soll diesen Gegenstand noch einmal ganz neu und aus staatlicher Sicht für verschiedene föderative Systeme genauer ausleuchten. Erst im 5. Kapitel wird dann nach den Implikationen für die Finanzkraftmessung im kommunalen Finanzausgleich der Bundesrepublik Deutschland gefragt.

\section{B. Ausgabenbedarf und Finanzkraft der Gemeinden in Systemen mit unterschiedlicher vertikaler Kompetenzverteilung bei der Aufgabenerfillung und der Einnahmebeschafiung}

\section{Die Auswahl der betrachteten Fälle}

Zwischen den extremen Polen einer vollkommenen Zentralisierung staatlicher Aktivitäten und einer vollständigen Dezentralisierung öffentlicher Aufgabenerfüllung sind eine Vielzahl von Systemen mit unterschiedlichem föderativen Gehalt und unterschiedlicher Kompetenzverteilung auf die staatlichen Ebenen denkbar. Obwohl diese Mischsysteme wesentlich realitätsnäher als die Extremfälle sind, sollen die Extremfälle hier am Anfang der Betrachtung stehen, weil an ihrem Beispiel einige Grundfragen der Messung von kommunalem Ausgabenbedarf und kommunaler Finanzkraft diskutiert werden können.

Im ersten Fall wird ein völlig zentralisiertes System untersucht, in dem die Gemeinden als ausführende Organe des Zentralstaates fungieren. Gegen die Untersuchung dieses Falles könnte eingewendet werden, daß eine Gemeindeebene als eigenständige Stufe staatlicher Organisation hier gar nicht existiert und folglich auch jede Messung ihres Finanzbedarfs und ihrer Finanzkraft überflüssig sei. Dieser Auffassung kann jedoch - wie in Punkt II zu belegen sein wird - nicht uneingeschränkt beigepflichtet werden. Überdies finden sich in den in der Realität existierenden föderalistischen Systemen - wie in dem der Bundesrepublik Deutschland - recht umfangreiche Aufgabengebiete, die die Gemeinden auf Weisung übergeordneter Gebietskörperschaften 
übernehmen. Die reine Betrachtung weisungsgebundener Aufgaben und ihrer Finanzierung kann deshalb für den Entwurf eines differenzierten, auch für Mischsysteme geeigneten Konzepts der Messung kommunaler Finanzkraft, das auch auf die Bedingungen in der Bundesrepublik Deutschland angewendet werden kann, hilfreich sein.

Demgegenüber liegt das andere Extrem der vollständigen Dezentralisation staatlicher Aufgabenerfüllung außerhalb des hier interessierenden Spektrums interner Organisationsformen des Staates, da keine übergeordnete Ebene existiert, aus deren Sicht die Messung von Finanzbedarf und Finanzkraft vorgenommen werden könnte. Trotzdem soll ein Fall weitgehender gemeindlicher Autonomie bei der Aufgabenwahrnehmung und der Einnahmebeschaffung betrachtet werden, um den Begriff der gemeindlichen Finanzkraft für frei gestaltbare Einnahmebeschaffung zu präzisieren. Deshalb wird davon ausgegangen, daß jene Aufgabenbereiche und Einnahmequellen, bei deren Wahrnehmung bzw. Inanspruchnahme staatliches Eingreifen notwendig werden könnte - etwa aufgrund externer Effekte oder meritorischer Vorstellungen der übergeordneten Ebene - von den übergeordneten Ebenen selbst übernommen werden und den Gemeinden nur der "Restbereich" zur völlig selbständigen Gestaltung überlassen bleibt.

Im Anschluß an die Betrachtung der beschriebenen Extremfälle werden verschiedenen Zwischenformen, insbesondere Mischsysteme mit geteilter Kompetenzzuweisung (mit und ohne Minimumstandard bei der Aufgabenerfüllung) untersucht. Hier wird gefragt, inwieweit die Messung von Finanzbedarf und Einnahmemöglichkeiten aus den für die Extremformen entwickelten Konzepten zusammengesetzt werden kann oder ob dazu qualitativ andersartige Konzepte erforderlich sind.

Anders als die zuerst betrachteten Extremformen lassen zumindest einige der anschließend untersuchten Mischsysteme eine Vielfalt von Finanzierungsformen zu, bei deren Ausschöpfung durch die Gemeinden staatliche Zielsetzungen mehr oder minder explizit begrenzend, aber auch fördernd eingebracht werden können. Ebenso vielfältig wie die möglichen Finanzierungssysteme sind die entsprechenden Konzepte zur Messung der kommunalen Finanzkraft wie auch des Finanzbedarfs. Ihrer Betrachtung in verschiedenen Mischsystemen wird daher relativ breiter Raum gewidmet. 
II. Das Extrem einer vollständig weisungsgebundenen Kommunalebene

In einem normativen Begründungszusammenhang können verschiedene Argumente für die Zentralisierung staatlicher Entscheidungen über Aufgabenerfüllung und Einnahmebeschaffung sprechen. Aus ökonomischer Sicht werden vor allem die unzulängliche Erfüllbarkeit von Stabilisierungs- und Distributionszielen genannt. ${ }^{3)}$ Daneben sind es aber besonders meritorische Vorstellungen über die regionale Versorgung mit öffentlichen Gütern und Leistungen, die eine zentralisierte Entscheidungsfindung nahelegen. Sofern davon ausgegangen werden kann, daß sich die Präferenzen der Wirtschaftssubjekte hinsichtlich des Angebots an öffentlichen Gütern interregional und interpersonell kaum unterscheiden, tritt das Allokationsproblem ohnehin in den Hintergrund. Die Meritorisierung des öffentlichen Angebots durch den Zentralstaat setzt aber darüber hinaus eventuell bestehende Präferenzunterschiede mit Präferenzverzerrungen gleich, die es durch die zentralisierte Entscheidung über öffentliche Aufgaben und Aufgabenerfüllung zu entzerren gilt: Letztlich werden allen Wirtschaftssubjekten die gleichen, öffentlich zu befriedigenden Kollektivbedürfnisse unterstellt.

Dementsprechend sind für die einzelnen regionalen Einheiten - die im Optimalfall dem räumlichen Nutzungsradius einer Leistung entsprechen - gesamtstaatliche Bedarfsnormen festzulegen. Diese Normen zielen nicht auf gleiche Ausgaben (etwa je Einwohner) ab, sondern auf gleiche Bedarfsdekkung.

Die alleinige Entscheidungskompetenz übergeordneter Gebietskörperschaften in bezug auf die Aufgabenerfüllung und Ausgabentätigkeit der Gemeinden erfordert ein Einnahmesystem, in dem die Verantwortlichkeit der Mittelbeschaffung ebenfalls von übergeordneten Ebenen übernommen wird. ${ }^{4}$ )

3) Vgl. etwa Oates, Wallace E., Ein ökonomischer Ansatz zum Föderalismusproblem, aus Oates, Wallace E., Fiscal Federalism, New York 1972, Kap. 1, S. 3 ff, in deutscher Ubersetzung abgedruckt in: Kirsch, Guy, Hrsg., Föderalismus, Stuttgart-New York 1977, S. 15-26, hier S. 15 ff, und Zimmermann, Horst, Föderalismus und "Einheitlichkeit der Lebensverhältnisse", a.a.O., S. 36.

4) In der rechtssystematischen Literatur wird dieser Grundsatz, der prinzipiell auch für das in der Bundesrepublik Deutschland bestehende System Geltung beansprucht, als Konnexitätsprinzip bezeichnet. Es umschreibt das Prinzip "getrennter, mit der Verwaltungskompetenz korrespondierender Finanzverantwortung". Vgl. Rosenschon, Jürgen Ekkehard, Gemeindefinanzsystem und Selbstverwaltungsgarantie, Schriften zur öffentlichen Verwaltung, Bd. 19, Köln u.a. 1980, S. 37 ff. 
Da es bei einer vollständigen Vorgabe der zu deckenden Bedarfe nicht darauf ankommen kann, ob die einzelnen Gemeinden zur Finanzierung der anfallenden Ausgaben selbst in der Lage sind oder nicht, muß dafür Sorge getragen werden, daß die erforderlichen Mittel in allen Gemeinden zur Verfügung stehen. Prinzipiell sind dazu verschiedene Einnahmesysteme geeignet. So könnte etwa versucht werden, sämtliche kommunale Aufgaben und Ausgaben über individuelle oder gruppenmäßige Äquivalenz, also über Gebühren und Beiträge zu finanzieren. Allerdings müßte in diesem Fall entweder ein Zwang zur Inanspruchnahme bei kostendeckenden Entgelten vorgesehen werden oder es müßten gegebenenfalls unvermeidliche Unterdeckungen von übergeordneten Gebietskörperschaften ausgeglichen werden. Allerdings wird mitunter hervorgehoben, daß die notwendigen Mittel sinnvoller über ein Erstattungs- oder Zweckzuweisungssystem bereitgestellt werden sollten, da diese Finanzierungsformen dem Status der Gemeinden als "staatlichen Zweigstellen" am ehesten entsprechen. ${ }^{5)}$

Um den Gemeinden die notwendigen Finanzierungsmöglichkeiten zur Verfügung stellen zu können, muß zunächst ermittelt werden, was eine qualitativ und quantitativ gleiche Bedarfsdeckung in den einzelnen Gemeinden kostet, und zwar auch dann, wenn eine Entgeltfinanzierung vorgesehen ist, damit staatlicherseits die richtige Entgelthöhe festgelegt werden kann. Zur Festsetzung der Kosten sind die jeweiligen regionalen Bedingungen im einzelnen zu untersuchen: die Zahl der Bedarfsfälle, die Kosten der Bedarfsdeckung in Abhängigkeit von bereits bestehenden Bedarfsdeckungsmöglichkeiten und dem regionalen Preisniveau sowie weitere Bedarfs- und Kosteneinflußgrößen.

Würde man den Gemeinden die Kompetenz zusprechen, selbständig die richtigen, und das heißt auch die kostengünstigsten Maßnahmen zur Bedarfsdeckung ergreifen zu können, so wäre die Messung des Ausgabenbedarfs überflüssig. Die Finanzierung könnte in Form von Erstattungen der getätigten Ausgaben durch die übergeordnete Ebene gesichert werden, bzw. den Gemeinden wäre das Recht zuzugestehen, die entsprechenden Entgeltsätze selbständig festzusetzen. Die Messung des Bedarfs entspringt hier also nur einem Kontroll- und Lenkungsbedürfnis gegenüber der untergeordneten Ebene.

5) Vgl. auch Zimmermann, Horst, Probleme und Methoden des kommunalen Finanzausgleichs, a.a.O., S. 38 . 
Theoretische Probleme werden in dieser Konzeption nicht aufgeworfen, allerdings treten wahrscheinlich insofern Umsetzungsschwierigkeiten auf, als die notwendigen Daten zur Kostenberechnung kaum vollständig zur Verfügung stehen dürften.

Erfolgt die Finanzierung des festgestellten Ausgabenbedarfs über Zuweisungen oder Erstattungen, so entfällt von vornherein jede Veranlassung zur Messung der Einnahmemöglichkeiten bzw. der Finanzkraft: Sie entsprechen genau der Summe, die die Gemeinden von übergeordneten Gebietskörperschaften erhalten.

Zu einer Notwendigkeit der Messung kommt es erst dann, wenn trotz der Fremdbestimmung der Ausgabentätigkeit eine Äquivalenz bei der Finanzierung durchgesetzt werden soll. Für eine solche Lösung könnte etwa der Tatbestand sprechen, daß die privaten Aktivitäten in den einzelnen Räumen sehr unterschiedliche kommunale Handlungsbedarfe und Ausgabenniveaus hervorrufen und den Verursachern diese Kosten angelastet werden sollen, um eine Verhaltensänderung herbeizuführen. Als Beispiel können etwa Ballungskosten dienen, sofern sie sich über die vorgegebene kommunale Aufgabenerfüllung in den Ausgaben der Ballungsgemeinden niederschlagen. Aber auch in diesem Fall müßte die kommunale Mittelbeschaffung nach dem Konnexitätsprinzip von übergeordneten Ebenen geregelt werden ${ }^{6}$, etwa indem eine besondere, mit den Kosten der Aufgabenerfüllung in Zusammenhang stehende Steuer erhoben und das Aufkommen den Gemeinden zugewiesen wird, oder indem Entgelte für die potentielle oder die tatsächliche Inanspruchnahme festgesetzt werden, deren Aufkommen ebenfalls den Gemeinden zustehen müßte.

Sofern diese Einnahmen, deren Erfassung unproblematisch ist, da die Abgabesätze übergemeindlich - wenn auch möglicherweise regional differenziert - festzulegen wären, zur Aufgabenerfüllung nicht ausreichen, muß ein zusätzlicher Ausgleich von Finanzbedarf und Einnahmen erfolgen. ?) Aber auch in diesem Fall wäre eine gesonderte Messung des gemeindlichen Einnahmepotentials überflüssig; solange die Höhe der Steuer- und Entgeltsätze nicht

6) Vgl. Fußnote 4 auf S. 255.

7) In der Umkehrung müBte man natürlich fordem, daB eventuelle Uberschüsse an die übergeordnete Ebene zurückgegeben werden. 
regional entscheidbar ist, sind Einnahmen und Einnahmemöglichkeiten bzw. Finanzkraft identisch.

Die vollständige Kompetenzzuweisung an die übergeordneten Gebietskörperschaften bedeutet aber auch, daß der gegebenenfalls notwendige Ausgleich von Ausgabenbedarf und Einnahmen unabhängig von der Wirtschaftskraft oder dem Einkommensniveau der Gemeinde vorgenommen werden muß. Hier handelt es sich also nicht um einen horizontalen Ausgleich von Finanzierungsmöglichkeiten - wenngleich auch dieses System natürlich horizontale Wirkungen hat, die aber nicht Zweck des Ausgleichs sind - sondern allein um die vertikale Abdeckung von Finanzierungserfordernissen, über die auf übergeordneter Ebene entschieden worden ist.

Zusammenfassend ist noch einmal folgendes festzuhalten:

In einem System vollkommen weisungsgebundener Gemeinden besteht aus gemeindlicher Sicht überhaupt keine Veranlassung zur Messung von Ausgabenbedarf oder Finanzkraft. Dagegen ist aus übergeordneter Sicht die Messung des Ausgabenbedarfs zur Umrechnung vorgegebener Bedarfsdeckungsnormen in monetäre Größen erforderlich, um den Gemeinden die entsprechenden Mittel zur Verfügung stellen zu können.

\section{Der Extremfall unbeschränkt autonomer Gemeinden}

Im zweiten Extremfall - der als "Tiebout-Fall" bezeichnet werden könnte werden sowohl hinsichtlich der Aufgabenerfüllung als auch der Einnahmebeschaffung autonome Gemeinden betrachtet. Die Begründung für die dezentrale Entscheidungsfindung wird vor allem aus dem Allokationsziel abgeleitet: Die Abstimmung des öffentlichen Angebots auf regional unterschiedliche Präferenzen erfolgt danach am besten durch einen dezentralen Preismechanismus. $^{8)}$

Allerdings kann vom Wirken dieses Preismechanismus nur unter bestimmten Bedingungen ein optimales Allokationsergebnis erwartet werden.

8) Vgl. Zimmermann, Horst, Probleme und Methoden des Finanzausgleichs, a.a.O., S. $10 \mathrm{f}$. 
Die wichtigsten Bedingungen sind im Tiebout-Model1 ${ }^{\text {9) }}$ formuliert. Sie stellen sicher, daß sich über vollständige Mobilität und Information der Wirtschaftssubjekte Gemeinden in Form von Gruppen mit homogenen Präferenzen herausbilden und das gemeindliche Angebot keine externen Effekte aufweist. Unter diesen idealisierten Bedingungen werden in den einzelnen Gemeinden Leistungen nach Maßgabe der Präferenzen und der Zahlungsbereitschaft der Bürger erbracht, die Finanzierung hätte nach dem Prinzip der "fiskalischen Äquivalenz" ${ }^{10)}$ und nach dem Trennsystem ${ }^{11)}$ zu erfolgen.

Aus übergeordneter Sicht können weder die konkrete Gestalt der Leistungen und der Umfang der Ausgabentätigkeit noch die jeweilige Finanzierungsform bestimmt werden. Qualität und Quantität von Ausgaben und Einnahmen können sich daher im Prinzip von Gemeinde zu Gemeinde stark unterscheiden. Eine Messung kommunaler Ausgabenbedarfe wäre in diesem Fall nicht möglich, da ex ante nicht abschätzbar ist, was sich in den einzelnen Gemeinden konkret als Bedarf - oder besser: als Nachfrage - äußert. Eine Bedarfsberechnung könnte allenfalls nachträglich, d.h. nachdem die Gemeinden Ausgaben für bestimmte Zwecke getätigt haben, erfolgen. Der Bedarfsbegriff beschreibt dann die regionalen Präferenzen, zu deren Befriedigung und Finanzierung die Gemeindebürger bereit und fähig waren.

Demgegenüber ist eine Erfassung der gemeindlichen Finanzierungsmöglichkeiten aus übergeordneter Sicht durchaus angebracht, wenn regionale Disparitäten in der finanziellen Leistungsfähigkeit als Ursache unterschiedlicher Aufgabenwahrnehmung quantifiziert und gegebenenfalls abgemildert oder beseitigt werden sollen. Ein solcher horizontaler "Ausgleich" hätte konsequenterweise nicht in Form von Zuweisungen an die Gemeinden zu erfolgen, sondern als Politik der personalen Einkommensumverteilung, damit die Gestaltungsmöglichkeit der Kommunalhaushalte auch in bezug auf ihren

9) Vgl. Tiebout, Charles M., A Pure Theory of Local Expenditures, in: Journal of Political Economy, October 1956, S. 416-424.

10) Mit diesem Prinzip soll sichergestellt werden, "daß jene, die aus dem öffentlichen Gut einen Nutzen erhalten, auch die sind, die dafür bezahlen." Olson, Mancur Jr., The Principle of "Fiscal Equivalence": The Division of Responsibilities among different Levels of Government, in: The American Economic Review, Vol. 59 (1969), S. 479-487; in deutscher Ubersetzung abgedruckt in: Kirsch, Guy, Hrsg., Föderalismus, Stuttgart-New York 1977, S. 66-76, hier S. 71.

11) So äußern sich auch Brennan, Geoffrey und Buchanan, James M., The Power to Tax, a.a.O., S. 183. 
Umfang voll erhalten bleibt. ${ }^{12)}$ Die Messung der Finanzierungsmöglichkeiten der Gemeinden hätte dementsprechend an der finanziellen Leistungsfähigkeit der im Gemeindegebiet angesiedelten Wirtschaftssubjekte und - mit Blick auf die Grenzen der Besteuerung beim Existenzminimum - an der Einkommensverteilung im Gemeinderaum anzusetzen.

In diesem System kann es also nicht Ziel der Finanzkraftmessung sein zu bestimmen, was eine einzelne Gemeinde zu einem gegebenen Zeitpunkt und unter den konkreten Bedingungen ihrer Ausgabentätigkeit einnehmen kann, da die aktuellen Einnahmemöglichkeiten jeweils an die von den Bürgern durchgesetzte Ausgabentätigkeit gekoppelt sind. ${ }^{13)}$ Vielmehr wäre zu ermitteln, wieviele kommunale Leistungen und die damit verbundenen Ausgaben die Gemeindebürger sich leisten könnten, wenn sie wollten. $\mathrm{Zu}$ messen wäre also das gesamte, für die Aufteilung auf private und kommunale Güter verfügbare Einkommen im Gemeindegebiet.

Anders als aus gemeindlicher Sicht interessiert es aus übergeordneter Sicht nicht, ob die Einnahmemöglichkeiten durch die Präferenzen der Wirtschaftssubjekte begrenzt werden. Wenn von einer Gemeinde tatsächlich nur $10 \%$ des gesamten verfügbaren Einkommens für die Deckung kommunaler Leistungen abgeschöpft werden können, weil die Wirtschaftssubjekte ein höheres Budget nicht finanzieren wollen und ihre Präferenzen notfalls über Abwanderung durchsetzen, so hat die "mikroökonomische" Sicht der Gemeinden diese Grenze des Einnahmepotentials zu berücksichtigen. Aus übergeordneter Sicht und für die Bestimmung der Finanzkraft spielt sie hingegen keine Rolle.

12) Regionale Verteilungspolitik in Form von Zuweisungen an die Gemeinden wäre nur dann "haushaltsneutral", wenn die Gemeinden die empfangenen Mittel über Steuersenkungen oder Transfers an die Bürger weitergäben, sofern diese keine weiteren kommunalen Leistungen wünschen. Vgl. dazu Musgrave, Richard Abel, Musgrave, Peggy B. und Kullmer, Lore, Die öffentlichen Finanzen in Theorie und Praxis, Bd.4, 1. Aufl., Tübingen 1980, S. 147. Im Ergebnis kommt dabei allerdings ebenfalls eine Politik der personalen Verteilung heraus, bei der die Gemeinden praktisch nur als Vermittler auftreten.

13) Vgl. dazu die Ausführungen im 3. Kapitel. 
IV. Grundfragen der Bestimmung von Ausgabenbedarf und Finanzkraft in Systemen mit geteilter Kompetenzzuweisung

Eine Aufteilung von Kompetenzen bei der Aufgabenwahrnehmung, der Ausgabentätigkeit und der Einnahmebeschaffung ist vor allem aus den Unzulänglichkeiten eines dezentral wirkenden Preismechanismus abzuleiten. Zwar sollen dessen allokative Vorteile erhalten und so weit wie möglich genutzt werden, andererseits aber unerwünschte Ergebnisse des Marktprozesses vermieden werden. ${ }^{14)}$

$\mathrm{Zu}$ diesem Zweck können übergeordnete Gebietskörperschaften in den dezentralen Preismechanismus eingreifen und über eine Veränderung der kommunalen Handlungsbedingungen die Ergebnisse hinsichtlich Aufgabenerfüllung, Ausgabentätigkeit und Einnahmenbeschaffung beeinflussen. Diese Eingriffe des Staates müssen nicht unbedingt in einer vollständigen Reglementierung des gesamten kommunalen Handelns oder einzelner gemeindlicher Aktivitäten bestehen, sondern die Regulierung kann auch über gezielte Rahmengesetzgebung und Incentives oder Disincentives erfolgen, die den Gemeinden größere Spielräume belassen.

Welche Formen des Eingriffs in die Aufgabenwahrnehmung und die Ausgabengestaltung einerseits sowie in die Einnahmebeschaffung andererseits im Einzelfall sinnvoll sind, hängt vor allem von den angestrebten Wirkungen ab. Deshalb wird zunächst gefragt, welche Ursachen aus staatlicher Sicht Regulierungen erforderlich erscheinen lassen, in welcher Weise in die Aufgabenund Ausgabenkompetenzen einzugreifen wäre und welche Konsequenzen daraus für die Finanzierung bzw. die Finanzierungskompetenzen abzuleiten sind. Jeweils anschließend wird untersucht, inwieweit die Bestimmung von Finanzbedarf und Finanzkraft durch die jeweiligen Eingriffe berührt wird.

Dabei bewegt sich die Betrachtung hier noch auf relativ hohem Abstraktionsniveau; es sollen nur grundsätzliche Fragen geklärt werden. Eine detaillierte Klärung von wichtigen Einzelproblemen wie etwa der zielgerechten

14) Systeme dieser Art stellen letztlich immer einen KompromiB dar, in dem allokative und nicht allokative - insbesondere verteilungspolitische Zielsetzungen abgestimmt werden. Vgl. Brennan, Geoffrey und Buchanan, James M., The Power to Tax, a.a.O., S. 182 ff. sowie Zimmermann, Horst, Föderalismus und "Einheitlichkeit der Lebensverhältnisse", a.a.O., S. 36 f. und S. 57. 
Ausformulierung einer aus staatlicher Sicht optimalen kommunalen Einnahmepolitik, also der "Normierung", erfolgt dann in Abschnitt C, der sich intensiver mit verschiedenen Systemen geteilter Kompetenz beschäftigt.

a. Externe Effekte als Ursache für Eingriffe des Staates in die kommunale Haushaltspolitik

Optimale Allokation von lokal anzubietenden (steuerfinanzierten) öffentlichen Gütern und Leistungen setzt voraus, daß der Nutzenradius der angebotenen Güter den räumlichen Grenzen der Gemeinde entspricht; nur in diesem Fall treten keine Nutzen-Spillovers auf, das Prinzip der fiskalischen Äquivalenz kann durchgesetzt werden. Sofern die Gruppe der Wirtschaftssubjekte, die simultan über das kommunale Angebot und dessen Finanzierung entscheidet, kleiner ist als die Gruppe der Nutznießer des Angebots, werden die Gesamtnutzen, die ein bestimmtes Angebot stiften würde, unterschätzt, und es kommt in der Folge zu einem Unterangebot an kommunalen Leistungen. ${ }^{15)}$

$\mathrm{Da}$ die Nutzenradien öffentlicher Leistungen von Aufgabenbereich zu Aufgabenbereich unterschiedlich ausfallen, sind externe Effekte unausweichlich, wenn nicht eine Vielzahl jeweils mit nur einer Aufgabe befaßter örtlicher Kollektive entstehen sollen. ${ }^{16)}$

Auf der Ebene der lokalen Gebietskörperschaften ist das Problem der externen Effekte nicht lösbar und macht deshalb den Eingriff übergeordneter Gebietskörperschaften erforderlich, sofern ein allokativ optimales kommunales Angebot durchgesetzt werden soll.

Für die übergeordnete Ebene bieten sich als Instrument ein horizontaler Ausgleich zwischen den durch Spillovers verflochtenen Gemeinden bzw. die

15) Vgl. Oates, Wallace E., Fiscal Federalism. New York u.a. 1972, S. 66 ff.

16) Selbst in diesem Fall ist jedoch vom Auftreten externer Effekte auszugehen, da geographisch bestimmte Kollektive nicht hermetisch abgeschlossen sind und kaum verhindert werden kann, daß sich Entscheidungen innerhalb des Kollektivs über die räumlichen Grenzen hinaus auswirken. Vgl. Tullock, Gordon, Föderalismus: Zum Problem der optimalen Kollektivgröße, in: Kirsch, Guy, Hrsg., Föderalismus, Stuttgart-New York 1977, S. 27-35, hier S. 27. 
zusätzliche Bereitstellung zweckgebundener Mittel (Pigou-Transfers) zur Ausdehnung des als zu gering eingeschätzten Angebots an. ${ }^{17}$

Zweckzuweisungen zum Ausgleich externer Effekte haben jedoch nur unter der - praktisch nicht erfüllbaren - Bedingung keine allokativ verzerrenden Wirkungen, daß die zu internalisierenden Nutzen und Kosten genau ermittelt werden können. ${ }^{18)}$ Damit wird das gleiche Ergebnis erzielt, das sich ergeben hätte, wenn die Gemeinden bei einer fiktiven Übereinstimmung von Zahlern und Nutzern die zusätzlichen Mittel selbst aufgebracht und die zusätzliche Leistung von sich aus angeboten hätten.

Anders als im oben betrachteten Fall der uneingeschränkt autonom wirtschaftenden Gemeinden stellt sich in diesem Fall auch aus übergeordneter Sicht die Notwendigkeit, Finanzbedarf und Finanzkraft oder besser: die Wünsche nach kommunalen Leistungen und die Finanzierungsfähigkeit und -willigkeit der Nachfrager, die von der zusätzlichen Leistung profitieren würden, zu messen und - soll dem Grundsatz der fiskalischen Äquivalenz weiterhin Rechnung getragen werden - die Mittel in der begünstigten Region aufzubringen. Werden staatlicherseits keine eigenen Vorstellungen über das wünschenswerte Angebot eingebracht, so besteht das eigentliche (und wohl kaum lösbare) Meßproblem in der Aufdeckung der Präferenzen und der Finanzierungsbereitschaft innerhalb und außerhalb der jeweiligen Gemeindegrenzen. Wäre es lösbar, so entfiele damit zumindest die ökonomische Begründung für die Einrichtung lokaler Gebietskörperschaften, der Zentralstaat könnte die Aufgaben der Gemeinden regionalisiert übernehmen. Die Messung der Finanzkraft würde dann aus jeweiliger lokaler Sicht als Messung des "Einnahmepotentials", also als Messung der Zahlungswilligkeit und Fähigkeit der Bürger und Unternehmen im Rahmen der bestehenden Gesetze, vorgenommen.

17) Vgl. dazu Breton, Albert, Eine Theorie staatlicher Finanzzuweisungen, in: Kirsch, Guy, Hrsg., Föderalismus. Stuttgart-New York 1977, S. 77-89, sowie Pollak, Helga und VoB, Wolfgang, Die Berücksichtigung von Spillover-Effekten im kommunalen Finanzausgleich, in: Räumliche Aspekte des kommunalen Finanzausgleichs, hrsg. von der ARL, Forschungsund Sitzungsberichte, Band 159, Hannover 1985. S. 133-158, hier S. 136.

18) So auch Smekal, Christian, Transfers zwischen Gebietskörperschaften (TG), in: Probleme des Finanzausgleichs II, von Karl-Heinrich Hansmeyer, Gerhard Seiler und Christian Smekal, hrsg. von Dieter Pohmer, Schriften des Vereins für Socialpolitik, Neue Folge, Band 96/II, Berlin 1980, S. 151-220, hier S. 188. 
Praktisch ist diese Möglichkeit jedoch nicht gegeben, der Staat ist daher gezwungen, eigene Vorstellungen über die zusätzlich zu erbringenden Leistungen und die erwünschten externen Effekte zu entwickeln und danach die Höhe der Zweckzuweisungen zu bemessen. In diesem Fall ist der betreffende Bereich - sowohl der Bedarf als auch seine Finanzierung - gesondert von der autonomen Aktivität der Gemeinden, für die die Finanzkraft allein im Sinne der finanziellen Leistungsfähigkeit zu bestimmen wäre, zu betrachten.

\section{b. Meritorisierung lokaler öffentlicher Angebote als Ursache staatlicher Eingriffe in die kommunale Autonomie}

Werden den Wirtschaftssubjekten staatlicherseits Präferenzverzerrungen unterstellt, so kann die Verantwortlichkeit für das lokale öffentliche Leistungsangebot nicht allein den untergeordneten Gebietskörperschaften überlassen bleiben, selbst wenn keine externen Effekte auftreten. Vielmehr ist von übergeordneten Gebietskörperschaften berichtigend in das kommunale Angebotsverhalten einzugreifen. Dabei sind verschiedene Möglichkeiten staatlicher Regulierung denkbar.

1. Vollständige Zuordnung einzelner Aufgaben-, Ausgaben- und Einnahmekompetenzen zu einer Gebietskörperschaftsebene

Zunächst sei von dem einfachen Fall ausgegangen, daß einige gemeindliche Aufgabenbereiche, in denen Präferenzverzerrungen vermutet werden, zentralstaatlich festgelegt, ihre Ausfüllung den Gemeinden pflichtig und nach Weisung überlassen bleibt und die notwendigen Einnahmen von der übergeordneten Ebene erstattet werden. Darüber hinausgehende Aufgaben sollen die Gemeinden selbständig wahrnehmen und die erforderlichen Einnahmen in eigener Verantwortung beschaffen.

Im geschilderten Fall sind zwei Bereiche kommunaler Aktivität zu unterscheiden, in denen die Entscheidungskompetenzen über Aufgabenwahrnehmung, Ausgabentätigkeit und Einnahmebeschaffung jeweils einer Ebene zugeordnet werden können. Für den pflichtigen Aufgabenbereich liegen alle Kompetenzen bei der übergeordneten Ebene, hier wäre allein die Ermittlung 
des kommunalen Finanzbedarfs im Sinne einer Kostenkalkulation notwendig. Die kommunale Finanzkraft wäre demgegenüber nur mit Blick auf die Fähigkeit zur Finanzierung selbstverantwortlicher Aufgabenwahrnehmung von Interesse; sie wäre als Leistungsfähigkeit unabhängig von der konkreten Ausgabentätigkeit der Gemeinden zu erfassen.

Bei der Ermittlung beider Größen geht es nicht um deren Gegenüberstellung - sie stehen in keinerlei Zusammenhang miteinander - sondern um ihre gesonderte Erfassung und zwei verschiedene, voneinander unabhängige Ausgleichsvorgänge. Der staatlicherseits vorgegebene Finanzbedarf aus pflichtigen Aufgaben ist in einem rein vertikalen Ausgleich anzupassen, wobei die erforderlichen Mittel durchaus in der Region erhoben worden sein können, in die sie dann - etwa als Erstattung - zurückfließen. ${ }^{19)}$ Der Ausgleich kommunaler Leistungsfähigkeit muß hingegen über einen Prozeß $B$ der horizontalen Umverteilung - entweder direkt zwischen Personen bzw. Regionen oder indirekt als vertikaler Ausgleich mit horizontalem Effekt - erfolgen.

Hier wird deutlich, daß eine Messung und Gegenüberstellung von Finanzbedarf und Einnahmemöglichkeiten der Gemeinden nur dann notwendig ist, wenn eine saubere Trennung von Bereichen ausschließlich kommunaler oder ausschließlich übergemeindlicher Kompetenz hinsichtlich der Aufgabenerfüllung und ihrer Finanzierung nicht mehr möglich ist; wenn also die übergeordnete Ebene Mitspracherechte bei der ansonsten selbständigen Aufgaben-, Ausgaben- und Einnahmetätigkeit der Gemeinde hat. Allerdings sind auch hier noch Fälle denkbar, in denen die beschriebenen Konzepte zur Bestimmung von Finanzbedarf und Finanzkraft isoliert angewendet werden können.

Geht man von einem Fall aus, in dem staatlicherseits Mindestgrenzen für die Aufgabenerfüllung festgelegt werden, so wären die anfallenden Ausgaben

19) Eine solche lokale Äquivalenz ist allerdings dann ausgeschlossen, wenn mit der Meritorisierung gleichzeitig Verteilungsziele verfolgt werden, d.h. wenn davon ausgegangen wird, $\mathrm{da} B$ das erwünschte Angebot außer aufgrund von Präferenzverzerrungen auch aufgrund mangelnder Finanzierungsfähigkeit nicht erstellt wird. In diesem Fall - der hinter der Konstruktion des kommunalen Finanzausgleichs in der Bundesrepublik stehen dürfte - ist jedoch eine alleinige Regelung über die personale Einkommensverteilung unmöglich; hier müssen Meritorisierung und interregionale Umverteilung verknüpft werden. 
der Gemeinden entsprechend anteilig zu finanzieren. ${ }^{20)}$ Zumindest theoretisch kann auch hier noch eine klare Zuordnung einzelner Aktivitäten auf die verschiedenen Gebietskörperschaftsebenen vorgenommen und die entsprechenden horizontalen und vertikalen Ausgleichsvorgänge getrennt werden. Dies ist nicht mehr möglich, wenn sich die "Mitsprache" der übergeordneten Gebietskörperschaften über eine indirekte Einflußnahme auf kommunale Aktivitäten mit Hilfe von in den Finanzausgleich eingebauter Anreizinstrumente vollzieht.

2. Geteilte Entscheidungen bei der Aufgaben-, Ausgaben- und Einnahmetätigkeit

Soll den Gemeinden zwar einerseits keine vollständige Handlungsfreiheit bei der Aufgaben- und Ausgabentätigkeit belassen werden, sollen aber andererseits auch keine genauen Vorgaben für die zu entfaltenden Aktivitäten gemacht werden, so bestehen verschiedene Möglichkeiten, die gemeindlichen handlungen in die Erfüllung staatlicher Zielsetzung einzubinden.

Eine erste Möglichkeit bestände in einer gemeinschaftlichen Entscheidung, Durchführung und Finanzierung von Aufgaben und Ausgaben durch Gemeinden und übergeordnete Gebietskörperschaften. In diesem System sind die Handlungen der staatlichen Ebenen Ergebnis von Verhandlungen, die mit oder ohne vereinbarte Rahmensetzungen ablaufen können. Inwieweit hier Finanzbedarf und Finanzkraft der Gemeinden zu bestimmen sind, hängt von der Gestaltung des Verhandlungsgegenstandes im einzelnen ab. Die vielfältigen Möglichkeiten bei der Ausgestaltung von Gemeinschaftsaufgaben sollen hier jedoch nicht weiter betrachtet werden. ${ }^{21)}$

20) Vgl. Zimmermann, Horst, Allgemeine Probleme und Methoden des Finanzausgleichs, a.a.O., S. 38.

21) Überdies werden die verschiedenen Formen von Politikverflechtung im föderativen Staat häufig für wenig leistungsfähig und nicht problemadäquat gehalten: "Politikverflechtung erweist sich lediglich gegenüber den weniger kontroversen politischen Problemen und Aufgaben als eine begrenzt leistungsfähige Modalität der aktiven staatlichen Problemverarbeitung." Scharpf, Fritz W., Reissert, Bernd und Schnabel, Fritz, Politikverflechtung: Theorie und Empirie des kooperativen Föderalismus in der Bundesrepublik, Kronberg 1976, S. 236. Insofern fallen kooperative Lösungen ohnehin nicht unter die hier zu betrachtenden "rationalen" Lösungen der Kompetenzverteilung im föderativen Staat und bleiben deshalb außer acht. 
Eine zweite Variante der übergemeindlichen Beeinflussung kommunaler Aktivitäten besteht in der Vorgabe von Minimumstandards bei der Aufgabenerfüllung und der Übernahme der Finanzierungszuständigkeit für diesen Teil der kommunalen Aufgabenerfüllung. Selbst wenn für alle kommunal erfüllbaren Aufgabenbereiche solche Mindestversorgungswerte festgelegt würden, handelt es sich hier genau genommen noch um einen Fall ungeteilter Kompetenz, die zwar keinen Aufgabenbereich insgesamt einer der staatlichen Ebenen überläßt, jedoch eine klare Zuordnung sowohl von Entscheidung als auch Finanzierung bestimmter Aktivitäten auf die einzelnen Ebenen erlaubt. Der beschriebene Fall wurde daher bereits unter Punkt 1 behandelt.

Eine dritte Möglichkeit der Einflußnahme könnte über den Einsatz indirekt wirksamer Anreize zur Erfüllung staatlicher Zielsetzungen wahrgenommen werden. Dabei spielt die Konstruktion des Finanzsystems eine besondere Rolle als Anreizinstrument.

Die Anforderungen an die Ausgestaltung des Gesamtsystems sind nun komplexer:

- Zunächst sind den Gemeinden jene Aufgabenbereiche zuzuweisen, in denen sie tätig werden sollen. Darüber hinaus ist aber eine "optimale Aufgabenerfüllung" festzulegen und der daraus folgende Ausgabenbedarf zu bestimmen.

- Im nächsten Schritt müssen den Gemeinden geeignete Finanzierungsquellen zugewiesen werden. Diese sollen sich einerseits durch eine gewisse, in Abschnitt $\mathrm{C}$ näher zu betrachtende Flexibilität auszeichnen, da die Gemeinden nicht zur oben festgelegten "optimalen Aufgabenerfüllung" gezwungen, sondern nur angereizt werden sollen. Andererseits sollten die Einnahmequellen so mit der erwünschten Aufgabenerfüllung verknüpft sein, daß von der Finanzierungsseite ein Anreiz zur Verwirklichung der "Optimalpolitik" ausgeht.

- In einem weiteren Schritt muß festgelegt werden, welche Einnahmepolitik aus staatlicher Sicht für angemessen gehalten wird. Daraus läßt sich dann eine normierte Finanzkraft berechnen, die selbst als Anreizinstrument zur Durchsetzung der Optimalpolitik dienen kann. 
- Zuletzt ist festzustellen, ob sich Ausgabenbedarf und Finanzkraft entsprechen oder ob ein Ausgleich durch übergeordnete Ebenen erfolgen muß. Ein solcher Ausgleich wird in der Regel dann als notwendig gelten, wenn die erwünschte Aufgabenerfüllung mit der ermittelten normierten Finanzkraft nicht geleistet werden kann. Allerdings kann man sich auch vorstellen, daß bei einer Überdeckung in einzelnen Gemeinden ein "negativer Ausgleich" in dem Sinne vorgenommen wird, daß Zahlungen der betreffenden Gemeinden an die übergeordnete Ebene fällig werden.

Wie die in den einzelnen Schritten angesprochenen Anforderungen erfüllt werden können, ist damit allerdings noch nicht vollständig festgelegt; hier bestehen - je nach den verfolgten Zielvorstellungen - durchaus verschiedene Ausgestaltungsmöglichkeiten. Ihrer Betrachtung ist der gesamte Abschnitt C gewidmet.

\section{Einzelfragen der Normierung von Finanzbedarf und Finanzkraft in verschiedenen Einnahmesystemen mit geteilten}

Entscheidungskompetenzen

I. Die übergemeindliche Festlegung des normierten Ausgabenbedarfs

\section{a. "Originäre" Bedarfsfestlegungen}

Die Besonderheit der hier zu betrachtenden Bedarfsfestlegung besteht darin, daß auf der einen Seite den Gemeinden bestimmte Ausgabenerfordernisse unterstellt werden, damit sie ihre Funktionen erfüllen können, andererseits aber der Aufgabenbedarf nicht in dem Sinne konkretisiert werden soll, daß den Gemeinden die Erfüllung der einzelnen Bedarfe vorzuschreiben wäre.

Soll den Gemeinden die Entscheidung über die Wahrnehmung und Dekkung von Bedarfen offengehalten werden, so ist eine direkte, auf die individuelle Gemeinde bezogene Umrechnung von Bedarfen in den kommunalen Finanzbedarf im Sinne einer reinen Kostenkalkulation nicht mehr ohne weiteres möglich. Vielmehr ist von einem veränderten Bedarfsbegriff auszugehen, der nicht wie in den bisher beschriebenen Fällen eine unverzichtbare und deshalb pflichtig auferlegte Summe von Leistungen und deren Kosten umschreibt, sondern einen "gesellschaftlich durchschnittlichen" Bedarf nach einem Korb 
kommunaler Leistungen. Dieser Bedarfsbegriff legt die Gemeinden nicht auf bestimmte in Art und Umfang festgelegte Leistungen fest, sondern faßt einen Korb von Leistungen zusammen ${ }^{22}$, die eine Gemeinde nach staatlichen Vorstellungen zu erbringen hätte, wenn sie alle die ihr zugedachten Funktionen erfüllt. Ein solcher Leistungskorb kann auf ähnliche Weise zusammengestellt werden wie die Warenkörbe, mit deren Hilfe die Bedarfe einzelner Personen ermittelt werden und ebenfalls die Verwendung der empfangenen Mittel nicht direkt vorgeben: "Ein Warenkorb als Bedarfsmengenschema ist nichts anderes als der Versuch, die Vielfalt individuellen Tagesbedarfs idealtypisch und damit vereinfachend zu gruppieren, die Gruppierungen wiederum mit beschriebenen Waren und Dienstleistungen zu belegen. Bewertet man den Inhalt des Warenkorbs mit Preisen, so ergibt sich die Basis für die Festsetzung der monetären Bedarfsdeckungspauschale...." ${ }^{23)}$ Dabei erfüllt der Warenkorb allein die Funktion des Maßstabs, nicht aber der "Gebrauchsanweisung". ${ }^{24)}$

Ein Normalkorb kommunaler Leistungen läßt sich nun nicht einfach aus den jeweils gegebenen Bedingungen ableiten, sondern kann nur aus den übergemeindlichen Zielsetzungen heraus konkretisiert werden. Zwar bestehen gewisse raum- und zeitabhängige "Funktionserfordernisse", die sich jedoch nicht scharf abgrenzen lassen. "Was "funktionsnotwendig" ist oder nicht, wird damit nicht eindeutig bestimmbar: es gibt Situationen, in denen sich beides die Waage halten kann. Ihre konkrete Entscheidung läßt sich nicht wissenschaftlich sozialtechnologisch begründen. Daraus ergibt sich ihr fundamental politischer Charakter". 25)

Je nach Ausprägung und Gewichtung der übergeordneten Zielsetzungen wird daher auch der Normalbedarf in den einzelnen Gemeinden unterschiedlich festzulegen sein. ${ }^{26)}$

22) Verschiedenen Konzepten originärer Bedarfsmessung liegt implizit ein solcher Leistungskorb zugrunde. Vgl. z.B. Hanusch, Horst, Messung des kommunalen Finanzbedarfs, a.a.O., sowie Zimmermann, Horst, Hardt, Ulrike und Postlep, Rolf-Dieter, Bestimmungsgründe der kommunalen Finanzsituation, a.a.O., S. 377 ff.

23) Galperin, Peter, Sozialhilfe und Bedarfsprinzip - Zum Streit um die Konkretisierung der Bedarfsdeckung - in: Leibfried, Stephan und Tennstedt, Florian, Politik und Armut und die Spaltung des Sozialstaats, Frankfurt 1985, S. 153-168, hier S. 156.

24) Ebenda, S. 157.

25) Matzner. Egon, Typologie der "öffentlichen Aufgaben", in: derselbe, Hrsg., Offentliche Aufgaben und Finanzausgleich, Wien 1977, S. 10-12, hier S. 11.

26) Vgl. Seiler, Gerhard. Ziele und Mittel des kommunalen Finanzausgleichs, a.a.O., S. 27 ff. 
Steht aus staatlicher Sicht eine möglichst gleichmäßige Versorgung mit kommunalen Leistungen im Vordergrund, so wäre von einem Standardbedarf jedes einzelnen Wirtschaftssubjektes auszugehen, der je nach den ökonomischen, sozialen oder anderen Bedingungen auf die eine oder andere Weise befriedigt werden müßte. Gleichmäßige Versorgungssituation ist dabei nicht unbedingt gleichbedeutend mit einheitlichen kommunalen Aktivitäten. So könnte etwa der Warenkorb nach zentralörtlichen Rängen der Gemeinden differenziert werden.

Anders sähe der Waren- bzw. Leistungskorb aus, wenn aus staatlicher Sicht die gemeindlichen Aktivitäten in hohem Maße an wachstumspolitischen Zielsetzungen ausgerichtet werden sollen. In diesem Fall könnte gefragt werden, welche Gemeinden überhaupt zur Förderung des gesamtwirtschaftlichen Wachstums in der Lage wären, wenn sie ihr Leistungsangebot ausdehnen und bestimmte Zusatzleistungen anbieten würden. Wo solche Zusatzleistungen zieladäquate Wachstumswirkungen erwarten lassen, wären sie deshalb in den Normalkorb aufzunehmen und würden sich dementsprechend in einem höheren, an staatlichen Zielsetzungen orientierten Normal-Finanzbedarf niederschlagen.

Weitere für die Gestaltung des bedarfsrelevanten Leistungskorbes wichtige Zielsetzungen könnten raumordnungspolitischer ${ }^{27}$ oder umweltpolitischer Art sein oder andere politisch angestrebte Wirkungen betreffen. Hier soll jedoch nicht weiter untersucht werden, in welcher Form sie in die Bestimmung des kommunalen Finanzbedarfs integriert werden können.

Festzuhalten bleibt zunächst nur, daß vielfältige Zielsetzungen in die Bestimmung des Bedarfs aufgenommen werden können, ohne daß damit gegen das Kriterium der Objektivität der Bestimmung verstoßen würde. Da der zu messende Bedarf nicht unmittelbar aus den in den einzelnen Gemeinden herrschenden Bedingungen resultiert, sondern erst aus der Beurteilung dieser Bedingungen nach Maßgabe der staatlichen Ziele, ist die Konzeption eines "Normalbedarfs" kommunaler Leistungen und des daraus ableitbaren Finanz-

27) Zur Feststellung eines regionalpolitisch ausgerichteten Normalbedarfs vgl. auch Rottmann, Joseph, Regionalpolitik und kommunaler Finanzausgleich, Beiträge zum Siedlungs- und Wohnungswesen und zur Raumplanung, Bd. 83, hrsg. von Werner Emst, Werner Happe und Rainer Thoss, Münster 1982, S. $175 \mathrm{ff}$. 
bedarfs eine Angelegenheit der politischen Entscheidung und kann insofern sehr unterschiedliche Gestalt annehmen. ${ }^{28)}$

In jedem Fall wird mit der Normierung jedoch eine Abkoppelung der staatlichen von der gemeindeindividuellen Sicht vorgenommen: Aus staatlicher Sicht ist nicht unbedingt ausschlaggebend, welches Angebot eine Gemeinde zu einem gegebenen Zeitpunkt realisiert oder welches Angebot sie bei einem Budget-Maximierungs-Verhalten realisieren könnte, sondern ein fiktives, für zieladäquat gehaltenes Leistungsbündel wird zur Grundlage des Finanzausgleichs gemacht. Damit relativiert sich gegenüber der gemeindlichen Sicht prinzipiell auch die Frage nach der zeitlichen Perspektive: Der Basis eines fiktiven Angebots entspricht die Abstraktion von Anpassungsfristen an Veränderungen kommunaler Politik; es wären alle fiskalisch relevanten Anpassungen an die gemeindliche Ausgaben- und Einnahmenentscheidungen bei "normaler" Politik in die Bestimmung von Finanzbedarf und Finanzkraft einzubeziehen.

\section{b. Die Problematik einer pauschalierten Bedarfsfestlegung}

Aus den zielgerecht zusammengesetzten Warenkörben lassen sich natürlich auf den Einwohner bezogene monetäre Bedarfsrichtwerte ermitteln, die wiederum nach Gemeindetypen - etwa unterschieden nach Gemeindegröße, Zentralität, Wirtschafts- und Bevölkerungsstruktur oder anderen für wichtig gehaltenen Merkmalen - differenziert werden können. Solange solche ProKopf-Werte aus der "originären", d.h. aus einer für zieladäquat gehaltenen Aufgabenerfüllung abgeleitet werden, ist dagegen kaum etwas einzuwenden.

Solche Pro-Kopf-Pauschalen sind allerdings dann problematisch, wenn monetäre Beträge ohne den direkten Rückgriff auf die Kosten bestimmter Aufgabenbereiche festgelegt werden. Sie bilden weder den Ausgabenbedarf aus gemeindlicher Sicht noch aus staatlicher Sicht ab und können letztlich nur als ein Instrument zur mehr oder weniger willkürlichen horizontalen Verteilung von staatlichen Zuschüssen dienen. Da sie ohne eine konkrete Grundlage

28) Zu einem ähnlichen Ergebnis kommt Hanusch, wenn er jedes Verfahren der Bedarfsmessung als letztlich willkürlich charakterisiert. Vgl. Hanusch, Horst, Messung des kommunalen Finanzbedarfs, a.a.O., S. 70. 
festgesetzt werden, lassen sie sich beliebig für ad hoc aufgestellte Verteilungszwecke einsetzen. Daß diese Verteilung unter wirtschafts- und verteilungspolitischen Zielen angreifbar ist, kann dann nicht verwundern.

Eine andere Möglichkeit der pauschalierten Bedarfsfestlegung bestände in der Orientierung an den zum Zeitpunkt der Betrachtung realisierten Ausgaben bzw. an den im Durchschnitt eines Gemeindetyps realisierten Ausgaben. Damit wird letztlich zum Ausdruck gebracht, daß die gemeindlichen Aktivitäten in ihrer beobachteten Quantität und Qualität zumindest im Durchschnitt für "normal" oder für erwünscht gehalten werden. ${ }^{29)}$ Allerdings ist dieses Verfahren durchaus angreifbar. Durchschnittswerte für die kommunalen Gesamtausgaben setzen sich aus höchst unterschiedlichen Einzelausgaben zusammen, deren Höhe im Gemeindevergleich zudem stark streut. Als eine der Hauptdeterminanten des Ausgabenumfangs sind dabei die Finanzierungsmöglichkeiten der Gemeinden einschließlich der im Finanzausgleich ver'eilten Schlüsselzuweisungen anzusehen. Mit einer staatlicherseits in den einzelnen Gemeinden für richtig bzw. angemessen gehaltenen Politik hat diese Größe jedoch wenig zu tun. Sollte sich bei einem empirischen Vergleich von durchschnittlichen Ausgaben und einem originär bestimmten Normalbedarf eine Übereinstimmung finden, so wäre sie vermutlich rein zufällig.

Die Bedarfsmeßzahlen des in der Bundesrepublik Deutschland praktizierten kommunalen Finanzausgleichs werden ähnlich unfundiert festgelegt. Ohnehin ist auf ihrer Basis nur ein horizontaler Ausgleich von Finanzierungsmitteln möglich: Der Gesamtansatz selbst wird als nicht-monetäre Größe erst über den Grundbetrag in DM-Werte umgerechnet. Dabei wird der Grundbetrag so bestimmt, daß die verfügbare Schlüsselmasse restlos unter den Gemeinden aufgeteilt werden kann - was in einem iterativen, und, da immer verschiedene Lösungen möglich sind, ad-hoc-Verfahren geschieht. Eine absolute Ermittlung des Finanzbedarfs der Gemeinden ist damit von vornherein ausgeschlossen. Davon abgesehen wird die Veredelung der Einwohnerzahlen zum Gesamtansatz aus dem Hauptansatz - dem in der Mehrzahl der

29) Ähnlich argumentierte etwa die Finanzausgleichskommission in Baden-Württemberg, man ging davon aus, daß die statistischen Durchschnittswerte des Ist-Aufwandes, "die auf einer Erfassung aller Gemeinden beruhen, einen zuverlässigen Schluß auf den objektiven Bedarf zulassen". Siehe den Bericht der gemeinsamen Kommission zur Erarbeitung von Grundlagen und Vorschlägen für eine Neuregelung des kommunalen Finanzausgleichs (Finanzausgleichskommission), Stuttgart, Juni 1977, S. 22. 
Bundesländer eine Einwohnerstaffelung zugrunde liegt - und den aus besonderen Aufgabenbereichen abgeleiteten Nebenansätzen ohne direkten Bezug zu den Kosten der Aufgabenerfüllung vorgenommen. Vielmehr dürften die Staffelungen letztlich allein Ergebnis politischer Verhandlungsprozesse sein und sind insofern unter Zielaspekten zu kritisieren. ${ }^{30}$ )

\section{c. Konsequenzen für die Finanzkraftmessung in der abstrakten Betrachtung}

Der Betrachtung der Bedarfsbestimmung wurde hier deswegen relativ breiter Raum gewidmet, weil sich die Finanzierung der als erwünscht zu kennzeichnenden gemeindlichen Aufgabenerfüllung an der Bestimmung des Bedarfs zu orientieren hat. Besonders dann, wenn die Finanzierung der Ausgabentätigkeit über "gemeindeeigene" Quellen erfolgen soll und wenn für den Fall, daß die Einnahmemöglichkeiten zur Deckung des Bedarfs nicht ausreichen, eine staatliche Zufinanzierung vorgesehen ist, muß die Messung der gemeindlichen Finanzkraft ein bestimmtes Leistungsangebot in den einzelnen Gemeinden voraussetzen. Nur unter dieser Bedingung läßt sich - wie im 3. Kapitel begründet wurde - die Ergiebigkeit der Einnahmequellen theoretisch exakt bestimmen. Dies gilt natürlich auch dann, wenn der Ausschöpfungsgrad der Einnahmequellen staatlicherseits normiert wird.

Wegen ihrer pauschalen und undifferenzierten Festlegung ist die Bedarfsmeßzahl des kommunalen Finanzausgleichs als Grundlage einer zielorientierten Finanzkraftbestimmung deshalb nicht geeignet.

Wenn anschließend die Frage nach geeigneten Finanzierungsmöglichkeiten eines staatlich normierten gemeindlichen Angebots und der gegebenenfalls notwendigen Feststellung gemeindeeigener Finanzierungsmöglichkeiten gestellt wird, so wird dabei ein bestimmter - hier nicht näher zu konkretisierender - Warenkorb gemeindlicher Leistungen als Bedarfsgrundlage unterstellt. Die genauere inhaltliche Bestimmung des Warenkorbes erübrigt sich hier deshalb noch, weil nicht auf ein genau umrissenes Einnahmesystem

30) Vgl. dazu etwa Hansmeyer, Karl-Heinrich, Der kommunale Finanzausgleich als Instrument zur Förderung Zentraler Orte, in: Probleme des Finanzausgleichs II, hrsg. von Dieter Pohmer, Schriften des Vereins für Socialpolitik, Neue Folge Bd. 96/II, Berlin 1980, S. 83150, hier S. 113 ff. sowie Zabel, Gerhard, Ist die Hauptansatzstaffel noch zeitgemäß? a.a.O. 
abgestellt wird, sondern zunächst nur die Möglichkeiten der Konstruktion von Einnahmesystemen und der entsprechenden Konzeption zur Bestimmung der gemeindlichen Finanzkraft untersucht werden.

Sollen die Ergebnisse auf die bestehenden Bedingungen und Regelungen in der Bundesrepublik Deutschland angewendet werden, so ist die Frage nach einer aus staatlicher Sicht adäquaten gemeindlichen Aufgabenerfüllung und der daraus abzuleitenden Bedarfsbestimmung allerdings noch einmal zu stellen.

II. Möglichkeiten der Finanzierung des "Normfinanzbedarfs" und Konsequenzen für die Bestimmung der kommunalen Finanzkraft

Sobald festgelegt ist, welche Aufgaben die Gemeinden erfüllen sollen, ist die Frage der Finanzierung zu klären. Hier kommen verschiedene Finanzierungsformen in Betracht, die einerseits einen Anreiz bieten sollen, vor allem die im Warenkorb zusammengestellten Aufgaben zu erfüllen, die andererseits aber so gewählt werden müssen, daß den Gemeinden ein gewisser Entscheidungsspielraum über Art und Umfang der Aufgabenerfüllung verbleibt. Dazu kommen Zweckzuweisungen, Schlüsselzuweisungen oder die Einrichtung eines auf die ermittelten Bedarfe abgestimmten Abgabesystems in Frage. Diese Möglichkeiten sollen anschließend näher betrachtet werden, wobei der Frage nach der Notwendigkeit und gegebenenfalls nach der Konzeption zur Bestimmung der gemeindlichen Finanzkraft besonderes Augenmerk gewidmet wird.

\section{a. Finanzierung über Zweckzuweisungen}

Die Finanzierung über ein reines Zweckzuweisungssystem wäre so auszugestalten, daß für jede bei der Bedarfsermittlung zugrunde gelegte Aufgabe der den Kosten der Aufgabenerfüllung entsprechende Betrag an Zuweisungen zu gewähren ist, sofern sich die Gemeinde für die Erfüllung der Aufgabe in der vom Staat vorgesehenen Weise entscheidet. Für die Gemeinden verbleibt hier also nur insofern ein Handlungsspielraum, als die über Zweckzuweisungen finanzierbaren Aufgaben nicht unbedingt wahrgenommen werden müssen. Al- 
lerdings sind die zugewiesenen Mittel nicht übertragbar; wird eine Aufgabe nicht oder nicht im vorgesehenen Umfang erfüllt, so werden dadurch keine Mittel für andere Zwecke frei, sondern sie entfallen. Wollte man in diesem System die kommunale Finanzkraft bestimmen, so wäre sie als Summe der $\mathrm{Zu}$ weisungen, die eine Gemeinde in Anspruch nehmen kann, wenn sie das "Normalangebot" erstellt, zu berechnen. Aus übergeordneter Sicht besteht zu einer solchen Bestimmung jedoch kaum eine Veranlassung, da der Ausgleich von Bedarf und Finanzierungsmöglichkeiten über die Zweckzuweisungen bereits vollständig erfolgt.

\section{b. Finanzierung über Schlüsselzuweisungen}

Die Finanzierung über Schlüsselzuweisungen in Höhe der Kosten des Normalangebots beläßt den Gemeinden anders als im Falle der Zweckzuweisungen die Entscheidung über die Verwendung der Mittel. Allerdings ist die Einnahmen- bzw. die Ausgabensumme festgelegt. Weder aus gemeindlicher noch aus staatlicher Sicht stellt sich hier deshalb die Frage nach Einnahmemöglichkeiten oder Finanzkraft.

c. Finanzierung über gemeindeeigene Einnahmequellen

1. Die Festlegung der Einnahmequellen

Größere Spielräume bei der Einnahmebeschaffung und damit auch bei der Ausgabengestaltung verbleiben den Gemeinden dann, wenn zur Finanzierung des "Normbedarfs" ein Abgabensystem eingerichtet wird, das den Gemeinden die Möglichkeit mehr oder weniger intensiver Ausschöpfung beläßt. Weder Umfang noch Struktur der Aktivitäten werden dadurch genau festgelegt, beides bleibt gemeindlich entscheidbar.

Der Vorteil dieses Finanzierungssystems liegt vor allem in der Allokationseffizienz; die Möglichkeit, das gemeindliche Angebot an die Präferenzen und die Zahlungsbereitschaft der Nutzer anzupassen, bleibt hier stärker als bei der Finanzierung über Zuweisungssysteme erhalten. 
Soll trotz dieser Entscheidungsspielräume ein Anreiz gegeben werden, das staatlich bestimmte "Normangebot" zu erstellen, so sind die Einnahmequellen möglichst an der gewünschten Aufgabenerfüllung und Ausgabentätigkeit auszurichten. Anders formuliert: Wenn den Gemeinden bestimmte Einnahmequellen zur Verfügung gestellt werden, so sind diese so zu bestimmen, daß sie um so höhere Einnahmemöglichkeiten eröffnen, je näher die gemeindlichen Aktivitäten dem übergemeindlich bestimmten Normbedarf kommen. Damit wird die interkommunale Konkurrenz und damit auch die Allokationseffizienz des Systems teilweise wieder eingeschränkt. ${ }^{31)}$ Allerdings ist dieser Effekt unvermeidlich, wenn zwischen der Ausrichtung des Angebots auf die Präferenzen der Nachfrager und der Entzerrung dieser Präferenzen durch den Staat ein Kompromiß gefunden werden soll.

Aus den angesprochenen Lenkungszielen lassen sich zwei Anforderungen an das kommunale Finanzierungssystem ableiten:

- Die Finanzierung aus "gemeindeeigenen" Quellen sollte nach dem Äquivalenzprinzip erfolgen.

- Die Einnahmen, die bei der Erstellung des Normangebots erzielbar wären, müssen auch dann in die Ermittlung der Finanzkraft eingehen, wenn eine Gemeinde ein anderes als das Normangebot realisiert und die tatsächlichen Einnahmemöglichkeiten nicht mit der Finanzkraft übereinstimmen. Eine solche Regelung kann für die jeweilige Gemeinde negative, aber auch positive Effekte haben. Positive Wirkungen sind dann zu erwarten, wenn es der Gemeinde gelingt, in einer Weise vom Normalangebot abzuweichen, die höhere Einnahmemöglichkeiten verspricht. Die positive Differenz zwischen Einnahmepotential und Finanzkraft würde die staatlichen Ausgleichszahlungen nicht mindern. Umgekehrt hätten Gemeinden, die aufgrund einer Ausgabenpolitik, die den staatlichen Zielsetzungen nicht entspricht, niedrigere Einnahmen erzielen können, nicht mit einem Ausgleich der negativen Differenz zwischen normierter Finanzkraft und tatsächlichen Einnahmemöglichkeiten zu rechnen. Diese Differenz müßte als "selbstverschuldet" in dem Sinn gelten, daß von der

31) Vgl. Auch Brennan, Geoffrey und Buchanan, James M., The Power to Tax, a.a.O., S. 182 f. 
Normalpolitik abgewichen wird und die finanziellen Konsequenzen deshalb auch von der jeweiligen Gemeinde selbst zu tragen sind. ${ }^{32}$ )

Als erste Einnahmequelle, die in das beschriebene Finanzierungssystem eingepaßt werden kann, sind die Entgelte zu nennen. Zahlen sollen diejenigen Wirtschaftssubjekte, die die kommunalen Leistungen und Einrichtungen in Anspruch nehmen (Gebühren) oder potentiell nutzen könnten und allein daraus schon einen Vorteil ziehen (Beiträge). Einen direkten Lenkungseffekt in Richtung auf das Normangebot hat dieses Finanzierungssystem dann, wenn die übergeordnete Ebene die Entgeltfinanzierung auf die Leistungen des Normangebots beschränken und andere Finanzierungsformen nicht zulassen würde. Damit ginge jedoch der allokative Vorteil des Systems verloren; es wäre einer Finanzierung durch Zweckzuweisungen ähnlich. Wird eine Entgeltfinanzierung hingegen auch für andere Leistungen zugelassen, so stellt sich nur insofern ein direkter Lenkungseffekt ein, als entgeltfähige gegenüber nicht entgeltfähigen Leistungen einen Finanzierungsvorteil aufweisen. Die Finanzkraft wäre im beschriebenen System aus der Menge der im gemeindeindividuellen Normangebot enthaltenen Leistungen und einem normierten, d.h. aus staatlicher Sicht für angemessen gehaltenen Entgeltsatz zu errechnen. ${ }^{33)}$

Nimmt man einmal an, der Normalkorb gemeindlicher Leistungen solle ausschließlich solche Leistungen enthalten, die langfristig eine volle Entgeltfinanzierung über einen Kostendeckungsgrad von $100 \%$ erlauben, so wäre ein Ausgleich zwischen Finanzbedarf und Finanzkraft streng genommen nicht notwendig. Sofern sich die Gemeinden an die staatliche Leistungsnorm halten, ist eine Deckung der Ausgabentätigkeit immer gewährleistet. Allerdings wäre zu überprüfen, ob dieses Finanzierungssystem nicht durch ein gemeindliches Verschuldungsrecht ergänzt werden müßte, damit notwendige Investitionsausgaben vorfinanziert werden können.

32) Diese Forderung wurde im 2. Kapitel bereits betrachtet, als die Ansprüche an die Finanzkraftmessung formuliert wurden. Allerdings ließ sich dort noch kein Weg aufzeigen, auf dem ihr Rechnung getragen werden konnte, weil die Finanzkraftmessung des kommunalen Finanzausgleichs in der Bundesrepublik nicht bei der Bestimmung eines gemeindlichen Normalangebots ansetzt, sondern allein von der Einnahmeseite des Budgets ausgeht.

Zur genauen Formulierung des genannten Anspruchs vgl. etwa Kirchhof, Paul, Der Finanzausgleich als Grundlage kommunaler Selbstverwaltung, a.a.O.

33) Den Schwierigkeiten einer zieladäquaten Normierung von Entgelt- und Steuersätzen wird in Punkt 2 gesondert nachgegangen. 
Die alleinige Entgelterhebung reicht zur Deckung des normierten Finanzbedarfs nicht mehr aus, wenn einige kommunale Leistungen innerhalb des Normangebots nicht oder nur teilweise entgeltfähig sind. Dies kann der Fall sein, weil es sich um öffentliche Güter handelt und Nicht-Zahler vom Genuß der Leistung nicht ausgeschlossen werden können, weil die ökonomischen Reaktionen der potentiellen Nutzer eine volle Kostendeckung nicht zulassen im 3. Kapitel wird etwa der Kulturbereich diesem Leistungstyp mit elastischem Nachfrageverlauf zugerechnet - oder weil aus übergeordneter Sicht eine Entgeltfestsetzung in kostendeckender Höhe nicht erwünscht ist - etwa weil mittels niedrigerer Preise die Nachfrage stimuliert werden soll. In den genannten Fällen sind den Gemeinden weitere Finanzierungsmittel zur Verfügung zu stellen. Dabei müßte es sich um allgemeine Deckungsmittel handeln, sofern den Gemeinden ein Entscheidungsspielraum verbleiben soll (vgl. oben).

Die über Entgelte nicht beschaffbaren, zur Deckung des Normbedarfs notwendigen Mittel können durch den Ausgleich, also über Schlüsselzuweisungen, aber auch durch eine vor den Ausgleich geschaltete Zuweisung bestimmter Steuerquellen an die Gemeinden bereitgestellt werden.

Neben allgemeinen wirtschafts- und finanzpolitischen Grundsätzen, denen gemeindliche Steuern wie alle anderen Steuern entsprechen sollen, sollten die Gemeindesteuern darüber hinaus dem Kriterium der gruppenmäßigen Äquivalenz genügen. "Gelingt es, für einige der wichtigsten Bestimmungsgründe kommunaler Bedarfe einigermaßen zuverlässige Indikatoren zu finden, die zugleich einen Anknüpfungspunkt für Gemeindesteuern abgeben, dann scheint sich ein grober Regelungsmechanismus zur Steuerung der kommunalen Aktivitäten entwickeln zu lassen: Die durch Einwohner bedingten kommunalen Bedarfe sind beispielsweise mit Aufkommen aus einer Bürgersteuer oder aus einer kommunalen Einkommensteuer zu lenken; die durch die örtlichen Produktionen entstandenen kommunalen Bedarfe werden durch Steuern geregelt, die an die Produktion anknüpfen. ${ }^{\text {"34) }}$ Anders formuliert: Wenn Steuern an jenen Tatbeständen anknüpfen, die den staatlicherseits definierten Bedarf verursachen, und wenn die Steuerzahlung gegenüber den Zen-

34) Wissenschaftlicher Beirat beim Bundesministerium der Finanzen, Gutachten zur Reform der Gemeindesteuern in der Bundesrepublik Deutschland, a.a.O., S. 31. 
siten mit Äquivalenzüberlegungen begründet wird, dann kann eine Kommune es sich auf Dauer nicht leisten, andere als die diese Bedarfe deckenden Aktivitäten mit dem Steueraufkommen zu finanzieren. Ausweichreaktionen der Zensiten wären die unvermeidliche Folge.

Insofern werden also nicht primär die Bedarfe, sondern zunächst die Bedarfsdeckung geregelt oder gesteuert. Als Beispiel für die - wenn auch wenig zielgerichtete - Wirksamkeit solcher Regelungsmechanismen kann das Gemeindesteuersystem vor der Gemeindesteuerreform von 1969 gelten. Einer der gegen dieses Steuersystem vorgebrachten Kritikpunkte lautete, daß es die Gemeinden aufgrund der Dominanz der Gewerbesteuer in besonderem Maße zur Deckung der Unternehmensbedarfe veranlaßt habe, Bedarfe der Bürger hingegen tendenziell eher vernachlässigt worden seien, weil mit der Deckung ihrer Bedarfe keine kommunale "Bemessungsgrundlagenpolitik" betrieben werden konnte. Mit der Beteiligung der Gemeinden am Aufkommen der Einkommensteuer wurde dieser Mangel zumindest teilweise behoben. ${ }^{35)}$

Ein "gutes" gemeindliches Steuersystem müßte sich dadurch auszeichnen, daß die Steuerkraft - also jene Steuereinnahmen, die eine Gemeinde bei zieladäquatem Verhalten aus den zugewiesenen Quellen erzielen kann- mit den Normaufgaben und den daraus resultierenden Ausgaben korreliert und hoch genug ist, um den Finanzbedarf zu decken.

Die Konstruktion eines Steuersystems mit den beschriebenen Qualitäten scheint allerdings nur in Grenzen möglich zu sein, weil einige der erwünschten kommunalen Aktivitäten kaum mit Indikatoren verknüpft werden können, die auch als Ansatzpunkt für eine Steuer dienen können. So weist der Wissenschaftliche Beirat beim BMF etwa auf Ausgabenbedarfe hin, die durch die Übernahme zentralörtlicher Funktionen oder durch rasches Bevölkerungswachstum hervorgerufen werden ${ }^{36}$ ), die sich aber kaum mit einer speziellen Steuer verknüpfen lassen.

35) Dabei ist allerdings zu berücksichtigen, daß den Gemeinden z.Z. kein Hebesatzrecht bei der Einkommensteuer zugestanden wird, und der gemeindliche Einkommensteueranteil insofern gewisse Merkmale einer allgemeinen Zuweisung aufweist. Vgl. dazu auch Pagenkopf, Hans, Das Gemeindefinanzsystem und seine Problematik, Schriften zum deutschen Kommunalrecht, Bd. 15, Siegburg 1978, S. 69 ff.

36) Wissenschaftlicher Beirat beim Bundesministerium der Finanzen, Gutachten zur Reform der Gemeindesteuern, a.a.O., S. $30 \mathrm{ff}$. 
Deshalb wird auch bei einem Steuersystem, das den gesetzten Anforderungen im Durchschnitt der Gemeinden genügt, häufig der Fall eintreten, daß die zur Verfügung gestellten Einnahmequellen selbst bei einer den staatlichen Zielsetzungen entsprechenden Ausschöpfung nicht ergiebig genug sind, um den Normbedarf zu decken. Finanzbedarf und Finanzkraft sind deshalb gegenüberzustellen und gegebenenfalls auszugleichen.

Die Messung der gemeindlichen Finanzkraft setzt nun voraus, daß zuvor die kommunale Einnahmepolitik innerhalb des einnahmepolitischen Spielraums normiert worden ist. Es ist also zu klären, welches Verhalten von den Gemeinden erwartet und zur Grundlage des Ausgleichs gemacht werden soll. Konkret bedeutet dies eine übergemeindliche Festlegung erwünschter Entgelt- und Steuersätze, die dann als "fiktive" Abgabensätze in die Finanzkraftmessung eingehen. Dabei besteht eines der Hauptprobleme darin, die staatlicherseits gestellten Anforderungen mit den kommunalen Möglichkeiten der Entgelt- und Steuersatzgestaltung in Einklang zu bringen.

\section{Zur Frage der zieladäquaten Normierung gemeindlicher Entgelt- und Steuersatzgestaltung}

Im 3. Kapitel wurde abgeleitet, daß für jede Abgabe fiskalisch optimale Steuersätze bestimmt werden können, bei deren Realisierung in den Gemeinden die jeweiligen Einnahmen durch eine Variation der Abgabesätze nicht weiter gesteigert werden können. Diese fiskalisch optimalen Abgabesätze können in den einzelnen Gemeinden durchaus unterschiedlich hoch ausfallen. Aus gemeindlicher Sicht sind diese Sätze - unabhängig davon, ob sie allein aus den Reaktionen der Wirtschaftssubjekte oder aus weiter einschränkenden Maximal-Abgabesatz-Vorschriften des Staates resultieren - entscheidend für die Bestimmung des Einnahmepotentials im Sinne der maximal erzielbaren Einnahmen.

Einige der im 2. Kapitel zitierten Kritiker der Finanzkraftmessung werfen dem praktizierten Konzept der Steuerkraftmessung vor, daß eben diese "optimalen" Sätze nicht erfaßt werden, sondern daß statt dessen auf fiktive, in der Regel einheitliche Sätze zurückgegriffen wird. Diese Kritik wäre insofern schlagkräftig, wenn sie nachweisen könnte, daß die normierten Sätze den 
staatlichen Zielsetzungen und Anforderungen, die an die kommunale Einnahmepolitik herangetragen werden, nicht genügen. Sie ist hingegen nicht berechtigt, wenn allein die Abweichung zwischen fiktiver und "optimaler" Einnahmepolitik angegriffen wird. Diese Abweichung kann - wie oben bereits herausgestellt - durchaus gewollt sein.

Aus staatlicher Sicht sind die für die Gemeinden fiskalisch optimalen Abgabesätze vor allem als Obergrenze möglicher Norm-Abgabesätze relevant. In besonderen Fällen - wenn es etwa um eine Nachfragelenkung mittels der Abgabensätze geht - sind auch Norm-Sätze denkbar, die über denen liegen, die für die Gemeinden maximale Einnahmen erbringen. ${ }^{37}$ ) Sieht man von solchen Fällen einmal ab, so können die der Ermittlung der Finanzkraft zugrunde gelegten Abgabesätze innerhalb des Bereichs, der von den fiskalisch optimalen Sätzen abgesteckt wird, verschiedene Höhe und interkommunale Struktur aufweisen, je nachdem, welche Ziele die übergeordneten Gebietskörperschaften mit ihrer Festlegung verfolgen. Auf die Gestaltungsmöglichkeiten wird im folgenden näher einzugehen sein.

\subsection{Normierung von Entgeltsätzen ${ }^{38)}$}

Eine erste Möglichkeit der Normierung der in die Finanzkraftmessung einzubeziehenden Entgeltsätze entspricht einer marktmäßigen Äquivalenz bei vorgegebenem Leistungsangebot: Das Normangebot wird sich in der Regel auf ein bestimmtes Versorgungsniveau je Kopf der Bevölkerung, oder genereller, je Nutzer beziehen. Soll genau diese Leistungsmenge zu Marktbedingungen abgesetzt werden, so läßt sich aus dem Verlauf der Nachfragefunktion der entsprechende Marktpreis ableiten. Unter der Bedingung, daß bei diesem

37) Als Beispiel könnten etwa "abschreckend" gestaltete Gebühren für die Müllbeseitigung genannt werden, wenn damit eine Reduktion des Müllaufkommens erreicht werden soll. Im Prinzip handelt es sich dabei um Instrumente zur Lenkung des Konsumentenverhaltens und nicht mehr (nur) um ein Mittel zur Finanzierung öffentlicher Angebote. Zur Wirkungsweise dieser Instrumente vgl. etwa Hansmeyer, Karl-Heinrich, Abgaben und steuerliche Instrumente der Umweltpolitik - Wirkungsweise. Erfahrungen. Möglichkeiten - in: Zeitschrift für Umweltpolitik und Umweltrecht, Nr. 3/ September 1987, S. 251-266.

38) Normierungsprinzipien werden detaillierter aus ökonomischer Sicht von Hansmeyer und Fürst, aus rechtlicher Perspektive von Kirchhof besprochen. Vgl. Hansmeyer, Karl-Heinrich und Fürst, Dietrich, Die Gebühren, a.a.O., insbesondere S. 45 ff. sowie Kirchhof, Ferdinand, Die Höhe der Gebühr, a.a.O., S. 61 ff. 
Preis die Struktur der Nutzer der angestrebten entspricht, insbesondere die Einkommensverteilung also nicht für korrekturbedürftig gehalten wird, wäre die versorgungspolitische Zielsetzung erreicht.

Für eine solche Normierung könnten zunächst allokative Vorteile sprechen: Die Leistungsabgabe müßte zu einem Preis erfolgen, der der Wertschätzung dieser Leistung entspricht; damit würde "Verschwendungstendenzen" entgegengewirkt werden. Überdies würde der Zuschußbedarf auf Gemeindeebene minimiert, weil von jeder Gemeinde verlangt würde, daß sie die marktlichen Einnahmespielräume innerhalb der staatlichen Rahmensetzung beim vorgegebenen Normangebot voll ausschöpft. ${ }^{39)}$

Gegen die Normierung nach marktmäßiger Äquivalenz sprechen vor allem verteilungspolitische Argumente, aber auch - besonders bei starrer Nachfrage nach der betreffenden Leistung - die vom privaten Sektor zu unterscheidende Zielsetzung des öffentlichen Angebots, die eben nicht in der Gewinnerzielung, sondern in der Versorgung mit nicht-privaten Gütern besteht. Zwar wird mit der Normierung den Gemeinden ein solches Verhalten gestattet, die Norm selbst regt jedoch nicht unmittelbar dazu an.

Die Normierung nach der marktmäßigen Äquivalenz wirft darüber hinaus einige unüberwindliche Schwierigkeiten für die Finanzkraftbestimmung auf. Als normierte Entgeltsumme wären jene Einnahmen zu erfassen, die die Gemeinde erzielen kann, wenn sie das Normangebot realisiert. Dabei kann wie im 3. Kapitel gezeigt - im Ergebnis die Finanzkraft über, aber auch unter den Kosten der Bedarfsdeckung liegen. Stimmen Normangebot und tatsächliches Angebot überein, so sind die staatlicherseits zu erfassende Finanzkraft und das aus gemeindlicher Sicht interessierende Einnahmepotential identisch. Die Ermittlung dieser Größe erschien im 3. Kapitel bereits als extrem schwierig. Sie wird zusätzlich kompliziert, wenn aus staatlicher Sicht von einem anderen als dem realisierten Angebot ausgegangen werden muß, weil letzteres dem Normangebot nicht entspricht. In diesem Fall müßten die Nachfrageverläufe mit ihren jeweiligen Preis-Mengen-Komponenten sogar für nicht angebotene Leistungen vollständig bekannt sein, um die Finanzkraft exakt erfassen zu können.

39) Vgl. dazu die Ausführungen im 3. Kapitel, Abschnitt B IV, sowie Münch, Klaus N., Kollektive Güter und Gebühren, a.a.O., S. 114 ff. 
Da die den Gemeinden vorgegebenen Normen, sollen sie die erwarteten Anreizwirkungen hervorbringen, staatlicherseits auch auf ihre Einhaltung überprüfbar sein müssen, eignet sich eine marktmäßige Äquivalenz als Grundlage der Finanzkraftmessung daher schon aus technischen Gründen wenig.

Eine zweite Möglichkeit der Normierung bestände darin, daß kostendekkende Entgelte bei der Ermittlung der Finanzkraft zugrundegelegt werden. Von dieser Variante werden übergeordnete Gebietskörperschaften insbesondere dann Gebrauch machen, wenn damit gerechnet wird, daß die Gemeinden niedrigere Entgeltsätze fordern, obwohl das Normangebot zu kostendeckenden Preisen abgesetzt werden könnte und aus übergeordneter Sicht den Nutzern die Kosten der Leistungserstellung angelastet werden sollen. Ein solches Ergebnis könnte sich etwa in der Parteienkonkurrenz aus wahlpolitischen Gründen einstellen.

Auch in diesem Fall ist von der normierten Leistungsabgabe als "Mengengerüst" der Finanzkraftbestimmung auszugehen. Die Kosten dieses Angebots - als Durchschnittswerte auf die Nutzer umgelegt - bilden als Normentgelt die Preiskomponente. In den einzelnen Gemeinden können diese Entgeltsätze in Abhängigkeit von den Kosten der Leistungserstellung durchaus unterschiedliche Höhe aufweisen.

Geht man zunächst von einer Kostendeckungsnorm in Höhe von $100 \%$ aus, so entfällt die Notwendigkeit eines Ausgleichs zwischen Finanzbedarf und Finanzkraft, wenn sichergestellt ist, daß volle Kostendeckung in allen Gemeinden durchsetzbar ist.

Eine solche Normierung ist also nur dann sinnvoll, wenn die Nachfrageverläufe prinzipiell eine Kostendeckung erlauben, d.h. die erwünschte Absatzmenge zu kostendeckenden Preisen abgesetzt werden kann, und dies in allen Gemeinden, für die das jeweilige Angebot in den Korb des Normalangebots aufgenommen worden ist. Das beschriebene Normierungsverfahren kommt deshalb nur für bestimmte - im 3. Kapitel gekennzeichnete - Leistungsbereiche mit relativ unelastischer Nachfrage in Betracht.

Lassen die jeweiligen Nachfrageverläufe eine vollständige Kostendeckung des Normangebots nicht zu, so sind die normierten Entgeltsätze entsprechend 
niedriger anzusetzen. Die Differenz zwischen Ausgabenbedarf (Kosten) und normierter Finanzkraft ist dann aus Mitteln übergeordneter Gebietskörperschaften zu decken. Die Höhe der auszugleichenden Differenz spiegelt dabei den "Meritorisierungsgrad" wider.

Bei allen der bisher betrachteten Normierungsvarianten können bzw. sollen die Entgeltsätze im interkommunalen Vergleich unterschiedlich hoch sein.

Eine dritte Variante der Normierung von Entgeltsätzen könnte für ein gegebenes Angebot überregional einheitliche Sätze festlegen. Diese Form der Normierung könnte etwa aus dem Ziel der Einheitlichkeit der Lebensverhältnisse begründet werden. Von den Gemeinden würde gefordert, daß sie zur Deckung des Ausgabenbedarfs eine je Leistungseinheit überall gleiche Summe über Entgelte selbst aufbringen; je höher die Kosten der Leistungserstellung, desto größer wäre auch die gegebenenfalls auszugleichende Differenz zwischen normiertem Finanzbedarf und normierter Finanzkraft. Wenn also in bestimmten Gemeinden besonders hohe Kosten der Leistungserstellung auftreten und in die Normkosten eingehen, so sind sie nach diesem Konzept der Normierung zu geringeren Anteilen äquivalent zu finanzieren als in Gemeinden, die das betreffende Angebot kostengünstiger erbringen können.

Welche der angesprochenen Normen letztlich durchgesetzt werden, ist Sache politischer Prioritätensetzung zugunsten bestimmter Ziele; sie soll hier nicht weiter untersucht werden. Vielmehr geht es allein um die Konsequenzen der Normierung für die Bestimmung der gemeindlichen Finanzkraft: Die Betrachtung hat gezeigt, daß nur im Fall der Entgeltnormierung nach der marktmäßigen Äquivalenz - die durchaus, wie im privaten Sektor auch, mit staatlich festgesetzten Höchstpreisen kombiniert werden kann - kommunales Entgeltpotential und staatlicherseits zu ermittelnde Finanzkraft übereinstimmen.

Allerdings gibt es auch bei der Festlegung anderer Normen wenig Sinn, die Finanzkraft höher als das Einnahmepotential anzusetzen, d.h. von einer Gemeinde einen höheren eigenen Finanzierungsbeitrag zu fordern, als sie bei der Leistungsabgabe tatsächlich erbringen kann. Will man dies ausschließen, so hat sich die Normierung der Entgeltsätze jeweils an den Einnahmemög- 
lichkeiten der "schwächsten" Gemeinde zu orientieren. Daraus folgt zwangsläufig, sowohl für die Normierung auf im Gemeindevergleich einheitliche Entgelthöhe als auch für die Normierung auf einheitliche Kostendeckungsgrade, daß einigen Gemeinden, insbesondere jenen mit überdurchschnittlichem Einkommensniveau und Nachfragepotential, ein Teil der objektiv vorhandenen Finanzierungsmöglichkeiten nicht als Finanzkraft angerechnet wird. Vielmehr sind die zusätzlich erzielbaren Einnahmen vollständig dem "autonomen Bereich" der kommunalen Aktivitäten zuzurechnen; sie induzieren deshalb keinen Ausgleichs- oder gar Abschöpfungsvorgang. Will man die interkommunalen Unterschiede in den Finanzierungsmöglichkeiten reduzieren, so ist entweder der Autonomiespielraum einzuschränken, in dem die Abgabesätze unmittelbar begrenzt bzw. indem die kommunalen Aktivitäten direkt gesteuert werden, oder die Finanzierungsmöglichkeiten sind über eine Politik zur Angleichung der sozioökonomischen Bedingungen in der gewünschten Weise zu beeinflussen. Eine stärkere Angleichung über die Manipulation der Finanzkraftmessung wäre hingegen nicht system-konform und würde den der Normierung zugrundeliegenden Zielsetzungen teilweise widersprechen.

Im 3. Kapitel wurde abgeleitet, daß sich die Nachfrageverläufe für ein und dieselbe Leistung im interkommunalen Vergleich wenig unterscheiden dürften; insofern unterscheiden sich die einzelnen Entgeltpotentiale und die nicht ausgeglichene Differenz zwischen möglichen und aus staatlicher Sicht zu fordernden Entgelten im interkommunalen Vergleich vermutlich ebenfalls kaum, es sei denn, daß die Kosten der Leistungserstellung und -abgabe stark differieren.

Demgegenüber weisen die Steuer- bzw. Hebesatzspielräume sicherlich gravierendere Disparitäten auf. Wie zu zeigen sein wird, kann es jedoch über die Normierung der Steuerkraft nicht zu einem vollständigen Ausgleich der Einnahmemöglichkeiten, oder besser: der Bedarfsdeckungsmöglichkeiten, kommen. Dieser Effekt resultiert aus dem Charakter der Normierung und kann nicht als fehlerhafte Messung der Steuerkraft interpretiert werden. 


\subsection{Die Normierung der Steuersätze}

Im 3. Kapitel wurden Entgelte als ein Unterfall der Besteuerung klassifiziert. Daraus könnte der Schluß gezogen werden, daß für die Festsetzung normierter Steuersätze die gleichen oder ähnliche Konzepte zur Anwendung gelangen könnten wie für die Normierung der Entgelte. Praktisch sind jedoch einige Unterschiede zu berücksichtigen:

- Zum ersten sind die im Zusammenhang mit der Besteuerung durch die Gemeindeebene üblicherweise angestellten Äquivalenzüberlegungen anderer Art als bei der Entgeltfinanzierung, vor allem weil sich keine direkte Zuordnung bestimmter Leistungen und ihrer Inanspruchnahme zu den Einnahmen aus bestimmten Steuerquellen oder gar bestimmter Zensiten herstellen läßt. Mit Blick auf die Besteuerung und die Steuersätze kann das Äquivalenzprinzip deshalb bestenfalls als lokale gruppenmäßige Äquivalenz angewendet werden, das heißt eine, oder mehrere Gruppen potentieller oder tatsächlicher Nutzer können zur Finanzierung eines ganzen, für sie erbrachten Leistungsbündels herangezogen werden. Allerdings scheint eine exakte Zuordnung kaum möglich. ${ }^{40)}$ Ausgangspunkt der Steuersatznormierung können deshalb nicht die Kosten einzelner Leistungen sein.

- Zum zweiten werden mit der Frage der Besteuerung und der "angemessenen" Steuersätze in der Regel weitergehende und umfangreichere Zielkataloge verbunden als mit der Festsetzung von Leistungsentgelten. Während für Entgelte Äquivalenzüberlegungen und zum Teil auch noch Verteilungsüberlegungen vorrangig sind, werden die Steuersätze darüber hinaus und vor allem unter konjunktur- und wachstumspolitischen Gesichtspunkten und nicht zuletzt nach raumordnungspolitischen Kriterien beurteilt. $^{41)}$ Diese Kriterien spielen deshalb auch bei der Festsetzung normierter Steuersätze eine besonders wichtige Rolle. Zwar könnten solche Beurteilungsmaßstäbe auch für die Entgeltnormierung herangezogen

40) In diesem Zusammenhang weist der Wissenschaftliche Beirat beim Bundesministerium der Finanzen auf die Bedeutung des "Vorteils- und Interessenausgleichs zwischen Kollektiven einer Gemeinde" hin. Wissenschaftlicher Beirat beim Bundesministerium der Finanzen, Gutachten zur Reform der Gemeindesteuern, a.a.O., S. 25 u. S. 32 ff.

41) Vgl. auch Zimmermann, Horst und Postlep, Rolf-Dieter. Beurteilungsmaßstäbe für Gemeindesteuern, in: Wirtschaftsdienst V/1980, S. 248-253. 
werden, in der Regel werden die genannten Zielsetzungen in diesem $\mathrm{Zu}$ sammenhang jedoch vernachlässigt.

Zum Zweck der Normierung der Steuersätze sind nun zwei Fragen zu beantworten:

- In bezug auf jede einzelne Steuer ist zu entscheiden, ob von allen Gemeinden die Durchsetzung gleich hoher Steuersätze gefordert werden soll oder ob nach Gemeindetypen differenzierte Steuersätze als Norm vorgegeben werden sollen.

- Ebenfalls für jede einzelne Steuer ist das Niveau des bzw. der Steuersätze festzulegen. Damit wird auch eine Entscheidung über das Verhältnis der Steuersätze verschiedener Steuern zueinander gefällt.

Mit Blick auf die Frage, ob einheitliche oder differenzierte Steuersätze als Norm gelten sollen, lassen sich mehrere Argumente und Zielsetzungen anführen.

Für einheitliche Norm-Steuersätze spricht zunächst die Forderung nach "Einheitlichkeit der Lebensverhältnisse" und mit Blick auf die Produktionsverhältnisse das Postulat der Wettbewerbsneutralität der Besteuerung. ${ }^{42}$ ) $\mathrm{Da}$ nach müßte die Steuerbelastung der in den Bemessungsgrundlagen abgebildeten, identischen Aktivitäten regional unterschiedslos sein. So wäre für dem Wert nach gleiche Einkommen in verschiedenen Regionen der gleiche Steuersatz zu fordern, weil sie zum einen gleiche Leistungsfähigkeit der Einkommensbezieher begründen und zum anderen - unter Äquivalenzaspekten - das Steueraufkommen zur Finanzierung eines Normalangebots der Gemeinden dienen soll, das überall einen bestimmten, gleichen Versorgungsstandard sichert. $^{43)}$

Legt die Steuersatznormierung zum Zweck der Finanzkraftbestimmung interkommunal unterschiedslose Steuersätze zugrunde, so wird damit das Recht der Gemeinden auf eine autonome Steuersatzgestaltung nicht beschnitten. Solche selbständig zu verantwortenden Steuersatzdifferenzen finden

42) Vgl. auch Haller, Heinz, Gründe für besondere gliedstaatliche Steuertarife in Bundesstaaten, in: Hanusch, Horst, Roskamp, Karl W., und Wiseman, Jack, Hrsg., Staat und politische Ökonomie heute, Horst Claus Recktenwald zum 65. Geburtstag, Stuttgart-New York 1985, S. 209-221, hier S. 210 f.

43) Vgl. auch Kirchhof, Paul, die kommunale Finanzhoheit, a.a.O., S. 12. 
ihre Rechtfertigung in unterschiedlicher kommunaler Haushaltspolitik, die über das Normalgebot hinausgehende Präferenzen der angesiedelten Wirtschaftssubjekte durch zusätzliche Angebote befriedigt.

Will man solche Steuersatzunterschiede vermeiden oder minimieren, so gilt auch hier, daß dies nicht durch die Normierung, sondern nur durch staatliche Begrenzung der kommunalen Autonomie erreicht werden kann.

Für den interkommunalen Vergleich bedeutet die Festsetzung gleicher Norm-Steuersätze natürlich eine "Schlechterstellung" jener Gemeinden, deren Konkurrenzposition ungünstiger und deren Steuersatzspielräume insgesamt geringer sind. Unterstellt man, daß sich alle Gemeinden sowohl bei der Ausgaben- als auch bei der Einnahmenpolitik der Norm entsprechend verhalten, so müßten Gemeinden mit geringeren Steuersatzspielräumen c.p. ihr gesamtes Steuerpotential stärker ausschöpfen als Gemeinden in einer vorteilhafteren Konkurrenzposition. ${ }^{44)}$ Die Möglichkeiten zur Finanzierung von über dem Normalbedarf hinausgehenden Angeboten wird also von vornherein stärker eingeschränkt. Dies gilt besonders dann, wenn die eigenverantwortliche Haushaltsgestaltung finanzierungsseitig auf das Recht der Steuersatzgestaltung bei denjenigen Steuern begrenzt ist, die auch zur Finanzierung des Normalangebots dienen sollen. Hier können deshalb weitere ausgleichsorientierte Maßnahmen notwendig werden, die jedoch vom Ausgleich zum Zweck der Finanzierung des Normalangebots zu trennen sind und idealerweise direkt an der Wirtschafts- und Einkommenskraft der Region ansetzen (vgl. oben).

Gegen die Festsetzung gleicher normierter Steuersätze können vor allem Äquivalenzüberlegungen angeführt werden. Identischen Normsteuersätzen liegt die Vorstellung zugrunde, daß Kostenunterschiede bei der Deckung des Normalbedarfs nicht von den Nutzern des Angebots verursacht und ihnen daher auch nicht anzulasten, sondern vielmehr durch den Finanzausgleich abzudecken seien. ${ }^{45)}$ Dem kann allerdings entgegengehalten werden, daß eine Nicht-Anlastung von Kostendifferenzen zu einer Verzerrung der Standortund Wohnortwahl führt. Sofern gemeindliche Vorleistungen im Normalange-

44) Vgl. dazu im einzelnen die Ausführungen im 3. Kapitel.

45) Diese Überlegung scheint der Steuerkraftermittlung in der Mehrzahl der Bundesländer zugrundezuliegen: Mit Ausnahme von Nordrhein-Westfalen sind die fiktiven Hebesätze für alle Gemeinden identisch. Vgl. die Aufstellung im 2. Kapitel. 
bot unterschiedlich teuer sind, sollten die Nutzer also auch zur Finanzierung des "Mehrbedarfs" herangezogen werden und die entsprechenden gemeindlichen Mehr- oder Mindereinnahmen in die Ermittlung der Finanzkraft eingehen. ${ }^{46)}$

Als Beispiel können etwa die Kosten der Verkehrsinfrastruktur angeführt werden, die - je Einwohner - sowohl in schwach verdichteten Räumen als auch in Ballungen besonders hoch sind. Würden diese Mehrkosten zwar bei der Ermittlung des Normfinanzbedarfs berücksichtigt, bei der Finanzkraftmessung jedoch eine Gleichbehandlung der Verursacher - der Unternehmen und Haushalte - im Sinne identischer normierter Steuersätze vorgenommen, so würde damit zum Ausdruck gebracht, daß die Gemeinden nicht zur Anlastung der jeweiligen Kostenunterschiede angereizt werden sollen. Mehrkosten wären über den Ausgleich von Finanzbedarf und Finanzkraft von der Allgemeinheit, nicht unbedingt von den lokalen Verursachern zu tragen. Die Produktions- und Reproduktionskosten würden auf diese Weise in den betreffenden Regionen künstlich verbilligt. Sofern ein solcher Effekt beabsichtigt ist, sind gleiche normierte Steuersätze in die Finanzkraftbestimmung aufzunehmen; dem Äquivalenzprinzip entspricht jedoch eine Staffelung der Sätze nach Maßgabe der Kosten der Bedarfsdeckung.

Allerdings stellt sich in diesem Zusammenhang die Frage, ob eine Internalisierung von Kostenunterschieden - sofern sie aus staatlicher Sicht erwünscht ist - nur in die Normierung aufgenommen und damit den Gemeinden letztlich anheim gestellt werden soll. Den Gemeinden würde damit die Option offengehalten, die Nicht-Internalisierung höherer Kosten als Instrument in der interkommunalen Konkurrenz zu nutzen, wenn die Finanzierungsmöglichkeiten dazu ausreichen. Wenn ein solches Verhalten in jedem Fall verhindert werden soll, so hätte die Internalisierung von Kostenunterschieden über Sonderabgaben des Zentralstaates, also etwa über eine "Ballungsraumsteuer" ${ }^{47)} \mathrm{zu}$ erfolgen.

46) So argumentieren auch Kirchhof, Paul, Die kommunale Finanzhoheit, a.a.O., S. 25 und Deubel, Ingolf, Der kommunale Finanzausgleich in Nordrhein-Westfalen, a.a.O., S. 122.

47) Vgl. Biehl, Dieter und Münzer, Urban, Agglomerationsoptima und Agglomerationsbesteuerung - Finanzpolitische Konsequenzen aus der Existenz agglomerationsbedingter sozialer Kosten, in: Ballung und öffentliche Finanzen, hrsg. von der ARL, Forschung- und Sitzungsberichte, Band 134, Hannover 1980, S. 113-150. 
Generell ist im Zusammenhang mit der Ermittlung der normierten Finanzkraft zwischen der Internalisierung von Kostenunterschieden bei der Deckung des Normbedarfs und der umfassenderen Internalisierung sämtlicher externer Kosten (und Vorteile) zu unterscheiden. Die Forderung, daß Gemeinden mit ihren Einnahmen höhere Kosten selbst aufbringen und entsprechend höhere Einnahmen erzielen sollen, kann sich nur auf jene Kosten beziehen, die auch in den Norm-Bedarf eingehen. So wären etwa siedlungsstrukturell bedingte Mehrkosten des Polizeischutzes in bestimmten Gemeinden (einer nicht-kommunalen Aufgabe) weder dem Norm-Bedarf zuzurechnen, noch wäre eine Internalisierung über höhere Gemeindesteuersätze und eine Einbeziehung potentieller Mehreinnahmen in die Norm-Finanzkraft sinnvoll.

Anders als im Falle identischer normierter Steuersätze lassen sich die interkommunalen Verteilungswirkungen "kostenorientierter" Steuersätze auf Gemeinden mit unterschiedlichen Steuersatzspielräumen nicht mehr eindeutig ableiten. Wenn man davon ausgehen könnte, daß gerade in jenen Gemeinden, die insgesamt höhere Steuersatzspielräume haben, auch die Kosten der Bedarfsdeckung höher sind, so wäre die kostenorientierte Steuersatznormierung "verteilungsfreundlicher" als die Normierung auf gleiche Steuersätze.

Zunächst hängt es natürlich von der Formulierung des Normbedarfs ab, ob und wie die Kosten der Bedarfsdeckung mit der Konkurrenzposition und dem Einnahmepotential einer Gemeinde zusammenhängen. Man könnte etwa dann einen solchen Zusammenhang vermuten, wenn die günstige Konkurrenzposition bestimmter Gemeinden daraus resultieren würde, daß sich hier ein größerer Anteil der privaten Kosten im normierten Finanzbedarf niederschlägt. Verlangt man nun gleichzeitig von den Gemeinden, daß sie zur Dekkung ihres Bedarfs die angesiedelten Wirtschaftssubjekte höher belasten, wäre damit eine "Rückinternalisierung" gefordert; die günstigere Konkurrenzposition würde geschwächt. Allerdings erscheint die These vom Zusammenhang zwischen Konkurrenzposition und privaten Externalisierungsmöglichkeiten doch recht gewagt.

Eine andere Argumentation könnte einen Zusammenhang zwischen Gemeindegröße, den Kosten der Bedarfsdeckung und den konkurrenzbedingten Möglichkeiten der Steuersatzanspannung postulieren. Eine ähnliche Bezie- 
hung wurde bereits von Arnold Brecht formuliert. ${ }^{48)}$ Aber auch in dieser Form ist die These kaum für alle Gemeinden haltbar, wenn sie auch für den Durchschnitt zutreffen mag. Als Gegenbeispiele können sowohl altindustrialisierte Ballungsgemeinden gelten, in denen zwar ebenso wie in anderen Ballungsraumgemeinden Ballungskosten bei der kommunalen Bedarfsdeckung durchschlagen, in denen die Steuersatzspielräume jedoch deutlich enger als in gut strukturierten kleineren Gemeinden sein dürften. Aber auch in ländlichen, konkurrenzschwachen Gemeinden mit sehr begrenzten Steuersatzspielräumen treten - zumindest in einigen Aufgabenbereichen, wie etwa dem Bereich Verkehr - siedlungsstrukturell bedingt überdurchschnittliche Kosten auf. ${ }^{49}$ )

Wenn die Festlegung der normierten Steuersätze nach Maßgabe der Kosten der Bedarfsdeckung vorgenommen würde, könnten also Fälle auftreten, in denen Gemeinden mit engen Steuersatz-Spielräumen hohe Norm-Steuersätze angerechnet, konkurrenzstarken Gemeinden jedoch nur niedrige oder durchschnittliche Steuersätze angerechnet würden.

Unter Allokationsgesichtspunkten ist der beschriebenen Form der Steuersatznormierung bei der Ermittlung der normierten Finanzkraft der Gemeinden sicher der Vorzug zu geben. Raumordnungspolitische Zielsetzungen, die besonders darauf ausgerichtet sind, konkurrenzschwache Gemeinden dadurch zu fördern, daß zur Finanzierung des Normbedarfs von ihnen die Durchsetzung eines niedrigeren Steuersatzes gefordert wird als von anderen Gemeinden, lassen sich mit der beschriebenen äquivalenzorientierten Normierung allerdings nur zum Teil realisieren.

Eine solche Zielsetzung wird nur dann gesichert erfüllt, wenn die Normierung der Steuersätze direkt nach Maßgabe der Steuersatzspielräume erfolgt. Damit würde gegenüber den Gemeinden eine Art "Leistungsfähigkeitsprinzip" geltend gemacht: Zur Finanzierung des festgelegten Normalbedarfs wäre von den Gemeinden die Durchsetzung eines um so höheren Steuersatzes zu fordern, je größer ihr Steuersatzspielraum und je höher der fiskalisch optimale Hebesatz ist. Dabei wären verschiedene Varianten denkbar:

48) Brecht, Arnold, Internationaler Vergleich der öffentlichen Ausgaben, Leipzig und Berlin 1932.

49) Vgl. auch Zimmermann, Horst, Hardt, Ulrike und Postlep, Rolf-Dieter, Bestimmungsgründe der kommunalen Finanzsituation, a.a.O., S. 278 ff. 
- So könnte etwa angestrebt werden, daß in allen Gemeinden der gleiche Prozentsatz der Einnahmemöglichkeiten aus den zur Deckung des Normbedarfs vorgesehenen Steuern zu dessen Finanzierung selbst aufgebracht werden soll.

- Eine andere Variante könnte so aussehen, daß allen Gemeinden nach Abzug ihres Finanzierungsbeitrags die gleiche Summe (etwa je Einwohner) zur Finanzierung freiwilliger Aufgaben verbleiben soll.

Weitere Varianten können, je nach Zielsetzung, entwickelt werden.

Die Orientierung der normierten Steuersätze an den kommunalen Steuersatzspielräumen wird von einigen der Kritiker der Steuerkraftmessung im kommunalen Finanzausgleich der Bundesrepublik Deutschland als ideale, wenn auch - wegen der Schwierigkeiten der empirischen Ermittlung - praktisch nicht realisierbare Konzeption der Steuerkraftmessung angesehen. ${ }^{50}$ ) Betrachtet man allein das Verhältnis der Gebietskörperschaften zueinander und die relativen Positionen der einzelnen Gemeinden auf der untersten Gebietskörperschaftsebene, so erscheint dieser Ansatz vor allem unter "Gerechtigkeitsüberlegungen" als plausibel. Problematisch ist hier allerdings die Rechtfertigung eines solchen "kommunalen Normalverhaltens" gegenüber den Steuerzahlern.

Die Übertragung der marktmäßigen Äquivalenz - um nichts anderes handelt es sich hier - auf den Bereich der Steuern hätte zur Konsequenz, daß selbst für den Fall, daß die Kosten der Bedarfsdeckung in allen Gemeinden gleich wären, unter Normbedingungen in der einen Gemeinde höhere Steuern zu zahlen wären als in der anderen, nur weil sie sich in den von kommunalen Aktivitäten unabhängigen Standort- und Wohnortbedingungen wie etwa der räumlichen Lage oder dem Verdichtungsgrad unterscheiden. Grundlage der differenzierten Norm-Steuersätze ist allein das Ausweichverhalten der Zensiten.

Differenzierungen dieser Art werden vom Steuerrecht, aber auch in der Diskussion um die Grundsätze der Besteuerung üblicherweise für unzulässig

50) Vgl. 2. Kapitel. 
gehalten $^{51)}$, weil sie dem Prinzip der horizontalen Steuergerechtigkeit widersprechen. Überdies sprechen wirtschaftspolitische Gründe gegen die Verwirklichung des beschriebenen Konzepts: Zum einen würden die in den konkurrenzstarken Gemeinden ansässigen Wirtschaftssubjekte besonders hart betroffen, ohne gleichzeitig einen Vorteil aus den erhöhten Steuerforderungen zu haben, zum anderen kann gerade die höhere Belastung der standortgebundenen, besonders leistungsfähigen Wirtschaftssubjekte in wenigen, besonders konkurrenzstarken Gemeinden unerwünscht sein. Zumindest unter Wachstumsaspekten empfiehlt sich eine so geartete Differenzierung der Normsteuersätze daher nicht.

Mit der zweiten oben gestellten Frage wurde das Niveau der Steuersätze angesprochen. Mit ihrer Festlegung wurden der Selbstfinanzierungsgrad der Gemeinden und - soweit den Gemeinden mehrere Steuerquellen zugewiesen werden - die Struktur der gruppenspezifischen Beiträge zur Deckung des normierten Finanzbedarfs bestimmt.

Der Selbstfinanzierungsgrad der Gemeinden ergibt sich als Anteil der normierten Finanzkraft am normierten Finanzbedarf. Je niedriger also die Normsteuersätze angesetzt werden, desto größer ist bei gegebener Aufgabenverteilung das Finanzierungsdefizit, das von übergeordneten Gebietskörperschaften anerkannt wird und dementsprechend auszugleichen ist. Damit kann zum einen ein Lenkungseffekt hinsichtlich des Ausgabenumfangs erzielt werden, zum anderen wirkt sich die Steuersatznormierung auf die horizontalen Verteilungspositionen der einzelnen Gemeinden aus.

Der genannte Lenkungseffekt besteht darin, daß - zumindest im hier betrachteten Normbereich der Haushaltsgestaltung - mit der Höhe der normierten Steuersätze der Entscheidungsspielraum der Gemeinden mehr oder weniger beschnitten werden kann. Niedrige Soll-Anspannung der Steuersätze führt zu einer anteilig entsprechend höheren Finanzierung über Schlüsselzuweisungen. Anders als über die Steuereinnahmen können die Gemeinden über Schlüsselzuweisungen nicht entscheiden; zwar bleibt ihnen die Entschei-

51) Vgl. auch Birk, Dieter, Das Leistungsfähigkeitsprinzip als Maßstab der Steuernormen, Köln 1983, S. 170 ff. Mit Bezug auf die Entgelte wird eine marktmäßige Äquivalenz hingegen nicht abgelehnt, weil die Zahler eine Gegenleistung in Anspruch nehmen (können), die ihnen einen Sondervorteil gegenüber der Gesamtheit der Staatsbürger verschafft. 
dung über die Art, nicht aber über die Höhe der Ausgabetätigkeit. Mit der Normierung der Steuersätze und mittels Schlüsselzuweisungen kann über eine Regelung des pflichtig zu erfüllenden Aufgabenbereichs hinaus ein Mindestniveau kommunaler Aktivität gesichert werden.

Horizontale Wirkungen der Steuersatznormierung ergeben sich aus der Gewichtung von Bemessungsgrundlagenunterschieden zwischen den Gemeinden. Je niedriger die normierten Steuersätze angesetzt werden, um so stärker werden die Bemessungsgrundlagenunterschiede verwischt:

Geht man etwa von zwei Gemeinden mit gleichem normierten Bedarf von $300 \mathrm{DM}$ und Steuerbemessungsgrundlagen von $1000 \mathrm{DM}$ in Gemeinde A, $500 \mathrm{DM}$ in Gemeinde B aus, so errechnet sich bei einem einheitlichen normierten Steuersatz von $10 \%$ eine Steuerkraft von 100 DM in A und von 50 DM in B. Bei vollem Ausgleich des Finanzierungsdefizits erhielte A 200 DM Schlüsselzuweisungen, B 250 DM. Bei einem normierten Steuersatz von $20 \%$ verringert sich naturgemäß der Zuschußbedarf insgesamt, da von den Gemeinden eine höhere Eigenfinanzierung erwartet würde. Außerdem verändert sich das Zuschußverhältnis auf 100 DM in A zu 200 in B. Bei einem normierten Steuersatz von $30 \%$ erhält Gemeinde A gar keine Schlüsselzuweisungen mehr, der Normbedarf kann allein aus eigenen Steuermitteln der Gemeinden finanziert werden. Gemeinde B wäre hingegen erst bei einem Steuersatz von $60 \%$ zur Selbstfinanzierung in der Lage. ${ }^{52)}$ Dabei wird unterstellt, daß sich mit steigendem Steuersatz die Summe der Bemessungsgrundlagen nicht verändert. Stellt man die Anpassungsreaktionen der Wirtschaftssubjekte in Rechnung, so läge der zur Selbstfinanzierung notwendige Steuersatz in beiden Gemeinden höher, weil der Verlust an Bemessungsgrundlagen durch die Steueranspannung kompensiert werden muß.

Der dargestellte horizontale Verteilungseffekt der Steuersatznormierung stellt sich in seinem Grundmuster auch in jenen Fällen ein, in denen die Normsätze zwischen Gemeinden differenziert werden.

52) Zu einem ähnlichen Ergebnis gelangt Postlep für den Fall, daß die Schlüsselmasse festliegt und nach Vergleich der Zuschußbedarfe der Gemeinden verteilt wird. Vgl. Postlep, RolfDieter, Räumliche Effekte der Steuerkraft- und Finanzbedarfsermittlung bei den Schlüsselzuweisungen, a.a.O., S. 220. 
Die horizontalen Verteilungseffekte können zum Zweck raumordnungspolitischer Steuerung eingesetzt werden, allein aus den interkommunalen Verteilungswirkungen läßt sich das Niveau der Steuersatznormierung jedoch nicht rechtfertigen. Ebenso wie für die Frage der Differenzierung zwischen Gemeinden muß auch die Höhe der normierten Steuersätze gegenüber den Steuerzahlern begründet werden. Dabei ist sowohl die absolute Steuerbelastung einer Zensitengruppe als auch das Verhältnis, in dem verschiedene Gruppen von Steuerzahlern zur Finanzierung des Normbedarfs herangezogen werden sollen, von Interesse.

Betrachtet man zunächst nur eine einzelne Steuer, so wären, ausgehend vom Äquivalenzprinzip, die normierten Steuersätze um so niedriger anzusetzen, je größer die externen Effekte, das heißt hier: die Vorteile der Allgemeinheit aus dem für die steuerzahlende Gruppe erbrachten Leistungsbündel eingeschätzt werden. Die Kosten jener Leistungen, die von besonderem überörtlichen Interesse sind, dürften dementsprechend nicht vollständig der jeweiligen Nutzergruppe - also den Steuerzahlern in den einzelnen Gemeinden angelastet werden, sondern wären $\mathrm{zu}$ mehr oder minder großen Teilen von der Allgemeinheit zu finanzieren.

Je größer dieser über allgemeine Zuweisung zu finanzierende Anteil des gemeindlichen Normbedarfs ist, desto schwächer fallen natürlich - wie oben gezeigt - die interkommunalen Ausgleichswirkungen der Steuersatznormierung aus. $\mathrm{Da}$ ein hoher Anteil überörtlich finanzierten Normbedarfs prinzipiell einnahmekräftige Gemeinden "begünstigt", ist dabei durchaus systemadäquat; dieses Ergebnis ist beabsichtigt und deckt sich mit der in Punkt B. II abgeleiteten Forderung, daß im Falle pflichtiger kommunaler Aufgabenerfüllung die erforderlichen Mittel vollständig von der übergeordneten Ebene und unabhängig vom Einnahmepotential der jeweiligen Gemeinde bereitgestellt werden müssen. Welche Prinzipien der Zentralstaat für die Aufbringung dieser Mittel zugrundelegt und welche regionalen Wirkungen sich daraus ergeben, ist dann eine andere, hier nicht zu klärende Frage.

Ebenfalls aus Äquivalenzüberlegungen wäre die Verteilung der gesamten, innerhalb der Gemeinden aufzubringenden normierten Steuereinnahmen auf verschiedene Gruppen von Steuerzahlern abzuleiten. Genau ge- 
nommen müßte sie sich aus der Normierung der einzelnen Steuersätze ergeben.

Als Norm könnte vorgesehen werden, daß jede Zensitengruppe für die Finanzierung derjenigen Leistungen und Einrichtungen des Normangebots aufkommen soll, die speziell für sie erbracht werden und aus denen sie Vorteile zieht (vgl. oben). Dabei tritt, wie angedeutet, ein Zurechnungsproblem auf. Viele kommunale Leistungen werden nicht nur von einer, sondern von mehreren Gruppen genutzt, wobei sich die Anteile kaum festlegen lassen; überdies stellt sich hier häufig die Frage, welchen Gruppen letztlich Vorteile aus der Nutzung erwachsen. Als Beispiel sei auf die Bereitstellung von Verkehrsinfrastruktur hingewiesen, die sowohl von Unternehmen als auch von Haushalten genutzt wird. Betrachtet man etwa die Fahrten der Arbeitnehmer zum Arbeitsplatz, so ist zwar die Frage der direkten Inanspruchnahme der Verkehrseinrichtungen relativ problemlos zu klären - es sind die Arbeitnehmer, die die Straßen und sonstigen Einrichtungen nutzen - allerdings profitieren die Unternehmen ebenfalls von der Existenz der Verkehrsinfrastruktur. Sogar wenn sie selbst kaum auf Transportleistungen angewiesen sind, ziehen sie Vorteile aus einer ausgebauten Verkehrsinfrastruktur, da die Standortbindung an die Wohnorte der Arbeitskräfte abgeschwächt wird und die stärkere Berücksichtigung anderer Kriterien der Standortwahl erlaubt.

Die Kosten solcher nicht zurechenbaren Leistungen - die betriebswirtschaftlich als Gemeinkosten gekennzeichnet werden - lassen sich nicht nach Äquivalenzüberlegungen auf die jeweiligen Verursacher verteilen, eine reine Äquivalenznorm ist deshalb nicht praktikabel. Vielmehr müssen hier anders festzulegende Verteilungskriterien gefunden werden.

Der Wissenschaftliche Beirat beim Bundesministerium der Finanzen schlägt als Normierungsmaßstab den Grundsatz des Interessenausgleichs vor. ${ }^{53)}$ "Der Gedanke des Interessenausgleichs geht (...) über die ökonomisch bestimmten Äquivalenzvorstellungen hinaus. Er basiert zwar auch auf dem Ansatz der Kompensation, bezieht sich jedoch auf den politischen Zusammenhang. ${ }^{\text {"54) }}$ So wird etwa darauf hingewiesen, daß (unvermeidbare) Beein-

53) Wissenschaftlicher Beirat beim Bundesministerium der Finanzen, Gutachten zur Reform der Gemeindesteuern, a.a.O., S. $32 \mathrm{ff}$.

54) Ebenda, S.35. 
trächtigungen der Lebensbedingungen der Bevölkerung durch die Produktion dadurch kompensiert werden könnten, daß für die Bevölkerung bestimmte kommunale Leistungen durch die Steuerzahlungen der Unternehmen finanziert werden. Wie ein solcher Interessenausgleich im einzelnen zu gestalten ist, ist damit allerdings noch klärungsbedürftig; er kann sehr unterschiedlich ausgestaltet und je nach den sozioökonomischen Bedingungen auch nach Gemeindetypen differenziert werden.

Neben den genannten Kompensationsargumenten können - besonders aus übergeordneter Sicht - gesamtwirtschaftliche Ziele und Wirkungen für die Steuersatznormierung eine Rolle spielen. So kann etwa Rücksicht auf die Reaktionsempfindlichkeit von Wirtschaftssubjekten und Steuerbemessungsgrundlagen genommen werden: Wird von bestimmten Steuerzahlern eine besonders intensive, aber unerwünschte sachliche Anpassung erwartet, so empfiehlt es sich unter diesem Aspekt, die Soll-Anspannung der Steuersätze durch die Gemeinden möglichst tief anzusetzen. Die dadurch bedingte Schmälerung der von den Gemeinden selbst erwarteten Finanzierung ist allerdings bei vorgegebenem Normbedarf zu kompensieren; entweder indem den Gemeinden nahegelegt wird, andere Gruppen und Bemessungsgrundlagen stärker zu belasten oder indem ein größerer Anteil des gesamten Finanzierungsbedarfs von übergeordneten Ebenen übernommen wird.

\section{Zusammenfassung}

Anders als mitunter angenommen, dient die Normierung von Entgelt- und Steuereinnahmen der Gemeinden im Rahmen der Finanzkraftmessung aus übergeordneter Sicht weniger der Vereinfachung und der Handhabbarkeit des Meßinstrumentariums. Vielmehr erfolgt sie mit dem Zweck, festzulegen, wieviele Einnahmen die Gemeinden unter den jeweiligen sozioökonomischen Verhältnissen und unter der Bedingung, daß sie sich bei der Haushaltsgestaltung gemäß übergeordneten Zielvorstellungen verhalten, aus eigenen Quellen zur Finanzierung des Normangebots aufbringen sollen. Dabei sind der Gestaltung der Normen kaum Grenzen gesetzt; im einzelnen hängt es allein von den Zielvorstellungen der übergeordneten Ebenen ab, welche Gestalt die 
Normierung hat bzw. haben sollte, sofern die verfolgten Ziele widerspruchsfrei sind.

Zielkonflikte lassen sich allerdings auch durch die Normierung nicht auflösen. Wird etwa ein für alle Zensiten gleicher Steuersatz angestrebt, so sind überall gleiche Normsteuersätze zur Ermittlung der kommunalen Finanzkraft zu verwenden. $\mathrm{Da}$ in diesem Fall die unterschiedlichen Steuersatzspielräume der Gemeinden nicht abgebildet werden, kann nicht auf die Art der Messung zurückgeführt werden, sondern resultiert aus den der Normierung zugrundeliegenden Zielsetzungen, die solche unterschiedlichen Spielräume von vornherein akzeptieren und ihren Ausgleich gar nicht vorsehen.

\section{Integration unterschiedlicher Kompetenzverteilungen und segmentierte Messung von Finanzbedarf und Finanzkraft}

Sowohl bei der vollständig autonomen kommunalen Aufgabenerfüllung und der eigenständigen Finanzierung der anfallenden Ausgaben als auch bei alleiniger Zuständigkeit übergeordneter Ebenen für die lokale Aufgabenerfüllung wird ein Teil der wirtschafts- und finanzpolitischen Ziele des Staates nicht angemessen erfüllt.

So dürften im Falle unreglementierter gemeindlicher Aktivitäten unerwünschte regionale Disparitäten auftreten und - soweit mit dem Leistungsangebot räumlich externe Effekte auftreten - ein unter Allokationsgesichtspunkten insgesamt zu niedriges Angebot öffentlicher Güter erstellt werden. Die Finanzierung nach dem Trennsystem verhindert überdies die Schaffung eines geschlossenen Steuersystems und erschwert eine Einbeziehung der Gemeinden in staatliche Politik, wie etwa die konjunktur- und wachstumspolitische Lenkung. ${ }^{55)}$

Andererseits sind bei vollständig zentraler Aufgabenerfüllung vor allem allokative Nachteile zu erwarten. Die Normierung kommunaler Aufgaben-, Ausgaben- und Einnahmetätigkeit stellt nun einen Kompromiß zwischen die-

55) Vgl. auch Limbert-Michaelis, Helga, Neuere Entwicklung in der Theorie des Finanzausgleichs, a.a.O., S. 19 f. 
sen beiden extremen Varianten der Kompetenzzuweisung dar, der die Vorteile beider vereinigen soll.

Allerdings ist eine isolierte Verwirklichung des "Normkonzepts" nicht denkbar; es schließt die Existenz eines autonomen Bereichs kommunaler Aktivitäten von vornherein ein:

Eine Normierung der Finanzierungsaktivitäten in der beschriebenen Weise ist nur dann sinnvoll, wenn den Gemeinden außerhalb des Normbereichs weitere, vollständig selbstverantwortlich zu gestaltende Aufgabenbereiche belassen werden. Ein Verbot anderer als der Normaktivitäten käme dem weiter oben untersuchten Zweckzuweisungssystem gleich, in dem die Gemeinden zwar auch nicht zur Erfüllung der einzelnen Aufgaben gezwungen werden, die eingenommenen Mittel aber nur den übergemeindlichen Vorstellungen entsprechend verwenden können, bzw. überhaupt nur dann Mittel einnehmen, wenn sie sich zielkonform verhalten. Damit wird der Entscheidungsspielraum der Gemeinden stark eingeschränkt, wenn nicht sogar beseitigt, was mit der Normierung jedoch nicht beabsichtigt ist.

Bei der Normierung der kommunalen Aktivitäten ist - damit der Kompromißcharakter dieser Lösung erhalten bleibt - deshalb sowohl bei der Vorgabe von Leistungsanforderungen an die Gemeinden als auch bei der Konstruktion des Einnahmesystems auf den Einbau gemeindlicher Freiräume zu achten. Wie groß die kommunalen Entscheidungsspielräume sind, hängt also nicht zuletzt von der Gestaltung des Einnahmesystems innerhalb des Normbereichs ab.

Hier kann bereits eine Kombination der verschiedenen Finanzierungsmittel mit unterschiedlichem Autonomiegrad vorgesehen werden, ohne daß damit der gemeindliche Entscheidungsspielraum wesentlich eingeengt wird. So können eigene Einnahmequellen etwa dann durch Zweckzuweisungen ergänzt werden, wenn anzunehmen ist, daß der Finanzbedarf im Zeitablauf stark schwankt und unregelmäßig anfallende Spitzenbelastungen, wie sie beispielsweise durch Investitionen hervorgerufen werden, nur mit Schwierigkeiten abgedeckt werden können. ${ }^{56)}$ Ebenso kann ein Teil des Normbedarfs über

56) So werden in Nordrhein-Westfalen offenbar in kleinen Gemeinden eigene Einnahmen teilweise durch Zweckzuweisungen ersetzt, um die sporadisch anfallende Investitionstätigkeit zu erleichtern. Vgl. dazu Wienen, Horst-Jürgen, Räumliche Verteilung der Zuweisun- 
Schlüsselzuweisungen finanziert werden, wenn den Gemeinden ein Mindestsockel an Ausgabentätigkeit - etwa im Bereich funktionsnotwendiger Verwaltungsdienste - gesichert werden soll, unabhängig davon, welche Präferenzen die angesiedelten Wirtschaftssubjekte hinsichtlich des spezifischen kommunalen Leistungsangebots äußern.

Neben dem normierten und dem autonomen Bereich wird aber aus einem Mißtrauen in die Funktionstüchtigkeit des dezentralen Preismechanismus heraus ein Bereich pflichtiger, übergemeindlich reglementierter Aufgabenerfüllung treten.

Festzuhalten bleibt, daß der Einteilung der Aufgaben nach ihrem Autonomiegrad in einer rationalen förderativen Kompetenzverteilung eine ebensolche Zuteilung von Einnahmen bzw. Einnahmekompetenzen entspräche. Oder anders formuliert: "Ausgangspunkt einer rechtlichen Eingrenzung der Finanzhoheit muß dabei sein, daß sie als Korrelat zur Aufgabenverantwortlichkeit keinen über die reale, abgestufte Verantwortung im Aufgabenvollzug hinaus gehenden Gestaltungsanspruch erheben kann. ${ }^{\text {.57) }}$

In einem solchen abgestuften System ist die gesamthafte umfassende $\mathrm{Be}$ stimmung der gemeindlichen Einnahmemöglichkeiten ebenso wie eine umfassende Bedarfsbestimmung aus übergeordneter Sicht nicht möglich, aber auch überhaupt nicht erforderlich. Sinnvoll ist nur eine Betrachtung der einzelnen, nach dem Autonomiegrad einzuteilenden Segmente kommunaler Aktivitäten.

- Im Bereich der Pflichtaufgaben ist die Erstattung der anfallenden Ausgaben angemessen. Unabhängig von der eigenen Leistungsfähigkeit der Gemeinde wäre hier nur der aus der Aufgabenvorgabe resultierende Ausgabenbedarf zu bestimmen und den Gemeinden die notwendigen Einnahmen zur Verfügung zu stellen. Eine Messung kommunaler Einnahmemöglichkeiten ist in diesem Bereich überflüssig; die zur Erfüllung von Auftragsangelegenheiten und Pflichtaufgaben nach Weisung an die Gemeinden weitergeleiteten Einnahmen wären bei der Messung der Fi-

gen und Baumaßnahmen des Landes Nordrhein-Westfalen 1969-1975, in: Ballung und öffentliche Finanzen, hrsg. von der ARL, Forschungs- und Sitzungsberichte, Bd. 134, Hannover 1980, S. 97-111, hier S. $101 \mathrm{f}$.

57) Rosenschon, Jürgen Ekkehard, Gemeindefinanzsystem und Selbstverwaltungsgarantic, a.a.O., 1980, S. 18. 
nanzkraft zur Deckung der normierten Ausgabenanforderungen natürlich auch nicht zu berücksichtigen.

Horizontale Wirkungen sind hier allein durch die Festlegung des Bedarfs bedingt und allein darüber zu steuern. Die Zuweisung von Einnahmen dient ausschließlich dem vertikalen Ausgleich, der der Bedarfsfestlegung folgt.

- Für den Bereich der Normaufgaben, deren Wahrnehmung durch die Gemeinden eine gewisse Normalausstattung sichern soll, wären den Gemeinden geeignete Einnahmequellen zuzuweisen, über deren Ausschöpfung ihnen ein Mitspracherecht zugestanden und ihnen damit die Möglichkeit eingeräumt werden sollte, die Aufgabenwahrnehmung an die regionalen Präferenzen anzupassen. Die Finanzkraft umfaßt dabei die Erträge jener Einnahmequellen, die den Gemeinden zur Deckung des Normalbedarfs zugewiesen worden sind, wenn diese Quellen in einem als normal oder erwünscht festgelegten Ausmaß in Anspruch genommen werden. Allein in diesem Bereich ist eine Gegenüberstellung von Finanzbedarf und Finanzkraft sinnvoll; sie dient gleichzeitig der Erfassung vertikaler und horizontaler Wirkungen.

- Für den autonom zu gestaltenden Bereich könnte den Gemeinden die Finanzierung selbst überlassen bleiben. Aus übergeordneter Sicht wird die Finanzierungsfähigkeit der Gemeinden - im Rahmen der bestehenden Gesetze - durch die Leistungsfähigkeit der Wirtschaftssubjekte begrenzt. Soll hier ein - allein der Veränderung horizontaler Positionen dienender Ausgleich erfolgen, so ist er ausschließlich an dieser Leistungsfähigkeit auszurichten; eine Bedarfsmessung ist nicht notwendig. Alle aus gemeindeindividueller Sicht vorhandenen Einnahmemöglichkeiten, die dem autonomen Bereich zuzuordnen sind - etwa Gebühren für die Inanspruchnahme freiwillig erbrachter kommunaler Leistungen - sind bei der Bestimmung des "Normpotentials" außer acht zu lassen.

In den drei hier grob eingeteilten Segmenten hätte also jeweils eine eigenständige Messung zu erfolgen. Die Zwecke sind dabei höchst unterschiedlich und die ermittelten Werte sind nicht aufsummierbar.

Von den drei genannten, nach dem Grad kommunaler Autonomie unterteilten Aufgabenbereichen ist - zumindest hinsichtlich der Bestimmung von 
Finanzbedarf und Finanzkraft - der zweite, in dem die Kompetenzen der Aufgabenwahrnehmung und der Finanzierung zwischen über- und untergeordneter Ebene geteilt sind, derjenige, der die größten Probleme aufwirft. Unter der Voraussetzung einer symmetrischen Kompetenzverteilung zwischen den föderativen Ebenen - unter der die übergeordnete Ebene jeweils in dem Maße, wie sie über Aufgabenerfüllung und Ausgabetätigkeit entscheidet, auch für die Finanzierung zuständig ist - könnten jedoch jedem der nach dem Rechtscharakter gegliederten Aufgabenbereiche bestimmte Einnahmearten mit gleichem Autonomiegrad zugeordnet werden. Die Frage, welche der kommunalen Einnahmequellen bei der Ermittlung der "normierten Finanzkraft" einzubeziehen wären, könnte damit ohne weitere Schwierigkeiten beantwortet werden: diejenigen, die zur Deckung des Normbedarfs dienen sollen.

Zur Messung der Finanzkraft wäre dann festzustellen, wo der staatlicherseits erwünschte Ausschöpfungsgrad der den Gemeinden zur Deckung des Normbedarfs zugewiesenen Einnahmequellen liegen soll und bei welchen Abgabesätzen eben dieser Ausschöpfungsgrad in den einzelnen Gemeinden erreicht ist.

Bei kongruenter Kompetenzverteilung ließe sich das dargestellte Konzept der segmentierten Bestimmung der kommunalen Finanzkraft allein mit Blick auf die Einnahmen bzw. die Einnahmekompetenzen umsetzen. Zusätzliche Probleme treten allerdings bei inkongruenter Kompetenzverteilung auf: zum ersten ist einzelnen Einnahmearten nicht mehr ohne weiteres anzusehen, in welches Segment sie einzuordnen sind; zum zweiten wird durch eine inkongruente Kompetenzverteilung der Zusammenhang zwischen Aufgaben-, Ausgaben- und Einnahmeentscheidung durchbrochen. Dies wirkt sich auch auf die Meßanforderungen aus. So kann etwa der pflichtige Bereich kommunaler Aktivitäten mit Blick auf die Bestimmung der normierten Finanzkraft dann nicht außer acht gelassen werden, wenn pflichtige Aufgaben und Ausgaben aus Mitteln gedeckt werden müssen, die die Gemeinden in eigener Verantwortung beschaffen müssen. In diesem Fall treten Meßprobleme auf, die auf die - letztlich "irrationale" - Konstruktion des Gesamtsystems zurückzuführen sind und die sich allein mit der Modifikation der Meßkonzepte nicht lösen lassen. 
Im 5. Kapitel wird für den Fall der Bundesrepublik Deutschland diese Problematik aufzuzeigen versucht.

\section{E. Ein Rückblick: "Kommunales Einnahmepotential" und "normierte Finanzkraft" - Ein Vergleich der gemeindlichen und der staatlichen Interessen bei der Bestimmung der kommunalen Einnahmemöglichkeiten}

Aus gemeindlicher Sicht dient die Messung des Einnahmepotentials dazu, abzuschätzen, wieviele Einnahmen zur Finanzierung von Ausgaben insgesamt aufgebracht werden können. Eine solche Messung müssen natürlich nur $\mathrm{Ge}$ meinden vornehmen, die selbständig Einnahmen beschaffen, über deren Höhe sie mitentscheiden können. Liegen alle Einnahmen von vornherein fest, so erübrigt sich zwar nicht unbedingt deren Vorausberechnung, wohl aber die Abschätzung potentieller Finanzierungskapazitäten.

Die Sicht ganz oder teilweise selbstverantwortlich wirtschaftender Gemeinden ähnelt der eines Unternehmens: Ausgehend von den jeweiligen konkreten Bedingungen - der Konkurrenzposition gegenüber anderen Gemeinden, der Leistungsfähigkeit und den Dispositionen der angesiedelten Wirtschaftssubjekte sowie den Restriktionen des Staates - werden Finanzierungsspielräume ausgelotet. Dieser Vorgang ist prinzipiell für alle Einnahmearten und -quellen der gleiche, wenn auch mit unterschiedlichen Schwierigkeiten verbunden. Welche Ziele der Staat mit der Zuweisung von bestimmten Einnahmen an die Gemeinden verfolgt, bestimmt zwar mit über die Grenzen der Einnahmetätigkeit und die Höhe des Einnahmepotentials, spielt aber für dessen Messung aus der Sicht der Gemeinden keine Rolle.

Aus staatlicher Sicht sind diese Ziele hingegen entscheidend für das, was unter der gemeindlichen Finanzkraft verstanden und was gemessen werden soll. Das Objekt, für das sich übergeordnete Gebietskörperschaften interessieren, ist je nach Zielsetzung und der daran anknüpfenden internen Staatsorganisation ein ganz anderes als das, was die Gemeinden zum Zweck der Haushaltsplanung zu messen oder abzuschätzen versuchen.

Ziel einer staatlichen Messung der "kommunalen Finanzkraft" ist nicht, die gesamten Einnahmemöglichkeiten der einzelnen Gemeinde in vollem 
Umfang und der tatsächlich realisierbaren Höhe zu quantifizieren. Vielmehr soll ermittelt werden, was die Gemeinden an Mitteln aufbringen können, wenn sie sich den staatlichen Zielsetzungen gegenüber konform verhalten.

Für den Fall vollständig autonomer Gemeinden bestehen keine bestimmten Anforderungen des Staates gegenüber der untergeordneten Ebene. Hier wird die angestrebte Allokation von Kollektivgütern allein dem Wirken eines regionalen Preismechanismus überlassen und den Gemeinden keinerlei Beschränkung - direkter oder indirekter Art - auferlegt. Aus staatlicher Sicht stößt daher auch das zu ermittelnde "kommunale Einnahmepotential" nur an die materielle Grenze des regional vorhandenen Reichtums: des produzierten Einkommens und des angesammelten Vermögens, sofern es nicht bereits für staatliche Zwecke beansprucht wird. Tatsächlich ist für die Kommunen diese Summe nicht verfügbar, solange die angesiedelten Wirtschaftssubjekte nicht sämtliche Bedürfnisse kollektiv befriedigen wollen. Die gemeindeindividrellen Präferenzstrukturen und die daraus resultierenden Beschränkungen kommunaler Einnahmemöglichkeiten sind jedoch aus übergeordneter Sicht nicht von Interesse.

Im zweiten Extrem einer völlig weisungsgebundenen Kommunalebene ist eine Messung des Einnahmepotentials weder aus gemeindlicher noch aus übergeordneter Sicht notwendig. Hier ist allein die Bestimmung des Finanzbedarfs erforderlich, dessen Deckung der übergeordneten Ebene überlassen bleibt.

Eine Bestimmung und Gegenüberstellung von Finanzbedarf und "Finanzkraft" erweist sich allein im Falle einer Normierung kommunaler Ausgaben- und Einnahmetätigkeit als sinnvoll. Ausgaben- bzw. Finanzbedarf und Einnahmemöglichkeiten sind hier allerdings anders definiert als für die beiden oben genannten Extremfälle. Die normierte Finanzkraft umfaßt jene Einnahmen, die die Gemeinden aus den ihnen zur Deckung des "Normbedarfs" besonders zugewiesenen Einnahmequellen erwirtschaften können, wenn sie sich den staatlichen Zielsetzungen hinsichtlich der Aufgabenerfüllung, der Ausgabentätigkeit und der Inanspruchnahme der genannten Einnahmequellen unterwerfen. Die Normierung der Einnahmetätigkeit schließt auch eine Normierung der Steuersätze und der Entgeltsätze ein, die die Gemeinden nach staatlichen Vorstellungen realisieren sollen. 
Mit der Normierung wird nicht ausgeschlossen, daß sich die Gemeinden anders verhalten und sich etwa aus zusätzlichen anderen Quellen oder durch stärkere Anspannung der Steuersätze weitere Mittel beschaffen. In die Messung der normierten Finanzkraft werden diese Einnahmemöglichkeiten allerdings nicht aufgenommen.

Das aus gemeindlicher Sicht zu ermittelnde Einnahmepotential jeder einzelnen Einnahmeart stellt für die jeweilige Gemeinde eine nicht zu ubberschreitende Obergrenze der Einnahmen dar und bildet deshalb auch die Obergrenze jeder Normierung. Da es unsinnig wäre, von den Gemeinden einen über ihre tatsächliche Finanzierungsfähigkeit hinausgehenden Beitrag zur Abdeckung des Normbedarfs zu fordern, muß sich die Normierung der Einnahmepolitik an den Möglichkeiten der schwächsten Gemeinden orientieren. Hier besteht deshalb auch aus staatlicher Sicht ein Interesse an der Erfassung des kommunalen Einnahmepotentials. Allerdings bezieht es sich nicht auf sämtliche Einnahmearten, sondern nur auf jene, die zur Deckung des Normbedarfs dienen sollen.

In der Regel wird die normierte Finanzkraft jedoch unterhalb dieses Potentials anzusetzen sein und die für angemessen gehaltenen Steuer- und Entgeltsätze entsprechend niedrig festgelegt, damit allen Gemeinden ein gewisser Spielraum zur Modifikation der Bedarfsdeckung verbleibt.

Anders sieht das staatliche Interesse an den gemeindlichen Finanzierungsmöglichkeiten aus, wenn es um die Erklärung kommunalen Handelns geht: $\mathrm{Da}$ das Einnahmepotential eine wesentliche Determinante gemeindlicher Aktivitäten ist, kann seine Erfassung in diesem Zusammenhang auch aus staatlicher Sicht durchaus sinnvoll sein. 
Ulrike Hardt - 978-3-631-75265-4

Downloaded from PubFactory at 01/11/2019 06:31:57AM

via free access 


\section{Kapitel: Probleme einer konsistenten Bestimmung der Finanzkraft im System des kommunalen Finanzausgleichs in der Bundesrepublik Deutschland}

\section{A. Ansatzpunkte der Finanzkraftbestimmung}

Im 2. Kapitel, das sich der Kritik der praktizierten Steuerkraftmessung widmete, wurden verschiedene vermeintliche Unzulänglichkeiten dieses Meßkonzepts herausgestellt. Danach

- bleiben mit der Steuerkraftmessung wichtige kommunale Einnahmearten unberücksichtigt,

- werden bei den einbezogenen Steuern insgesamt zu niedrige (nämlich durchweg unter den Durchschnittshebesätzen liegende) Hebesätze zugrundegelegt,

- werden interkommunale Unterschiede in den Steuersatzspielräumen vernachlässigt.

Nach den Ausführungen im 3. und 4. Kapitel können diese Kritikpunkte jedoch nicht mehr uneingeschränkt aufrecht erhalten werden. Sie beziehen sich in ihrer Mehrzahl darauf, daß die ermittelte Steuerkraft das gemeindliche Einnahmepotential nicht richtig abbildet. Nun wurde in den vorausgegangenen Kapiteln herausgearbeitet, was mit den Begriffen des "Einnahmepotentials" und der "Finanzkraft" gemeint ist und daß beide - von Ausnahmefällen einmal abgesehen - nicht den gleichen Gegenstand bezeichnen. Insofern ist es also auch nicht von vornherein als ein Mangel zu kennzeichnen, wenn die Steuerkraftmeßzahl die gemeindlichen Einnahmemöglichkeiten nicht richtig mißt.

Als Anforderung muß vielmehr geltend gemacht werden, daß sie die normierte Finanzkraft, eine durch staatliche Ziele bestimmte Größe, richtig abbildet. Zur Beurteilung ihrer Qualitäten als Maßstab ist deshalb die ge- 
meindliche "Finanzkraft" aus dem System des kommunalen Finanzausgleichs der Bundesrepublik Deutschland heraus zu konkretisieren.

In den Grundzügen entspricht das hier institutionalisierte System dem im 4. Kapitel beschriebenen, aus unterschiedlich stark reglementierten Teilsegmenten zusammengesetzten System. Für die Messung der Finanzkraft wären deshalb diese Segmente zu unterscheiden und darauf die jeweiligen besonderen Meßkonzepte anzuwenden.

Allerdings läßt sich die Segmentierung nicht allein anhand der Merkmale der Einnahmequellen vornehmen. Bereits im 2. Kapitel wurde darauf hingewiesen, daß ihnen - zumindest soweit es sich um allgemeine Deckungsmittel handelt - nicht unmittelbar anzusehen ist, für welche Zwecke sie nach staatlichen Vorstellungen verwendet werden sollen.

Ein Versuch zur Bestimmung der gemeindlichen Finanzkraft müßte deshalb für das konkrete föderative System der Bundesrepublik von der vertikalen Aufgabenverteilung ausgehen, um mit mehreren Schritten, die den Aufbau des 5. Kapitels vorgeben, zu einer Annäherung an den zu ermittelnden Gegenstand zu gelangen:

(1) Zunächst ist festzustellen, ob, und wenn ja, welche Segmente kommunaler Aktivitäten nach dem Autonomiegrad gemeindlichen Handelns zu unterscheiden sind und welche Aufgabenbereiche den einzelnen Segmenten zuzuordnen sind.

(2) In einem weiteren Schritt müßte versucht werden, die verschiedenen Einnahmearten ebenfalls den abgegrenzten Segmenten zuzuordnen. Damit kann eine Aussage darüber getroffen werden, welche der kommunalen Einnahmequellen bei der Erfassung der normierten Finanzkraft zu berücksichtigen sind. Für die zweckgebundenen Einnahmen ist eine solche Zuordnung unproblematisch, schwierig ist sie hingegen für die allgemeinen Deckungsmittel. Gerade ihre Zuordnung ist jedoch für eine zielgerechte Normierung - vor allem der Steuersätze - unabdingbar.

(3) Zuletzt wäre nach jenen Richtwerten zu fragen, die innerhalb des Normbereichs für die kommunale Einnahmepolitik, insbesondere die Hebesatzpolitik aufgestellt werden, oder, soweit dies bisher nicht geschieht, aufgestellt werden könnten. Diese Werte zeigen, welches gemeindliche 
Verhalten als zielgerecht angesehen wird und sind bei der Finanzkraftermittlung als Normalsätze zugrunde zu legen.

Wie sich zeigen wird, lassen sich diese Schritte kaum oder nur mit großen Problemen vollziehen. Eine konsistente, unter den vorgegebenen Zielen "richtige" Messung der normierten gemeindlichen Finanzkraft kann letztlich nicht entwickelt werden. Andererseits werden jedoch einige Komponenten der Steuerkraftzumessung plausibel, die allein aus dem Blickwinkel der Gemeinden, die ihre gesamten Einnahmemöglichkeiten zu bestimmen versuchen, aber auch aus der Perspektive jener Kritiker, die das gemeindliche Einnahmepotential als den richtigen Maßstab für die Finanzkraftmessung im Rahmen des kommunalen Finanzausgleichs ansehen, völlig unverständlich bleiben.

\section{B. Zur Abgrenzung normierter gemeindlicher Aktivitäten im geltenden Finanzausgleich}

I. Die Gliederung der Aufgabenbereiche nach ihrem Autonomiegrad

Nach Art. 28 Abs. 2 GG wird den Gemeinden das Recht gewährleistet,

- alle Angelegenheiten der örtlichen Gemeinschaft (Kompetenzgegenstand)

- im Rahmen der Gesetze (Kompetenzgrenze für die Gemeinden und Kompetenzzusicherung an Bund und Länder)

- $\quad$ in eigener Verantwortung zu regeln (Kompetenzinhalt). ${ }^{1)}$

Damit wird kein enumerativ festgelegter Aufgabenkatalog umschrieben $^{2)}$, sondern das Feld kommunaler Aktivitäten offengehalten, wobei einige Aufgaben durch übergeordnete Gebietskörperschaften - durch den Bund und vor allem die Länder - geregelt und in ihrer Art oder in Art und Umfang vorgegeben werden. Die Aufteilungen vorgegebener Aufgaben auf die Gemein-

1) Zur Auslegung des Art 28 Abs. 2 GG vgl. etwa Pagenkopf, Hans, Kommunalrecht, Band 1, Verfassungsrecht, 2., neubearbeitete Auflage, Köln u.a. 1975, S. 57 ff.

2) Vgl. dazu Schmidt-Eichstädt, Gerd, Bundesgesetze und Gemeinden - Die Inanspruchnahme der Kommunen durch die Ausführung von Bundesgesetzen - Schriften des Deutschen Instituts für Urbanistik, Bd. 65, Stuttgart u.a. 1981, S. 34. 
den einerseits und das Land andererseits weichen allerdings von Bundesland zu Bundesland voneinander $\mathrm{ab}$. 3 )

Diese Unterschiede resultieren vor allem aus der Tatsache, daß die Aufgaben des Gesamtstaates zunächst nur zwischen den beiden Ebenen des Bundes und der Länder aufgeteilt werden. Die Gesetzgebungskompetenz liegt vielfach beim Bund, die Aufgabenerfüllung ist hingegen vorwiegend Angelegenheit der Länder. ${ }^{4)}$ Die Länder können nun ihre Aufgaben der Kommunalebene zuteilen, und zwar als Auftragsangelegenheiten oder als pflichtige Selbstverwaltungsaufgaben. Von dieser Möglichkeit wird jedoch in unterschiedlichem Umfang Gebrauch gemacht.

Betrachtet man die jeweiligen Bundesländer gesondert (hier wird nur die Kompetenzverteilung in Nordrhein-Westfalen zugrunde gelegt), so gibt die Unterteilung der gemeindlichen Aufgaben nach dem Pflichtigkeitsgrad einen Anhaltspunkt für die Zuordnung der Aufgaben-, Ausgaben und Einnahmeaktivitäten in die für die Finanzkraftmessung relevanten Segmente.

Die übliche Unterscheidung trennt nach Pflichtaufgaben, pflichtigen Selbstverwaltungsaufgaben und freiwilligen Selbstverwaltungsaufgaben. $\left.{ }^{5}\right)$ Während die Gemeinden bei den Pflichtaufgaben weitgehend weisungsgebunden sind, erfüllen sie die freiwilligen Selbstverwaltungsaufgaben eher autonom; der Zwischenbereich - die pflichtigen Selbstverwaltungsaufgaben könnten dann, wie im 4. Kapitel ausführlicher begründet, als "Normbereich" interpretiert werden, für den Finanzbedarf und Finanzkraft gemessen werden müssen:

- Für die Pflichtaufgaben, die die Auftragsangelegenheiten bzw. die Pflichtaufgaben nach Weisung sowie die Aufgaben zur Sicherung der verfassungsmäßigen Ordnung umfassen, ist die Aufgabenwahrnehmung

3) Die fiskalischen Wirkungen werden im einzelnen dargestellt bei Scheel, Werner, Untersuchung der Leistungen der Bundesländer an die Gemeinden (GV) in den Jahren 1970-77, hrsg. vom Finanzminister des Landes Nordrhein-Westfalen, Düsseldorf 1979, S. 16 ff.

4) Vgl. im einzelnen Schreyer, Michaele, Transferpolitik im Föderalismus - Probleme der Kompetenzverteilung, Frankfurt a.M.-Bern-New York 1983, S. 25 ff.

5) Zum Überblick vgl. Postlep, Rolf-Dieter, Wirtschaftsstruktur und großstädtische Finanzen, a.a.O., S. $230 \mathrm{ff}$. Je nach Bundesland unterscheidet sich auch die Terminologie zur gesetzlichen Trennung der genannten Bereiche. Dies ändert jedoch nichts an ihrer grundsätzlichen Einordnung. Vgl. dazu Schmidt-Jortzig, Edzard, Probleme der kommunalen Fremdverwaltung, a.a.O., S. 394 und Pagenkopf, Hans, Kommunalrecht, Bd. 1, Verfassungsrecht, a.a.O. S. $170 \mathrm{ff}$. 
weitgehend übergemeindlich festgelegt. Dabei handelt es sich zum Teil um Mindeststandards, die in allen Gemeinden realisiert weren müssen, auf freiwilliger Basis aber auch überschritten werden können (etwa im Einzelplan 4, Abschnitt 41/Sozialhilfe). Innerhalb des staatlich reglementierten Aufgabenbereichs verbleibt den Gemeinden jedoch kaum ein Entscheidungsspielraum. Bei kongruenter Kompetenzverteilung müßten die Kosten der entsprechenden Maßnahmen ebenfalls von übergeordneten Ebenen - je nach dem, wo die Gesetzgebungskompetenz liegt, also von Bund oder Land - übernommen werden. In diesem Bereich ist dann allenfalls eine Bedarfsmessung, aber keine Finanzkraftmessung erforderlich.

- Über die Wahrnehmung freiwilliger Selbstverwaltungsaufgaben können die Gemeinden "im Rahmen ihrer finanziellen Leistungsfähigkeit nach eigenem Ermessen - die Staatsaufsicht erfaßt i.d.R. lediglich eine Rechtmäßigkeitskontrolle - entsprechend den lokalen Bedürfnissen"6) entscheiden. Die Finanzierungskompetenz sollte hier ebenfalls auf Gemeindeebene angesiedelt sein; dies setzt die rechtliche Möglichkeit zur eigenverantwortlichen Einnahmebeschaffung voraus. Wie im 4. Kapitel beschrieben, kommt es hier aus staatlicher Sicht nicht auf die exakte Bestimmung gemeindeindividueller Finanzierungsmöglichkeiten (also des Einnahmepotentials) an, sondern nur auf die Erfassung der Leistungsfähigkeit, die überdies auch nicht nach Maßgabe einer Bedarfsgröße auszugleichen wäre. Der Bereich der freiwilligen Selbstverwaltungsaufgaben bzw. die dazugehörigen Finanzierungsinstrumente sind bei der Erfassung der normierten Finanzkraft daher ebenfalls auszuklammern.

- Als letzter zu nennender Bereich verbleibt der der pflichtigen Selbstverwaltungsaufgaben, die von den Gemeinden grundsätzlich wahrzunehmen sind, bei deren Ausgestaltung jedoch mehr oder weniger große, jeweils durch Einzelgesetze geregelte Entscheidungsspielräume bestehen. Die hier zusammengefaßten Aufgaben, die auch vom Ausgabevolumen her den Kernbereich kommunaler Aktivitäten bilden (siehe unten), entsprechen am ehesten dem im 4. Kapitel beschriebenen Normbereich. Seine Finanzierbarkeit ist durch übergeordnete Gebietskörperschaften sicher-

6) Postlep, Rolf-Dieter, Wirtschaftsstruktur und großstädtische Finanzen, a.a.O., S. 234. 
zustellen, möglichst ohne dadurch die Entscheidungsspielräume bei der Aufgabenwahrnehmung weiter einzuschränken, als dies durch die bestehenden gesetzlichen Regelungen zur Aufgabenerfüllung bereits geschieht.

Mit der Untersuchung dieser drei Bereiche ist natürlich keine Aussage darüber zu treffen, inwieweit die Gemeinden ihre Ausgabentätigkeit kurzfristig beeinflussen oder gegebenenfalls einschränken können. So ist es aus gemeindlicher Sicht von Bedeutung, ob langfristige vertragliche Bindungen eingegangen wurden, die die Variabilität der Aufgabenwahrnehmung und der Ausgabentätigkeit stark einschränken, unabhängig davon, ob es sich im Einzelfall um eine Pflichtaufgabe oder eine freiwillige Selbstverwaltungsaufgabe handelt. Aus staatlicher Sicht ist zunächst jedoch nur die Unterscheidung nach dem Pflichtigkeitsgrad von Bedeutung. Sofern die Gemeinden ihren Entscheidungsspielraum über die staatlichen Beschränkungen hinaus selbst einengen, fällt dies prinzipiell in ihre eigene, nicht aber in übergeordnete Zuständigkeit.

In Tabelle 10 wurden die kommunalen Aufgabenbereiche nach ihrem Pflichtigkeitsgrad untergliedert und den genannten drei Segmenten zugeordnet. Allerdings ist diese Zuordnung nicht ganz unproblematisch, und zwar sowohl grundsätzlich als auch für die besonderen Zwecke der hier verfolgten Fragestellung.

Zunächst bildet die rein rechtliche Klassifizierung nicht unbedingt den Grad der Fremdbestimmung einer Aufgabe insgesamt ab. ${ }^{7}$ So konstatiert auch Schmidt-Jortzig, "daß bei der praktischen Verwaltungshandhabung die Unterschiede zwischen kommunalen Selbst- und Fremdverwaltungsbereich nurmehr gering sind, zumal umgekehrt der Selbstverwaltungsbereich zunehmend sachlich-zweckhaft ebenso eingebunden erscheint wie der übertragene Wirkungskreis." ${ }^{8)}$

Zum zweiten gehen in die rechtliche Klassifizierung nicht vollständig jene Ziele ein, die in der Regel an die kommunalen Aktivitäten und den Finanzausgleich herangetragen werden. So wird etwa einhellig die Meinung vertreten, daß zentralörtliche Funktionen einer Gemeinde im kommunalen Finanz-

7) Ebenda, S. $234 \mathrm{ff}$.

8) Schmidt-Jortzig, Edzard, Probleme der kommunalen Fremdverwaltung, a.a.O., S. 395. 
ausgleich berücksichtigt werden sollen 9); zentralitätsbedingte Aufgaben müßten dementsprechend auch dem normierten Bedarf zugerechnet werden. Nach ihrem Rechtscharakter wird ein großer Teil dieser Aufgaben jedoch dem freiwilligen Bereich zugeordnet.

Eine konsistente und zieladäquate Neukonzeption des kommunalen Finanzausgleichs hätte deshalb zuerst eine durch die verfolgten Zielsetzungen geleitete Einordnung und Normierung der gemeindlichen Aufgaben vorzunehmen. Hier muß allerdings mit der Einteilung der Aufgaben nach ihrem Rechtscharakter vorlieb genommen werden.

Wurde die Finanzierung der abgegrenzten Aufgabenbereiche nach der im 4. Kapitel entwickelten oder einer ähnlichen Systematik vorgenommen, so könnte sich die Erfassung der gemeindlichen Finanzkraft - soweit sie dem Finanzbedarf gegenübergestellt werden soll - auf die Finanzierung der pflichtigen Selbstverwaltungsaufgaben beschränken. Voraussetzung dafür ist jedoch wie oben bereits ausgeführt - eine kongruente und gleichermaßen segmentierbare Kompetenzverteilung bei der Aufgabenformulierung, der Ausgabentätigkeit und der Einnahmebeschaffung. $\left.{ }^{10}\right)$ Danach müßten die Ausgaben für Pflichtaufgaben aus staatlichen zweckgebundenen Zuweisungen bzw. Erstattungen finanziert werden, diese Zuweisungen wären dann bei der Finanzkraftbestimmung außer acht zu lassen. Ebenso könnten jene Quellen unberücksichtigt bleiben, die den Gemeinden allein zur autonomen Ausschöpfung überlassen werden und zur Finanzierung rein freiwilliger Aufgaben dienen.

Im nächsten Schritt soll deshalb überprüft werden, inwieweit im kommunalen Finanzsystem der Bundesrepublik eine solche Zuordnung getroffen worden ist.

Dabei tritt insofern eine Schwierigkeit auf, als nur die speziellen, nicht aber die allgemeinen Deckungsmittel direkt nach Pflichtigkeitsgraden der Aufgabenerfüllung zugerechnet werden können. Deshalb muß ein Zwischenschritt eingefügt werden: Wenn gezeigt werden kann, daß die Kompetenzver-

9) Vgl. u.a. Deubel, Ingolf, Der kommunale Finanzausgleich in Nordrhein-Westfalen, a.a.O., S. 109, sowie Hansmeyer, Karl-Heinrich, Der kommunale Finanzausgleich als Instrument zur Förderung zentraler Orte, a.a.O., S. 83.

10) So auch Hansmeyer, Karl-Heinrich und Kops, Manfred, Die Kompetenzen der Aufgabenzuständigkeit und deren Verteilung im föderativen Staat, in: Hamburger Jahrbücher für Wirtschafts- und Gesellschaftspolitik, 29. Jg., 1984, S. 127-140. 
teilung bei der Aufgabenerfüllung und der Ausgabentätigkeit insgesamt durch die Kompetenzverteilung bei der Finanzierung konterkariert wird, kann nicht mehr eindeutig bestimmt werden, was der Gegenstand einer Messung der "normierten Finanzkraft" sein soll.

$\mathrm{Zu}$ diesem Zweck wurden in Tabelle 10 die Ausgaben in den einzelnen Aufgabenbereichen und die jeweiligen Haushaltsanteile ausgewiesen; sie werden in Punkt II mit der Finanzierungsstruktur des Budgets verglichen. Damit sollen mögliche Inkongruenzen in der Kompetenzverteilung abzuschätzen versucht werden.

Nach Tabelle 10 nehmen die Pflichtaufgaben in der Gesamtheit der Gemeinden und Gemeindeverbände in Nordrhein-Westfalen nur etwa 17,2\% der Gesamtaufgaben in Anspruch. Dabei ist zu berücksichtigen, daß die Gemeinden auch im Pflichtbereich zusätzliche, über die staatlichen Anforderungen hinausgehende Leistungen erbringen können. Überdies wird betont, daß sich eine Relation zum Gesamtaufgabenfächer nur schwer bestimmen lasse, "weil der Bestand an Selbstverwaltungsangelegenheiten nicht abschließend feststeht, ja aufgrund des schöpferischen Aufgabenfindungsmandates unbegrenzt ist."11)

Andererseits sind die Ausgabenzahlen für die freiwilligen Aufgaben in besonderem Maße bereits durch die Einnahmesituation mitbestimmt. Sowohl die Ausgaben- und Aufgabenanteile in den pflichtigen Selbstverwaltungsbereichen (in NW etwa 20,5 Mrd. DM und $45 \%$ der Gesamtausgaben) und die freiwilligen Selbstverwaltungsaufgaben (etwa 9 Mrd. DM und 19,8\% der Gesamtausgaben) können also nur als grobe Orientierungspunkte für die anschließende Untersuchung und Beurteilung der Finanzierungsstruktur dienen.

11) Schmidt-Jortzig, Edzard, Probleme der kommunalen Fremdverwaltung, a.a.O., S. 397. 
Tabelle 10: Aufgaben und Ausgaben der Gemeinden und Gemeindeverbände nach ihrem Autonomiegrad, Nordrhein-Westfalen 1983

Gliederungs-
nummer gemäß
Haushalts-

systematik

I.

$1 \% \quad$ Offentliche Sicherheit und Ordnung

13

41

44

487

49

zusammen

II.

0

13

2

400

45

47

心

Auf gabenbereich

abzüglich Feuerschutz

Sozialhilfe nach dem BSHG

Sonstige soziale Angelegenheiten

Allgemeine Verwaltung

Feuerschutz. und Jugendhilfe

Bau- und Wohnungswesen, Verkehr
Kriegsopferfürsorge und ähnliche Maßnahmen

Hilfe für Heimkehrer und politische Häftlinge

Pflichtige Selbstverwaltungsaufgaben

$3.484,7$

650,6

4.114,0

585,4

845,0

250,5

$0.961,2$

Verwaltung der sozialen Angelegenheiten

$1.119,8$

$.587,6$

581,0

472,2

$7.760,7$

Jugendhilfe nach dem JWG

Förderung von anderen Trägern der Wohlfahrtspflege
Ausgaben 1)

in v.H. der

Gesamtausgaben ${ }^{2)}$

12,4

1,3

0,0

1,0 
noch Tabelle 10: Aufgaben und Ausgaben der Gemeinden und Gemeindeverbănde mach ihrem Autonomiegrad, Nordrhein-Westfalen 1983

\author{
Gliederungs- \\ nummer gemäß \\ Haushalts- \\ systematik
}

\section{Aufgabenbereich}

Ausgaben

\section{thas}

70

72

75

77

815

zusammen

III.

3
Abwasserbeseitigung

Abfallbeseitigung

Bestattungswesen

Hilfsbetriebe der Verwaltung

Wasserversorgung

Freiwillige Selbstverwaltungsaufgaben

Wissenschaft, Forschung, Kulturpflege

Einrichtungen der Sozialhilfe und der K riegsopferfürsorge

Kindergärten und sonstige Einrichtungen der Jugendhilfe

Gesundheit, Sport, Erholung

Märkte

Schlacht - und Viehhöfe

Sonstige öffentliche Einsichtungen

Förderung der Land- und Forstwirtschaft

Fremdenverkehr, Sonstige Förderung von Wirtschaft und Verkehr in v.H. der

Gesamtausgaben $^{2)}$

in Mio. DM

4,5

$2.019,8$

2,0

889,0

0,7

331,9

1,0

456,4

0,1

$20.624,8$

45,6

$1.689,4$

3,7

444,1

1,0

627,0

1,4

$2.622,5$

5,8

$37,9 \quad 0,1$

65,7

0,1

107,2

0,2

42,3

0,1

245,7 
noch Tabelle 10: Aufgaben und Ausgaben der Gemeinden und Gemeindeverbände nach ihrem Autonomiegrad, Nordrhein-West falen 1983

Gliederungsnummer gemäß Haushaltssystematik

$8 . \%$

815

zusammen

IV.

insgesamt
Aufgabenbereich

in Mio. DM

$3.054,3$

$8.936,1$ und Sondervermögen abzüglich

Wasserversorgung

Nicht zugeordnete Aufgaben

Allgemeine Finanzwirtschaft

$6.570,3$

$43.891,9$

97,1

1) Bereinigte Ausgaben; Abweichungen durch Rundung.

2) Anteile am Gesamthaushalt.

Quellen: Zuordnung übernommen aus Zimmermann, Horst, Hardt, Ulrike und Postlep, Rolf-Dieter, Bestimmungsgründe der kommunalen Finanzsituation, a.a.O., S. 65 ff.

Bereinigte Ausgaben für die Gemeinden und Gemeindeverbände in Nordrhein-Westfalen, aus: Statistische Berichte, Die staatlichen und kommunalen Finanzen in Nordrhein-Westfalen, Rechnungsjahr 1983, hrsg. vom Landesamt für Datenverarbeitung und Statistik Nordrhein-Westfalen, Düsseldorf 1985, S. 23 und 27. 
II. Die Finanzierungsstruktur und die Kompetenzverteilung bei der Einnahmebeschaffung nach Aufgabenbereichen

a. Eine Unterscheidung der gemeindlichen Einnahmen nach Autonomiespielräumen und Folgerungen für die Messung der Finanzkraft

In Tabelle 11 werden noch einmal die kommunalen Einnahmen, die bereits im 2. und 3. Kapitel eingehender beschrieben wurden, aufgelistet und nach den Spielräumen, die den Gemeinden bei der Einnahmebeschaffung offen gelassen werden, gegliedert. Zusätzlich werden sie nach dem Kriterium der Zweckbindung unterteilt; sofern das Problem der Aufgaben- und Ausgabensegmentierung befriedigend gelungen wäre, könnten zweckgebundene Einnahmen ohne größere Schwierigkeiten den Segmenten zugeordnet werden und damit eine Auswahl der in die Finanzkraftmessung einzubeziehenden Einnahmequellen getroffen werden. Sie werden hier daher nicht mehr im einzelnen behandelt.

Die Einteilung trennt vor allem zwei Bereiche voneinander: Einnahmen, die in ihrer Höhe durch übergemeindlich festgelegte Regeln bestimmt werden und von den Gemeinden kurzfristig kaum beeinflußt werden können, werden von Einnahmen, auf deren Umfang die Gemeinden in gewissen Grenzen selbst einwirken können, unterschieden.

Daneben werden einige Einnahmen gesondert ausgewiesen, die nicht plausibel dem einen oder anderen Bereich zugeordnet werden können. Dabei handelt es sich vor allem um die kommunale Schuldaufnahme. Diese Einnahmequelle wird vom Kommunalrecht als subsidär eingestuft - so bestimmt etwa $\S 63 \mathrm{GO}$ NW, daß die Gemeinden erforderliche Einnahmen aus speziellen Entgelten und in zweiter Linie aus Steuern beschaffen sollen, soweit die sonstigen Einnahmen nicht ausreichen. Zur Verschuldung wird hingegen eine Genehmigung, und dies auch nur unter bestimmten Bedingungen, erteilt. Sie können daher nicht als normale, von den Gemeinden erwartete Einnahmen gekennzeichnet werden und sind bei der Finanzkraftmessung deshalb von vornherein ausgeklammert. 
Ein Bereich völlig autonomer Einnahmebeschaffung, bei dem den $\mathrm{Ge}$ meinden sowohl die volle Gesetzgebungs- bzw. Satzungshoheit als auch die Ertragshoheit zukäme, ist - anders als für die Aufgabenerfüllung - kaum auszumachen, weil die entsprechenden Quellen nahezu unbedeutende Erträge bringen. Sie werden hier deshalb der Abteilung II zugeschlagen.

\section{Tabelle 11: Kommunale Einnahmen nach ihrem Autonomiegrad,} Nordrhein-Westfalen 1983

Einnahmeart

Einnahmen

Anteil an in Mio DM den Gesamtein-1 nahmen

I. Einnahmen mit geringem

Gestaltungsspielraum

a. Zweckgebundene Einnahmen

Erstattungen von Ausgaben des Verwaltungshaushalts

Zuweisungen und Zuschüsse für laufende Zwecke

Zuweisungen und Zuschüsse für Investitionen

Ersatz von sozialen

Leistungen

Schuldendiensthilfen

b. Allgemeine Deckungsmittel

Gemeindeanteil an der

Einkommensteuer

Zuschlag zur Grunderwerbsteuer

Vergnügungsteuer 


\section{noch Tabelle 11:}

Einnahmeart

Einnahmen

Anteil an

in Mio DM

den Ge-

samtein-

nahmen

Schlüsselzuweisungen

$6.514,5$

14,5

Bedarfszuweisungen

165,4

0,4

Sonstige allgemeine

Zuweisungen

85,9

Einnahmen ohne kommunalen

Gestaltungsspielraum insgesamt

$25.498,1$

56,8

II. Einnahmen mit größerem kommunalen Gestaltungsspielraum

a. Zweckgebundene Einnahmen

Gebühren, zweckgebundene

Abgaben

$5.185,5$ i. 6

Sonstige Verwaltungs-

und Betriebseinnahmen

561,7

Beiträge und ähnl.

Entgelte

784,9

1,7

Zusammen

$6.532,1$

14,5

b. Allgemeine Deckungsmittel

Gewerbesteuer (netto)

Grundsteuer A

Grundsteuer B

Örtl. Aufwand- und

Verbrauchsteuern

67,5

Einnahmen aus Verkauf

Zinseinnahmen

218,3

Mieten und Pachten

Gewinnanteile, Kon-

zessionsabgaben

$1.429,9 \quad 3,2$

Entnahmen aus Rücklagen

449,4

Veräußerung von Beteili-

gungen und Sachvermögen

Zusammen

Summe II. 
noch Tabelle 11:

Einnahmeart

Einnahmen

Anteil an

in Mio DM den Gesamtein $_{\overline{1}}$ nahmen

III. Nicht eindeutig zuzu-

ordnende Einnahmen

Darlehensrückflüsse

Schuldaufnahme im öffentlichen Bereich und besondere Finanzierungsvorgänge

Zusammen

Summe I. - III. ${ }^{2}$

1 Bereinigte Einnahmen

2 Abweichung von den bereinigten Einnahmen durch Einbeziehung der Zahlung von Gemeinden (GV)

Quellen: Zuordnung übernommen aus Zimmermann, Horst, Hardt, Ulrike und Postlep, Rolf-Dieter, Bestimmungsgründe der kommunalen Finanzsituation, a.a.O., S. 69 ff.; Statistisches Bundesamt, Hrsg., Fachserie 14, Reihe 3.3., Rechnungsergebnisse der kommunalen Haushalte 1983, S. 44, 54 und 80, sowie eigene Berechnungen.

Im Bereich I sind jene Einnahmen aufgelistet, die bei gegebenem kommunalen Angebot festliegen. Sofern das Leistungsangebot ausgedehnt wird, ist ein Teil von ihnen ebenfalls variabel, wie etwa die Zuschüsse und Zuweisungen für Investitionen. Hier soll jedoch vom gegebenen Angebot des Haushaltsjahres 1983 ausgegangen werden, für dessen Finanzierung die aufgelisteten Einnahmen gedient haben.

Die Bewertung der verschiedenen Einnahmequellen erfolgt mit den tatsächlichen, nicht etwa mit normierten Einnahmen. Für diese Vorgehens- 
weise spricht, daß eine Normierung nur für jene Einnahmearten sinnvoll ist, die in die Finanzkraftmessung aufgenommen werden sollen. Eine fundierte Einteilung kann hier jedoch noch nicht getroffen werden.

Unter den Einnahmen, über deren Höhe die Gemeinden mitentscheiden können, wird überdies in der Praxis des kommunalen Finanzausgleichs nur für eine Gruppe explizit eine staatliche Normierung der kommunalen Einnahmepolitik vorgenommen: die Realsteuern. Sie gehen als einzige aus dem Bereich II in die Finanzkraft- bzw. Steuerkraftmessung des kommunalen Finanzausgleichs ein; dabei werden normierte Hebesätze zugrunde gelegt. Da die Entgelte - als zweite bedeutende Gruppe - bei der Finanzkraftmessung keine Berücksichtigung finden, wird die jeweilige Entgelthöhe auch nicht normiert; praktisch wäre dies jedoch kaum problematisch. Das zeigt sich etwa in jenen Fällen, in denen über den eigentlichen Finanzausgleich hinaus zusätzliche Zuweisungen erforderlich werden. Dann wird vor der Gewährung überprüft, ob die betreffenden Gemeinden ihre Entgeltfinanzierungsmöglichkeiten angemessen ausgeschöpft haben. Dabei wird allerdings vom realisierten, und nicht, wie es für die Zwecke des Finanzausgleichs genau genommen erforderlich wäre, vom erwünschten Leistungsangebot ausgegangen.

Für die Zwecke des kommunalen Finanzausgleichs würde möglicherweise eine andere politische Wertung und Normierung vorgenommen als für den oben genannten Zweck, deshalb wird sie nicht übernommen. Auf eine eigene Normierung wird hier indessen bewußt ebenfalls verzichtet, weil sie zwar zahlenmäßige Ergebnisse, aber keinen Erkenntnisfortschritt in der Fragestellung liefern könnte.

Betrachtet man den Anteil der nicht beeinflußbaren Einnahmen an den Gesamteinnahmen, so ist zunächst festzustellen, daß dieser bereits ohne die allgemeine Zuweisungen übergeordneter Ebenen mit etwa $42 \%$ weit über dem Anteil der pflichtigen Aufgaben an den Gesamtausgaben liegt. Einschließlich der durch den Finanzausgleich verteilten allgemeinen Zuweisungen werden sogar mehr als die Hälfte aller Ausgaben durch nicht unmittelbar beeinflußbare Einnahmen abgedeckt. Andererseits reichen diese festliegenden Einnahmen zusammen nicht aus, um sämtliche Ausgaben für Pflichtaufgaben und pflichtige Selbstverwaltungsaufgaben zu finanzieren. 
Dabei ist noch nicht berücksichtigt, daß unter den nicht gestaltbaren Einnahmen auch solche subsumiert sind, die zum Teil für die Finanzierung freiwilliger Aufgaben zu verwenden sind. Dies ist etwa bei einem Teil der Investitionszuweisungen der Fall.

Insgesamt kann damit bereits eine Inkongruenz in der Aufgaben-, Ausgaben- und Einnahmenzuweisung an die Gebietskörperschaften vermutet werden. Zumindest hat es den Anschein, als seien die kommunalen Entscheidungsspielräume bei der Einnahmebeschaffung wesentlich stärker eingeengt als die rechtlichen Spielräume bei der Aufgaben- und Ausgabengestaltung. Das heißt, daß durch die für die Gemeinde festliegenden Einnahmen ein Mindestmaß an Ausgabentätigkeit im Aufgabenbereich II, den pflichtigen Selbstverwaltungsaufgaben, vorgegeben wird, ohne damit dessen Struktur vollständig zu bestimmen. Ein Struktureffekt wird hier allenfalls durch die Zuweisung zweckgebundener Mittel erzielt, sofern kein Mitnahmeeffekt vorliegt.

Beide Formen der Steuerung der normierten Ausgabentätigkeit wurden im 4. Kapitel für bestimmte, wenn auch weniger allokativ orientierte Zwecke als durchaus adäquat eingestuft. Deshalb muß aus der Tatsache, daß die Einnahmetätigkeit der Gemeinden stärker reglementiert wird als die Ausgabentätigkeit, noch keine besondere Schwierigkeit für die Finanzkraftmessung resultieren. Die festliegenden, für die Finanzierung des Normbedarfs vorgesehenen Einnahmen wären dem Finanzbedarf zusammen mit normierten, jedoch von den Gemeinden mitgestaltbaren Einnahmen gegenüberzustellen. Voraussetzung dafür ist allerdings, daß sie von den für die Abteilungen I und III vorgesehenen Einnahmen zu trennen sind. Inwieweit eine solche Trennung zwischen Pflichtbereich, pflichtigem Selbstverwaltungsbereich - der hier für den Normbereich steht - und freiwilligem Selbstverwaltungsbereich möglich ist, kann anhand der Kompetenzverteilung auf der Einnahmeseite nicht abschließend abgeschätzt werden.

Problematisch erscheint allerdings bereits an dieser Stelle der Tatbestand, daß die Gemeinden kaum über ein "Einnahmefindungsrecht" verfügen, um die Ausgaben des freiwilligen Aufgabenbereichs zu finanzieren. Das bedeutet, daß die entsprechenden Ausgaben aus Überschüssen der festliegenden und der normierten Einnahmen über die für den Normbereich ermittelten 
Normausgaben abdeckbar sein müssen. ${ }^{12)}$ Aus staatlicher Perspektive sind deshalb bei der Normierung der Einnahmepolitik von vornherein Einnahmespielräume auszugrenzen, die die Finanzkraftmessung nicht erfassen soll, weil die hier erzielbaren Mittel allein zur Finanzierung autonom bestimmmbarer Gemeindeaktivitäten dienen sollen.

Genau dies geschieht faktisch mit der Normierung der Realsteuersätze unterhalb der Durchschnittshebesätze. Werden jedoch im Normbereich niedrigere Hebesätze festgelegt, als dies ohne die Überlappung von Normbereich und freiwilligem Bereich auf der Finanzierungsseite notwendig wäre, so folgt daraus eine relative Begünstigung finanzkräftiger Gemeinden beim Ausgleichsvorgang innerhalb des Normbereichs. Dieser Effekt wurde im einzelnen im zweiten Kapitel beschrieben. Hier richtet sich die Kritik allerdings nicht auf jene Begünstigung, sondern allein darauf, daß durch die Verkopplung der Bereiche nur noch entweder eine zieladäquate Normierung der $\mathrm{He}$ besätze innerhalb des Normbereichs oder aber die Gewährung eines gewissen Finanzierungsspielraums außerhalb des Normbereichs möglich ist. Beide Zielsetzungen widersprechen sich, sofern die Segmente kommunaler Aufgabenerfüllung nicht auch auf der Finanzierungsseite getrennt werden können.

\section{b. Inkongruente Finanzierungsstrukturen in den Aufgabensegmenten als besonderes Problem der Finanzkraftmessung}

Nach den Ausführungen im 4. Kapitel sollte jede staatliche Ebene die Finanzierung für die in ihre Kompetenz fallenden Aufgabenbereiche übernehmen; dies gilt sowohl aus finanzwissenschaftlicher, vor allem allokativ orientierter Sicht, wird aber prinzipiell auch unter rechtlichen Aspekten des föderativen Staatsaufbaus in der Bundesrepublik Deutschland anerkannt. ${ }^{13)}$ Hier hat das Konnexitätsprinzip Eingang in Art. 104a Abs. 1 GG gefunden, wenn auch mit der Besonderheit, "daß die zur Aufgabendurchführung herangezogenen In-

12) Ewringmann konstatiert insbesondere aufgrund der finanziellen Abhängigkeit auch für den "formal freiwilligen Bereich" eine "von zentralistischen Kalküls vorgeformte" Ordnung. Ewringmann, Dieter, Der staatliche Einfluß auf die kommunale Aufgabenerfüllung, in: Reform kommunaler Aufgaben, Studien zur Kommunalpolitik des Instituts für Kommunalwissenschaften (Konrad-Adenauer-Stiftung), Bd. 19, Bonn 1978, S. 116-139, hier S. 132.

13) Vgl. Kirchhof, Paul, Rechtliche Rahmenbedingungen des kommunalen Finanzausgleichs, a.a.O., S. $8 \mathrm{ff}$. 
stanzen auch in ihren eigenen Kräften (also auf ihre Rechnung) herangezogen werden können". ${ }^{14)}$ Die Ausgabenlast wird nach Gestaltungsfeldern aufgeteilt: Während den Gemeinden die Verwaltungskosten in ihrer eigenen Organisationshoheit zufallen, haben die übergeordneten Ebenen, speziell die Länder, die eigentlichen Zweckausgaben zu tragen. ${ }^{15}$ )

Damit wird das Konnexitätsprinzip bereits partiell durchbrochen, es kommt zu einem unsichtbaren Finanzausgleich zwischen den Gebietskörperschaftsebenen. ${ }^{16)}$

Darüber hinaus ist es inzwischen jedoch üblich geworden, die Unterscheidung zwischen Verwaltungs- und Zwecckosten im Finanzausgleich weitgehend außer acht $\mathrm{zu}$ lassen und die Zweckausgaben durch pauschalierte $\mathrm{Zu}$ weisungen abzugelten. Offenbar "sieht es auch die Rechtsprechung als unbedenklich an, daß im Finanzausgleich keinerlei gesonderte Kostenerstattung für die Wahrnehmung übertragener Aufgaben stattfinden, 'sondern die Abgeltung pauschal und im Rahmen eines allgemeinen Finanzausgleichs erfolgt". ${ }^{17}$ ) Dabei begründen die Mehrzahl der Länderverfassungen nicht einmal eine Verpflichtung des Landes zur völligen oder teilweisen Finanzierung von übertragenen Aufgaben. ${ }^{18)}$ In der Folge konstatiert Schmidt-Jortzig "eine deutliche Unterfinanzierung der Fremdverwaltung". ${ }^{19)}$ Der Forderung nach "scharf konturierten Maßstäben, die den Verlauf der Kompetenzgrenzen eindeutig festlegen", wird das gesetzliche Regelwerk damit nicht mehr gerecht. ${ }^{20}$ )

14) Schmidt-Jortzig, Edzard, Probleme der kommunalen Fremdverwaltung, a.a.O., S. 395; ebenso Schreyer, Michaele, Transferpolitik im Föderalismus, a.a.O., S. 28.

15) Ebenda, S. 30; Schmidt-Jortzig, Probleme der kommunalen Fremdverwaltung, a.a.O., S. 395.

16) Vgl. Zimmermann, Horst, Allgemeine Probleme und Methoden des Finanzausgleichs, a.a.O., S. 38.

17) Schmidt-Jortzig, Edzard, Probleme der kommunalen Fremdverwaltung, a.a.O., S. 400.

18) Schreyer, Michaele, Transferpolitik im Föderalismus, a.a.O., S. 98.

19) Schmidt-Jortzig, Edzard, a.a.O., S. 401.

20) Fischer-Menshausen, Herbert, Unbestimmte Rechtsbegriffe in der bundesstaatlichen Finanzverfassung, in: Probleme des Finanzausgleichs I, von Dieter Bös, Herbert FischerMenshausen, Guy Kirsch und Fritz Neumark, hrsg. von Wilhelmine Dreißig, Schriften des Vereins für Socialpolitik, Neue Folge, Band 96/1, Berlin 1978, S. 135-164, hier S. 144 f. 


\section{Gliederungsnr. Aufgabenbereich \\ gemäß \\ Ilaushalts- \\ systematik}

I.

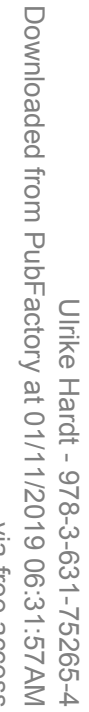

abzüglich

13 Feuerschutz
II.

400

45

47
Bereinigte

Einnahmen

in $x$ der be-

reinigten

Ausgaben 1)

insgesamt
Pflichtaufgaben (zur Erfüllung nach Weisung, Auftragsangelegenheiten)

$1 \%$ Offentliche Sicherheit und Ordnung

41 Sozialhilfe nach dem BSHG

57,6

26,9

33,1

200,1

48,7

36,9

12,2

11,5

12,4

5,7

11,7

161,0
Allgemeine Verwaltung

Feuerschutz

Schulen

Verwaltung der sozialen Angelegenheiten

Jugendhilfe nach dem JWG

Förderung von anderen Trägern der Wohlfahrtspflege und Jugendhilfe

\section{darunter}

Entgelte $^{2)} \quad$ Erstattungen ${ }^{3)}$
37,8

$\begin{array}{ll}- & 1,3\end{array}$


noch Tabelle 12: Bereinigte Einnahmen nach Aufgabenbereichen, Nordrhein-West falen 1983

\begin{tabular}{|c|c|c|c|c|}
\hline \multirow{2}{*}{$\begin{array}{l}\text { Gliederungsnr. } \\
\text { gemäß } \\
\text { Haushalts- } \\
\text { syst ematik }\end{array}$} & \multirow[t]{2}{*}{ Aufgabenbereich } & \multirow{2}{*}{$\begin{array}{l}\text { Bereinigte } \\
\text { Einnahmen } \\
\text { in } \% \text { der be- } \\
\text { reinigten } \\
\text { Ausgaben } 1 \text { ) } \\
\text { insgesamt }\end{array}$} & \multicolumn{2}{|c|}{ darunter } \\
\hline & & & Entgelte $^{2)}$ & Erstattungen ${ }^{3)}$ \\
\hline 6 & Bau- und Wohnungswesen, Verkehr & 56,4 & 16,6 & - \\
\hline 70 & Abwasserbeseitigung & 113,3 & 97,9 & - \\
\hline 72 & Abfallbeseitigung & 117,9 & 114,4 & - \\
\hline 75 & Best attungswesen & 77,2 & 72,1 & - \\
\hline 77 & Hilfsbetriebe der Verwaltung & 12,4 & 6,3 & - \\
\hline 815 & Wasserversorgung & 193,3 & 36,4 & - \\
\hline zusammen & & 44,9 & 21,8 & - \\
\hline III. & Freiwillige Selbstverwaltungsaufgaben & & & \\
\hline 3 & Wissenschaft, Forschung, Kulturpflege & 25,7 & 11,2 & - \\
\hline 43 & $\begin{array}{l}\text { Einrichtungen der Sozialhilfe und der } \\
\text { Kriegsopferfürsorge }\end{array}$ & 90,1 & 75,5 & 3,2 \\
\hline 46 & $\begin{array}{l}\text { Kindergärten und sonstige Einrichtungen } \\
\text { der Jugendhilfe }\end{array}$ & 36,2 & 26,9 & - \\
\hline 5 & Gesundheit, Sport, Erholung & 24,0 & 8,2 & - \\
\hline 7 & Märkte & 140,4 & 91,8 & - \\
\hline 74 & Schlacht - und Viehhöfe & 99,7 & 78,3 & 1,8 \\
\hline 76 & Sonstige öffentliche Einrichtungen & 52,7 & 5,5 & - \\
\hline 78 & Förderung der Land- und Forst wirtschaft & 15,4 & 2,1 & - \\
\hline
\end{tabular}




\begin{tabular}{|c|c|c|c|c|}
\hline \multirow{2}{*}{$\begin{array}{l}\text { Gliederungsnr. } \\
\text { gemäß } \\
\text { Haushalts- } \\
\text { systematik }\end{array}$} & \multirow[t]{2}{*}{ Aufgabenbereich } & \multirow{2}{*}{$\begin{array}{l}\text { Bereinigte } \\
\text { Einnahmen } \\
\text { in } \% \text { der be- } \\
\text { reinigten } \\
\text { Ausgaben } 1 \text { ) } \\
\text { insgesamt }\end{array}$} & \multicolumn{2}{|c|}{ darunter } \\
\hline & & & Entgelte $^{2)}$ & Erstattungen ${ }^{3)}$ \\
\hline 79 & $\begin{array}{l}\text { Fremdenverkehr, Sonstige Förderung von } \\
\text { Wirtschaft und Verkehr }\end{array}$ & 39,3 & 4,0 & - \\
\hline $8 . /$ & $\begin{array}{l}\text { Wirtschaftliche Unternehmen, Allgemeines } \\
\text { Grund- und Sondervermögen abzüglich }\end{array}$ & & & \\
\hline 815 & Wasserversorgung & 129,8 & 1,3 & - \\
\hline zusammen & & 66,4 & 11,8 & 0,5 \\
\hline IV. & Nicht zugeordnete Aufgaben & 405,4 & (Berechnung & wenig sinnvoll) \\
\hline 9 & $\begin{array}{l}\text { Allgemeine Finanzwirtschaft (Steuern, } \\
\text { allgemeine Zuweisungen und Umlagen) }\end{array}$ & & & \\
\hline I., II., III. & zusammen & $101,6^{3)}$ & & \\
\hline
\end{tabular}

1) nach Tabelle 10

2) Gebühren, zweckgebundene Abgaben, Beiträge und ähnliche Entgelte; Steuern, soweit zurechenbar

3) Abweichung von $100 \%$ bereits in den Ursprungstabellen

Quellen: Tabelle 10, sowie Statistische Berichte, Die staatlichen und kommunalen Finanzen in Nordrhein-Westfalen, a.a.O., S. $134 \mathrm{ff}$. und eigene Berechnungen 
$\mathrm{Zu}$ einem ähnlichen Ergebnis führen die Berechnungen in Tabelle 12. Hier wurden den jeweiligen Aufgaben- und Ausgabenbereichen sämtliche zweckgebundenen bzw. zurechenbaren Einnahmen einschließlich der zweckgebundenen Zuweisungen zugeordnet. Der Saldo zwischen bereinigten Ausgaben und bereinigten Einnahmen ist durch allgemeine Deckungsmittel zu finanzieren. Entgegen den aufgestellten Forderungen besteht ein solcher Saldo auch im Bereich der Pflichtaufgaben, hier ist er anteilsmäßig sogar am größten, am niedrigsten ist er dagegen im Bereich der freiwilligen Selbstverwaltungsaufgaben.

Sowohl pflichtige Aufgaben als auch freiwillige Selbstverwaltungsaufgaben müssen damit teilweise aus allgemeinen Mitteln finanziert werden, die im kommunalen Finanzausgleich vorwiegend dem Normbereich zugerechnet werden: den Realsteuern, dem gemeindlichen Einkommensteueranteil (der zwar nicht mit einem Hebesatzrecht ausgestattet ist, in der Regel jedoch als Pendant zur Gewerbesteuer interpretiert wird und auch in die Steuerkraftmeßzahl eingeht) sowie aus den vorweg vergebenen und den zum Ausgleich zwischen Finanzbedarf und Finanzkraft verteilten allgemeinen Zuweisungen.

Oben wurde gezeigt, daß die Verkopplung von freiwilligem und normiertem Bereich über die Finanzierungsseite tendenziell zugunsten finanzstarker Gemeinden wirkt. Die Verkopplung von Pflichtbereich und Normbereich und der unsichtbare Finanzausgleich zu Lasten der Gemeindeebene führen indessen zu einer relativen Begünstigung der finanzschwachen Gemeinden. Dies soll anhand eines Beispiels verdeutlicht werden:

In Tabelle 13 werden zwei Fälle unterschieden:

- Im ersten Fall wird der Finanzbedarf für pflichtige Aufgaben und normierte Aufgaben gemeinsam (durch Addition) ermittelt; ein Ausgleich findet statt, wenn die normierte Finanzkraft zur Finanzierung beider Bereiche nicht ausreicht. Es wird unterstellt, daß die Pflichtausgaben in beiden Gemeinden 200.000 DM, die normierten Ausgaben 500.000 DM betragen sollen. Beim normierten Hebesatz $300 \%$ erhält die Gemeinde 1 Zuweisungen in Höhe von 400.000 DM, Gemeinde 2 nur 100.000 DM. Im Fall 2 wird von den gleichen Bedarfen ausgegangen, jedoch übernimmt nun der Staat die Ausgaben des Pflichtbereichs in beiden Gemeinden. Weil aus den Einnahmequellen des Normbereichs nur noch die normierten Aufgaben zu finanzieren sind, kann 
ein niedrigerer normierter Hebesatz zugrundegelegt werden, die finanzielle Belastung verschiebt sich von der lokalen auf eine übergeordnete Ebene. Daraus ergibt sich der oben bereits dargestellte Effekt zugunsten der finanzstarken Gemeinde; sie erhält nach wie vor 100.000 DM an Zuweisungen, Gemeinde 1 mit niedrigerer Finanzkraft jedoch nur 300.000 DM. Die Übernahme der Pflichtausgaben durch den Staat im Fall 2 wirkt also zugunsten "reicher" Gemeinden, eine Finanzierung im Rahmen eines "Gesamtfinanzierungspakets" begünstigt dagegen verglichen mit dem Fall der gemeinsamen Bedarfsermittlung eher finanzschwache Gemeinden.

Auch dieser Effekt der Gesamtfinanzierung soll hier nicht kritisiert werden; aus verteilungspolitischen Überlegungen kann er durchaus für angemessen gehalten werden. Allerdings werden auf diese Weise die allokativ möglicherweise erwünschten interkommunalen Unterschiede in den Finanzierungsspielräumen, die mit ein Anlaß für die Formulierung eines normierten Aufgabenbereichs sind, teilweise wieder eingeebnet.

Darüber hinaus bewirkt der unsichtbare Finanzausgleich aber auch allokative Verzerrrungen zwischen gleich finanzstarken Gemeinden, wenn sich das Verhältnis von Pflichtausgaben zu Normausgaben unterscheidet. Dies läßt sich leicht für 2 Gemeinden verdeutlichen, die beide einen Gesamtbedarf von 100.000 DM aufweisen, wobei in Gemeinde 1 davon 80.000 DM auf Pflichtaufgaben entfallen, in Gemeinde 2 jedoch nur 20.000 DM. Damit wird in Gemeinde 1 der Dispositionsspielraum wesentlich stärker eingeschränkt; sie kann je nach Normierung gezwungen werden, einen bestimmten, recht hohen Hebesatz zu fordern, um die Pflichtausgaben abzudecken. Gemeinde 2 kann ihren Hebesatz unabhängig vom Normhebesatz dagegen wesentlich tiefer ansetzen und ein weitaus geringeres Haushaltsvolumen realisieren. Damit werden unter den gegebenen Bedingungen der Aufgabenverteilung in besonderem Maße jene Gemeinden einem Konkurrenznachteil ausgesetzt, die eine ungünstige Sozialstruktur und in der Regel gleichzeitig eine ungünstige Wirtschaftsstruktur aufweisen und deshalb aus regionalpolitischer Perspektive eher auf eine Stärkung ihrer Konkurrenzposition angewiesen wären. 
Tabelle 13: Relationen des Ausgleichs in steuerstarken und steuerschwachen Gemeinden bei gemeinsamer und getrennter Finanzierung pflichtiger und normierter Aufgaben

Hebesatz eigene Einnahmen

Gemeinde 1

$\begin{array}{lll}\text { Fall 1: } & 100 & 100.000 \\ \text { gemein- } & 200 & 200.000 \\ \text { same } & 300 & 300.000 \\ \text { Finan- } & 400 & 400.000 \\ \text { zierung } & 500 & 500.000\end{array}$

Gemeinde 2

\section{Ausgleichsbetrag}

bei $\mathrm{H}_{\text {norm }}=\mathbf{3 0 0}$

Gemeinde 1 Gemeinde 2

100.000
100.000
100.000
100.000
100.000

Ausgleichsbetrag

bei $\mathrm{H}_{\text {norm }}=200$

$\begin{array}{llll}\text { Fall 2: } & 100 & 100.000 & 200.000 \\ \text { get rennte } & 200 & 200.000 & 400.000 \\ \text { Finan- } & 300 & 300.000 & 600.000 \\ \text { zierung } & 400 & 400.000 & 800.000\end{array}$

Ausgaben; Norm-Aufgaben $=500.000$

Pflichtaufgaben Norm-Aufgaben Norm-Aufgaben $\mathrm{G}_{1}, \mathrm{G}_{2}$ Gemeinde 1 Gemeinde 2

$\begin{array}{lll}200.000 & 300.000 & 100.000 \\ 200.000 & 400.000 & 300.000 \\ 200.000 & 500.000 & 500.000 \\ 200.000 & 600.000 & 700.000 \\ 200.000 & 700.000 & 900.000\end{array}$

$\begin{array}{lllll}300.000 & 100.000 & 200.000 & 400.000 & 300.000 \\ 300.000 & 100.000 & 200.000 & 500.000 & 500.000 \\ 300.000 & 100.000 & 200.000 & 600.000 & 700.000 \\ 300.000 & 100.000 & 200.000 & 700.000 & 900.000\end{array}$


Zusammenfassend ist damit festzuhalten, daß die Gesamtfinanzierung der drei unterschiedlichen Aufgabenbereiche durch eigene und zugewiesene allgemeine Deckungsmittel die Zielsetzungen, die mit der Trennung dieser Aufgabenbereiche und mit der Einführung des Konnexitätsprinzips als eine der Hauptregeln der Finanzverfassung in das Grundgesetz verfolgt werden, konterkariert oder zumindest unkalkulierbare und widersprüchliche Effekte hervorruft. Die dargestellten ungeplanten Verteilungseffekte lassen sich in ihrem Saldo wohl nicht einmal abschätzen.

Aus den vorangegangenen Überlegungen muß der Schluß gezogen werden, daß die Kompetenzverteilung und die Finanzierung der kommunalen Aufgabenerfüllung im gegenwärtigen föderativen System eine konsistente und zieladäquate Bestimmung der zu messenden Finanzkraft unmöglich machen. Eine solche Bestimmung kann sich wegen der Unterschiedlichkeit der im Gesamtsystem verfolgten Zielsetzungen und der Verschränkung seiner Einzelbestandteile jeweils nur auf einen - mit Blick auf den Entscheidungsspielraum relativ homogenen Aufgabenbereich und, soweit klar abgrenzbar, auf seine Finanzierung, nicht aber auf alle Bereiche gleichzeitig beziehen.

Innerhalb des beschriebenen Systems erscheint es daher wenig sinnvoll zu sein, weiter nach einer "richtigen" Erfassung der gemeindlichen Finanzkraft, unter Einschluß auch spezieller Deckungsmittel, zu suchen. Dazu fehlen entscheidende politische Vorgaben und vor allem die konsistente Formulierung und Umsetzung der jeweils verfolgten Zielsetzungen, ohne die nicht einmal eine theoretische Bestimmung des Meßgegenstandes "gemeindliche Finanzkraft" möglich ist.

Allenfalls ließe sich aus Gerechtigkeitsüberlegungen die Forderung aufstellen, daß neben den Steuern auch die speziellen Deckungsmittel in die Finanzkraftberechnung aufgenommen werden sollten. Allerdings wäre ihre Einbeziehung wiederum der Willkür unterworfen, weil weder ein Kriterium existiert, welche Mittel im einzelnen berücksichtigt werden sollen, noch welche Höhe dabei angesetzt werden soll.

Damit würden dann möglicherweise die Verteilungsergebnisse - gemessen an einer ad hoc aufgestellten Verteilungsnorm - verbessert, "richtiger" und letztlich zielführender wird die Messung der kommunalen Finanzkraft auf diese Weise jedoch nicht. 


\section{Zur Neuformulierung der Kritik an der Steuerkraftmessung im System des kommunalen Finanzausgleichs}

Die im 2. Kapitel referierte Kritik an der Messung der Steuerkraft als Maß gemeindlicher Finanzkraft lautete wie folgt: Die Steuerkraftmeßzahl ist nicht in der Lage, jenen Beitrag zu ermitteln, den die Gemeinden selbst, das heißt ohne den Ausgleich von Finanzbedarf und Finanzkraft, zur Deckung des Finanzbedarfs beitragen können. Damit wurde - zumindest von einigen Autoren - das kommunale Einnahmepotential abzüglich der Schlüsselzuweisungen als ideales Finanzkraftmaß für den kommunalen Finanzausgleich charakterisiert.

Diese Kritik hat sich insofern als nicht treffend erwiesen, als sie nicht zwischen der gemeindlichen Perspektive bei der Messung von Einnahmemöglichkeiten und den staatlichen, hinter der Finanzkraftmessung stehenden Fragestellung unterscheidet.

Aus staatlicher Sicht soll gemessen werden, was die Gemeinden aufbringen können, wenn sie eine staatlich definierte, zielgerechte Bedarfsdeckungspolitik und eine ebenso zielgerechte Einnahmepolitik betreiben.

Als ein erster, großer Mangel des kommunalen Finanzausgleichs, der auch für die Messung der Finanzkraft nicht folgenlos bleibt, ist deshalb hervorzuheben, daß eine solche gemeindliche "Optimalpolitik" nicht ausreichend konkret und überdies nicht konsistent formuliert wird. Zunächst wäre zu bestimmen, was als "lokaler Bedarf" anerkannt werden soll; ob, wie und von welcher Ebene die Bedarfsdeckung erfolgen soll. ${ }^{21)}$

Dabei entscheidet das Gewicht der verschiedenen Zielsetzungen darüber, welche Gestaltungsspielräume den Gemeinden belassen werden. Von besonderer Bedeutung ist hier die Ausrichtung des Ausgleichsziels: Soll ein interregionaler Ausgleich über das kommunale Leistungsangebot erfolgen, so sind die Gemeinden an staatliche Vorgaben zur Angebotspolitik zu binden; ist ein ursachenbezogener Ausgleich über die direkte Beeinflussung der Einkommenssituation vorgesehen, so kann dem dezentralen Preismechanismus zur Steuerung des lokalen öffentlichen Angebots ein größerer Wirkungsgrad ein-

21) So auch Hansmeyer, Karl-Heinrich und Kops, Manfred, Finanzwissenschaftliche Grundsätze für die Ausgestaltung des kommunalen Finanzausgleichs, in: Hoppe, Werner, Hrsg., Reform des kommunalen Finanzausgleichs, Köln u.a. 1985, S. 31-60, hier S. 36 ff. 
geräumt werden. Dazwischen sind Kompromisse möglich, die eine Normierung gemeindlicher Aufgabenerfüllung vornehmen, ohne sie unmittelbar festzulegen.

Damit die Bedarfsdeckung zieladäquat erfolgen kann, müssen, wie auch im Konnexitätsprinzip zum Ausdruck gebracht wird, entsprechende Finanzierungssysteme für jeden der Teilbereiche kommunaler Aufgabenerfüllung gefunden werden (vgl. 4. Kapitel).

Den kommunalen Auftragsangelegenheiten entspricht eine Finanzierung durch übergeordnete Ebenen; praktisch wird hier die Gemeindeebene nur vermittelnd für den Staat tätig. An der finanzkraftunabhängigen Zuweisung der erforderlichen Mittel wird mitunter die Kritik geäußert, daß sie zugunsten finanzstarker Gemeinden wirke und insofern verteilungspolitisch nicht erwünscht sei. ${ }^{22)}$ Diese Argumentation vernachlässigt jedoch vollkommen die Aufbringung der zugewiesenen Mittel durch die übergeordnete Ebene; Verteilungswirkungen auch regionaler Art lassen sich hier erst bestimmen, wenn geklärt werden kann, wo diese Mittel abgeschöpft worden sind. Überdies muß noch einmal festgehalten werden, daß es sich bei der Aufgabenerfüllung und bei der Finanzierung der Ausgaben um staatliche Angelegenheiten handelt, die letztlich unabhängig von der Gemeindeebene, also selbst ohne deren Existenz, verfolgt würden. In die Finanzkraftmessung dürfen die hier zuzuordnenden Mittel deshalb nicht eingehen.

Ebenfalls außer acht zu lassen wären jene Finanzierungsmöglichkeiten, die allein zur Deckung autonom zu gestaltender gemeindlicher Ausgabetätigkeit dienen. Sie sind rein kommunale Angelegenheit und geben in einem rationalen System keinen Anlaß für staatliche Ausgleichsleistungen im Rahmen des kommunalen Finanzausgleichs.

Damit reduziert sich das Spektrum der in der Finanzkraftmessung zu berücksichtigenden Einnahmequellen auf jene, die den Gemeinden zur Deckung des Normalbedarfs zugewiesen worden sind. Im Gemeindefinanzsystem der Bundesrepublik Deutschland sind dies - soweit man hier einen Normbereich ausmachen kann - zum einen die großen Gemeindesteuern, die in der Steuer-

22) So argumentiert etwa Deubel mit Blick auf die bis 1982 in Nordrhein-Westfalen vergebene Auftragspauschale. Deubel, Ingolf, Der kommunale Finanzausgleich in Nordrhein-Westfalen, a.a.O., S. $171 \mathrm{f}$. 
kraftmessung berücksichtigt werden. Darüber hinaus dienen aber insbesondere Entgelte, die nach den Gemeindeordnungen sogar Priorität bei der Einnahmebeschaffung haben sollen, sowie weitere allgemeine und spezielle Dekkungsmittel zur Finanzierung dieser Normausgaben.

Während sich die im 2. Kapitel formulierte Kritik noch mit dem Problem beschäftigte, ob nicht grundsätzlich die gesamten Einnahmemöglichkeiten aus allen Einnahmearten in die Finanzkraftmessung einzubeziehen wären, muß die Frage nun umformuliert werden: Welche Segmente der einzelnen Einnahmearten, etwa welche Entgelte, sind zu berücksichtigen? Innerhalb der gleichen Einnahmeart wäre nach den Ergebnissen des 4. Kapitels noch einmal zu differenzieren: Es sollten nur solche Entgelte einbezogen werden, die für die Inanspruchnahme von Leistungen zu entrichten sind, die gleichzeitig Bestandteil des staatlicherseits normierten Leistungskorbes sind. Die gleiche Differenzierung wäre für die übrigen Einnahmearten, also auch für die Zweckzuweisungen vorzunehmen. Eine Ausnahme bildet allein die Verschuldung, die von den Gemeindeordnungen explizit und gesamthaft aus dem Spektrum der "normalen" Einnahmearten ausgegrenzt wird.

Aufgrund der unterschiedlichen Kompetenzzuweisung bei Aufgaben, Ausgaben und Einnahmen und der "Querfinanzierung" der gemeindlichen Ausgabentätigkeit kann eine solche Zuordnung jedoch nicht vorgenommen werden. Damit liegt der eigentlich angreifbare Schwachpunkt jedoch nicht mehr bei der Finanzkraftmessung, sondern bei der vorgelagerten Entscheidung über die Kompetenzzuweisung und der Nichteinhaltung des Prinzips der "fiskalischen Äquivalenz".

Der dritte, im 2. Kapitel formulierte Kritikpunkt bezog sich auf die Höhe der Abgabesätze bzw. der Steuersätze, die bei der Ermittlung der Steuerkraftmeßzahl zugrunde gelegt werden. Es wurde bemängelt, daß die normierten Hebesätze die tatsächlichen Hebesatzspielräume der Gemeinden weder absolut noch im interkommunalen Vergleich richtig wiedergeben. Diese Kritik ist in verschiedener Hinsicht zu relativieren: Zum ersten wurde erklärt, wie die erstaunlich niedrigen normierten Hebesätze im geltenden kommunalen Finanzausgleich zustandekommen; innerhalb des vorgegebenen Systems erscheinen sie durchaus angemessen, weil aus den Steuereinnahmen weit mehr als das Normalangebot finanziert werden muß. Verläßt man jedoch die- 
ses System und bezieht sich auf eine rationale Neukonzeption, so sind - je nach staatlichen Zielsetzungen - höchst unterschiedliche Normierungsgrundsätze denkbar. Sie wurden im 4. Kapitel eingehender diskutiert. Letztlich ist über ihre Ausgestaltung politisch zu entscheiden. Eine Gewichtungsregel für das Verhältnis der in eine rationale Finanzkraftmeßzahl einzubeziehenden Steuern und Abgaben - wie sie etwa die Forderung nach der Normierung auf durchschnittliche Hebesätze darstellt ${ }^{23}$ ) - ist wissenschaftlich nicht ableitbar. Es kann (und sollte) nur geprüft werden, inwieweit die getroffenen Entscheidungen in sich konsistent sind und ob sie mit allgemeinen Prinzipien der Abgabepolitik übereinstimmen.

Auch dies leistet die Gesamtkonzeption des kommunalen Finanzausgleichs - wie gezeigt werden konnte - nicht; das Problem der "richtigen" Festsetzung normierter Abgabesätze ist deshalb kein Problem der Finanzkraftmessung, sondern es resultiert daraus, daß man sich hinsichtlich der Ziele des Finanzausgleichs und ihrer Gewichtung letztlich nicht entschieden hat.

Als Fazit bleibt damit festzuhalten, daß die Kritik an der Messung kommunaler Finanzkraft, die sich ursprünglich auf bloße technische Unzulänglichkeiten aufgrund von Informationsmängeln zu richten schien, in eine umfassendere Kritik des gesamten vertikalen und horizontalen Finanzausgleichs münden muß. Solange sich die Kritikpunkte nur auf die einmal mit der vertikalen Aufgaben-, Ausgaben- und Einnahmezuweisung vorentschiedenen Verteilungswirkungen beziehen, diese Vorentscheidungen selbst aber nicht als Ursache unerwünschter und zum Teil widersprüchlicher Wirkungen angreifen, bleibt jede punktuelle Änderung des Gesamtsystems letztlich ein Kurieren von Symptomen, das gleichzeitig neue unerwünschte Wirkungen hervorruft. Solche punktuellen Veränderungen sind jedoch - gegenüber grundsätzlichen Neukonzeptionen - die Regel, was die Auffassung stützt, "daß Finanzausgleichsregelungen nicht gemacht werden, daß sie vielmehr gleichsam geschehen, daß die föderalistische Aufgliederung eines Gemeinwesens weniger das Resultat eines globalen Staatsentwurfs als die Resultante einer Vielzahl und einer Vielfalt von Partikularentscheidungen ist." ${ }^{24)}$ Die Wirkung einzelner

23) Vgl. ebenfalls Deubel, Ingolf, Der kommunale Finanzausgleich in Nordrhein-Westfalen, a.a.O., S. 121, sowie die im 2. Kapitel zitierten Kritiker Bös, Ehrlicher, Gläser und andere.

24) Kirsch, Guy, Über zentrifugale und zentripetale Kräfte im Föderalismus, in: Beiträge zu ökonomischen Problemen des Föderalismus, von Guy Kirsch, Christian Smekal, Horst 
Maßnahmen hängt dabei jedoch maßgeblich von der Funktionsweise des Gesamtsystems ab; es ist keineswegs gesichert, daß gerade die erwünschte Wirkung und nur sie eintritt. Oder wie Kirsch formuliert: "Möge ein jeder von ihnen wollen, daß etwas Bestimmtes gemacht wird, so mag im Ergebnis geschehen, was niemand gewollt hat."25)

Im Zusammenhang mit der Finanzkraftmessung wäre deshalb die Einsicht erforderlich, daß - zumindest wenn Finanzbedarf und Finanzkraft verglichen und damit vertikaler und horizontaler Finanzausgleich kombiniert werden sollen, die horizontalen Verteilungswirkungen nicht unabhängig von der vertikalen Kompetenzverteilung auftreten. Mit der jeweils isolierten Betrachtung der horizontalen, aber auch der vertikalen Effekte und den entsprechenden Änderungsversuchen werden dagegen Anforderungen an das praktizierte System herangetragen, die es in ihrer Komplexität und Widersprüchlichkeit kaum erfüllen kann.

Damit werden letztlich vor allem die Kommunen überfordert, die sich dem geltenden Finanzsystem in ihren Handlungen anpassen und dadurch regelmäßig in Konflikt mit staatlichen Zielsetzungen geraten.

Zimmermann. hrsg. von Kurt Schmidt, Schriften des Vereins für Socialpolitik, Neue Folge, Band 166, Berlin 1987, S. 13-34, hier S. 14.

25) ebenda. 
Ulrike Hardt - 978-3-631-75265-4

Downloaded from PubFactory at 01/11/2019 06:31:57AM

via free access 


\section{Literaturverzeichnis}

Advisory Commission on Intergovernmental Relations, The Tax Capacity of the Fifty States: Methology and Estimates, Washington 1982

Advisory Commission on Intergovernmental Relations, Measuring the Fiscal Capacity and Effort of State and Local Areas, Washington 1971

Albers, Willi, Finanzausgleich (III), Deutschland, in: Handwörterbuch der Sozialwissenschaften, dritter Band, Tübingen-Göttingen 1961, S. 553-575

Alonso, William, Zur Ökonomie der Stadtgröße, in: Fürst, Dietrich, Hrsg., Stadtökonomie, Stuttgart-New York 1977, S. 50-67

Akademie für Raumforschung und Landesplanung, Hrsg., Räumliche Aspekte des kommunalen Finanzausgleichs, Forschungs- und Sitzungsberichte Band 159, Hannover 1985

Amedick, Klaus, Die Besteuerung der gewerblichen Unternehmen als Standortfaktor, Diss., Köln 1959

Backhaus, Jürgen, Öffentliche Unternehmen, 2., durchgesehene und erweiterte Auflage, Frankfurt 1980

Bade, Franz-Josef, Die Beschäftigungsentwicklung in den Regionen der Bundesrepublik Deutschland 1976-1983, 2. Zwischenbericht zum Gutachten "Die regionale Verteilung von Wirtschaftsaktivitäten" im Auftrage des Niedersächsischen Ministers für Wirtschaft und Verkehr, Schriften des Deutschen Instituts für Wirtschaftsforschung, Berlin 1985

Bade, Franz-Josef, Funktionale Aspekte der regionalen Wirtschaftsstruktur, in: Raumforschung und Raumordnung, Heft 4, 37. Jg. 1979, S. 253-268 
Bade, Franz-Josef, Sektorale und räumliche Aspekte der Mobilität industrieller Betriebe, discussion papers des International Institute of Management - Wissenschaftszentrum Berlin, 1977

Bayer, Herrmann-Willfried, Die Kleinen Gemeindesteuern, in: Handbuch der kommunalen Wissenschaft und Praxis, Band 6, Kommunale Finanzen, hrsg. von Günter Püttner, 2., völlig neu bearbeitete Auflage, Berlin u.a. 1985, S. 156-245

Bericht der gemeinsamen Kommission zur Erarbeitung von Grundlagen und Vorschlägen für eine Neuregelung des kommunalen Finanzausgleichs (Finanzausgleichskommission), Stuttgart, Juni 1977

Bertelmann, Brigitte D., Regionalpolitisch relevante Wirkungen von Steuern, Frankfurt 1982

Biehl, Dieter und Münzer, Urban, Agglomerationsoptima und Agglomerationsbesteuerung - Finanzpolitische Konsequenzen aus der Existenz agglomerationsbedingter sozialer Kosten, in: Ballung und öffentliche Finanzen, hrsg. von der ARL, Forschungs- und Sitzungsberichte, Band 134, Hannover 1980, S. 113-150

Biehl, Dieter und Mitarbeiter, Bestimmungsgründe des regionalen Entwicklungspotentials, Kieler Studien Nr. 133, hrsg. von Herbert Giersch, Tübingen 1975

Birk, Dieter, Das Leistungsfähigkeitsprinzip als Maßstab der Steuernormen, Köln 1983

Bloch, Arthur, Entwicklung und Standortorientierung des Dienstleistungsbereichs aus der Sicht der Landesplanung, Schriftenreihe Landes- und Stadtentwicklungsforschung des Landes Nordrhein-Westfalen, Band 1.021, ILS Dortmund 1980 
Bohley, Peter, Gebühren und Beiträge - ihre wirtschaftspolitischen Funktionen und ihr Platz im System der öffentlichen Einnahmen -, Tübingen 1977

Brecht, Arnold, Internationaler Vergleich der öffentlichen Ausgaben, Leipzig und Berlin 1932

Brennan, Geoffrey und Buchanan, James M., The Power to Tax - Analytical foundations of a fiscal constitution, Cambridge u.a. 1980

Breton, Albert, Eine Theorie staatlicher Finanzzuweisungen, in: Kirsch, Guy, Hrsg., Föderalismus, Stuttgart-New York 1977, S. 77-89

Budäus, Dietrich, Betriebswirtschaftliche Instrumente zur Entlastung kommunaler Haushalte, Baden-Baden 1982

Bullinger, Dieter, Die Einnahmesituation und Strategien zur Einnahmesteigerung, in: Handbuch der kommunalen Wissenschaft und Praxis, Band 6, Kommunale Finanzen, hrsg. von Günter Püttner, 2., völlig neu bearbeitete Auflage, Berlin u.a. 1984, S. 106-116

Bundesministerium der Finanzen, Hrsg., Unsere Steuern von A-Z, Ausgabe 1986, Bonn 1986

Bundesministerium der Finanzen, Hrsg., Finanzbericht 1987, Bonn 1986

Bundesministerium der Finanzen, Hrsg., Finanzbericht 1980, Bonn 1979

Deubel, Ingolf, Der kommunale Finanzausgleich in Nordrhein-Westfalen Eine ökonomische und statistische Analyse, Köln u.a. 1984

Dietrich, H.P. und Straus, H.-G., Das Standortverhalten im Dienstleistungsbereich - Ergebnisse von Betriebs- und Expertenbefragungen - 3. Bericht zum Forschungsprojekt "Raumordnerische Standortbeeinflussung bei Arbeitsstätten des Dienstleistungsbereichs", hrsg. von der Gesellschaft für Wohnungs- und Siedlungswesen mbH Hamburg 1979 
Ehrlicher, Werner, Kommunaler Finanzausgleich und Raumordnung, Veröffentlichungen der ARL, Abhandlungen Band 51, Hannover 1967

Ewringmann, Dieter, Der staatliche Einfluß auf die kommunale Aufgabenerfüllung, in: Reform kommunaler Aufgaben, Studien zur Kommunalpolitik des Instituts für Kommunalwissenschaften (Konrad-Adenauer-Stiftung), Band 19, Bonn 1978, S. 116-139

Färber, Robert und Schneider, Ekkehard, Wanderungsmotivuntersuchung in der Region Oberfranken-Ost und im Landkreis Kronach, in: Tendenzen der Wanderungen und der Bevölkerungs- und Siedlungsentwicklung in Bayern, Veröffentlichungen der ARL, Forschungs- und Sitzungsberichte, Band 136, Hannover 1981, S. 35-66

Fischer, Ralf Joachim; Gschwind, Friedemann und Henkel, Dietrich, Siedlungsstrategien und kommunale Einnahmen, Schriftenreihe "Städtebauliche Forschung" des Bundesministers für Raumordnung, Bauwesen und Städtebau, Bonn 1980

Fischer-Menshausen, Herbert, Unbestimmte Rechtsbegriffe in der bundesstaatlichen Finanzverfassung, in: Probleme des Finanzausgleichs I, von Dieter Bös, Herbert Fischer-Menshausen, Guy Kirsch und Fritz Neumark, hrsg. von Wilhelmine Dreißig, Schriften des Vereins für Socialpolitik, Neue Folge, Band 96/I, Berlin 1978, S. 135-164

Flämig, Christian, Gemeindefinanzen und kommunale Wirtschaftsentwicklungsplanung, Baden-Baden 1974

Freund, Ulrich und Zabel, Gerhard, Zur Effizienz der regionalpolitischen Industrieförderung, in: Raumforschung und Raumordnung, Heft 3, 36. Jg. 1978, S. 99-106

Fürst, Dietrich und Zimmermann, Klaus (unter Leitung von Karl-Heinrich Hansmeyer), Standortwahl industrieller Unternehmen, Schriftenreihe der Gesellschaft für Regionale Strukturentwicklung, Band 1, Bonn 1973 
Galperin, Peter, Sozialhilfe und Bedarfsprinzip - Zum Streit um die Konkretisierung der Bedarfsdeckung - in: Leibfried, Stephan und Tennstedt, Florian, Politik und Armut und die Spaltung des Sozialstaates, Frankfurt 1985, S. 153-168

Gandenberger, Otto, Das Finanzmonopol - Fiskalische und außerfiskalische Wirkungen im Vergleich zur Verbrauchsteuer-, Veröffentlichungen des Forschungsinstituts für Wirtschaftspolitik an der Universität Mainz, Band 24, Heidelberg 1968

Gee, Colin; Keller, Ulrike und Treuner, Peter, Infrastrukturelle und wirtschaftsstrukturelle Bestimmungsgründe der industriellen Standortwahl, Schriftenreihe des IREUS, Band 4, Stuttgart 1980

Gerloff, Wilhelm, Allgemeine Steuerlehre, in: Handbuch der Finanzwissenschaft, 2., völlig neu bearbeitete Auflage, hrsg. von Wilhelm Gerloff und Fritz Neumark, zweiter Band, Tübingen 1956, S. 239-325

Gesetz über den kommunalen Finanzausgleich in der Fassung vom 23. Januar 1984, Gesetzblatt für das Land Baden-Würthemberg 1984, S. 129-143

Gesetz über den Finanzausgleich zwischen Staat, Gemeinden und Gemeindeverbänden in der Fassung der Bekanntmachung vom 10. Januar 1984, Bayerisches Gesetz- und Verordnungsblatt Nr. 2/1984, S. 6-12

Gesetz zur Regelung des Finanzausgleichs in der Fassung vom 29. Juni 1984, Gesetz- und Verordnungsblatt für das Land Hessen, Teil I, Ausgegeben zu Wiesbaden am 31. Juli 1984, S. 193-206

Gesetz zur Änderung des Gesetzes über den Finanzausgleich und des Niedersächsischen Gesetzes zum Bundesgesetz zur wirtschaftlichen Sicherung der Krankenhäuser und zur Regelung der Krankenhauspflegesätze vom 4. Oktober 1983,

Niedersächsiches Gesetz- und Verordnungsblatt, Ausgegeben in Hannover am 7. Oktober 1983, 37. Jahrgang, Nr. 34, S. 253-254 
Gesetz über den Finanzausgleich in der Fassung vom 11. November 1981,

Niedersächsisches Gesetz- und Verordnungsblatt, Ausgegeben in Hannover am 17. November 1981, 35. Jahrgang, Nr. 42, S. 340-345

Gesetz zur Regelung der Zuweisungen des Landes Nordrhein-Westfalen an die Gemeinden und Gemeindeverbände im Haushaltsjahr 1984 vom 21. Februar 1984,

Gesetz- und Verordnungsblatt für das Land Nordrhein-Westfalen - Nr. 8 vom 28. Februar 1984, S. 55-63

Landesgesetz zur Änderung des Finanzausgleichsgesetzes vom 6. April 1984, Gesetz- und Verordnungsblatt für das Land Rheinland-Pfalz, Nr. 10, Ausgegeben zu Mainz, den 19. April 1984, S. 77-78

Gesetz zur Änderung des Finanzausgleichsgesetzes vom 18. Dezember 1981, Gesetz- und Verordnungsblatt für das Land Rheinland-Pfalz, Nr. 28, Ausgegeben zu Mainz, den 30. Dezember 1981, S. 332-333

Gesetz zur Änderung des Finanzausgleichsgesetzes vom 2. Juli 1980, Gesetzund Verordnungsblatt für das Land Rheinland-Pfalz, Nr. 14, Ausgegeben zu Mainz, den 11. Juli 1980, S. 147

Änderung des Finanzausgleichsgesetzes im Landesgesetz vom 22. März 1978,

Gesetz- und Verordnungsblatt für das Land Rheinland-Pfalz, Nr. 9, Ausgegeben zu Mainz, den 1. April 1978, S. 145

Landesgesetz über den Finanzausgleich in Rheinland-Pfalz vom 28. Oktober 1977,

Gesetz- und Verordnungsblatt für das Land Rheinland-Pfalz, Nr. 31, Ausgegeben zu Mainz, den 9. November 1977, S. 353-362

Gesetz Nr. 1163 über die Haushaltsfinanzierung vom 15. Dezember 1983, 
Amtsblatt des Saarlandes vom 5. Januar 1984, S. 4

Gesetz Nr. 1157 "Kommunalfinanzausgleichsgesetz - KFAG -" vom 12. Juli 1983, Amtsblatt des Saarlandes vom 11. August 1983, S. 462-471

Gesetz über den Finanzausgleich in Schleswig-Holstein in der Fassung vom 16. Januar 1984,

Gesetz- und Verordnungsblatt für Schleswig-Holstein 1984, Tag der Ausgabe: Kiel, den 25. Januar 1984, S. 27-35

Gläser, Martin, Die staatlichen Finanzzuweisungen an die Gemeinden - Gestaltungskriterien, Effektivität, Reform -Thun-Frankfurt/Main 1981

Glaser, Gundolf, Lohnt sich die Neuansiedlung von Industrie aus fiskalischer Sicht der Kommunen?, in: Ballung und öffentliche Finanzen, Veröffentlichungen der ARL, Forschungs- und Sitzungsberichte, Band 134, Hannover 1980, S. $185-202$

Göb, Rüdiger, Kommunale Wirtschaftspolitik, in: Archiv für Kommunalwissenschaften, 26. Jg., 1987, Erster Halbjahresband, S. 66-87

Goetz, Charles, J., What is Revenue Sharing?, Washington D.C. 1972

Grewal, B.S. und Mathews, R.L., Intergovernmental Tax Competition and CoOrdination, in: Mathews, R.L., Hrsg., State and Local Taxation, Canberra 1977, S. 83-93

Grimme, Leonhard, Neuberechnung der Steuerkraft im kommunalen Finanzausgleich - dargestellt am Beispiel des Landes Bayern, in: Räumliche Aspekte des kommunalen Finanzausgleichs, Veröffentlichungen der ARL, Forschungs- und Sitzungsberichte, Band 159, Hannover 1985, S. $159-200$

Gutenberg, Erich, Zur Diskussion der polypolistischen Absatzkurve, in: Jahrbücher für Nationalökonomie und Statistik, Band 177, Heft 4 (1965), S. 289-303 
Hahne, Ulf, Regionalentwicklung durch Aktivierung intraregionaler Potentiale, Schriften des Instituts für Regionalforschung der Universität Kiel, Band 8, hrsg. von Karin Peschel, München 1985

Haller, Heinz, Gründe für besondere gliedstaatliche Steuertarife in Bundesstaaten, in: Hanusch, Horst, Roskamp, Karl W. und Wiseman, Jack, Hrsg., Staat und politische Ökonomie heute, Horst Claus Recktenwald zum 65. Geburtstag, Stuttgart-New York 1985, S. 209-221

Haller, Heinz, Die Steuern, 3., überarbeitete Auflage, Tübingen 1981

Handelskammer Trier - Industrie- und Handelskammer Trier, Hrsg., Sparen und rationalisieren? ... privatisieren!, Trier 1983

Hansmeyer, Karl-Heinrich, Abgaben und steuerliche Instrumente der Umweltpolitik - Wirkungsweise, Erfahrungen, Möglichkeiten -, in: Zeitschrift für Umweltpolitik und Umweltrecht, Nr. 3/September 1987, S. 251-266

Hansmeyer, Karl-Heinrich und Kops, Manfred, Finanzwissenschaftliche Grundsätze für die Ausgestaltung des kommunalen Finanzausgleichs, in: Hoppe, Werner, Hrsg., Reform des kommunalen Finanzausgleichs, Köln u.a. 1985 , S. $31-60$

Hansmeyer, Karl-Heinrich und Kops, Manfred, Die Kompetenzen der Aufgabenzuständigkeit im föderativen Staat, in: Hamburger Jahrbücher für Wirtschafts- und Gesellschaftspolitik, 29. Jg., 1984, S. 127-140

Hansmeyer, Karl-Heinrich, Der kommunale Finanzausgleich als Instrument zur Förderung zentraler Orte, in: Probleme des Finanzausgleichs II, hrsg. von Dieter Pohmer, Schriften des Vereins für Socialpolitik, Neue Folge, Band 96/II, Berlin 1980, S. 83-150

Hansmeyer, Karl-Heinrich und Fürst, Dietrich, Die Gebühren - zur Theorie eines Instrumentariums der Nachfragelenkung bei öffentlichen Leistungen - Schriften des Vereins für Kommunalwissenschaften e.V., Band 18, Stuttgart u.a. 1968 
Hanusch, Horst und Kuhn, Thomas, Messung des kommunalen Finanzbedarfs - Ein alternativer Ansatz für die Schlüsselzuweisungen, in: Räumliche Aspekte des kommunalen Finanzausgleichs, Veröffentlichungen der ARL, Forschungs- und Sitzungsberichte Band 159, Hannover 1985, S. 5574

Haverkamp, Franz, Die Grundsteuer, in: Handbuch der kommunalen Wissenschaft und Praxis, Band 6, Kommunale Finanzen, hrsg. von Günter Püttner, 2., völlig neu bearbeitete Auflage, Berlin u.a. 1985, S. 117-127

Haverkamp, Franz, Die Gemeindefinanzreform, in: Die Finanzbeziehungen zwischen Bund, Ländern und Gemeinden, hrsg. vom Bundesminister der Finanzen, Bonn 1982, S. 527-560

Heller, Wolfgang, Die Grenzen der Besteuerung, in: Festgabe für Georg Schanz, Band II, hrsg. von Hans Teschemacher, Tübingen 1928, S. 87-111

Hessler, Heinz-Dieter, Finanzwissenschaftliches System der Besteuerung, Tübingen-Düsseldorf 1976

Heuer, Hans, Instrumente kommunaler Gewerbepolitik - Ergebnisse empirischer Erhebungen - Schriften des Deutschen Instituts für Urbanistik, Band 73, Stuttgart u.a. 1985

Hidien, Jürgen, Staatliche Wirtschaftsaufsicht und Besteuerung kommunaler Unternehmen, in: Handbuch der kommunalen Wissenschaft und Praxis, Band 5, kommunale Wirtschaft, hrsg. von Günter Püttner, 2., völlig neu bearbeitete Auflage, Berlin u.a. 1984, S. 98-118

Hirschmann, Albert O., Abwanderung und Widerspruch, Tübingen 1974

Kaiser, Karl-Heinz, Industrielle Standortfaktoren und Betriebstypenbildung, Berlin 1979

Karrenberg, Hanns, Die Bedeutung der Gewerbesteuer für die Städte, neue Schriften des Deutschen Städtetages, Heft 52, Stuttgart u.a. 1985 
Karrenberg, Hanns und Münstermann, Engelbert, Gemeindefinanzbericht 1987, in: Der Städtetag, Sonderdruck 2/1987

Karrenberg, Hanns und Münstermann, Engelbert, Gemeindefinanzbericht 1985, in: Der Städtetag, Sonderdruck 2/1985, S. 72-117

Karrenberg, Hanns und Münstermann, Engelbert, Gemeindefinanzbericht 1984, in: Der Städtetag, Sonderdruck 2/1984

Kentmann, Konrad, Das Äquivalenzprinzip in den Gemeinden, Frankfurt 1978

Kilger, Wolfgang, Die quantitative Ableitung polypolistischer Preisabsatzfunktionen aus den Heterogenitätsbedingungen atomistischer Märkte, in: Koch, Helmut, Hrsg., Zur Theorie der Unternehmung, Festschrif zum 65. Geburtstag von Erich Gutenberg, Wiesbaden 1962, S. 269-312

Kirchhof, Ferdinand, Die Höhe der Gebühr - Grundlagen der Gebührenbemessung - Schriften zum öffentlichen Recht, Band 399, Berlin 1981

Kirchhof, Paul, Die kommunale Finanzhoheit, in: Handbuch der kommunalen Wissenschaft und Praxis, Band 6, Kommunale Finanzen, hrsg. von Günter Püttner, 2., völlig neu bearbeitete Auflage, Berlin u.a. 1985, S. 3-28

Kirchhof, Paul, Rechtliche Rahmenbedingungen des kommunalen Finanzausgleichs, in: Hoppe, Werner, Hrsg., Reform des kommunalen Finanzausgleichs, Schriftenreihe des Landkreistages Nordrhein-Westfalen, Köln u.a. 1985, Band 4, S. 1-30

Kirchhof, Paul, Der Finanzausgleich als Grundlage kommunaler Selbstverwaltung, in: Deutsches Verwaltungsblatt, Jg. 1980, S. 711-719

Kirsch, Bernd und Schußmann, Klaus, Kommunale Gewerbesteuerpolitik, in: Informationen zur Raumentwicklung, hrsg. von der BfLR, Heft 2/3, 1978, S. 105-114. 
Kirsch, Guy, Über zentrifugale und zentripetale Kräfte im Föderalismus, in: Beiträge zu ökonomischen Problemen des Föderalismus, von Guy Kirsch, Christian Smekal, Horst Zimmermann, hrsg. von Kurt Schmidt, Schriften des Vereins für Socialpoltik, neue Folge, Band 166, Berlin 1987, S. 13-34

Kirsch, Guy, Neue politische Ökonomie, 2., neu bearbeitete und erweiterte Auflage, Düsseldorf 1983

Kirsch, Guy, Hrsg., Föderalismus, Stuttgart-New York 1977

Klein, Richard R., Kommunale Schuldenpolitik, Schriften des Deutschen Instituts für Urbanistik, Band 61, Stuttgart u.a. 1977

Klemmer, Paul, Ermittlung von Basisdienstleistungsbereichen, hrsg. von der ARL, Beiträge, Band 38, Hannover 1980

Klemmer, Paul, Abgrenzung strukturgefährdeter ländlicher Räume - Indikatoren für die Arbeitsmarktsituation, Arbeitsmaterialien der ARL, Nr. 8, Hannover 1978

Köhne, Manfred, Die steuerliche Sonderstellung der Landwirtschaft, in: Köhne, Manfred, Hrsg., Beiträge zur Agrarökonomie, Festschrift zum 80. Geburtstag von Emil Woermann, Hamburg und Berlin 1979, S. 153-168

Kommunalabgabengesetz Nordrhein-Westfalen; abgedruckt in: Landkreistag Nordrhein-Westfalen, Hrsg., Verfassungs- und Verwaltungsvorschriften in Nordrhein-Westfalen, Düsseldorf 1985, S. 519-540

Kops, Manfred, Die regionalen Verteilungswirkungen des kommunalen Finanzausgleichs, Thun-Frankfurt 1983

Laffer, Arthur B. and Miles, Marc A., International Economics in an Integrated World, Dallas, Tex. u.a. 1982

Landesamt für Datenverarbeitung und Statistik Nordrhein-Westfalen, Hrsg., Statistische Berichte, Die staatlichen und kommunalen Finanzen in Nordrhein-Westfalen, Rechnungsjahr 1983, Düsseldorf 1985 
Lauschmann, Elisabeth, Grundlagen einer Theorie der Regionalpolitik, 3., völlig neu bearbeitete Auflage, Hannover 1976

Lehmann, Matthias, Beiträge, in: Handbuch der kommunalen Wissenschaft und Praxis, Band 6, Kommunale Finanzen, hrsg. von Günter Püttner, 2., völlig neu bearbeitete Auflage, Berlin u.a. 1984, S. 260-279

Limbert-Michaelis, Helga, Neuere Entwicklung in der Theorie des Finanzausgleichs, Diss., Reinheim 1973

Littmann, Konrad, Überblick über die Ertragssteuern, in: Handbuch der Finanzwissenschaft, Band II, gänzlich neu bearbeitete Auflage, hrsg. von Fritz Neumark, Tübingen 1980, S. 565-574

Lösch, August, Die räumliche Ordnung der Wirtschaft, 2., Auflage, Jena 1944

Massat, Dieter, Die ökonomische Problematik von Zuweisungen, ThunFrankfurt/Main 1984

Matzner, Egon, Typologie der "öffentlichen Aufgaben", in: derselbe, Hrsg., Öffentliche Aufgaben und Finanzausgleich, Wien 1977, S. 10-12

Metz, Hans-Günter, Der Ausgleich von Finanzkraft- und Bedarfsunterschieden im föderalistischen Staat, Frankfurt 1979

Milbradt, Georg H., Die Gewerbesteuer, in: Handbuch der kommunalen Wissenschaft und Praxis, Band 6, Kommunale Finanzen, hrsg. von Günter Püttner, 2., völlig neu bearbeitete Auflage, Berlin u.a. 1985, S. 128-140

Müller, Wolfgang-Hans, Wirtschaftsförderung, in: Handbuch der kommunalen Wissenschaft und Praxis, Band 4, Die Fachaufgaben, hrsg. von Günter Püttner, 2., völlig neu bearbeitete Auflage, Berlin u.a. 1983, S. 625-641

Münch, Klaus N., Kollektive Güter und Gebühren - Elemente einer Gebührentheorie für Kollektivgüter, Göttingen 1976 
Münstermann, Engelbert, Der kommunale Finanzausgleich I - Begriff und Funktion, in: WISU 6/1976, S. 275-279

Münstermann, Engelbert, Die Berücksichtigung zentralörtlicher Funktionen im kommunalen Finanzausgleich, Forschungsberichte des Landes Nordrhein-Westfalen, Nr. 2520, Opladen 1975

Münstermann, Engelbert und Becker, Hubert, Finanzausgleichsleistungen an Kommunen - Ein Vergleich der Finanzausgleichssysteme in den Bundesländern - DST - Beiträge zur Finanzpolitik, Heft 7, hrsg. vom Deutschen Städtetag, Köln 1978

Musgrave, Richard Abel; Musgrave, Peggy B. und Kullmer, Lore, Die öffentlichen Finanzen in Theorie und Praxis, 1. Band, 3., völlig überarbeitete Auflage, Tübingen 1984

Musgrave, Richard Abel; Musgrave, Peggy B. und Kullmer, Lore, Die öffentlichen Finanzen in Theorie und Praxis, 3. Band, 1. Auflage, Tübingen 1977

Musgrave, Richard Abel, Musgrave Peggy B. und Kullmer, Lore, Die öffentlichen Finanzen in Theorie und Praxis, 4. Band, 1. Auflage, Tübingen 1980

Neumark, Fritz, Gedanken zur Steuer- und Finanzreform, in: Wirtschaftsdienst IX/1978, S. 446-450

Neumark, Fritz, Vom Wesen der Besteuerung, in: Voigt, Fritz, Hrsg., Beiträge zur Finanzwissenschaft und zur Geldtheorie, Festschrift für Rudolf Stucken, Göttingen 1953, S. 7-20

Niskanen, W.A., Ein ökonomisches Modell der Bürokratie, in: Pommerehne, Werner W. und Frey, Bruno S., Hrsg., Ökonomische Theorie der Politik, Berlin-Heidelberg-New York 1979, S. 349-368

Oates, Wallace E., Ein ökonomischer Ansatz zum Föderalismusproblem, aus: Oates, Wallace E., Fiscal Federalism, New York 1972, Kap. 1, S. 3 ff; in deutscher Übersetzung abgedruckt in: Kirsch, Guy, Hrsg., Föderalismus, Stuttgart-New York 1977, S. 15-26 
Olson, Mancur Jr., The Principle of "Fiscal Equivalence": The Division of Responsabilities among different Levels of Government, in: The American Economic Review, Vol. 59 (1969), S.479-487

Ott, Alfred E., Preistheorie, in: Kompendium der Volkswirtschaftslehre, Band 1, 5. ergänzte und überarbeitete Auflage, Göttingen 1975, S. 114-181

Pagenkopf, Hans, Das Gemeindefinanzsystem und seine Problematik, Schriften zum deutschen Kommunalrecht, Band 15, Siegburg 1978

Pagenkopf, Hans, Kommunalrecht, Band 1, Verfassungsrecht, 2., neu bearbeitete Auflage, Köln u.a. 1975

Pagenkopf, Hans, Kommunalrecht, Band 2, Wirtschaftsrecht, 2., neu bearbeitete und erweiterte Auflage, Köln u.a. 1976

Patzig, Werner, Strukturprobleme des kommunalen Finanzausgleichs, in: Deutsches Verwaltungsblatt, 94. Jg., Heft 13, Juli 1979, S. 477-490

Pohl, Martha, Wirtschaftsförderung in Großstädten, Teil I: Arbeitsmarkt und Wirtschaftsstruktur, in: Bremer Zeitschrift für Wirtschaftspolitik, Heft 5/1982, S. 5-199

Pohmer, Dieter und Saile, Peter, Das "ideale" Einnahmensystem, in: Handbuch der kommunalen Wissenschaft und Praxis, Band 6, Kommunale Finanzen, hrsg. von Günter Püttner, 2., völlig neu bearbeitete Auflage, Berlin u.a. 1985, S. 25-105

Pollak, Helga und Voß, Wolfgang, Die Berücksichtigung von Spillover-Effekten im kommunalen Finanzausgleich, in: Räumliche Aspekte des kommunalen Finanzausgleichs, hrsg. von der ARL, Forschungs- und Sitzungsberichte, Band 159, Hannover 1985, S. 133-158

Popitz, Johannes, Der künftige Finanzausgleich zwischen Reich, Ländern und Gemeinden, Berlin 1932 
Postlep, Rolf-Dieter, Räumliche Effekte der Steuerkraft- und Finanzbedarfsermittlung bei den Schlüsselzuweisungen, in: Räumliche Aspekte des kommunalen Finanzausgleichs, Veröffentlichungen des ARL, Forschungs- und Sitzungsberichte Band 159, Hannover 1985, S. 201-234

Postlep, Rolf-Dieter, Wirtschaftsstruktur und großstädtische Finanzen - Einflüsse höherwertiger Dienstleistungen auf die kommunalen Steuereinnahmen und Ausgaben in verschiedenen Großstädten - Veröffentlichungen der ARL, Abhandlungen Band 87, Hannover 1985

Postlep, Rolf-Dieter, Regionale Effekte höherwertiger Dienstleistungen, Schriftenreihe der Gesellschaft für Regionale Strukturentwicklung, Band 10, Bonn 1982

Prognos AG, Struktur und Motive der Wanderungsbewegungen in der Bundesrepublik Deutschland, hrsg. vom Bundesminister des Inneren, Basel 1968

Recktenwald, Horst Claus, Steuerüberwälzungslehre, 2., überarbeitete und ergänzte Auflage, Berlin 1966

Rehm, Hannes, Beteiligung der Gemeinden an der Umsatzsteuer?, in: Wirtschaftsdienst XII/1978, S. 624-632

Reissert, Bernd, Finanzielle Spielräume für kommunale Beschäftigungspolitik?, in: Maier Hans E. und Wollmann, Hellmut, Hrsg., Lokale Beschäftigungspolitik, Basel-Boston-Stuttgart 1986, S. 35-63

Rohr, Hans-Gottfried von, Intraregionale Wanderungen in ihrem Einfluß auf die Entwicklung in Verdichtungsräumen - Erklärungsansätze und Trendszenarien - Schriftenreihe "Raumordnung" des Bundesministers für Raumordnung, Bauwesen und Städtebau, Mr. 06029, Bonn 1978

Roppel, Ulrich, Ökonomische Theorie der Bürokratie, Freiburg 1979

Rose, Gerd, Betrieb und Steuer, 1. Buch, Die Ertragsteuern, 9., erheblich überarbeitete Auflage, Wiesbaden 1986 
Rosenschon, Jürgen Ekkehard, Gemeindefinanzsystem und Selbstverwaltungsgarantie, Schriften zur öffentlichen Verwaltung, Band 19, Köln u.a. 1980

Rottmann, Joseph, Regionalpolitik und kommunaler Finanzausgleich, Beiträge zum Siedlungs- und Wohnungswesen und zur Raumplanung, Band 83, hrsg. von Werner Ernst, Werner Happe und Rainer Thoss, Münster 1982

Sälzer, Bruno E., Standortdynamik von Industrieunternehmungen, FrankfurtBern-New York 1985

Schaab, Bodo, Örtliche Wirtschaftsstruktur und kommunale Steuerkraft, Diss., Konstanz 1972

Scharpf, Fritz W., Reissert, Bernd und Schnabel, Fritz, Politikverflechtung: Theorie und Empirie des kooperativen Föderalismus in der Bundesrepublik, Kronberg 1976

Scheel, Werner, Untersuchung der Leistungen der Bundesländer an die Gemeinden (GV) in den Jahren 1970-77, hrsg. vom Finanzminister des Landes Nordrhein-Westfalen, Düsseldorf 1979

Schliebe, Klaus, Industrieansiedlungen, Forschungen zur Raumentwicklung, Band 11, hrsg. von der BfLR, Bonn 1982

Schliebe, Klaus, Zur regionalen Verteilung höherwertiger Unternehmensfunktionen und Arbeitskräfteressourcen, in: Informationen zur Raumentwicklung, hrsg. von der BfLR, Heft 7, 1978, S. 545-554

Schmidt-Eichstädt, Gerd, Bundesgesetze und Gemeinden. - Die Inanspruchnahme der Kommunen durch die Ausführung von Bundesgesetzen Schriften des Deutschen Instituts für Urbanistik, Band 65, Stuttgart u.a. 1981 
Schmidt-Eichstaedt, Gerd; Stade, Isabell und Borchmann, Michael, Die Gemeindeordnungen in der Bundesrepublik Deutschland, Schriften des Deutschen Instituts für Urbanistik, Band 47, Loseblattsammlung, Stuttgart u.a.

Schmidt-Jortzig, Edzard, Die Zulässigkeit kommunaler wirtschaftlicher Unternehmen im einzelnen, in: Handbuch der kommunalen Wissenschaft und Praxis, Band 5, Kommunale Wirtschaft, hrsg. von Günter Püttner, 2., völlig neu bearbeitete Auflage, Berlin u.a. 1984, S. 50-70

Schmidt-Jortzig, Edzard, Probleme der kommunalen Fremdverwaltung Fehlentwicklungen in der Struktur einer bewährten Verwaltungsform in: Die öffentliche Verwaltung, 34. Jg., Heft 11, Juni 1981, S. 393-401

Schmölders, Günter und Hansmeyer, Karl-Heinrich, Allgemeine Steuerlehre, 5. Auflage, neu bearbeitet von Karl-Heinrich Hansmeyer, Berlin 1980

Schmölders, Günter, Finanzpolitik, 3., neu bearbeitete Auflage, Berlin-Heidelberg-New York 1970

Schmölders, Günter, "Finanzstarke" und "finanzschwache" Gemeinden, in: Archiv für Kommunalwissenschaften, 1. Halbjahresband 1965, S. 30-46

Scholler, Heinrich und Broß, Siegfried, Grundzüge des Kommunalrechts in der Bundesrepublik Deutschland, 3., neu bearbeitete Auflage, Heidelberg 1984

Schreyer, Michaele, Transferpolitik im Föderalismus - Probleme der Kompetenzverteilung, Frankfurt a.M.-Bern-New York 1983

Seiler, Gerhard, Ziele und Mittel des kommunalen Finanzausgleichs, in: Probleme des Finanzausgleichs II, von Karl-Heinrich Hansmeyer, Gerhard Seiler und Christian Smekal, hrsg. von Dieter Pohmer, Schriften des Vereins für Socialpolitik, Neue Folge, Band 96/III, Berlin 1980, S. 11-82 
Seiler, Gerhard, Zur Bedeutung der Gebühren, Beiträge und anderer Verwaltungsabgaben für den Haushaltsausgleich der Gebietskörperschaften, Institut "Finanzen und Steuern", Hrsg., Brief 180, Bonn 1978

Smekal, Christian, Transfers zwischen Gebietskörperschaften (TG), in: Probleme des Finanzausgleichs II, von Karl-Heinrich Hansmeyer, Gerhard Seiler und Christian Smekal, hrsg. von Dieter Pohmer, Schriften des Vereins für Socialpolitik, Neue Folge, Band 96/II, Berlin 1980, S. 151-220

Spehl, Harald; Töpfer, Klaus und Töpfer, Peter, Folgewirkungen von Industrieansiedlungen, Schriftenreihe der Gesellschaft für Regionale Sturkturentwicklung, Band 3, Bonn 1975

Statistisches Bundesamt, Hrsg., Statistisches Jahrbuch für die Bundesrepublik Deutschland, Stuttgart und Mainz 1984

Statistisches Bundesamt, Hrsg., Fachserie 14, Reihe 3.3., Rechnungsergebnisse der kommunalen Haushalte 1983

Statistisches Bundesamt, Gewerbesteuer nach Ertrag und Kapital 1970, Wiesbaden 1978

Strauß, Wolfgang, Räumliche Wirkungen der Gewerbesteuer, in: Informationen zur Raumentwicklung, hrsg. von der BfLR, Heft 6/7, 1983, S. 405-418

Tetsch, Friedemann, Raumwirkungen des Finanzsystems in der Bundesrepublik Deutschland, Frankfurt 1978

Tipke, Klaus, Steuerrecht - Ein systematischer Grundriß, 9., völlig überarbeitete Auflage, Köln 1983

Tofaute, Hartmut, Die Übertragung öffentlicher Leistungen und Funktionen auf Private (Privatisierung) - Eine Darstellung politischer, ökonomischer, gesellschaftlicher und arbeitnehmerorientierter Gesichtspunkte, Schriften zur Privatisierung öffentlicher Dienstleistungen Nr. 5, hrsg. vom Hauptvorstand der Gewerkschaft ÖTV, Reutlingen 1977 
Tiebout, Charles M., A Pure Theory of Local Expenditures, in: Journal of Political Economy, October 1956, S. 416-424

Tullock, Gordon, Föderalismus: Zum Problem der optimalen Kollektivgröße, in: Kirsch, Guy, Hrsg., Föderalismus, Stuttgart-New York 1977, S. 27-35

Voigtländer, Hubert, Zur Problematik der Gestaltung eines selbstverwaltungsgerechten und aufgabenorientierten Finanzausgleichs, in: Informationen zur Raumentwicklung, hrsg. von der BfLR, Heft 2/3, 1978, S. 185192

Wanniski, Jude, Taxes, Revenues, and the "Laffer Curve", in: Laffer, Arthur B. und Seymour, Jan P., The Economics of the Tax Revolt: A Reader, New York u.a. 1979, S. 7-12

Weber, Alfred, Über den Standort der Industrien, Teil 1: Reine Theorie des Standorts, Tübingen 1909

Wienen, Horst-Jürgen, Räumliche Verteilung der Zuweisungen und Baumaßnahmen des Landes Nordrhein-Westfalen 1969-1975, in: Ballung und öffentliche Finanzen, hrsg. von der ARL, Forschungs- und Sitzungsberichte, Band 134, Hannover 1980, S. 97-111

Wilke, Dieter, Gebühren, in: Handwörterbuch der kommunalen Wissenschaft und Praxis, Band 6, Kommunale Finanzen, hrsg. von Günter Püttner, 2., völlig neu bearbeitete Auflage, Berlin u.a. 1985, S. 246-259

Willeke, Franz-Ulrich, Wettbewerbspolitik, Tübingen 1980

Wissenschaftlicher Beirat beim Bundesministerium der Finanzen, Gutachten zur Reform der Gemeindesteuern in der Bundesrepublik Deutschland, Schriftenreihe des BMF, Heft 31, Bonn 1982

Zabel, Gerhard, Ist die Hauptansatzstaffel noch zeitgemäß?, in: Information zur Raumentwicklung, hrsg. von der BfLR, Heft 6/7, 1983, S. 445-459 
Zimmermann, Horst, Föderalismus und "Einheitlichkeit der Lebensverhältnisse", in: Beiträge zu ökonomischen Problemen des Föderalismus, von Guy Kirsch, Christian Smekal, Horst Zimmermann, hrsg. von Kurt Schmidt, Schriften des Vereins für Socialpolitik, Neue Folge, Band 166, Berlin 1987, S. 35-70

Zimmermann, Horst; Hardt, Ulrike und Postlep, Rolf-Dieter, Determinanten der kommunalen Finanzsituation - unter besonderer Berücksichtigung der Gemeinden in Ballungsgebieten - Schriftenreihe der Gesellschaft für Regionale Strukturentwicklung, Band 15, Bonn 1987

Zimmermann, Horst und Henke, Klaus-Dirk, Finanzwissenschaft - Eine Einführung in die Lehre von der öffentlichen Finanzwirtschaft - 4., überarbeitete und ergänzte Auflage, München 1985

Zimmermann, Horst, Allgemeine Probleme und Methoden des Finanzausgleichs, in: Handbuch der Finanzwissenschaft, 3., gänzlich neu bearbeitete Auflage, hrsg. von Fritz Neumark, Band IV, Tübingen 1983, S. 3-52

Zimmermann, Horst und Postlep, Rolf-Dieter, Beurteilungsmaßstäbe für Gemeindesteuern, in: Wirtschaftsdienst V/1980, S. 248-253

Zimmermann, Horst und Mitarbeiter, Regionale Präferenzen, Schriftenreihe der Gesellschaft für Regionale Strukturentwicklung, Band 2, Bonn 1973 


\section{FINANZWISSENSCHAFTLICHE SCHRIFTEN}

Band 1 Werner Steden: Finanzpolitik und Einkommensverteilung. Ein Wachstums- und Konjunkturmodell der Bundesrepublik Deutschland. 1979.

Band 2 Rainer Hagemann: Kommunale Finanzplanung im foderativen Staat. 1976.

Band 3 Klaus Scherer: Maßstäbe zur Beurteilung von konjunkturellen Wirkungen des bffentlichen Haushalts. 1977.

Band 4 Brita Steinbach: "Formula Flexibility" - Kritische Analyse und Vergleich mit diskretionärer Konjunkturpolitik. 1977.

Band 5 Hans-Georg Petersen: Personelle Einkommensbesteuerung und Inflation. Eine theoretischempirische Analyse der Lohn- und veranlagten Einkommensteuer in der Bundesre-publik Deutschland. 1977.

Band 6 Friedemann Tetsch: Raumwirkungen des Finanzsystems der Bundesrepublik Deutschland. Eine Untersuchung der Auswirkungen der Finanzreform von 1969 auf die Einnahmenposition der untergeordneten Gebietskorperschaften und inrer regionalpolitischen Zieladăquanz. 1978.

Band 7 Wilhelm Pfăhler: Normative Theorie der fiskalischen Besteuerung. Ein methodologischer und theoretischer Beitrag zur Integration der normativen Besteuerungstheorie in der Wohlfahrtstheorie. 1978.

Band 8 Wolfgang Wiegard: Optimale Schattenpreise und Produktionsprogramme für offentliche Unternehmen. Second-Best Modelle im finanzwirtschaftlichen Staatsbereich. 1978.

Band 9 Hans P. Fischer: Die Finanzierung des Umweltschutzes im Rahmen einer rationalen Umweltpolitik. 1978.

Band 10 Rainer Paulenz: Der Einsatz finanzpolitischer Instrumente in der Forschungs- und Entwicklungspolitik. 1978.

Band 11 Hans-Joachim Hauser: Verteilungswirkungen der Staatsverschuldung. Eine kreislauftheoretische Inzidenzbetrachtung. 1979.

Band 12 Gunnar Schwarting: Kommunale investitionen. Theoretische und empirische Untersuchungen der Bestimmungsgründe kommunaler Investitionstătigkeit in Nordrhein-Westfalen 1965-1972. 1979.

Band 13 Hans-Joachim Conrad: Stadt-Umland-Wanderung und Finanzwirtschaft der Kernstădte. Amerikanische Ertahrungen, grundsătzliche Zusammenhänge und eine Fallstudie für das Ballungsgebiet Frankfurt am Main. 1980.

Band 14 Cay Folkers: Vermógensverteilung und staatliche Aktivităt. Zur Theorie distributiver Prozesse im Interventionsstaat. 1981.

Band 15 Helmut Fischer: US-amerikanische Exporttörderung durch die DISC-Gesetzgebung. 1981.

Band 16 Günter Ott: Einkommensumverteilungen in der gesetzlichen Krankenversicherung. Eine quantitative Analyse. 1981.

Band 17 Johann Hermann von Oehsen: Optimale Besteuenung. (Optimal Taxation). 1982.

Band 18 Richard Kóssler: Sozialversicherungsprinzip und Staatszuschüsse in der gesetzlichen Rentenversicherung. 1982.

Band 19 Hinrich Steffen: Zum Handlungs- und Entscheidungsspielraum der kommunalen Investitionspolitik in der Bundesrepublik Deutschland. 1983. 
Band 20 Manfred Scheuer: Wirkungen einer Auslandsverschuldung des Staates bei flexiblen Wechselkursen. 1983.

Band 21 Christian Schiller: Staatsausgaben und crowding-out-Effekte. Zur Effizienz einer Finanzpolitik keynesianischer Provenienz. 1983.

Band 22 Hannelore Weck: Schattenwirtschaft: Eine Möglichkeit zur Einschrănkung der offentlichen Venwaltung? Eine Okonomische Analyse. 1983.

Band 23 Wolfgang Schmitt: Steuern als Mittel der Einkommenspolitik. Eine Ergânzung der Stabilitătspolitik? 1984.

Band 24 Wolfgang Laux: Erhöhung staatswirtschaftlicher Effizienz durch budgetăre Selbstbeschrănkung? Zur Idee einer verfassungsmaßig verankerten Ausgabengrenze. 1984.

Band 25 Brita Steinbach-van der Veen: Steuerinzidenz. Methodologische Grundlagen und empirischstatistische Probleme von Lănderstudien. 1985.

Band 26 Albert Peters: Okonomische Kriterien für eine Aufgabenverteilung in der Marktwirtschaft. Eine deskriptive und normative Betrachtung für den Allokationsbereich. 1985.

Band 27 Achim Zeidler: Moglichkeiten zur Fortsetzung der Gemeindefinanzreform. Eine theoretische und empirische Analyse. 1985.

Band 28 Peter Bartsch: Zur Theorie der lăngerfristigen Wirkungen 'expansiver Fiskalpolitik. Eine dynamische Analyse unter besonderer Berücksichtigung der staatlichen Budgetbeschränkung und ausgewăhlter Möglichkeiten der offentlichen Defizitfinanzierung. 1986.

Band 29 Konrad Beiwinkel: Wehrgerechtigkeit als finanzpolitisches Verteilungsproblem. Moglichkeiten einer Kompensation von Wehrungerechtigkeit durch monetäre Transfers. 1986.

Band 30 Wolfgang Kitterer: Effizienz- und Verteilungswirkungen des Steuersystems. 1986.

Band 31 Heinz Dieter Hessler: Theorie und Politik der Personalsteuern. Eine Kritik ihrer Einkommensund Vermogensbegriffe. 1987.

Band 32 Wolfgang Scherf: Die beschăftigungspolitische und fiskalische Problematik der Arbeitgeberbeitrăge zur Rentenversicherung. Eine Auseinandersetzung mit der Kritik an der lohnbezogenen Beitragsbemessung. 1987.

Band 33 Andreas Măstle: Die Steuerunion. Probleme der Harmonisierung spezifischer Gütersteuern. 1987.

Band 34 Günter Ott: Internationale Verteilungswirkungen im Finanzausgleich der Europăischen Gemeinschaften. 1987.

Band 35 Heinz Haller: Zur Frage der zweckmäßigen Gestalt gemeindlicher Steuern. Ein Diskussionsbeitrag zur Gemeindesteuerreform. 1987.

Band 36 Thomas Kuhn: Schlüsselzuweisungen und fiskalische Ungleichheit. Eine theoretische Analyse der Verteilung von Schlüsselzuweisungen an Kommunen. 1988.

Band 37 Walter Hahn: Steuerpolitische Willensbildungsprozesse in der Europäischen Gemeinschaft. Das Beispiel der Umsatzssteuer-Harmonisierung. 1988.

Band 38 Ulrike Hardt: Kommunale Finanzkraft. Die Problematik einer objektiven Bestimmung kommunaler Einnahmemoglichkeiten in der gemeindlichen Haushaltsplanung und im kommunalen Finanzausgleich. 1988. 
Ulrike Hardt - 978-3-631-75265-4

Downloaded from PubFactory at 01/11/2019 06:31:57AM

via free access 
Ulrike Hardt - 978-3-631-75265-4

Downloaded from PubFactory at 01/11/2019 06:31:57AM

via free access 